Utah State University

DigitalCommons@USU

\title{
Particle Size, CaCO3, Chemical, Magnetic, and Age Data from Surficial Deposits in and around Canyonlands National Park, Utah
}

\author{
Harland Goldstein \\ Richard Reynolds \\ Marith Reheis \\ James Yount \\ Paul Lamothe \\ Helen Roberts
}

See next page for additional authors

Follow this and additional works at: https://digitalcommons.usu.edu/crc_research

Part of the Natural Resources and Conservation Commons

\section{Recommended Citation}

Goldstein, Harland; Reynolds, Richard; Reheis, Marith; Yount, James; Lamothe, Paul; Roberts, Helen; and McGeehin, John, "Particle Size, CaCO3, Chemical, Magnetic, and Age Data from Surficial Deposits in and around Canyonlands National Park, Utah" (2005). Canyonlands Research Bibliography. Paper 1.

https://digitalcommons.usu.edu/crc_research/1

This Report is brought to you for free and open access by the Canyonlands Research Center at DigitalCommons@USU. It has been accepted for inclusion in Canyonlands Research Bibliography by an authorized administrator of DigitalCommons@USU. For more information, please contact digitalcommons@usu.edu.

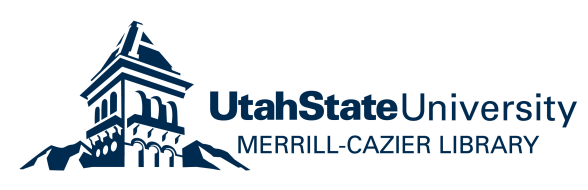


Authors

Harland Goldstein, Richard Reynolds, Marith Reheis, James Yount, Paul Lamothe, Helen Roberts, and John McGeehin 


\section{Particle-Size, $\mathrm{CaCO}_{3}$, Chemical, Magnetic, and Age Data from Surficial Deposits in and around Canyonlands National Park, Utah}

By Harland Goldstein, ${ }^{1}$ Richard Reynolds, ${ }^{1}$ Marith Reheis, ${ }^{1}$ James Yount, ${ }^{1}$ Paul Lamothe, ${ }^{2}$ Helen Roberts, ${ }^{3}$ and John McGeehin ${ }^{4}$

Open-File Report 2005-1186 


\section{Particle-Size, $\mathrm{CaCO}_{3}$, Chemical, Magnetic, and Age Data from Surficial Deposits in and around Canyonlands National Park, Utah}

By Harland Goldstein, ${ }^{1}$ Richard Reynolds, 'Marith Reheis, ' James Yount, ${ }^{1}$ Paul Lamothe, ${ }^{2}$ Helen Roberts, ${ }^{3}$ and John McGeehin ${ }^{4}$

${ }^{1}$ U.S. Geological Survey, MS-980, Federal Center, Box 25046, Denver, CO 80225

${ }^{2}$ U.S. Geological Survey, MS-964, Federal Center, Box 25046, Denver, CO 80225

${ }^{3}$ Institute of Geography and Earth Sciences, University of Wales, Aberystwyth, SY23 3DB, Wales, U.K.

${ }^{4}$ U.S. Geological Survey, MS-926A, National Center, 12201 Sunrise Valley Dr., Reston, VA 20192

Open-File Report 2005-1186 


\section{U.S. Department of the Interior \\ Gale A. Norton, Secretary \\ U.S. Geological Survey \\ Charles G. Groat, Director}

U.S. Geological Survey, Reston, Virginia: 2005

For sale by U.S. Geological Survey, Information Services
Box 25286, Denver Federal Center
Denver, CO 80225
For more information about the USGS and its products:
Telephone: 1-888-ASK-USGS
World Wide Web: http://www.usgs.gov/

Any use of trade, product, or firm names in this publication is for descriptive purposes only and does not imply endorsement by the U.S. Government.

Although this report is in the public domain, permission must be secured from the individual copyright owners to reproduce any copyrighted materials contained within this report. 


\section{Contents}

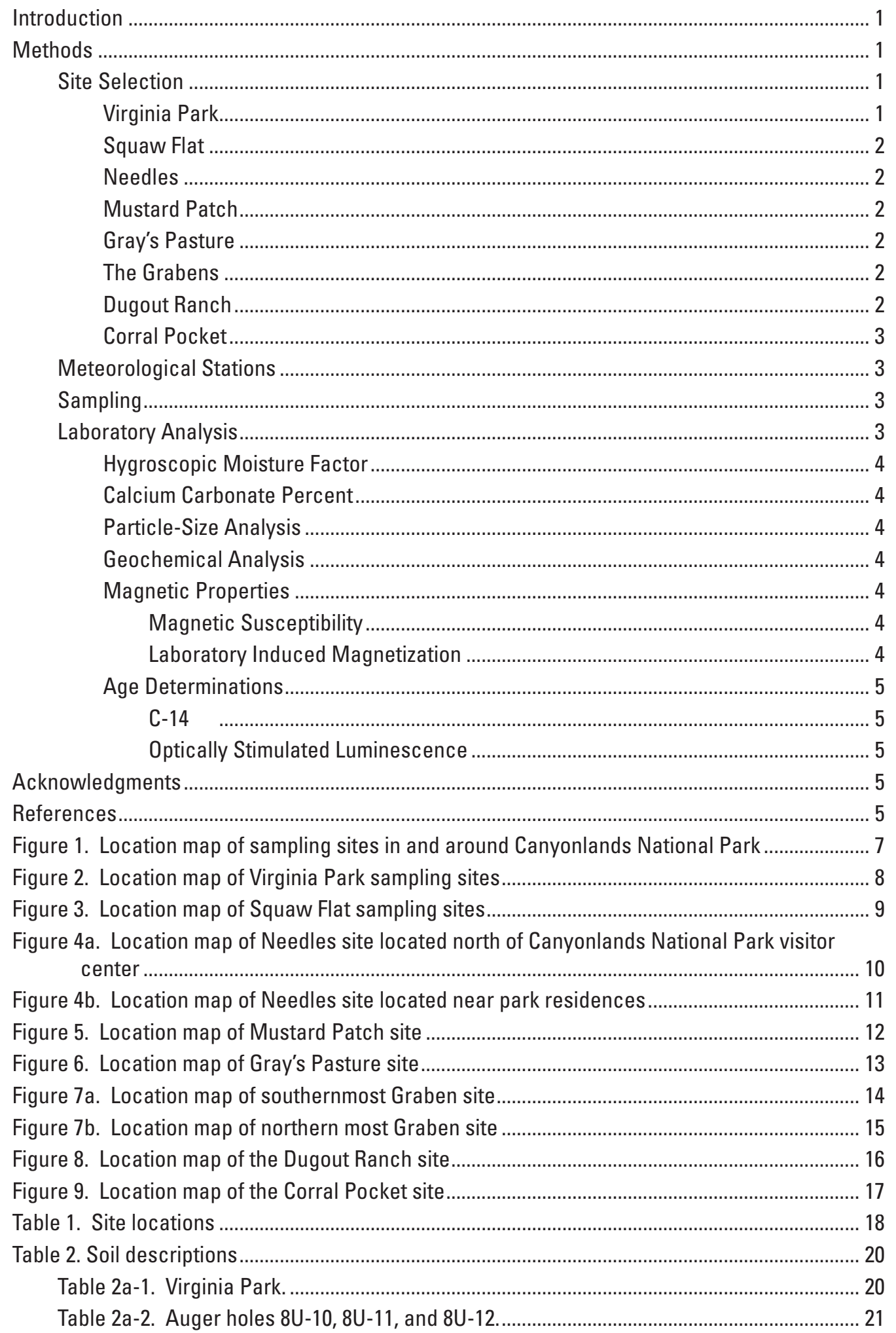




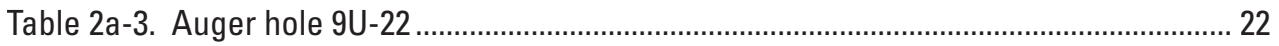

Table 2a-4. Auger hole 9U-23 ...................................................................................... 23

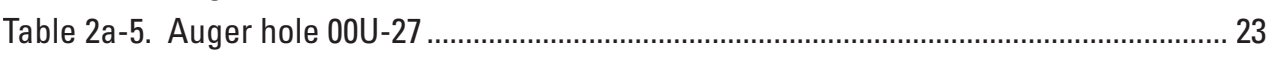

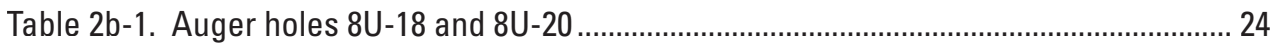

Table 2c-1. 9U-21 graben fill exposure ……………….................................................. 25

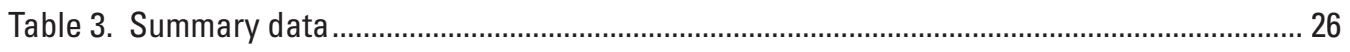

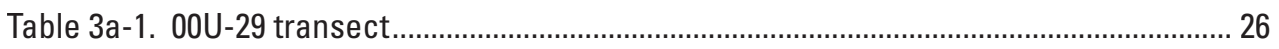

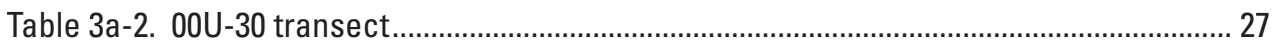

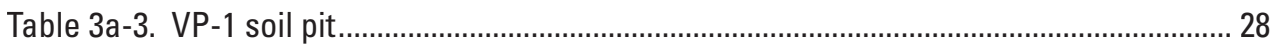

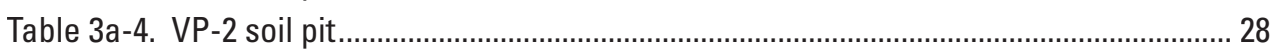

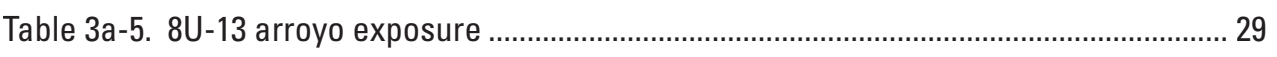

Table 3a-6. 8U-14 arroyo exposure ………………….................................................. 29

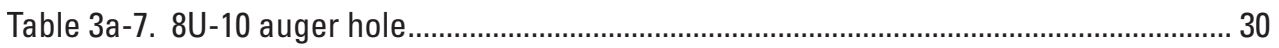

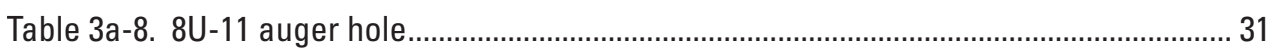

Table 3a-9. 8U-12 auger hole....................................................................................... 32

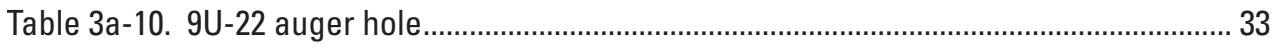

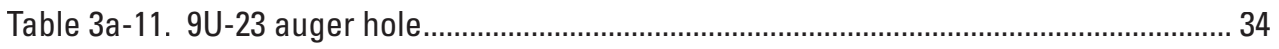

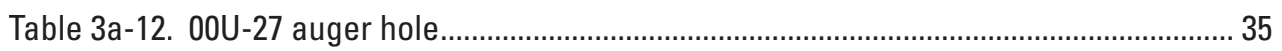

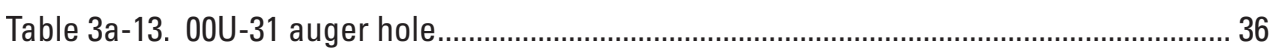

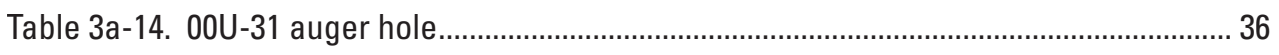

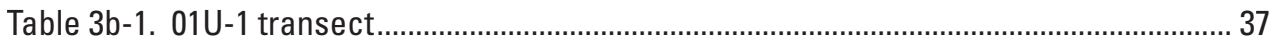

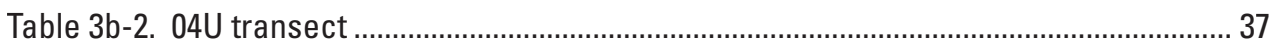

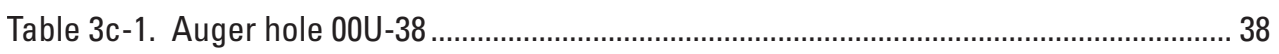

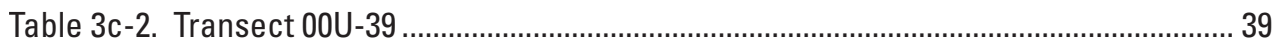

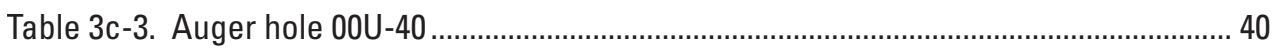

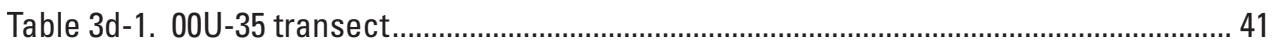

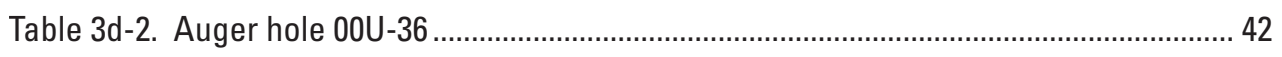

Table 3d-3. Auger hole 00U-37 ..................................................................................... 42

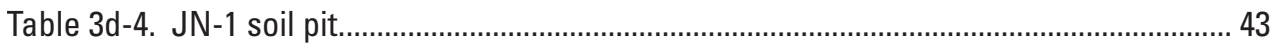

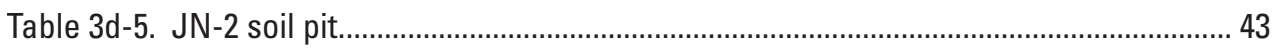

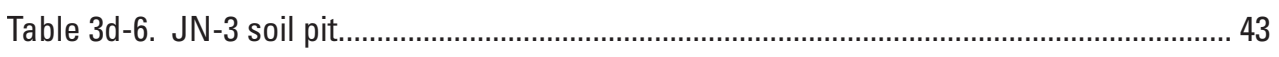

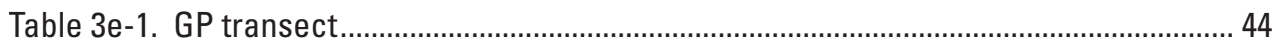

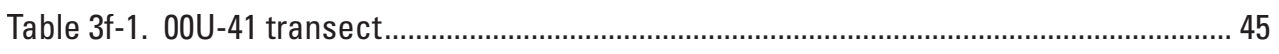

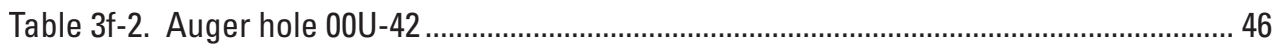

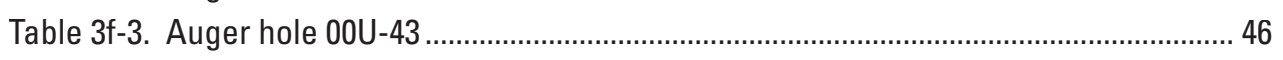

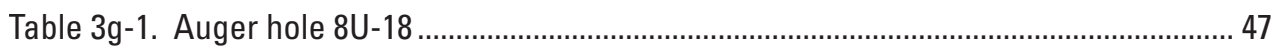

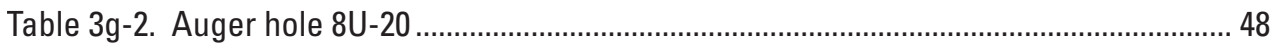

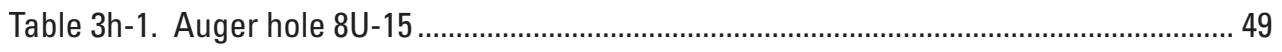

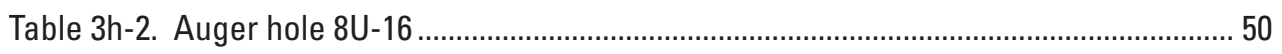

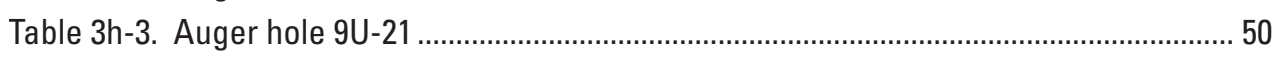

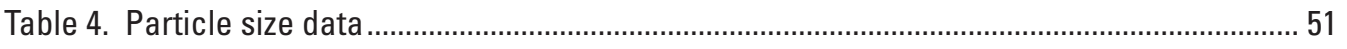

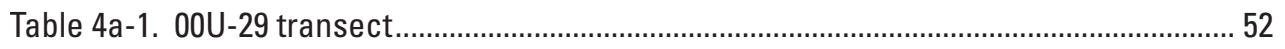

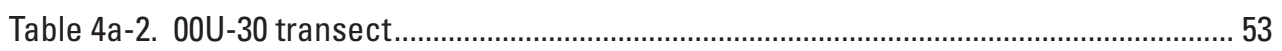

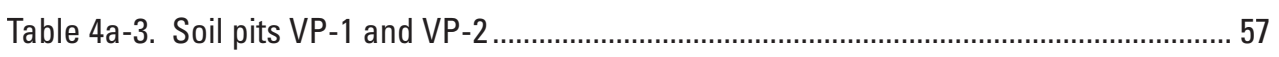

Table 4a-4. Arroyo exposure sites 8U-13 and 8U-14 ....................................................... 58 


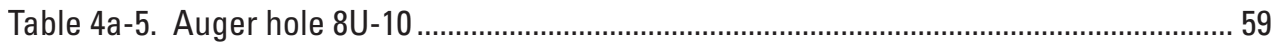

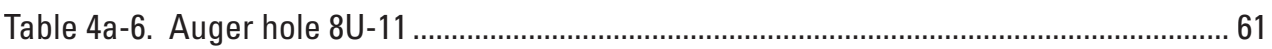

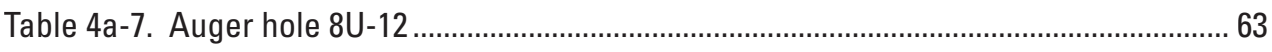

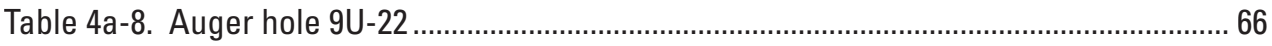

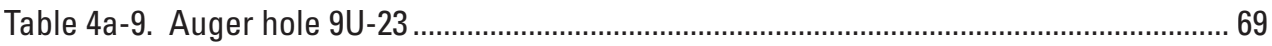

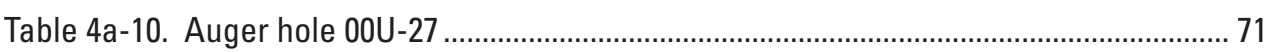

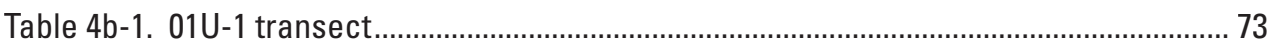

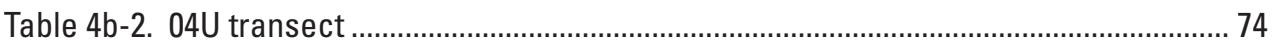

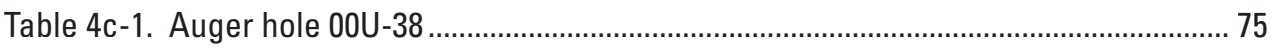

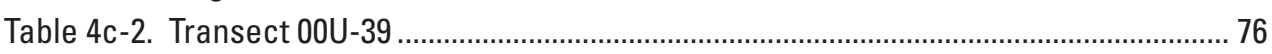

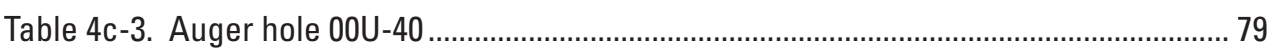

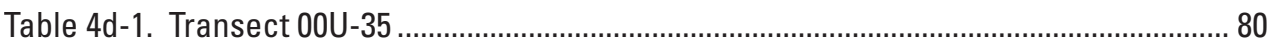

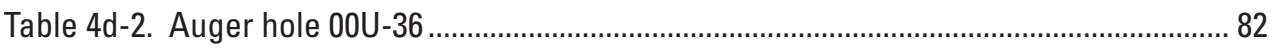

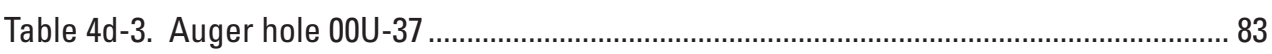

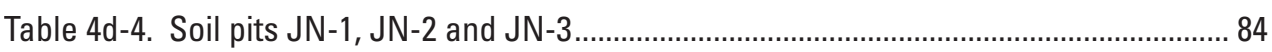

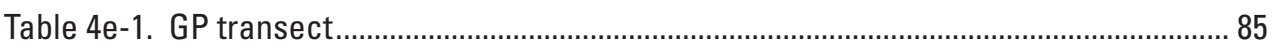

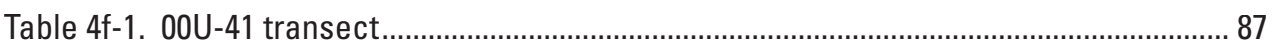

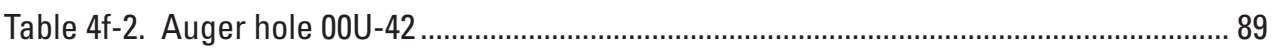

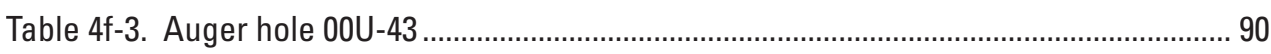

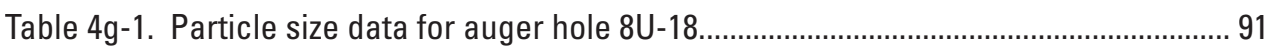

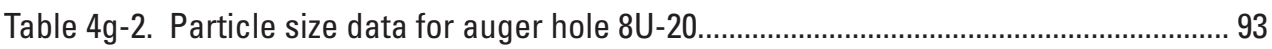

Table 4h-1. Particle size data for arroyo exposure site 9U-21. Data is displayed in

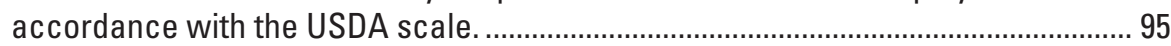

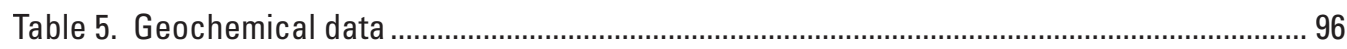

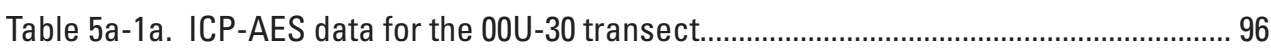

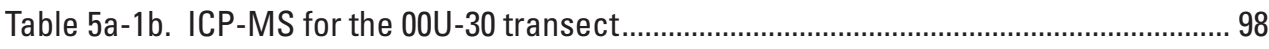

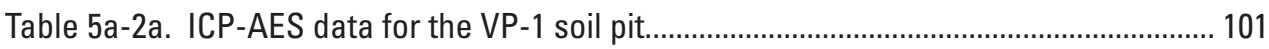

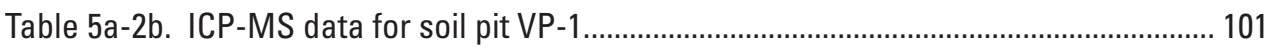

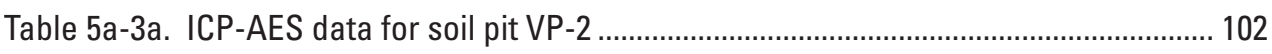

Table 5a-3b. ICP-MS data for soil pit VP-2

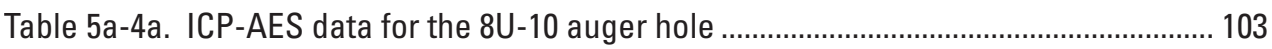

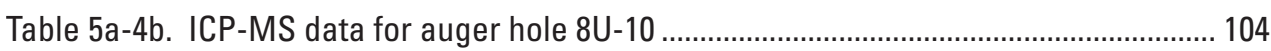

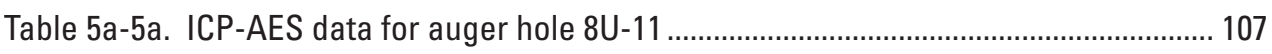

Table 5a-5b. ICP-MS data for auger hole 8U-11 ............................................................ 108

Table 5a-6a. ICP-AES data for auger hole 8U-12 ….................................................... 111

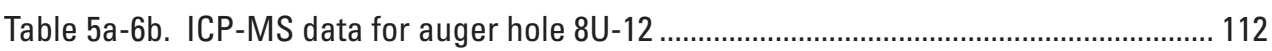

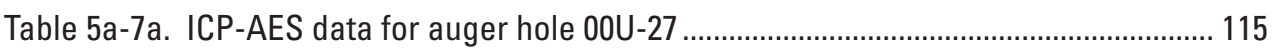

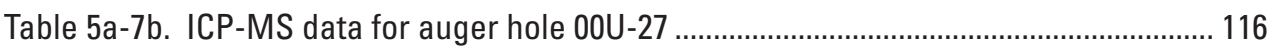

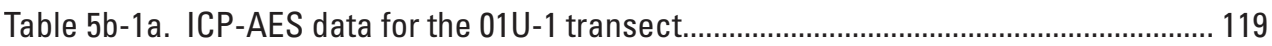

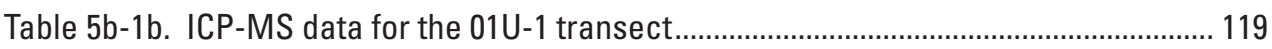

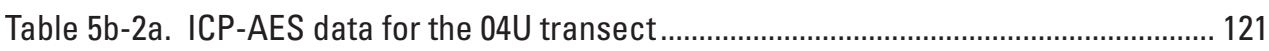

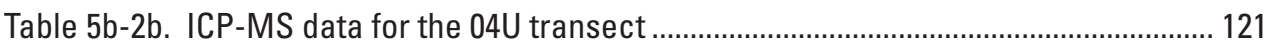

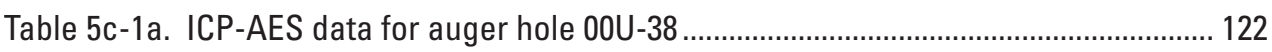

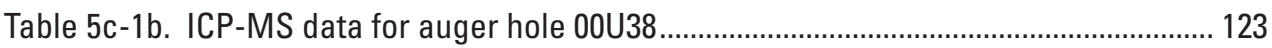

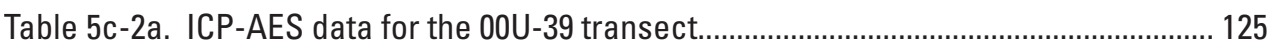

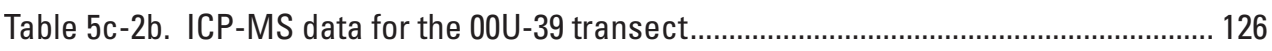




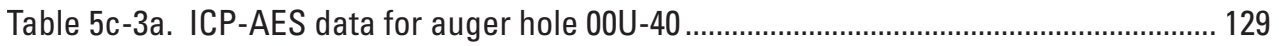

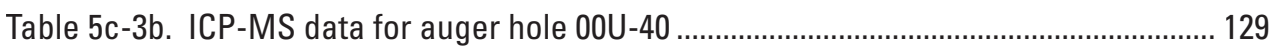

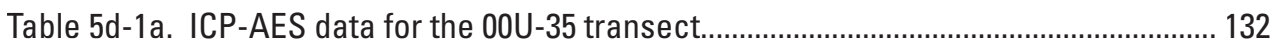

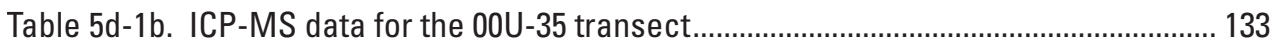

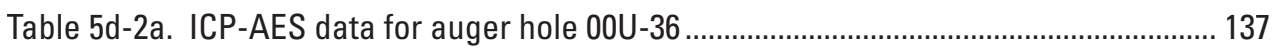

Table 5d-3a. ICP-AES data for auger hole 00U-37 ……....................................................... 139

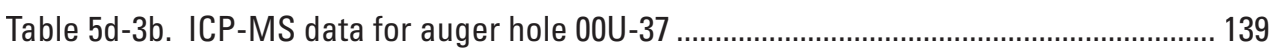

Table 5e-1a. ICP-AES data for the GP transect................................................................. 142

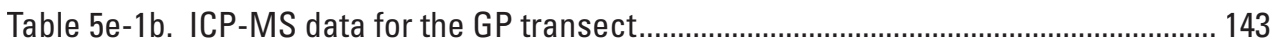

Table 5f-1a. ICP-AES data for the 00U-41 transect........................................................... 146

Table 5f-1b. ICP-MS data for the 00U-41 transect.............................................................. 147

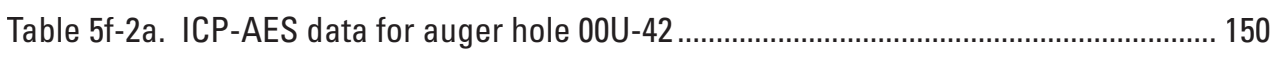

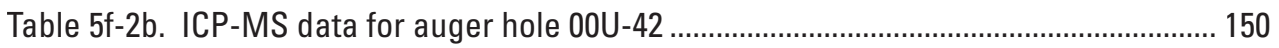

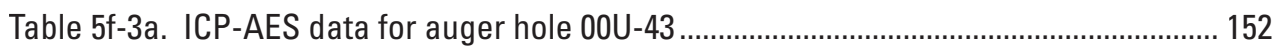

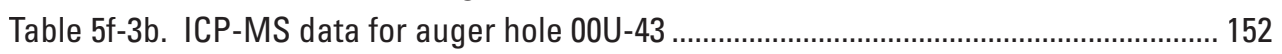

Table 6. Magnetic properties ............................................................................................ 154

Table 6a-1. Magnetic properties of the less than 2-mm size fraction for the 00U-29

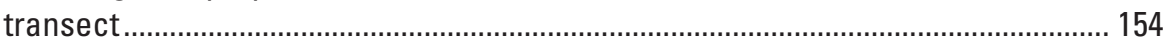

Table 6a-2a. Magnetic properties of the less than 2-mm size fraction for the $00 \mathrm{U}-30$ transect...... 155

Table 6a-2b. Magnetic properties of the less than 63-micron size fraction for the 00U-30 transect

Table 6a-3a. Magnetic properties of the less than 2-mm size fraction for soil pit VP-1 ..... 157

Table 6a-3b. Magnetic properties of the less than 63-micron size fraction for soil pit VP-1

Table 6a-4a. Magnetic properties of the less than 2-mm size fraction for soil pit VP-2 .... 158

Table 6a-4b. Magnetic properties of the less than 63-micron size fraction for soil pit VP-2

Table 6a-5. Magnetic properties of the less than 2-mm size fraction for auger hole $8 \mathrm{U}-10$.

Table 6a-6. Magnetic properties of the less than 2-mm size fraction for auger hole $8 \mathrm{U}-11$

Table 6a-7. Magnetic properties of the less than 2-mm size fraction for auger hole $8 \mathrm{U}-12$

Table 6a-8. Magnetic properties of the less than 2-mm size fraction for auger hole $9 \mathrm{U}-22$

Table 6a-9. Magnetic properties of the less than 2-mm size fraction for auger hole $9 \mathrm{U}-23$

Table 6a-10a. Magnetic properties of the less than 2-mm size fraction for auger hole $00 \mathrm{U}-27$.

Table 6a-10b. Magnetic properties of the less than 63-micron size fraction for auger hole 00U-27.

Table 6a-11a. Magnetic properties of the less than 2-mm size fraction for auger hole 00U-31

Table 6a-11b. Magnetic properties of the less than 63-micron size fraction for auger hole $00 \mathrm{U}-31$

Table 6a-12a. Magnetic properties of the less than 2-mm size fraction for auger hole $00 \mathrm{U}-33$. 
Table 6a-12b. Magnetic properties of the less than 63-micron size fraction for auger hole $00 \mathrm{U}-33$

Table 6b-1a. Magnetic properties of the less than 2-mm size fraction for the 01U-1 transect 168

Table 6b-1b. Magnetic properties of the less than 63-micron size fraction for the 01U-1 transect 169

Table 6b-2. Magnetic properties of the less than 63-micron size fraction for the $04 \mathrm{U}$ transect

Table 6c-1. Magnetic properties of the less than 2-mm size fraction for auger hole 00U-38

Table 6c-2a. Magnetic properties of the less than 2-mm size fraction for the 00U-39 transect

Table 6c-2b. Magnetic properties of the less than 63-micron size fraction for the 00U-39 transect

Table 6c-3a. Magnetic properties of the less than 2-mm size fraction for auger hole $00 \mathrm{U}-40$.

Table 6c-3b. Magnetic properties of the less than 63-micron size fraction for the $00 \mathrm{U}-40$ transect

Table 6d-1a. Magnetic properties of the less than 2-mm size fraction for the $00 \mathrm{U}-35$ transect.

Table $6 \mathrm{~d}-1 \mathrm{~b}$. Magnetic properties of the less than 63-micron size fraction for the 00U-35 transect

Table 6d-2a. Magnetic properties of the less than 2-mm size fraction for auger hole 00U-36

Table $6 \mathrm{~d}-2 \mathrm{~b}$. Magnetic properties of the less than 63-micron size fraction for auger hole 00U-36

Table 6d-3a. Magnetic properties of the less than 2-mm size fraction for auger hole 00U-37

Table 6d-3b. Magnetic properties of the less than 63-micron size fraction for auger hole 00U-37

Table 6d-4a. Magnetic properties of the less than 2-mm size fraction for soil pit JN-1 .... 178

Table 6d-4b. Magnetic properties of the less than 63-micron size fraction for soil pit JN-1

Table 6d-5a. Magnetic properties of the less than 2-mm size fraction for soil pit JN-2 $\ldots . .179$

Table 6d-5b. Magnetic properties of the less than 63-micron size fraction for soil pit JN-2

Table 6d-6a. Magnetic properties of the less than 2-mm size fraction for soil pit JN-3 .... 180

Table $6 \mathrm{~d}-6 \mathrm{~b}$. Magnetic properties of the less than 63-micron size fraction for soil pit JN-3

Table 6e-1. Magnetic properties of the less than 2-mm size fraction for the GP transect. 181

Table 6f-1a. Magnetic properties of the less than 2-mm size fraction for the 00U-41 transect

Table 6f-1b. Magnetic properties of the less than 63-micron size fraction for the 00U-41 transect

Table 6f-2a. Magnetic properties of the less than 2-mm size fraction for auger hole 00U-42

Table 6f-2b. Magnetic properties of the less than 63-micron size fraction for auger hole 00U-42 
Table 6f-3a. Magnetic properties of the less than 2-mm size fraction for auger hole $00 \mathrm{U}-43$.

Table 6f-3b. Magnetic properties of the less than 63-micron size fraction for auger hole $00 \mathrm{U}-43$.

Table $6 \mathrm{~g}-1$. Magnetic properties of the less than 2-mm size fraction for auger hole $8 \mathrm{U}-18$.

Table $6 \mathrm{~g}-2$. Magnetic properties of the less than 2-mm size fraction for auger hole $8 \mathrm{U}-20$.

Table 6h-1. Magnetic properties of the less than 2-mm size fraction for auger hole $8 \mathrm{U}-15$

Table 6h-2. Magnetic properties of the less than 2-mm size fraction for auger hole $8 \mathrm{U}-16$

Table 6h-3. Magnetic properties of the less than 2-mm size fraction for arroyo exposure $9 \mathrm{U}-21$

Table 7. Age data 190

Table 7-1. ${ }^{14} \mathrm{C}$ age from Virginia Park................................................................................. 190

Table 7-2. Optically stimulated luminescence (OSL) ages 


\section{Particle-Size, $\mathrm{CaCO}_{3}$, Chemical, Magnetic, and Age Data from Surficial Deposits in and around Canyonlands National Park, Utah}

By Harland Goldstein, Richard Reynolds, ${ }^{1}$ Marith Reheis, ${ }^{1}$ James Yount, ${ }^{1}$ Paul Lamothe, ${ }^{2}$ Helen Roberts, ${ }^{3}$ and John McGeehin ${ }^{4}$

\section{Introduction}

This report presents data and describes the methodology for magnetic, geochemical, and textural measurements of sediment and soil, as well as age determinations of sites located in and around Canyonlands National Park, on the Colorado Plateau, southeastern Utah (fig. 1).

The data presented in this report are results of ongoing studies of American drylands, where numerous sites have been investigated to assess how climate change and land use affect the landscape, largely through geomorphic processes. In particular, the influence of eolian and fluvial processes on ecosystem dynamics, ranging from nutrient inputs to landscape evolution, is a central focus of these studies (Neff and others, in press; Reheis and others, in press; Reynolds and others, 2001; Reynolds and others, in press).

\footnotetext{
${ }^{1}$ U.S. Geological Survey, MS-980, Federal Center, Box 25046, Denver, CO 80225

${ }^{2}$ U.S. Geological Survey, MS-964, Federal Center, Box 25046, Denver, CO 80225

${ }^{3}$ Institute of Geography and Earth Sciences, University of Wales, Aberystwyth, SY23 3DB, Wales, U.K.

${ }^{4}$ U.S. Geological Survey, MS-926A, National Center, 12201 Sunrise Valley Dr., Reston, VA 20192
}

\section{Methods}

\section{Site Selection}

Many sites in and around Canyonlands National Park, Utah, were selected to include different geomorphic settings that contain sand sheets/dunes, playas, and fluvial/alluvial deposits (table 1; fig.1). Site selection was determined by strategies to examine a variety of plant communities, ecotones, occurrences of plant invasion, especially by cheat grass (Bromus tectorum), as well as different land-use histories and local bedrock.

\section{Virginia Park}

Virginia Park is located in Canyonlands National Park (figs. 1 and 2). It is a sheltered basin of vegetated, stabilized sand dunes surrounded on three sides by walls of the Cedar Mesa Sandstone and bounded on the southwest by a canyon. This site was sampled from the fall of 1998 through the spring of 2000. The deposits sampled at this site consist of multiple sequences of locally derived sand separated by poorly to moderately developed soil that formed in the sand layers. The shallowest samples $(0-10 \mathrm{~cm})$ represent the most recent episode of significant eolian modification of the landscape, including dune formation, which likely ceased about $2 \mathrm{ka}$. Difficult access and lack of water have precluded livestock grazing at this site (Kleiner and Harper, 1972). It is the only such ungrazed site in this study. 


\section{Squaw Flat}

The Squaw Flat site is located in Canyonlands National Park (figs. 1 and 3). The transect sampling and the soil pit sampling at this site occurred during the spring of 2000 and the fall of 2001, respectively. The upper sampling sites in the transect are in a low-angle $\left(1-3^{\circ}\right)$ sand-sheet that slopes away from the Cedar Mesa Sandstone. The lower sampling sites are from mixed alluvial-eolian deposits in a poorly drained area near the middle of a meadow. This site was grazed until 1974 (M.E. Miller, U.S. Geological Survey, oral commun., 2004).

\section{Needles}

The Needles area is located in Canyonlands National Park (figs. 1, 4a, and 4b). The sites in this area are in playa deposits interbedded with eolian sand sheets and were sampled in the fall of 1998. These sites were auger sampled to about 4 meters in depth. The bedrock in this area is the Cedar Mesa Sandstone. This site was grazed until 1974 (M.E. Miller, U.S. Geological Survey, oral commun., 2004).

\section{Mustard Patch}

The Mustard Patch site (figs. 1 and 5) is near the eastern boundary of Canyonlands National Park and consists of eolian sand on a surface sloping away from Cedar Mesa Sandstone. This site was sampled during the spring of 2000. The upper part of this transect occupies a surface characterized by small coppice dunes representing recent eolian activity. The surface over the middle part of the transect generally is featureless, thereby representing a sand-sheet environment. The lowest sampling sites are in a mixed eolian sand-alluvial setting at the margin of a poorly drained valley oriented nearly $90^{\circ}$ to the trend of the sampling transect. Grazing by domestic livestock ceased at this site in 1974 (M.E. Miller, U.S. Geological Survey, oral commun., 2004).

\section{Gray's Pasture}

Gray's Pasture (figs. 1 and 6) is located in the Island in the Sky area of Canyonlands National Park. Samples were collected in the spring of 2003 along a shallow-gradient slope away from the Navajo Sandstone. The sampled deposits consist mainly of eolian sand in small dune-forms in the upper half of the transect and in a mostly featureless surface in the lower half. The site previously was grazed until 1974 (M.E. Miller, U.S. Geological Survey, oral commun., 2004).

\section{The Grabens}

The Grabens sites (figs. 1 and 7) are located in Canyonlands National Park and consist of alluvium, colluvium, and eolian sediment deposited within closed, fault-related depressions formed in the Cedar Mesa Sandstone. The auger hole samples were taken during the fall of 1998. The arroyo exposure was sampled during the fall of 1999, and the optically stimulated luminescence samples were collected during the fall of 2002. Grazing practices for the Grabens area are not directly known; however, nearby Chesler Park was previously grazed until 1964, and the Grabens area grazing practices likely followed those at Chesler Park (M.E. Miller, U.S. Geological Survey, oral commun., 2004).

\section{Dugout Ranch}

The Dugout Ranch site (figs. 1 and 8) is located approximately $7 \mathrm{~km}$ east of the Canyonlands National Park boundary. This site consists mainly of eolian sand deposited on a nearly flat surface. The bedrock in this area is the Chinle Formation, the Wingate Sandstone, and the Kayenta Formation. This site initially was sampled during the fall of 2001 and was resampled in the spring 2004 to assess the influence of documented wind erosion between sampling times. This site and the surrounding areas have been grazed by domestic livestock since the arrival of Anglo-American settlers in the 1880s (Loope, 1977) and is currently grazed, mostly in the spring. 


\section{Corral Pocket}

The Corral Pocket site (figs. 1 and 9) is located approximately $4 \mathrm{~km}$ east of the Canyonlands National Park boundary and consists of a sandsheet and alluvial fan complex. The local bedrock consists of small exposures of Cedar Mesa Sandstone and overlying cliffs of Organ Rock Shale. The Organ Rock Shale contributes nearly all of the locally derived sediment to the sampled slope. This transect was sampled during the fall of 2000. The site is on Bureau of Land Management land and is currently grazed (M.E. Miller, U.S. Geological Survey, oral commun., 2004).

\section{Meteorological Stations}

The majority of sites presented in this report are in close proximity to climate monitoring equipment. Virginia Park, Corral Pocket, and Dugout Ranch sampling sites have Climate Impact Meteorological Stations (CLIM-MET) on site that are operated and maintained by the Central Region Earth Surface Processes team of the U.S. Geological Survey (USGS) (http://climchange.cr.usgs. gov/info/sw/clim-met/). Another USGS CLIM-MET station is located in the Needles district of Canyonlands National Park near the staff residence area, approximately $1-2 \mathrm{~km}$ from the Needles sampling sites (http://climchange.cr.usgs.gov/info/sw/climmet/needles.html). The closest CLIM-MET station to the Squaw Flat and Mustard Patch sampling sites is located in Needles; however, the Mustard Patch sampling site also is in close proximity $(\sim 6 \mathrm{~km})$ to the Corral Pocket CLIM-MET station (http://climchange.cr.usgs.gov/info/sw/clim-met/corral.html). The Virginia Park CLIM-MET station (http://climchange.cr.usgs.gov/info/sw/clim-met) virgpark.html) is closest to the Grabens sampling sites. The nearest weather monitoring station to the Gray's Pasture sampling site is located in the Island in the Sky area of Canyonlands National Park and is operated and maintained by the National Park Service (http://www.wrcc.dri.edu/cgi-bin/cliMAIN. pl? utcank).

\section{Sampling}

Three sampling strategies were employed throughout the study area: (1) isolated deep auger holes, (2) transects that consist of several shallow $(50 \mathrm{~cm})$, small soil pits anchored by deep auger holes, and (3) soil pits (table 1). In addition, when possible, arroyo exposures were utilized for stratigraphic relations and sampling.

The isolated deep auger holes were sampled approximately every 10 to $20 \mathrm{~cm}$ and ranged from 2.5 to 4.0 meters deep. The shallow, small soil pits and deep auger hole transects are the most common sampling arrangement. The shallow, small soil pits and the top of the deep auger holes were sampled fairly consistently at $0-10 \mathrm{~cm}, 10-30 \mathrm{~cm}$, and $30-50 \mathrm{~cm}$. The deep auger holes that anchor the transects are as much as 2.29 meters deep and below $50 \mathrm{~cm}$ were sampled approximately every 10 $\mathrm{cm}$. Five larger soil pits (VP-1, VP-2, JN-1, JN-2, JN-3) were excavated at selected sites within the study area. The soil pits are all approximately 1 meter deep and were channel sampled based on soil horizon designations. Arroyo exposures also were sampled based on soil horizon designations.

The arroyo and graben exposures and the VPsoil pits were described (table 2) following Birkeland (1999). In some cases, the soil auger sequences were described similarly, but with less precision due to the lack of visible exposure and to mixing when the auger was reinserted into the hole. Approximate soil horizon boundaries were identified during the augering using changes in soil color, carbonate morphology, effervescence in $\mathrm{HCl}$, and sediment texture.

\section{Laboratory Analysis}

Samples were analyzed at the USGS Earth Surface Processes soils and magnetics laboratories (Denver, Colo., United States of America) for hygroscopic moisture factor, calcium carbonate percent, particle size, and magnetic properties (table 3 ). The soil analyses followed the protocols outlined in Singer and Janitzky, (1986). Geochemical analysis was performed by the USGS Geologic Division geochemistry lab (Denver, Colo., United States of 
America). All samples were analyzed at $<2$-mm size fraction and in some cases magnetic properties were measured on the $<63-\mu \mathrm{m}$ size fraction (silt + clay).

Age determinations were made by optically stimulated luminescence (OSL) and Carbon-14 $\left({ }^{14} \mathrm{C}\right)$ methods. Optically stimulated luminescence ages were determined at the Aberystwyth Luminescence Research Laboratory (Wales, United Kingdom). Radiocarbon samples were prepped at the ${ }^{14} \mathrm{C}$ laboratory of the U.S. Geological Survey (Reston, Va.), and ages were determined by accelerator mass spectrometry (AMS) at the NSF-University of Arizona AMS laboratory (Tucson, Ariz.). Age data presented in this report, as well as other age data from the area, have been placed in stratigraphic and geomorphic context and can be found in Reheis and others, (in press).

\section{Hygroscopic Moisture Factor}

The hygroscopic moisture factor (table 3 ) is a measure of the moisture content of a sample at or near field conditions and is calculated by:

$\mathrm{H}=\mathrm{OD} / \mathrm{AD}$, where

$\mathrm{H}=$ Hydroscopic Moisture Factor

$\mathrm{OD}=$ Oven-dry sample weight $\left(105^{\circ} \mathrm{C} ; 16 \mathrm{hrs}\right)$

$\mathrm{AD}=$ Air-dry sample weight

\section{Calcium Carbonate Percent}

Calcium carbonate percent (table 3) was measured using a Chittick apparatus (Dreimanis, 1962) as described by Machette (1986) whereby $6 \mathrm{~N} \mathrm{HCl}$ is applied to the sample and the gas evolved from the reaction displaces fluid within the Chittick apparatus. The volume of liquid displaced is used to calculate the percent of calcium carbonate in the sample.

$\mathrm{CaCO}_{3} \%=\left(\mathrm{V}_{\mathrm{CO} 2} * \mathrm{C}\right) / \mathrm{W}_{\mathrm{S}}$, where

$\mathrm{CaCO}_{3} \%=$ Calcium carbonate percent

$\mathrm{V}_{\mathrm{CO} 2}=$ The volume of liquid displaced during reaction in presence of $\mathrm{HCl}$

$\mathrm{C}=$ Constant $;\left(\mathrm{P}_{\mathrm{B}} / \mathrm{T}_{\mathrm{K}}\right) * 0.16$, where

$\mathrm{P}_{\mathrm{B}}=$ Barometric Pressure in $\mathrm{Hg} \mathrm{mm}$

$\mathrm{T}_{\mathrm{K}}=$ Temperature in degrees Kelvin

$\mathrm{W}_{\mathrm{S}}=$ Oven-dry sample weight $\left(105^{\circ} \mathrm{C} ; 16 \mathrm{hrs}\right)$

\section{Particle-Size Analysis}

Particle-size analysis was performed on the $<2$-mm size fraction using a Malvern laser analyzer (tables 3 and 4). Prior to analysis, all samples were prepared by digesting organic matter and $\mathrm{CaCO}_{3}$ using $30 \% \mathrm{H}_{2} \mathrm{O}_{2}$ and $15 \% \mathrm{HCl}$, respectively. Prior to analysis, sodium hexametaphosphate was added to each sample to deflocculate clays.

\section{Geochemical Analysis}

Samples were analyzed for chemical properties by inductively coupled plasma-atomic emission spectrometry (ICP-AES) and inductively coupled plasma-mass spectrometry (ICP-MS) techniques (tables 3 and 5) (Lichte and others, 1987). All samples were analyzed on the $<2-\mathrm{mm}$ size fraction and were finely ground using a shatterbox.

\section{Magnetic Properties}

Magnetic properties were determined on a majority of samples, both $<2-\mathrm{mm}$ and, in some cases, $<63-\mu \mathrm{m}$ size fractions (tables 3 and 6). Magnetic properties determined included low frequency and high frequency magnetic susceptibility $\left(\mathrm{MS}_{\mathrm{If}}\right.$, $\mathrm{MS}_{\mathrm{hf}}$ ), anhysteretic remanent magnetization (ARM), and isothermal remanent magnetization (IRM).

\section{Magnetic Susceptibility}

Magnetic susceptibility (MS) was measured by using a susceptometer with a sensitivity better than about $4 \times 10^{-7} \mathrm{~m}^{3} / \mathrm{kg}$. Samples were measured in a $0.1 \mathrm{mT}$ induction at a low frequency of $600 \mathrm{~Hz}$ $\left(\mathrm{MS}_{\mathrm{lf}}\right)$ and high frequency of $6000 \mathrm{~Hz}\left(\mathrm{MS}_{\mathrm{hf}}\right)$. For each sample, the MS value was determined as the mean of four measurements. Frequency dependent susceptibility was calculated as:

$$
\mathrm{FDMS}=\left(\mathrm{MS}_{\mathrm{lf}}-\mathrm{MS}_{\mathrm{hf}}\right) / \mathrm{MS}_{\mathrm{lf}}
$$

Laboratory Induced Magnetization

Measurements of anhysteretic remanent magnetization (ARM) and isothermal remanent magnetization (IRM) experiments were made by using a high-speed spinner magnetometer. Anhysteretic remanent magnetization was imparted in a decreas- 
ing alternating field from a peak induction of 100 $\mathrm{mT}$ and a DC bias of $0.1 \mathrm{mT}$. IRM magnetizations were generated at room temperature by using an impulse magnetizer. First, IRM was imparted in a $1.2 \mathrm{~T}$ induction $\left(\mathrm{IRM}_{1.2 \mathrm{~T}}\right)$. The samples then were magnetized in the opposite direction by using an induction of $0.3 \mathrm{~T}\left(\mathrm{IRM}_{-0.3 \mathrm{~T}}\right)$. Hard isothermal remanent magnetization (HIRM) and the S-parameter were calculated as follows (King and Channel, 1991):

$$
\begin{aligned}
& \mathrm{HIRM}=\left(\operatorname{IRM}_{1.2 \mathrm{~T}}+\mathrm{IRM}_{-0.3 \mathrm{~T}}\right) / 2 ; \\
& \mathrm{S}=\mathrm{IRM}_{-0.3 \mathrm{~T}} / \mathrm{IRM}_{1.2 \mathrm{~T}}
\end{aligned}
$$

\section{Age Determinations}

\section{C-14}

Charcoal within the alluvial deposits at the Virginia Park site was sampled and placed into plastic bags using metal trowels. The charcoal received a standard acid-alkali-acid pretreatment to remove post-depositional contamination. One milligram of carbon was collected from the charcoal by converting it to graphite over an iron catalyst. The graphite was pressed into a target and ${ }^{14} \mathrm{C}$ dated using accelerator mass spectrometry. The raw ${ }^{14} \mathrm{C}$ ages later were converted to calibrated years B.P. (cal yr B.P.) by using the CALIB program of Stuiver and others (2003) (table 7). Calibration of raw ${ }^{14} \mathrm{C}$ ages corrects for known time intervals when atmospheric carbon concentrations deviated from modern values causing the appearance of an age that was too old or too young.

\section{Optically Stimulated Luminescence}

For OSL dating, samples were taken from outcrops and pits using PVC pipe hammered horizontally into freshly cleaned faces. Laboratory OSL measurements were performed using an automated Ris $\varnothing$ TL/OSL reader, equipped with blue LEDs (470 $\mathrm{nm}$ ) providing about $17 \mathrm{~mW} \mathrm{~cm} \mathrm{~cm}^{-2}$ power density and a beta source for irradiations. Three $3 \mathrm{~mm}$ Hoya U340 filters were used to detect the OSL signal. To determine the equivalent dose $\left(\mathrm{D}_{\mathrm{e}}\right)$ of each sample, a minimum of 24 aliquots were examined for a range of preheat temperatures between $160-300^{\circ} \mathrm{C}$ using the single-aliquot regenerative-dose (SAR) measurement procedure of Murray and Wintle (2000), which corrects for changes in the luminescence sensitivity. All OSL measurements were made at $125^{\circ} \mathrm{C}$. Each aliquot yields an independent estimate of $\mathrm{D}_{\mathrm{e}}$; the mean $\mathrm{D}_{\mathrm{e}}$ of between 13-23 aliquots was used to determine each $90-125-\mu \mathrm{m}$ coarse-grained OSL age, and the 4-11- $\mu$ m finegrained OSL ages were calculated using the mean $\mathrm{D}_{\mathrm{e}}$ of between 19-22 aliquots (table 7). The uncertainty on $\mathrm{D}_{\mathrm{e}}$ was calculated as the standard error of the mean for all samples. For further discussion of OSL methods, see Reheis and others, (in press).

\section{Acknowledgments}

Many people have contributed to the compilation of data presented in this report. We thank Rich Tigges, Marie Bernatchez, and the Bobby's Hole crew for field assistance; Eric Fisher, Jiang Xiao, Isla Castaneda, Yarrow Axford, and Ken Takagi for sediment analyses; Darren "Paco" Van Sistine for GIS work; and Jeremy Havens and Lisa Rukstales for their stellar formatting and editorial assistance. Thanks to Gary Skipp and Jossh Beann for review of this report and helpful suggestions for improvement.

\section{References}

Birkeland, P.W., 1999, Soils and Geomorphology [third ed.]: New York, Oxford University Press, 430 p.

Dreimanis, Aleksis, 1962, Quantitative gasometric determination of calcite and dolomite by using Chittick apparatus: Journal of Sedimentary Petrology, v. 32, no. 3, p. 520-529.

King, J.W., and Channel, J.E.T., 1991, Sedimentary magnetism, environmental magnetism, and magnetostratigraphy: Reviews of Geophysics, Supplement, p. 358-370.

Kleiner, E.F., and Harper, K.T., 1972. Environment and community organization in grasslands of Canyonlands National Park: Ecology, 53, p. 299-309. 
Lichte, F.E., Golightly, D.W., Lamothe, P.J., 1987, Inductively coupled plasma-atomic spectrometry, in Baedecker, P.A., ed., Methods for geochemical analysis, U.S. Geological Survey Bulletin 1770, p. B1-B10.

Loope, W.L., 1977, Relationships of vegetation to environment in Canyonlands National Park, Ph.D. Thesis: Utah State University, Logan, Utah, USA, 142 p.

Machette, Michael, 1986, Calcium and magnesium carbonates, in Singer, M.J., and Janitzky, Peter, eds., Field and laboratory procedures used in a soil chronosequence study: U.S. Geological Survey Bulletin 1648, p. 30-33.

Murray, A.S., and Wintle, A.G., 2000, Luminescence dating of quartz using an improved single-aliquot regenerative-dose protocol: Radiation Measurements, v. 32, p. 57-73.

Neff, J.C., Reynolds, R.L., Belnap, Jayne, and Lamothe, Paul, in press, Multi-decadal impacts of grazing on soil physical and biogeochemical properties in Southeast Utah: Ecological Applications.

Prescott, J.R. and Hutton, J.T., 1994, Cosmic ray contributions to dose rates for luminescence and ESR dating: large depths and long-term time variations: Radiation Measurements, v. 23, p. 497-500.

Rees-Jones, Julie, 1995, Optical dating of young sediments using fine-grain quartz: Ancient TL, v. 13, p. 9-13.

Reheis, M.C., Reynolds, R.L., Goldstein, Harland, Roberts, H.M., Yount, J.C., Axford, Yarrow, Cummings, L.S., and Shearin, N., in press, Late Quaternary eolian and alluvial response to paleoclimate, Canyonlands, southeastern Utah: Geological Society of America Bulletin.

Reynolds, R.L., Neff, J.C., Reheis, M.C., Lamothe, Paul, in press, Atmospheric dust in modern soil on aeolian sandstone, Colorado Plateau (USA): Variation with landscape position and contribution to potential plant nutrients: Geoderma.

Reynolds, R.L., Belnap, Jayne, Reheis, M.C., Lamothe, Paul, and Luiszer, Fred, 2001, Aeolian dust in Colorado Plateau soils: Nutrient inputs and recent change in source: Proceedings of the National Academy of Sciences, v. 98, no. 13, p. 7123-7127.

Singer, Michael J., and Janitzky, Peter, eds., 1986, Field and laboratory procedures used in a soil chronosequence study: U.S. Geological Survey Bulletin 1648, 49 pp.

Soil Survey Staff, 1975, Soil taxonomy: A basic system of soil classification for making and interpreting soil surveys: USDA-SCS Agricultural Handbook 436 (2nd Edition), U.S. Government Printing Office, Washington D.C.
Stuiver, Minze, Reimer, P.J., and Reimer, Ron, 2003, CALIB Radiocarbon Calibration: http://radiocarbon.pa.qub.ac.uk/ calib/.

Wentworth, C.K., 1922, A scale of grade and class terms for clastic sediments: Journal of Geology, v. 30, p. 377-392. 


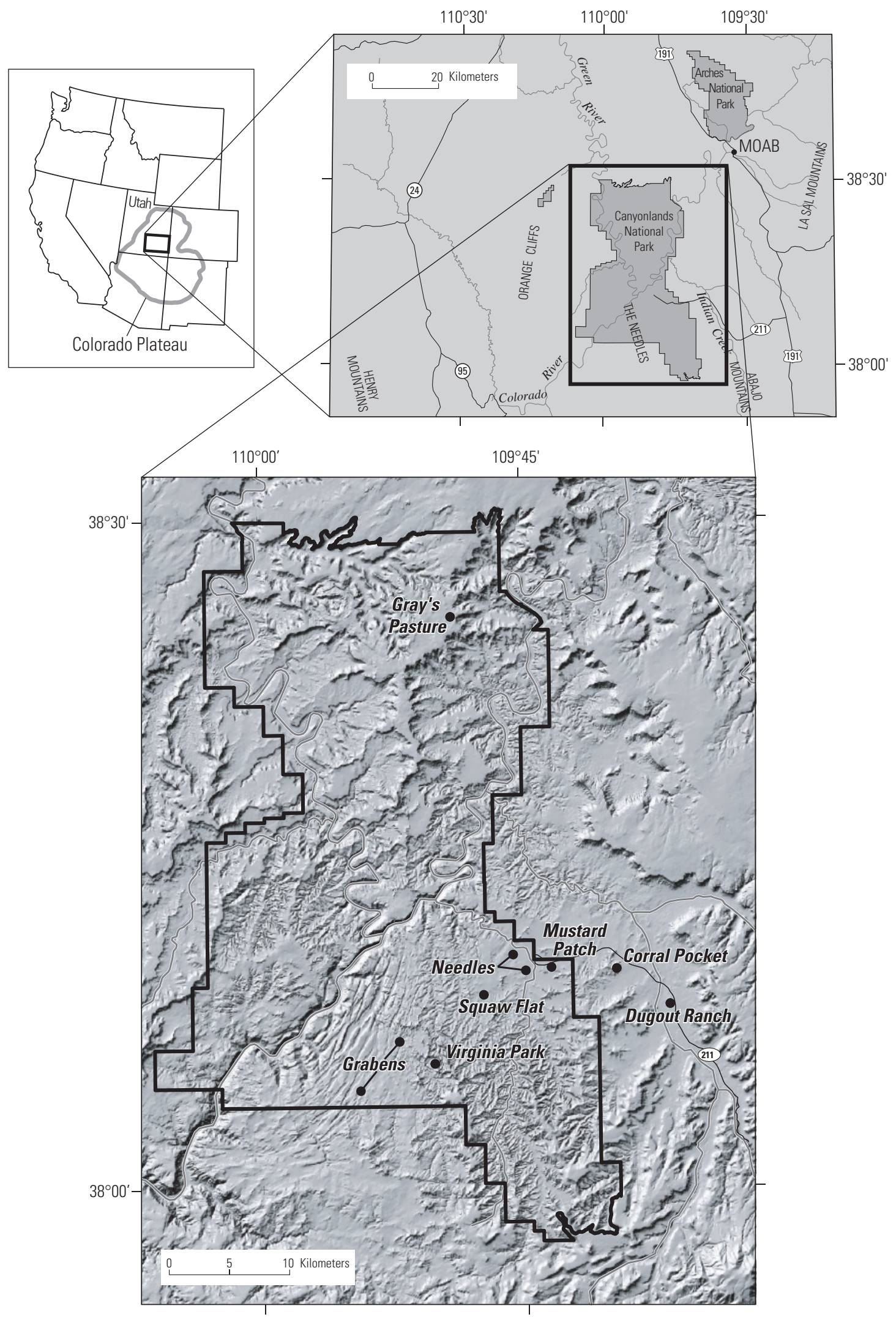

Figure 1. Location map of sampling sites in and around Canyonlands National Park. 


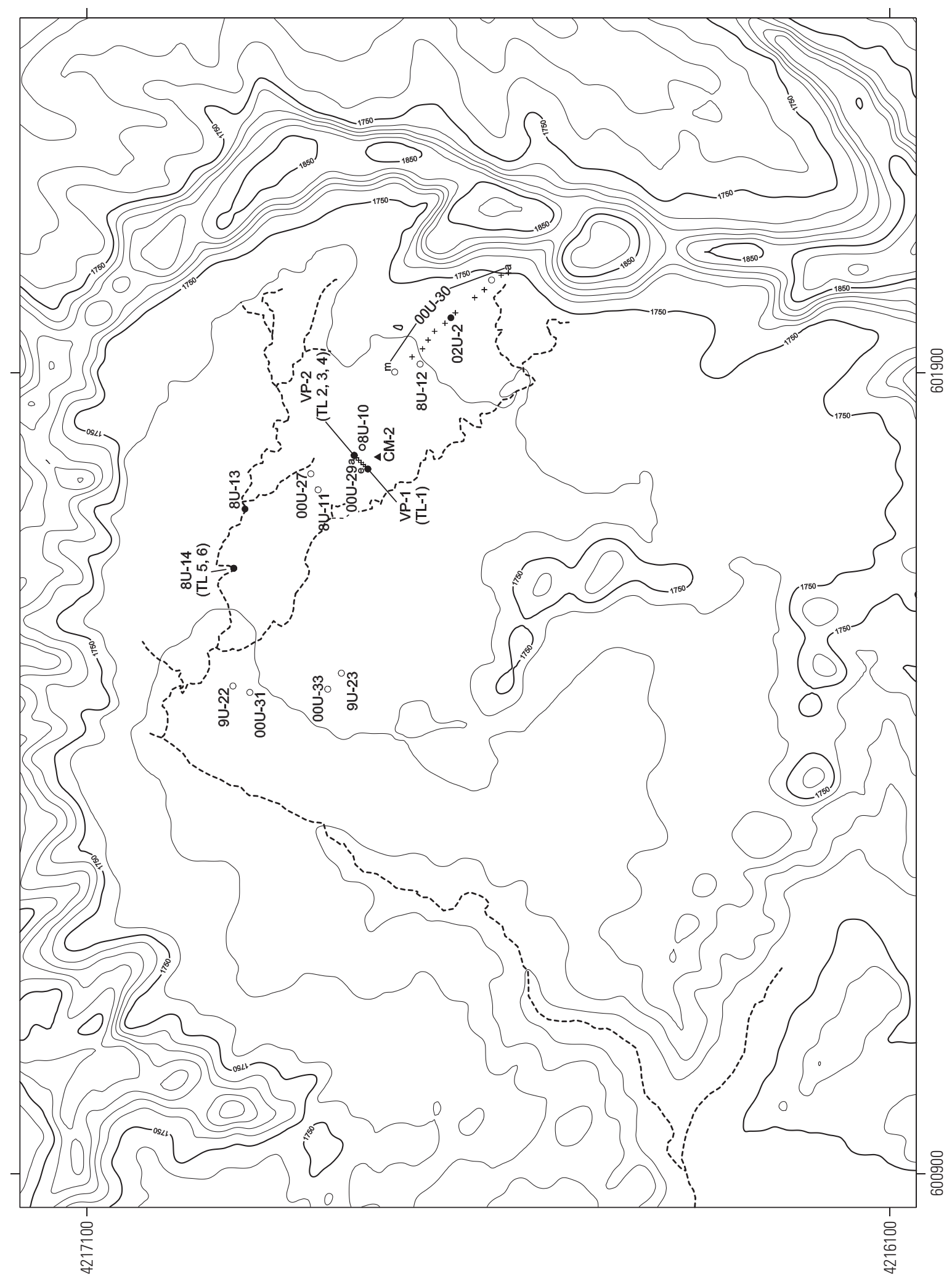




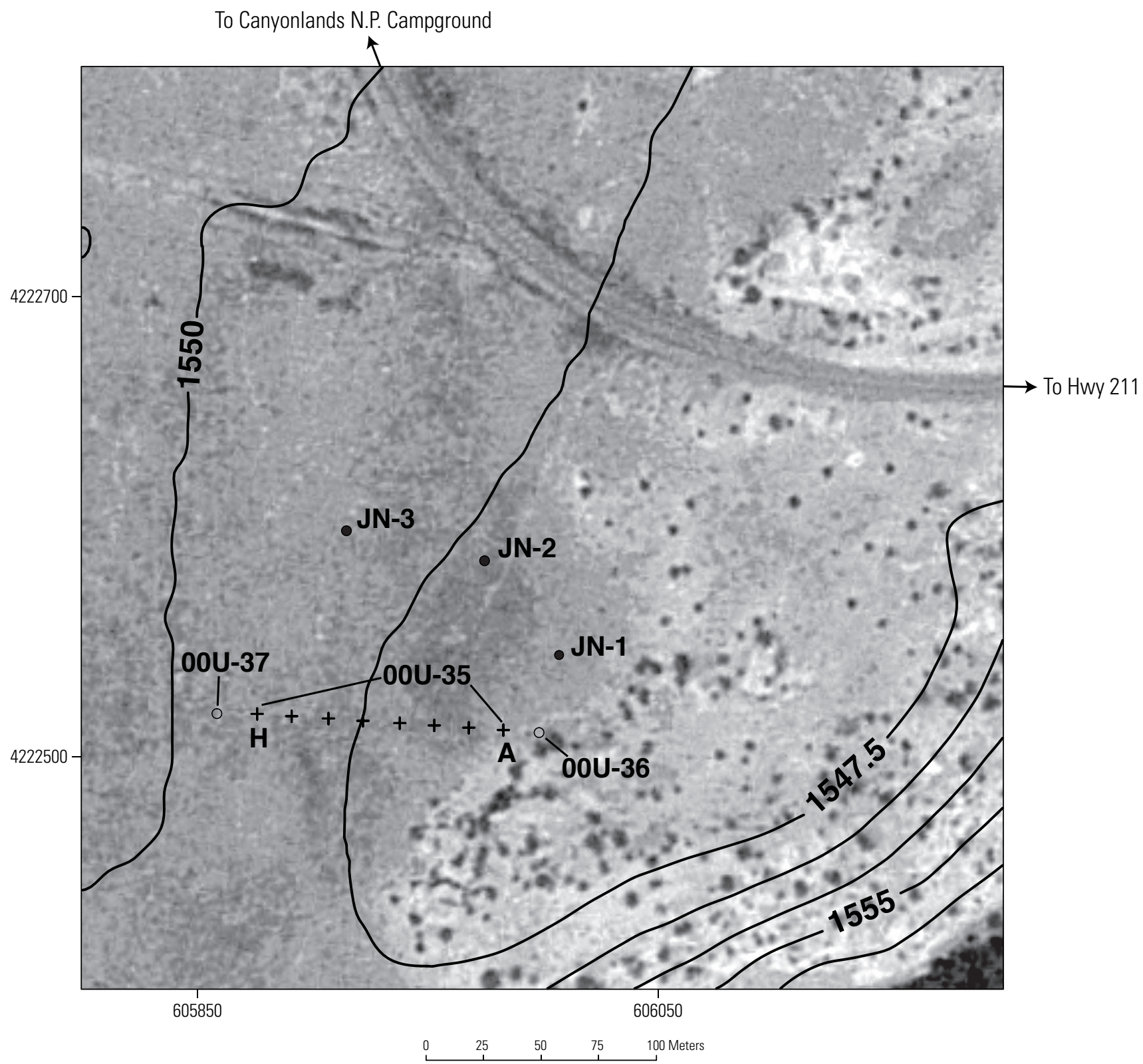

Figure 3. Location map of Squaw Flat sampling sites. Open circles represent auger holes, closed circles represent soil pits, and plus symbols represent sampling points $(\mathrm{A}-\mathrm{H})$ along the transect. Contour interval is 2.5 meters. Base Universal Tranverse Mercator (m) Zone 12; NAD 27 horizontal datum; elevation data contoured from The Loop 10 meter DEM; orthophotograph base from The Loop southeast DOQ. 


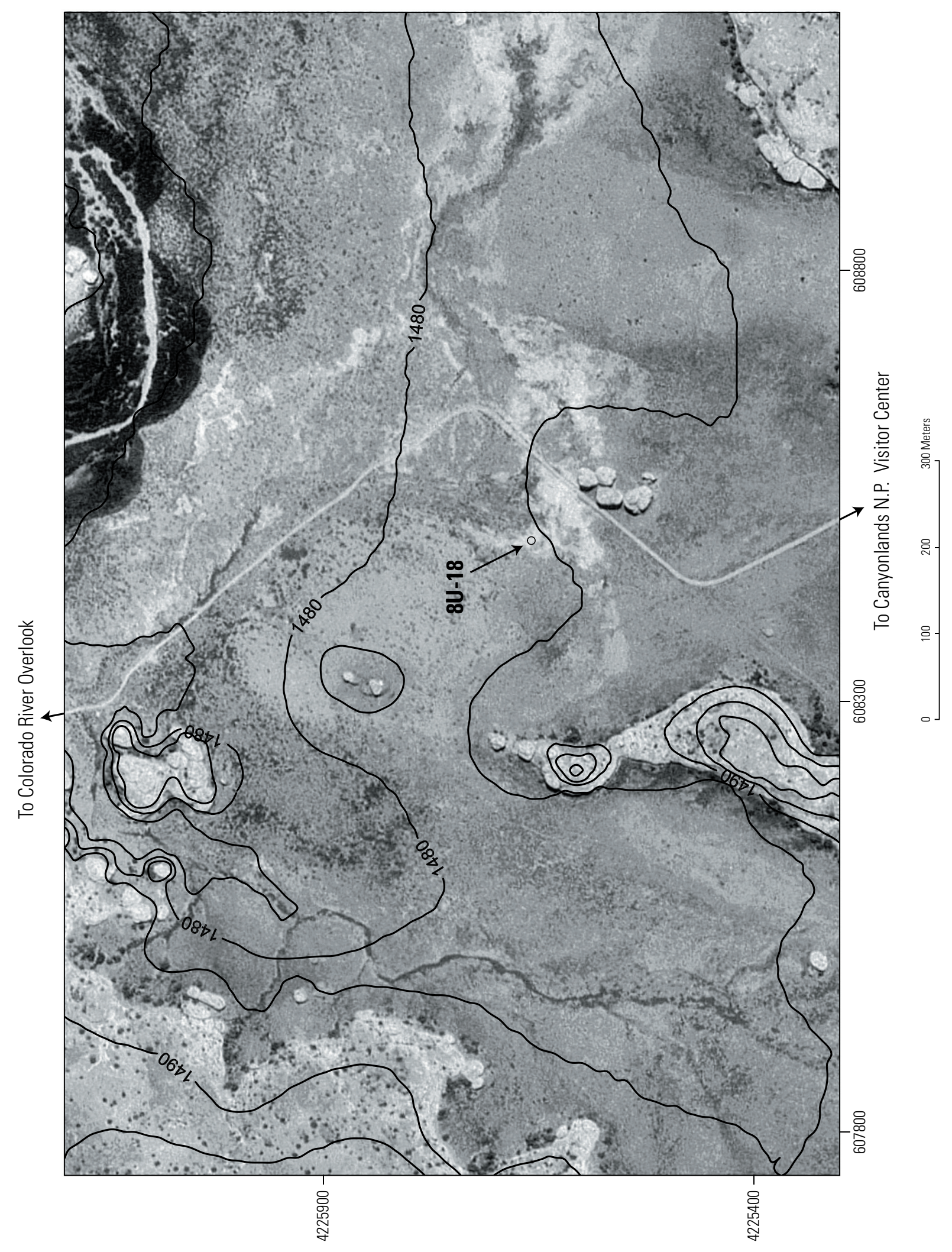

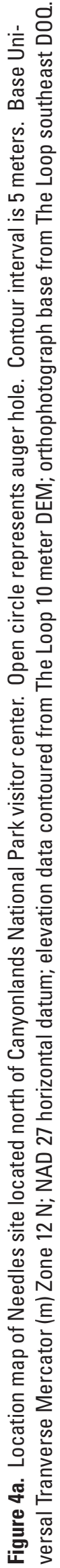




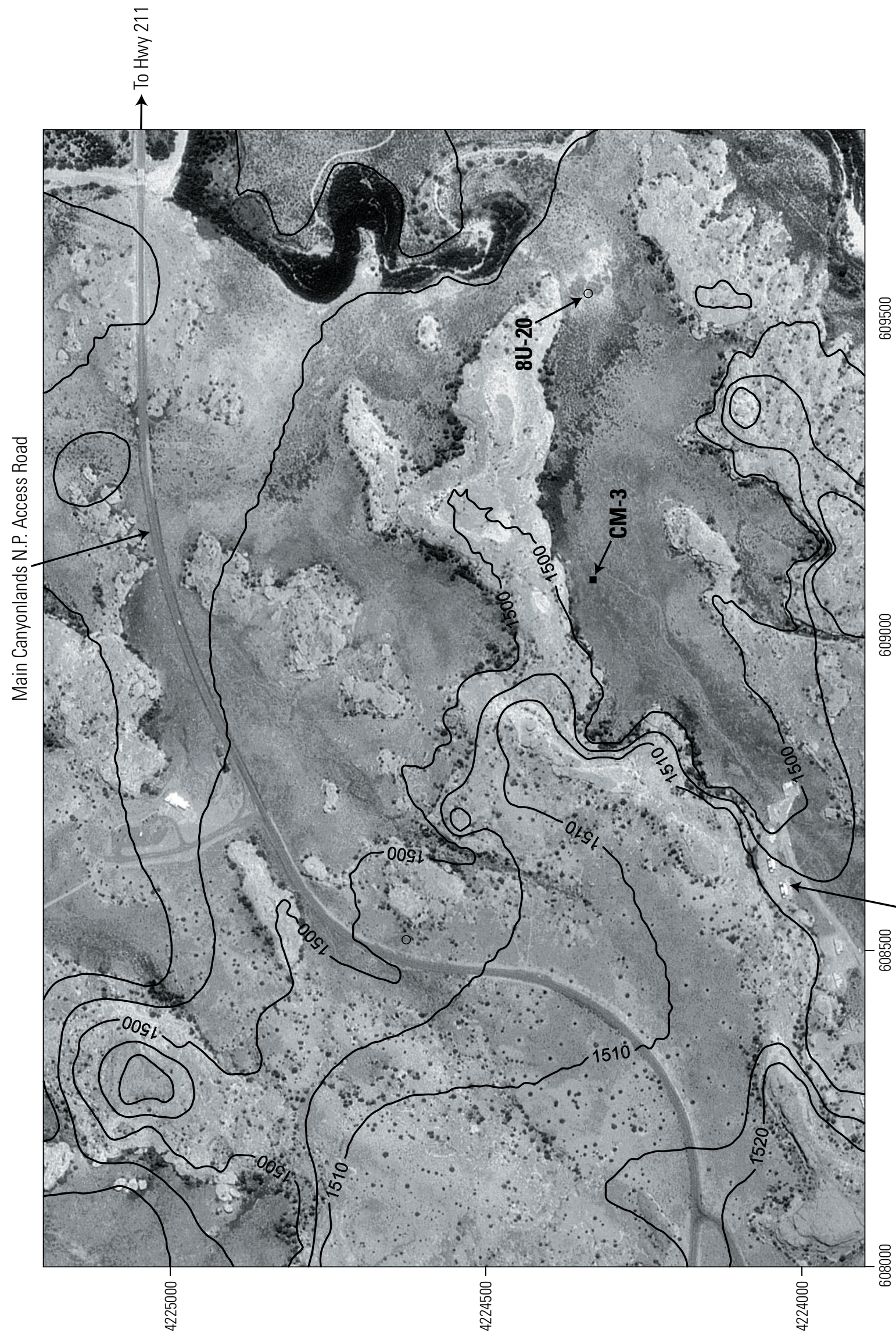

言

产 흉

m

느 을

崖京

峁

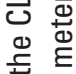

음

ํํ응

凹

흔 을

인

즘

엉

은



总

1 형

든

암 일 힘

峁

으-

बृ

흥 중

은 은

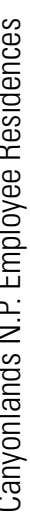

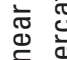

돌

总

으 힐

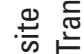

Ð

일

文

造

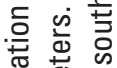

ठु 屯

동 응

寸

을 竞 


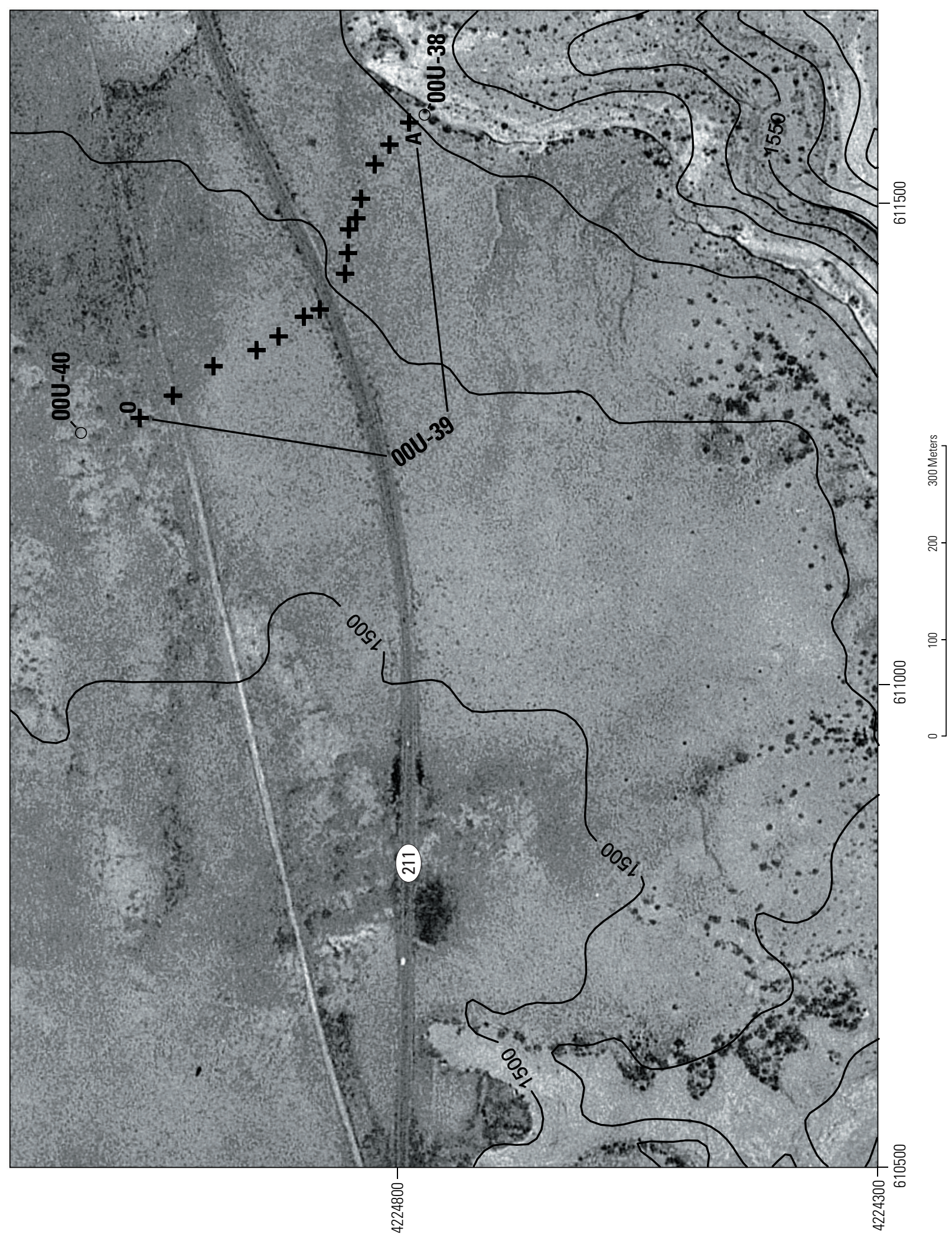

苞

으

$\underline{\underline{s}} \underline{\underline{\underline{t}}}$

垔

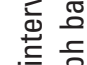

든

혿

한 등

倞

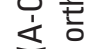

范

造

(ָ)

要

흠음

:

흠은

함

高希

怘 눙

范衣

递

$\frac{2}{0} \frac{2}{0}$

Ф

농 문

है 잉

के

금 흥

is 흐

임

힝

을

艺 要

边

흥

는

흠

.ำ

웅

닝

के

Nㅗㄴ

tह

응 흥

늄

$\sum^{\infty} \sum^{\infty}$

$\sum_{0} \sum_{0}$

은

ॠ

을 흘

异 元

웧

เ

농

言怘 


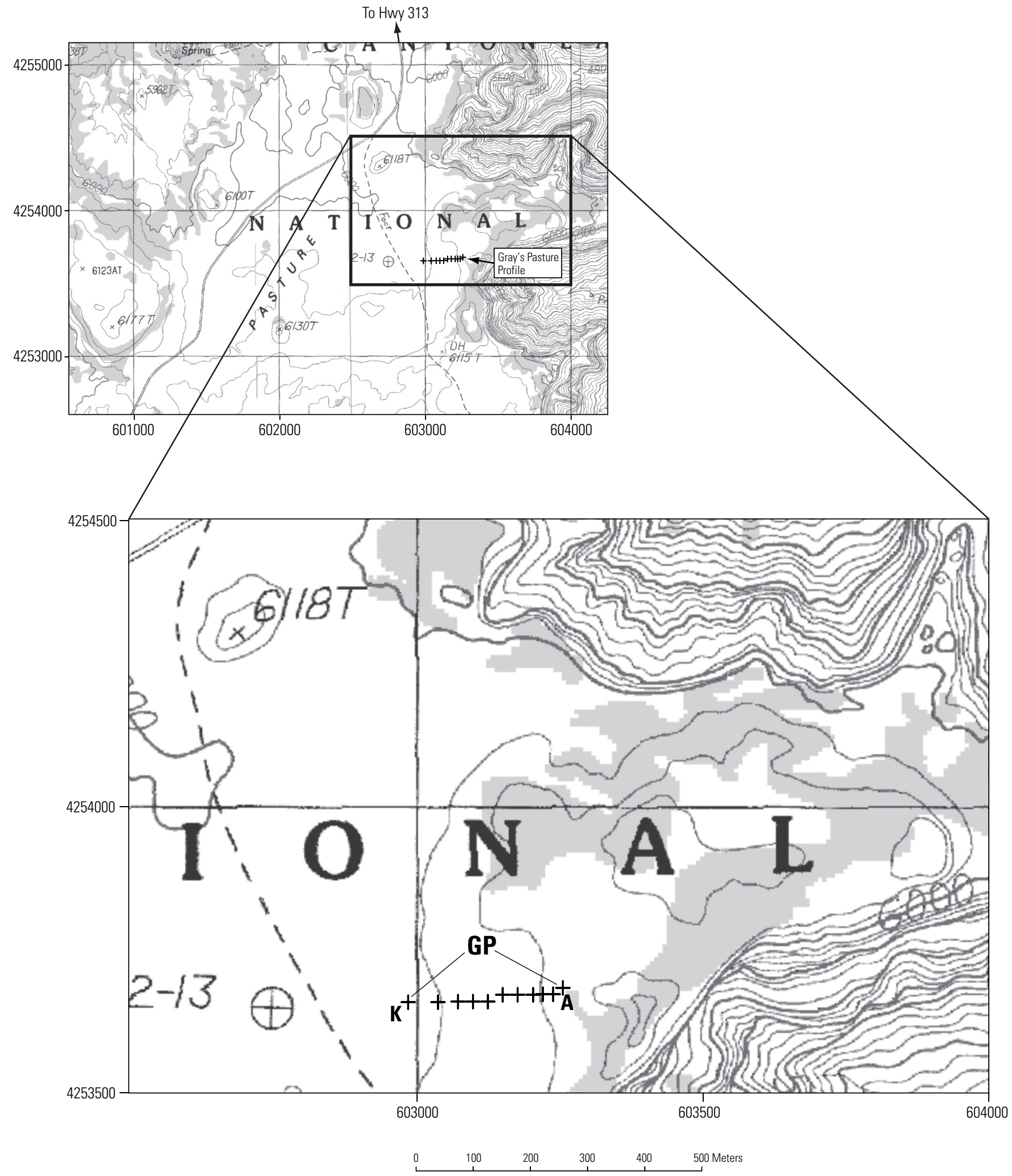

Figure 6. Location map of Gray's Pasture site. Plus symbols represent sampling points along the transect (A-K). Contour interval is 20 meters. Base Universal Tranverse Mercator (m) Zone 12; Musselman Arch Digital Raster Graphic. 


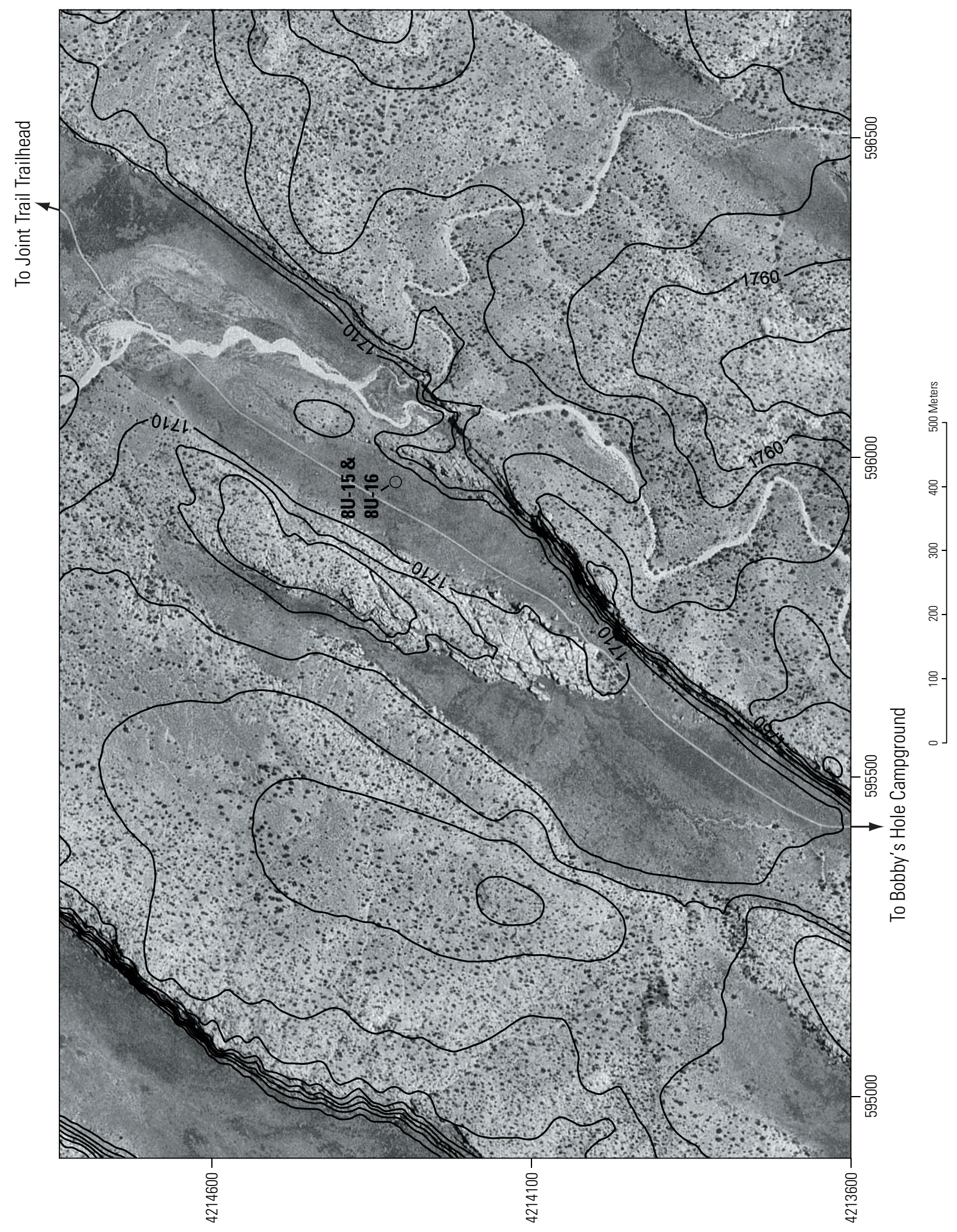

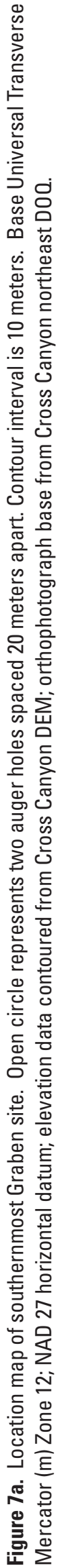




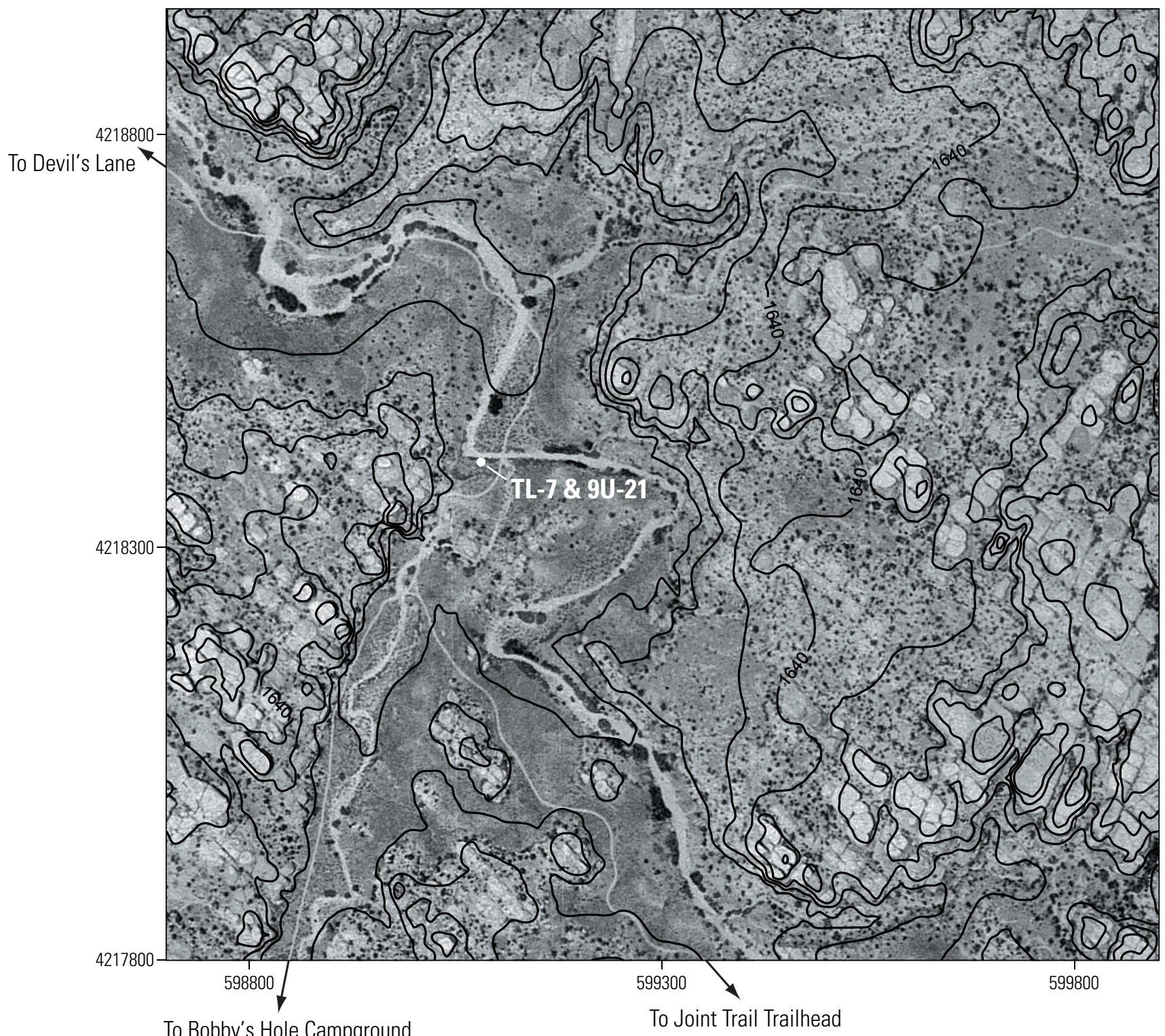

To Bobby's Hole Campground

To Joint Trail Trailhead

$\begin{array}{llll}0 & 200 & 300 & 400 \text { Meters }\end{array}$

Figure 7b. Location map of northern most Graben site. The closed white circle represents the arroyo cut. Contour interval is 10 meters. Base Universal Transverse Mercator (m) Zone 12; NAD 27 horizontal datum; elevation data contoured from Cross Canyon and Druid Arch 10 meter DEMs; orthophotograph base from Cross Canyon Northeast and Druid Arch northwest DEMs. 


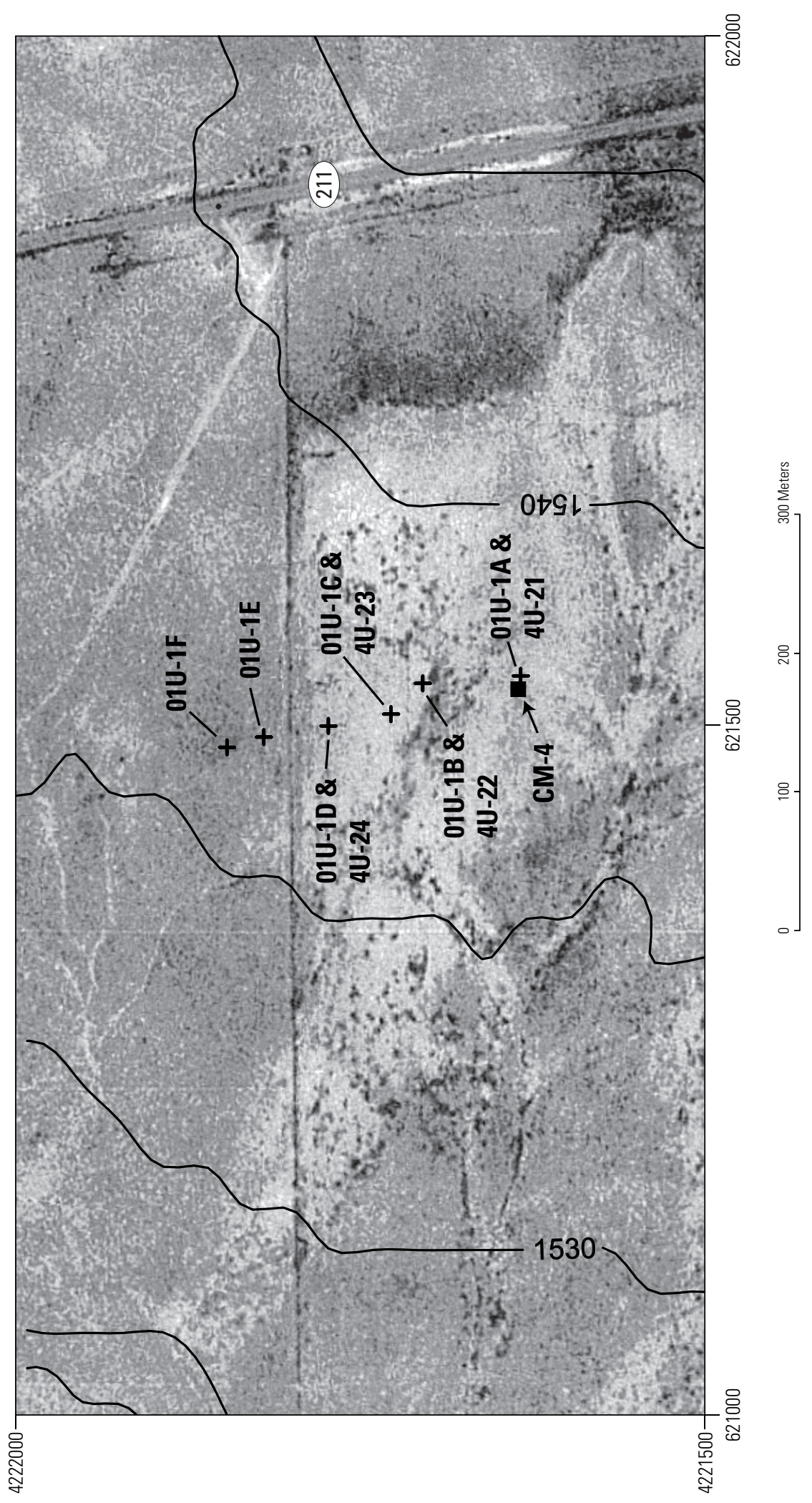

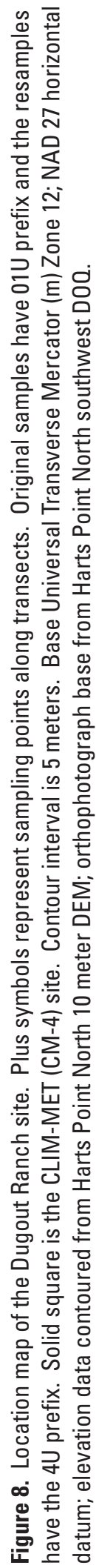




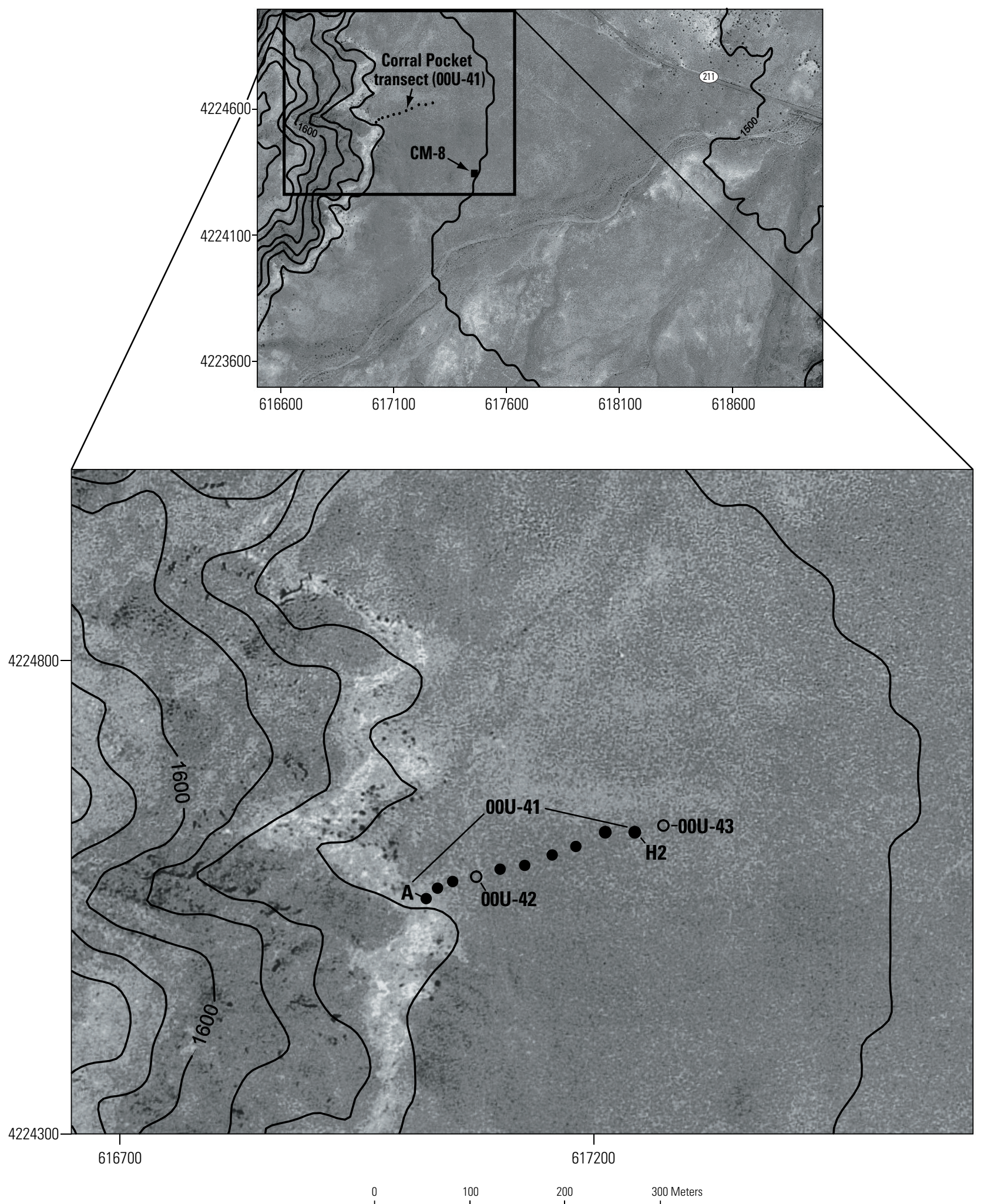

Figure 9. Location map of the Corral Pocket site. Open circles represent auger holes, closed circles represent sampling points along transect (A-H2). Square (top map) represents the CLIM-MET (CM-8) site. Contour interval is 20 meters. Base Universal Tranverse Mercator $(\mathrm{m})$ Zone 12; NAD 27 horizontal datum; elevation data contoured from North Six-Shooter 10 meter DEM; orthophotograph base from North Six-Shooter southeast DEM. 


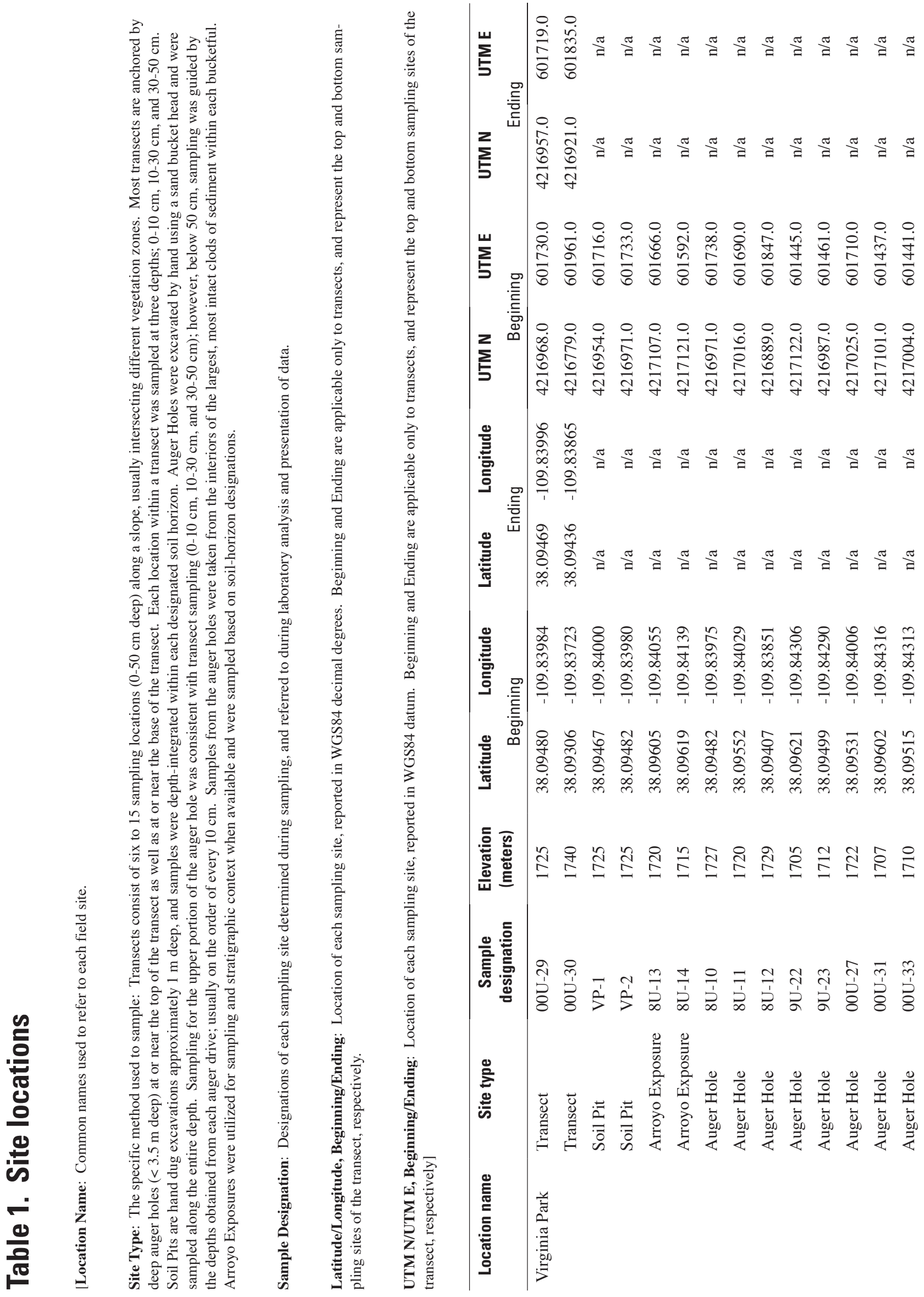




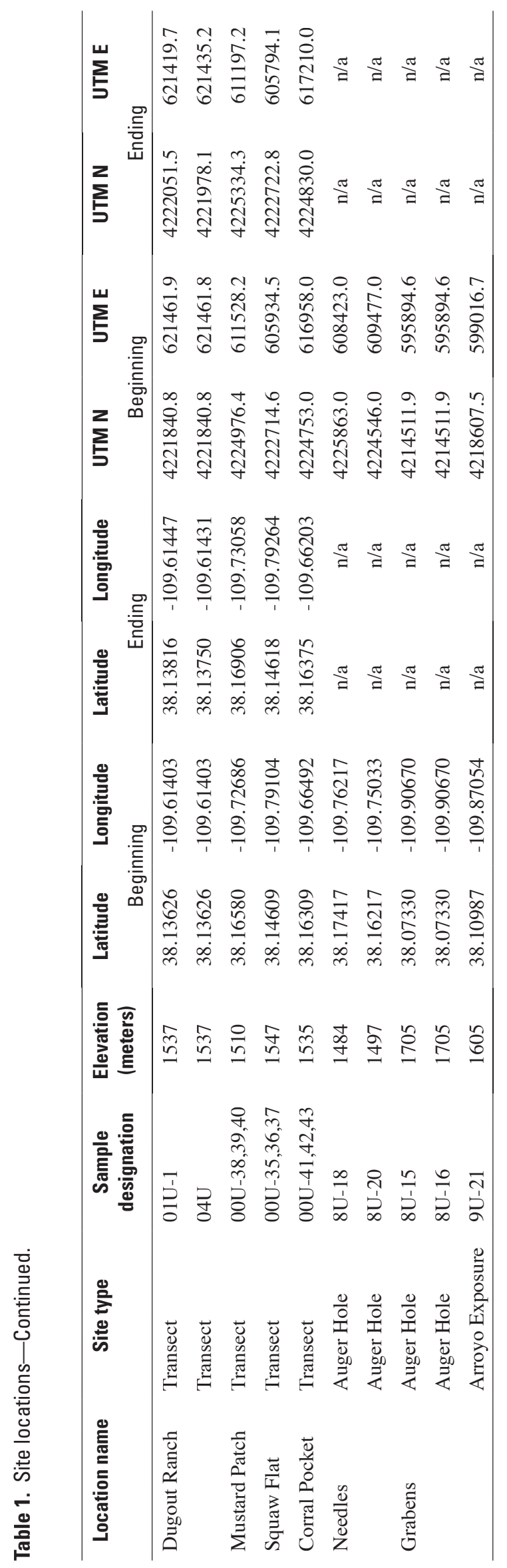




\section{Table 2. Soil descriptions}

In some cases, exposures, soil pits, and auger holes were described following Birkeland (1999). Descriptions were made only for sites in Virginia Park, Needles, and the Grabens. All other sites lack descriptions. Explanations of description notations are listed with the descriptions.

Table 2a-1. Soil decriptions of soil pits in Virginia Park.

\begin{tabular}{|c|c|c|c|c|c|c|c|c|}
\hline Horizon' ${ }^{1}$ & $\begin{array}{l}\text { Depth } \\
\text { (cm) }\end{array}$ & $\begin{array}{l}\text { Dry } \\
\text { color }\end{array}$ & $\begin{array}{l}\text { Moist } \\
\text { color }\end{array}$ & Structure $^{2}$ & $\begin{array}{c}\text { Dry } \\
\text { consitence }^{3}\end{array}$ & $\begin{array}{c}\text { Wet } \\
\text { consitence }^{4}\end{array}$ & Clay films ${ }^{5}$ & $\begin{array}{l}\text { Stage } \mathrm{CaCO}_{3}{ }^{1} \text {, } \\
\text { other cements }\end{array}$ \\
\hline \multicolumn{9}{|c|}{ VP-1, hand-dug soil pit in dune crest, Virginia Park site } \\
\hline Bwk & $6-19$ & $5 \mathrm{YR} 6 / 5$ & $5 \mathrm{YR} 4 / 6$ & $2 \mathrm{~m}-\mathrm{c}$ sbk & so & vss, vps & 0 & 0 , es \\
\hline B1tk & $19-30$ & $5 Y R 7 / 5$ & $5 Y R 5 / 6$ & $2 \mathrm{f}-\mathrm{m} \mathrm{sbk}$ & so & vss, vps & $1 \mathrm{nbr}$ & 0 , es \\
\hline B31tkb & $55-66$ & 5 YR6/5 & $5 Y R 5 / 6$ & $2 \mathrm{f}-\mathrm{m}$ abk & sh & vss, vps & $1 \mathrm{npf}$ & $\mathrm{I}+, \mathrm{ev}$ \\
\hline B32tkb & $66-78$ & $5 Y R 6 / 4$ & $5 Y R 5 / 6$ & $2 \mathrm{f}-\mathrm{m}$ abk & $\mathrm{h}$ & s, ps & $2 \mathrm{npf}$ & III, ev \\
\hline $\mathrm{Bkb}$ & $78-90$ & n.d. & n.d. & n.d. & $\mathrm{h}$ & n.d. & n.d. & III, ev \\
\hline \multicolumn{9}{|c|}{ VP-2, hand-dug soil pit in dune swale, Virginia Park site } \\
\hline Btk1b & $44-55$ & 5YR7/4 & 5YR5/6 & $2 \mathrm{f}-\mathrm{m} \mathrm{sbk}$ & sh & ss, vps & $1 \mathrm{nbr}$ & II, ev \\
\hline Btk2b & $55-85$ & 5YR6/5 & 5YR5/6 & $2 \mathrm{~m}-\mathrm{c}$ abk & sh & ss, vps & $1 \mathrm{nbr}, 1 \mathrm{npf}$ & I, es \\
\hline $\mathrm{Bkb}$ & $98-115$ & n.d. & n.d. & n.d. & $\mathrm{h}$ & n.d. & n.d. & n.d. \\
\hline
\end{tabular}

${ }^{1}$ Horizon nomenclature and carbonate stage follow Birkeland (1999) and references therein.

${ }^{2}$ Structure codes: Strength/abundance-1-3. Size-f, fine; m, medium; c, coarse. Shape - sbk, subangular blocky; abk, angular blocky; pl, platy. S.g., single grain.

${ }^{3}$ Dry consistence codes: lo, loose; so, soft; sh, slightly hard; h, hard; vh, very hard.

${ }^{4}$ Wet consistence codes: so, non-sticky; vss, very slightly sticky; ss, slightly sticky; s, sticky; vs, very sticky; po, non-plastic; pss, very slightly plastic; ps, slightly plastic; p, plastic; vp, very plastic.

${ }^{5}$ Clay film codes: Abundance-1-3; v1, very few. Thickness -n, thin. Location-br, grain bridge; po, pore filling; pf, ped face; co, grain coat.

${ }^{6}$ Carbonate codes: e, effervesces weakly; es, effervesces strongly; ev, effervesces violently. 
Table 2a-2. Soil descriptions of auger holes 8U-10, 8U-11, and 8U-12.

\begin{tabular}{|c|c|c|c|c|c|c|c|c|}
\hline Horizon ${ }^{1}$ & $\begin{array}{l}\text { Depth } \\
\text { (cm) }\end{array}$ & $\begin{array}{l}\text { Dry } \\
\text { color }\end{array}$ & $\begin{array}{l}\text { Moist } \\
\text { color }\end{array}$ & Structure $^{2}$ & $\begin{array}{c}\text { Dry } \\
\text { cositence }^{3} \\
\end{array}$ & $\begin{array}{c}\text { Wet } \\
\text { consistence }^{4}\end{array}$ & Clay films ${ }^{5}$ & $\begin{array}{c}\text { Stage } \mathrm{CaCO}_{3}{ }^{1}, \\
\text { other cements }{ }^{6}\end{array}$ \\
\hline \multicolumn{9}{|c|}{ Auger hole $8 \mathrm{U}-10$} \\
\hline $\mathrm{A} / \mathrm{Bw}$ & $0-22$ & 5YR6/5 & $5 Y R 4 / 6$ & $2 \mathrm{f}-\mathrm{m} \mathrm{sbk}$ & so & so, po & 0 & $0, \mathrm{e}$ \\
\hline $\mathrm{Bw}^{*}$ & $22-47$ & $5 Y R 6 / 4$ & $5 Y R 5 / 6$ & $1 \mathrm{f}-\mathrm{m} \mathrm{sbk}$ & lo-so & so, po & 0 & 0 , es \\
\hline Btb1 & $47-58$ & $5 Y R 7 / 6$ & 5YR6/6 & $1 \mathrm{f}-\mathrm{m} \mathrm{sbk}$ & so-sh & ss, ps & 0 & $\mathrm{I}, \mathrm{ev}$ \\
\hline Btkb1* & $58-74$ & $5 Y R 7 / 4$ & $5 \mathrm{YR} 4 / 6$ & $2 \mathrm{f}-\mathrm{m} \mathrm{sbk}$ & sh-h & vss, vps & 0 & II, ev \\
\hline $\mathrm{Bk} 1 \mathrm{~b} 1 *$ & $74-99$ & $5 Y R 7 / 3$ & 5YR6/5 & $3 \mathrm{~m} \mathrm{sbk}, 1 \mathrm{pl}$ & h-vh & vss, vps & 0 & $\mathrm{II}+, \mathrm{ev}$ \\
\hline $\mathrm{Bk} 2 \mathrm{~b} 1 *$ & $99-154$ & $5 Y R 7 / 4-7 / 5$ & 5YR6/5-6/6 & $2 \mathrm{~m} \mathrm{sbk}$ & h-vh & so, po & 0 & II, ev \\
\hline Btkqb2* & $154-220$ & $5 \mathrm{YR} 7 / 4-7 / 5$ & $5 Y R 5 / 7$ & $2 \mathrm{~m}$ abk & h-vh & ss, po & 0 & I, e-es; silica \\
\hline Bkb2* & $220-284$ & 5 YR7/4-8/4 & 5YR6/6 & n.d. & h-so & so, po & 0 & I, e-es; silica \\
\hline \multicolumn{9}{|c|}{ Auger hole 8U-11 } \\
\hline $\mathrm{A} / \mathrm{Bw}$ & $0-22$ & $5 Y R 6 / 5$ & $5 Y R 4 / 6$ & $2 \mathrm{f}-\mathrm{m} \mathrm{sbk}$ & so & so, po & 0 & 0, e-es \\
\hline $\mathrm{Bw}^{*}$ & $22-50$ & 5YR6/5 & $5 Y R 5 / 6$ & $2 \mathrm{f}-\mathrm{m} \mathrm{sbk}$ & so-sh & vss, vps & 0 & 0 -I, es-ev \\
\hline Btk1b1* & $50-86$ & 5YR6/6 & $5 Y R 5 / 6$ & $2 \mathrm{f}-\mathrm{m} \mathrm{sbk}$ & sh-h & vss, vps & 0 & $\mathrm{I}+, \mathrm{ev}$ \\
\hline Btk2b1* & $86-118$ & $5 Y R 7 / 4$ & $5 Y R 6 / 5$ & $3 f-m$ sbk & $\mathrm{h}$ & vss, vps & 0 & II,+ ev \\
\hline $\mathrm{Bkb} 1 *$ & $118-151$ & $5 Y R 7 / 4$ & $5 Y R 5 / 6$ & $2 \mathrm{f}-\mathrm{m} \mathrm{sbk}$ & sh-h & so, po & 0 & II, ev \\
\hline $\mathrm{Bkb} 2 *$ & $151-172$ & $5 Y R 7 / 5$ & $5 Y$ Y $6 / 6$ & n.d. & sh-so & so, po & 0 & I, es-ev \\
\hline Ckb2* & $172-251$ & $5 Y R 7 / 5$ & $5 Y R 6 / 6$ & Single grain & lo-so & so, po & 0 & I, es-ev \\
\hline Cyb2* & $251-278$ & $5 Y R 7 / 5$ & $5 Y R 6 / 6$ & Single grain & lo-so & so, po & 0 & $\begin{array}{l}0, \text { es; gypsum } \\
\text { rosettes }\end{array}$ \\
\hline \multicolumn{9}{|c|}{ Auger hole 8U-12 } \\
\hline A & $0-12$ & $5 Y R 4 / 4$ & $5 Y R 6 / 4$ & $2 \mathrm{f}-\mathrm{m} \mathrm{sbk}$ & so & so, po & 0 & $0, \mathrm{e}-$ \\
\hline Bw & $12-27$ & $5 Y R 6 / 5$ & $5 Y R 5 / 6$ & $3 \mathrm{f}-\mathrm{m} \mathrm{sbk}$ & so & vss, vps & 0 & 0 , es-ev \\
\hline Btk1b1 & $27-55$ & $5 Y R 7 / 4$ & $5 Y$ Y 5/6 & 1f sbk & lo-so & vss, po & 0 & $\mathrm{I}, \mathrm{ev}$ \\
\hline Btk2b1 & $55-80$ & $5 Y R 6 / 6$ & $5 \mathrm{YR} 5 / 8$ & $2 \mathrm{f}-\mathrm{m} \mathrm{sbk}$ & lo-so & so, po & 0 & $\mathrm{I}+, \mathrm{ev}$ \\
\hline Btk3b1 & $80-106$ & $5 Y R 8 / 3$ & 5YR6/6 & $3 \mathrm{f}-\mathrm{m}$ sbk & sh & ss, ps & 0 & $\mathrm{II}+, \mathrm{ev}$ \\
\hline Bk1b1 & $106-147$ & $5 Y R 8 / 3$ & 5YR6/6 & $2 \mathrm{f}-\mathrm{m} \mathrm{sbk}$ & h-so & so, po & 0 & II, ev-es \\
\hline $\mathrm{Bk} 2 \mathrm{~b} 1$ & $147-180$ & $5 Y R 7 / 4$ & 5YR6/6 & n.d. & lo-so & so, po & 0 & I, ev-e \\
\hline Btkqb2 & $180-216$ & 5YR6/5 & $5 Y R 5 / 6$ & n.d. & so-h & so, po & 0 & $\mathrm{I}, \mathrm{ev}$ \\
\hline $\mathrm{Bk} 1 \mathrm{~b} 2$ & $216-273$ & $5 Y R 6 / 5$ & $5 Y$ Y 5/6 & n.d. & lo-sh & so, po & 0 & $\mathrm{I}, \mathrm{ev}$ \\
\hline $2 \mathrm{Bk} 2 \mathrm{~b} 2$ & $273-280$ & $5 Y R 7 / 4$ & $5 Y R 6 / 6$ & Single grain & lo & so, po & 0 & I, ev \\
\hline $3 \mathrm{Bk} 3 \mathrm{~b} 2$ & 280-299 & $5 Y R 7 / 5$ & $5 Y R 6 / 6$ & Single grain & lo-sh & so, po & 0 & $\mathrm{I}$, ev-es \\
\hline 3Bk4b2 & 299-317 & $5 Y R 7 / 5$ & 5YR6/6 & Single grain & lo & n.d. & 0 & $\mathrm{I}, \mathrm{ev}$ \\
\hline
\end{tabular}

*Represents average or range of properties of 2-5 samples within given depth range

${ }^{1}$ Horizon nomenclature and carbonate stage follow Birkeland (1999) and references therein

${ }^{2}$ Structure codes: Strength/abundance-1-3. Size -f, fine; m, medium; c, coarse. Shape — sbk, subangular blocky; abk, angular blocky; pl, platy. S.g., single grain.

${ }^{3}$ Dry consistence codes: lo, loose; so, soft; sh, slightly hard; h, hard; vh, very hard.

${ }^{4}$ Wet consistence codes: so, non-sticky; vss, very slightly sticky; ss, slightly sticky; s, sticky; vs, very sticky; po, non-plastic; pss, very slightly plastic; ps, slightly plastic; p, plastic; vp, very plastic.

${ }^{5}$ Clay film codes: Abundance-1-3; v1, very few. Thickness - n, thin. Location - br, grain bridge; po, pore filling; pf, ped face; co, grain coat.

${ }^{6}$ Carbonate codes: e, effervesces weakly; es, effervesces strongly; ev, effervesces violently. 
Table 2a-3. Soil description of auger hole 9U-22.

\begin{tabular}{|c|c|c|c|c|c|c|c|c|}
\hline Horizon' ${ }^{1}$ & $\begin{array}{l}\text { Depth } \\
\text { (cm) }\end{array}$ & Dry color & Moist color & Structure $^{2}$ & $\begin{array}{c}\text { Dry } \\
\text { consistence }^{3}\end{array}$ & $\begin{array}{c}\text { Wet } \\
\text { consistence }^{4}\end{array}$ & Clay films ${ }^{5}$ & $\begin{array}{l}\text { Stage } \mathrm{CaCO}_{3}{ }^{1} \text {, } \\
\text { other cements }{ }^{6}\end{array}$ \\
\hline \multicolumn{9}{|c|}{ Auger hole 9U-22 } \\
\hline A & $0-15$ & n.d. & n.d. & n.d. & n.d. & ss, po & n.d. & 0 , es \\
\hline $\mathrm{Bt}$ & $15-41$ & n.d. & n.d. & 1f sbk & n.d. & ss, po & n.d. & 0 , es \\
\hline Btk1 & $41-67$ & n.d. & n.d. & $2 \mathrm{f} \mathrm{sbk}$ & n.d. & s, ps & n.d. & 0 , es \\
\hline Btk2 & $67-87$ & n.d. & n.d. & $2 \mathrm{f} \mathrm{sbk}$ & n.d. & s, ps & n.d. & $\mathrm{I}+$, es \\
\hline $\mathrm{Bk} 1$ & $87-97$ & n.d. & n.d. & $2 \mathrm{f} \mathrm{sbk}$ & n.d. & ss, po & n.d. & II, ev \\
\hline $\mathrm{Bk} 2$ & $97-124$ & n.d. & n.d. & $2 \mathrm{f} \mathrm{sbk}$ & n.d. & so, po & n.d. & II, ev \\
\hline $\mathrm{Bk} 3$ & $124-146$ & n.d. & n.d. & 1f sbk & n.d. & so, po & n.d. & $\mathrm{I}, \mathrm{ev}$ \\
\hline $\mathrm{C}$ & $146-156$ & n.d. & n.d. & single grain & n.d. & so, po & n.d. & 0 , es \\
\hline Btb1 & $156-178$ & n.d. & n.d. & 1f sbk & n.d. & so, po & n.d. & 0 , es \\
\hline Btb2 & $178-216$ & n.d. & n.d. & 1f sbk & n.d. & so, po & n.d. & 0 , es \\
\hline Bwb2 & $216-240$ & n.d. & n.d. & 1f sbk & n.d. & so, po & n.d. & 0 , es \\
\hline $\mathrm{Cb}$ & $240-265$ & n.d. & n.d. & single grain & n.d. & so, po & n.d. & 0 , es \\
\hline Cyb & $265-274$ & n.d. & n.d. & single grain & n.d. & so, po & n.d. & $\begin{array}{l}0, \text { es; gypsum } \\
\text { rosettes }\end{array}$ \\
\hline Btyb3 & $274-295$ & n.d. & n.d. & 1f sbk & n.d. & ss, ps & n.d. & $\begin{array}{l}0, \text { es; gypsum } \\
\text { rosettes }\end{array}$ \\
\hline Btky1b3 & 295-305 & n.d. & n.d. & $2 \mathrm{f} \mathrm{sbk}$ & n.d. & $\mathrm{s}, \mathrm{ps}$ & n.d. & $\begin{array}{l}0, \text { es; gypsum } \\
\text { rosettes }\end{array}$ \\
\hline Btky2b3 & $305-325$ & n.d. & n.d. & 3f sbk & n.d. & ss, ps & n.d. & $\begin{array}{l}\text { I, ev; gypsum } \\
\text { rosettes }\end{array}$ \\
\hline Bk1b3 & $325-364$ & n.d. & n.d. & 3f sbk & n.d. & ss, ps & n.d. & $\mathrm{II}+, \mathrm{ev}$ \\
\hline $\mathrm{Bk} 2 \mathrm{~b} 3$ & $364-383$ & n.d. & n.d. & 1f sbk & n.d. & so, po & n.d. & I, es \\
\hline
\end{tabular}

*Represents average or range of properties of 2-5 samples within given depth range

${ }^{1}$ Horizon nomenclature and carbonate stage follow Birkeland (1999) and references therein

${ }^{2}$ Structure codes: Strength/abundance-1-3. Size-f, fine; m, medium; c, coarse. Shape-sbk, subangular blocky; abk, angular blocky; pl, platy. S.g., single grain.

${ }^{3}$ Dry consistence codes: lo, loose; so, soft; sh, slightly hard; h, hard; vh, very hard.

${ }^{4}$ Wet consistence codes: so, non-sticky; vss, very slightly sticky; ss, slightly sticky; s, sticky; vs, very sticky; po, non-plastic; pss, very slightly plastic; ps, slightly plastic; $p$, plastic; vp, very plastic.

${ }^{5}$ Clay film codes: Abundance-1-3; v1, very few. Thickness $-\mathrm{n}$, thin. Location - br, grain bridge; po, pore filling; pf, ped face; co, grain coat.

${ }^{6}$ Carbonate codes: e, effervesces weakly; es, effervesces strongly; ev, effervesces violently. 
Table 2a-4. Soil description of auger hole 9U-23.

\begin{tabular}{|c|c|c|c|c|c|c|c|c|}
\hline Horizon $^{1}$ & $\begin{array}{l}\text { Depth } \\
\text { (cm) }\end{array}$ & Dry color & Moist color & Structure $^{2}$ & $\begin{array}{c}\text { Dry } \\
\text { consistence }^{3}\end{array}$ & $\begin{array}{c}\text { Wet } \\
\text { consistence }^{4}\end{array}$ & Clay films ${ }^{5}$ & $\begin{array}{l}\text { Stage } \mathrm{CaCO}_{3}{ }^{1} \text {, } \\
\text { other cements }\end{array}$ \\
\hline \multicolumn{9}{|c|}{ Auger hole 9U-23 } \\
\hline $\mathrm{A} / \mathrm{Bw}$ & $0-33$ & n.d. & n.d. & 1f sbk & n.d. & so, po & 0 & 0 , es \\
\hline Btj & $33-46$ & n.d. & n.d. & 1f sbk & n.d. & so, po & 0 & $0, \mathrm{ev}$ \\
\hline Btk1b1 & $46-67$ & n.d. & n.d. & 2f sbk & n.d. & ss, ps & 0 & $\mathrm{I}$, ev \\
\hline Btk2b1 & $67-96$ & n.d. & n.d. & 3f sbk & n.d. & ss, ps & 0 & $\mathrm{II}+, \mathrm{ev}$ \\
\hline Bkb1 & $96-115$ & n.d. & n.d. & 3f sbk & n.d. & ss, ps & 0 & II, ev \\
\hline Bkb2 & $115-138$ & n.d. & n.d. & 3f sbk & n.d. & so, po & 0 & II, ev \\
\hline Bkyb1 & $138-171$ & n.d. & n.d. & 1f sbk & n.d. & so, po & 0 & $\begin{array}{l}\text { I, es; gypsum } \\
\text { rosettes }\end{array}$ \\
\hline Btb2 & $171-185$ & n.d. & n.d. & $2 \mathrm{f} \mathrm{sbk}$ & n.d. & ss, ps & 2npo & 0 , es \\
\hline Btkb2 & $185-218$ & n.d. & n.d. & 2f sbk & n.d. & ss, ps & 2 npo & I, es \\
\hline $\mathrm{Bk} 1 \mathrm{~b} 2$ & $218-248$ & n.d. & n.d. & 1f sbk & n.d. & so, po & 0 & II, es \\
\hline $\mathrm{Bk} 2 \mathrm{~b} 2$ & $248-285$ & n.d. & n.d. & 1f sbk & n.d. & so, po & 0 & I, es \\
\hline Btkb3 & $285-298$ & n.d. & n.d. & $2 \mathrm{f} \mathrm{sbk}$ & n.d. & vss, po & 1 npo & II-, ev \\
\hline rock & $298+$ & n.d. & n.d. & n.d. & n.d. & n.d. & n.d. & n.d. \\
\hline
\end{tabular}

Table 2a-5. Soil description of auger hole 00U-27.

\begin{tabular}{|c|c|c|c|c|c|c|c|c|}
\hline Horizon' 1 & $\begin{array}{l}\text { Depth } \\
\text { (cm) }\end{array}$ & Dry color & Moist color & Structure $^{2}$ & $\begin{array}{c}\text { Dry } \\
\text { consistence }^{3}\end{array}$ & $\begin{array}{c}\text { Wet } \\
\text { consistence }^{4}\end{array}$ & Clay films $^{5}$ & $\begin{array}{l}\text { Stage } \mathrm{CaCO}_{3}{ }^{1}, \\
\text { other cements }{ }^{6}\end{array}$ \\
\hline \multicolumn{9}{|c|}{ Auger hole 00U-27 } \\
\hline A & $0-10$ & n.d. & n.d. & $\mathrm{sg}$ to $1 \mathrm{f}-\mathrm{m} \mathrm{sbk}$ & so, sh & so, ps & 0 & 0,0 \\
\hline Bwk & $10-30$ & n.d. & n.d. & $1 \mathrm{f}-\mathrm{m} \mathrm{sbk}$ & so & so, po & 0 & $\mathrm{I}, \mathrm{e}$ \\
\hline Btb1 & $30-84$ & n.d. & n.d. & $2 \mathrm{~m} \mathrm{sbk}$ & so & ss, ps & 1 vnco & $0, \mathrm{e}$ \\
\hline Btkb1 & $84-116$ & n.d. & n.d. & $2 \mathrm{~m} \mathrm{sbk}$ & so, sh & ss, ps & 0 & I, es \\
\hline Btkqb2 & $116-156$ & n.d. & n.d. & $2 \mathrm{~m} \mathrm{sbk}$ & $\mathrm{vh}$ & ss, p & 0 & II+, ev-es \\
\hline Bkq1b2 & $156-194$ & n.d. & n.d. & $2 \mathrm{f}-\mathrm{m} \mathrm{sbk}$ & so-vh & so, ps & 0 & II, ev-es \\
\hline Bkq2b2 & $194-233$ & n.d. & n.d. & $1 \mathrm{f}-\mathrm{m} \mathrm{sbk}$ & so-vh & so, ps & 0 & II-, ev-es \\
\hline Bkq3b2 & $233-274$ & n.d. & n.d. & 1f-m sbk & so-h & vss, po & 0 & It, es-e \\
\hline Bkq1b3 & $274-301$ & n.d. & n.d. & $1 \mathrm{f}-\mathrm{m} \mathrm{sbk}$ & lo-h & vss, po & 0 & I-, es-e \\
\hline Bkq2b3 & $301-324$ & n.d. & n.d. & $1 \mathrm{f}-\mathrm{m} \mathrm{sbk}$ & lo-h & ss, pss & 0 & I-, es-e \\
\hline
\end{tabular}

${ }^{1}$ Horizon nomenclature and carbonate stage follow Birkeland (1999) and references therein

${ }^{2}$ Structure codes: Strength/abundance-1-3. Size-f, fine; m, medium; c, coarse. Shape - sbk, subangular blocky; abk, angular blocky; pl, platy. S.g., single grain.

${ }^{3}$ Dry consistence codes: lo, loose; so, soft; sh, slightly hard; h, hard; vh, very hard.

${ }^{4}$ Wet consistence codes: so, non-sticky; vss, very slightly sticky; ss, slightly sticky; s, sticky; vs, very sticky; po, non-plastic; pss, very slightly plastic; ps, slightly plastic; p, plastic; vp, very plastic.

${ }^{5}$ Clay film codes: Abundance-1-3; v1, very few. Thickness - n, thin. Location-br, grain bridge; po, pore filling; pf, ped face; co, grain coat.

${ }^{6}$ Carbonate codes: e, effervesces weakly; es, effervesces strongly; ev, effervesces violently. 
24 Particle-Size, $\mathrm{CaCO}_{3}$, Chemical, Magnetic, and Age Data from Surficial Deposits around Canyonlands N.P., Utah

Table 2b-1. Soil descriptions of auger holes 8U-18 and 8U-20.

\begin{tabular}{|c|c|c|c|c|c|c|c|c|}
\hline Horizon ${ }^{1}$ & $\begin{array}{l}\text { Depth } \\
\text { (cm) }\end{array}$ & Dry color & Moist color & Structure $^{2}$ & $\begin{array}{c}\text { Dry } \\
\text { consistence }^{3}\end{array}$ & $\begin{array}{c}\text { Wet } \\
\text { consistence }^{4}\end{array}$ & Clay films ${ }^{5}$ & $\begin{array}{l}\text { Stage } \mathrm{CaCO}_{3}{ }^{1} \text {, } \\
\text { other cements }\end{array}$ \\
\hline \multicolumn{9}{|c|}{ Auger hole 8U-18 } \\
\hline \multirow{5}{*}{$\begin{array}{l}\text { Horizons not } \\
\text { differenti- } \\
\text { ated due } \\
\text { to Clayey } \\
\text { nature of } \\
\text { deposit }\end{array}$} & $95-155$ & $5 Y R 7 / 4$ & $5 Y R 5 / 4$ & Massive & n.d. & vs, vp & v1npf & $0, \mathrm{ev}$ \\
\hline & $155-187$ & 5YR6/4 & $5 \mathrm{YR} 5 / 6$ & Massive & n.d. & $\mathrm{vs}, \mathrm{vp}$ & v1npf & $0, \mathrm{ev}$ \\
\hline & $205-333$ & $5 Y R 7 / 5$ & 5YR5/6 & Single grain & n.d. & so, po & 0 & $0, \mathrm{ev}$ \\
\hline & $333-391$ & $5 Y R 7 / 4$ & $5 Y R 6 / 6$ & Single grain & n.d. & ss, ps & 0 & $0, \mathrm{ev}$ \\
\hline & $391-400$ & 5YR7/5 & 5YR6/6 & Single grain & n.d. & so, po & 0 & $0, \mathrm{ev}$ \\
\hline \multicolumn{9}{|c|}{ Auger hole 8U-20 } \\
\hline $\mathrm{C}$ & $0-65$ & 5YR6/5 & 5YR5/6 & $\begin{array}{c}\text { massive to } \\
\text { bedded }\end{array}$ & $\mathrm{sh}$ & $\mathrm{vs}, \mathrm{vp}$ & pressure films & $0, \mathrm{ev}$ \\
\hline $2 \mathrm{Cb} 1$ & $102-165$ & $5 Y R 7 / 4$ & 5YR6/6 & $\mathrm{sg}$ & lo & so, po & 0 & $0, \mathrm{e}$ \\
\hline 2Bwb2 & $165-190$ & 5YR6/4 & 5YR5/4 & $2 \mathrm{~m} \mathrm{sbk}$ & so-sh & ss, ps & 0 & $0, \mathrm{ev}$ \\
\hline $2 \mathrm{Cb} 2$ & $190-215$ & $5 Y R 7 / 4$ & $5 Y R 5 / 4$ & $\mathrm{sg}$ & so-lo & so, po & 0 & 0 , es \\
\hline $3 \mathrm{C} 1 \mathrm{~b} 3$ & $215-365$ & 5YR6/6 & 5YR5/6 & massive & $\mathrm{sh}$ & vs, vp & pressure films & 0 , es \\
\hline $3 \mathrm{C} 2 \mathrm{~b} 3$ & $365-389$ & 5YR6/7 & $5 \mathrm{YR} 5 / 6$ & massive & $\operatorname{sh}$ & vs, vp & pressure films & 0 , es \\
\hline $3 \mathrm{C} 3 \mathrm{~b} 3$ & $389-400$ & n.d. & $5 Y R 5 / 5$ & massive & $\mathrm{sh}$ & $\mathrm{vs}, \mathrm{vp}$ & pressure films & 0, es \\
\hline
\end{tabular}

${ }^{1}$ Horizon nomenclature and carbonate stage follow Birkeland (1999) and references therein.

${ }^{2}$ Structure codes: Strength/abundance-1-3. Size-f, fine; m, medium; c, coarse. Shape-sbk, subangular blocky; abk, angular blocky; pl, platy. S.g., single grain.

${ }^{3}$ Dry consistence codes: lo, loose; so, soft; sh, slightly hard; h, hard; vh, very hard.

${ }^{4}$ Wet consistence codes: so, non-sticky; vss, very slightly sticky; ss, slightly sticky; s, sticky; vs, very sticky; po, non-plastic; pss, very slightly plastic; ps, slightly plastic; p, plastic; vp, very plastic.

${ }^{5}$ Clay film codes: Abundance-1-3; v1, very few. Thickness $-\mathrm{n}$, thin. Location - br, grain bridge; po, pore filling; pf, ped face; co, grain coat.

${ }^{6}$ Carbonate codes: e, effervesces weakly; es, effervesces strongly; ev, effervesces violently. 
Table 2c-1. Soil description of the 9U-21 graben fill exposure.

\begin{tabular}{|c|c|c|c|c|c|c|c|c|}
\hline Horizon $^{1}$ & $\begin{array}{l}\text { Depth } \\
\text { (cm) }\end{array}$ & Dry color & Moist color & Structure $^{2}$ & $\begin{array}{c}\text { Dry } \\
\text { consistence }^{3}\end{array}$ & $\begin{array}{c}\text { Wet } \\
\text { consistence }^{4}\end{array}$ & Clay films ${ }^{5}$ & $\begin{array}{l}\text { Stage } \mathrm{CaCO}_{3}{ }^{1} \text {, } \\
\text { other cements }\end{array}$ \\
\hline \multicolumn{9}{|c|}{ Graben fill exposure 9U-21 } \\
\hline Bwk & $5-25$ & n.d. & n.d. & 1f sbk & n.d. & n.d. & n.d. & 0 , es \\
\hline 2Btk1b1 & $25-70$ & n.d. & n.d. & $2 \mathrm{f}-\mathrm{m} \mathrm{sbk}$ & n.d. & n.d. & n.d. & II+ \\
\hline 2Btk3b1 & $90-115$ & n.d. & n.d. & $1-2 \mathrm{f} \mathrm{sbk}$ & n.d. & n.d. & n.d. & $\mathrm{I}, \mathrm{e}$ \\
\hline 2Bwk1b1 & $115-128$ & n.d. & n.d. & $2 \mathrm{f} \mathrm{sbk}$ & n.d. & n.d. & n.d. & II \\
\hline 2Bwk2b1 & $128-148$ & n.d. & n.d. & $2 \mathrm{f} \mathrm{sbk}$ & n.d. & n.d. & n.d. & I \\
\hline 2Bkb1 & $148-168$ & n.d. & n.d. & s.g. & n.d. & n.d. & n.d. & I-II \\
\hline $2 \mathrm{Ckb} 1$ & $168-210$ & n.d. & n.d. & s.g. & n.d. & n.d. & n.d. & $0, \mathrm{e}$ \\
\hline $3 \mathrm{Cb} 2$ & $250-300$ & n.d. & n.d. & single grain & n.d. & n.d. & n.d. & 0 \\
\hline 3Btjkb3 & $300-375$ & n.d. & n.d. & n.d. & n.d. & n.d. & present & I-, es \\
\hline 3Bkb3 & $375-440+$ & n.d. & n.d. & n.d. & n.d. & n.d. & n.d. & I \\
\hline
\end{tabular}

${ }^{1}$ Horizon nomenclature and carbonate stage follow Birkeland (1999) and references therein.

${ }^{2}$ Structure codes: Strength/abundance-1-3. Size-f, fine; m, medium; c, coarse. Shape-sbk, subangular blocky; abk, angular blocky; pl, platy. S.g., single grain.

${ }^{3}$ Dry consistence codes: lo, loose; so, soft; sh, slightly hard; h, hard; vh, very hard.

${ }^{4}$ Wet consistence codes: so, non-sticky; vss, very slightly sticky; ss, slightly sticky; s, sticky; vs, very sticky; po, non-plastic; pss, very slightly plastic; ps, slightly plastic; $p$, plastic; vp, very plastic.

${ }^{5}$ Clay film codes: Abundance-1-3; v1, very few. Thickness $-\mathrm{n}$, thin. Location - br, grain bridge; po, pore filling; pf, ped face; co, grain coat.

${ }^{6}$ Carbonate codes: e, effervesces weakly; es, effervesces strongly; ev, effervesces violently. 


\section{Table 3. Summary data}

This table lists common soils laboratory data and chemical and magnetic properties completed.

[Sample \#: Unique sample number (e.g., 00U-41A/0-10); presented as sample designation (00U-41), transect sampling location (A), and depth in centimeters $(0-10)$.

Hydroscopic moisture factor: A laboratory measure of soil moisture at or near field conditions (driest conditions when values are closest to 1).

$\mathrm{CaCO}_{3} \%$ : Calcium Carbonate percent measured using a Chittick Apparatus (Singer and Janitzky, 1986).

Sand, Silt, Clay \%: Sand, Silt and Clay percents determined using a laser particle size analyzer. For detailed particle size data, see Table 4.

Chemistry completed: Samples for which chemical analysis has been completed. Samples were analyzed by ICP-AES and ICP-MS techniques. Refer to appropriate table for specific chemistry data.

Magnetic properties completed: Samples for which magnetic properties were determined. One column lists the magnetic properties that were determined for the $<2 \mathrm{~mm}$ size fraction and the other column lists the magnetic properties that were determined for the $<63$-mm size fraction. Refer to appropriate table for specific magnetic property data.

n.d.: Not determined]

Table 3a-1. Summary data for 00U-29 transect.

\begin{tabular}{lcccccccc}
\hline \multicolumn{1}{c}{ Sample \# } & $\begin{array}{c}\text { Hygroscopic } \\
\text { moisture factor }\end{array}$ & $\begin{array}{c}\mathbf{C a C O}^{2} \\
(\%)\end{array}$ & $\begin{array}{c}\text { Sand } \\
(\%)\end{array}$ & $\begin{array}{c}\text { Silt } \\
(\%)\end{array}$ & $\begin{array}{c}\text { Clay } \\
(\%)\end{array}$ & $\begin{array}{c}\text { Chemistry } \\
(<2 \mathrm{~mm}) \\
\text { completed }\end{array}$ & $\begin{array}{c}\text { Magnetic } \\
\text { properties }(<2 \mathrm{~mm}) \\
\text { completed } \\
(\text { see Table 6) }\end{array}$ & $\begin{array}{c}\text { Magnetic } \\
\text { properties } \\
\text { (<63-Microns) } \\
\text { completed }\end{array}$ \\
\hline 00U-29A/0-10 & 0.99 & 0.95 & 62.29 & 31.13 & 6.58 & n.d. & MS & n.d. \\
00U-29A/10-30 & 0.99 & 5.08 & 61.93 & 31.44 & 6.63 & n.d. & MS & n.d. \\
00U-29A/30-50 & 0.99 & 4.55 & 55.86 & 34.75 & 9.39 & n.d. & MS & n.d. \\
00U-29B/0-10 & 0.99 & 1.11 & 54.11 & 37.57 & 8.32 & n.d. & MS & n.d. \\
00U-29B/10-30 & 0.99 & 3.22 & 55.47 & 35.64 & 8.89 & n.d. & MS & n.d. \\
00U-29B/30-50 & 0.99 & 4.28 & 52.89 & 37.08 & 10.03 & n.d. & MS & n.d. \\
00U-29C/0-10 & 0.99 & 0.56 & 60.86 & 32.66 & 6.48 & n.d. & MS & n.d. \\
00U-29C/10-30 & 0.99 & 2.85 & 56.89 & 34.82 & 8.30 & n.d. & MS & n.d. \\
00U-29C/30-50 & 0.99 & 3.61 & 60.55 & 31.38 & 8.07 & n.d. & MS & n.d. \\
00U-29D/0-10 & 0.99 & 0.91 & 60.34 & 33.06 & 6.60 & n.d. & MS & n.d. \\
00U-29D/10-30 & 0.99 & 3.74 & 60.27 & 32.02 & 7.71 & n.d. & MS & n.d. \\
00U-29D/30-50 & 0.99 & 4.37 & 58.04 & 34.26 & 7.70 & n.d. & MS & n.d. \\
00U-29E/0-10 & 0.99 & 0.76 & 61.98 & 32.39 & 5.63 & n.d. & MS & n.d. \\
00U-29E/10-30 & 0.99 & 3.45 & 55.89 & 34.79 & 9.32 & n.d. & MS & n.d. \\
00U-29E/30-50 & 0.99 & 3.77 & 53.71 & 36.28 & 10.02 & n.d. & MS & n.d. \\
\hline
\end{tabular}


Table 3a-2. Summary data for 00U-30 transect

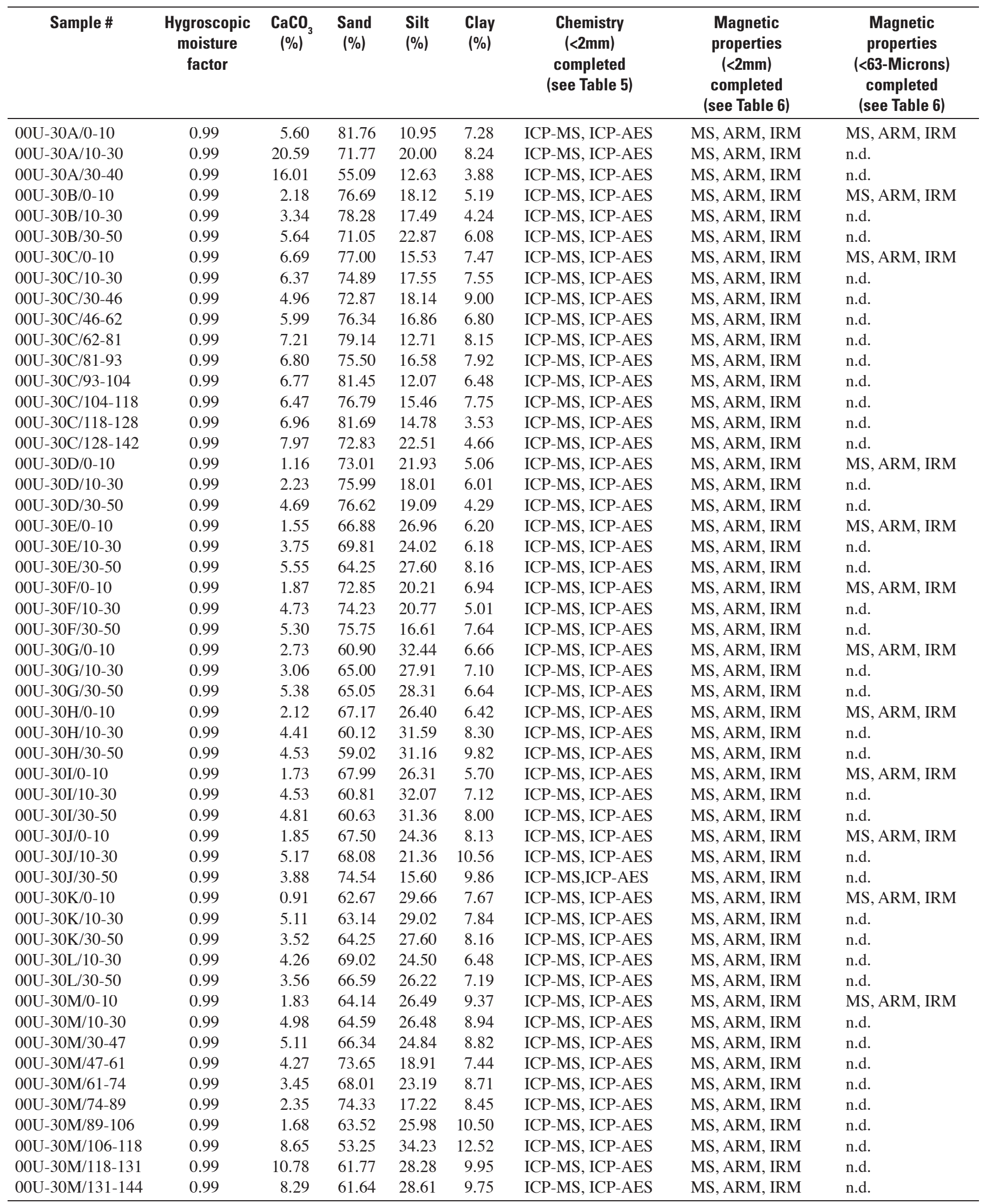


Table 3a-3. Summary data for VP-1 soil pit.

\begin{tabular}{lcccccccc}
\hline Sample \# & $\begin{array}{c}\text { Hygroscopic } \\
\text { moisture } \\
\text { factor }\end{array}$ & $\begin{array}{c}\text { CaC0 } \\
(\%)\end{array}$ & $\begin{array}{c}\text { Sand } \\
(\%)\end{array}$ & $\begin{array}{c}\text { Silt } \\
(\%)\end{array}$ & $\begin{array}{c}\text { Clay } \\
(\%)\end{array}$ & $\begin{array}{c}\text { Chemistry } \\
\text { (<2mm) } \\
\text { completed } \\
\text { (see Table 5) }\end{array}$ & $\begin{array}{c}\text { Magnetic } \\
\text { properties } \\
\text { (<2mm) } \\
\text { completed } \\
\text { (see Table 6) }\end{array}$ & $\begin{array}{c}\text { Magnetic } \\
\text { properties } \\
\text { (<63-Microns) } \\
\text { completed } \\
\text { (see Table 6) }\end{array}$ \\
\hline VP1/0-6 & 0.99 & 4.63 & 70.80 & 23.84 & 5.36 & ICP-AES, ICP-MS & MS, IRM, ARM & MS, IRM, ARM \\
VP1/6-19 & 0.99 & 6.21 & 62.41 & 28.43 & 9.16 & ICP-AES, ICP-MS & MS, IRM, ARM & MS, IRM, ARM \\
VP1/19-30 & 0.99 & 6.32 & 65.64 & 26.09 & 8.27 & ICP-AES, ICP-MS & MS, IRM, ARM & MS, IRM, ARM \\
VP1/30-55 & 0.99 & 7.77 & 75.18 & 18.60 & 6.22 & ICP-AES, ICP-MS & MS, IRM, ARM & MS, IRM, ARM \\
VP1/55-66 & 0.99 & 7.02 & 63.42 & 28.36 & 8.22 & ICP-AES, ICP-MS & MS, IRM, ARM & MS, IRM, ARM \\
VP1/66-78 & 0.99 & 7.02 & 63.97 & 28.20 & 7.83 & ICP-AES, ICP-MS & MS, IRM, ARM & MS, IRM, ARM \\
VP1/80-90 & 0.99 & 14.59 & 80.13 & 15.48 & 4.39 & ICP-AES, ICP-MS & MS, IRM, ARM & MS, IRM, ARM \\
\hline
\end{tabular}

Table 3a-4. Summary data for VP-2 soil pit.

\begin{tabular}{lcccccccc}
\hline Sample \# & $\begin{array}{c}\text { Hygroscopic } \\
\text { moisture } \\
\text { factor }\end{array}$ & $\begin{array}{c}\mathbf{C a C O}_{3} \\
(\%)\end{array}$ & $\begin{array}{c}\text { Sand } \\
(\%)\end{array}$ & $\begin{array}{c}\text { Silt } \\
(\%)\end{array}$ & $\begin{array}{c}\text { Clay } \\
(\%)\end{array}$ & $\begin{array}{c}\text { Chemistry } \\
\text { (<2mm) } \\
\text { completed } \\
\text { (see Table 5) }\end{array}$ & $\begin{array}{c}\text { Magnetic } \\
\text { properties } \\
\text { (<2mm) } \\
\text { completed } \\
\text { (see Table 6) }\end{array}$ & $\begin{array}{c}\text { Magnetic } \\
\text { properties } \\
\text { (<63-Microns) } \\
\text { completed } \\
\text { (see Table 6) }\end{array}$ \\
\hline VP2/0-5 & 0.99 & 5.86 & 64.80 & 29.41 & 5.79 & ICP-AES, ICP-MS & MS, IRM, ARM & MS, IRM, ARM \\
VP2/5-14 & 0.99 & 7.19 & 60.07 & 31.04 & 8.89 & ICP-AES, ICP-MS & MS, IRM, ARM $\quad$ MS, IRM, ARM \\
VP2/14-44 & 0.99 & 7.10 & 65.89 & 26.46 & 7.65 & ICP-AES, ICP-MS & MS, IRM, ARM & MS, IRM, ARM \\
VP2/44-55 & 0.99 & 7.04 & 68.69 & 23.71 & 7.60 & ICP-AES, ICP-MS & MS, IRM, ARM & MS, IRM, ARM \\
VP2/55-85 & 0.99 & 7.67 & 61.42 & 28.55 & 10.03 & ICP-AES, ICP-MS & MS, IRM, ARM & MS, IRM, ARM \\
VP2/110-115 & 0.99 & 10.41 & 68.52 & 23.05 & 8.43 & ICP-AES, ICP-MS & MS, IRM, ARM & MS, IRM, ARM \\
\hline
\end{tabular}


Table 3a-5. Summary data for 8U-13 arroyo exposure.

\begin{tabular}{|c|c|c|c|c|c|c|c|c|}
\hline Sample \# ${ }^{1}$ & $\begin{array}{l}\text { Hygroscopic } \\
\text { moisture } \\
\text { factor }\end{array}$ & $\begin{array}{c}\mathrm{CaCO}_{3} \\
(\%)\end{array}$ & $\begin{array}{c}\text { Sand } \\
(\%)\end{array}$ & $\begin{array}{l}\text { Silt } \\
(\%)\end{array}$ & $\begin{array}{l}\text { Clay } \\
(\%)\end{array}$ & $\begin{array}{l}\text { Chemistry } \\
(<2 \mathrm{~mm}) \\
\text { completed }\end{array}$ & $\begin{array}{c}\text { Magnetic } \\
\text { properties } \\
(<2 \mathrm{~mm}) \\
\text { completed }\end{array}$ & $\begin{array}{c}\text { Magnetic } \\
\text { properties } \\
\text { (<63-Microns) } \\
\text { completed }\end{array}$ \\
\hline $8 \mathrm{U}-13 / 0-5$ & 0.99 & 3.73 & 76.26 & 19.00 & 4.74 & n.d. & n.d. & n.d. \\
\hline $8 U-13 / 35$ & 0.98 & 7.74 & 89.11 & 9.06 & 1.82 & n.d. & n.d. & n.d. \\
\hline $8 \mathrm{U}-13 / 45-65$ & 0.98 & 7.20 & 91.16 & 5.90 & 2.94 & n.d. & n.d. & n.d. \\
\hline $8 U-13 / 90$ & 0.99 & 3.03 & 73.33 & 24.68 & 1.98 & n.d. & n.d. & n.d. \\
\hline $8 \mathrm{U}-13 / 130$ & 0.99 & 9.19 & 76.94 & 20.98 & 2.08 & n.d. & n.d. & n.d. \\
\hline
\end{tabular}

${ }^{1}$ Samples were collected over depth intervals or at discreet depths, in centimeters.

Table 3a-6. Summary data for 8U-14 arroyo exposure

\begin{tabular}{|c|c|c|c|c|c|c|c|c|}
\hline Sample \#1 & $\begin{array}{l}\text { Hygroscopic } \\
\text { moisture } \\
\text { factor }\end{array}$ & $\begin{array}{c}\mathrm{CaCO}_{3} \\
(\%)\end{array}$ & $\begin{array}{c}\text { Sand } \\
(\%)\end{array}$ & $\begin{array}{l}\text { Silt } \\
(\%)\end{array}$ & $\begin{array}{l}\text { Clay } \\
(\%)\end{array}$ & $\begin{array}{c}\text { Chemistry } \\
(<2 \mathrm{~mm}) \\
\text { completed }\end{array}$ & $\begin{array}{c}\text { Magnetic } \\
\text { properties } \\
(<2 \mathrm{~mm}) \\
\text { completed }\end{array}$ & $\begin{array}{c}\text { Magnetic } \\
\text { properties } \\
\text { (<63-Microns } \\
\text { completed }\end{array}$ \\
\hline $8 U-14 / 15$ & 0.99 & 8.84 & 86.64 & 9.32 & 4.04 & n.d. & n.d. & n.d. \\
\hline $8 \mathrm{U}-14 / 60$ & 0.98 & 7.95 & 86.01 & 12.71 & 1.28 & n.d. & n.d. & n.d. \\
\hline $8 U-14 / 75$ & 0.99 & 7.29 & 84.84 & 14.02 & 1.14 & n.d. & n.d. & n.d. \\
\hline
\end{tabular}

${ }^{1}$ Samples were collected at discreet depths, in centimeters. 
Table 3a-7. Summary data for $8 \mathrm{U}-10$ auger hole.

\begin{tabular}{|c|c|c|c|c|c|c|c|c|}
\hline Sample \# & $\begin{array}{l}\text { Hygroscopic } \\
\text { moisture } \\
\text { factor }\end{array}$ & $\begin{array}{c}\mathrm{CaCO}_{3} \\
(\%)\end{array}$ & $\begin{array}{c}\text { Sand } \\
(\%)\end{array}$ & $\begin{array}{l}\text { Silt } \\
(\%)\end{array}$ & $\begin{array}{l}\text { Clay } \\
(\%)\end{array}$ & $\begin{array}{c}\text { Chemistry } \\
\quad(<2 \mathrm{~mm}) \\
\text { completed } \\
\text { (see Table 5) }\end{array}$ & $\begin{array}{c}\text { Magnetic } \\
\text { properties }(<2 \mathrm{~mm}) \\
\text { completed } \\
\text { (see Table 6) }\end{array}$ & $\begin{array}{c}\text { Magnetic } \\
\text { properties } \\
\text { (<63-Microns) } \\
\text { completed }\end{array}$ \\
\hline $8 \mathrm{U}-10 / 0-22$ & 0.98 & 1.17 & 58.75 & 30.36 & 10.89 & ICP-AES, ICP-MS & MS, IRM, ARM & n.d. \\
\hline $8 \mathrm{U}-10 / 22-36$ & 0.97 & 5.17 & 59.63 & 29.15 & 11.23 & ICP-AES, ICP-MS & MS, IRM, ARM & n.d. \\
\hline $8 \mathrm{U}-10 / 36-47$ & 0.99 & 4.19 & 58.35 & 29.08 & 12.57 & ICP-AES, ICP-MS & MS, IRM, ARM & n.d. \\
\hline $8 \mathrm{U}-10 / 47-58$ & 0.96 & 3.69 & 55.63 & 32.08 & 12.28 & ICP-AES, ICP-MS & MS, IRM, ARM & n.d. \\
\hline $8 \mathrm{U}-10 / 58-65$ & 0.97 & 3.56 & 52.27 & 35.49 & 12.24 & ICP-AES, ICP-MS & MS, IRM, ARM & n.d. \\
\hline $8 \mathrm{U}-10 / 65-74$ & 0.98 & 9.28 & 50.93 & 35.39 & 13.68 & ICP-AES, ICP-MS & MS, IRM, ARM & n.d. \\
\hline $8 \mathrm{U}-10 / 74-83$ & 0.98 & 12.06 & 52.80 & 34.73 & 12.48 & ICP-AES, ICP-MS & MS, IRM, ARM & n.d. \\
\hline $8 \mathrm{U}-10 / 83-90$ & 0.97 & 11.43 & 52.87 & 34.48 & 12.65 & ICP-AES, ICP-MS & MS, IRM, ARM & n.d. \\
\hline 8U-10/90-99 & 0.97 & 10.28 & 54.19 & 33.04 & 12.77 & ICP-AES, ICP-MS & MS, IRM, ARM & n.d. \\
\hline 8U-10/99-112 & 0.97 & 10.69 & 60.20 & 27.82 & 11.99 & ICP-AES, ICP-MS & MS, IRM, ARM & n.d. \\
\hline $8 \mathrm{U}-10 / 112-121$ & 0.98 & 13.19 & 62.14 & 25.65 & 12.21 & ICP-AES, ICP-MS & MS, IRM, ARM & n.d. \\
\hline $8 \mathrm{U}-10 / 121-131$ & 0.98 & 12.01 & 63.41 & 24.83 & 11.76 & ICP-AES, ICP-MS & MS, IRM, ARM & n.d. \\
\hline $8 \mathrm{U}-10 / 131-142$ & 0.97 & 10.57 & 63.66 & 24.73 & 11.61 & ICP-AES, ICP-MS & MS, IRM, ARM & n.d. \\
\hline 8U-10142-154 & 0.97 & 11.27 & 64.57 & 26.26 & 9.17 & ICP-AES, ICP-MS & MS, IRM, ARM & n.d. \\
\hline $8 \mathrm{U}-10 / 154-165$ & 0.98 & 7.04 & 57.79 & 32.36 & 9.86 & ICP-AES, ICP-MS & MS, IRM, ARM & n.d. \\
\hline $8 \mathrm{U}-10 / 165-175$ & 0.98 & 7.49 & 56.76 & 30.52 & 12.72 & ICP-AES, ICP-MS & MS, IRM, ARM & n.d. \\
\hline $8 \mathrm{U}-10 / 175-184$ & 0.98 & 6.79 & 63.94 & 23.91 & 12.15 & ICP-AES, ICP-MS & MS, IRM, ARM & n.d. \\
\hline 8U-10/184-197 & 0.97 & 5.13 & 63.89 & 24.03 & 12.08 & ICP-AES, ICP-MS & MS, IRM, ARM & n.d. \\
\hline 8U-10/197-209 & 0.97 & 5.7 & 69.30 & 20.58 & 10.13 & ICP-AES, ICP-MS & MS, IRM, ARM & n.d. \\
\hline $8 \mathrm{U}-10 / 209-220$ & 0.99 & 6.95 & 73.84 & 17.18 & 8.98 & ICP-AES, ICP-MS & MS, IRM, ARM & n.d. \\
\hline $8 \mathrm{U}-10 / 220-230$ & 0.97 & 7.85 & 78.35 & 13.97 & 7.68 & ICP-AES, ICP-MS & MS, IRM, ARM & n.d. \\
\hline $8 \mathrm{U}-10 / 230-241$ & 0.99 & 9.72 & 81.85 & 11.96 & 6.19 & ICP-AES, ICP-MS & MS, IRM, ARM & n.d. \\
\hline $8 \mathrm{U}-10 / 241-249$ & 0.98 & 7.6 & 77.08 & 15.33 & 7.60 & ICP-AES, ICP-MS & MS, IRM, ARM & n.d. \\
\hline $8 \mathrm{U}-10 / 249-261$ & 0.98 & 11.22 & 81.01 & 12.62 & 6.38 & ICP-AES, ICP-MS & MS, IRM, ARM & n.d. \\
\hline $8 \mathrm{U}-10 / 261-270$ & 0.98 & 8.77 & 77.24 & 15.30 & 7.46 & ICP-AES, ICP-MS & MS, IRM, ARM & n.d. \\
\hline $8 \mathrm{U}-10 / 270-274$ & 0.96 & 6.94 & 81.47 & 11.90 & 6.63 & ICP-AES, ICP-MS & MS, IRM, ARM & n.d. \\
\hline $8 \mathrm{U}-10 / 274-284$ & 0.97 & 7.48 & 85.23 & 9.48 & 5.29 & ICP-AES, ICP-MS & MS, IRM, ARM & n.d. \\
\hline
\end{tabular}


Table 3a-8. Summary data for 8U-11 auger hole.

\begin{tabular}{|c|c|c|c|c|c|c|c|c|}
\hline Sample \# & $\begin{array}{l}\text { Hygroscopic } \\
\text { moisture } \\
\text { factor }\end{array}$ & $\begin{array}{c}\mathrm{CaCO}_{3} \\
(\%)\end{array}$ & $\begin{array}{c}\text { Sand } \\
(\%)\end{array}$ & $\begin{array}{l}\text { Silt } \\
(\%)\end{array}$ & $\begin{array}{l}\text { Clay } \\
(\%)\end{array}$ & $\begin{array}{c}\text { Chemistry } \\
(<2 \mathrm{~mm}) \\
\text { completed } \\
\text { (see Table 5) }\end{array}$ & $\begin{array}{c}\text { Magnetic } \\
\text { properties }(<2 \mathrm{~mm}) \\
\text { completed } \\
\text { (see Table 6) } \\
\end{array}$ & $\begin{array}{c}\text { Magnetic } \\
\text { properties } \\
\text { (<63-Microns) } \\
\text { completed }\end{array}$ \\
\hline $8 \mathrm{U}-11 / 0-22$ & 0.98 & 1.16 & 68.63 & 22.28 & 9.09 & ICP-AES, ICP-MS & MS, IRM, ARM & n.d. \\
\hline $8 \mathrm{U}-11 / 22-38$ & 0.98 & 4.87 & 73.06 & 17.27 & 9.67 & ICP-AES, ICP-MS & MS, IRM, ARM & n.d. \\
\hline $8 \mathrm{U}-11 / 38-50$ & 0.98 & 3.52 & 81.32 & 13.88 & 4.80 & ICP-AES, ICP-MS & MS, IRM, ARM & n.d. \\
\hline $8 \mathrm{U}-11 / 50-62$ & 0.98 & 2.6 & 45.88 & 35.01 & 19.11 & ICP-AES, ICP-MS & MS, IRM, ARM & n.d. \\
\hline $8 \mathrm{U}-11 / 62-71$ & 0.98 & 5.49 & 64.86 & 25.89 & 9.25 & ICP-AES, ICP-MS & MS, IRM, ARM & n.d. \\
\hline $8 \mathrm{U}-11 / 71-86$ & 0.98 & 11.16 & 66.67 & 24.47 & 8.86 & ICP-AES, ICP-MS & MS, IRM, ARM & n.d. \\
\hline 8U-11/86-98 & 0.98 & 11.6 & 73.03 & 18.53 & 8.44 & ICP-AES, ICP-MS & MS, IRM, ARM & n.d. \\
\hline 8U-11/98-109 & 0.96 & 10.82 & 73.64 & 16.47 & 9.89 & ICP-AES, ICP-MS & MS, IRM, ARM & n.d. \\
\hline $8 \mathrm{U}-11 / 109-118$ & 0.98 & 10.42 & 72.24 & 18.96 & 8.80 & ICP-AES, ICP-MS & MS, IRM, ARM & n.d. \\
\hline $8 \mathrm{U}-11 / 118-131$ & 0.98 & 12.19 & 80.64 & 15.91 & 3.46 & ICP-AES, ICP-MS & MS, IRM, ARM & n.d. \\
\hline 8U-11/131-139 & 0.97 & 6.96 & 83.21 & 11.80 & 4.98 & ICP-AES, ICP-MS & MS, IRM, ARM & n.d. \\
\hline $8 \mathrm{U}-11 / 139-151$ & 0.98 & 6.02 & 85.43 & 10.46 & 4.10 & ICP-AES, ICP-MS & MS, IRM, ARM & n.d. \\
\hline $8 \mathrm{U}-11 / 151-161$ & 0.98 & 4.91 & 77.95 & 11.22 & 10.84 & ICP-AES, ICP-MS & MS, IRM, ARM & n.d. \\
\hline $8 \mathrm{U}-11 / 161-172$ & 0.97 & 4.58 & 76.50 & 18.94 & 4.57 & ICP-AES, ICP-MS & MS, IRM, ARM & n.d. \\
\hline $8 \mathrm{U}-11 / 172-183$ & 0.97 & 4.14 & 72.16 & 19.96 & 7.88 & ICP-AES, ICP-MS & MS, IRM, ARM & n.d. \\
\hline 8U-11/183-196 & 0.98 & 3.96 & 71.82 & 18.46 & 9.72 & ICP-AES, ICP-MS & MS, IRM, ARM & n.d. \\
\hline $8 \mathrm{U}-11 / 196-210$ & 0.98 & 4.81 & 72.68 & 20.25 & 7.07 & ICP-AES, ICP-MS & MS, IRM, ARM & n.d. \\
\hline $8 \mathrm{U}-11 / 210-224$ & 0.98 & 6.44 & 73.65 & 14.44 & 11.91 & ICP-AES, ICP-MS & MS, IRM, ARM & n.d. \\
\hline $8 \mathrm{U}-11 / 224-237$ & 0.96 & 5.28 & 74.83 & 18.66 & 6.51 & ICP-AES, ICP-MS & MS, IRM, ARM & n.d. \\
\hline $8 \mathrm{U}-11 / 237-251$ & 0.98 & 4.98 & 72.57 & 18.76 & 8.67 & ICP-AES, ICP-MS & MS, IRM, ARM & n.d. \\
\hline $8 \mathrm{U}-11 / 251-265$ & 0.98 & 3.35 & 75.81 & 15.24 & 8.96 & ICP-AES, ICP-MS & MS, IRM, ARM & n.d. \\
\hline $8 \mathrm{U}-11 / 265-278$ & 0.98 & 4.35 & 76.05 & 16.51 & 7.43 & ICP-AES, ICP-MS & MS, IRM, ARM & n.d. \\
\hline
\end{tabular}


Table 3a-9. Summary data for $8 \mathrm{U}-12$ auger hole.

\begin{tabular}{|c|c|c|c|c|c|c|c|c|}
\hline Sample \# & $\begin{array}{l}\text { Hygroscopic } \\
\text { moisture } \\
\text { factor }\end{array}$ & $\begin{array}{c}\mathrm{CaCO}_{3} \\
(\%)\end{array}$ & $\begin{array}{c}\text { Sand } \\
(\%)\end{array}$ & $\begin{array}{l}\text { Silt } \\
(\%)\end{array}$ & $\begin{array}{c}\text { Clay } \\
(\%)\end{array}$ & $\begin{array}{l}\text { Chemistry } \\
(<2 \mathrm{~mm}) \\
\text { completed }\end{array}$ & $\begin{array}{c}\text { Magnetic } \\
\text { properties (<2mm) } \\
\text { completed } \\
\text { (see Table 6) }\end{array}$ & $\begin{array}{c}\text { Magnetic } \\
\text { properties } \\
\text { (<63-Microns) } \\
\text { completed } \\
\end{array}$ \\
\hline $8 \mathrm{U}-12 / 0-13$ & 0.99 & 2.16 & 88.87 & 9.48 & 1.66 & n.d. & MS, IRM, ARM & n.d. \\
\hline $8 \mathrm{U}-12 / 13-27$ & 0.99 & 5.17 & 87.16 & 11.27 & 1.58 & n.d. & MS, IRM, ARM & n.d. \\
\hline $8 \mathrm{U}-12 / 27-37$ & 0.98 & 5.61 & 67.49 & 27.13 & 5.39 & n.d. & MS, IRM, ARM & n.d. \\
\hline $8 \mathrm{U}-12 / 48-60$ & 0.98 & 4.91 & 61.60 & 25.90 & 12.50 & n.d. & MS, IRM, ARM & n.d. \\
\hline $8 \mathrm{U}-12 / 60-71$ & 0.99 & 3.77 & 91.47 & 7.48 & 1.05 & n.d. & MS, IRM, ARM & n.d. \\
\hline $8 \mathrm{U}-12 / 71-83$ & 0.99 & 7.5 & 89.99 & 8.92 & 1.08 & n.d. & MS, IRM, ARM & n.d. \\
\hline $8 U-12 / 83-96$ & 0.99 & 11.33 & 51.24 & 34.93 & 13.84 & n.d. & MS, IRM, ARM & n.d. \\
\hline $8 U-12 / 128-137$ & 0.99 & 10.19 & 82.79 & 13.38 & 3.83 & n.d. & MS, IRM, ARM & n.d. \\
\hline $8 U-12 / 137-147$ & 0.99 & 8.05 & 77.29 & 15.23 & 7.49 & n.d. & MS, IRM, ARM & n.d. \\
\hline $8 U-12 / 147-158$ & 0.99 & 6.37 & 82.23 & 14.66 & 3.12 & n.d. & MS, IRM, ARM & n.d. \\
\hline $8 U-12 / 158-170$ & 0.99 & 6.79 & 88.17 & 9.51 & 2.32 & n.d. & MS, IRM, ARM & n.d. \\
\hline $8 U-12 / 170-180$ & 0.99 & 6.05 & 87.38 & 9.88 & 2.74 & n.d. & MS, IRM, ARM & n.d. \\
\hline $8 U-12 / 180-191$ & 0.99 & 6.65 & 72.24 & 17.81 & 9.95 & n.d. & MS, IRM, ARM & n.d. \\
\hline $8 U-12 / 191-203$ & 0.99 & 5.89 & 74.17 & 19.08 & 6.75 & n.d. & MS, IRM, ARM & n.d. \\
\hline $8 U-12 / 203-216$ & 0.99 & 6.66 & 63.38 & 20.88 & 15.74 & n.d. & MS, IRM, ARM & n.d. \\
\hline $8 U-12 / 279-290$ & 0.99 & 13.09 & 73.23 & 19.03 & 7.74 & n.d. & MS, IRM, ARM & n.d. \\
\hline $8 U-12 / 290-299$ & 0.99 & 9.1 & 74.67 & 19.29 & 6.04 & n.d. & MS, IRM, ARM & n.d. \\
\hline $8 U-12 / 299-310$ & 0.99 & 7.65 & 75.06 & 11.05 & 13.89 & n.d. & MS, IRM, ARM & n.d. \\
\hline $8 \mathrm{U}-12 / 310-317$ & 0.99 & 7.74 & 74.99 & 13.34 & 11.67 & n.d. & MS, IRM, ARM & n.d. \\
\hline
\end{tabular}


Table 3a-10. Summary data for 9U-22 auger hole.

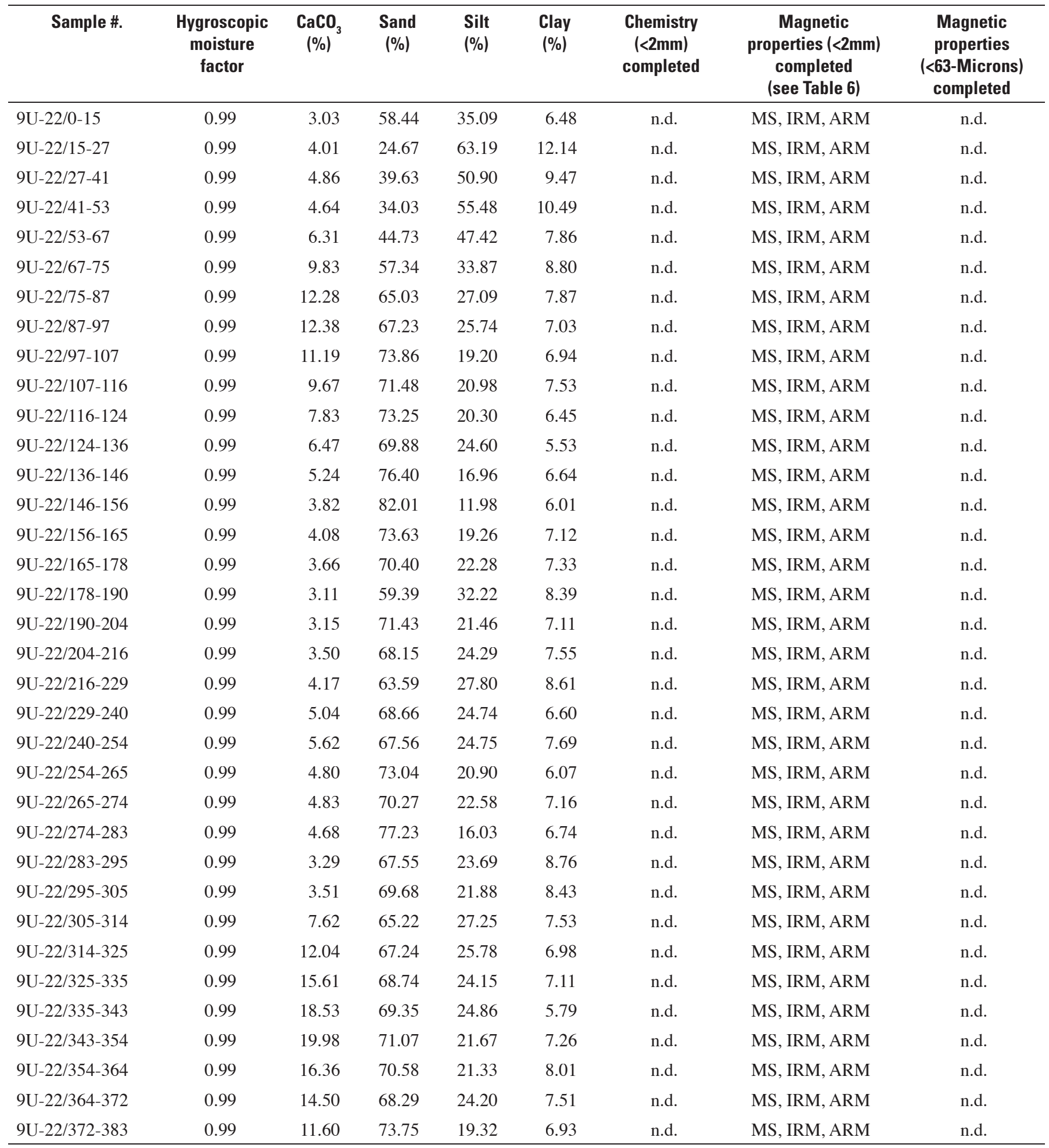


Table 3a-11. Summary data for 9U-23 auger hole.

\begin{tabular}{|c|c|c|c|c|c|c|c|c|}
\hline Sample \# & $\begin{array}{l}\text { Hygroscopic } \\
\text { moisture } \\
\text { factor }\end{array}$ & $\begin{array}{c}\mathrm{CaCO}_{3} \\
(\%)\end{array}$ & $\begin{array}{l}\text { Sand } \\
(\%)\end{array}$ & $\begin{array}{l}\text { Silt } \\
(\%)\end{array}$ & $\begin{array}{l}\text { Clay } \\
(\%)\end{array}$ & $\begin{array}{c}\text { Chemistry } \\
(<2 \mathrm{~mm}) \\
\text { completed }\end{array}$ & $\begin{array}{c}\text { Magnetic } \\
\text { properties }(<2 \mathrm{~mm}) \\
\text { completed } \\
\text { (see Table 6) }\end{array}$ & $\begin{array}{c}\text { Magnetic } \\
\text { properties } \\
\text { (<63-Microns) } \\
\text { completed }\end{array}$ \\
\hline $9 \mathrm{U}-23 / 0-22$ & 0.99 & 2.14 & 76.40 & 19.09 & 4.51 & n.d. & MS, IRM, ARM & n.d. \\
\hline $9 \mathrm{U}-23 / 22-23$ & 0.99 & 2.63 & 70.95 & 24.44 & 4.62 & n.d. & MS, IRM, ARM & n.d. \\
\hline 9U-23/33-46 & 0.99 & 5.04 & 74.14 & 20.73 & 5.14 & n.d. & MS, IRM, ARM & n.d. \\
\hline 9U-23/46-58 & 0.99 & 6.03 & 72.18 & 22.72 & 5.09 & n.d. & MS, IRM, ARM & n.d. \\
\hline 9U-23/58-67 & 0.99 & 10.58 & 69.48 & 25.59 & 4.93 & n.d. & MS, IRM, ARM & n.d. \\
\hline $9 \mathrm{U}-23 / 67-75$ & 0.99 & 13.49 & 69.62 & 25.87 & 4.51 & n.d. & MS, IRM, ARM & n.d. \\
\hline $9 \mathrm{U}-23 / 75-83$ & 0.99 & 14.03 & 72.66 & 22.27 & 5.06 & n.d. & MS, IRM, ARM & n.d. \\
\hline 9U-23/83-96 & 0.99 & 13.63 & 72.88 & 21.50 & 5.62 & n.d. & MS, IRM, ARM & n.d. \\
\hline 9U-23/96-105 & 0.99 & 12.58 & 76.78 & 19.05 & 4.17 & n.d. & MS, IRM, ARM & n.d. \\
\hline $9 \mathrm{U}-23 / 105-115$ & 0.99 & 10.42 & 79.13 & 16.94 & 3.93 & n.d. & MS, IRM, ARM & n.d. \\
\hline $9 \mathrm{U}-23 / 115-125$ & 0.99 & 7.97 & 77.23 & 17.63 & 5.14 & n.d. & MS, IRM, ARM & n.d. \\
\hline $9 U-23 / 125-138$ & 0.99 & 5.55 & 77.93 & 17.37 & 4.71 & n.d. & MS, IRM, ARM & n.d. \\
\hline $9 U-23 / 138-148$ & 0.99 & 6.27 & 72.92 & 19.65 & 7.44 & n.d. & MS, IRM, ARM & n.d. \\
\hline 9U-23/148-159 & 0.99 & 4.41 & 74.44 & 18.47 & 7.09 & n.d. & MS, IRM, ARM & n.d. \\
\hline $9 U-23 / 159-171$ & 0.99 & 3.10 & 75.17 & 16.27 & 8.56 & n.d. & MS, IRM, ARM & n.d. \\
\hline $9 \mathrm{U}-23 / 171-182$ & 0.99 & 4.37 & 68.66 & 25.65 & 5.69 & n.d. & MS, IRM, ARM & n.d. \\
\hline $9 \mathrm{U}-23 / 182-192$ & 0.99 & 3.21 & 70.39 & 23.96 & 5.65 & n.d. & MS, IRM, ARM & n.d. \\
\hline 9U-23/192-201 & 0.99 & 4.65 & 71.92 & 23.43 & 4.65 & n.d. & MS, IRM, ARM & n.d. \\
\hline $9 U-23 / 201-208$ & 0.99 & 9.37 & 75.41 & 19.09 & 5.51 & n.d. & MS, IRM, ARM & n.d. \\
\hline $9 \mathrm{U}-23 / 208-218$ & 0.99 & 10.35 & 75.26 & 19.97 & 4.77 & n.d. & MS, IRM, ARM & n.d. \\
\hline $9 \mathrm{U}-23 / 218-228$ & 0.99 & 12.36 & 78.17 & 16.32 & 5.51 & n.d. & MS, IRM, ARM & n.d. \\
\hline $9 \mathrm{U}-23 / 228-236$ & 0.99 & 12.06 & 78.46 & 16.53 & 5.01 & n.d. & MS, IRM, ARM & n.d. \\
\hline $9 \mathrm{U}-23 / 236-248$ & 0.99 & 5.64 & 75.68 & 17.68 & 6.64 & n.d. & MS, IRM, ARM & n.d. \\
\hline $9 U-23 / 248-260$ & 0.99 & 8.44 & 78.36 & 17.01 & 4.64 & n.d. & MS, IRM, ARM & n.d. \\
\hline $9 U-23 / 260-270$ & 0.99 & 7.19 & 77.94 & 15.40 & 6.67 & n.d. & MS, IRM, ARM & n.d. \\
\hline $9 \mathrm{U}-23 / 270-280$ & 0.99 & 5.73 & 77.74 & 17.18 & 5.08 & n.d. & MS, IRM, ARM & n.d. \\
\hline $9 U-23 / 280-290$ & 0.99 & 10.41 & 77.41 & 15.75 & 6.84 & n.d. & MS, IRM, ARM & n.d. \\
\hline 9U-23/290-298 & 0.99 & 11.11 & 82.89 & 12.75 & 4.36 & n.d. & MS, IRM, ARM & n.d. \\
\hline
\end{tabular}


Table 3a-12. Summary data for 00U-27 auger hole.

\begin{tabular}{|c|c|c|c|c|c|c|c|c|}
\hline Sample \# & $\begin{array}{l}\text { Hygroscopic } \\
\text { moisture } \\
\text { factor }\end{array}$ & $\begin{array}{c}\mathrm{CaCO}_{3} \\
(\%)\end{array}$ & $\begin{array}{c}\text { Sand } \\
(\%)\end{array}$ & $\begin{array}{l}\text { Silt } \\
(\%)\end{array}$ & $\begin{array}{l}\text { Clay } \\
(\%)\end{array}$ & $\begin{array}{c}\text { Chemistry } \\
(<2 \mathrm{~mm}) \\
\text { completed } \\
\text { (see Table 5) }\end{array}$ & $\begin{array}{c}\text { Magnetic } \\
\text { properties } \\
(<2 \mathrm{~mm}) \\
\text { completed }\end{array}$ & $\begin{array}{c}\text { Magnetic } \\
\text { properties } \\
\text { (<63-Microns) } \\
\text { completed }\end{array}$ \\
\hline 00U-27/0-10 & 0.99 & 1.24 & 64.20 & 29.24 & 6.56 & ICP-AES, ICP-MS & n.d. & n.d. \\
\hline 00U-27/10-30 & 0.99 & 3.91 & 83.79 & 11.45 & 4.76 & ICP-AES, ICP-MS & n.d. & n.d. \\
\hline 00U-27/30-50 & 0.99 & 4.88 & 63.34 & 28.44 & 8.22 & ICP-AES, ICP-MS & n.d. & n.d. \\
\hline 00U-27/50-65 & 0.99 & 4.25 & 52.90 & 38.14 & 8.96 & ICP-AES, ICP-MS & n.d. & n.d. \\
\hline 00U-27/65-84 & 0.99 & 3.33 & 51.66 & 38.48 & 9.85 & ICP-AES, ICP-MS & n.d. & n.d. \\
\hline 00U-27/84-100 & 0.99 & 2.30 & 51.86 & 38.51 & 9.63 & ICP-AES, ICP-MS & n.d. & n.d. \\
\hline 00U-27/100-116 & 0.99 & 2.26 & 51.05 & 38.12 & 10.83 & ICP-AES, ICP-MS & n.d. & n.d. \\
\hline 00U-27/116-128 & 0.99 & 4.49 & 54.09 & 36.14 & 9.77 & ICP-AES, ICP-MS & n.d. & n.d. \\
\hline 00U-27/128-141 & 0.99 & 10.52 & 61.12 & 31.23 & 7.65 & ICP-AES, ICP-MS & n.d. & n.d. \\
\hline 00U-27/141-156 & 0.99 & 9.62 & 66.23 & 26.58 & 7.19 & ICP-AES, ICP-MS & n.d. & n.d. \\
\hline 00U-27/156-168 & 0.99 & 11.01 & 68.18 & 24.88 & 6.93 & ICP-AES, ICP-MS & n.d. & n.d. \\
\hline 00U-27/168-179 & 0.99 & 11.60 & 68.03 & 24.90 & 7.07 & ICP-AES, ICP-MS & n.d. & n.d. \\
\hline 00U-27/179-194 & 0.99 & 11.24 & 70.29 & 22.65 & 7.07 & ICP-AES, ICP-MS & n.d. & n.d. \\
\hline 00U-27/194-208 & 0.99 & 10.06 & 73.00 & 20.57 & 6.43 & ICP-AES, ICP-MS & n.d. & n.d. \\
\hline 00U-27/208-221 & 0.99 & 9.90 & 72.96 & 19.82 & 7.22 & ICP-AES, ICP-MS & n.d. & n.d. \\
\hline 00U-27/221-233 & 0.99 & 10.41 & 73.06 & 19.92 & 7.03 & ICP-AES, ICP-MS & n.d. & n.d. \\
\hline 00U-27/233-242 & 0.99 & 9.98 & 73.96 & 19.12 & 6.92 & ICP-AES, ICP-MS & n.d. & n.d. \\
\hline 00U-27/242-252 & 0.99 & 10.95 & 75.21 & 18.02 & 6.77 & ICP-AES, ICP-MS & n.d. & n.d. \\
\hline 00U-27/242-265 & 0.99 & 9.27 & 73.22 & 18.83 & 7.95 & ICP-AES, ICP-MS & n.d. & n.d. \\
\hline 00U-27/265-274 & 0.99 & 8.07 & 76.41 & 17.18 & 6.42 & ICP-AES, ICP-MS & n.d. & n.d. \\
\hline 00U-27/274-283 & 0.99 & 7.28 & 77.10 & 16.66 & 6.24 & ICP-AES, ICP-MS & n.d. & n.d. \\
\hline 00U-27/283-291 & 0.99 & 6.14 & 68.40 & 23.42 & 8.18 & ICP-AES, ICP-MS & n.d. & n.d. \\
\hline 00U-27/291-301 & 0.99 & 8.16 & 61.47 & 26.87 & 11.67 & ICP-AES, ICP-MS & n.d. & n.d. \\
\hline 00U-27/301-313 & 0.99 & 9.07 & 59.22 & 26.86 & 13.92 & ICP-AES, ICP-MS & n.d. & n.d. \\
\hline 00U-27/313-324 & 0.99 & 6.41 & 71.54 & 20.37 & 8.09 & ICP-AES, ICP-MS & n.d. & n.d. \\
\hline
\end{tabular}


Table 3a-13. Summary data for 00U-31 auger hole.

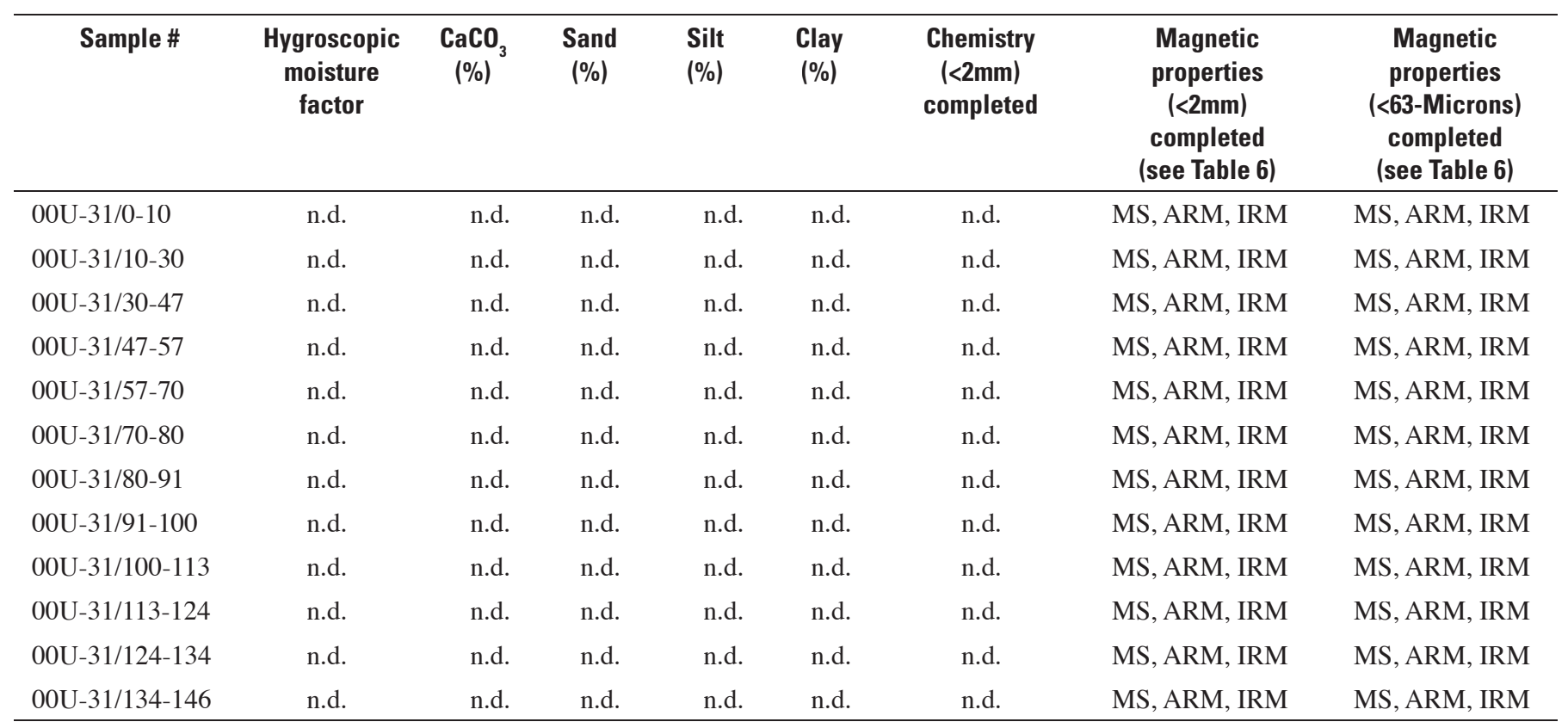

Table 3a-14. Summary data for 00U-31 auger hole.

\begin{tabular}{|c|c|c|c|c|c|c|c|c|}
\hline Sample \# & $\begin{array}{l}\text { Hygroscopic } \\
\text { moisture } \\
\text { factor }\end{array}$ & $\begin{array}{c}\mathrm{CaCO}_{3} \\
(\%)\end{array}$ & $\begin{array}{c}\text { Sand } \\
(\%)\end{array}$ & $\begin{array}{l}\text { Silt } \\
(\%)\end{array}$ & $\begin{array}{l}\text { Clay } \\
(\%)\end{array}$ & $\begin{array}{l}\text { Chemistry } \\
(<2 \mathrm{~mm}) \\
\text { completed }\end{array}$ & $\begin{array}{c}\text { Magnetic } \\
\text { properties } \\
\text { (<2mm) } \\
\text { pompleted } \\
\text { (see Table 6) }\end{array}$ & $\begin{array}{c}\text { Magnetic } \\
\text { properties } \\
\text { (<63-Microns) } \\
\text { completed } \\
\text { (see Table 6) }\end{array}$ \\
\hline 00U-33/0-10 & n.d. & n.d. & n.d. & n.d. & n.d. & n.d. & MS, ARM, IRM & MS, ARM, IRM \\
\hline 00U-33/10-30 & n.d. & n.d. & n.d. & n.d. & n.d. & n.d. & MS, ARM, IRM & MS, ARM, IRM \\
\hline 00U-33/47-61 & n.d. & n.d. & n.d. & n.d. & n.d. & n.d. & MS, ARM, IRM & MS, ARM, IRM \\
\hline 00U-33/61-73 & n.d. & n.d. & n.d. & n.d. & n.d. & n.d. & MS, ARM, IRM & MS, ARM, IRM \\
\hline 00U-33/73-89 & n.d. & n.d. & n.d. & n.d. & n.d. & n.d. & MS, ARM, IRM & MS, ARM, IRM \\
\hline
\end{tabular}


Table 3b-1. Summary data for 01U-1 transect.

\begin{tabular}{|c|c|c|c|c|c|c|c|c|}
\hline Sample \# & $\begin{array}{l}\text { Hygroscopic } \\
\text { moisture } \\
\text { factor }\end{array}$ & $\begin{array}{c}\mathrm{CaCO}_{3} \\
(\%)\end{array}$ & $\begin{array}{l}\text { Sand } \\
(\%)\end{array}$ & $\begin{array}{l}\text { Silt } \\
(\%)\end{array}$ & $\begin{array}{l}\text { Clay } \\
(\%)\end{array}$ & $\begin{array}{c}\text { Chemistry }(<2 \mathrm{~mm} \text { ) } \\
\text { completed } \\
\text { (see Table 5) }\end{array}$ & $\begin{array}{c}\text { Magnetic } \\
\text { properties } \\
\text { (<2mm) } \\
\text { completed } \\
\text { (see Table 6) }\end{array}$ & $\begin{array}{c}\text { Magnetic } \\
\text { properties } \\
\text { (<63-Microns) } \\
\text { completed } \\
\text { (see Table 6) }\end{array}$ \\
\hline 01U-1A/0-10 & 0.99 & 9.49 & 69.89 & 25.58 & 4.52 & ICP-AES, ICP-MS & MS, IRM, ARM & MS, IRM, ARM \\
\hline 01U-1A/10-30 & 0.99 & 8.44 & 73.93 & 21.81 & 4.26 & ICP-AES, ICP-MS & MS, IRM, ARM & MS, IRM, ARM \\
\hline 01U-1A/30-50 & 0.99 & 8.60 & 72.91 & 22.50 & 4.58 & ICP-AES, ICP-MS & MS, IRM, ARM & MS, IRM, ARM \\
\hline $01 \mathrm{U}-1 \mathrm{~B} / 0-10$ & 0.99 & 11.62 & 59.12 & 35.19 & 5.69 & ICP-AES, ICP-MS & MS, IRM, ARM & MS, IRM, ARM \\
\hline 01U-1B/10-30 & 0.99 & 8.80 & 73.51 & 21.72 & 4.77 & ICP-AES, ICP-MS & MS, IRM, ARM & MS, IRM, ARM \\
\hline 01U-1B/30-50 & 0.99 & 6.83 & 75.82 & 19.90 & 4.28 & ICP-AES, ICP-MS & MS, IRM, ARM & MS, IRM, ARM \\
\hline 01U-1C/0-10 & 0.99 & 9.50 & 69.55 & 25.50 & 4.95 & ICP-AES, ICP-MS & MS, IRM, ARM & MS, IRM, ARM \\
\hline 01U-1C/10-30 & 0.99 & 9.93 & 71.61 & 23.41 & 4.98 & ICP-AES, ICP-MS & MS, IRM, ARM & MS, IRM, ARM \\
\hline 01U-1C/30-50 & 0.99 & 12.81 & 81.64 & 11.90 & 6.46 & ICP-AES, ICP-MS & MS, IRM, ARM & MS, IRM, ARM \\
\hline $01 \mathrm{U}-1 \mathrm{D} / 0-10$ & 0.99 & 4.55 & 65.91 & 27.41 & 6.69 & ICP-AES, ICP-MS & MS, IRM, ARM & MS, IRM, ARM \\
\hline 01U-1D/10-30 & 0.99 & 9.85 & 75.56 & 20.80 & 3.65 & ICP-AES, ICP-MS & MS, IRM, ARM & MS, IRM, ARM \\
\hline 01U-1D/30-50 & 0.99 & 8.42 & 74.29 & 21.34 & 4.37 & ICP-AES, ICP-MS & MS, IRM, ARM & MS, IRM, ARM \\
\hline $01 \mathrm{U}-1 \mathrm{E} / 0-10$ & 0.99 & 7.12 & 78.97 & 18.03 & 3.00 & ICP-AES, ICP-MS & MS, IRM, ARM & MS, IRM, ARM \\
\hline 01U-1E/10-30 & 0.99 & 10.03 & 81.06 & 15.27 & 3.67 & ICP-AES, ICP-MS & MS, IRM, ARM & MS, IRM, ARM \\
\hline 01U-1E/30-50 & 0.99 & 26.99 & 88.23 & 9.29 & 2.48 & ICP-AES, ICP-MS & MS, IRM, ARM & MS, IRM, ARM \\
\hline 01U-1F/0-10 & 0.99 & 8.77 & 78.25 & 18.34 & 3.41 & ICP-AES, ICP-MS & MS, IRM, ARM & MS, IRM, ARM \\
\hline 01U-1F/10-30 & 0.99 & 18.22 & 83.40 & 13.69 & 2.91 & ICP-AES, ICP-MS & MS, IRM, ARM & MS, IRM, ARM \\
\hline $01 \mathrm{U}-1 \mathrm{~F} / 30-50$ & 0.99 & 24.04 & 81.54 & 15.41 & 3.05 & ICP-AES, ICP-MS & MS, IRM, ARM & MS, IRM, ARM \\
\hline
\end{tabular}

Table 3b-2. Summary data for the 04U transect.

\begin{tabular}{lcrrrrrrc}
\hline \multicolumn{1}{c}{ Sample \# } & $\begin{array}{c}\text { Hygroscopic } \\
\text { moisture } \\
\text { factor }\end{array}$ & \multicolumn{1}{c|}{$\begin{array}{c}\mathbf{C a C O}_{3} \\
(\%)\end{array}$} & $\begin{array}{c}\text { Sand } \\
(\%)\end{array}$ & \multicolumn{1}{c}{$\begin{array}{c}\text { Silt } \\
(\%)\end{array}$} & $\begin{array}{c}\text { Clay } \\
(\%)\end{array}$ & $\begin{array}{c}\text { Chemistry } \\
(<2 \mathrm{~mm}) \\
\text { completed } \\
\text { (see Table 5) }\end{array}$ & $\begin{array}{c}\text { Magnetic } \\
\text { properties (<2mm) } \\
\text { completed } \\
\text { (see Table 6) }\end{array}$ & $\begin{array}{c}\text { Magnetic } \\
\text { properties } \\
\text { (<63-Microns) } \\
\text { completed }\end{array}$ \\
\hline 04U-21A/0-10 & 0.99 & 11.39 & 51.59 & 37.60 & 10.81 & ICP-AES, ICP-MS & MS, IRM, ARM & n.d. \\
04U-21B/10-30 & 0.99 & 9.81 & 58.69 & 33.32 & 8.00 & ICP-AES, ICP-MS & MS, IRM, ARM & n.d. \\
04U-22A/0-10 & 0.99 & 10.66 & 53.12 & 33.98 & 12.91 & ICP-AES, ICP-MS & MS, IRM, ARM & n.d. \\
04U-22B/10-30 & 0.99 & 9.76 & 69.69 & 21.75 & 8.56 & ICP-AES, ICP-MS & MS, IRM, ARM & n.d. \\
04U-23A/0-10 & 0.99 & 12.73 & 42.87 & 43.07 & 14.06 & ICP-AES, ICP-MS & MS, IRM, ARM & n.d. \\
04U-23B/10-30 & 0.99 & 13.66 & 44.94 & 41.19 & 13.87 & ICP-AES, ICP-MS & MS, IRM, ARM & n.d. \\
04U-24A/0-10 & 0.99 & 12.59 & 44.77 & 42.18 & 12.42 & ICP-AES, ICP-MS & MS, IRM, ARM & n.d. \\
04U-24B/10-30 & 0.99 & 8.44 & 74.11 & 19.48 & 6.41 & ICP-AES, ICP-MS & MS, IRM, ARM & n.d. \\
\hline
\end{tabular}


Table 3c-1. Summary data for auger hole 00U-38. This auger hole anchors the 00U-39 transect.

\begin{tabular}{lcccccccc}
\hline \multicolumn{1}{c}{ Sample \# } & $\begin{array}{c}\text { Hygroscopic } \\
\text { Moisture } \\
\text { Factor }\end{array}$ & $\begin{array}{c}\mathbf{C a C O}_{3} \\
(\%)\end{array}$ & $\begin{array}{c}\text { Sand } \\
(\%)\end{array}$ & $\begin{array}{c}\text { Silt } \\
(\%)\end{array}$ & $\begin{array}{c}\text { Clay } \\
(\%)\end{array}$ & $\begin{array}{c}\text { Chemistry } \\
\text { (<2mm) } \\
\text { completed } \\
\text { (see Table 5) }\end{array}$ & $\begin{array}{c}\text { Magnetic } \\
\text { properties (<2mm) } \\
\text { completed } \\
\text { (see Table 6) }\end{array}$ & $\begin{array}{c}\text { Magnetic } \\
\text { properties } \\
\text { (<63-Microns) } \\
\text { completed }\end{array}$ \\
\hline 00U-38/0-10 & 0.99 & 3.72 & 83.43 & 10.03 & 6.55 & ICP-MS, ICP-AES & MS, IRM, ARM & n.d. \\
00U-38/10-30 & 0.99 & 4.44 & 87.51 & 6.22 & 6.27 & ICP-MS, ICP-AES & MS, IRM, ARM & n.d. \\
00U-38/30-44 & 0.99 & 4.44 & 84.54 & 8.28 & 7.17 & ICP-MS, ICP-AES & MS, IRM, ARM & n.d. \\
00U-38/44-57 & 0.99 & 3.87 & 74.13 & 18.28 & 7.60 & ICP-MS, ICP-AES & MS, IRM, ARM & n.d. \\
00U-38/57-74 & 0.99 & 3.50 & 81.55 & 11.74 & 6.72 & ICP-MS, ICP-AES & MS, IRM, ARM & n.d. \\
00U-38/74-96 & 0.99 & 5.29 & 81.90 & 11.52 & 6.58 & ICP-MS, ICP-AES & MS, IRM, ARM & n.d. \\
00U-38/96-115 & 0.99 & 6.35 & 79.08 & 11.67 & 9.25 & ICP-MS, ICP-AES & MS, IRM, ARM & n.d. \\
00U-38/115-140 & 0.99 & 6.02 & 82.64 & 14.29 & 3.07 & ICP-MS, ICP-AES & MS, IRM, ARM & n.d. \\
00U-38/140-156 & 0.99 & 7.35 & 82.57 & 11.11 & 6.33 & ICP-MS, ICP-AES & MS, IRM, ARM & n.d. \\
00U-38/156-185 & 0.99 & 6.61 & 91.58 & 5.64 & 2.78 & ICP-MS, ICP-AES & MS, IRM, ARM & n.d. \\
00U-38/185-207 & 0.99 & 7.62 & 92.43 & 5.22 & 2.35 & ICP-MS, ICP-AES & MS, IRM, ARM & n.d. \\
00U-38/207-229 & 0.99 & 6.51 & 92.85 & 5.50 & 1.65 & ICP-MS, ICP-AES & MS, IRM, ARM & n.d. \\
00U-38/229-242 & 0.99 & 7.95 & 92.39 & 5.72 & 1.90 & ICP-MS, ICP-AES & MS, IRM, ARM & n.d. \\
\hline
\end{tabular}


Table 3c-2. Summary data for transect 00U-39.

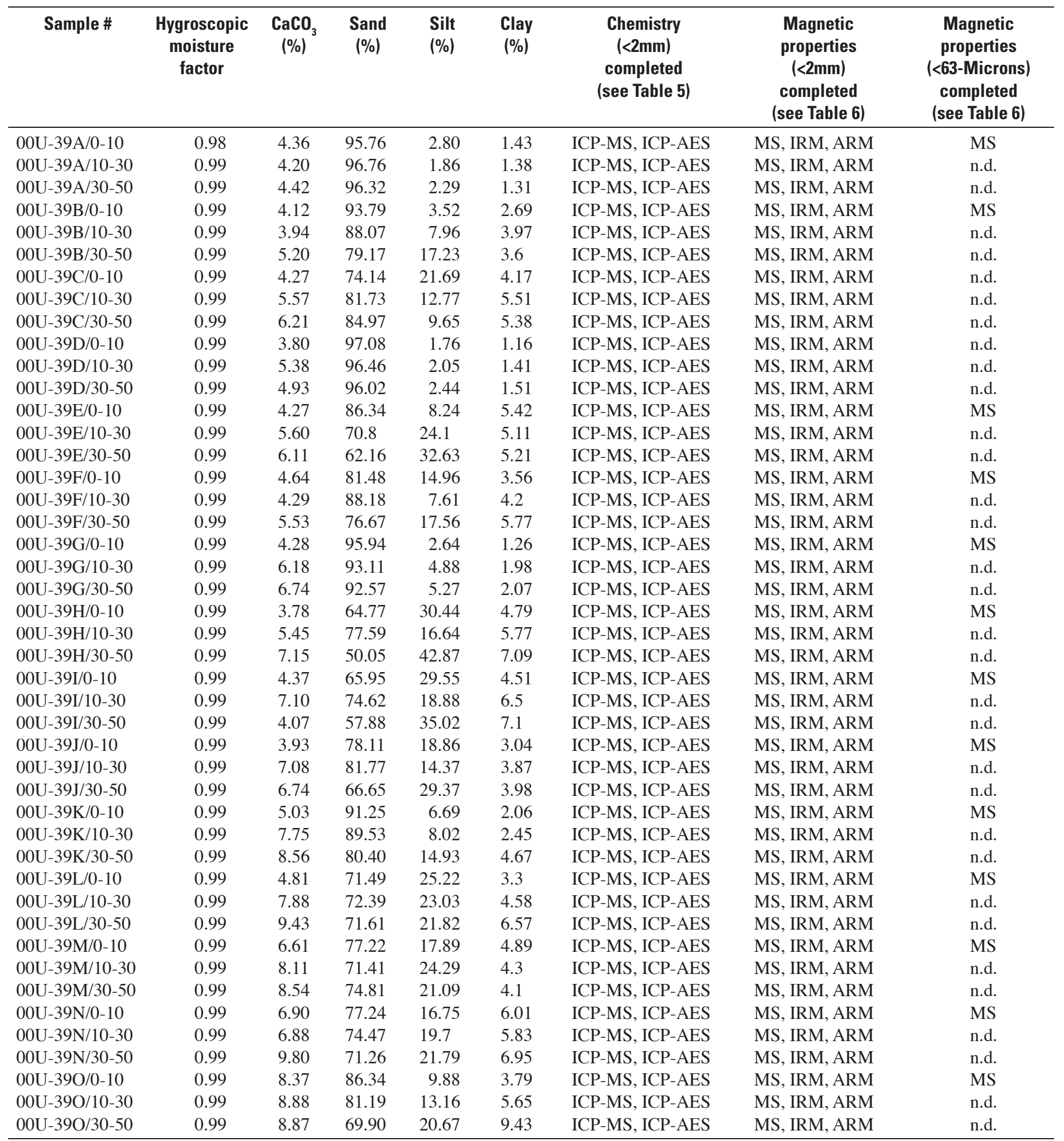


Table 3c-3. Summary data for auger hole 00U-40. This auger hole anchors the 00U-39 transect.

\begin{tabular}{lcccccccc}
\hline Sample \# & $\begin{array}{c}\text { Hygroscopic } \\
\text { moisture } \\
\text { factor }\end{array}$ & $\begin{array}{c}\mathbf{C}^{\text {CaCO }} \\
(\%)\end{array}$ & $\begin{array}{c}\text { Sand } \\
(\%)\end{array}$ & $\begin{array}{c}\text { Silt } \\
(\%)\end{array}$ & $\begin{array}{c}\text { Clay } \\
(\%)\end{array}$ & $\begin{array}{c}\text { Chemistry } \\
\text { (<2mm) } \\
\text { completed } \\
\text { (see Table 5) }\end{array}$ & $\begin{array}{c}\text { Magnetic } \\
\text { properties } \\
\text { (<2mm) } \\
\text { completed } \\
\text { (see Table 6) }\end{array}$ & $\begin{array}{c}\text { Magnetic } \\
\text { properties } \\
\text { <63-Microns) } \\
\text { completed } \\
\text { (see Table 6) }\end{array}$ \\
\hline 00U-40/0-10 & 0.99 & 10.34 & 62.52 & 26.56 & 10.92 & ICP-MS, ICP-AES & MS, IRM, ARM & MS \\
00U-40/10-30 & 0.99 & 11.69 & 57.05 & 30.48 & 12.48 & ICP-MS, ICP-AES & MS, IRM, ARM & n.d. \\
00U-40/30-48 & 0.99 & 10.29 & 64.88 & 24.11 & 11.01 & ICP-MS, ICP-AES & MS, IRM, ARM & n.d. \\
00U-40/48-64 & 0.99 & 11.42 & 62.62 & 27.21 & 10.17 & ICP-MS, ICP-AES & MS, IRM, ARM & n.d. \\
00U-40/64-82 & 0.97 & 14.01 & 52.70 & 35.94 & 11.36 & ICP-MS, ICP-AES & MS, IRM, ARM & n.d. \\
00U-40/82-102 & 0.99 & 14.76 & 47.69 & 37.69 & 14.62 & ICP-MS, ICP-AES & MS, IRM, ARM & n.d. \\
00U-40/102-121 & 0.99 & 8.36 & 65.46 & 26.00 & 8.54 & ICP-MS, ICP-AES & MS, IRM, ARM & n.d. \\
00U-40/121-142 & 0.99 & 5.72 & 86.85 & 9.79 & 3.36 & ICP-MS, ICP-AES & MS, IRM, ARM & n.d. \\
00U-40/142-154 & 0.99 & 6.09 & 88.68 & 8.48 & 2.84 & ICP-MS, ICP-AES & MS, IRM, ARM & n.d. \\
00U-40/154-168 & 0.99 & 7.61 & 91.64 & 6.33 & 2.03 & ICP-MS, ICP-AES & MS, IRM, ARM & n.d. \\
00U-40/168-183 & 0.99 & 5.28 & 88.56 & 9.19 & 2.26 & ICP-MS, ICP-AES & MS, IRM, ARM & n.d. \\
00U-40/183-193 & 0.99 & 11.09 & 91.84 & 6.02 & 2.14 & ICP-MS, ICP-AES & MS, IRM, ARM & n.d. \\
00U-40/192-204 & 0.99 & 34.34 & 93.01 & 5.01 & 1.99 & ICP-MS, ICP-AES & MS, IRM, ARM & n.d. \\
00U-40/204-217 & 0.99 & 25.40 & 91.97 & 5.31 & 2.72 & ICP-MS, ICP-AES & MS, IRM, ARM & n.d. \\
\hline
\end{tabular}


Table 3d-1. Summary data for the 00U-35 transect.

\begin{tabular}{|c|c|c|c|c|c|c|c|c|}
\hline Sample \# & $\begin{array}{l}\text { Hygroscopic } \\
\text { moisture } \\
\text { factor }\end{array}$ & $\begin{array}{c}\mathrm{CaCO}_{3} \\
(\%)\end{array}$ & $\begin{array}{c}\text { Sand } \\
(\%)\end{array}$ & $\begin{array}{l}\text { Silt } \\
(\%)\end{array}$ & $\begin{array}{l}\text { Clay } \\
(\%)\end{array}$ & $\begin{array}{c}\text { Chemistry } \\
\text { (<2mm) } \\
\text { completed } \\
\text { (see Table 5) }\end{array}$ & $\begin{array}{c}\text { Magnetic proper- } \\
\text { ties }(<2 \mathrm{~mm}) \\
\text { completed } \\
\text { (see Table 6) }\end{array}$ & $\begin{array}{c}\text { Magnetic } \\
\text { properties } \\
\text { (<63-Microns) } \\
\text { completed } \\
\text { (see Table 6) }\end{array}$ \\
\hline OOU-35A/0-10 & 0.99 & 6.91 & 82.61 & 12.46 & 4.92 & ICP-MS, ICP-AES & MS, IRM, ARM & MS, IRM, ARM \\
\hline OOU-35A/10-30 & 0.99 & 8.10 & 77.84 & 17.72 & 4.44 & ICP-MS, ICP-AES & MS, IRM, ARM & MS, IRM, ARM \\
\hline OOU-35A/30-50 & 0.99 & 8.33 & 82.45 & 12.98 & 4.57 & ICP-MS, ICP-AES & MS, IRM, ARM & MS, IRM, ARM \\
\hline OOU-35B/0-10 & 0.99 & 3.65 & 81.26 & 13.16 & 5.59 & ICP-MS, ICP-AES & MS, IRM, ARM & MS, IRM, ARM \\
\hline OOU-35B/10-30 & 0.99 & 5.73 & 81.15 & 14.35 & 4.50 & ICP-MS, ICP-AES & MS, IRM, ARM & MS, IRM, ARM \\
\hline OOU-35B/30-50 & 0.99 & 6.01 & 77.27 & 17.50 & 5.23 & ICP-MS, ICP-AES & MS, IRM, ARM & MS, IRM, ARM \\
\hline OOU-35C/0-10 & 0.99 & 6.05 & 81.67 & 13.83 & 4.50 & ICP-MS, ICP-AES & MS, IRM, ARM & MS, IRM, ARM \\
\hline OOU-35C/10-30 & 0.99 & 6.10 & 78.87 & 16.48 & 4.66 & ICP-MS, ICP-AES & MS, IRM, ARM & MS, IRM, ARM \\
\hline OOU-35C/30-50 & 0.99 & 7.23 & 76.94 & 17.67 & 5.39 & ICP-MS, ICP-AES & MS, IRM, ARM & MS, IRM, ARM \\
\hline OOU-35D/0-10 & 0.99 & 4.95 & 85.61 & 11.05 & 3.34 & ICP-MS, ICP-AES & MS, IRM, ARM & MS, IRM, ARM \\
\hline OOU-35D/10-30 & 0.99 & 5.67 & 78.11 & 17.41 & 4.48 & ICP-MS, ICP-AES & MS, IRM, ARM & MS, IRM, ARM \\
\hline OOU-35D/30-50 & 0.99 & 6.35 & 77.36 & 17.00 & 5.65 & ICP-MS, ICP-AES & MS, IRM, ARM & MS, IRM, ARM \\
\hline OOU-35E/0-10 & 0.99 & 5.07 & 74.91 & 18.48 & 6.61 & ICP-MS, ICP-AES & MS, IRM, ARM & MS, IRM, ARM \\
\hline OOU-35E/10-30 & 0.99 & 6.67 & 70.70 & 23.12 & 6.18 & ICP-MS, ICP-AES & MS, IRM, ARM & MS, IRM, ARM \\
\hline OOU-35E/30-50 & 0.97 & 8.48 & 82.25 & 13.76 & 3.99 & ICP-MS, ICP-AES & MS, IRM, ARM & MS, IRM, ARM \\
\hline OOU-35F/0-10 & 0.99 & 6.90 & 54.69 & 33.69 & 11.62 & ICP-MS, ICP-AES & MS, IRM, ARM & MS, IRM, ARM \\
\hline OOU-35F/10-30 & 0.99 & 8.60 & 51.59 & 34.83 & 13.58 & ICP-MS, ICP-AES & MS, IRM, ARM & MS, IRM, ARM \\
\hline OOU-35F/30-50 & 0.99 & 8.33 & 55.00 & 33.45 & 11.55 & ICP-MS, ICP-AES & MS, IRM, ARM & MS, IRM, ARM \\
\hline OOU-35G/0-10 & 0.99 & 5.53 & 62.21 & 26.30 & 11.50 & ICP-MS, ICP-AES & MS, IRM, ARM & MS, IRM, ARM \\
\hline OOU-35G/10-30 & 0.99 & 7.23 & 60.93 & 27.84 & 11.23 & ICP-MS, ICP-AES & MS, IRM, ARM & MS, IRM, ARM \\
\hline OOU-35G/30-50 & 0.99 & 7.79 & 59.17 & 28.83 & 11.99 & ICP-MS, ICP-AES & MS, IRM, ARM & MS, IRM, ARM \\
\hline OOU-35H/0-10 & 0.99 & 6.62 & 48.12 & 37.19 & 14.69 & ICP-MS, ICP-AES & MS, IRM, ARM & MS, IRM, ARM \\
\hline OOU-35H/10-30 & 0.99 & 6.44 & 40.02 & 39.50 & 20.47 & ICP-MS, ICP-AES & MS, IRM, ARM & MS, IRM, ARM \\
\hline OOU-35H/30-50 & 0.99 & 8.02 & 40.16 & 37.78 & 22.06 & ICP-MS, ICP-AES & MS, IRM, ARM & MS, IRM, ARM \\
\hline
\end{tabular}


Table 3d-2. Summary data for auger hole 00U-36. This auger hole anchors the 00U-35 transect.

\begin{tabular}{lcccccccc}
\hline Sample \# & $\begin{array}{c}\text { Hygroscopic } \\
\text { moisture } \\
\text { factor }\end{array}$ & $\begin{array}{c}\mathbf{C a C O}_{3} \\
(\%)\end{array}$ & $\begin{array}{c}\text { Sand } \\
(\%)\end{array}$ & $\begin{array}{c}\text { Silt } \\
(\%)\end{array}$ & $\begin{array}{c}\text { Clay } \\
(\%)\end{array}$ & $\begin{array}{c}\text { Chemistry } \\
\text { (<2mm) } \\
\text { completed } \\
\text { (see Table 5) }\end{array}$ & $\begin{array}{c}\text { Magnetic } \\
\text { properties } \\
\text { (<2mm) } \\
\text { completed } \\
\text { (see Table 6) }\end{array}$ & $\begin{array}{c}\text { Magnetic } \\
\text { properties } \\
\text { (<63-Microns) } \\
\text { completed } \\
\text { (see Table 6) }\end{array}$ \\
\hline OOU-36/0-10 & 0.99 & 5.09 & 87.19 & 9.11 & 3.69 & ICP-MS, ICP-AES & MS, IRM, ARM & MS, IRM, ARM \\
OOU-36/10-30 & 0.99 & 7.45 & 58.48 & 33.01 & 8.51 & ICP-MS, ICP-AES & MS, IRM, ARM & MS, IRM, ARM \\
OOU-36/30-46 & 0.99 & 7.56 & 81.55 & 13.56 & 4.88 & ICP-MS, ICP-AES & MS, IRM, ARM & MS, IRM, ARM \\
OOU-36/46-68 & 0.99 & 7.89 & 79.58 & 15.04 & 5.38 & ICP-MS, ICP-AES & MS, IRM, ARM & MS, IRM, ARM \\
OOU-36/68-86 & 0.99 & 8.20 & 78.41 & 15.78 & 5.81 & ICP-MS, ICP-AES & MS, IRM, ARM & MS, IRM, ARM \\
OOU-36/86-97 & 0.99 & 13.24 & 71.52 & 21.68 & 6.80 & ICP-MS, ICP-AES & MS, IRM, ARM & MS, IRM, ARM \\
OOU-36/97-108 & 0.99 & 11.85 & 77.62 & 17.06 & 5.32 & ICP-MS, ICP-AES & MS, IRM, ARM & MS, IRM, ARM \\
OOU-36/108-120 & 0.99 & 11.84 & 72.76 & 21.50 & 5.74 & ICP-MS, ICP-AES & MS, IRM, ARM & MS, IRM, ARM \\
OOU-36/120-130 & 0.99 & 14.97 & 81.08 & 14.01 & 4.92 & ICP-MS, ICP-AES & MS, IRM, ARM & MS, IRM, ARM \\
\hline
\end{tabular}

Table 3d-3. Summary data for auger hole 00U-37. This auger hole anchors the 00U-35 transect.

\begin{tabular}{lcrrrrrrr}
\hline Sample \# & $\begin{array}{c}\text { Hygroscopic } \\
\text { moisture } \\
\text { factor }\end{array}$ & \multicolumn{1}{c|}{$\begin{array}{c}\mathbf{C a C O}_{3} \\
(\%)\end{array}$} & $\begin{array}{c}\text { Sand } \\
(\%)\end{array}$ & $\begin{array}{c}\text { Silt } \\
(\%)\end{array}$ & $\begin{array}{c}\text { Clay } \\
(\%)\end{array}$ & $\begin{array}{c}\text { Chemistry } \\
\text { (<2mm) } \\
\text { completed } \\
\text { (see Table 5) }\end{array}$ & $\begin{array}{c}\text { Magnetic } \\
\text { properties } \\
\text { (<2mm) } \\
\text { completed } \\
\text { (see Table 6) }\end{array}$ & $\begin{array}{c}\text { Magnetic } \\
\text { properties } \\
\text { <63-Microns) } \\
\text { completed } \\
\text { (see Table 6) }\end{array}$ \\
\hline OOU-37/0-10 & 0.99 & 9.99 & 36.71 & 38.48 & 24.81 & ICP-MS, ICP-AES & MS, IRM, ARM & MS, IRM, ARM \\
OOU-37/10-30 & 0.99 & 10.41 & 38.09 & 37.85 & 24.06 & ICP-MS, ICP-AES & MS, IRM, ARM & MS, IRM, ARM \\
OOU-37/30-43 & 0.99 & 9.20 & 42.13 & 34.69 & 23.19 & ICP-MS, ICP-AES & MS, IRM, ARM & MS, IRM, ARM \\
OOU-37/43-55 & 0.99 & 6.23 & 56.59 & 25.66 & 17.75 & ICP-MS, ICP-AES & MS, IRM, ARM & MS, IRM, ARM \\
OOU-37/55-68 & 0.99 & 12.84 & 70.96 & 17.41 & 11.64 & ICP-MS, ICP-AES & MS, IRM, ARM & MS, IRM, ARM \\
OOU-37/68-80 & 0.99 & 7.81 & 80.40 & 11.90 & 7.70 & ICP-MS, ICP-AES & MS, IRM, ARM & MS, IRM, ARM \\
OOU-37/80-94 & 0.99 & 6.41 & 85.18 & 8.93 & 5.89 & ICP-MS, ICP-AES & MS, IRM, ARM & MS, IRM, ARM \\
OOU-37/94-110 & 0.99 & 33.28 & 61.47 & 26.08 & 12.46 & ICP-MS, ICP-AES & MS, IRM, ARM & MS, IRM, ARM \\
OOU-37/110-120 & 0.99 & 20.89 & 58.48 & 30.67 & 10.84 & ICP-MS, ICP-AES & MS, IRM, ARM & MS, IRM, ARM \\
OOU-37/120-131 & 0.99 & 11.63 & 81.41 & 12.78 & 5.81 & ICP-MS, ICP-AES & MS, IRM, ARM & MS, IRM, ARM \\
OOU-37/131-139 & 0.99 & 17.66 & 83.34 & 11.71 & 4.96 & ICP-MS, ICP-AES & MS, IRM, ARM & MS, IRM, ARM \\
OOU-37/139-148 & 0.99 & 12.28 & 82.92 & 11.62 & 5.47 & ICP-MS, ICP-AES & MS, IRM, ARM & MS, IRM, ARM \\
OOU-37/148-155 & 0.99 & 12.12 & 71.72 & 18.96 & 9.32 & ICP-MS, ICP-AES & MS, IRM, ARM & MS, IRM, ARM \\
\hline
\end{tabular}


Table 3d-4. Summary data for the JN-1 soil pit.

\begin{tabular}{lcccccccc}
\hline \multicolumn{1}{c}{ Sample \# } & $\begin{array}{c}\text { Hygroscopic } \\
\text { moisture } \\
\text { factor }\end{array}$ & $\begin{array}{c}\mathbf{C a C O}_{3} \\
(\%)\end{array}$ & $\begin{array}{c}\text { Sand } \\
(\%)\end{array}$ & $\begin{array}{c}\text { Silt } \\
(\%)\end{array}$ & $\begin{array}{c}\text { Clay } \\
(\%)\end{array}$ & $\begin{array}{c}\text { Chemistry } \\
\text { (<2mm) } \\
\text { completed }\end{array}$ & $\begin{array}{c}\text { Magnetic } \\
\text { properties } \\
\text { (<2mm) } \\
\text { completed } \\
\text { (see Table 6) }\end{array}$ & $\begin{array}{c}\text { Magnetic } \\
\text { properties } \\
\text { (<63-Microns) } \\
\text { completed } \\
\text { (see Table 6) }\end{array}$ \\
\hline $\mathrm{JN}-1 /$ 0-10 & 0.99 & 4.23 & 77.01 & 17.64 & 5.35 & n.d. & MS & MS \\
$\mathrm{JN}-1 / 10-26$ & 0.99 & 6.55 & 73.12 & 19.78 & 7.10 & n.d. & MS & MS \\
$\mathrm{JN}-1 / 26-77$ & 0.99 & 8.13 & 64.19 & 28.12 & 7.70 & n.d. & MS & MS \\
$\mathrm{JN}-1 / 77-93$ & 0.99 & 7.96 & 70.97 & 21.45 & 7.58 & n.d. & MS & MS \\
$\mathrm{JN}-1 / 93-110$ & 0.99 & 12.29 & 66.53 & 25.39 & 8.09 & n.d. & MS & MS \\
\hline
\end{tabular}

Table 3d-5. Summary data for the JN-2 soil pit.

\begin{tabular}{lcccccccc}
\hline Sample \# & $\begin{array}{c}\text { Hygroscopic } \\
\text { moisture } \\
\text { factor }\end{array}$ & $\begin{array}{c}\mathrm{CaCO}_{3} \\
(\%)\end{array}$ & $\begin{array}{c}\text { Sand } \\
(\%)\end{array}$ & $\begin{array}{c}\text { Silt } \\
(\%)\end{array}$ & $\begin{array}{c}\text { Clay } \\
(\%)\end{array}$ & $\begin{array}{c}\text { Chemistry } \\
(<2 \mathrm{~mm}) \\
\text { completed }\end{array}$ & $\begin{array}{c}\text { Magnetic } \\
\text { properties } \\
\text { (<2mm) } \\
\text { completed } \\
\text { (see Table 6) }\end{array}$ & $\begin{array}{c}\text { Magnetic } \\
\text { properties } \\
\text { (<63-Microns) } \\
\text { completed } \\
\text { (see Table 6) }\end{array}$ \\
\hline $\mathrm{JN}-2 / 0-7$ & 0.99 & 2.86 & 79.75 & 15.21 & 5.04 & n.d. & MS & MS \\
$\mathrm{JN}-2 / 7-14$ & 0.99 & 2.64 & 76.91 & 17.58 & 5.51 & n.d. & MS & MS \\
$\mathrm{JN}-2 / 14-41$ & 0.98 & 5.62 & 64.70 & 25.24 & 10.06 & n.d. & MS & MS \\
$\mathrm{JN}-2 / 41-64$ & 0.98 & 5.03 & 67.04 & 23.05 & 9.91 & n.d. & MS & MS \\
$\mathrm{JN}-2 / 64-86$ & 0.99 & 5.03 & 71.28 & 19.90 & 8.83 & n.d. & MS & MS \\
$\mathrm{JN}-2 / 86-100$ & 0.99 & 9.08 & 71.95 & 19.67 & 8.38 & n.d. & MS & MS \\
\hline
\end{tabular}

Table 3d-6. Summary data for the JN-3 soil pit.

\begin{tabular}{lcccccccc}
\hline \multicolumn{1}{c}{ Sample \# } & $\begin{array}{c}\text { Hygroscopic } \\
\text { moisture } \\
\text { factor }\end{array}$ & $\begin{array}{c}\mathrm{CaCO}_{3} \\
(\%)\end{array}$ & $\begin{array}{c}\text { Sand } \\
(\%)\end{array}$ & $\begin{array}{c}\text { Silt } \\
(\%)\end{array}$ & $\begin{array}{c}\text { Clay } \\
(\%)\end{array}$ & $\begin{array}{c}\text { Chemistry } \\
\text { (<2mm) } \\
\text { completed }\end{array}$ & $\begin{array}{c}\text { Magnetic } \\
\text { properties } \\
\text { (<2mm) } \\
\text { completed } \\
\text { (see Table 6) }\end{array}$ & $\begin{array}{c}\text { Magnetic } \\
\text { properties } \\
\text { (<63-Microns) } \\
\text { completed } \\
\text { (see Table 6) }\end{array}$ \\
\hline $\mathrm{JN}-3 /$ - 0-7 & 0.99 & 6.48 & 51.00 & 33.87 & 15.13 & n.d. & MS & MS \\
$\mathrm{JN}-3 / 7-36$ & 0.98 & 7.85 & 46.48 & 34.84 & 18.68 & n.d. & MS & MS \\
$\mathrm{JN}-3 / 36-52$ & 0.99 & 6.51 & 56.78 & 27.14 & 16.08 & n.d. & MS & MS \\
$\mathrm{JN}-3 / 52-83$ & 0.99 & 8.92 & 74.49 & 14.93 & 10.58 & n.d. & MS & MS \\
$\mathrm{JN}-3 / 83-100$ & 0.99 & 5.25 & 73.77 & 17.75 & 8.48 & n.d. & MS & MS \\
\hline
\end{tabular}


Table 3e-1. Summary data for the GP transect.

\begin{tabular}{|c|c|c|c|c|c|c|c|c|}
\hline Sample \# & $\begin{array}{l}\text { Hygroscopic } \\
\text { moisture } \\
\text { factor }\end{array}$ & $\begin{array}{c}\mathrm{CaCO}_{3} \\
(\%)\end{array}$ & $\begin{array}{l}\text { Sand } \\
(\%)\end{array}$ & $\begin{array}{l}\text { Silt } \\
(\%)\end{array}$ & $\begin{array}{l}\text { Clay } \\
(\%)\end{array}$ & $\begin{array}{c}\text { Chemistry } \\
(<2 \mathrm{~mm}) \\
\text { completed } \\
\text { (see Table 5) }\end{array}$ & $\begin{array}{c}\text { Magnetic } \\
\text { properties (<2mm) } \\
\text { completed } \\
\text { (see Table 6) }\end{array}$ & $\begin{array}{c}\text { Magnetic } \\
\text { properties } \\
\text { (<63-Microns) } \\
\text { completed }\end{array}$ \\
\hline GP-A/0-10 & 0.84 & 1.05 & 89.88 & 6.76 & 3.35 & ICP-AES, ICP-MS & MS, IRM, ARM & n.d. \\
\hline GP-A/10-30 & 0.99 & 0.34 & 90.67 & 6.51 & 2.82 & ICP-AES, ICP-MS & MS, IRM, ARM & n.d. \\
\hline GP-A/30-50 & 0.99 & 2.06 & 89.58 & 7.28 & 3.14 & ICP-AES, ICP-MS & MS, IRM, ARM & n.d. \\
\hline GP-B/0-10 & 0.99 & 0.81 & 86.55 & 8.49 & 4.96 & ICP-AES, ICP-MS & MS, IRM, ARM & n.d. \\
\hline GP-B/10-30 & 0.99 & 2.12 & 87.65 & 8.54 & 3.81 & ICP-AES, ICP-MS & MS, IRM, ARM & n.d. \\
\hline GP-B/30-50 & 0.99 & 4.51 & 89.58 & 7.11 & 3.31 & ICP-AES, ICP-MS & MS, IRM, ARM & n.d. \\
\hline GP-C/0-10 & 0.99 & 0.65 & 88.91 & 7.23 & 3.87 & ICP-AES, ICP-MS & MS, IRM, ARM & n.d. \\
\hline GP-C/10-30 & 0.99 & 0.96 & 84.25 & 10.81 & 4.94 & ICP-AES, ICP-MS & MS, IRM, ARM & n.d. \\
\hline GP-C/30-50 & 0.99 & 1.78 & 86.25 & 10.27 & 3.48 & ICP-AES, ICP-MS & MS, IRM, ARM & n.d. \\
\hline GP-D/0-10 & 0.99 & 0.68 & 84.76 & 10.64 & 4.60 & ICP-AES, ICP-MS & MS, IRM, ARM & n.d. \\
\hline GP-D/10-30 & 0.99 & 0.14 & 87.05 & 9.18 & 3.77 & ICP-AES, ICP-MS & MS, IRM, ARM & n.d. \\
\hline GP-D/30-50 & 0.99 & 2.27 & 85.74 & 10.04 & 4.23 & ICP-AES, ICP-MS & MS, IRM, ARM & n.d. \\
\hline GP-E/0-10 & 0.99 & 0.34 & 87.57 & 8.28 & 4.15 & ICP-AES, ICP-MS & MS, IRM, ARM & n.d. \\
\hline GP-E/10-30 & 0.99 & 0.81 & 84.99 & 10.61 & 4.40 & ICP-AES, ICP-MS & MS, IRM, ARM & n.d. \\
\hline GP-E/30-50 & 0.99 & 0.16 & 86.41 & 9.55 & 4.05 & ICP-AES, ICP-MS & MS, IRM, ARM & n.d. \\
\hline GP-F/0-10 & 0.99 & 0.62 & 85.17 & 9.61 & 5.22 & ICP-AES, ICP-MS & MS, IRM, ARM & n.d. \\
\hline GP-F/10-30 & 0.99 & 1.16 & 86.65 & 9.36 & 3.99 & ICP-AES, ICP-MS & MS, IRM, ARM & n.d. \\
\hline GP-F/30-50 & 0.99 & 2.67 & 87.30 & 9.13 & 3.57 & ICP-AES, ICP-MS & MS, IRM, ARM & n.d. \\
\hline GP-G/0-10 & 0.99 & 0.07 & 86.21 & 8.47 & 5.32 & ICP-AES, ICP-MS & MS, IRM, ARM & n.d. \\
\hline GP-G/10-30 & 0.99 & 1.71 & 85.78 & 9.67 & 4.56 & ICP-AES, ICP-MS & MS, IRM, ARM & n.d. \\
\hline GP-G/30-50 & 0.99 & 2.06 & 84.42 & 11.27 & 4.31 & ICP-AES, ICP-MS & MS, IRM, ARM & n.d. \\
\hline GP-H/0-10 & 0.99 & 1.59 & 82.31 & 11.66 & 6.04 & ICP-AES, ICP-MS & MS, IRM, ARM & n.d. \\
\hline GP-H/10-30 & 0.99 & 2.05 & 84.45 & 11.09 & 4.46 & ICP-AES, ICP-MS & MS, IRM, ARM & n.d. \\
\hline GP-H/30-50 & 0.99 & 2.29 & 86.30 & 9.54 & 4.16 & ICP-AES, ICP-MS & MS, IRM, ARM & n.d. \\
\hline GP-I/0-10 & 0.99 & 0.19 & 79.65 & 14.66 & 5.69 & ICP-AES, ICP-MS & MS, IRM, ARM & n.d. \\
\hline GP-I/10-30 & 0.99 & 1.52 & 82.92 & 12.37 & 4.70 & ICP-AES, ICP-MS & MS, IRM, ARM & n.d. \\
\hline GP-I/30-50 & 0.99 & 2.24 & 84.29 & 11.26 & 4.45 & ICP-AES, ICP-MS & MS, IRM, ARM & n.d. \\
\hline GP-J/0-10 & 0.99 & 0.60 & 86.85 & 7.95 & 5.21 & ICP-AES, ICP-MS & MS, IRM, ARM & n.d. \\
\hline GP-J/10-30 & 0.99 & 2.20 & 82.30 & 12.52 & 5.19 & ICP-AES, ICP-MS & MS, IRM, ARM & n.d. \\
\hline GP-J/30-50 & 0.99 & 1.84 & 81.70 & 13.07 & 5.24 & ICP-AES, ICP-MS & MS, IRM, ARM & n.d. \\
\hline GP-K/0-10 & 0.99 & 0.07 & 77.51 & 15.10 & 7.40 & ICP-AES, ICP-MS & MS, IRM, ARM & n.d. \\
\hline GP-K/10-30 & 0.99 & 0.58 & 79.40 & 14.64 & 5.96 & ICP-AES, ICP-MS & MS, IRM, ARM & n.d. \\
\hline GP-K/30-50 & 0.99 & 1.09 & 77.95 & 16.26 & 5.80 & ICP-AES, ICP-MS & MS, IRM, ARM & n.d. \\
\hline
\end{tabular}


Table 3f-1. Summary data for the 00U-41 transect.

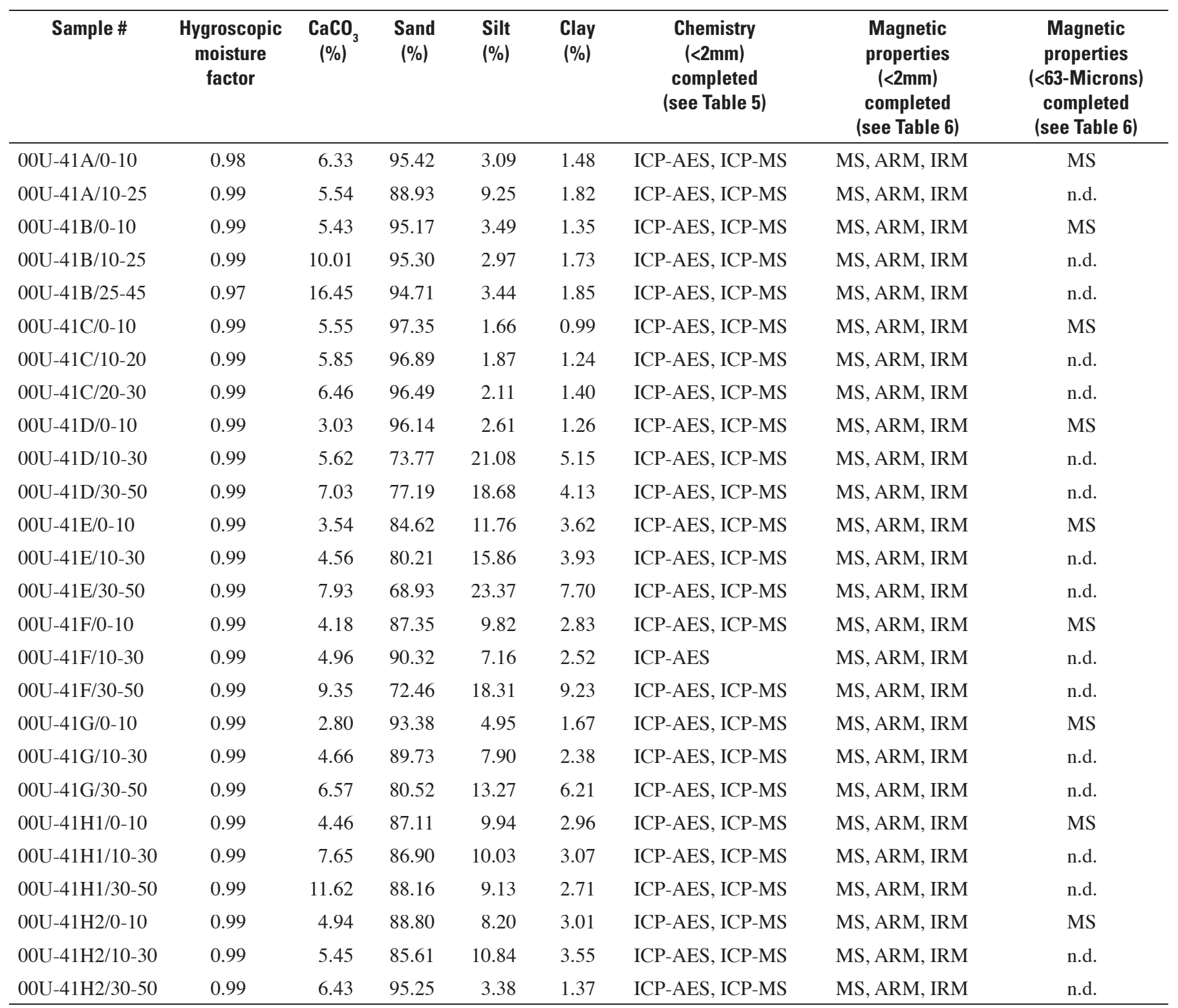


Table 3f-2. Summary data for auger hole 00U-42. This auger hole anchors the 00U-41 transect.

\begin{tabular}{lcrrrrccc}
\hline \multicolumn{1}{c}{ Sample \# } & $\begin{array}{c}\text { Hygroscopic } \\
\text { moisture } \\
\text { factor }\end{array}$ & $\begin{array}{c}\text { CaCO} \\
(\%)\end{array}$ & $\begin{array}{c}\text { Sand } \\
(\%)\end{array}$ & $\begin{array}{c}\text { Silt } \\
(\%)\end{array}$ & $\begin{array}{c}\text { Clay } \\
(\%)\end{array}$ & $\begin{array}{c}\text { Chemistry } \\
\text { (<2mm) } \\
\text { completed } \\
\text { (see Table 5) }\end{array}$ & $\begin{array}{c}\text { Magnetic } \\
\text { properties } \\
\text { (<2mm) } \\
\text { completed } \\
\text { (see Table 6) }\end{array}$ & $\begin{array}{c}\text { Magnetic } \\
\text { properties } \\
\text { (<63-Microns) } \\
\text { completed } \\
\text { (see Table 6) }\end{array}$ \\
\hline 00U-42/0-10 & 0.97 & 4.29 & 97.01 & 1.87 & 1.03 & ICP-AES, ICP-MS & MS, ARM, IRM & MS \\
00U-42/10-30 & 0.99 & 4.95 & 97.00 & 1.84 & 1.16 & ICP-AES, ICP-MS & MS, ARM, IRM & n.d. \\
00U-42/30-50 & 0.99 & 6.75 & 95.52 & 3.03 & 1.45 & ICP-AES, ICP-MS & MS, ARM, IRM & n.d. \\
00U-42/50-63 & 0.99 & 6.02 & 95.16 & 3.41 & 1.44 & ICP-AES, ICP-MS & MS, ARM, IRM & n.d. \\
00U-42/63-74 & 0.99 & 7.50 & 94.14 & 4.32 & 1.54 & ICP-AES, ICP-MS & MS, ARM, IRM & n.d. \\
00U-42/74-87 & 0.99 & 10.44 & 76.90 & 20.16 & 2.94 & ICP-AES, ICP-MS & MS, ARM, IRM & n.d. \\
00U-42/87-95 & 0.99 & 9.62 & 77.83 & 19.27 & 2.90 & ICP-AES, ICP-MS & MS, ARM, IRM & n.d. \\
00U-42/95-106 & 0.99 & 12.03 & 81.03 & 16.37 & 2.60 & ICP-AES, ICP-MS & MS, ARM, IRM & n.d. \\
00U-42/106-119 & 0.99 & 10.16 & 85.30 & 11.84 & 2.86 & ICP-AES, ICP-MS & MS, ARM, IRM & n.d. \\
00U-42/119-125 & 0.99 & 9.11 & 67.25 & 28.97 & 3.78 & ICP-AES, ICP-MS & MS, ARM, IRM & n.d. \\
00U-42/125-133 & 0.99 & 10.44 & 74.08 & 22.88 & 3.04 & ICP-AES, ICP-MS & MS, ARM, IRM & n.d. \\
\hline
\end{tabular}

Table 3f-3. Summary data for auger hole 00U-43. This auger hole anchors the 00U-41 transect.

\begin{tabular}{lcccccccc}
\hline Sample \# & $\begin{array}{c}\text { Hygroscopic } \\
\text { moisture } \\
\text { factor }\end{array}$ & $\begin{array}{c}\mathbf{C a C O}_{3} \\
(\%)\end{array}$ & $\begin{array}{c}\text { Sand } \\
(\%)\end{array}$ & $\begin{array}{c}\text { Silt } \\
(\%)\end{array}$ & $\begin{array}{c}\text { Clay } \\
(\%)\end{array}$ & $\begin{array}{c}\text { Chemistry } \\
\text { (<2mm) } \\
\text { completed } \\
\text { (see Table 5) }\end{array}$ & $\begin{array}{c}\text { Magnetic } \\
\text { properties } \\
\text { (<2mm) } \\
\text { completed } \\
\text { (see Table 6) }\end{array}$ & $\begin{array}{c}\text { Magnetic } \\
\text { roperties } \\
\text { (<63-Microns) } \\
\text { completed } \\
\text { (see Table 6) }\end{array}$ \\
\hline 00U-43/0-10 & 0.99 & 4.93 & 84.14 & 13.48 & 2.38 & ICP-AES, ICP-MS & MS, ARM, IRM & MS \\
00U-43/10-25 & 0.99 & 6.47 & 81.95 & 13.69 & 4.36 & ICP-AES, ICP-MS & MS, ARM, IRM & n.d. \\
00U-43/25-34 & 0.99 & 7.57 & 85.92 & 10.47 & 3.60 & ICP-AES, ICP-MS & MS, ARM, IRM & n.d. \\
00U-43/34-48 & 0.99 & 9.13 & 94.27 & 4.02 & 1.72 & ICP-AES, ICP-MS & MS, ARM, IRM & n.d. \\
00U-43/48-55 & 0.99 & 14.67 & 70.69 & 25.15 & 4.16 & ICP-AES, ICP-MS & MS, ARM, IRM & n.d. \\
00U-43/55-64 & 0.98 & 29.04 & 80.13 & 16.56 & 3.30 & ICP-AES, ICP-MS & MS, ARM, IRM & n.d. \\
00U-43/64-76 & 0.99 & 25.33 & 89.72 & 7.95 & 2.33 & ICP-AES, ICP-MS & MS, ARM, IRM & n.d. \\
00U-43/76-86 & 0.98 & 23.76 & 55.39 & 38.51 & 6.01 & ICP-AES, ICP-MS & MS, ARM, IRM & n.d. \\
00U-43/86-96 & 0.99 & 23.85 & 78.31 & 18.39 & 3.30 & ICP-AES, ICP-MS & MS, ARM, IRM & n.d. \\
00U-43/96-108 & 0.99 & 15.66 & 61.43 & 31.47 & 7.01 & ICP-AES, ICP-MS & MS, ARM, IRM & n.d. \\
00U-43/108-114 & 0.99 & 15.37 & 79.06 & 16.70 & 4.24 & ICP-AES, ICP-MS & MS, ARM, IRM & n.d. \\
\hline
\end{tabular}


Table 3g-1. Summary data for auger hole 8U-18.

\begin{tabular}{|c|c|c|c|c|c|c|c|c|}
\hline Sample \# & $\begin{array}{l}\text { Hygroscopic } \\
\text { moisture } \\
\text { factor }\end{array}$ & $\begin{array}{c}\mathrm{CaCO}_{3} \\
(\%)\end{array}$ & $\begin{array}{l}\text { Sand } \\
(\%)\end{array}$ & $\begin{array}{l}\text { Silt } \\
(\%)\end{array}$ & $\begin{array}{l}\text { Clay } \\
(\%)\end{array}$ & $\begin{array}{c}\text { Chemistry } \\
(<2 \mathrm{~mm}) \\
\text { completed }\end{array}$ & $\begin{array}{c}\text { Magnetic } \\
\text { properties }(<2 \mathrm{~mm}) \\
\text { completed } \\
\text { (see Table 6) }\end{array}$ & $\begin{array}{c}\text { Magnetic } \\
\text { properties } \\
\text { (<63-Microns) } \\
\text { completed }\end{array}$ \\
\hline $8 \mathrm{U}-18 / 0-18$ & 0.98 & 17.92 & 22.35 & 61.12 & 16.53 & n.d. & MS & n.d. \\
\hline $8 \mathrm{U}-18 / 18-28$ & 0.99 & 15.97 & 9.67 & 70.66 & 19.68 & n.d. & MS & n.d. \\
\hline $8 \mathrm{U}-18 / 28-37$ & 0.97 & 15.16 & 13.29 & 62.33 & 24.38 & n.d. & MS & n.d. \\
\hline $8 \mathrm{U}-18 / 37-50$ & 0.99 & 10.39 & 44.07 & 42.16 & 13.77 & n.d. & MS & n.d. \\
\hline $8 \mathrm{U}-18 / 50-61$ & 0.98 & 19.94 & 13.79 & 67.02 & 19.19 & n.d. & MS & n.d. \\
\hline $8 \mathrm{U}-18 / 61-72$ & 0.99 & 18.05 & 1.70 & 67.04 & 31.26 & n.d. & MS & n.d. \\
\hline $8 \mathrm{U}-18 / 72-84$ & 0.99 & 23.60 & 7.78 & 65.35 & 26.87 & n.d. & MS & n.d. \\
\hline 8U-18/84-95 & 0.99 & 19.72 & 4.54 & 68.68 & 26.78 & n.d. & MS & n.d. \\
\hline $8 \mathrm{U}-18 / 95-105$ & 0.99 & 19.17 & 0.75 & 67.26 & 31.99 & n.d. & MS & n.d. \\
\hline $8 U-18 / 105-119$ & 0.99 & 11.95 & 37.33 & 46.89 & 15.78 & n.d. & MS & n.d. \\
\hline 8U-18/119-131 & 0.99 & 16.46 & 2.00 & 71.73 & 26.27 & n.d. & MS & n.d. \\
\hline $8 \mathrm{U}-18 / 131-141$ & 0.99 & 16.62 & 0.56 & 61.98 & 37.46 & n.d. & MS & n.d. \\
\hline $8 U-18 / 141-155$ & 0.99 & 18.64 & 0.06 & 62.94 & 37.00 & n.d. & MS & n.d. \\
\hline $8 U-18 / 155-166$ & 0.99 & 19.87 & 1.63 & 71.59 & 26.78 & n.d. & MS & n.d. \\
\hline $8 U-18 / 166-173$ & 0.99 & 17.10 & 7.89 & 69.01 & 23.10 & n.d. & MS & n.d. \\
\hline $8 \mathrm{U}-18 / 173-187$ & 0.99 & 25.43 & 16.98 & 57.71 & 25.31 & n.d. & MS & n.d. \\
\hline $8 U-18 / 187-198$ & 0.99 & 13.58 & 59.84 & 28.89 & 11.27 & n.d. & MS & n.d. \\
\hline $8 U-18 / 198-212$ & 0.98 & 10.22 & 83.95 & 11.32 & 4.73 & n.d. & MS & n.d. \\
\hline $8 \mathrm{U}-18 / 212-228$ & 0.99 & 10.32 & 84.46 & 11.60 & 3.94 & n.d. & MS & n.d. \\
\hline $8 U-18 / 228-241$ & 0.99 & 11.19 & 80.12 & 16.61 & 3.28 & n.d. & MS & n.d. \\
\hline $8 \mathrm{U}-18 / 241-254$ & 0.99 & 10.18 & 79.87 & 14.69 & 5.44 & n.d. & MS & n.d. \\
\hline $8 \mathrm{U}-18 / 254-266$ & 0.99 & 10.21 & 76.08 & 18.10 & 5.82 & n.d. & MS & n.d. \\
\hline $8 \mathrm{U}-18 / 266-280$ & 0.99 & 10.88 & 79.13 & 14.93 & 5.94 & n.d. & MS & n.d. \\
\hline $8 U-18 / 280-290$ & 0.99 & 11.73 & 84.08 & 11.56 & 4.36 & n.d. & MS & n.d. \\
\hline $8 U-18 / 290-298$ & 0.99 & 9.33 & 81.77 & 14.45 & 3.78 & n.d. & MS & n.d. \\
\hline 8U-18/298-309 & 0.99 & 11.29 & 79.09 & 15.90 & 5.01 & n.d. & MS & n.d. \\
\hline $8 U-18 / 309-322$ & 0.99 & 11.33 & 79.10 & 16.64 & 4.26 & n.d. & MS & n.d. \\
\hline $8 \mathrm{U}-18 / 322-333$ & 0.99 & 13.05 & 59.70 & 30.34 & 9.96 & n.d. & MS & n.d. \\
\hline $8 U-18 / 333-344$ & 0.99 & 10.91 & 66.95 & 26.09 & 6.95 & n.d. & MS & n.d. \\
\hline $8 \mathrm{U}-18 / 344-355$ & 0.99 & 13.42 & 68.23 & 24.35 & 7.42 & n.d. & MS & n.d. \\
\hline $8 U-18 / 355-367$ & 0.99 & 14.38 & 73.56 & 19.98 & 6.46 & n.d. & MS & n.d. \\
\hline $8 U-18 / 367-378$ & 0.99 & 17.24 & 64.30 & 28.44 & 7.27 & n.d. & MS & n.d. \\
\hline $8 U-18 / 378-391$ & 0.98 & 15.82 & 74.05 & 20.89 & 5.06 & n.d. & MS & n.d. \\
\hline $8 U-18 / 391-400$ & 0.99 & 12.01 & 78.81 & 14.63 & 6.57 & n.d. & MS & n.d. \\
\hline
\end{tabular}


Table 3g-2. Summary data for auger hole 8U-20.

\begin{tabular}{|c|c|c|c|c|c|c|c|c|}
\hline Sample \# & $\begin{array}{l}\text { Hygroscopic } \\
\text { moisture } \\
\text { factor }\end{array}$ & $\begin{array}{c}\mathrm{CaCO}_{3} \\
(\%)\end{array}$ & $\begin{array}{c}\text { Sand } \\
(\%)\end{array}$ & $\begin{array}{l}\text { Silt } \\
(\%)\end{array}$ & $\begin{array}{l}\text { Clay } \\
(\%)\end{array}$ & $\begin{array}{c}\text { Chemistry } \\
\quad<2 \mathrm{~mm}) \\
\text { completed }\end{array}$ & $\begin{array}{c}\text { Magnetic } \\
\text { properties }(<2 \mathrm{~mm}) \\
\text { completed } \\
\text { (see Table 6) }\end{array}$ & $\begin{array}{c}\text { Magnetic } \\
\text { properties } \\
\text { (<63-Microns) } \\
\text { completed }\end{array}$ \\
\hline $8 \mathrm{U}-20 / 0-18$ & 0.99 & 20.01 & 5.71 & 64.30 & 29.99 & n.d. & MS & n.d. \\
\hline $8 U-20 / 18-26$ & 0.99 & 24.47 & 3.33 & 69.24 & 27.43 & n.d. & MS & n.d. \\
\hline 8U-2026-35 & 0.99 & 19.52 & 2.48 & 61.43 & 36.09 & n.d. & MS & n.d. \\
\hline $8 U-20 / 35-45$ & 0.99 & 22.28 & 1.19 & 63.19 & 35.63 & n.d. & MS & n.d. \\
\hline $8 U-20 / 45-54$ & 0.97 & 19.06 & 2.53 & 58.39 & 39.08 & n.d. & MS & n.d. \\
\hline $8 U-20 / 54-65$ & 0.99 & 17.25 & 30.66 & 43.04 & 26.30 & n.d. & MS & n.d. \\
\hline $8 U-20 / 65-76$ & 0.99 & 12.10 & 69.69 & 21.09 & 9.23 & n.d. & MS & n.d. \\
\hline $8 U-20 / 76-88$ & 0.99 & 16.47 & 63.20 & 31.83 & 4.98 & n.d. & MS & n.d. \\
\hline $8 U-20 / 88-102$ & 0.98 & 15.41 & 61.20 & 29.44 & 9.35 & n.d. & MS & n.d. \\
\hline $8 U-20 / 102-124$ & 0.99 & 9.29 & 76.89 & 16.72 & 6.39 & n.d. & MS & n.d. \\
\hline $8 U-20 / 124-136$ & 0.99 & 7.53 & 92.00 & 4.18 & 3.82 & n.d. & MS & n.d. \\
\hline $8 U-20 / 136-147$ & 0.99 & 8.00 & 92.17 & 4.29 & 3.54 & n.d. & MS & n.d. \\
\hline $8 U-20 / 147-159$ & 0.99 & 8.67 & 91.96 & 5.08 & 2.96 & n.d. & MS & n.d. \\
\hline $8 U-20 / 159-168$ & 0.99 & 13.18 & 74.76 & 20.43 & 4.81 & n.d. & MS & n.d. \\
\hline $8 U-20 / 168-182$ & 0.99 & 16.41 & 55.96 & 36.96 & 7.08 & n.d. & MS & n.d. \\
\hline 8U-20/182-194 & 0.99 & 16.43 & 76.05 & 18.08 & 5.87 & n.d. & MS & n.d. \\
\hline $8 U-20 / 194-207$ & 0.99 & 12.17 & 69.88 & 23.57 & 6.54 & n.d. & MS & n.d. \\
\hline $8 U-20 / 207-218$ & 0.99 & 24.08 & 40.02 & 45.11 & 14.87 & n.d. & MS & n.d. \\
\hline $8 \mathrm{U}-20 / 218-230$ & 0.99 & 21.44 & 11.03 & 65.38 & 23.59 & n.d. & MS & n.d. \\
\hline $8 U-20 / 230-240$ & 0.99 & 19.27 & 4.53 & 59.21 & 36.27 & n.d. & MS & n.d. \\
\hline $8 \mathrm{U}-20 / 240-251$ & 0.99 & 19.49 & 6.15 & 58.62 & 35.23 & n.d. & MS & n.d. \\
\hline $8 U-20 / 251-261$ & 0.99 & 15.82 & 33.07 & 49.26 & 17.67 & n.d. & MS & n.d. \\
\hline $8 U-20 / 261-272$ & 0.99 & 15.67 & 33.21 & 53.47 & 13.32 & n.d. & MS & n.d. \\
\hline $8 U-20 / 272-282$ & 0.98 & 18.13 & 13.66 & 63.56 & 22.78 & n.d. & MS & n.d. \\
\hline $8 U-20 / 282-290$ & 0.99 & 18.05 & 10.81 & 63.23 & 25.96 & n.d. & MS & n.d. \\
\hline $8 U-20 / 290-298$ & 0.99 & 17.17 & 19.42 & 58.97 & 21.61 & n.d. & MS & n.d. \\
\hline $8 U-20 / 298-311$ & 0.99 & 18.23 & 9.54 & 63.20 & 27.26 & n.d. & MS & n.d. \\
\hline $8 U-20 / 311-321$ & 0.98 & 23.28 & 10.58 & 63.60 & 25.82 & n.d. & MS & n.d. \\
\hline $8 U-20 / 321-335$ & 0.99 & 27.09 & 9.02 & 62.27 & 28.72 & n.d. & MS & n.d. \\
\hline $8 U-20 / 335-346$ & 0.99 & 17.48 & 20.45 & 65.45 & 23.10 & n.d. & MS & n.d. \\
\hline $8 U-20 / 346-358$ & 0.99 & 12.55 & 17.91 & 58.30 & 23.79 & n.d. & MS & n.d. \\
\hline $8 U-20 / 358-370$ & 0.99 & 20.54 & 33.17 & 49.01 & 17.82 & n.d. & MS & n.d. \\
\hline $8 U-20 / 370-380$ & 0.99 & 19.47 & 34.84 & 51.47 & 13.70 & n.d. & MS & n.d. \\
\hline $8 U-20 / 380-389$ & 0.99 & 15.01 & 48.28 & 41.79 & 9.93 & n.d. & MS & n.d. \\
\hline $8 \mathrm{U}-20 / 389-400$ & 0.99 & 22.23 & 20.90 & 59.15 & 19.94 & n.d. & MS & n.d. \\
\hline
\end{tabular}


Table 3h-1. Summary data for auger hole 8U-15.

\begin{tabular}{|c|c|c|c|c|c|c|c|c|}
\hline Sample \# & $\begin{array}{l}\text { Hygroscopic } \\
\text { moisture } \\
\text { factor }\end{array}$ & $\begin{array}{c}\mathrm{CaCO}_{3} \\
(\%)\end{array}$ & $\begin{array}{c}\text { Sand } \\
(\%)\end{array}$ & $\begin{array}{l}\text { Silt } \\
(\%)\end{array}$ & $\begin{array}{l}\text { Clay } \\
(\%)\end{array}$ & $\begin{array}{c}\text { Chemistry } \\
(<2 \mathrm{~mm}) \\
\text { completed }\end{array}$ & $\begin{array}{c}\text { Magnetic } \\
\text { properties }(<2 \mathrm{~mm}) \\
\text { completed } \\
\text { (see Table 6) }\end{array}$ & $\begin{array}{c}\text { Magnetic } \\
\text { properties } \\
\text { (<63-Microns) } \\
\text { completed }\end{array}$ \\
\hline $8 \mathrm{U}-15 / 0-19$ & n.d & n.d. & n.d. & n.d. & n.d. & n.d. & MS & n.d. \\
\hline $8 \mathrm{U}-15 / 19-29$ & n.d & n.d. & n.d. & n.d. & n.d. & n.d. & MS & n.d. \\
\hline $8 \mathrm{U}-15 / 29-42$ & n.d & n.d. & n.d. & n.d. & n.d. & n.d. & MS & n.d. \\
\hline $8 \mathrm{U}-15 / 42-53$ & n.d & n.d. & n.d. & n.d. & n.d. & n.d. & MS & n.d. \\
\hline $8 \mathrm{U}-15 / 53-64$ & n.d & n.d. & n.d. & n.d. & n.d. & n.d. & MS & n.d. \\
\hline $8 \mathrm{U}-15 / 64-77$ & n.d & n.d. & n.d. & n.d. & n.d. & n.d. & MS & n.d. \\
\hline $8 \mathrm{U}-15 / 77-94$ & n.d & n.d. & n.d. & n.d. & n.d. & n.d. & MS & n.d. \\
\hline $8 \mathrm{U}-15 / 94-103$ & n.d & n.d. & n.d. & n.d. & n.d. & n.d. & MS & n.d. \\
\hline $8 \mathrm{U}-15 / 103-114$ & n.d & n.d. & n.d. & n.d. & n.d. & n.d. & MS & n.d. \\
\hline $8 \mathrm{U}-15 / 114-124$ & n.d & n.d. & n.d. & n.d. & n.d. & n.d. & MS & n.d. \\
\hline $8 \mathrm{U}-15 / 124-137$ & n.d & n.d. & n.d. & n.d. & n.d. & n.d. & MS & n.d. \\
\hline $8 \mathrm{U}-15 / 137-143$ & n.d & n.d. & n.d. & n.d. & n.d. & n.d. & MS & n.d. \\
\hline $8 \mathrm{U}-15 / 143-152$ & n.d & n.d. & n.d. & n.d. & n.d. & n.d. & MS & n.d. \\
\hline $8 \mathrm{U}-15 / 152-162$ & n.d & n.d. & n.d. & n.d. & n.d. & n.d. & MS & n.d. \\
\hline $8 \mathrm{U}-15 / 162-171$ & n.d & n.d. & n.d. & n.d. & n.d. & n.d. & MS & n.d. \\
\hline $8 \mathrm{U}-15 / 171-182$ & n.d & n.d. & n.d. & n.d. & n.d. & n.d. & MS & n.d. \\
\hline $8 U-15 / 182-192$ & n.d & n.d. & n.d. & n.d. & n.d. & n.d. & MS & n.d. \\
\hline $8 U-15 / 192-202$ & n.d & n.d. & n.d. & n.d. & n.d. & n.d. & MS & n.d. \\
\hline $8 \mathrm{U}-15 / 202-213$ & n.d & n.d. & n.d. & n.d. & n.d. & n.d. & MS & n.d. \\
\hline $8 \mathrm{U}-15 / 213-221$ & n.d & n.d. & n.d. & n.d. & n.d. & n.d. & MS & n.d. \\
\hline $8 \mathrm{U}-15 / 221-228$ & n.d & n.d. & n.d. & n.d. & n.d. & n.d. & MS & n.d. \\
\hline $8 U-15 / 228-240$ & n.d & n.d. & n.d. & n.d. & n.d. & n.d. & MS & n.d. \\
\hline $8 \mathrm{U}-15 / 240-247$ & n.d & n.d. & n.d. & n.d. & n.d. & n.d. & MS & n.d. \\
\hline $8 \mathrm{U}-15 / 247-260$ & n.d & n.d. & n.d. & n.d. & n.d. & n.d. & MS & n.d. \\
\hline $8 U-15 / 260-267$ & n.d & n.d. & n.d. & n.d. & n.d. & n.d. & MS & n.d. \\
\hline $8 \mathrm{U}-15 / 267-278$ & n.d & n.d. & n.d. & n.d. & n.d. & n.d. & MS & n.d. \\
\hline $8 U-15 / 278-286$ & n.d & n.d. & n.d. & n.d. & n.d. & n.d. & MS & n.d. \\
\hline $8 \mathrm{U}-15 / 286-300$ & n.d & n.d. & n.d. & n.d. & n.d. & n.d. & MS & n.d. \\
\hline $8 \mathrm{U}-15 / 300-308$ & n.d & n.d. & n.d. & n.d. & n.d. & n.d. & MS & n.d. \\
\hline $8 \mathrm{U}-15 / 308-319$ & n.d & n.d. & n.d. & n.d. & n.d. & n.d. & MS & n.d. \\
\hline $8 \mathrm{U}-15 / 319-330$ & n.d & n.d. & n.d. & n.d. & n.d. & n.d. & MS & n.d. \\
\hline $8 U-15 / 330-339$ & n.d & n.d. & n.d. & n.d. & n.d. & n.d. & MS & n.d. \\
\hline $8 U-15 / 339-349$ & n.d & n.d. & n.d. & n.d. & n.d. & n.d. & MS & n.d. \\
\hline $8 \mathrm{U}-15 / 349-360$ & n.d & n.d. & n.d. & n.d. & n.d. & n.d. & MS & n.d. \\
\hline $8 U-15 / 360-369$ & n.d & n.d. & n.d. & n.d. & n.d. & n.d. & MS & n.d. \\
\hline $8 \mathrm{U}-15 / 369-378$ & n.d & n.d. & n.d. & n.d. & n.d. & n.d. & MS & n.d. \\
\hline $8 \mathrm{U}-15 / 379-387$ & n.d & n.d. & n.d. & n.d. & n.d. & n.d. & MS & n.d. \\
\hline $8 U-15 / 387-395$ & n.d & n.d. & n.d. & n.d. & n.d. & n.d. & MS & n.d. \\
\hline
\end{tabular}


Table 3h-2. Summary data for auger hole 8U-16.

\begin{tabular}{|c|c|c|c|c|c|c|c|c|}
\hline Sample \# & $\begin{array}{l}\text { Hygroscopic } \\
\text { moisture } \\
\text { factor }\end{array}$ & $\begin{array}{c}\mathrm{CaCO}_{3} \\
(\%)\end{array}$ & $\begin{array}{c}\text { Sand } \\
(\%)\end{array}$ & $\begin{array}{l}\text { Silt } \\
(\%)\end{array}$ & $\begin{array}{l}\text { Clay } \\
(\%)\end{array}$ & $\begin{array}{l}\text { Chemistry } \\
(<2 \mathrm{~mm}) \\
\text { completed }\end{array}$ & $\begin{array}{c}\text { Magnetic } \\
\text { properties }(<2 \mathrm{~mm}) \\
\text { completed } \\
\text { (see Table 6) } \\
\end{array}$ & $\begin{array}{c}\text { Magnetic } \\
\text { properties } \\
\text { (<63-Microns) } \\
\text { completed } \\
\end{array}$ \\
\hline $8 \mathrm{U}-16 / 0-22$ & n.d & n.d. & n.d. & n.d. & n.d. & n.d. & MS & n.d. \\
\hline $8 U-16 / 22-36$ & n.d & n.d. & n.d. & n.d. & n.d. & n.d. & MS & n.d. \\
\hline $8 U-16 / 36-51$ & n.d & n.d. & n.d. & n.d. & n.d. & n.d. & MS & n.d. \\
\hline $8 U-16 / 63-76$ & n.d & n.d. & n.d. & n.d. & n.d. & n.d. & MS & n.d. \\
\hline $8 U-16 / 76-89$ & n.d & n.d. & n.d. & n.d. & n.d. & n.d. & MS & n.d. \\
\hline $8 \mathrm{U}-16 / 89-103$ & n.d & n.d. & n.d. & n.d. & n.d. & n.d. & MS & n.d. \\
\hline 8U-16/103-117 & n.d & n.d. & n.d. & n.d. & n.d. & n.d. & MS & n.d. \\
\hline
\end{tabular}

Table 3h-3. Summary data for auger hole 9U-21.

\begin{tabular}{|c|c|c|c|c|c|c|c|c|}
\hline Sample \# & $\begin{array}{l}\text { Hygroscopic } \\
\text { moisture } \\
\text { factor }\end{array}$ & $\begin{array}{c}\mathrm{CaCO}_{3} \\
(\%)\end{array}$ & $\begin{array}{c}\text { Sand } \\
(\%)\end{array}$ & $\begin{array}{l}\text { Silt } \\
(\%)\end{array}$ & $\begin{array}{l}\text { Clay } \\
(\%)\end{array}$ & $\begin{array}{l}\text { Chemistry } \\
(<2 \mathrm{~mm}) \\
\text { completed }\end{array}$ & $\begin{array}{c}\text { Magnetic } \\
\text { properties }(<2 \mathrm{~mm}) \\
\text { completed } \\
\text { (see Table 6) }\end{array}$ & $\begin{array}{c}\text { Magnetic } \\
\text { properties } \\
\text { (<63-Microns) } \\
\text { completed }\end{array}$ \\
\hline $9 \mathrm{U}-21 / 0-5$ & 0.99 & 6.78 & 68.82 & 26.16 & 5.03 & n.d. & MS, ARM, IRM & n.d. \\
\hline $9 \mathrm{U}-21 / 5-25$ & 0.99 & 8.60 & 61.62 & 32.32 & 6.06 & n.d. & MS, ARM, IRM & n.d. \\
\hline $9 \mathrm{U}-21 / 25-70$ & 0.99 & 12.40 & 73.80 & 21.90 & 4.31 & n.d. & MS, ARM, IRM & n.d. \\
\hline $9 U-21 / 90-115$ & 0.99 & 9.48 & 75.77 & 19.40 & 4.83 & n.d. & MS, ARM, IRM & n.d. \\
\hline 9U-21/115-128 & 0.99 & 13.44 & 76.70 & 18.75 & 4.55 & n.d. & MS, ARM, IRM & n.d. \\
\hline $9 U-21 / 128-148$ & 0.99 & 10.65 & 74.92 & 20.37 & 4.72 & n.d. & MS, ARM, IRM & n.d. \\
\hline 9U-21/148-168 & 0.99 & 7.45 & 70.74 & 23.05 & 6.21 & n.d. & MS, ARM, IRM & n.d. \\
\hline $9 U-21 / 250-300$ & 0.99 & 6.45 & 84.60 & 13.49 & 1.91 & n.d. & MS, ARM, IRM & n.d. \\
\hline $9 \mathrm{U}-21 / 300-345$ & 0.99 & 8.85 & 82.65 & 15.31 & 2.04 & n.d. & MS, ARM, IRM & n.d. \\
\hline $9 U-21 / 345-375$ & 0.99 & 10.98 & 82.79 & 13.55 & 3.66 & n.d. & MS, ARM, IRM & n.d. \\
\hline $9 U-21 / 375-440$ & 0.99 & 10.03 & 84.58 & 13.44 & 1.98 & n.d. & MS, ARM, IRM & n.d. \\
\hline
\end{tabular}




\section{Table 4. Particle size data}

This table contains high-resolution particle-size data obtained using a laser particle size analyzer. In most cases, the particle size is displayed in accordance to the Wentworth scale (Wentworth, 1922); however, in a few instances the USDA scale (Soil Survey Staff, 1975) was used.

Values are reported as volume percent. Each value corresponds to the volume percent of the sample that has a grain size falling within that range. 


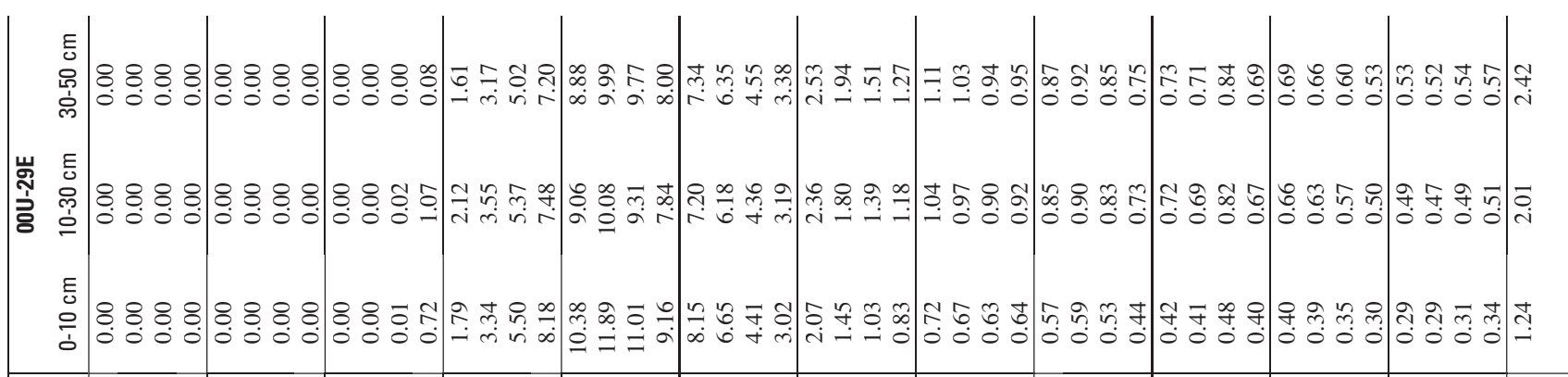

E

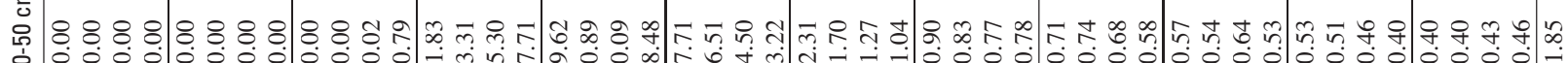

客

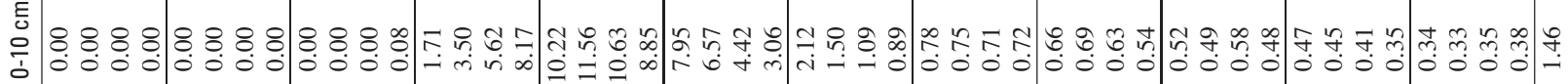

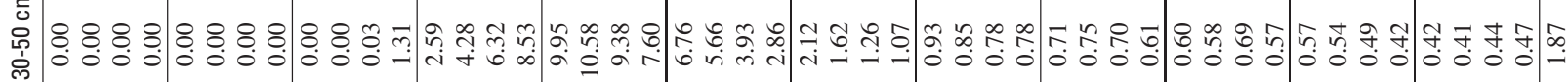

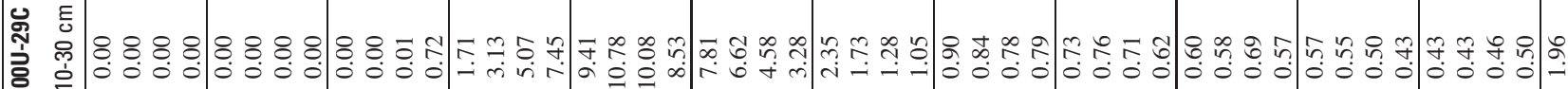

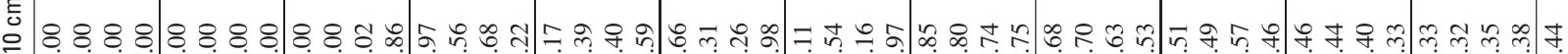

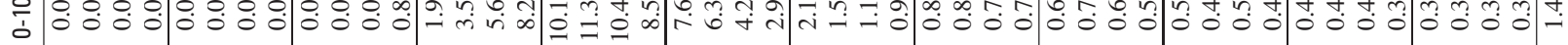

E

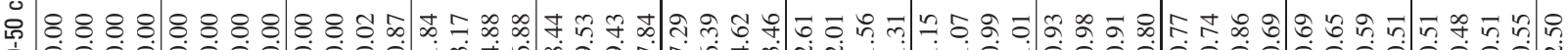

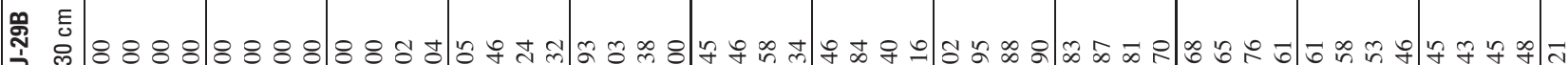

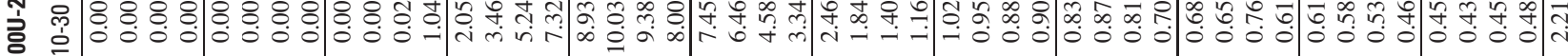

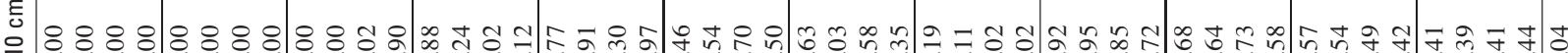

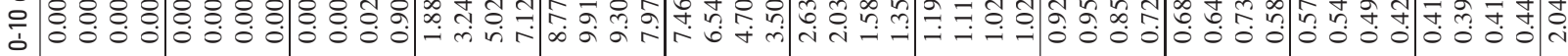

E

管

ฐ

8 8 o 8 o o

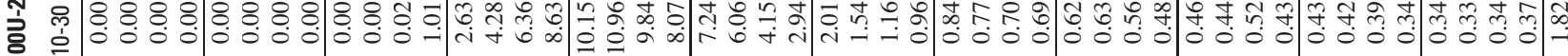
○一

竞

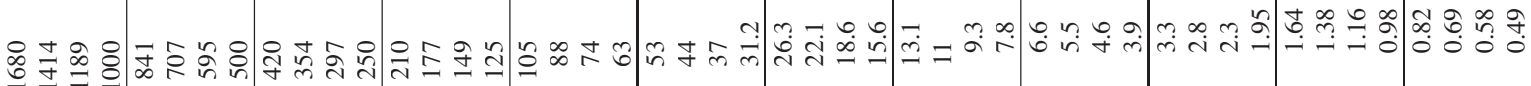

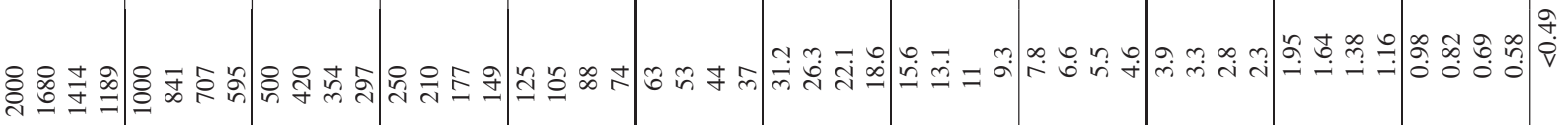

\begin{tabular}{|c|c|c|c|c|c|c|c|c|c|c|c|c|}
\hline 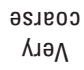 & әsıеoJ & un!рәW & әอ! & $\begin{array}{l}\text { әи!। } \\
\Lambda ! ә \Lambda\end{array}$ & әsАеo & ш!nрәW & әи! & $\begin{array}{l}\text { әи!y } \\
\Lambda\lfloor\text { ᄉ }\end{array}$ & әs.е० & un!pәW & әи! & 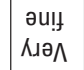 \\
\hline & & pues & & & & & & & & Ке|כ & & \\
\hline
\end{tabular}




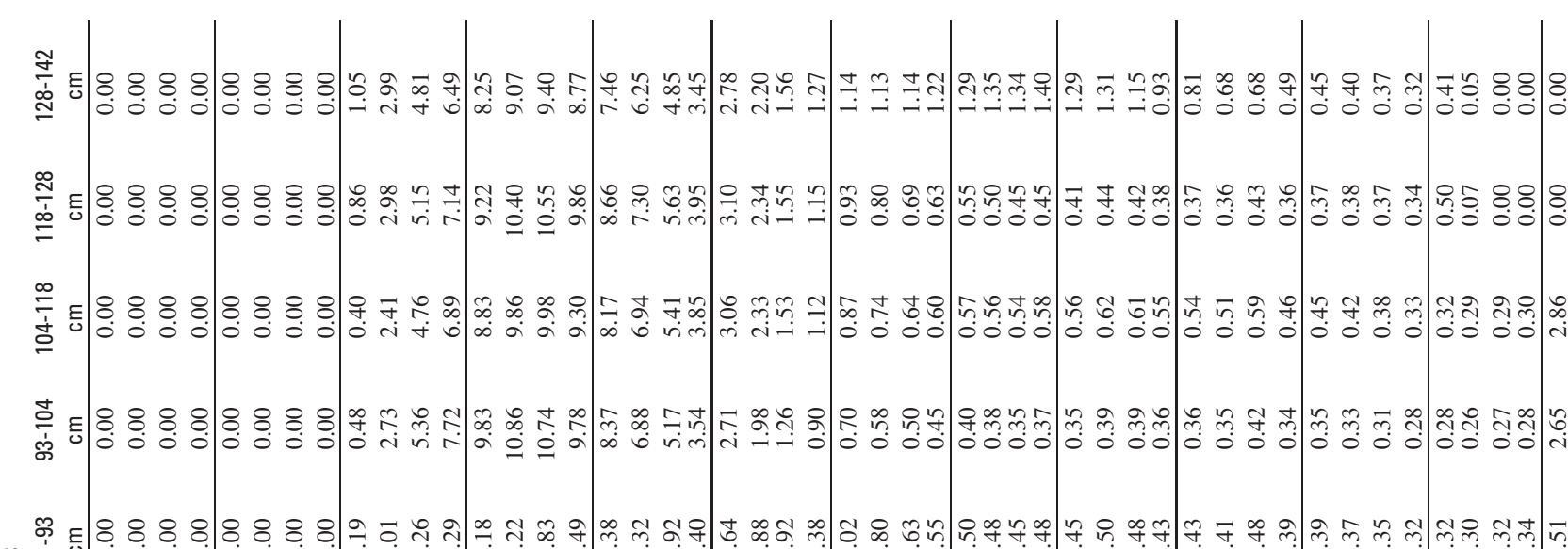

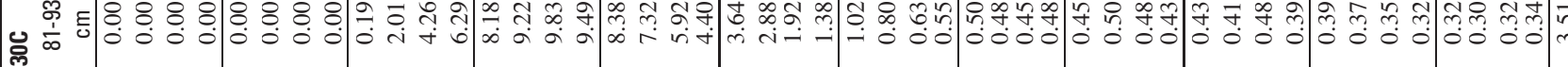
容

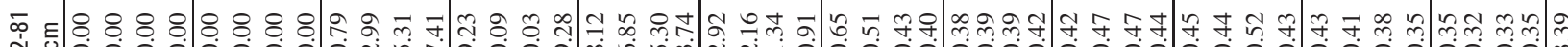
过 产

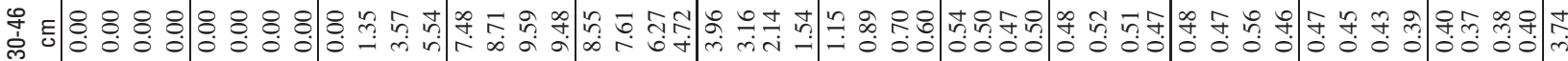
它

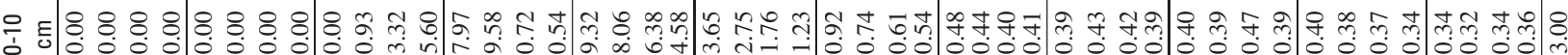

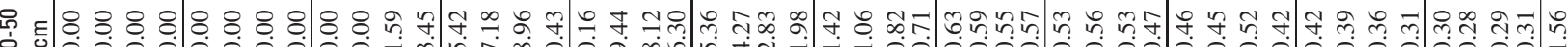

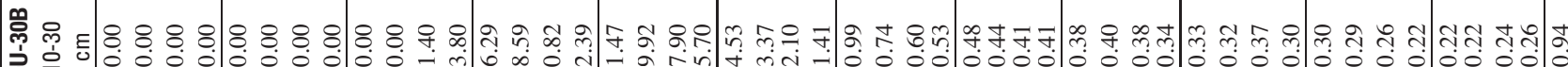
䒠白 0

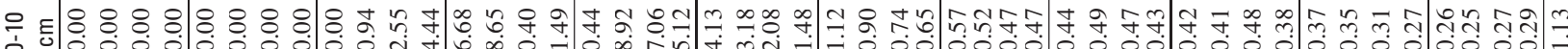

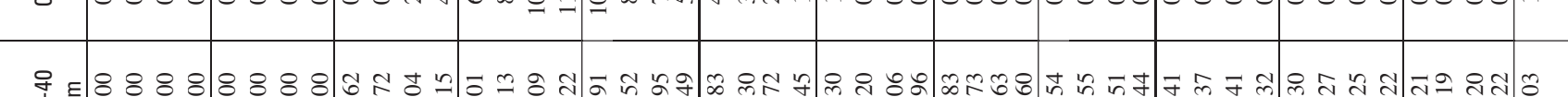
क्षे

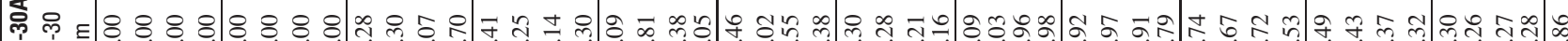

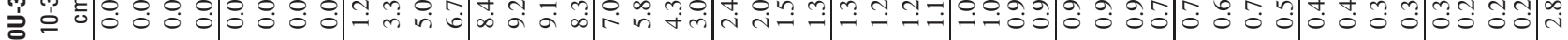

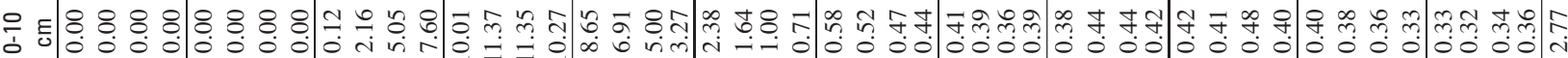

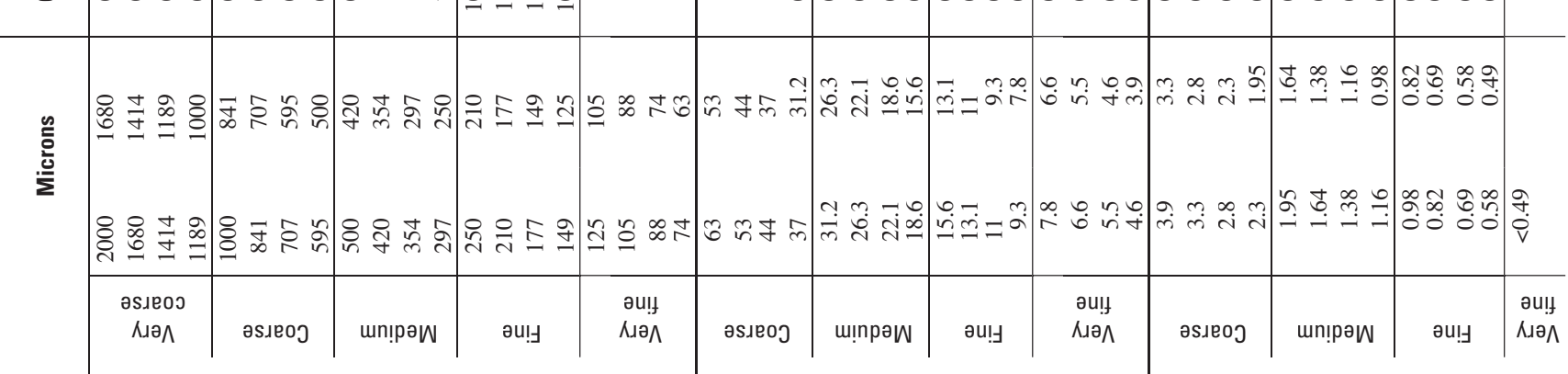
pues 


\begin{tabular}{|c|c|c|c|c|c|c|c|c|c|c|c|c|c|}
\hline 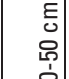 & ○̊̊ : & $\mid$ & 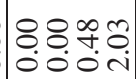 & 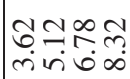 & $\begin{array}{llll}= & \infty & 0 \\
a & \infty & 0 & 0 \\
0 & \infty & 0\end{array}$ & 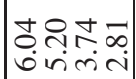 & 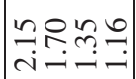 & 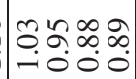 & $\left|\begin{array}{cccc} & \infty & n & \\
\infty & \infty & \infty & t \\
0 & 0 & 0 & 0 \\
0 & 0 & 0 & 0\end{array}\right|$ & 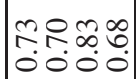 & 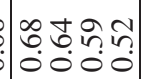 & ñ & $\frac{a}{i}$ \\
\hline 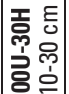 & 8.8:8 & $\begin{array}{l}888 \% \\
00000\end{array}$ & $\begin{array}{cccc}8 & 8 & 0 & 0 \\
0 & 0 & 0 & 0\end{array}$ & 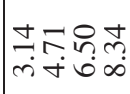 & 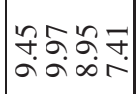 & 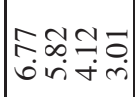 & 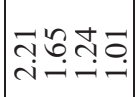 & 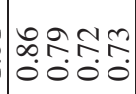 & 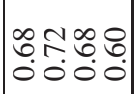 & 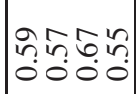 & ?: & 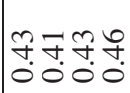 & $\frac{a}{i}$ \\
\hline 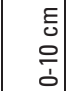 & ৪̊: & $8.8 \% 8$ & 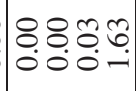 & 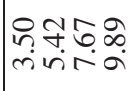 & $\begin{array}{l}m \infty n ̃ \\
=0 \\
=0\end{array}$ & 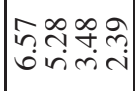 & $\mid$\begin{tabular}{lll}
0 & 0 \\
\hdashline & 0 & 0 \\
-0 & 0 & 0
\end{tabular} & 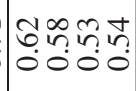 & 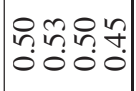 & 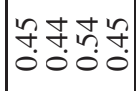 & 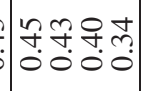 & 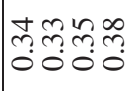 & $\stackrel{n}{n}$ \\
\hline 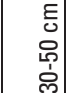 & ৪:৪ & $\begin{array}{l}888 \% \\
0000 \\
000\end{array}$ & $\mid \begin{array}{ccc}88 & 8 \\
0 & 0 & 0 \\
000 & 0\end{array}$ & 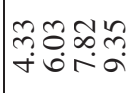 & 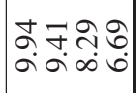 & 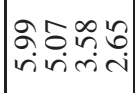 & 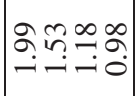 & $\begin{array}{llll}2 & n & 0 & 0 \\
0 & \ddots 0 & 0 \\
0 & 0 & 0 & 0\end{array}$ & 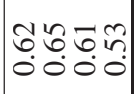 & 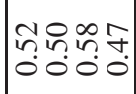 & 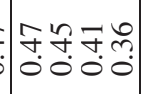 & 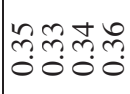 & $\sqrt[n]{?}$ \\
\hline 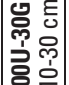 & 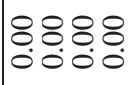 & 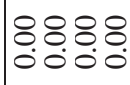 & 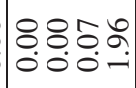 & 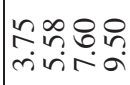 & 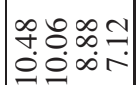 & 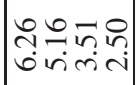 & 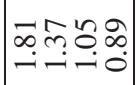 & 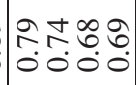 & $\left|\begin{array}{cccc} & 0 & 0 & 0 \\
0 & 0 & 0 \\
0 & 0 & 0 & 0\end{array}\right|$ & 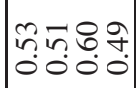 & 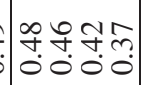 & 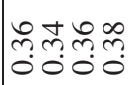 & $\stackrel{\infty}{\infty}$ \\
\hline 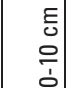 & ¿8 & 8:8:8: & $\begin{array}{llll}8 & 8 & 0 & 0 \\
0 & 0 & 0 \\
0 & 0 & 0\end{array}$ & 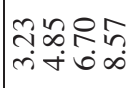 & 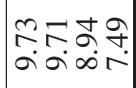 & 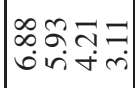 & 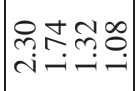 & 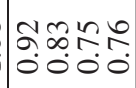 & 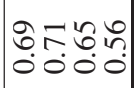 & 苼 & 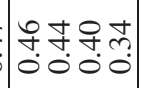 & 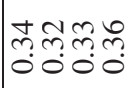 & $\stackrel{n}{n}$ \\
\hline 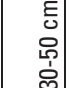 & ৪8: & 8888 & 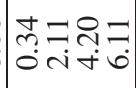 & 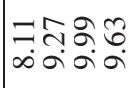 & 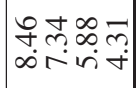 & 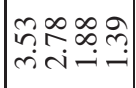 & $\mid \begin{array}{llll}\infty & 2 & 0 & 0 \\
0 & \infty & 0 & 0 \\
-0 & 0 & 0 & 0\end{array}$ & {$\left[\begin{array}{llll}0 & 0 & 0 \\
\hdashline & n & 0 & + \\
0 & 0 & 0 & 0 \\
\end{array}\right.$} & 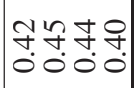 & 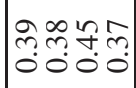 & 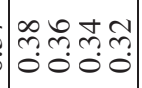 & 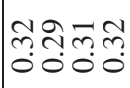 & $\vec{r}$ \\
\hline 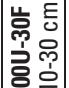 & 8:8:8: & 8:8:8: & 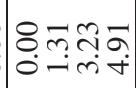 & 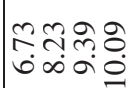 & 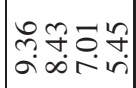 & 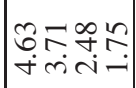 & 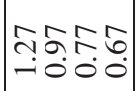 & 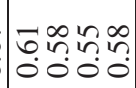 & 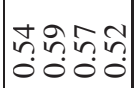 & $\mid \begin{array}{llll}n & 0 & 0 & 0 \\
n & n & 0 & 0 \\
0 & 0 & 0 & 0 \\
0\end{array}$ & 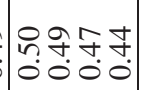 & 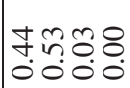 & 8 \\
\hline 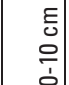 & 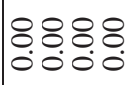 & 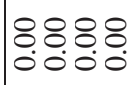 & 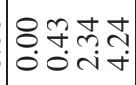 & 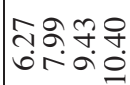 & 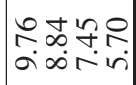 & 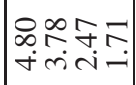 & 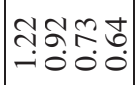 & 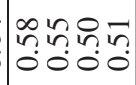 & 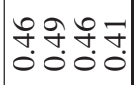 & 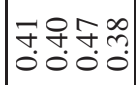 & 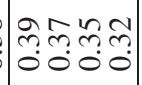 & 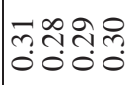 & î \\
\hline 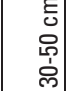 & \&̊: & \&̊: & 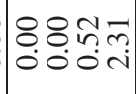 & 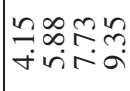 & $\begin{array}{cccc} & 0 & 0 & \infty \\
0 & + & \text { c } & n \\
0 & 0 & \infty & 0\end{array}$ & 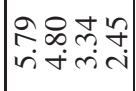 & 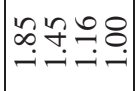 & $\mid \begin{array}{llll}\infty & 0 & N & N \\
0 & \infty & 0 & 1 \\
0 & 0 & 0 & 0 \\
0\end{array}$ & $\left|\begin{array}{llll} & 0 & 0 \\
0 & 0 & 0 & 0 \\
0 & 0 & 0 & 0 \\
0 & 0 & 0\end{array}\right|$ & 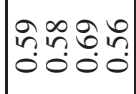 & 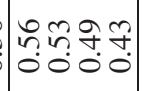 & 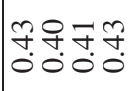 & $\stackrel{8}{i}$ \\
\hline 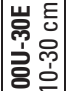 & 8.8:8: & 8.88 .8 & 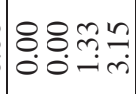 & 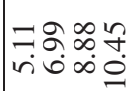 & 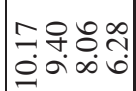 & 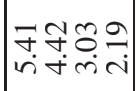 & 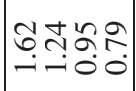 & 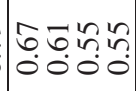 & 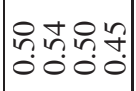 & 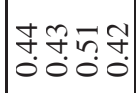 & 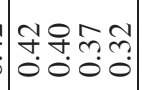 & 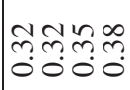 & $\stackrel{n}{n}$ \\
\hline $\begin{array}{l}\frac{E}{0} \\
0 \\
0\end{array}$ & 8:8:8: & 8.8:8 & 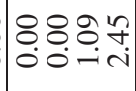 & 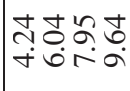 & 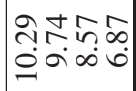 & 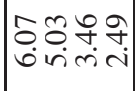 & 車 & 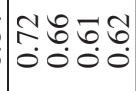 & 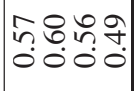 & 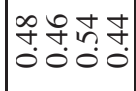 & 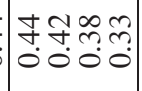 & 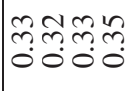 & ले \\
\hline 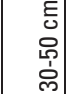 & $\begin{array}{l}8.8 \% \\
0.00 \\
000\end{array}$ & $\begin{array}{l}8888 \\
0 \\
0\end{array}$ & 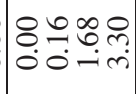 & 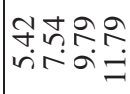 & 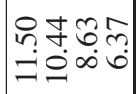 & 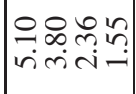 & 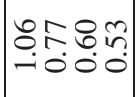 & fr. & 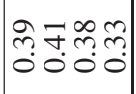 & 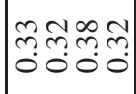 & 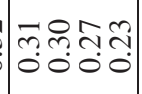 & 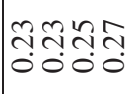 & $\infty$ \\
\hline 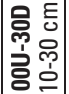 & ৪̊: & ¿̊: & 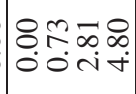 & 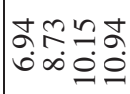 & 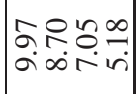 & 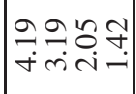 & 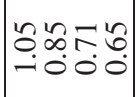 & ṇ̣̃̂ & ケัr & 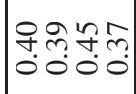 & 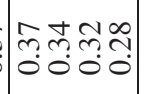 & 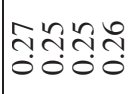 & ì \\
\hline $\begin{array}{l}\frac{E}{0} \\
0 \\
0\end{array}$ & \&̊̊ & :8:8 & 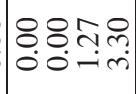 & 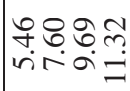 & 우옹 & 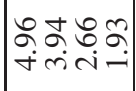 & 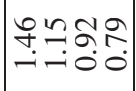 & 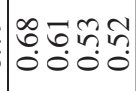 & 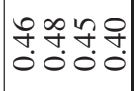 & 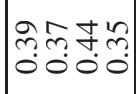 & mิ & 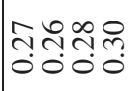 & $\stackrel{n}{=}$ \\
\hline 黑 & \& & ‡숭ํํㅇํำ & $\begin{array}{l}\text { ํํํํำ } \\
\text { ชñ }\end{array}$ & 으솜의 & 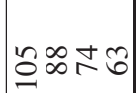 & 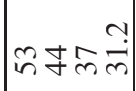 & 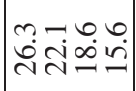 & $\vec{m}=\stackrel{m}{\sim} \stackrel{\infty}{r}$ & 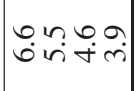 & må & 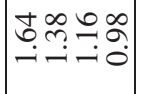 & 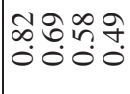 & \\
\hline$\Sigma$ & 8̊유: & 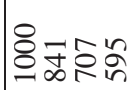 & ร̊ำษ & 을을워 & 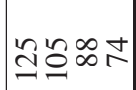 & ming & 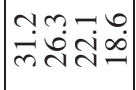 & $\ddot{\ddot{n}}=\ddot{\sigma}$ & 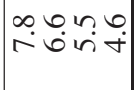 & mim mon & 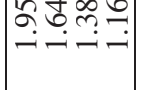 & 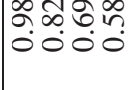 & 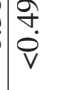 \\
\hline & 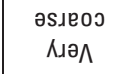 & əs & un!pәw & әи!」 & $\begin{array}{l}\text { әи!! } \\
\text { ᄉә ^ }\end{array}$ & әsıеoj & щ!nрәш & әи!」 & 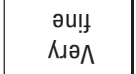 & әsıeoj & un!pәW & әи!」 & $\begin{array}{l}\text { әи! } \\
\text { Кәә }\end{array}$ \\
\hline & & & p & & & & & & & & 1) & & \\
\hline
\end{tabular}




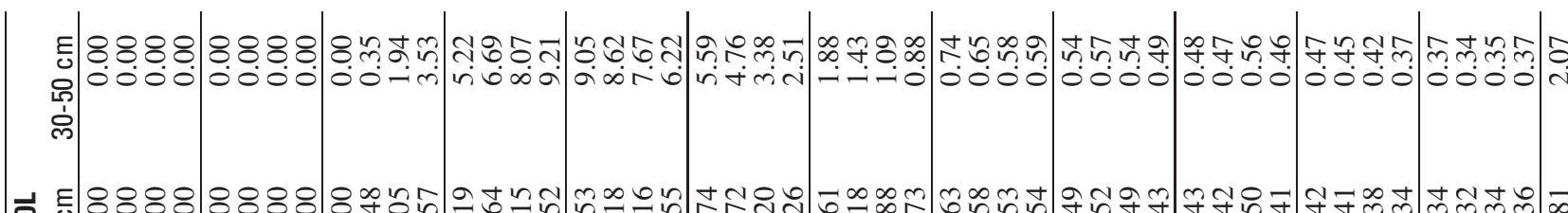

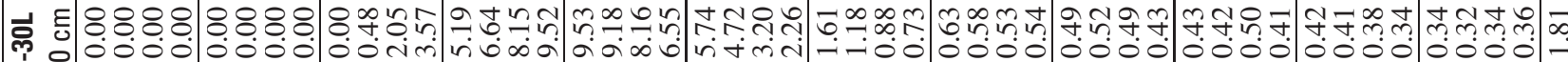
字 官

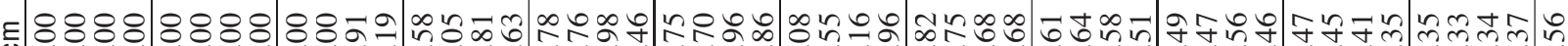

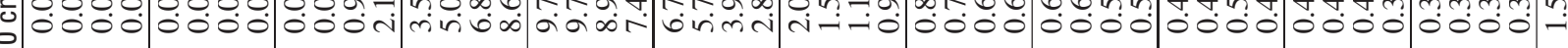
¿े

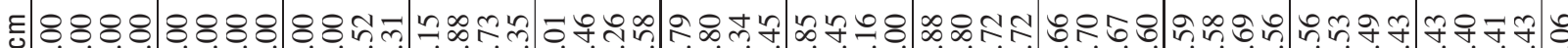

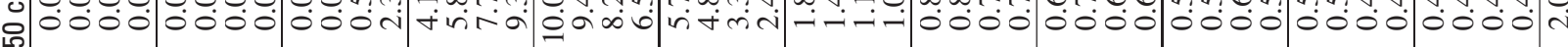
官

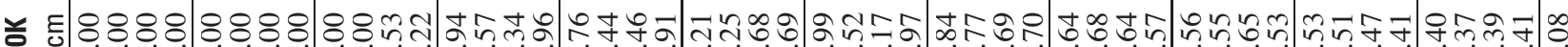

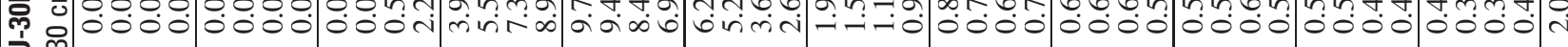
흥

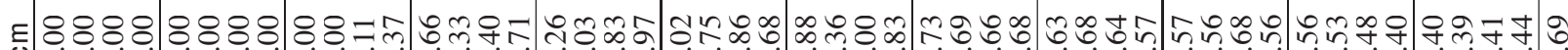

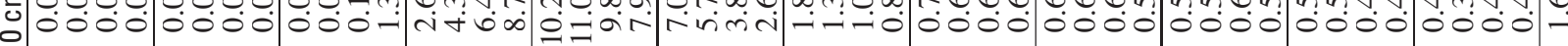
○े

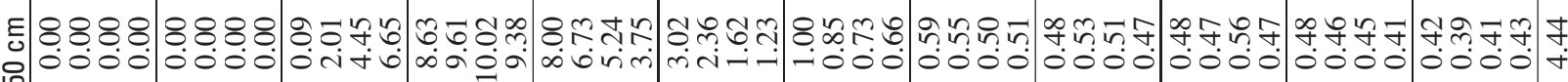
宛

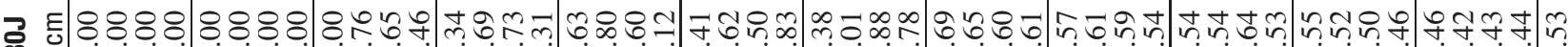
m. 홍

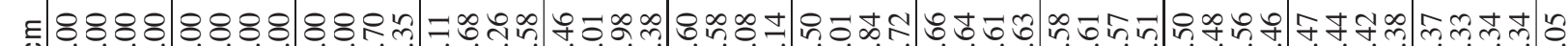

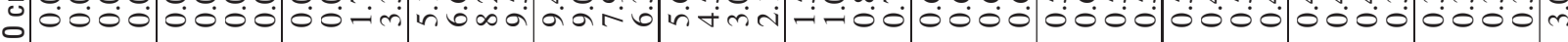
웅

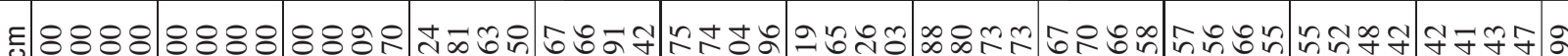

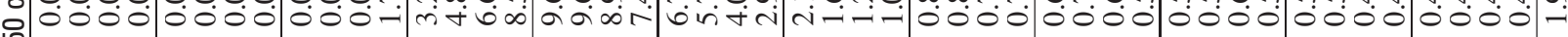
ले

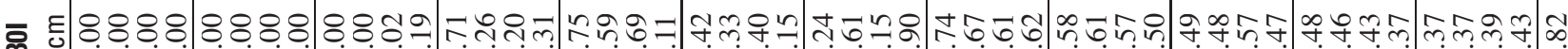

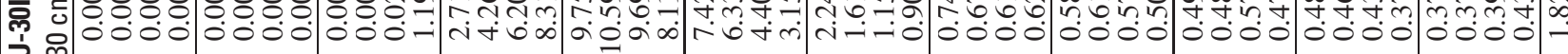
홍

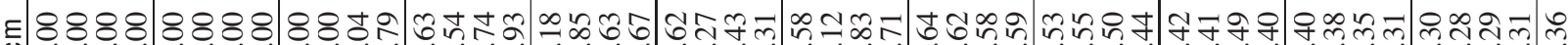

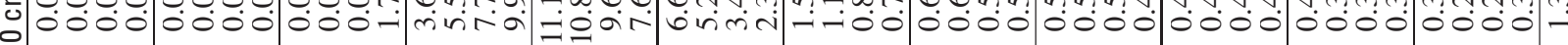
¿े

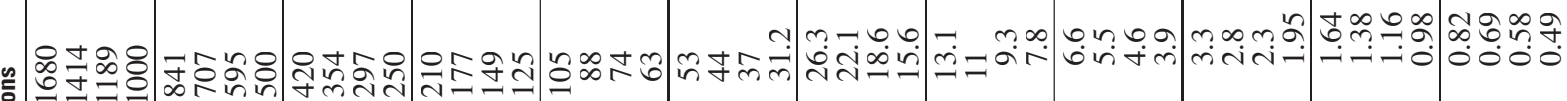
产

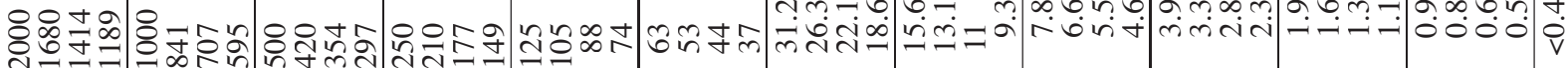

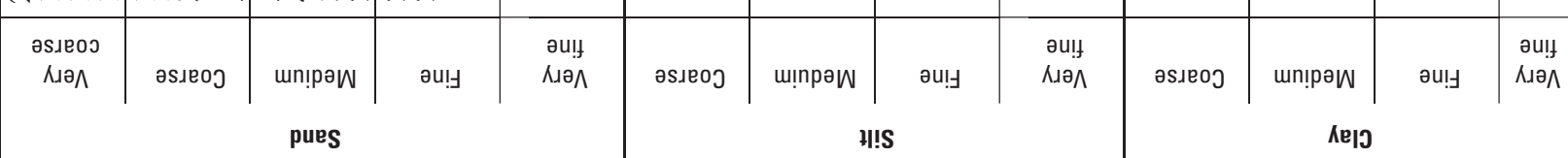




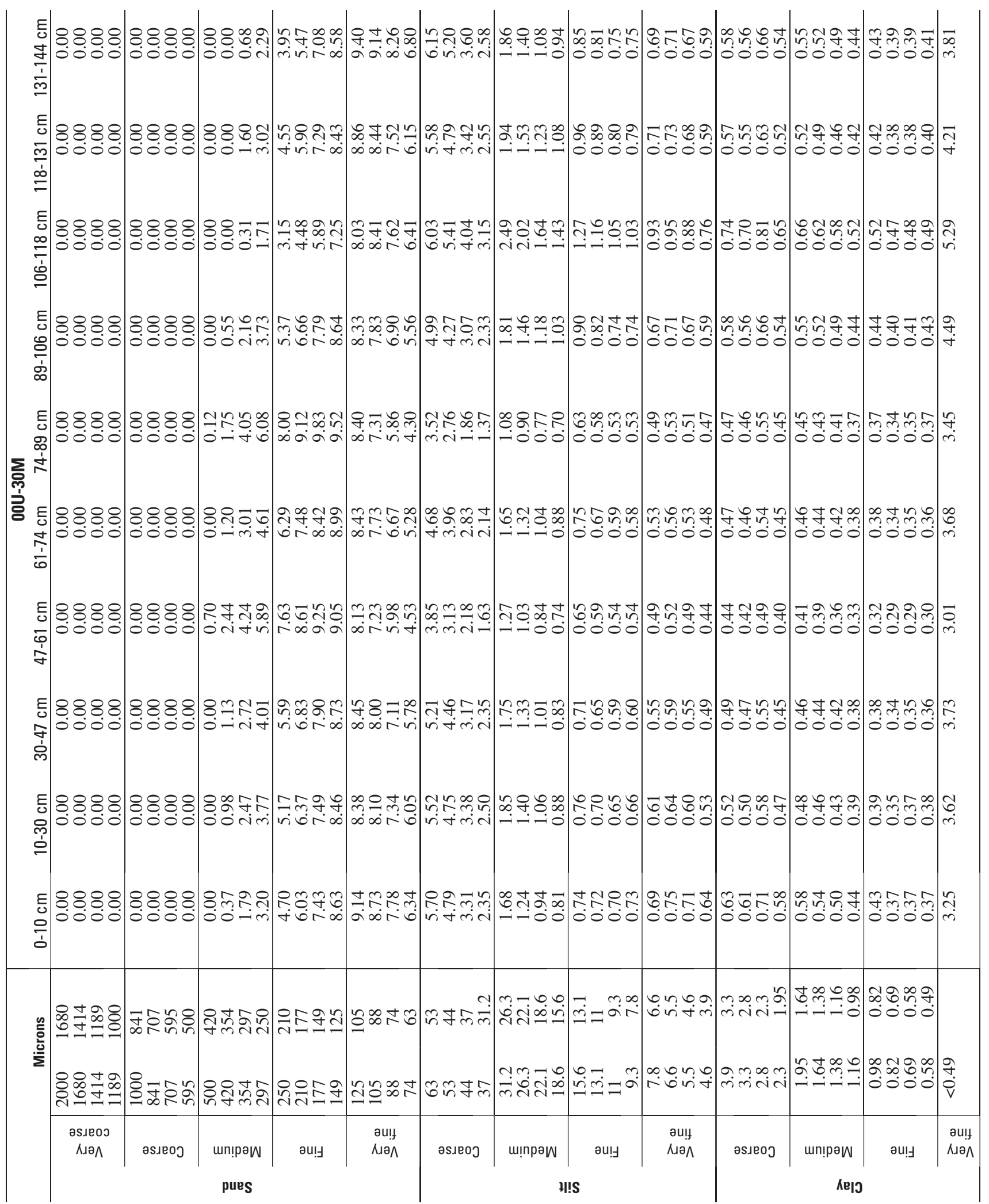




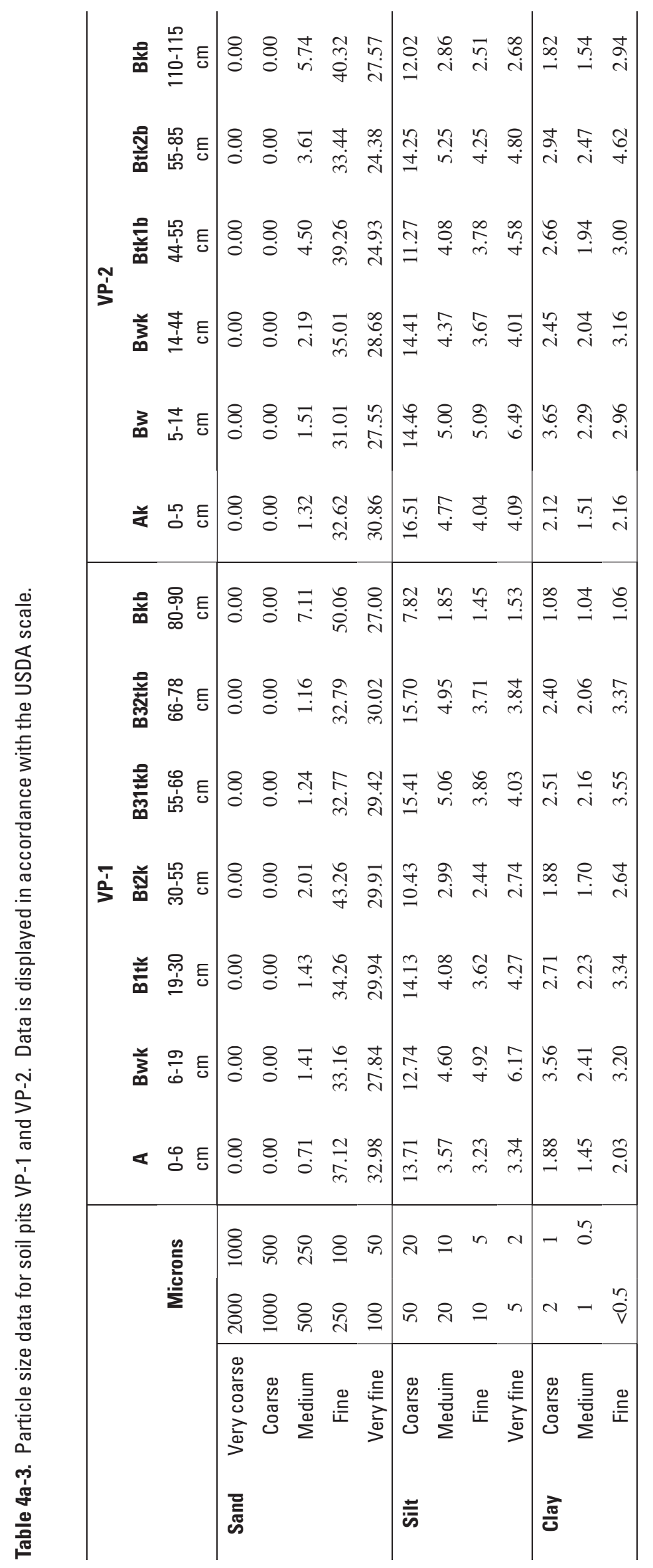




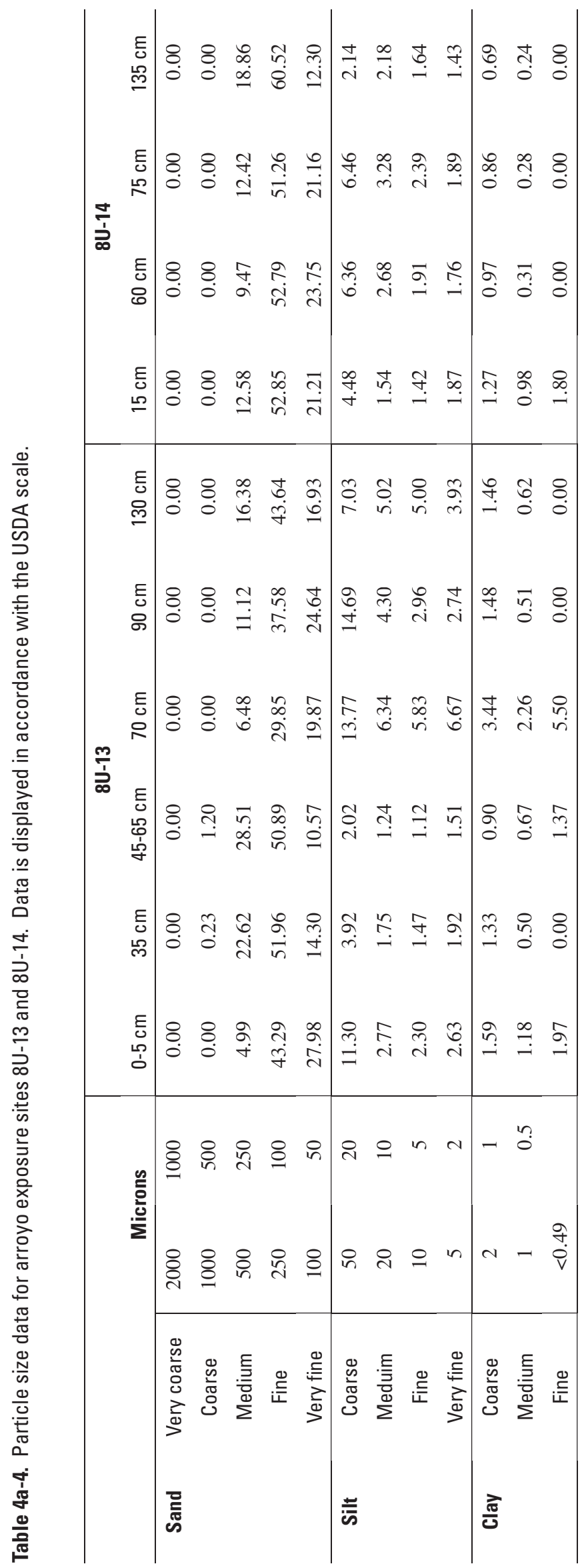


它

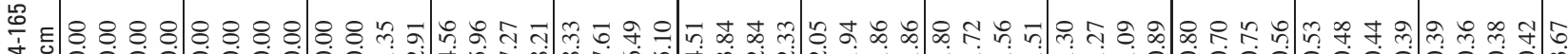

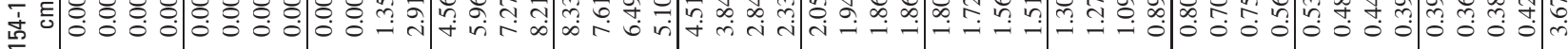
苟劳

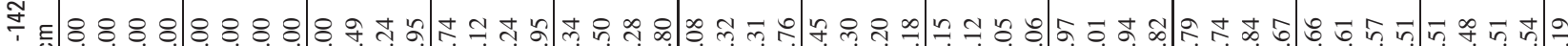

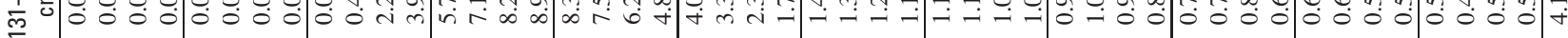

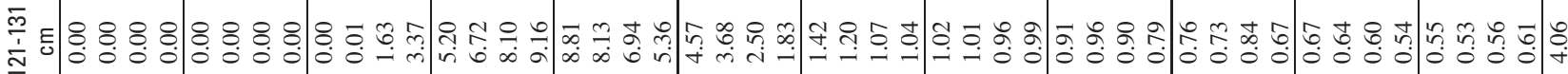

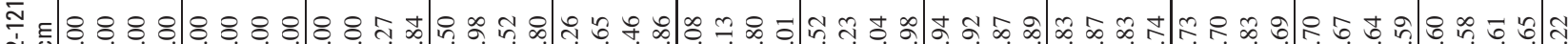
a

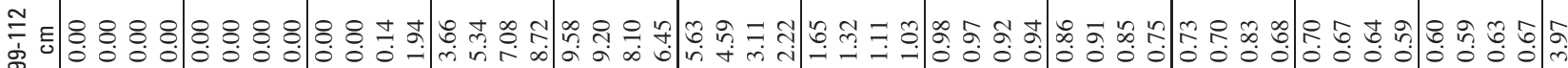

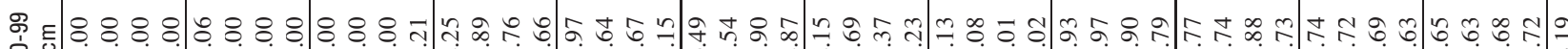
웋

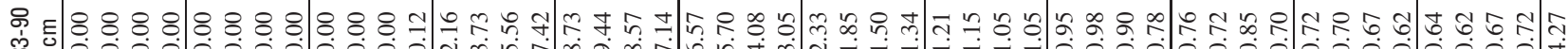

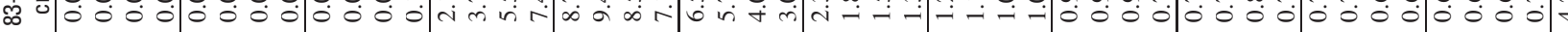

咅

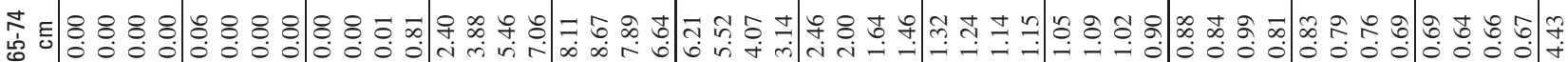

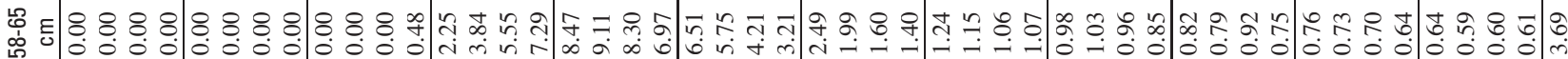

采

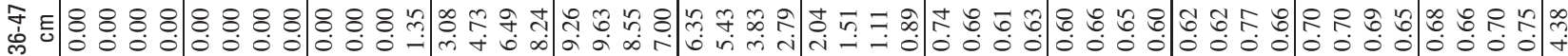

离

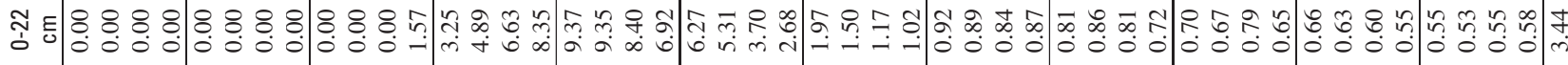

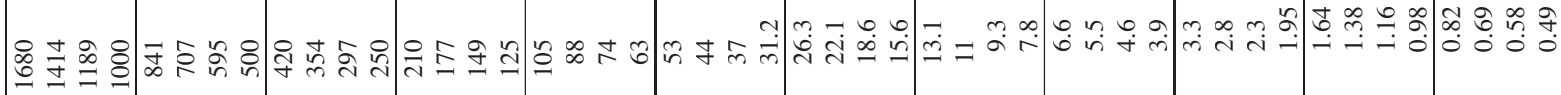

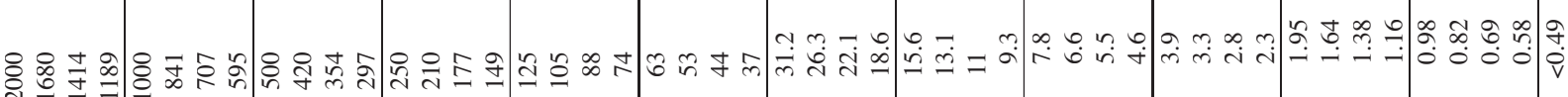

\begin{tabular}{|c|c|c|c|c|c|c|c|c|c|c|c|c|}
\hline & & & & & & & & & & & & \\
\hline $\begin{array}{c}\text { әs.еоo } \\
\text { КґәА }\end{array}$ & әs.ео & un!pәw & әи! & $\begin{array}{l}\text { әи!। } \\
\text { Кıә }\end{array}$ & әs.eoo & ш!̣рәш & әи! & 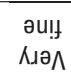 & әs.еoj & un!pәw & әи! & 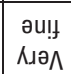 \\
\hline & & pues & & & & & & & & Kel| & & \\
\hline
\end{tabular}




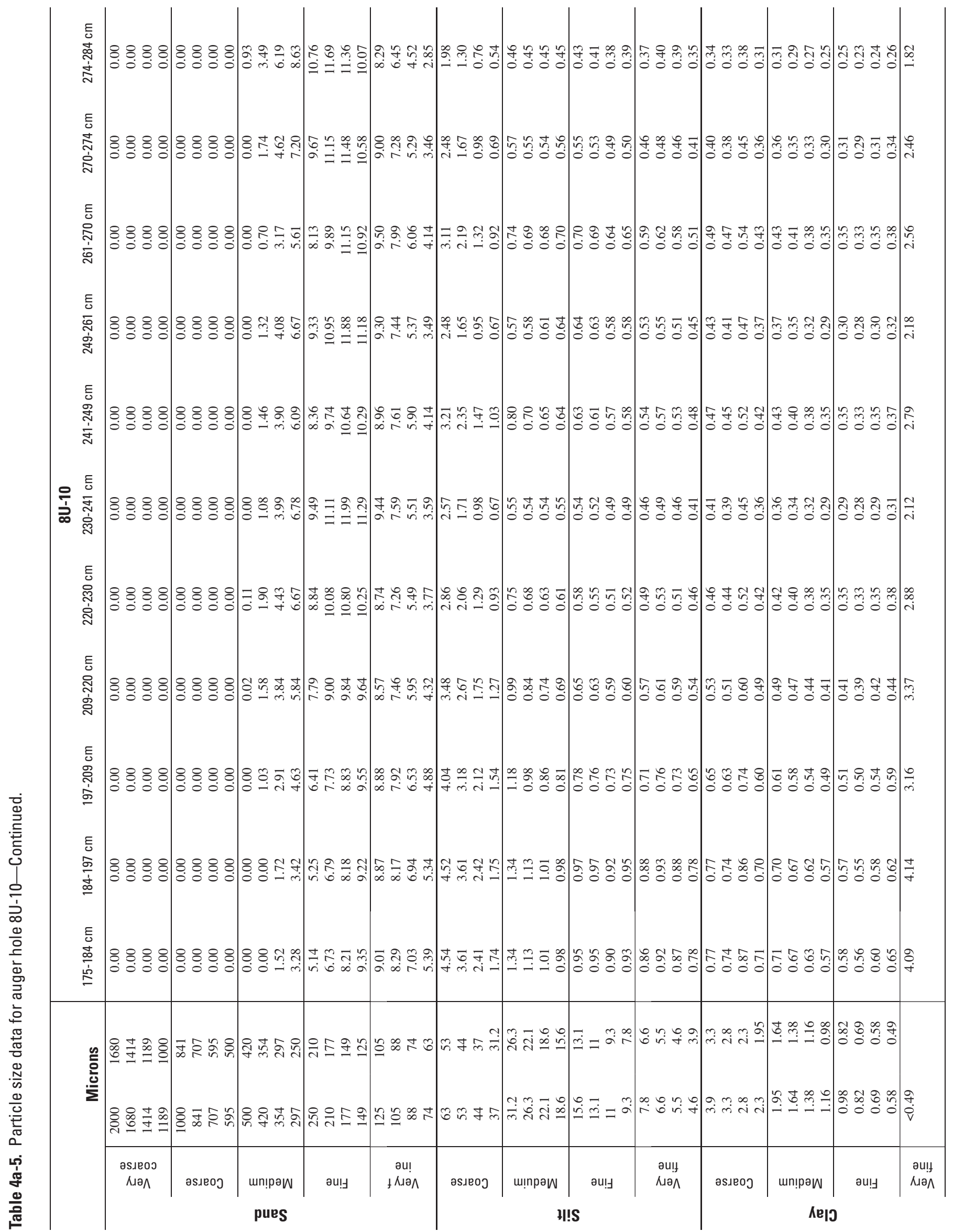




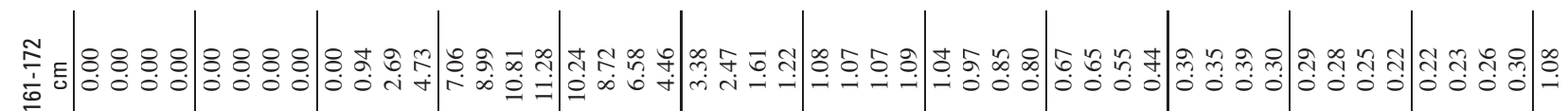

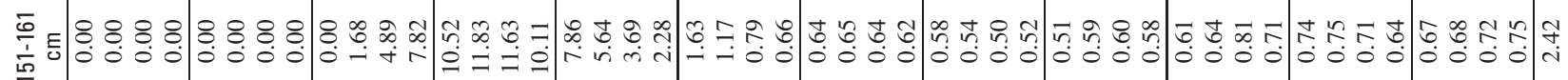
留 m $\bar{m}$
$\overline{1}$ m

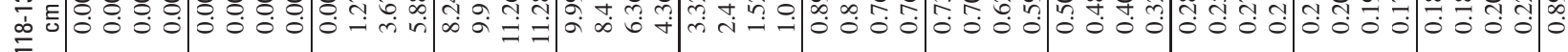
审

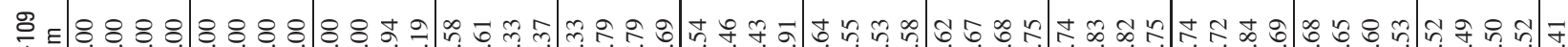
‡
\end{abstract}

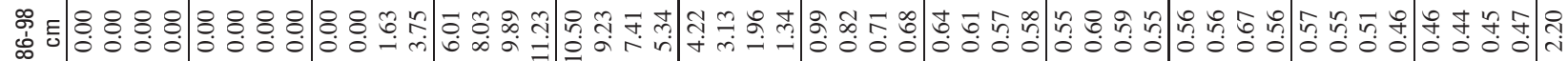

ஜ

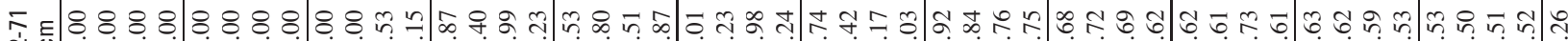
गु่

గ্ৰ

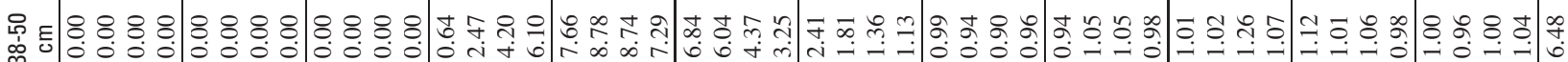

岪

స

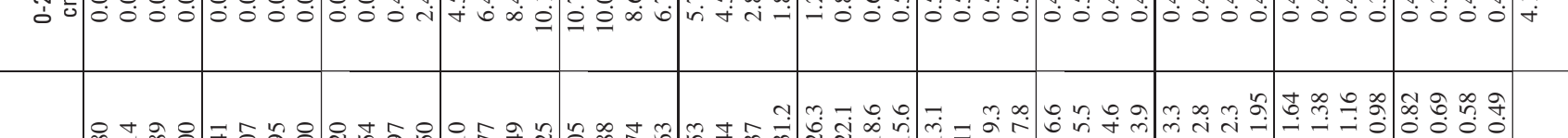

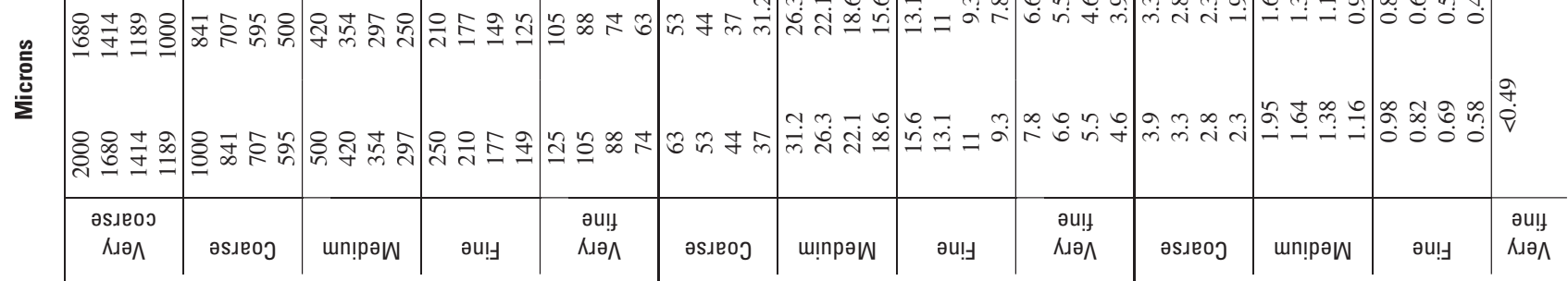

pues 


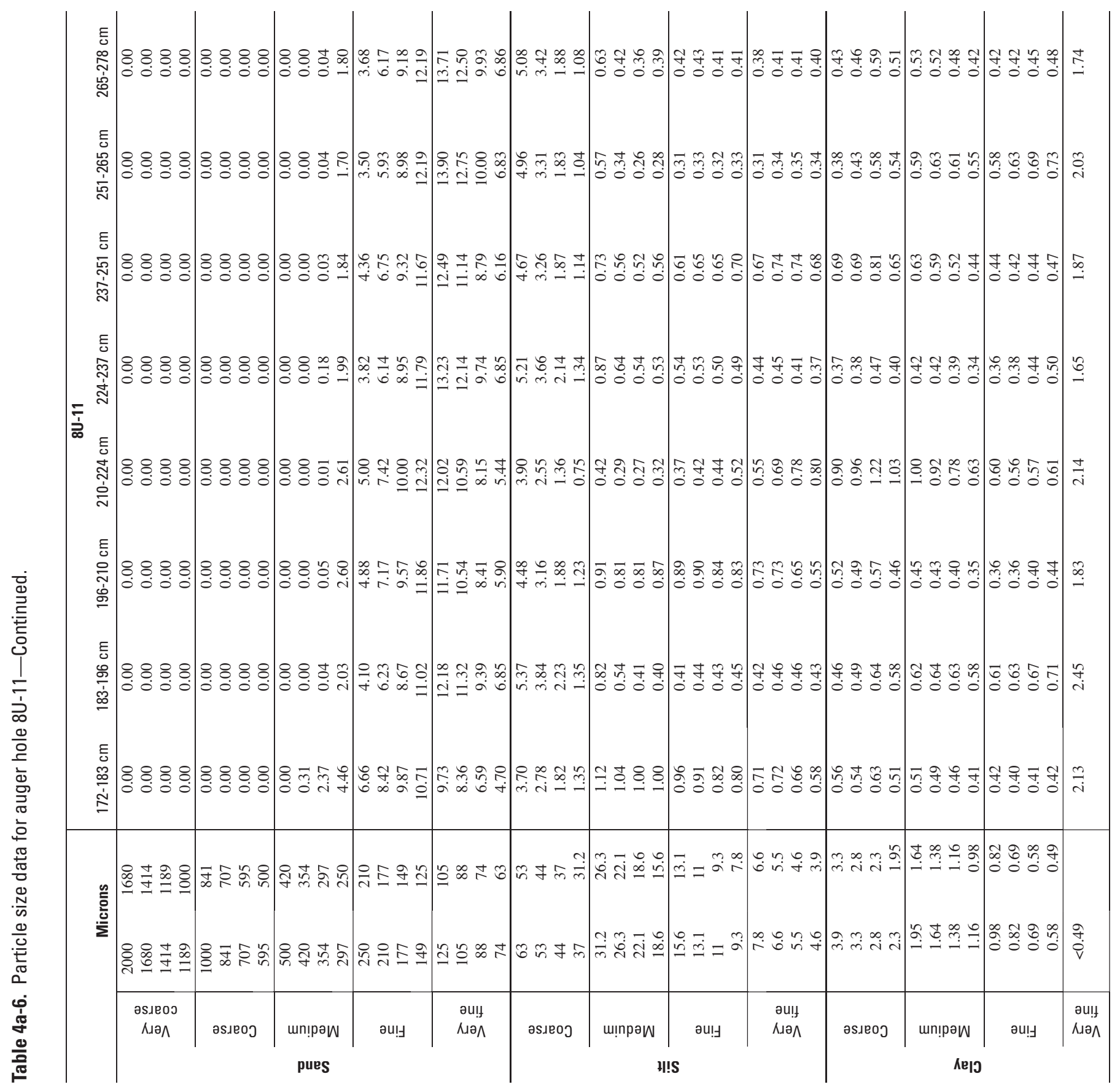




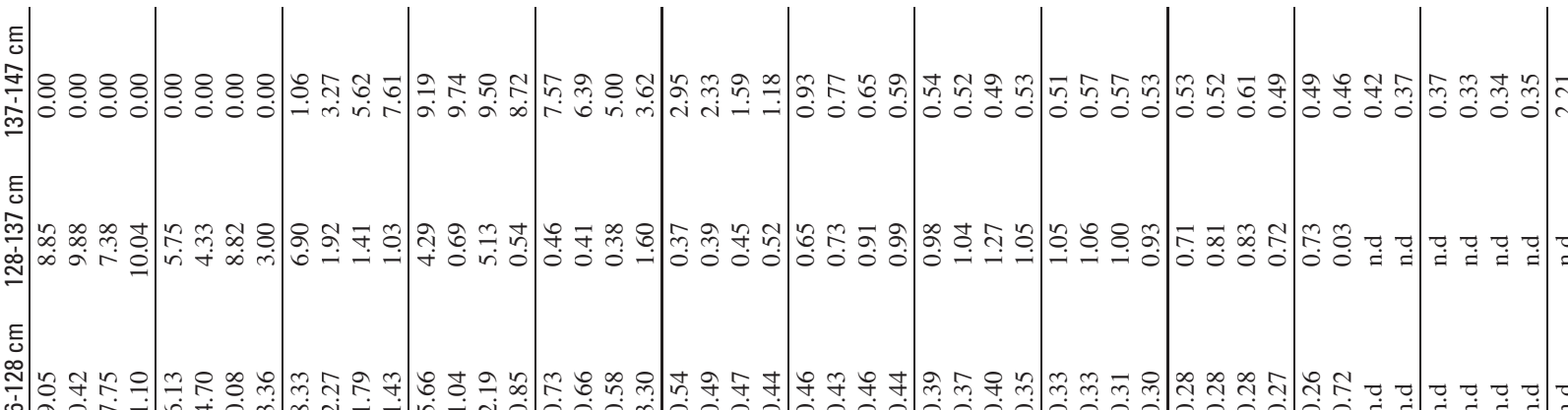

E

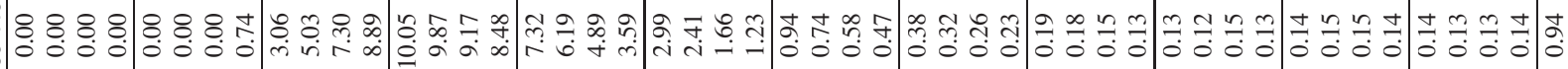

छ

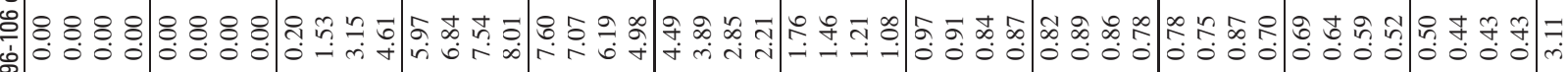

巨.

:

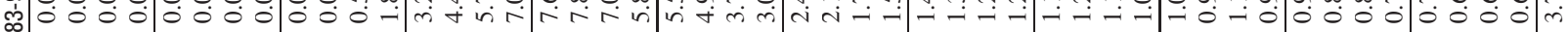

$\simeq$ 은

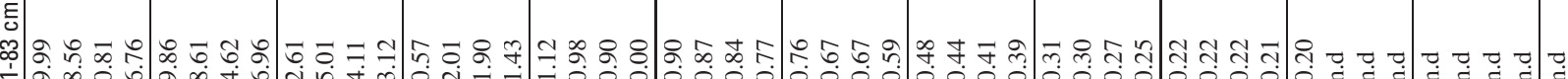

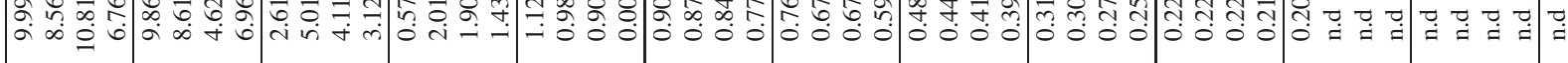

E

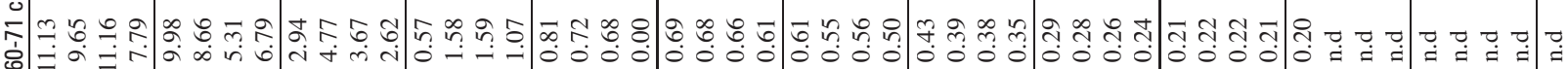

둥

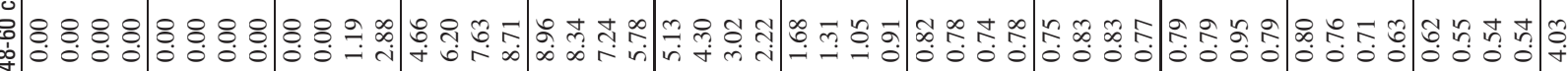

등

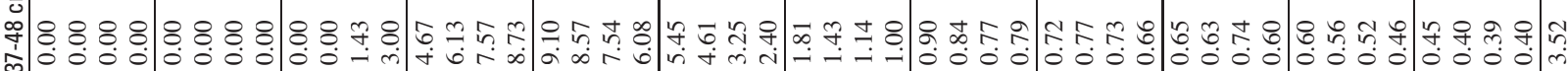

등

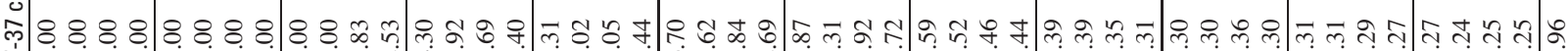

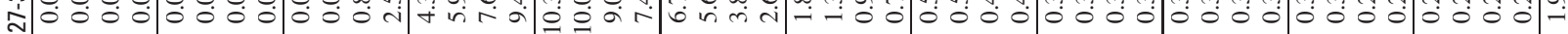

E్

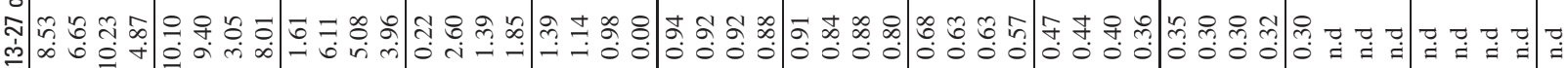

है

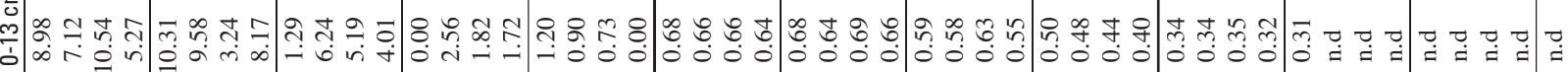

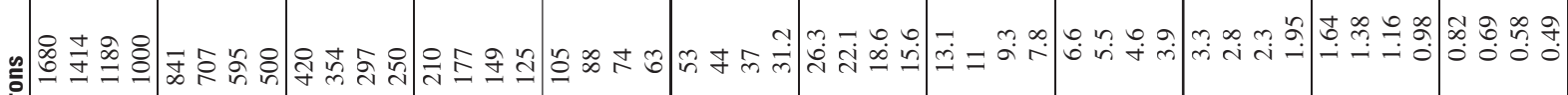

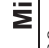

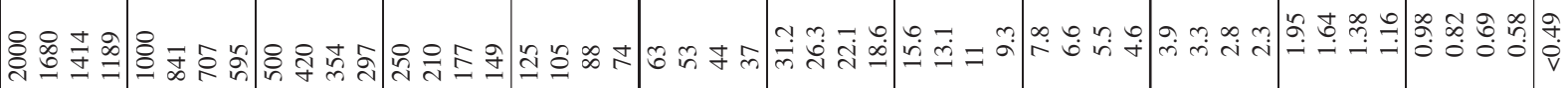

\begin{tabular}{|c|c|c|c|c|c|c|c|c|c|c|c|c|}
\hline 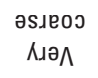 & әsıеoj & un!pәW & әи! & 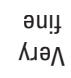 & әs.е० & ш!nрәW & әи! & 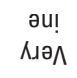 & әs.ео & un!pəw & әи! & \\
\hline & & pues & & & & & & & & & & \\
\hline
\end{tabular}




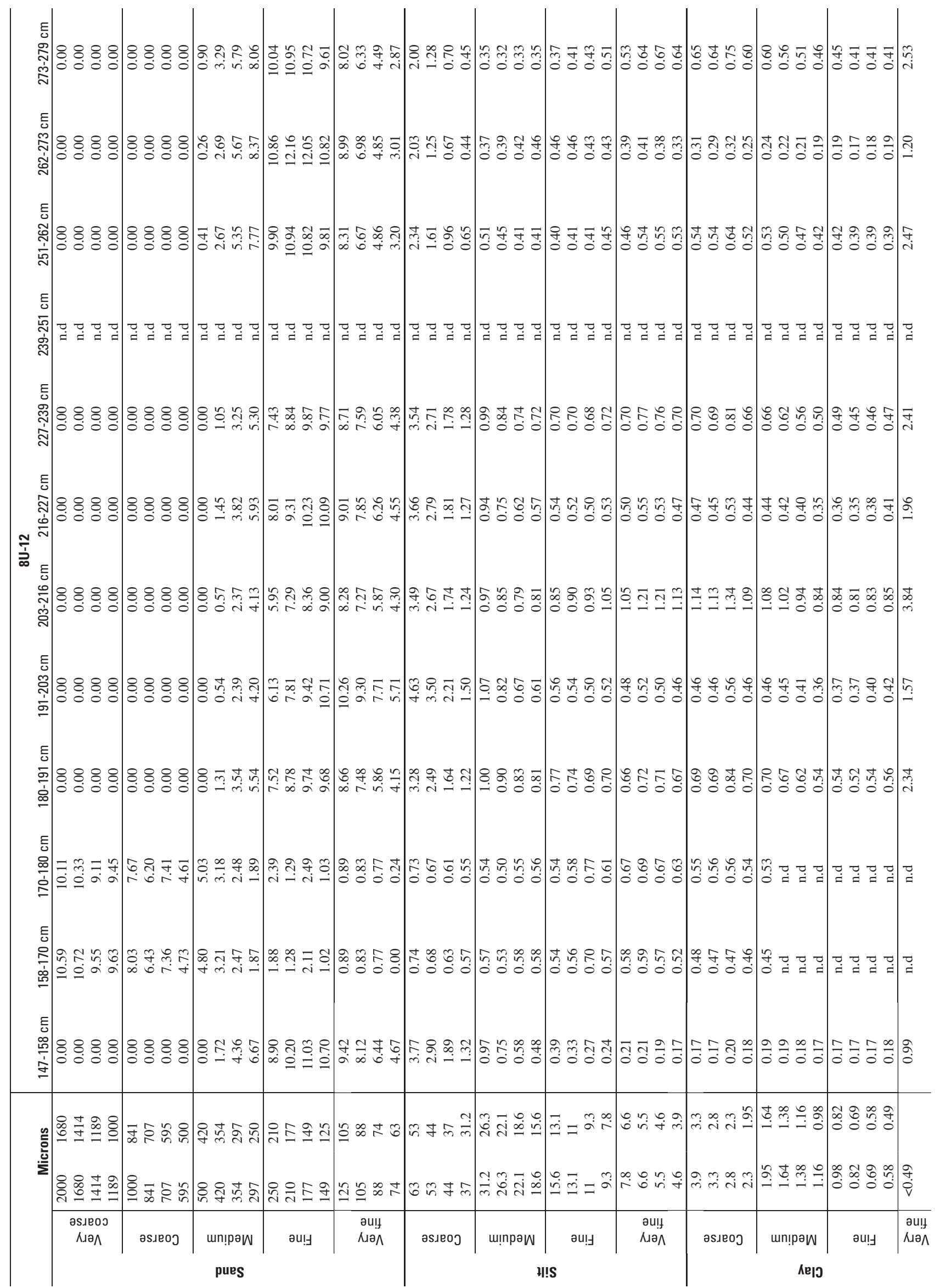




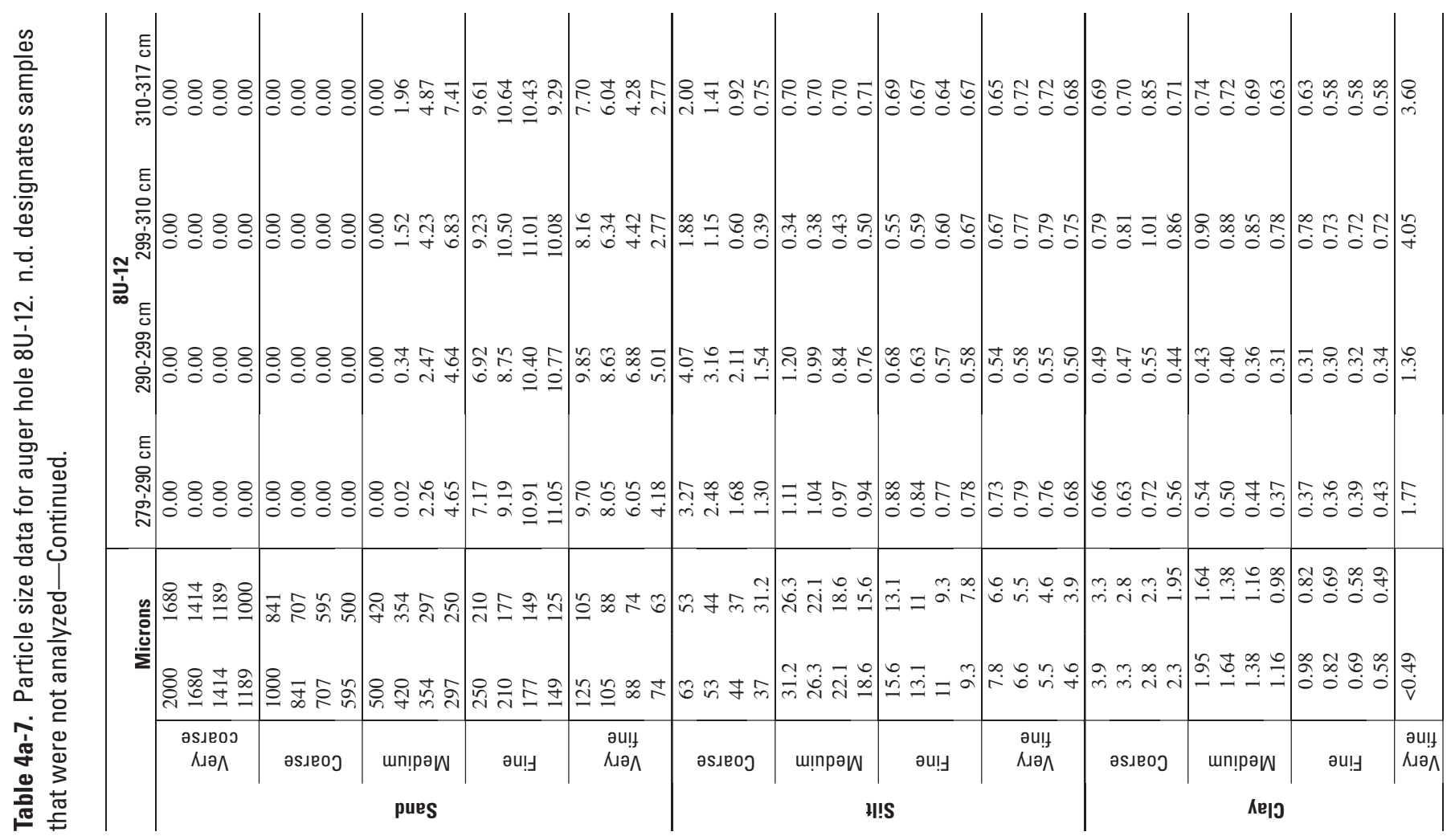


党 言 菅

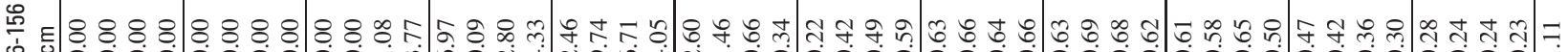

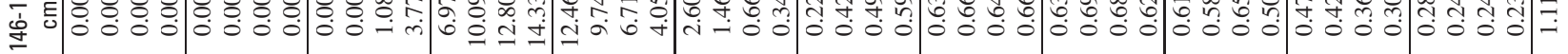
定 车 吾 䒧 운

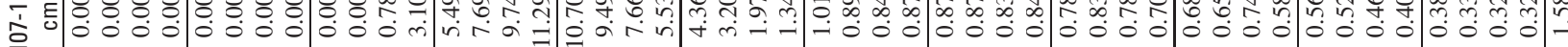

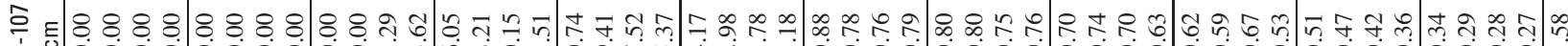

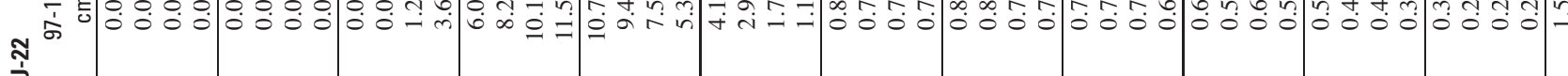
के

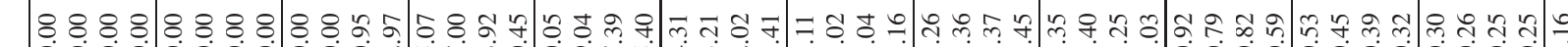

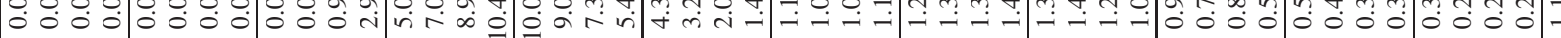

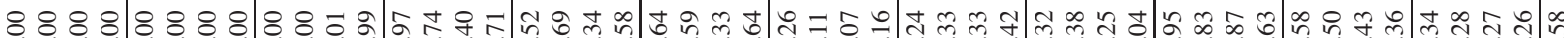

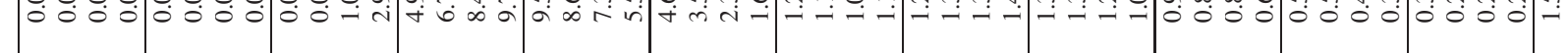

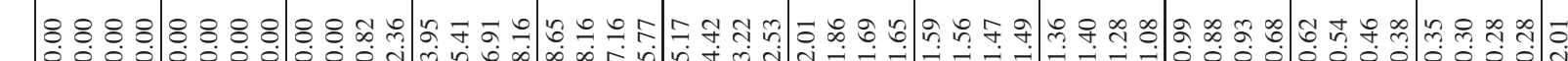

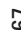

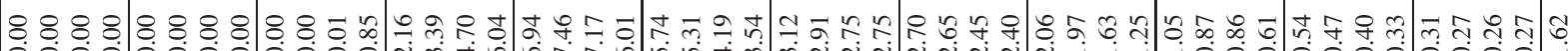
ֻ

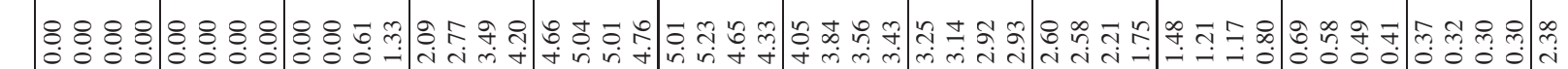

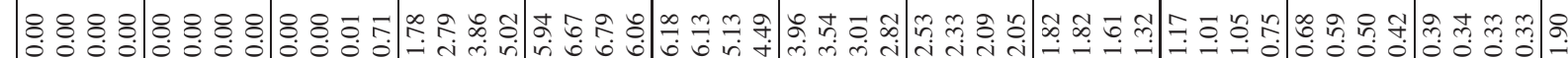

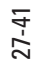
E

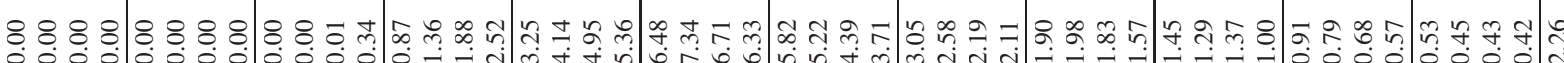

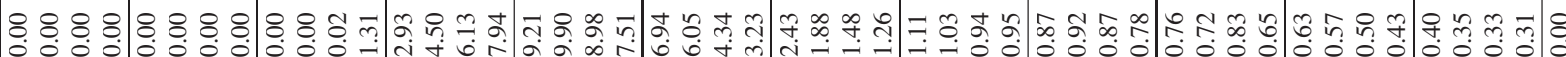

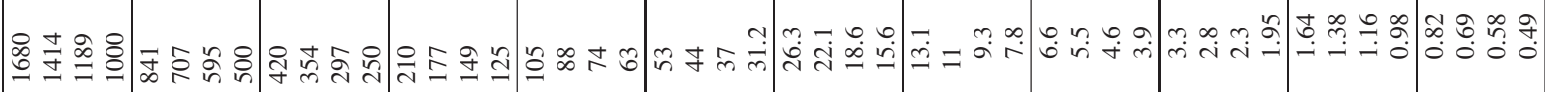
$\stackrel{\circ}{\Sigma}$

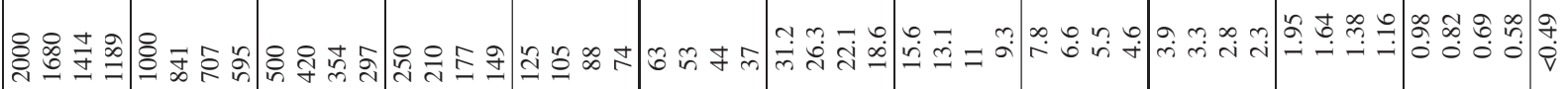

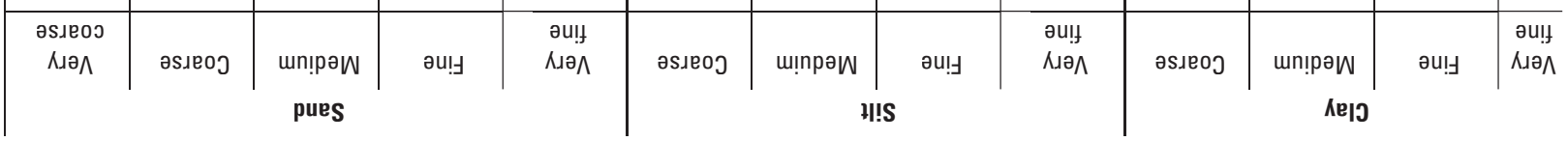


Table 4a-8. Particle size data for auger hole 9U-22—Continued.

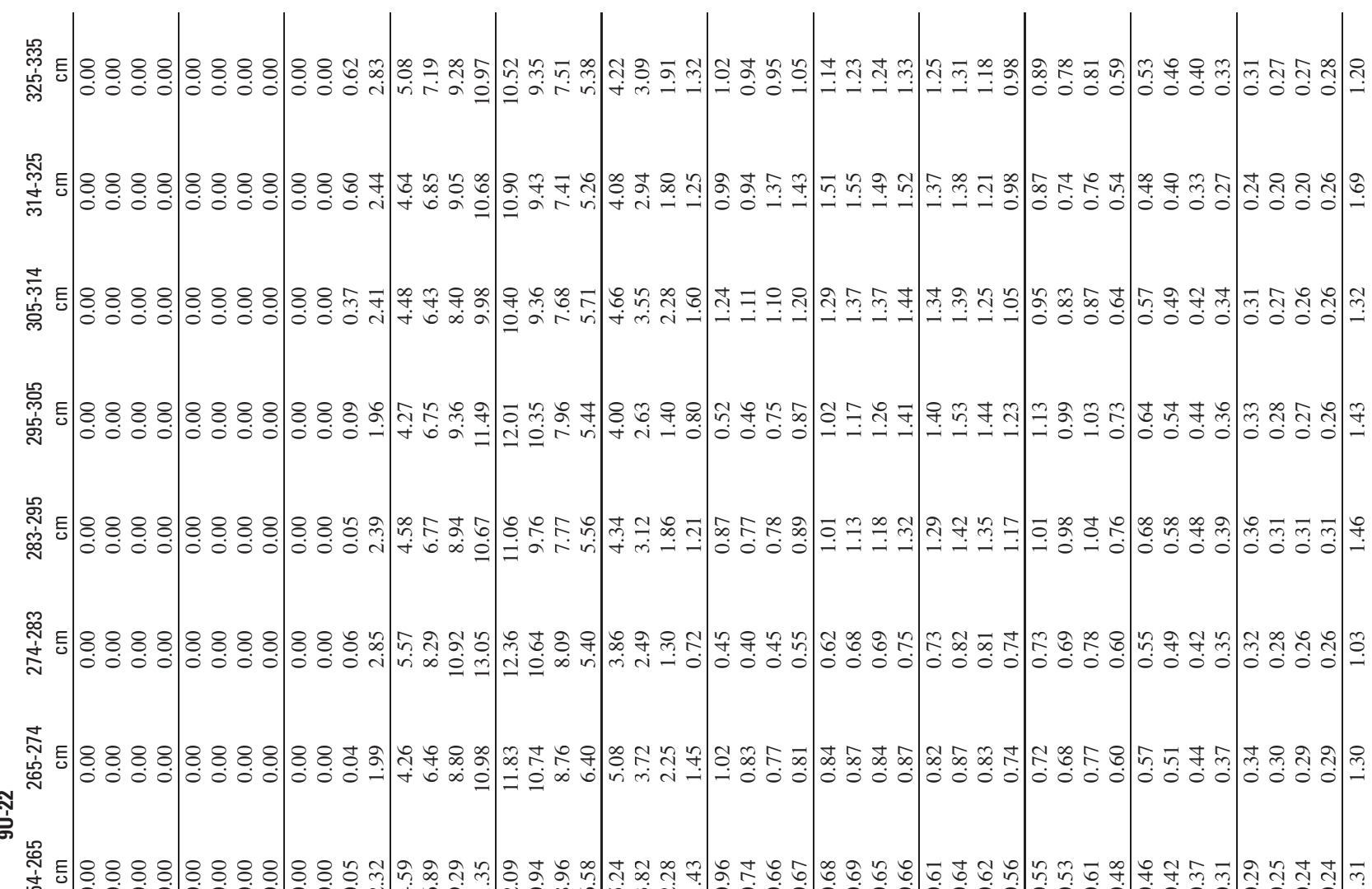

热 崔

竎 先 0 光

商

苛

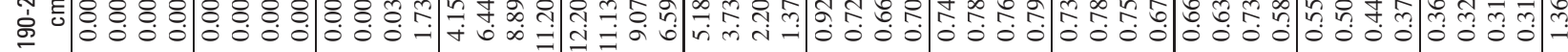

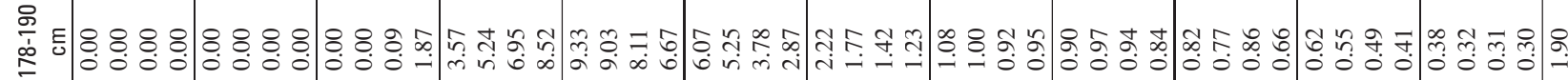

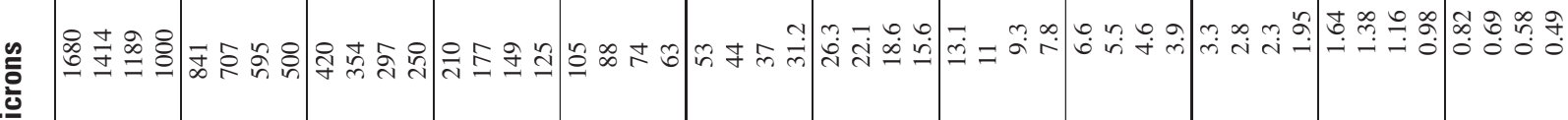

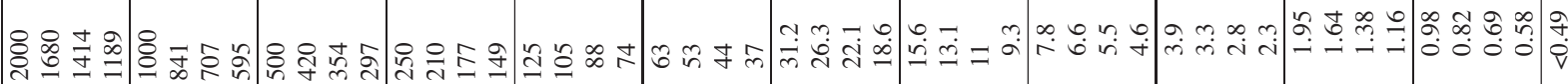

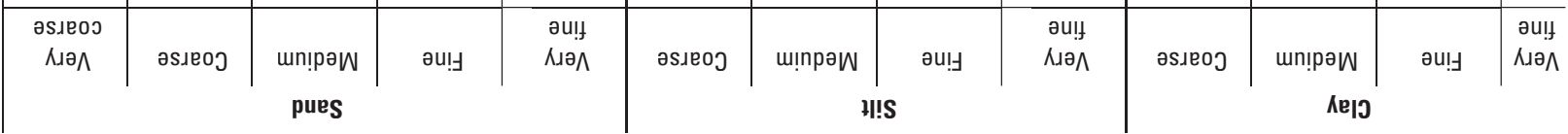




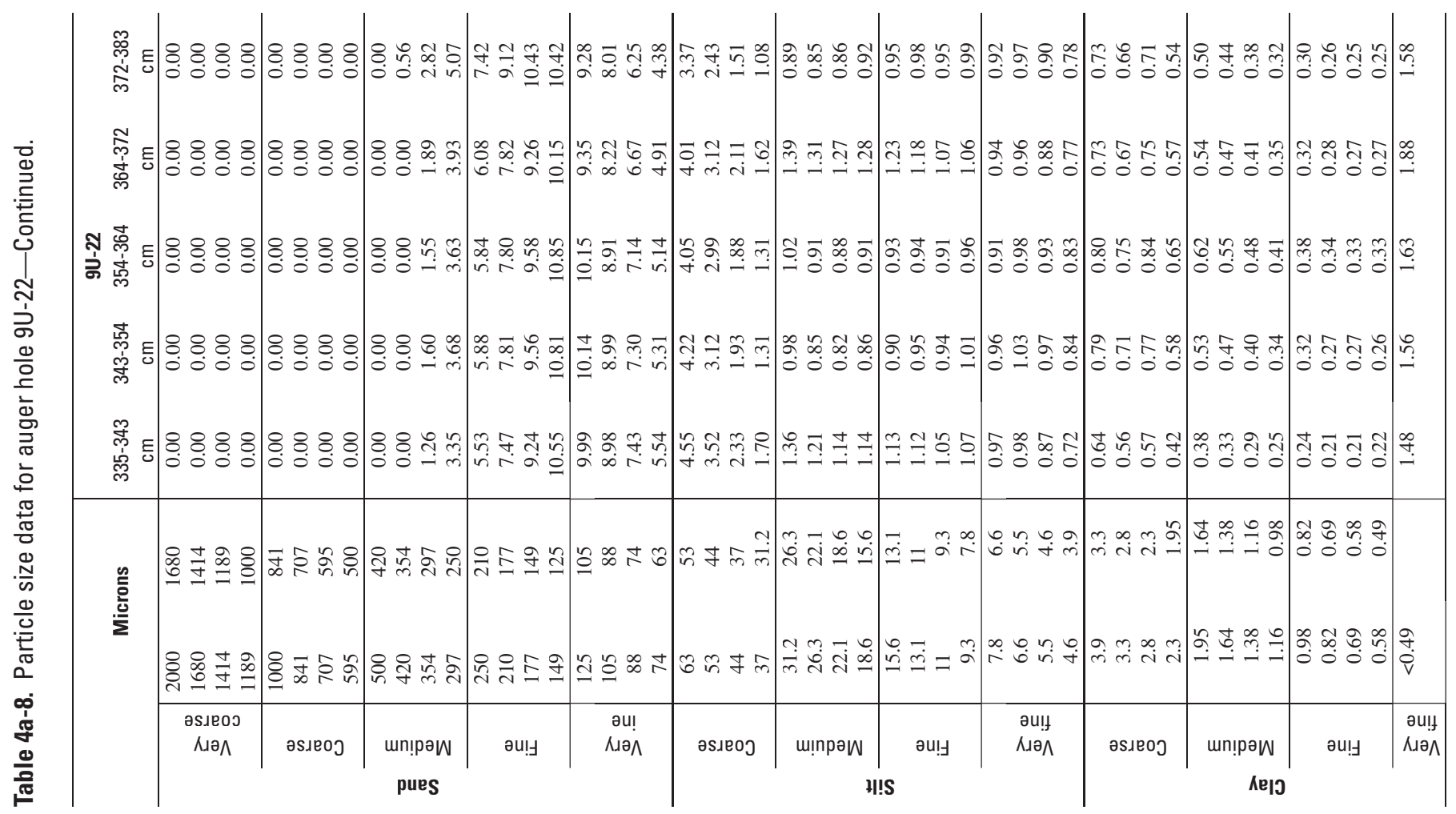



空

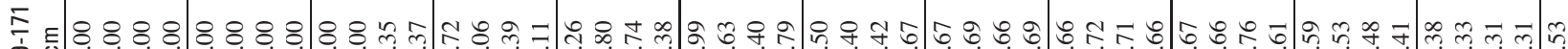

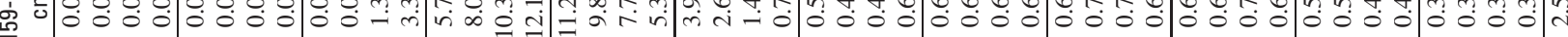

旁 守

吾

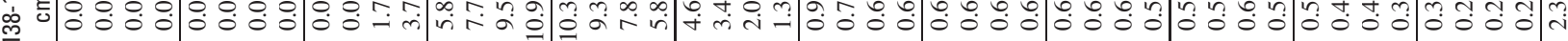
党

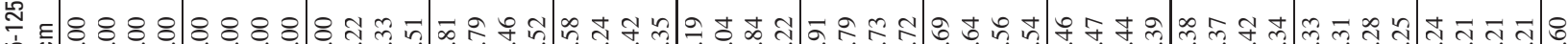

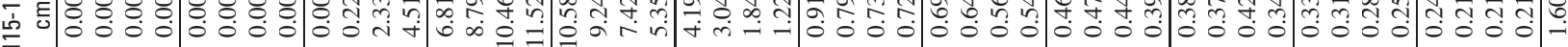

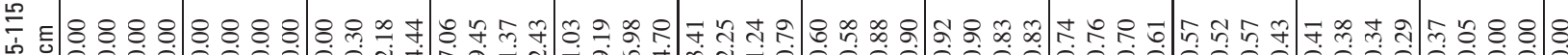

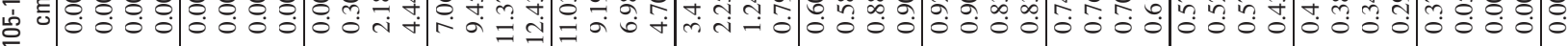

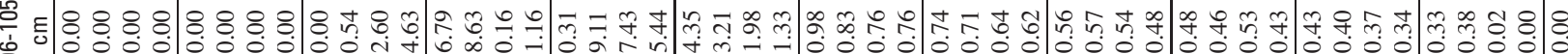
พั่

亏े ह

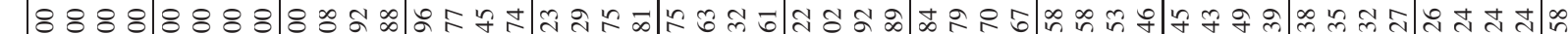

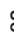

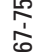

ह

co

(1)

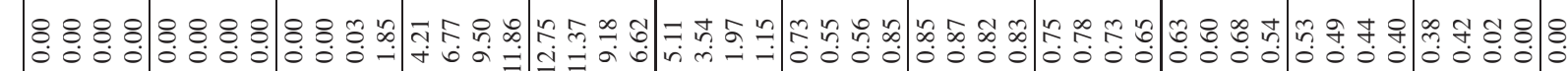

年

E 8

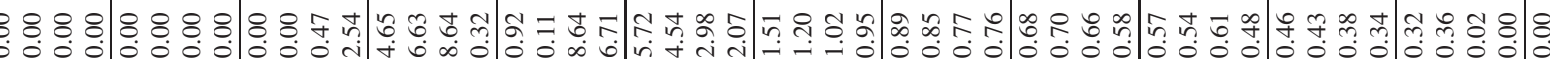

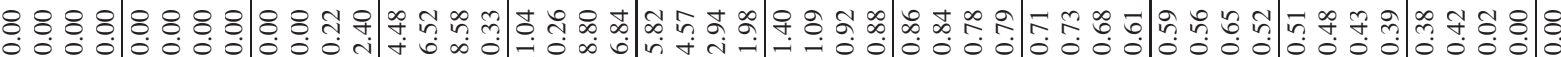

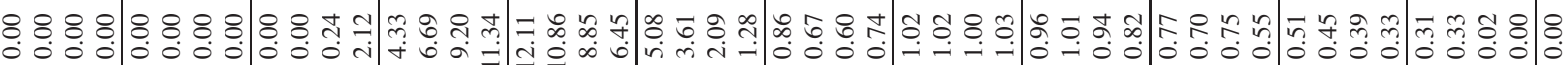

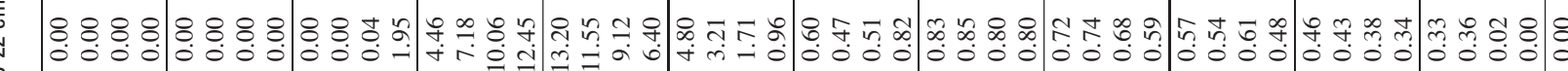

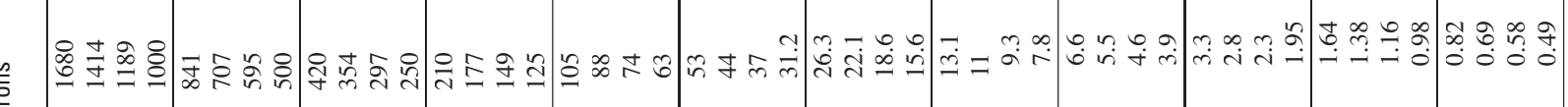

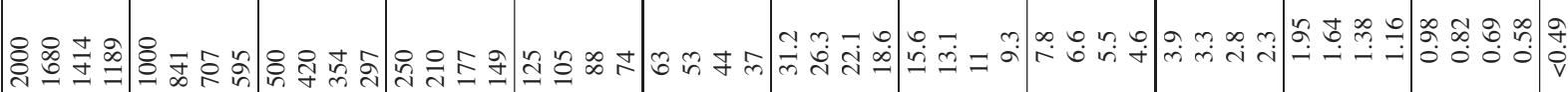

\begin{tabular}{|c|c|c|c|c|c|c|c|c|c|c|c|}
\hline $\begin{array}{c}\text { әsıeoJ } \\
\Lambda\lrcorner ә \Lambda\end{array}$ & әsıеoj & un!pәw & әц!- & $\begin{array}{l}\text { әu!! } \\
\text { ผ ^ }\end{array}$ & әsıeoว & щ!̣рәW & әи! & $\begin{array}{l}\text { әи!」 } \\
\Lambda \text { Кә }\end{array}$ & әsıeoJ & un!рәW & әи! \\
\hline
\end{tabular}

pues

I!!

Ке|כ 


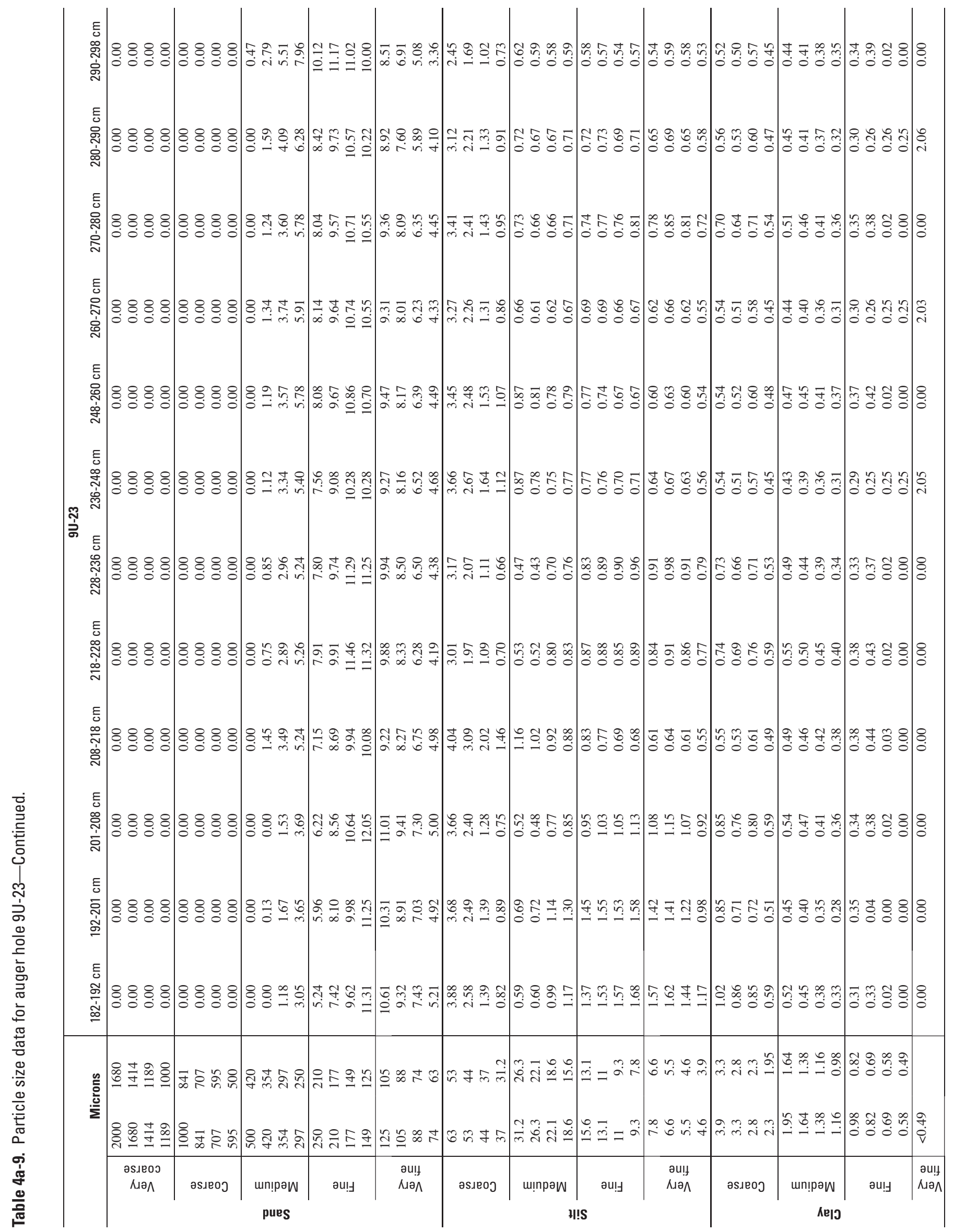


苗 每

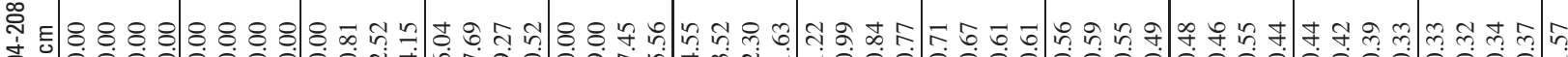

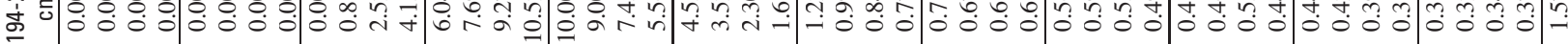

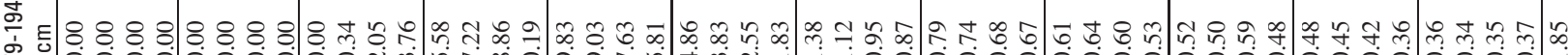

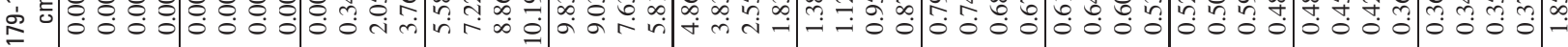

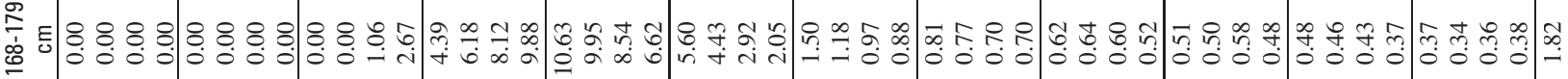

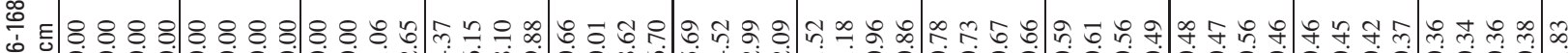

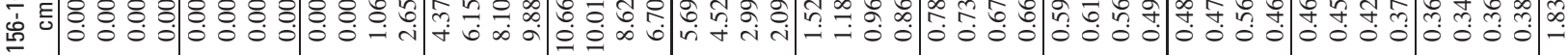

皇

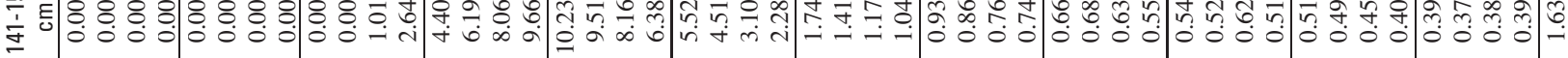
芴

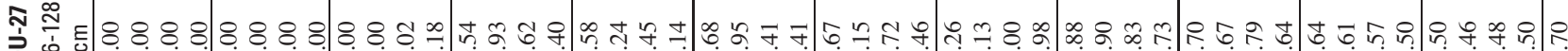

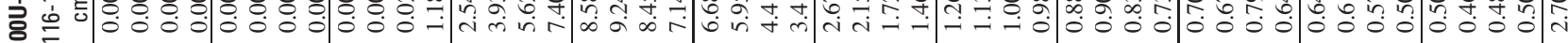

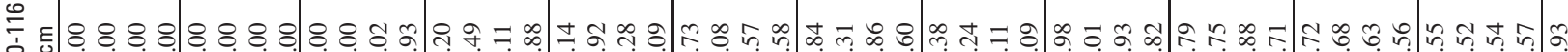

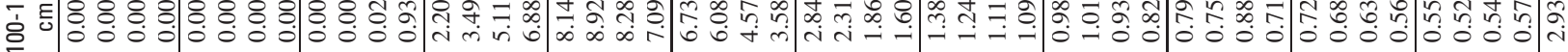

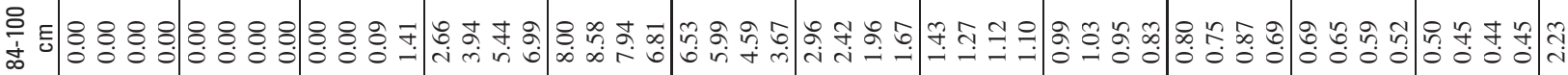

常

品

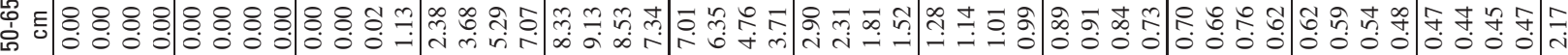

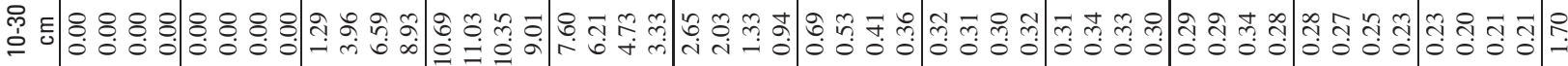

웅

$\stackrel{0}{\stackrel{0}{\underline{L}}}$

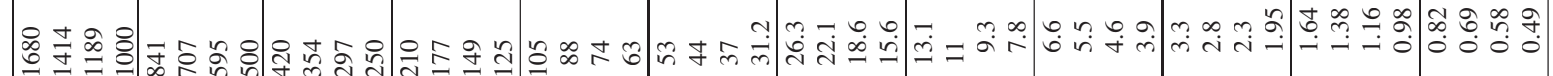

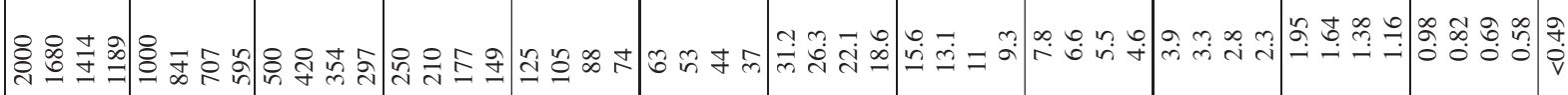

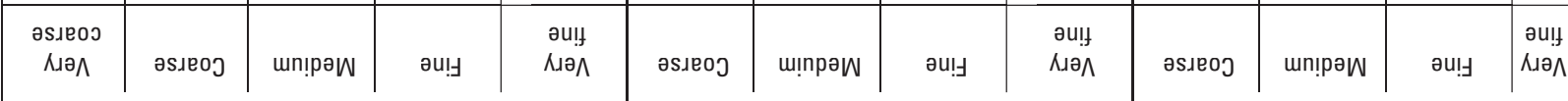

pues 


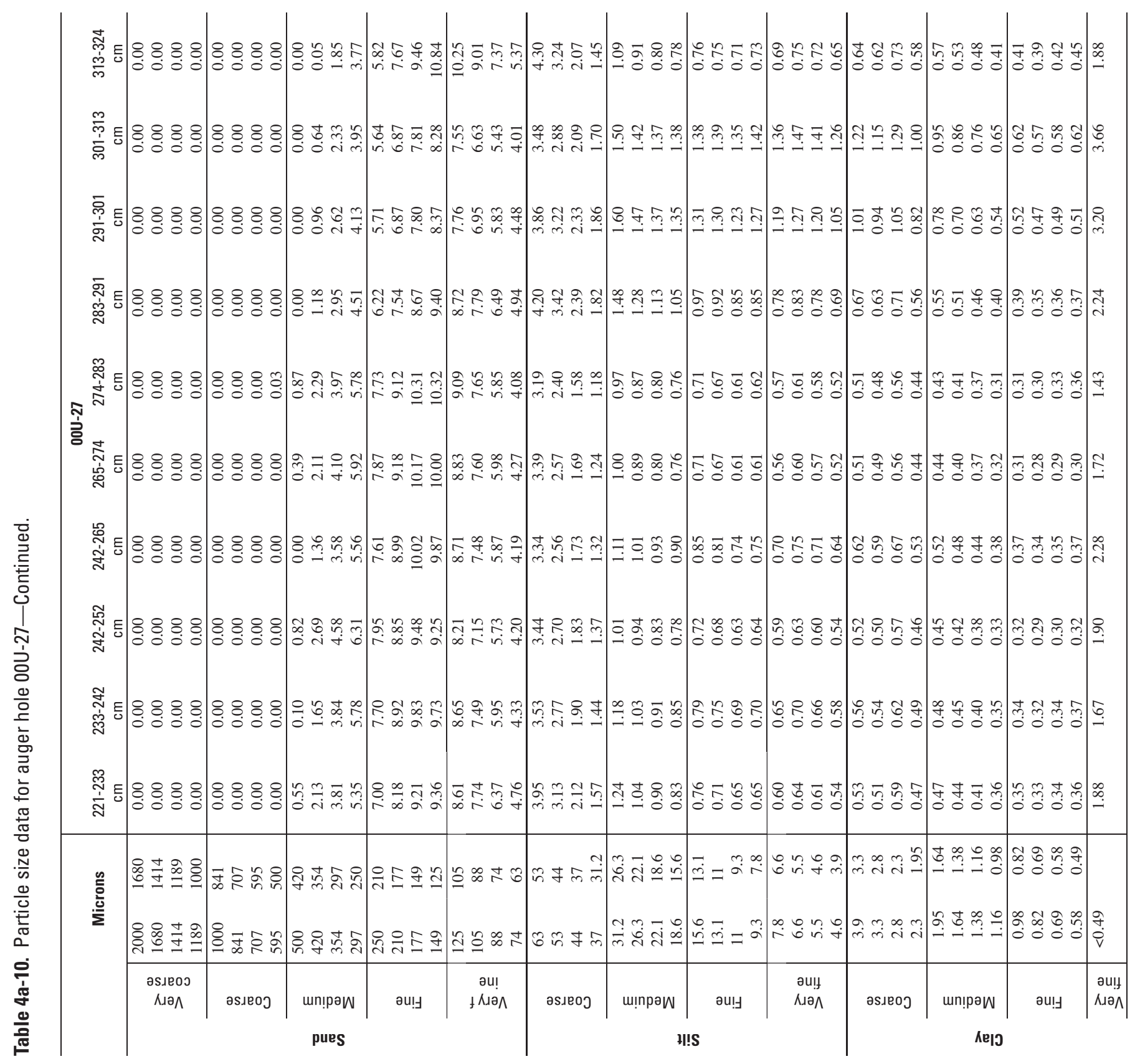




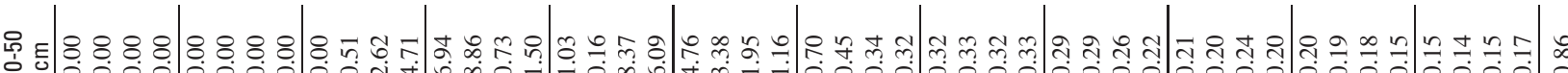

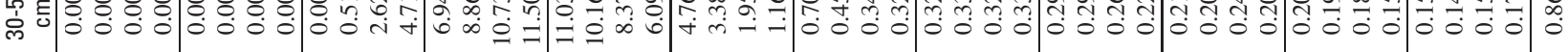

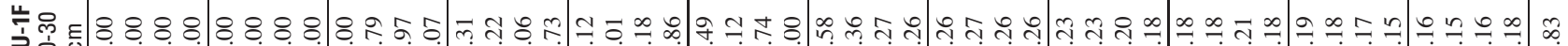

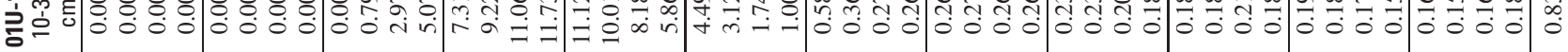

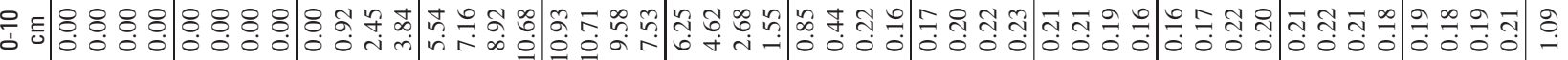

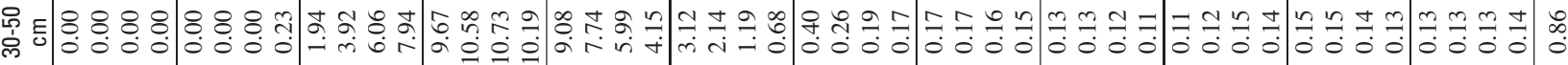

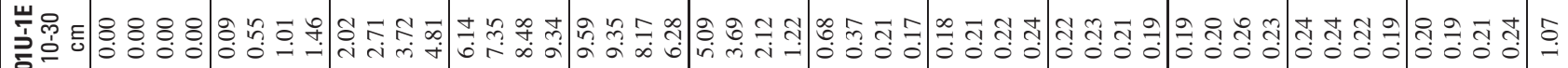

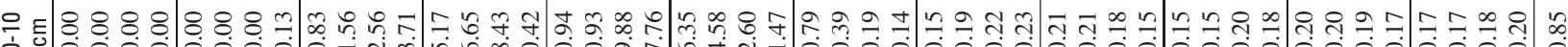

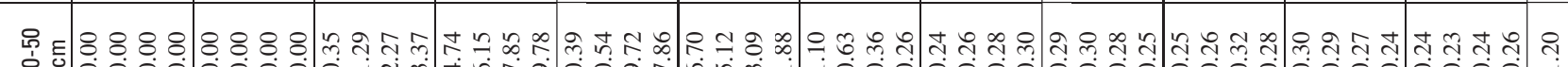

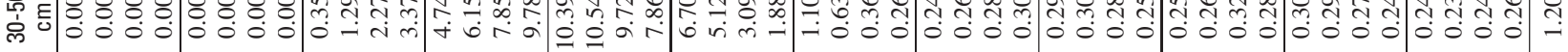

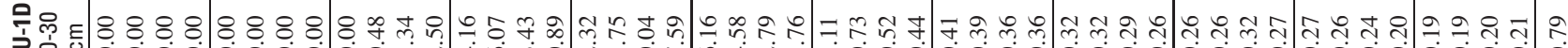

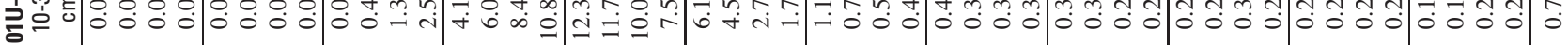

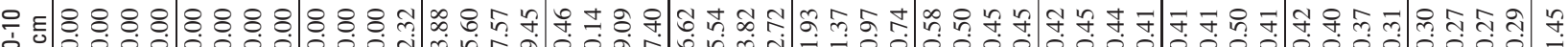

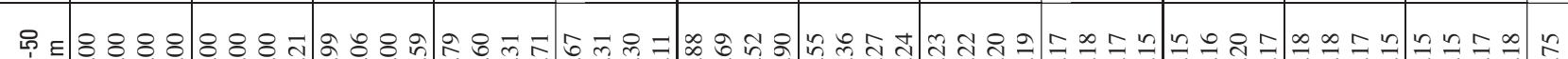

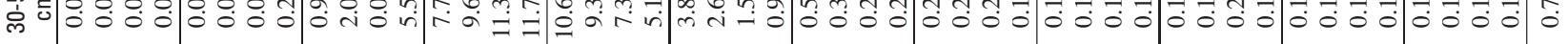
产旁

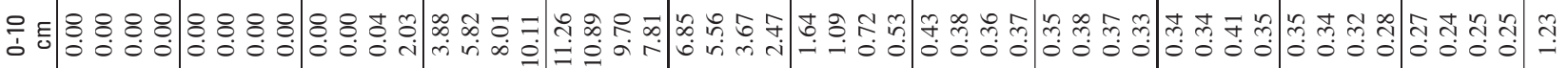

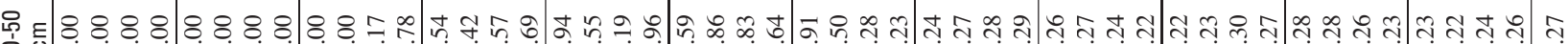
虫

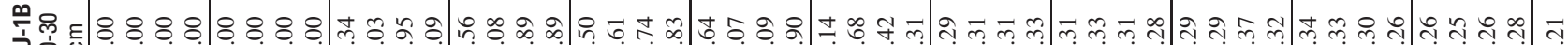

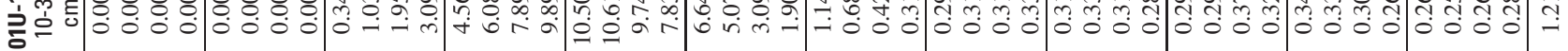

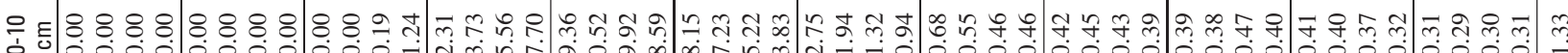
希

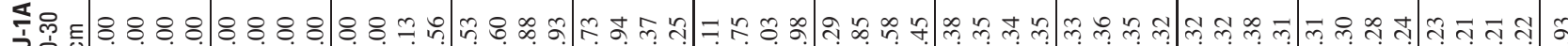

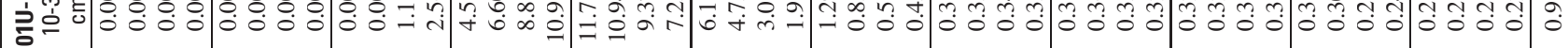

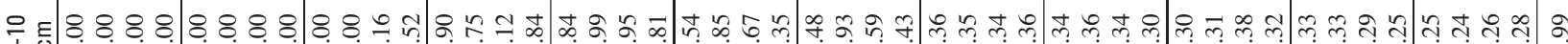
वं

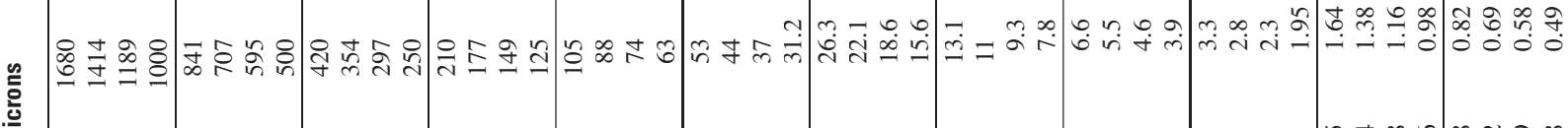

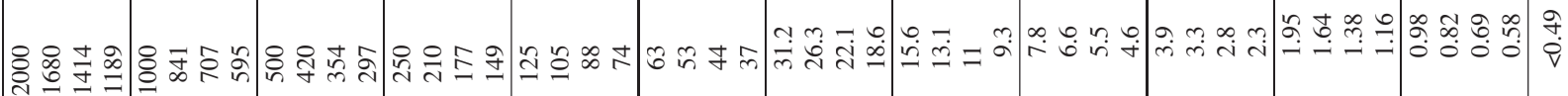

\begin{tabular}{|c|c|c|c|c|c|c|c|c|c|c|c|c|}
\hline 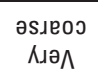 & әs.еоэ & un!pәW & әи!」 & $\begin{array}{l}\text { әи! } \\
\text { Аә }\end{array}$ & әsıеoj & ш!nрәW & әи! & $\begin{array}{l}\text { әи! } \\
\text { Һә }\end{array}$ & әs.ео & un!рәW & әи!」 & $\begin{array}{l}\text { әu!y } \\
\Lambda\lrcorner \curvearrowright\end{array}$ \\
\hline & & pues & & & & & & & & ${ }_{k e} \mid$ & & \\
\hline
\end{tabular}




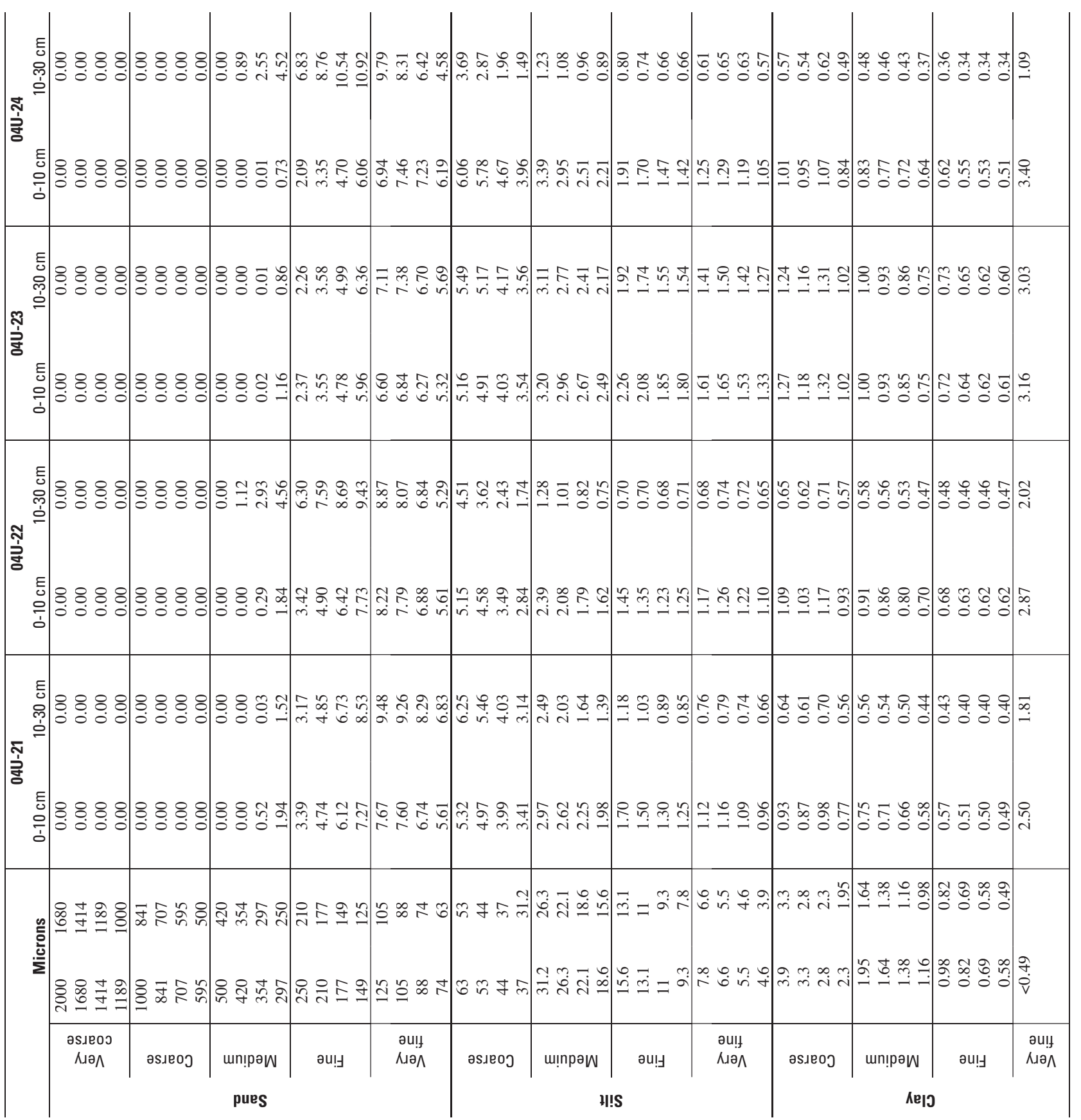




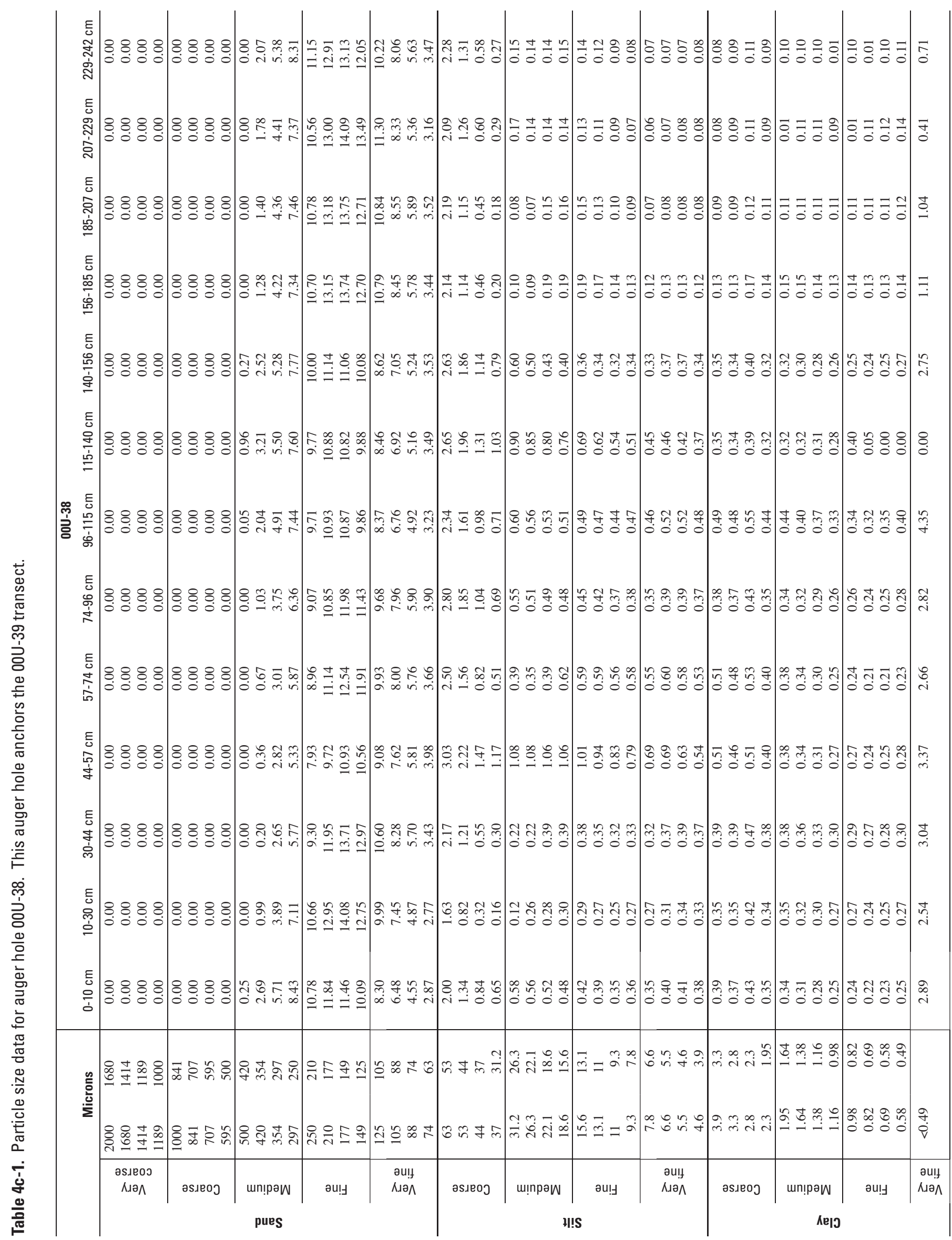




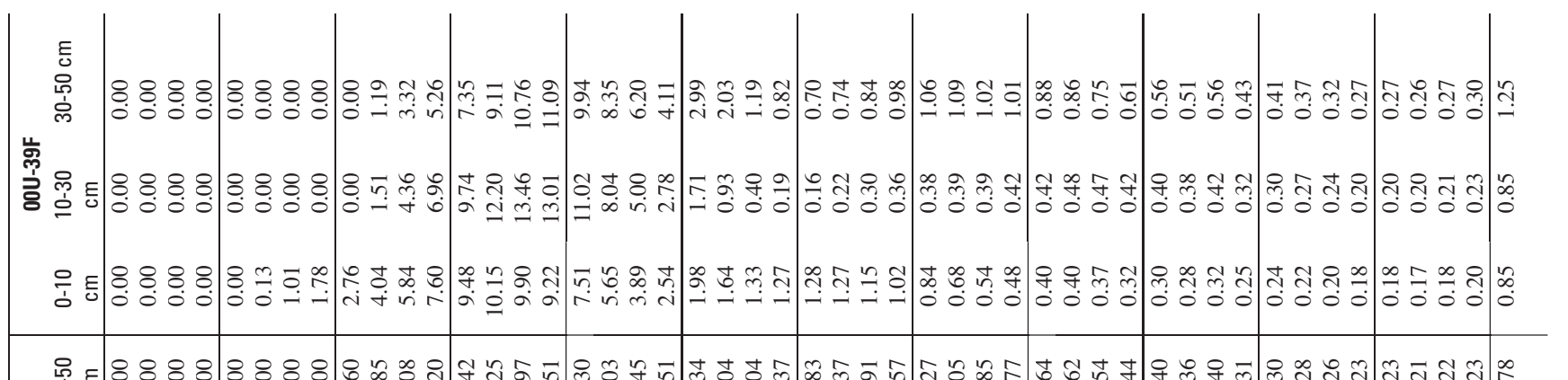

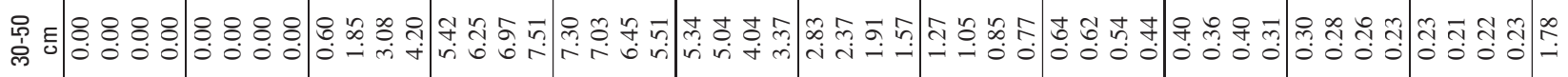

峞

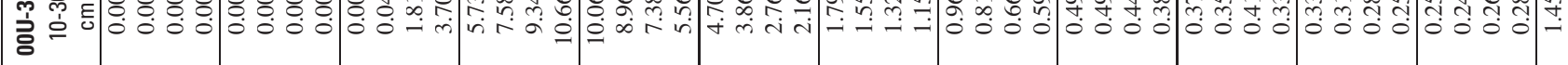

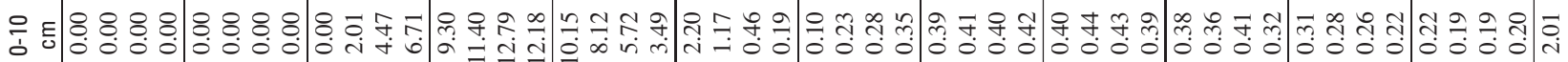

扂 尊品 高

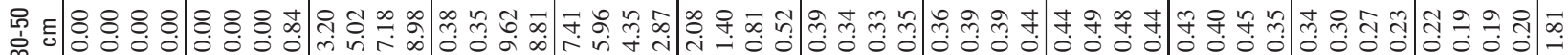
岸芦

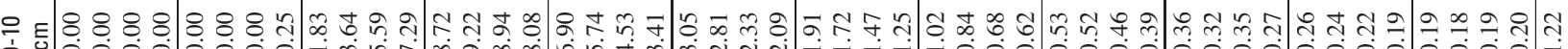

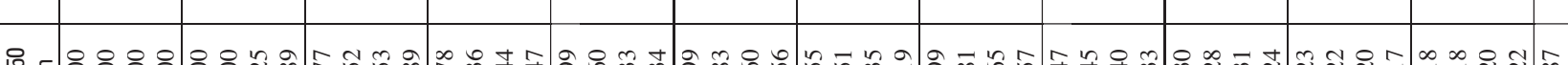

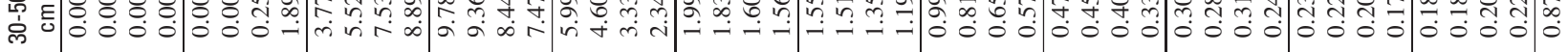
䖭 守至 웅

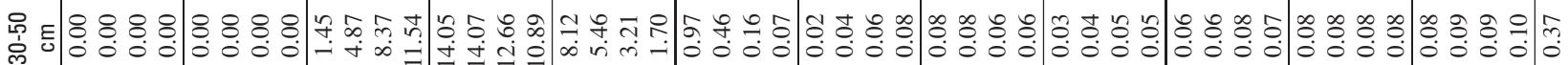

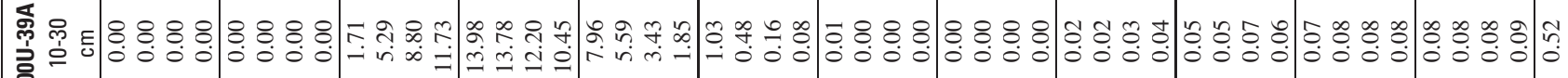

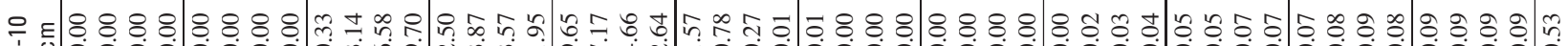

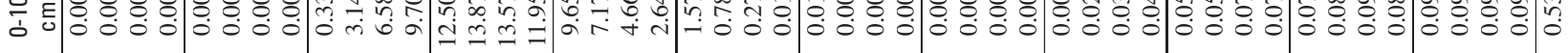

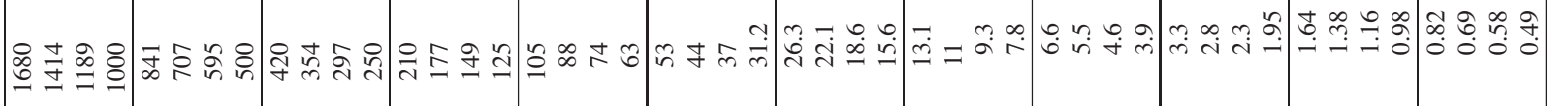

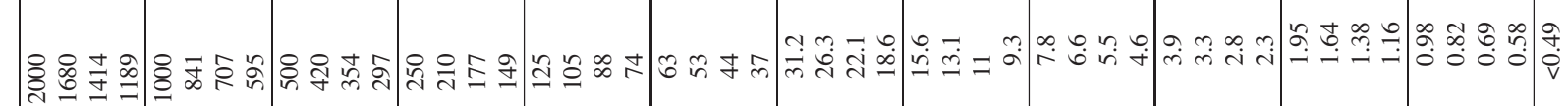

\begin{tabular}{|c|c|c|c|c|c|c|c|c|c|c|c|}
\hline $\begin{array}{c}\text { әs.ео } \\
\Lambda \text { « }\end{array}$ & әs.eoJ & un!̣әW & әи!」 & $\begin{array}{c}\text { әи!! } \\
\text { КАә }\end{array}$ & әs.eoj & ш!̣рәW & әи!」 & 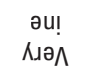 & әsıеоэ & un!pәW & әи! \\
\hline
\end{tabular}
pues 


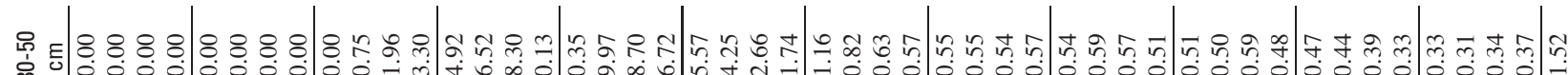

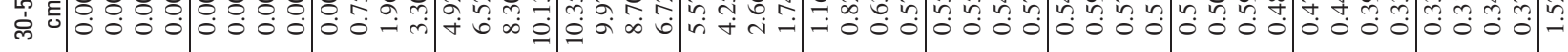

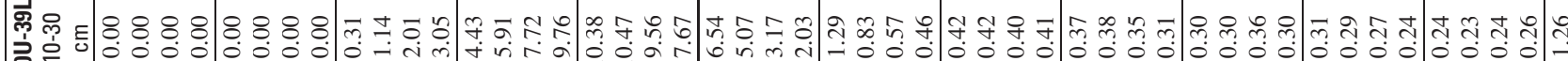

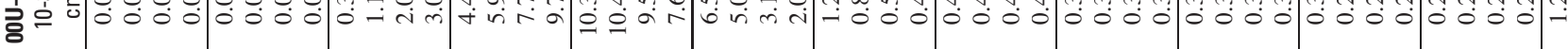

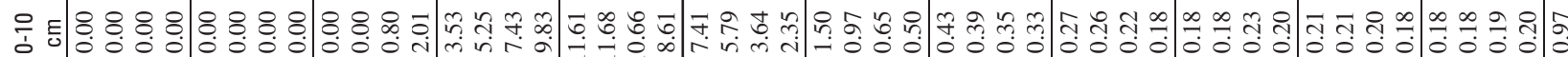

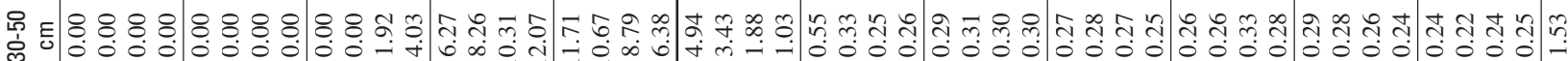

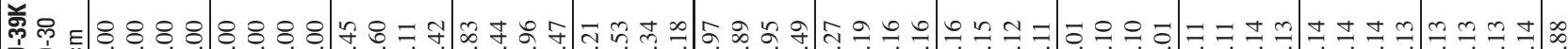

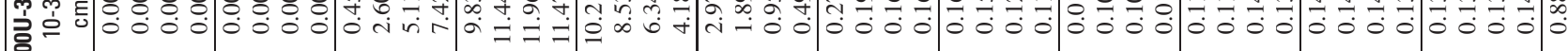

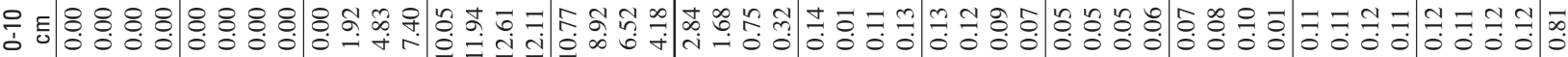
号 गु⿰彳

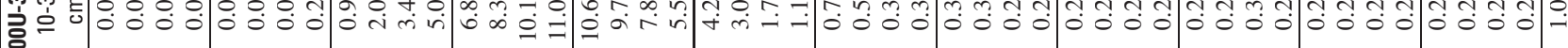

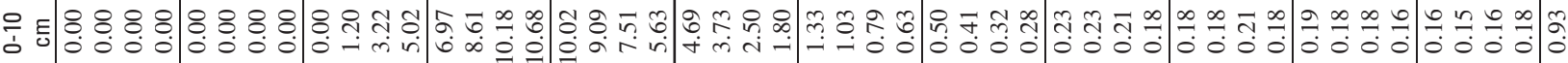

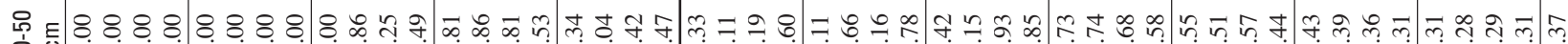
में

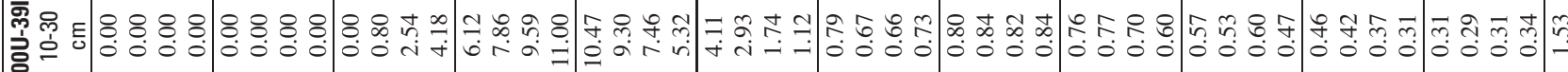

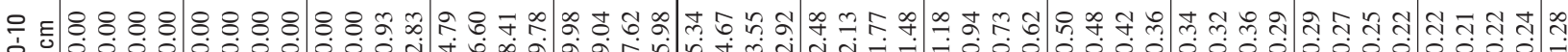

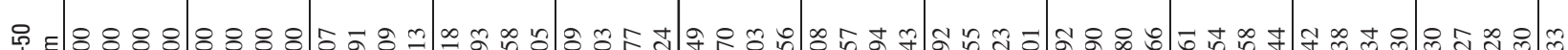

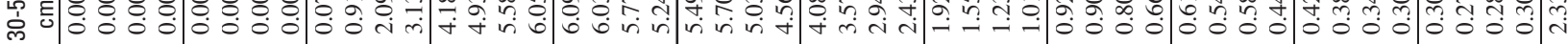

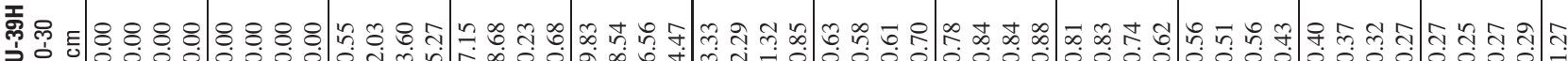

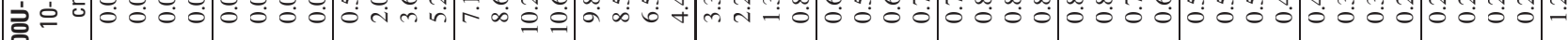

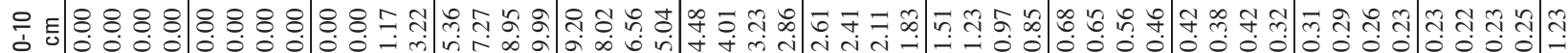

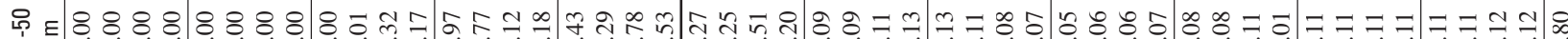

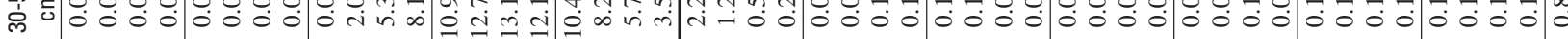

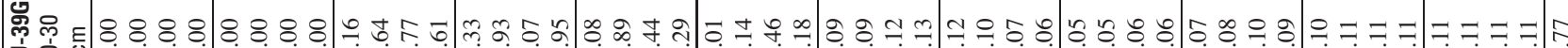
客至

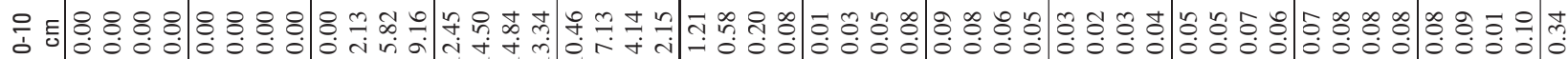

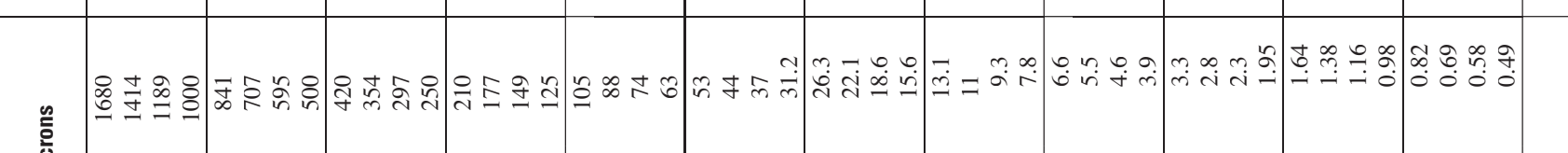

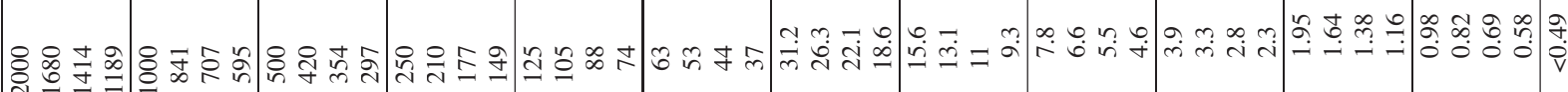

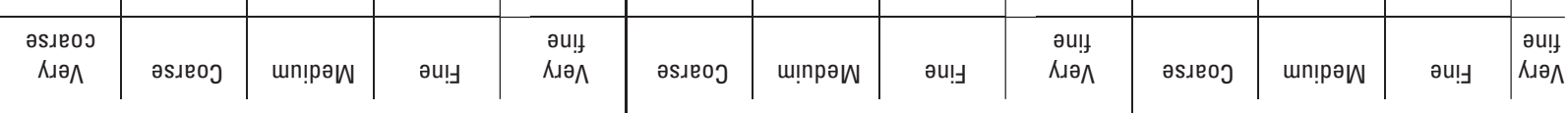

pues 


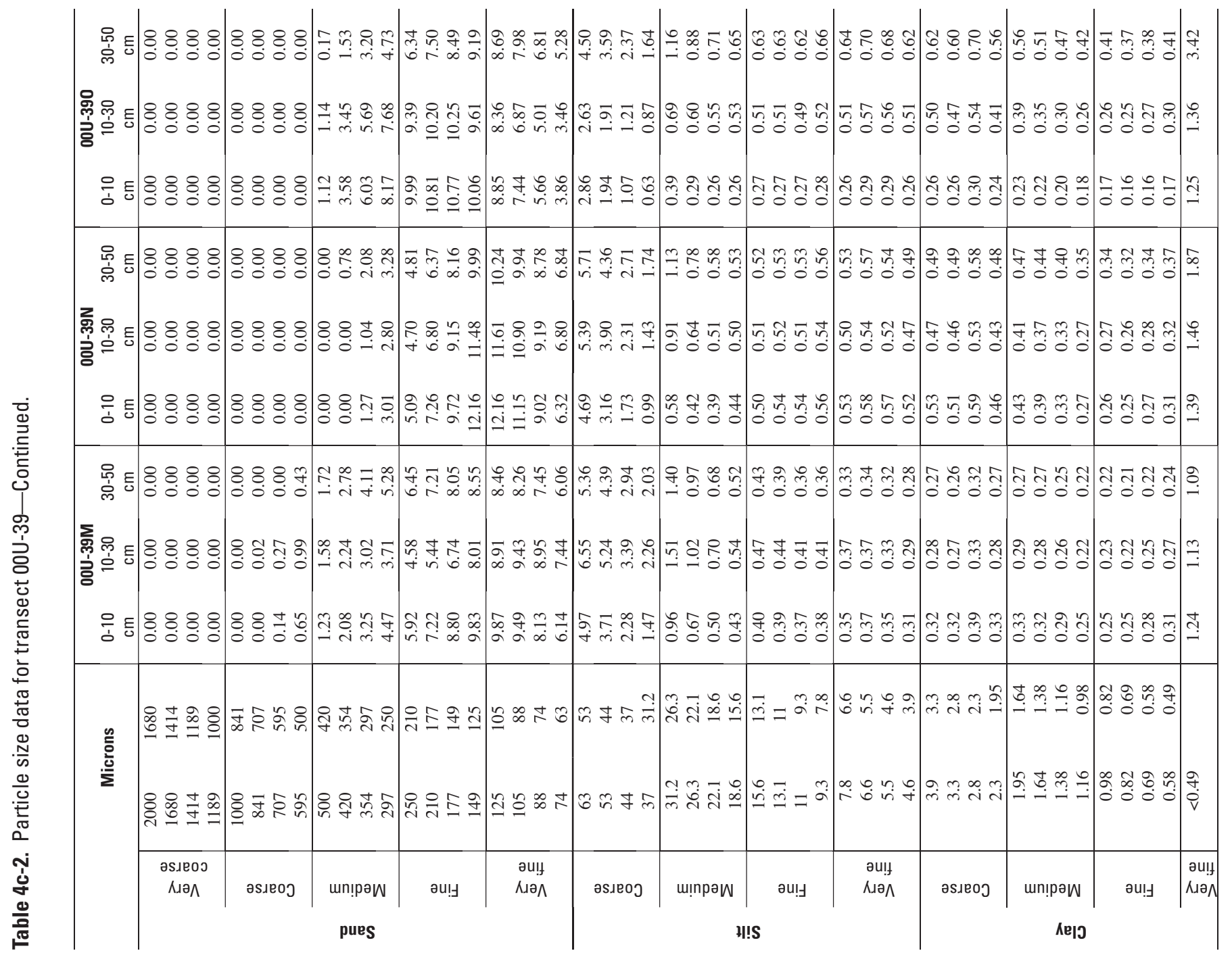




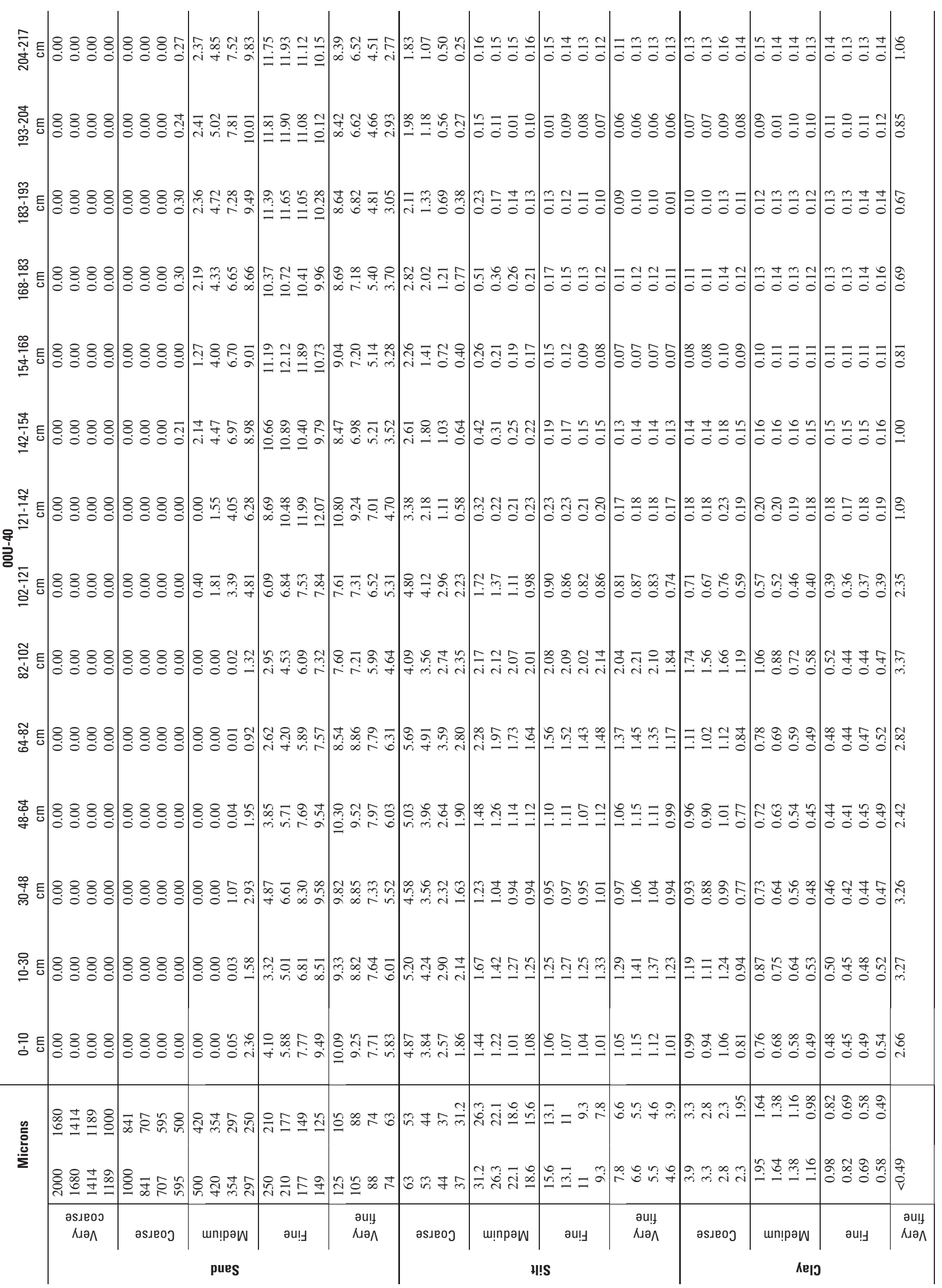




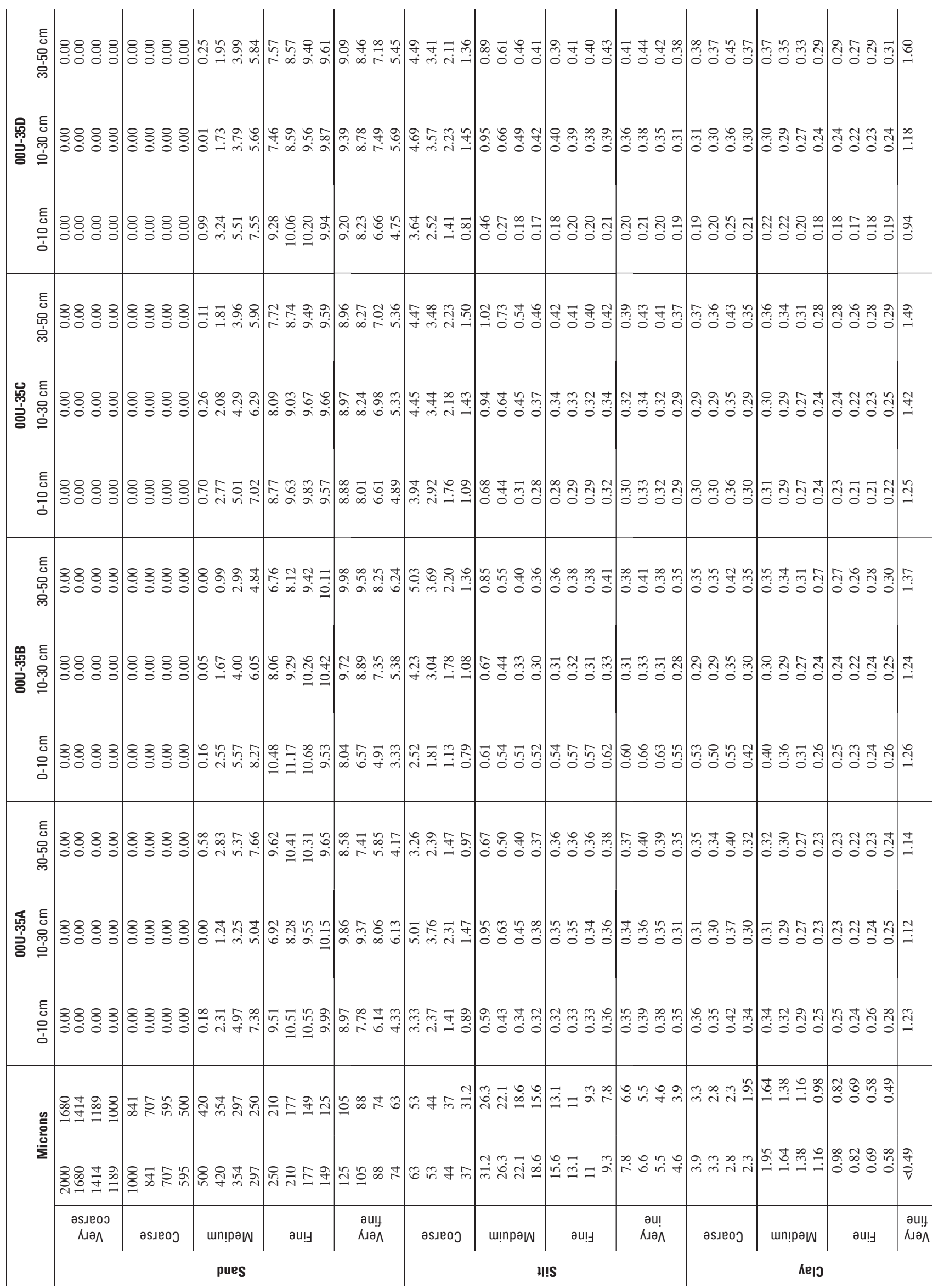




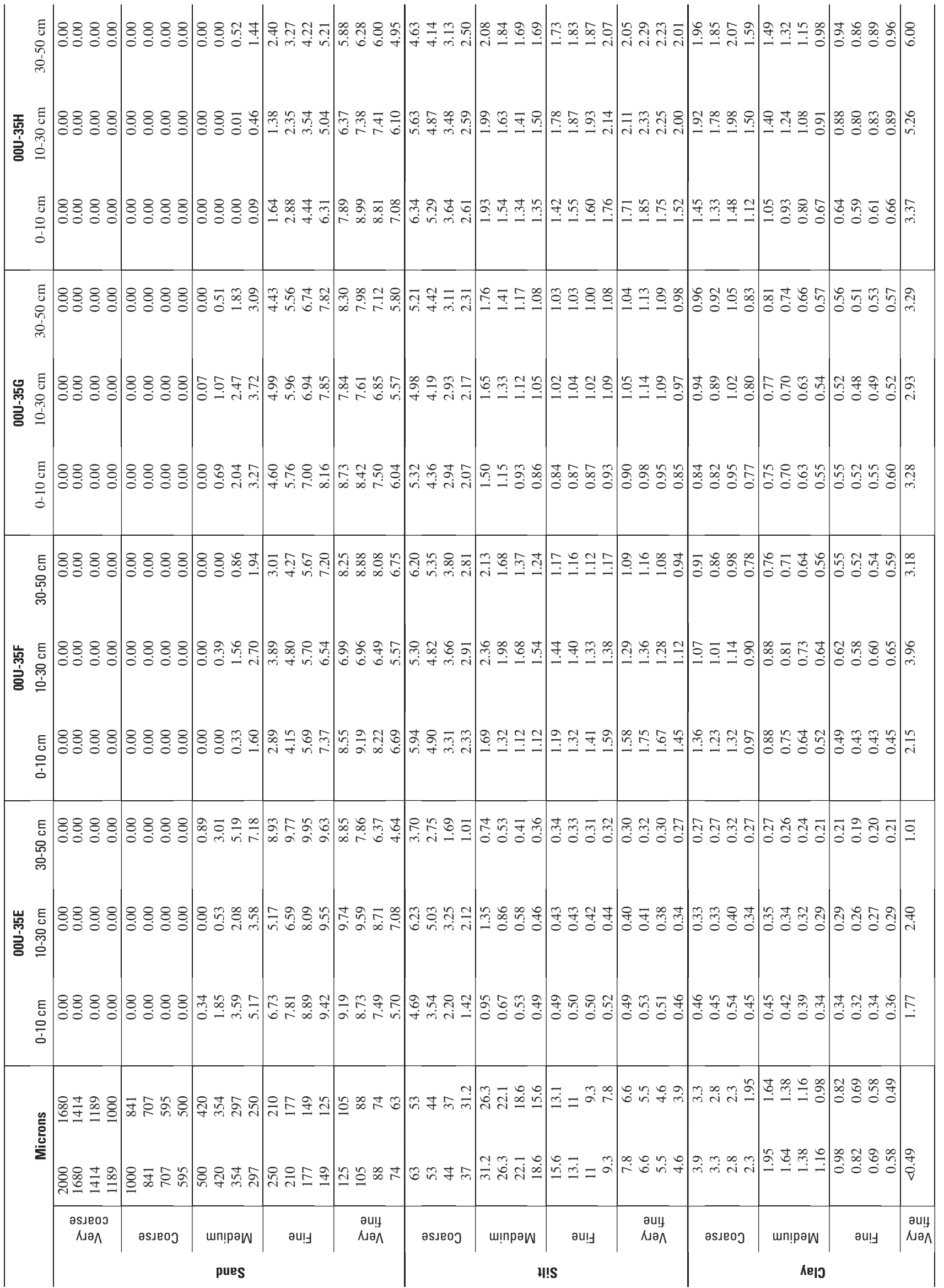




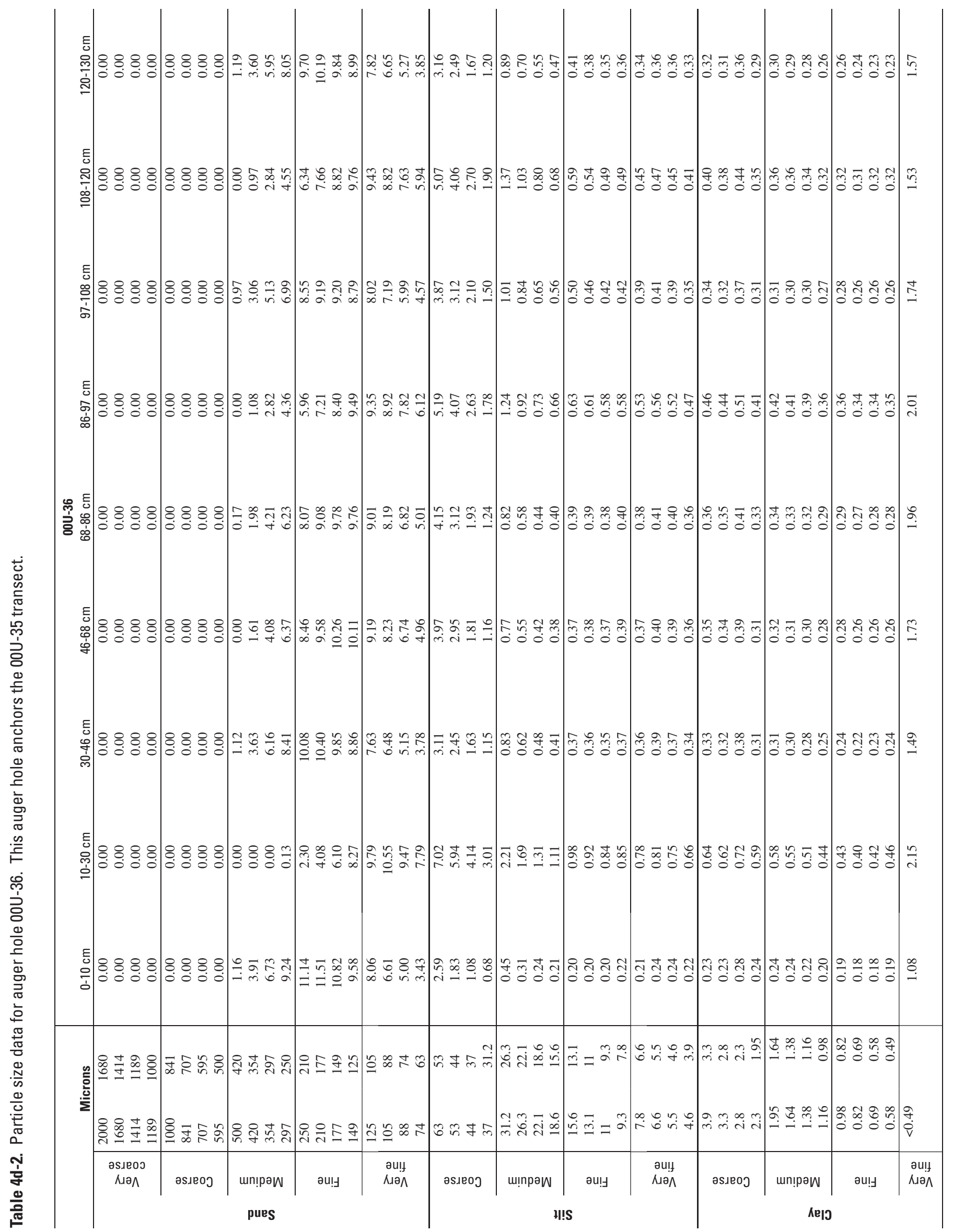




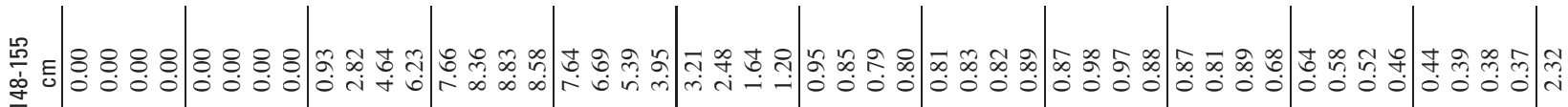

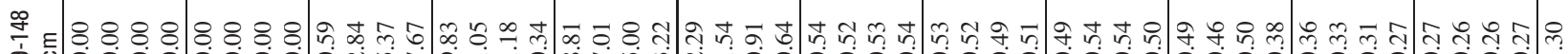

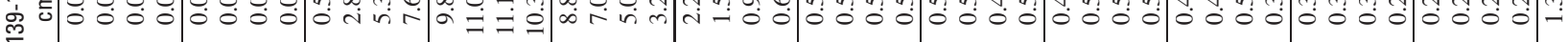

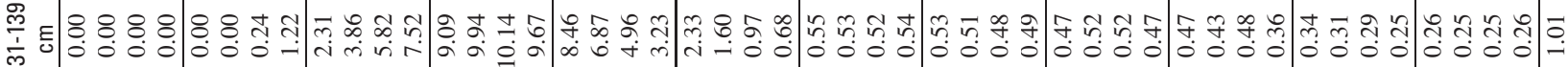

穴 0

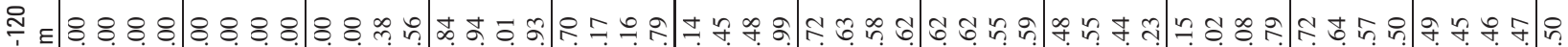
白

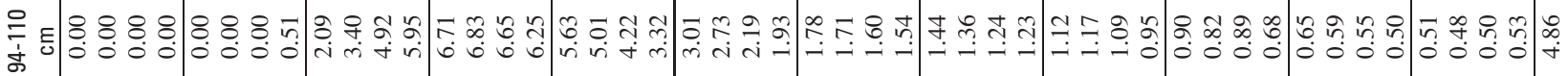

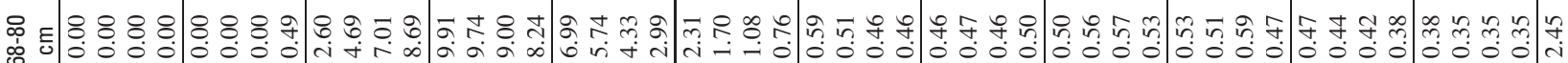

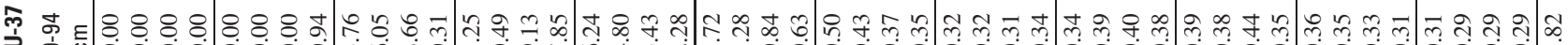
ठо

: 崫 虫

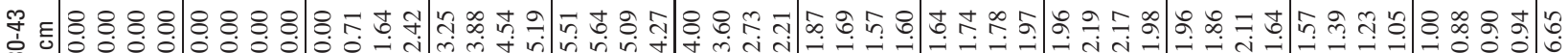

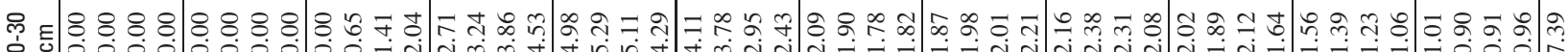

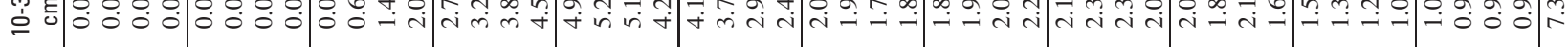

으 튼 혼

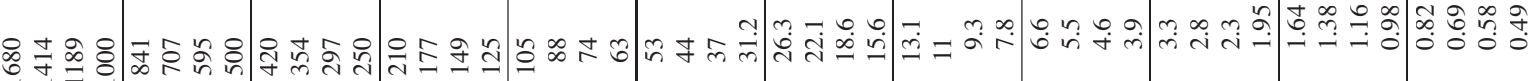

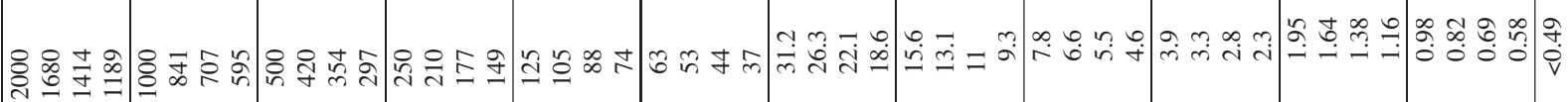

\begin{tabular}{|c|c|c|c|c|c|c|c|c|c|c|c|c|}
\hline $\begin{array}{c}\text { әsıеоo } \\
\Lambda\lrcorner ә \Lambda\end{array}$ & әsıeoj & шпірәw & әиІ- & 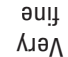 & әsıеоว & шıпрәW & әuIf & 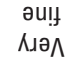 & әs.e00 & unıрәw & әul- & \begin{tabular}{|c|} 
әu! \\
Кıә
\end{tabular} \\
\hline & & pues & & & & & & & & Аер & & \\
\hline
\end{tabular}




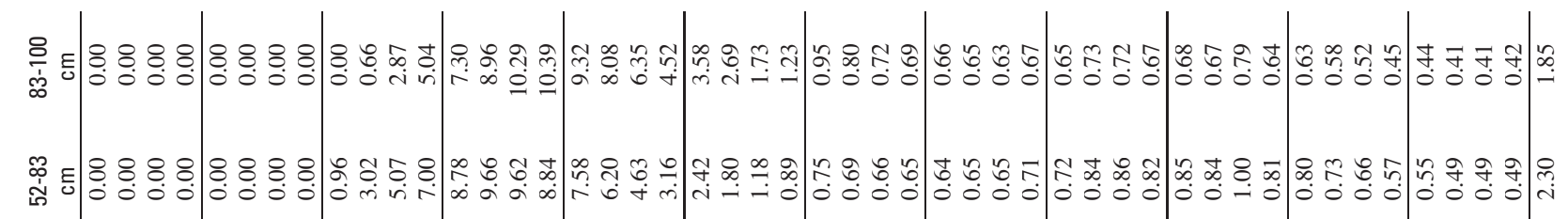

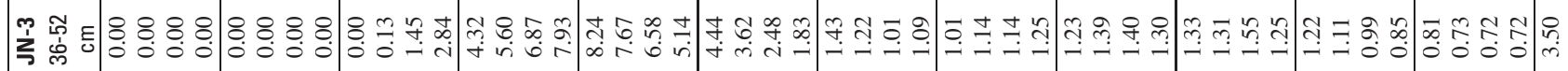

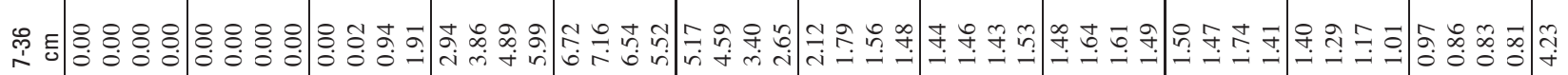
至 家 \&

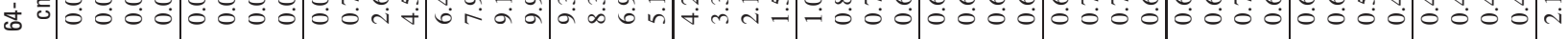

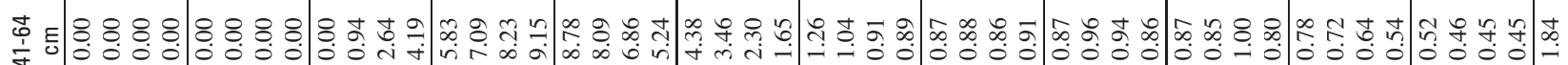

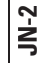

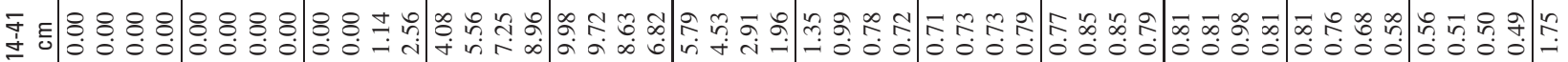

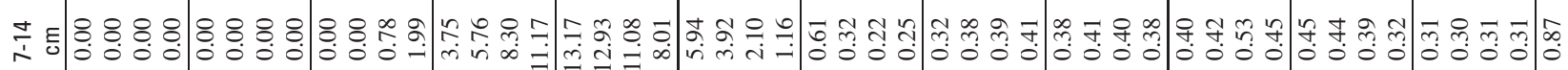

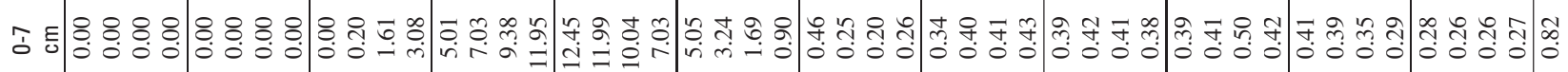

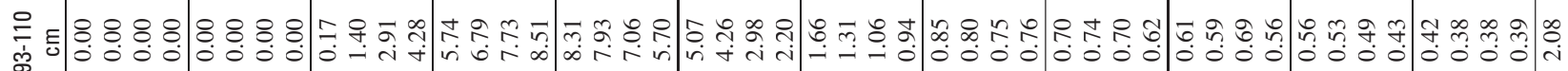
क

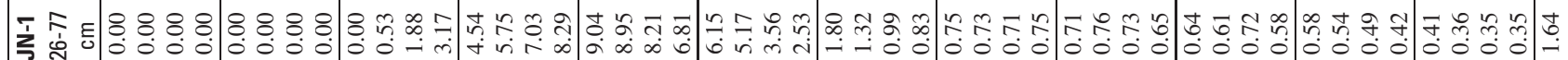

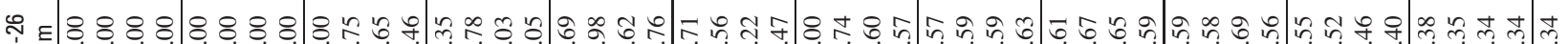

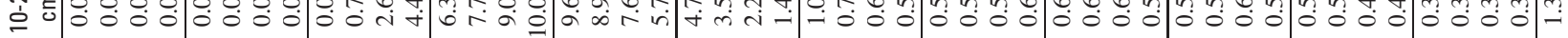

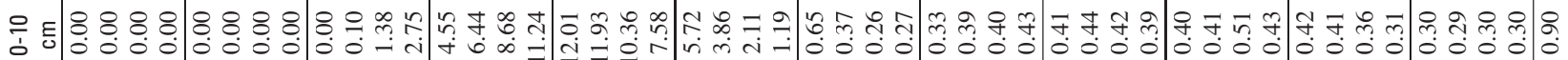

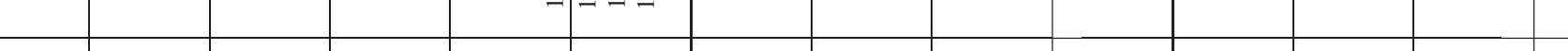

点

$\Sigma$

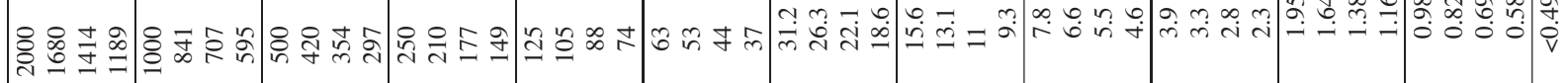

\begin{tabular}{|c|c|c|c|c|c|c|c|c|c|c|c|c|}
\hline $\begin{array}{c}\text { әsıео } \\
\Lambda\lrcorner ә \wedge\end{array}$ & әsАео & un!pəw & әи! & $\begin{array}{c}\text { әи! } \\
\text { «ґә }\end{array}$ & әsıеo了 & щ!nрәW & әи! & $\begin{array}{l}\text { әи!! } \\
\Lambda \text { « }\end{array}$ & әs.еоэ & un!̣рәW & әи! & 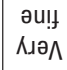 \\
\hline & & pues & & & & & & & & AE|O & & \\
\hline
\end{tabular}




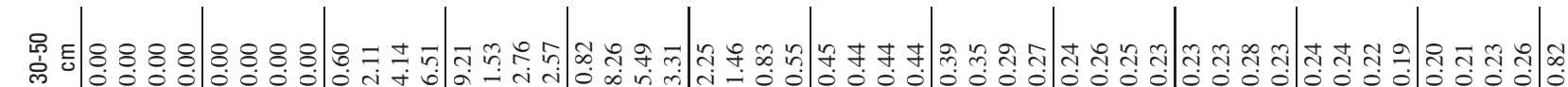

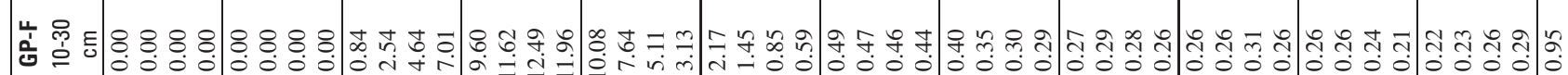

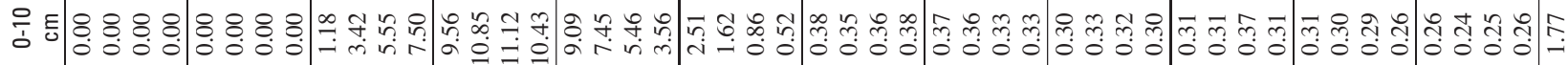

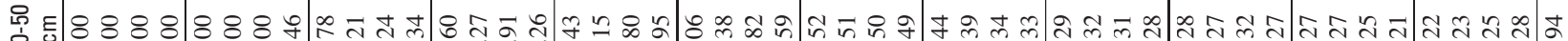
में 崔 产

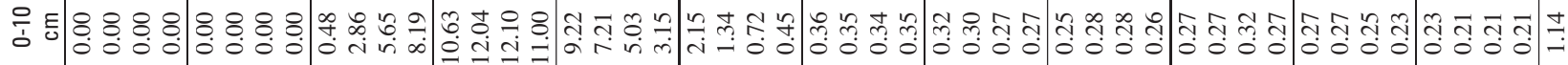

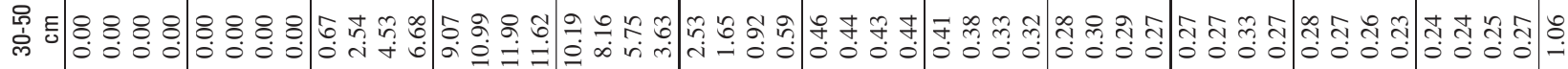
업 엄

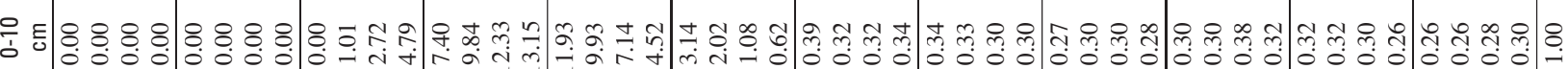
局

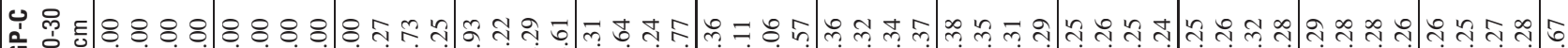

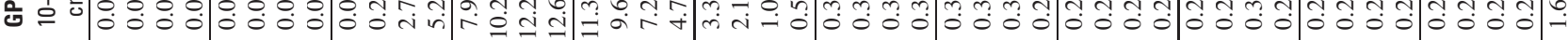

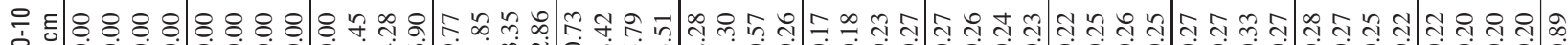
ó

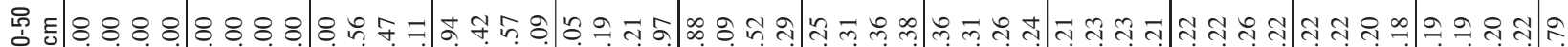

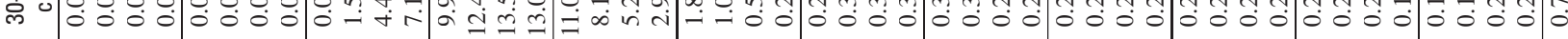
商 产

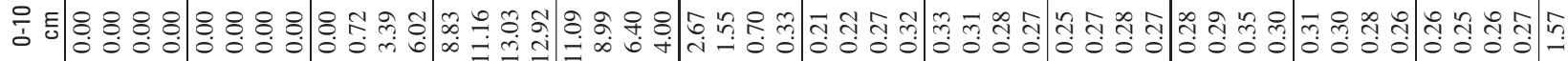

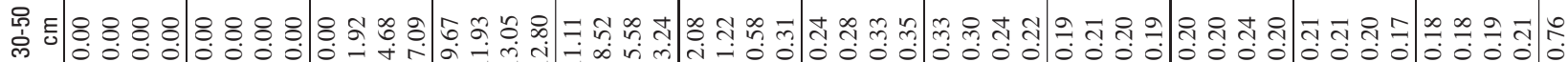
乐官

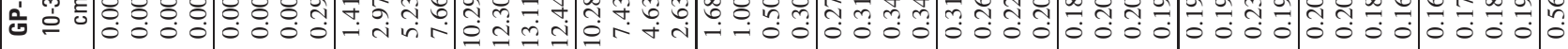

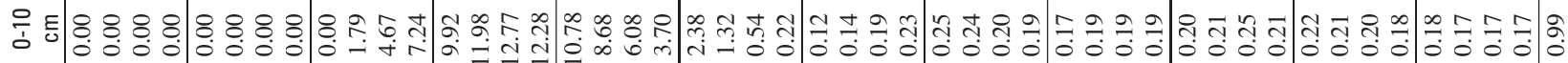

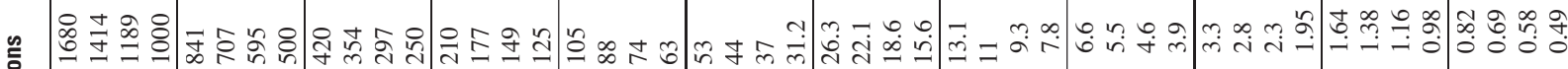

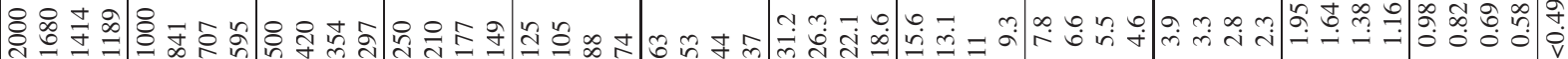

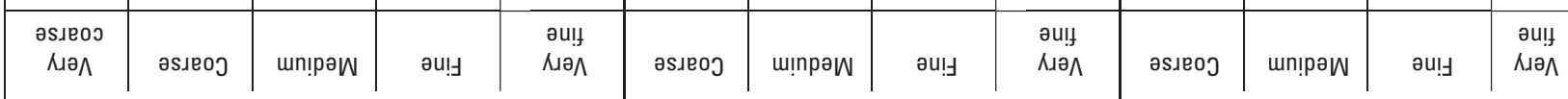

pues 


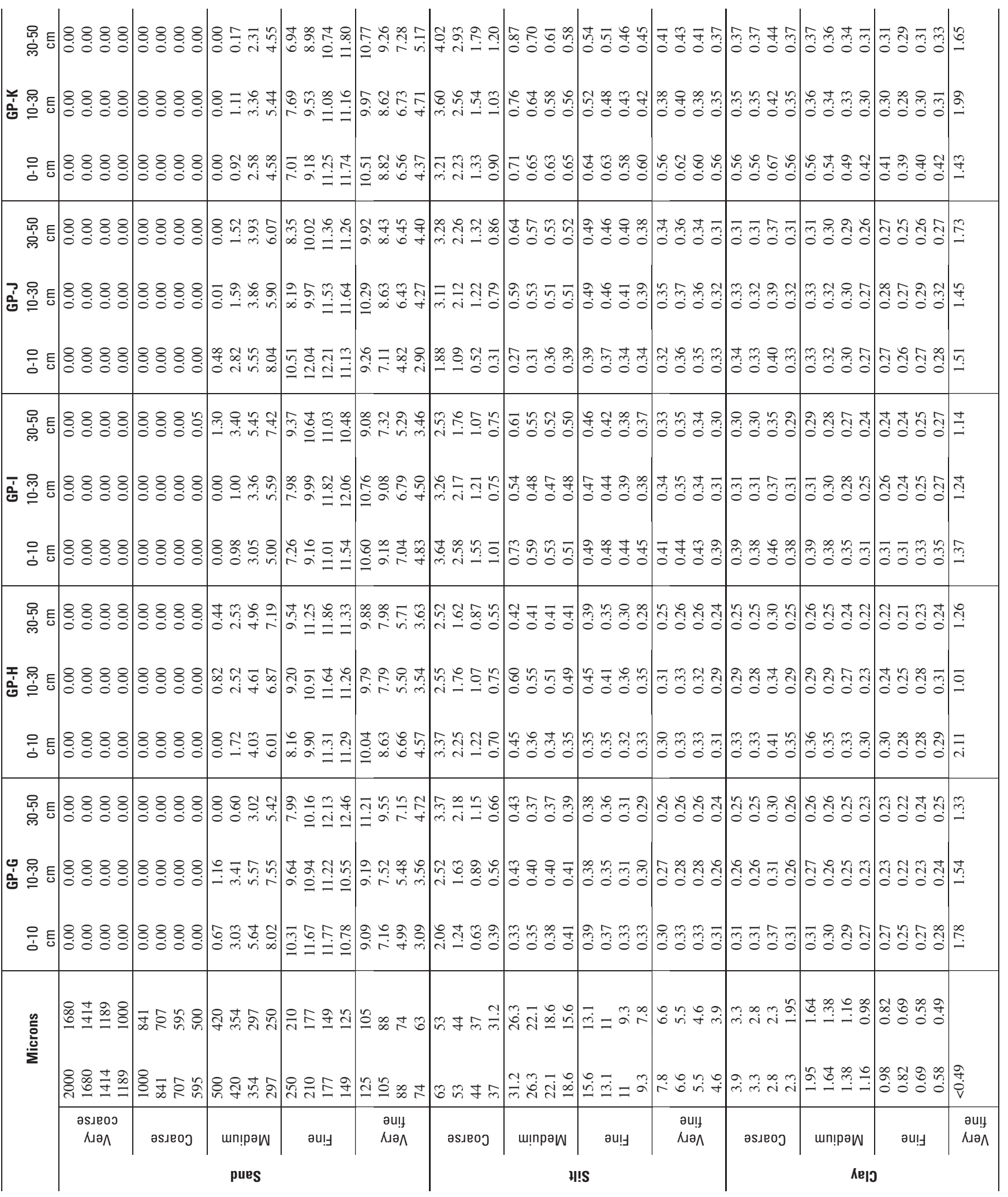




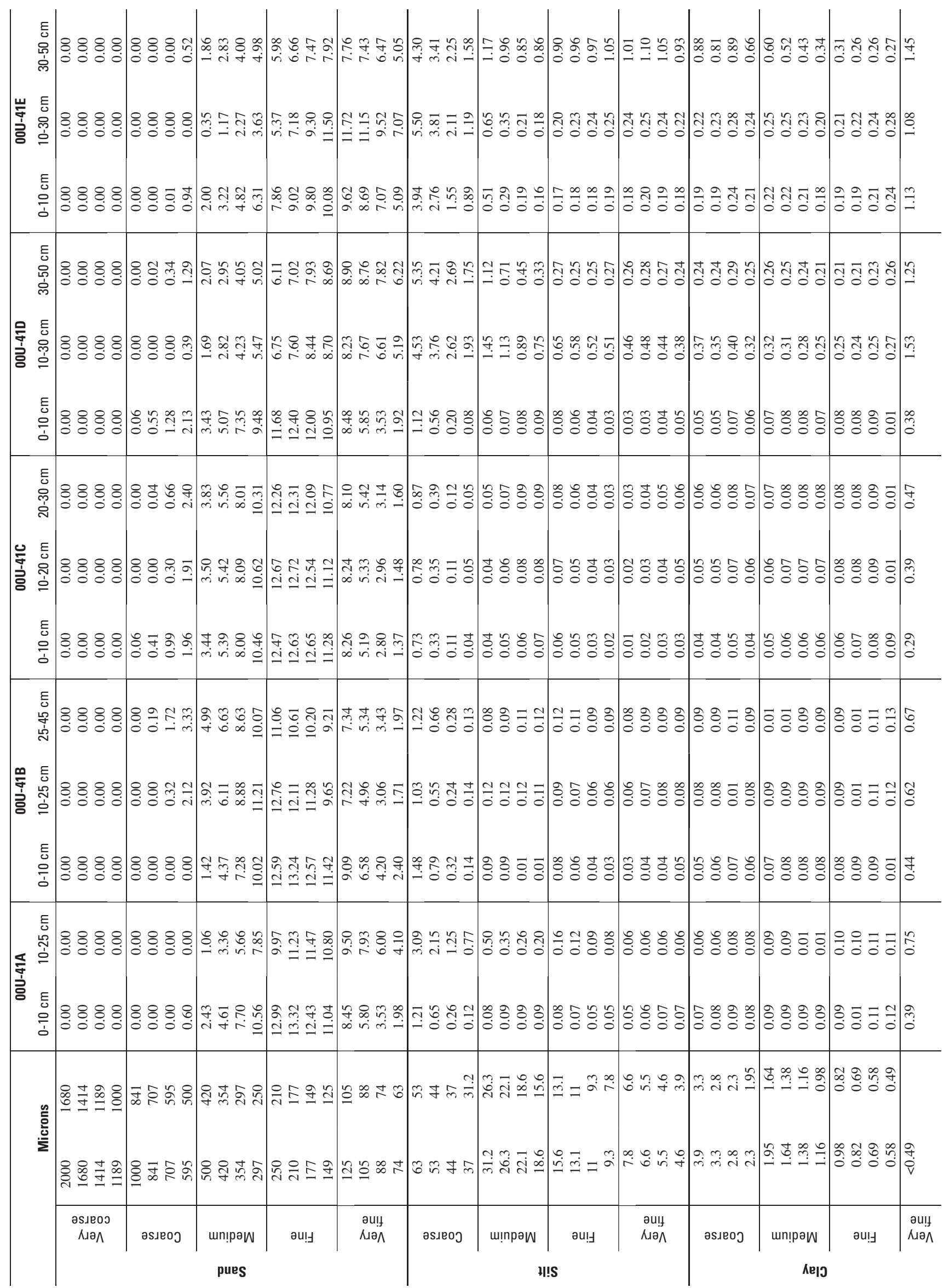




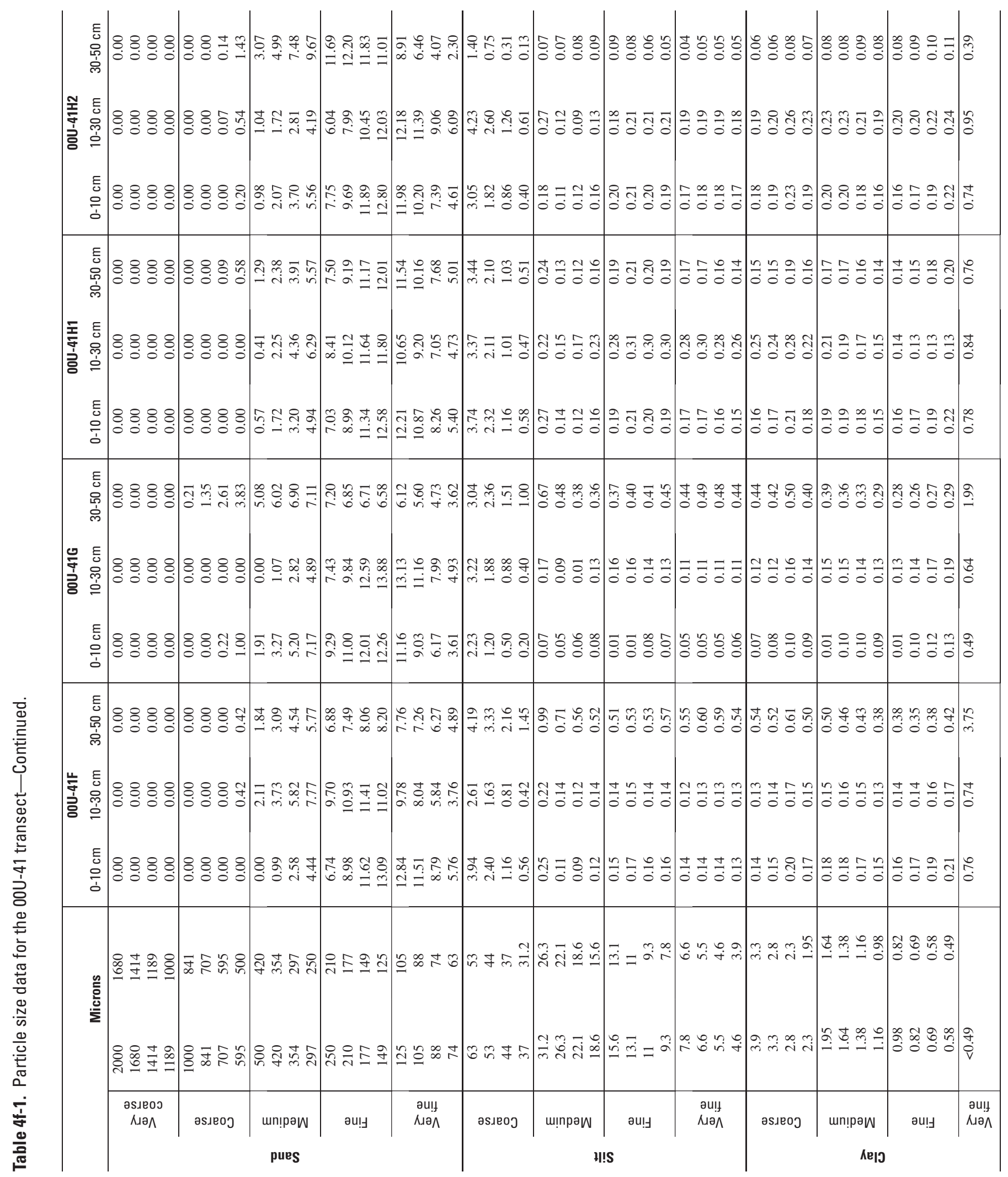




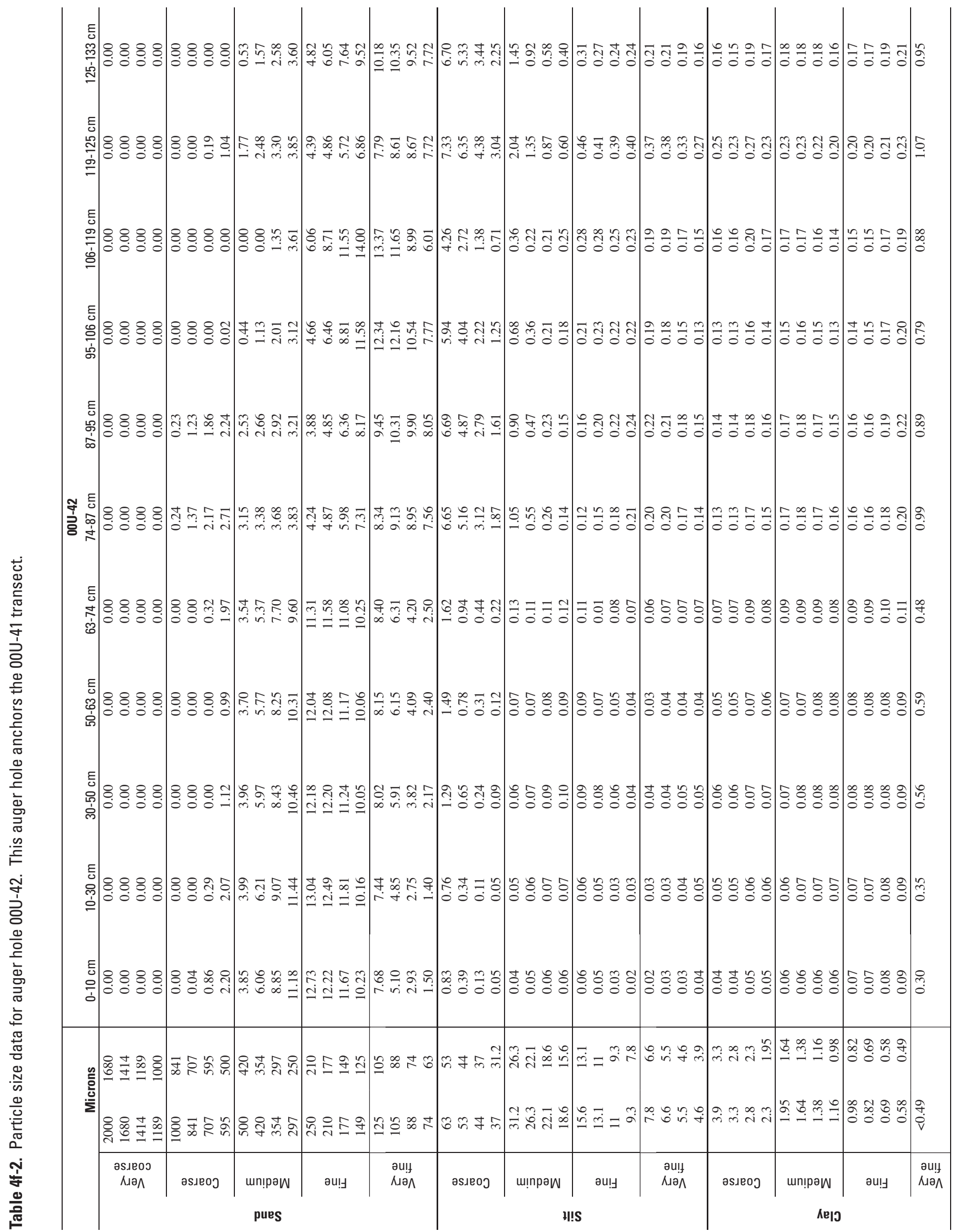




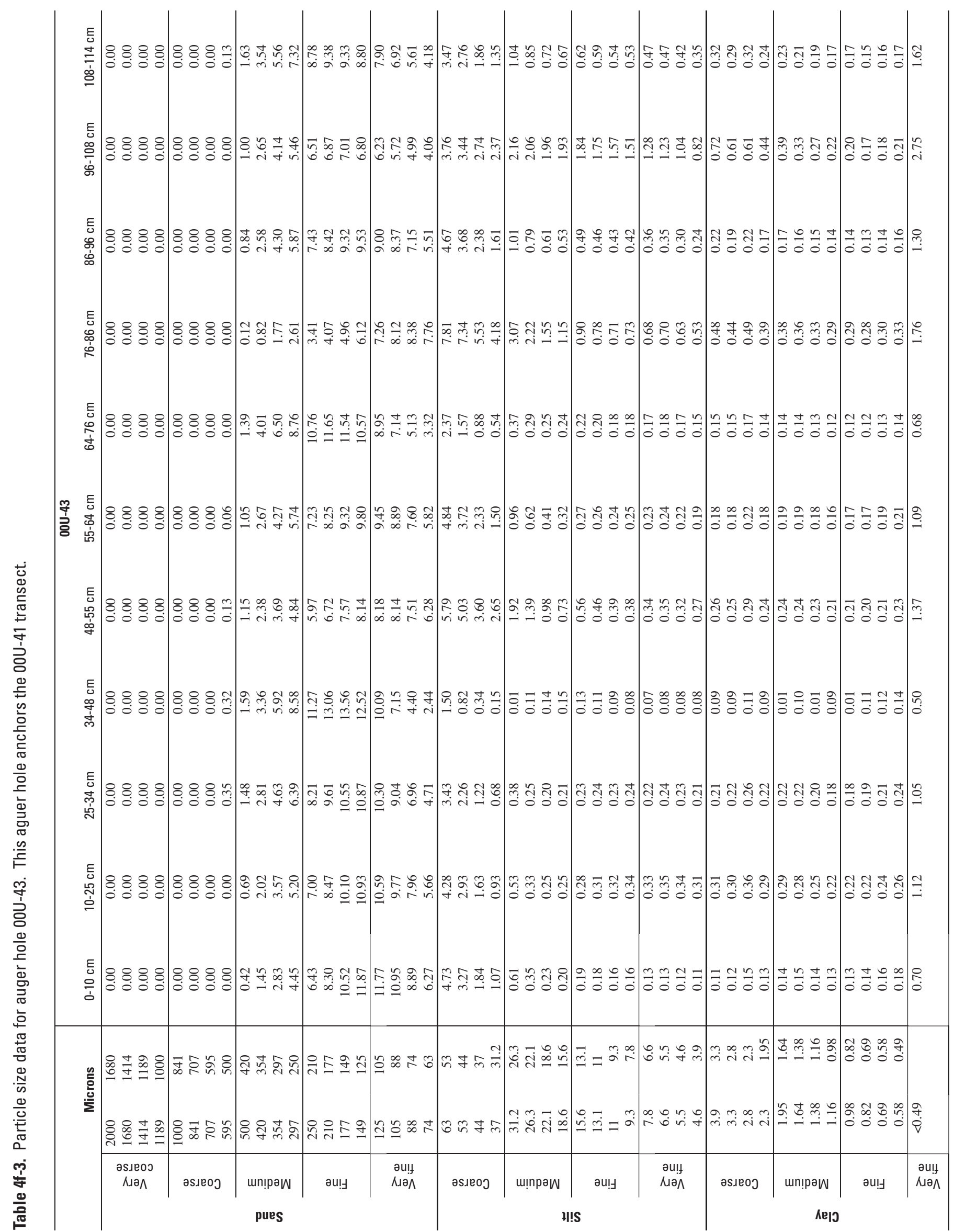


蒠

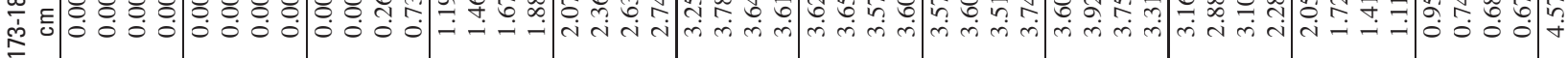

芒

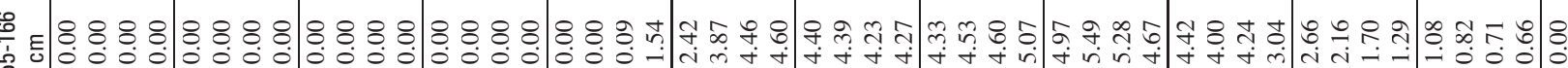

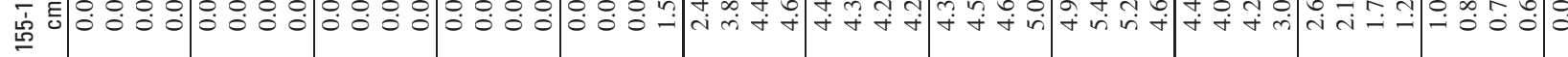
虽 至

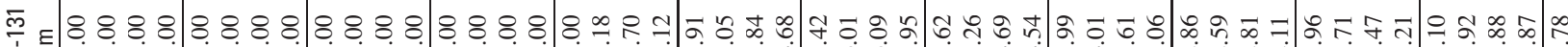
西 它 道 $\frac{\infty}{\overline{1}}$

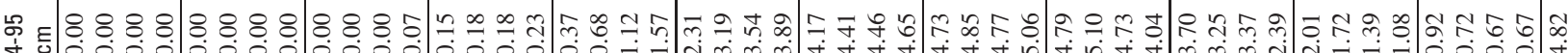

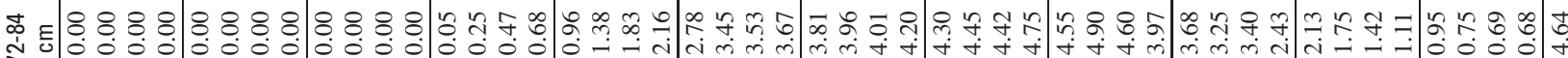

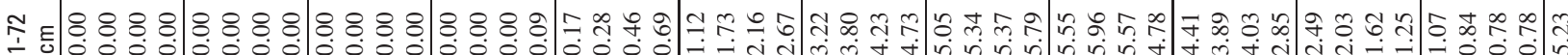
雇 둥 8.88 4

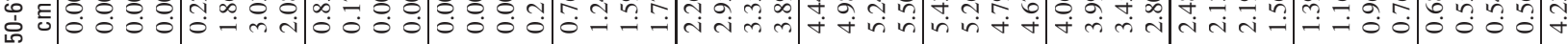

居 行888888888888888

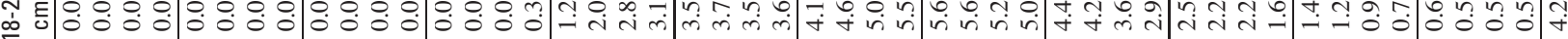

至

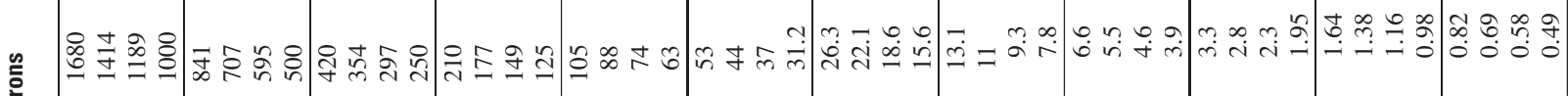

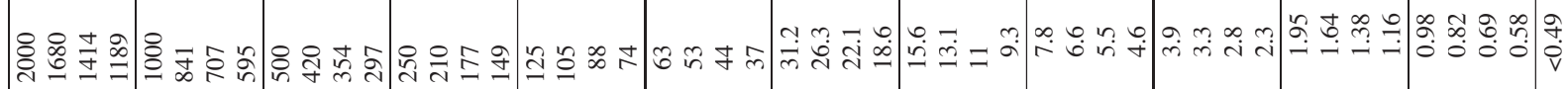

\begin{tabular}{|c|c|c|c|c|c|c|c|c|c|c|c|}
\hline 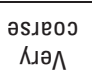 & әsıеoj & un!pәw & әи! & $\begin{array}{l}\text { әи!। } \\
\text { Кıә }\end{array}$ & әs.еоว & щ!nрәW & әи! & $\begin{array}{l}\text { әи! } \\
\Lambda \text { Кә }\end{array}$ & әs.еoว & un!pәw & әи! \\
\hline & & pues & & & & & & & & КЕ|ગ & \\
\hline
\end{tabular}




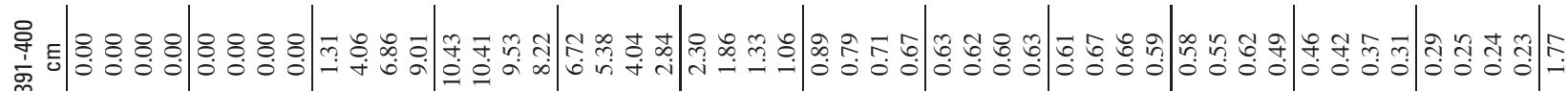

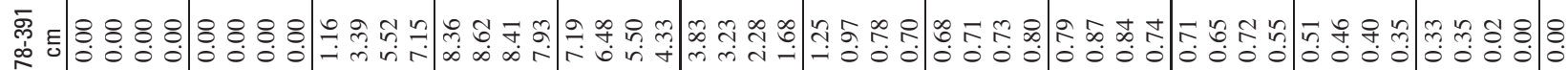
产

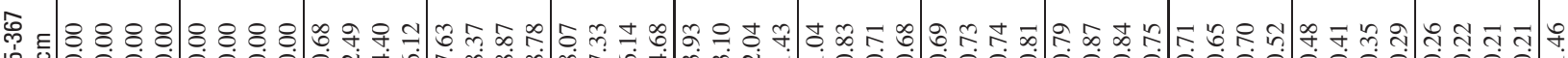

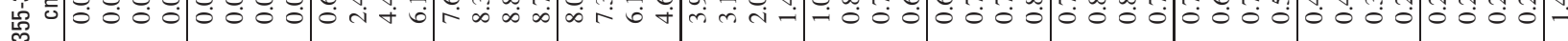

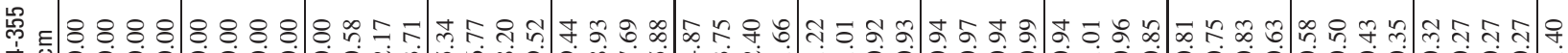

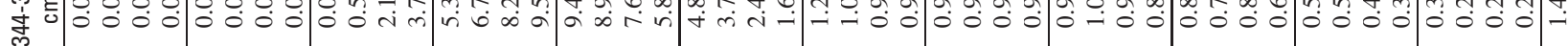
每

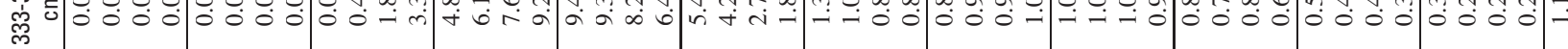
m 每

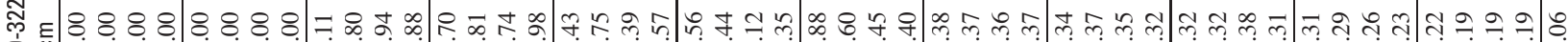

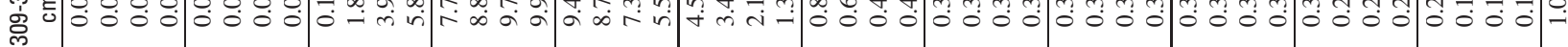

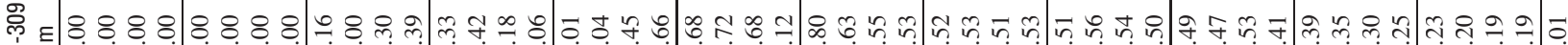

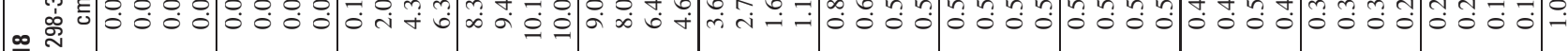

ळ

商

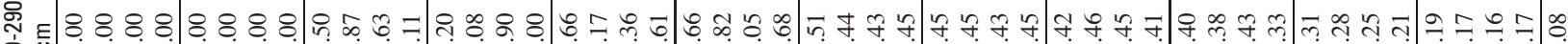
总 惫 商 $\varepsilon_{0}$

荣

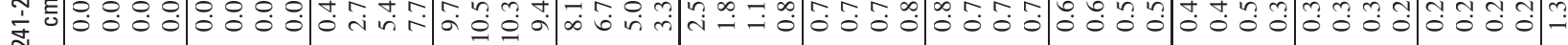

武

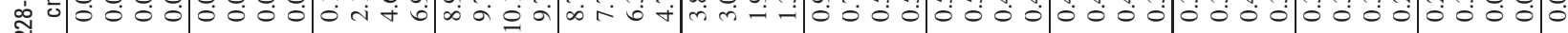

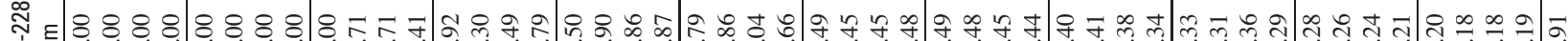

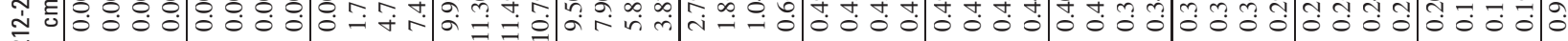

సัก

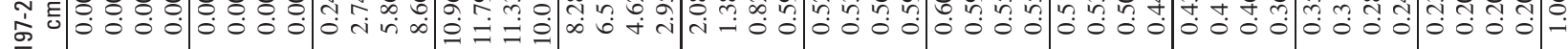
舟

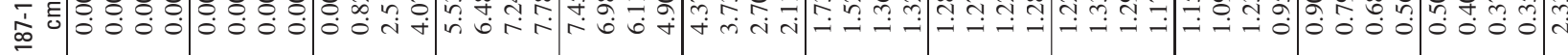

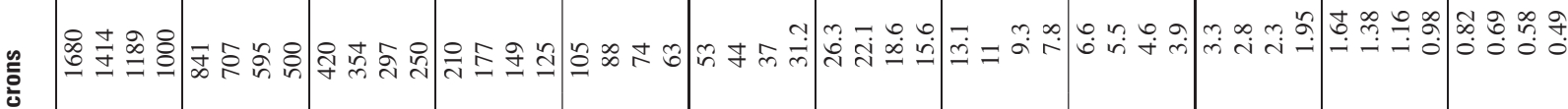

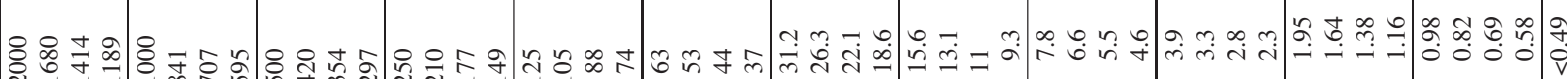

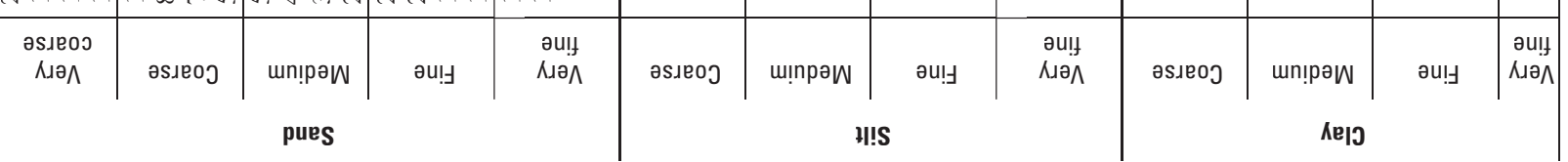


产 宫

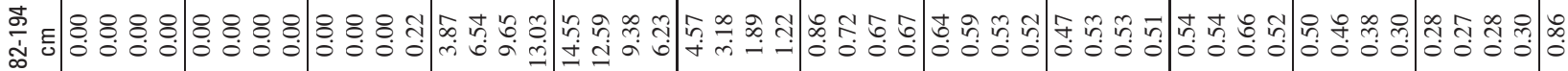

๗ 88888888888 ก बं 耍

星

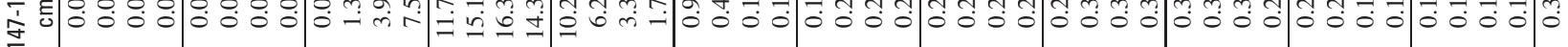

d 它

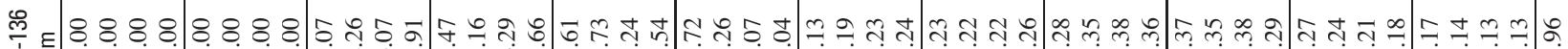

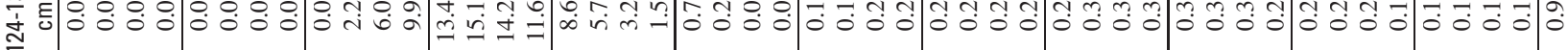

至

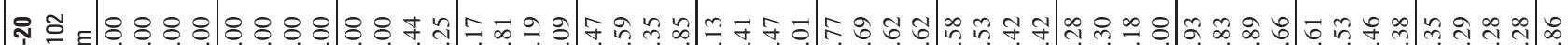

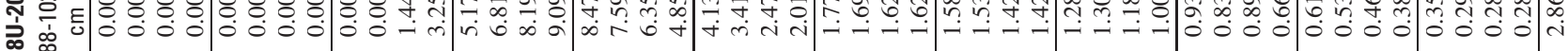
焉 店 $\mathrm{E}_{0}$ 년 $8888888888 ㄴ$

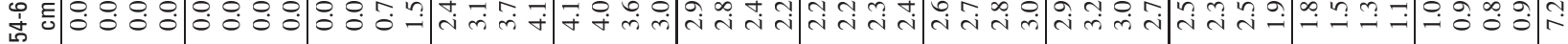
菅 守

出

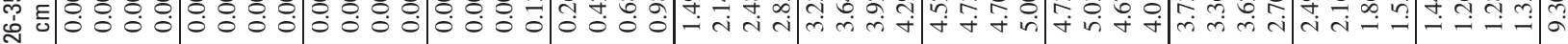
空 我

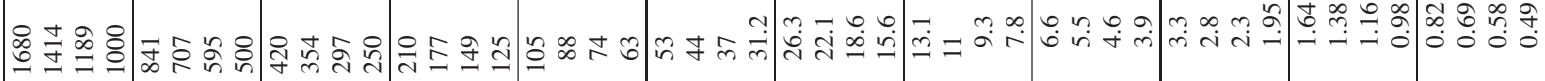

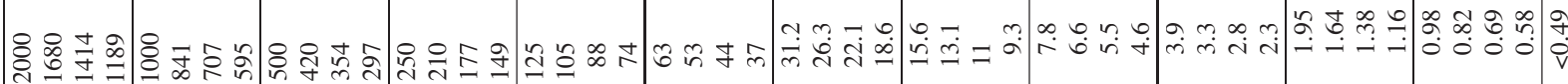

\begin{tabular}{|c|c|c|c|c|c|c|c|c|c|c|c|}
\hline 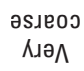 & әs.eoj & un!pәw & әи! & $\begin{array}{c}\text { әи!! } \\
\Lambda\lrcorner{ }^{\prime}\end{array}$ & әs.е०० & щ!̣рәw & әи! & $\begin{array}{l}\text { әи!! } \\
\text { Кә }\end{array}$ & әs.eoj & un!рәw & 14 \\
\hline
\end{tabular}

pues

H!S 


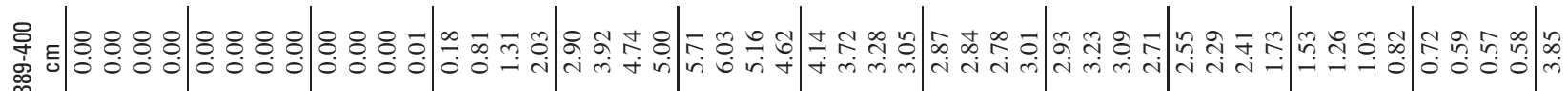

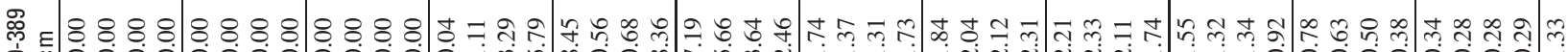

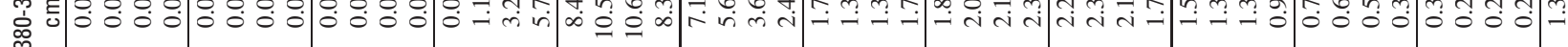

চ 을

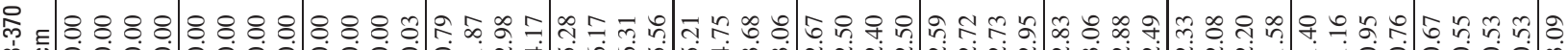

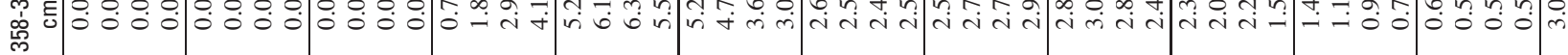

隽

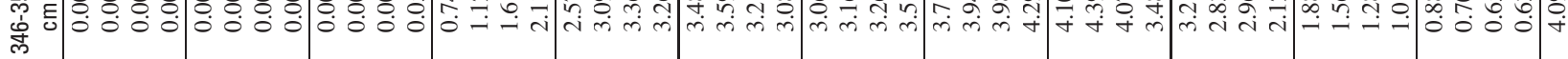

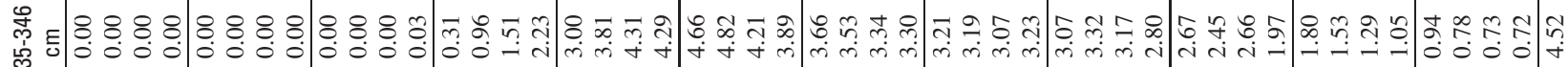

娓

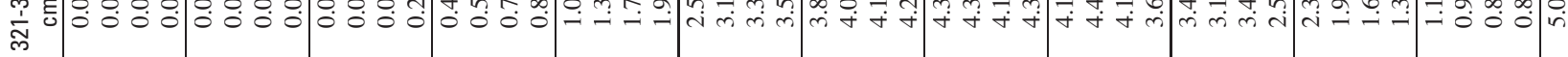

宔 宛

䍃 స్తి के

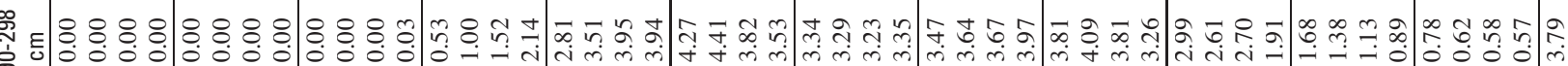
实

究 ळ̊ำ

๙ొ

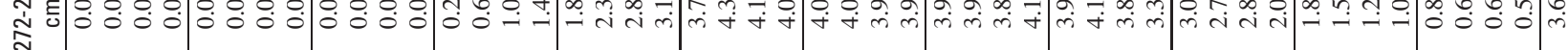

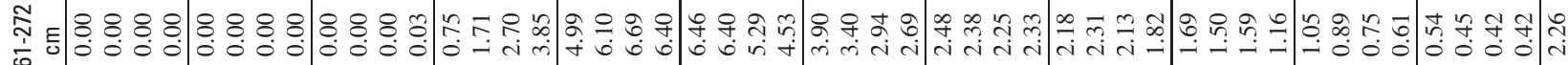

帘

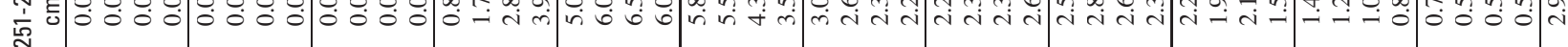

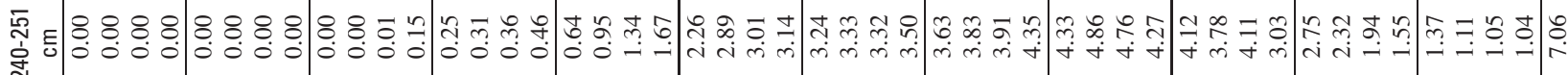
趈

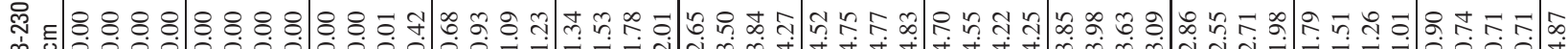

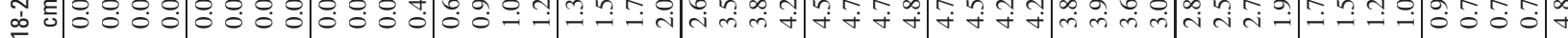

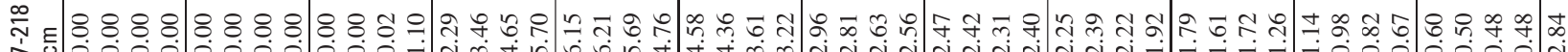
ลิ่

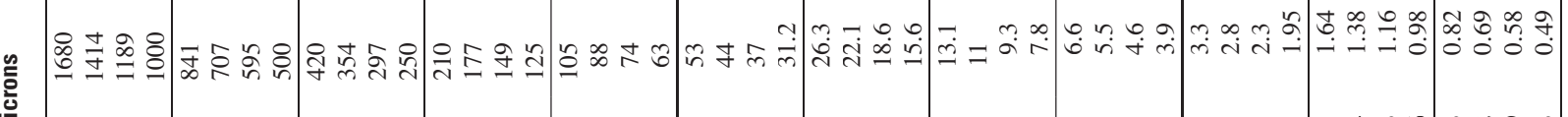

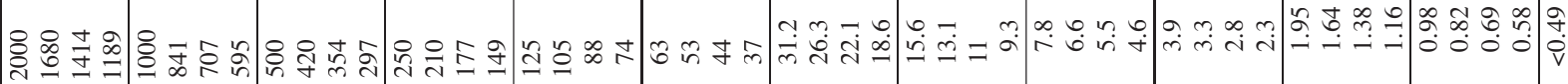

\begin{tabular}{|c|c|c|c|c|c|c|c|c|c|c|c|c|}
\hline $\begin{array}{c}\text { әsАво } \\
\text { КАә }\end{array}$ & әs.180 & un!pәW & әи! & 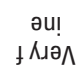 & әs.еoo & u!npәW & әu! & $\begin{array}{l}\text { әи!। } \\
\text { ᄉә }\end{array}$ & әs.еoj & un!̣әw & әи! & 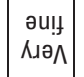 \\
\hline & & pues & & & & & & & & Ке|ગ & & \\
\hline
\end{tabular}




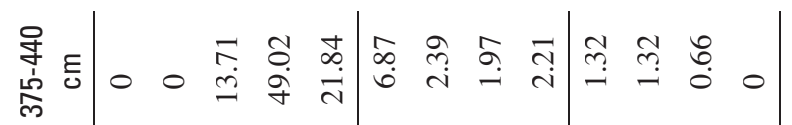

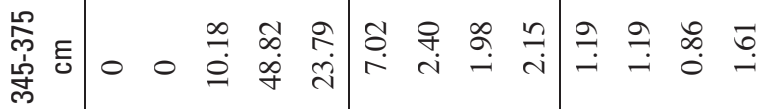

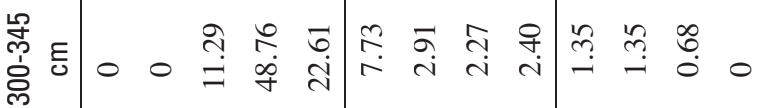

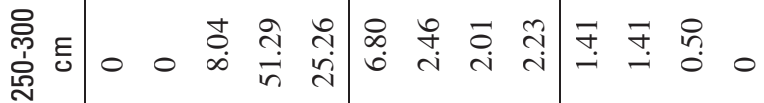

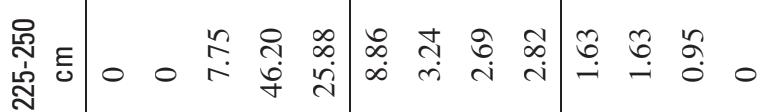

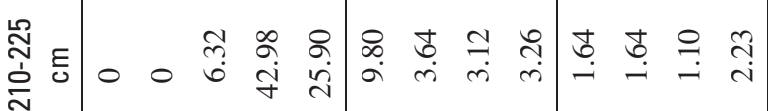

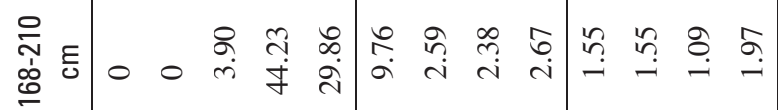

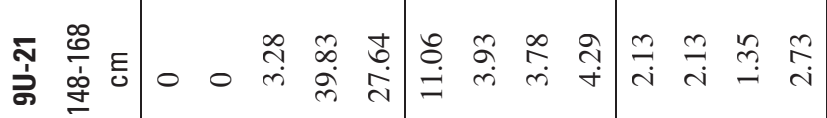

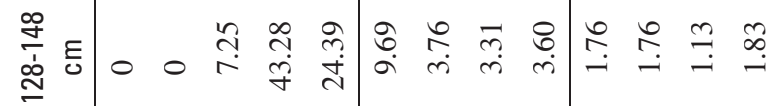

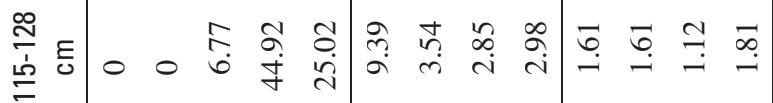

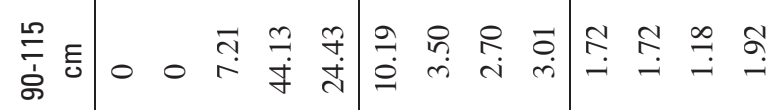

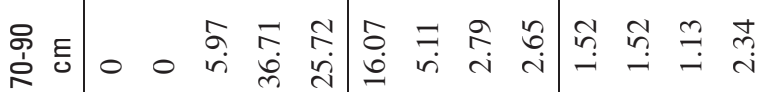

呙

虫

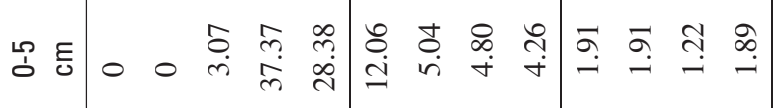

\begin{tabular}{|c|c|c|}
\hline 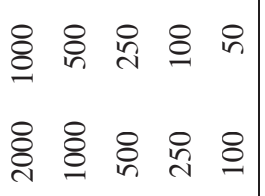 & $\begin{array}{l}\curvearrowright \circ n n \\
i \approx 0\end{array}$ & $\begin{array}{l}-r \tilde{0} \\
N \sim-\tilde{n} \\
N\end{array}$ \\
\hline 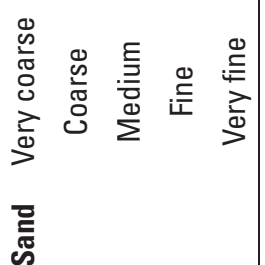 & 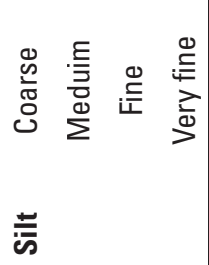 & 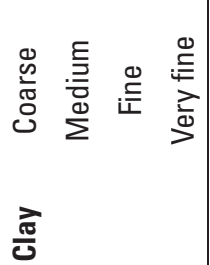 \\
\hline
\end{tabular}




\section{Table 5. Geochemical data}

This table contains geochemical data obtained by ICP-AES (Inductively coupled plasma - atomic emission spectrometry) and ICP-MS (Inductively coupled plasma - mass spectrometry) techniques (Lichte et al., 1987). All analyses were performed at the USGS Geologic Division geochemistry lab in Denver, CO and are reported in either parts per million (ppm) or percent (\%).

Table 5a-1a. ICP-AES data for the 00U-30 transect.

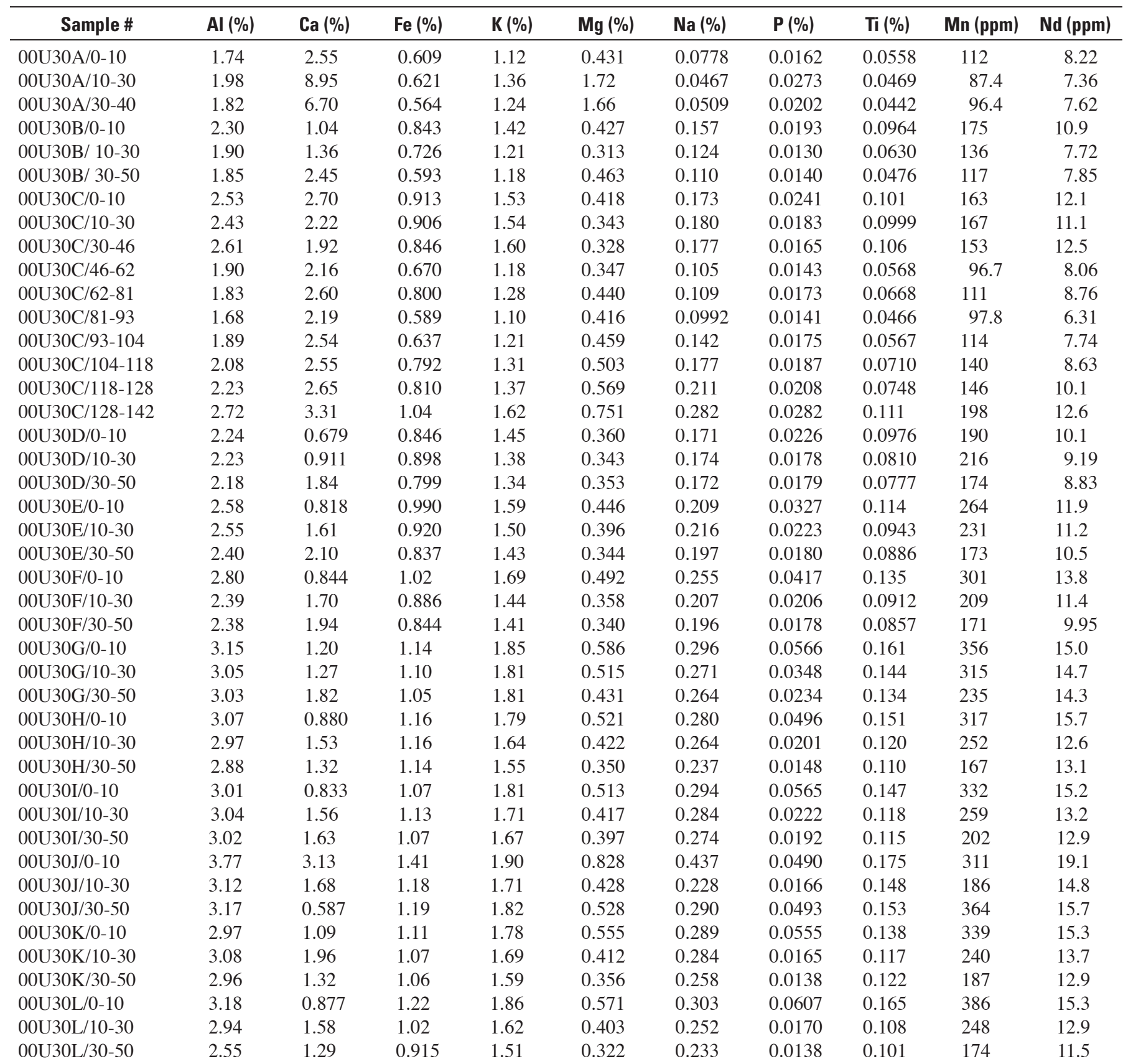


Table 5a-1a. ICP-AES data for the 00U-30 transect-Continued.

\begin{tabular}{llllllllllc}
\hline \multicolumn{1}{c}{ Sample \# } & Al (\%) & $\mathbf{C a}(\%)$ & $\mathbf{F e}(\%)$ & $\mathbf{K ~ ( \% )}$ & $\mathbf{M g}(\%)$ & $\mathbf{N a}(\%)$ & $\mathbf{P}(\%)$ & $\mathbf{T i}(\%)$ & $\mathbf{M n}(\mathbf{p p m})$ & $\mathbf{N d}(\mathbf{p p m})$ \\
\hline 00U30M/0-10 & 3.09 & 1.04 & 1.18 & 1.82 & 0.560 & 0.300 & 0.0502 & 0.170 & 328 & 15.0 \\
00U30M/10-30 & 3.18 & 2.06 & 1.16 & 1.85 & 0.502 & 0.306 & 0.0271 & 0.159 & 321 & 15.7 \\
00U30M/30-47 & 3.16 & 1.88 & 1.18 & 1.85 & 0.475 & 0.298 & 0.0252 & 0.148 & 274 & 15.0 \\
00U30M/47-61 & 2.68 & 1.41 & 0.959 & 1.57 & 0.342 & 0.260 & 0.0183 & 0.108 & 185 & 11.2 \\
00U30M/61-74 & 2.70 & 1.18 & 1.03 & 1.54 & 0.342 & 0.254 & 0.0183 & 0.110 & 182 & 11.9 \\
00U30M/74-89 & 2.76 & 0.762 & 1.13 & 1.51 & 0.397 & 0.216 & 0.0189 & 0.126 & 199 & 12.6 \\
00U30M/89-106 & 3.47 & 0.583 & 1.30 & 1.68 & 0.510 & 0.361 & 0.0227 & 0.138 & 242 & 15.6 \\
00U30M/106-118 & 3.86 & 3.46 & 1.46 & 1.91 & 0.879 & 0.453 & 0.0496 & 0.178 & 309 & 18.7 \\
00U30M/118-131 & 3.17 & 4.11 & 1.12 & 1.60 & 0.725 & 0.390 & 0.0364 & 0.118 & 224 & 13.3 \\
00U30M/131-144 & 3.30 & 3.61 & 1.15 & 1.81 & 0.748 & 0.398 & 0.0376 & 0.141 & 249 & 16.2 \\
\hline
\end{tabular}


Table 5a-1b. ICP-MS for the 00U-30 transect.

\begin{tabular}{|c|c|c|c|c|c|c|c|c|c|c|c|c|}
\hline Sample \# & $\begin{array}{c}\mathrm{Ag} \\
(\mathrm{ppm})\end{array}$ & $\begin{array}{c}\text { Al } \\
(\mathbf{p p m})\end{array}$ & $\begin{array}{c}\text { As } \\
\text { (ppm) }\end{array}$ & $\begin{array}{c}\mathrm{Ba} \\
\text { (ppm) }\end{array}$ & $\begin{array}{c}\text { Be } \\
(\mathrm{ppm})\end{array}$ & $\begin{array}{c}\mathbf{B i} \\
(\mathbf{p p m})\end{array}$ & $\begin{array}{c}\text { Ca } \\
\text { (ppm) }\end{array}$ & $\underset{(p p m)}{C d}$ & $\begin{array}{c}\mathrm{Ce} \\
\text { (ppm) }\end{array}$ & $\begin{array}{c}\text { Co } \\
\text { (ppm) }\end{array}$ & $\underset{(\mathbf{p p m})}{\mathrm{Cr}}$ & $\begin{array}{c}\text { Cs } \\
\text { (ppm) }\end{array}$ \\
\hline 00U30A/0-10 & $<2$ & 19200 & 4.0 & 239 & 0.54 & $<0.06$ & 28200 & 0.06 & 17.0 & 1.8 & 40.6 & 1.3 \\
\hline 00U30A/10-30 & $<2$ & 19000 & 3.8 & 291 & 0.88 & $<0.06$ & 81000 & 0.05 & 18.1 & 1.6 & 28.4 & 1.5 \\
\hline 00U30A/30-40 & $<2$ & 17800 & 3.4 & 264 & 0.83 & $<0.06$ & 61200 & 0.04 & 15.9 & 1.6 & 33.1 & 1.4 \\
\hline $00 \mathrm{U} 30 \mathrm{~B} / 0-10$ & $<2$ & 26300 & 2.9 & 311 & 0.68 & $<0.06$ & 12100 & 0.08 & 23.3 & 2.5 & 52.2 & 1.8 \\
\hline 00U30B/ 10-30 & $<2$ & 19400 & 2.7 & 260 & 0.74 & $<0.06$ & 12800 & 0.05 & 16.6 & 1.8 & 47.4 & 1.5 \\
\hline $00 \mathrm{U} 30 \mathrm{~B} / 30-50$ & $<2$ & 18500 & 3 & 256 & 0.64 & 0.06 & 23100 & 0.07 & 15 & 1.7 & 31.9 & 1.3 \\
\hline 00U30C/0-10 & $<2$ & 28300 & 3.5 & 330 & 0.89 & $<0.06$ & 30000 & 0.08 & 25.3 & 2.9 & 53.4 & 2.0 \\
\hline 00U30C/10-30 & $<2$ & 27500 & 3.5 & 338 & 0.86 & $<0.06$ & 25500 & 0.06 & 24.6 & 2.8 & 57.0 & 1.9 \\
\hline $00 \mathrm{U} 30 \mathrm{C} / 30-46$ & $<2$ & 30100 & 3.7 & 355 & 0.85 & $<0.06$ & 22200 & 0.05 & 25.6 & 3.1 & 31.2 & 2.1 \\
\hline 00U30C/46-62 & $<2$ & 18800 & 2.4 & 263 & 0.62 & $<0.06$ & 20200 & 0.05 & 15.9 & 2 & 29.3 & 1.5 \\
\hline 00U30C/62-81 & $<2$ & 22000 & 2.8 & 299 & 0.62 & $<0.06$ & 30300 & 0.05 & 18.7 & 2.4 & 61.0 & 1.5 \\
\hline 00U30C/81-93 & $<2$ & 16300 & 2.2 & 249 & 0.47 & $<0.06$ & 20500 & 0.04 & 13.2 & 1.6 & 29.4 & 1.2 \\
\hline 00U30C/93-104 & $<2$ & 18800 & 2.6 & 270 & 0.72 & $<0.06$ & 23600 & 0.05 & 16.1 & 1.9 & 25.5 & 1.4 \\
\hline 00U30C/104-118 & $<2$ & 20700 & 3.1 & 322 & 0.72 & $<0.06$ & 23800 & 0.05 & 18.8 & 2.2 & 40.5 & 1.6 \\
\hline 00U30C/118-128 & $<2$ & 22100 & 2.9 & 328 & 0.73 & $<0.06$ & 24200 & 0.05 & 19.7 & 2.3 & 35.7 & 1.6 \\
\hline 00U30C/128-142 & $<2$ & 31100 & 3.6 & 409 & 0.96 & $<0.06$ & 37300 & 0.08 & 27.8 & 3.4 & 52.7 & 2.0 \\
\hline 00U30D/0-10 & $<2$ & 26400 & 2.5 & 330 & 0.68 & $<0.06$ & 8000 & 0.08 & 23.3 & 2.5 & 51.6 & 1.7 \\
\hline 00U30D/10-30 & $<2$ & 22400 & 2.5 & 299 & 0.79 & $<0.06$ & 8450 & 0.06 & 19.8 & 2.3 & 50 & 1.8 \\
\hline 00U30D/30-50 & $<2$ & 22000 & 2.5 & 302 & 0.74 & $<0.06$ & 17400 & 0.06 & 20.4 & 2.1 & 40 & 1.7 \\
\hline 00U30E/0-10 & $<2$ & 31500 & 3.0 & 364 & 0.79 & $<0.06$ & 9860 & 0.11 & 27.2 & 3.1 & 63.1 & 2.1 \\
\hline 00U30E/10-30 & $<2$ & 26000 & 2.8 & 330 & 0.86 & 0.06 & 15300 & 0.14 & 24.9 & 2.6 & 36.6 & 2.1 \\
\hline 00U30E/30-50 & $<2$ & 24500 & 2.7 & 317 & 0.88 & 0.06 & 20000 & 0.06 & 26.4 & 2.4 & 34.2 & 2 \\
\hline 00U30F/0-10 & $<2$ & 35000 & 3.1 & 398 & 1.3 & $<0.06$ & 10300 & 0.14 & 30.9 & 3.4 & 48.8 & 2.4 \\
\hline 00U30F/10-30 & $<2$ & 24500 & 2.7 & 322 & 0.82 & 0.06 & 16100 & 0.08 & 23.5 & 2.4 & 41.6 & 1.9 \\
\hline $00 \mathrm{U} 30 \mathrm{~F} / 30-50$ & $<2$ & 24300 & 2.5 & 318 & 0.8 & $<0.06$ & 18900 & 0.05 & 22.8 & 2.4 & 35.6 & 1.9 \\
\hline 00U30G/0-10 & $<2$ & 38300 & 3.6 & 422 & 1.2 & $<0.06$ & 13900 & 0.16 & 34.6 & 3.8 & 50.4 & 2.7 \\
\hline 00U30G/10-30 & $<2$ & 37100 & 3.2 & 407 & 0.96 & $<0.06$ & 15200 & 0.10 & 34.0 & 3.8 & 45.0 & 2.6 \\
\hline 00U30G/30-50 & $<2$ & 37600 & 3.2 & 404 & 0.91 & $<0.06$ & 22000 & 0.07 & 32.2 & 3.8 & 43.3 & 2.6 \\
\hline 00U30H/0-10 & $<2$ & 38900 & 3.2 & 402 & 1.2 & $<0.06$ & 10600 & 0.12 & 37.0 & 4.0 & 48.8 & 2.7 \\
\hline 00U30H/10-30 & $<2$ & 29500 & 3.3 & 364 & 0.85 & 0.09 & 14900 & 0.07 & 27.1 & 3.3 & 57 & 2.3 \\
\hline 00U30H/30-50 & $<2$ & 28900 & 3.4 & 354 & 0.9 & 0.08 & 13000 & 0.04 & 26.3 & 3.4 & 55.9 & 2.2 \\
\hline 00U30I/0-10 & $<2$ & 37000 & 3.1 & 419 & 0.87 & $<0.06$ & 9970 & 0.14 & 33.8 & 3.6 & 44.8 & 2.5 \\
\hline 00U30I/10-30 & $<2$ & 29900 & 3 & 374 & 0.87 & 0.08 & 15100 & 0.08 & 28.2 & 3.4 & 54.3 & 2.4 \\
\hline 00U30I/30-50 & $<2$ & 30200 & 3.1 & 375 & 0.9 & 0.08 & 16000 & 0.06 & 28.2 & 3.4 & 39.8 & 2.4 \\
\hline 00U30J/0-10 & $<2$ & 46100 & 4.3 & 477 & 1.2 & $<0.06$ & 39800 & 0.11 & 42.5 & 5.7 & 48.1 & 3.1 \\
\hline $00 \mathrm{U} 30 \mathrm{~J} / 10-30$ & $<2$ & 38700 & 3.4 & 387 & 1.0 & $<0.06$ & 22500 & 0.06 & 34.3 & 4.4 & 53.5 & 2.8 \\
\hline $00 \mathrm{U} 30 \mathrm{~J} / 30-50$ & $<2$ & 40700 & 3.5 & 424 & 1.2 & $<0.06$ & 7210 & 0.14 & 37.3 & 4.3 & 44.3 & 2.8 \\
\hline 00U30K/0-10 & $<2$ & 37400 & 3.0 & 420 & 0.96 & $<0.06$ & 13200 & 0.17 & 33.8 & 3.8 & 51.2 & 2.5 \\
\hline 00U30K/10-30 & $<2$ & 30100 & 3.1 & 372 & 0.82 & 0.08 & 18900 & 0.06 & 29.4 & 3.4 & 38.2 & 2.4 \\
\hline $00 \mathrm{U} 30 \mathrm{~K} / 30-50$ & $<2$ & 30400 & 3.1 & 379 & 0.86 & 0.08 & 13200 & 0.04 & 28.8 & 3.5 & 36.2 & 2.4 \\
\hline $00 \mathrm{U} 30 \mathrm{~L} / 0-10$ & $<2$ & 40000 & 3.3 & 429 & 0.84 & $<0.06$ & 10700 & 0.17 & 36.0 & 4.3 & 54.9 & 2.7 \\
\hline 00U30L/10-30 & $<2$ & 29000 & 2.8 & 354 & 0.8 & 0.08 & 15200 & 0.08 & 27.9 & 3.2 & 34.5 & 2.3 \\
\hline $00 \mathrm{U} 30 \mathrm{~L} / 30-50$ & $<2$ & 26100 & 2.7 & 338 & 0.76 & 0.07 & 12500 & 0.05 & 24.8 & 2.8 & 32.7 & 2.1 \\
\hline $00 \mathrm{U} 30 \mathrm{M} / 0-10$ & $<2$ & 39300 & 3.4 & 426 & 0.99 & $<0.06$ & 12700 & 0.16 & 38.4 & 4.0 & 56.2 & 2.6 \\
\hline 00U30M/10-30 & $<2$ & 39800 & 3.5 & 422 & 0.73 & $<0.06$ & 24700 & 0.12 & 34.7 & 4.3 & 46.4 & 2.7 \\
\hline 00U30M/30-47 & $<2$ & 40100 & 3.3 & 428 & 1.0 & $<0.06$ & 22800 & 0.08 & 35.2 & 4.3 & 55.1 & 2.7 \\
\hline 00U30M/47-61 & $<2$ & 27600 & 2.6 & 364 & 0.83 & 0.08 & 13600 & 0.05 & 26 & 3 & 33.9 & 2.2 \\
\hline 00U30M/61-74 & $<2$ & 27900 & 2.8 & 360 & 0.76 & 0.08 & 11300 & 0.05 & 25.9 & 3.2 & 39.6 & 2.2 \\
\hline 00U30M/74-89 & $<2$ & 36200 & 3.4 & 376 & 0.97 & $<0.06$ & 9510 & 0.04 & 30.3 & 4.3 & 58.7 & 2.5 \\
\hline 00U30M/89-106 & $<2$ & 34700 & 3.6 & 406 & 1 & 0.1 & 5760 & 0.04 & 32.6 & 4.2 & 44.1 & 2.7 \\
\hline 00U30M/106-118 & $<2$ & 47800 & 4.5 & 471 & 0.93 & $<0.06$ & 40800 & 0.10 & 43.6 & 5.7 & 49.2 & 3.2 \\
\hline 00U30M/118-131 & $<2$ & 30900 & 4.1 & 460 & 0.92 & 0.08 & 40200 & 0.09 & 27.9 & 3.7 & 37.4 & 2.4 \\
\hline 00U30M/131-144 & $<2$ & 41200 & 4.4 & 468 & 1.1 & $<0.06$ & 43000 & 0.09 & 36.1 & 4.6 & 42.3 & 2.7 \\
\hline
\end{tabular}


Table 5a-1b. ICP-MS for the 00U-30 transect-Continued.

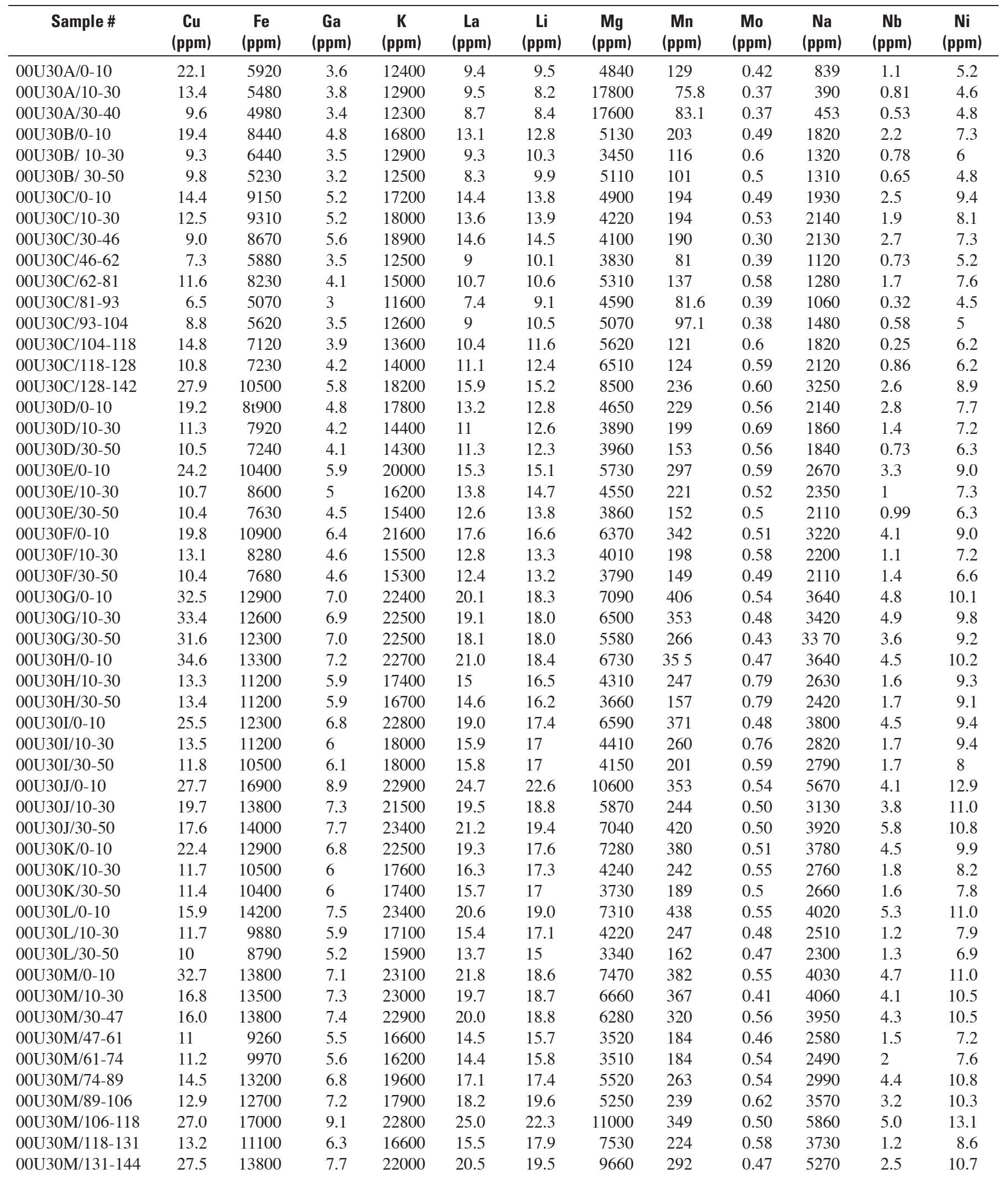


Table 5a-1b. ICP-MS for the 00U-30 transect-Continued.

\begin{tabular}{|c|c|c|c|c|c|c|c|c|c|c|c|c|c|}
\hline Sample \# & $\underset{(\mathbf{p p m})}{P}$ & $\begin{array}{c}\mathrm{Pb} \\
(\mathbf{p p m})\end{array}$ & $\begin{array}{c}\mathbf{R b} \\
(\mathbf{p p m})\end{array}$ & $\underset{(\mathbf{p p m})}{\mathbf{S b}}$ & $\begin{array}{c}\text { Sc } \\
(\mathbf{p p m})\end{array}$ & $\underset{(\mathbf{p p m})}{\mathrm{Sr}}$ & $\begin{array}{c}\text { Th } \\
\text { (ppm) }\end{array}$ & $\underset{(\mathbf{p p m})}{\mathrm{Ti}}$ & $\begin{array}{c}\mathrm{TI} \\
(\mathbf{p p m})\end{array}$ & $\underset{(\mathbf{p p m})}{\mathbf{U}}$ & $\begin{array}{c}\mathbf{V} \\
(\mathbf{p p m})\end{array}$ & $\begin{array}{c}Y \\
\text { (ppm) }\end{array}$ & $\underset{(p p m)}{\mathrm{Zn}}$ \\
\hline 00U30A/0-10 & 175 & 7.59 & 31.9 & 0.2 & 1.8 & 66.1 & 2.27 & 812 & 0.18 & 0.58 & 11.1 & 7.5 & 26.4 \\
\hline 00U30A/10-30 & 264 & 5.06 & 30.9 & 0.1 & 2.1 & 198 & 2.58 & 472 & 0.2 & 0.55 & 12 & 5.1 & 14 \\
\hline 00U30A/30-40 & 202 & 4.92 & 29.5 & 0.2 & 1.8 & 162 & 2.36 & 467 & 0.19 & 0.52 & 10.7 & 5.1 & 13.4 \\
\hline 00U30B/0-10 & 219 & 10.4 & 43.4 & 0.24 & 2.7 & 63.5 & 3.62 & 1420 & 0.24 & 0.78 & 16.5 & 10.2 & 26.5 \\
\hline 00U30B/ 10-30 & 124 & 6.68 & 33.5 & 0.2 & 1.8 & 44.7 & 2.76 & 574 & 0.22 & 0.61 & 10.9 & 6 & 13.2 \\
\hline $00 \mathrm{U} 30 \mathrm{~B} / 30-50$ & 153 & 6.52 & 31.8 & 0.2 & 1.7 & 55.2 & 2.56 & 478 & 0.23 & 0.56 & 10.6 & 5.4 & 26.6 \\
\hline $00 \mathrm{U} 30 \mathrm{C} / 0-10$ & 258 & 9.78 & 45.8 & 0.26 & 3.0 & 80.5 & 3.79 & 1490 & 0.27 & 0.87 & $\mathrm{t} 19.8$ & 11.6 & 22.7 \\
\hline 00U30C/10-30 & 192 & 9.28 & 47.0 & 0.25 & 2.9 & 82.6 & 3.60 & 1410 & 0.26 & 0.80 & 19.4 & 10.6 & 35.4 \\
\hline 00U30C/30-46 & 174 & 9.99 & 49.7 & 0.22 & 3.1 & 86.4 & 3.71 & 1520 & 0.28 & 0.80 & 22.1 & 11.0 & 20.0 \\
\hline 00U30C/46-62 & 146 & 6.86 & 34 & 0.2 & 2 & 56 & 2.65 & 539 & 0.23 & 0.58 & 13.6 & 5.8 & 14.1 \\
\hline 00U30C/62-81 & 193 & 8.23 & 39.4 & 0.2 & 2.2 & 79.3 & 2.77 & 986 & 0.22 & 0.63 & 14.4 & 8.2 & 14.4 \\
\hline 00U30C/81-93 & 140 & 6.37 & 31.3 & 0.2 & 1.6 & 53.1 & 2.16 & 436 & 0.21 & 0.53 & 10.5 & 4.9 & 11.6 \\
\hline 00U30C/93-104 & 167 & 6.87 & 33.8 & 0.2 & 1.9 & 64.7 & 2.73 & 572 & 0.23 & 0.7 & 13.7 & 6 & 13.9 \\
\hline 00U30C/104-118 & 177 & 7.39 & 36.7 & 0.2 & 2.2 & 72.8 & 3.18 & 641 & 0.25 & 0.77 & 16 & 6.8 & 16.8 \\
\hline 00U30C/118-128 & 206 & 7.62 & 38.1 & 0.22 & 2.4 & 80.7 & 3.39 & 688 & 0.26 & 0.84 & 17 & 7.2 & 17.9 \\
\hline 00U30C/128-142 & 312 & 10.5 & 48.5 & 0.26 & 3.4 & 120 & 4.22 & 1580 & 0.27 & 0.97 & 24.9 & 12.3 & 24.9 \\
\hline 00U30D/0-10 & 262 & 11.0 & 45.6 & 0.27 & 2.6 & 61.0 & 3.45 & 1430 & 0.24 & 0.80 & 16.3 & 10.3 & 19.8 \\
\hline 00U30D/10-30 & 171 & 7.8 & 39.2 & 0.26 & 2.3 & 47.9 & 3.34 & 764 & 0.27 & 0.74 & 14.2 & 7.4 & 17.4 \\
\hline 00U30D/30-50 & 180 & 7.5 & 38.7 & 0.22 & 2.3 & 54.3 & 3.33 & 710 & 0.27 & 0.75 & 14.2 & 7.4 & 16.3 \\
\hline 00U30E/0-10 & 395 & 11.0 & 52.1 & 0.32 & 3.3 & 71.2 & 4.03 & 1740 & 0.28 & 0.89 & 20.5 & 12.7 & 27.8 \\
\hline 00U30E/10-30 & 230 & 8.6 & 44.2 & 0.25 & 2.8 & 57.9 & 4.12 & 896 & 0.3 & 0.86 & 17.4 & 8.8 & 20.6 \\
\hline 00U30E/30-50 & 182 & 7.93 & 41.8 & 0.23 & 2.6 & 62.7 & 3.76 & 874 & 0.28 & 0.82 & 17 & 8.4 & 18.8 \\
\hline 00U30F/0-10 & 512 & 12.7 & 56.4 & 0.33 & 3.8 & 79.1 & 4.58 & 2120 & 0.31 & 1.07 & 24.0 & 14.7 & 29.2 \\
\hline 00U30F/10-30 & 198 & 8.08 & 42.3 & 0.24 & 2.6 & 57.8 & 3.83 & 845 & 0.28 & 0.82 & 16.4 & 8.4 & 19.6 \\
\hline 00U30F/30-50 & 192 & 7.95 & 42 & 0.26 & 2.5 & 62.6 & 3.74 & 817 & 0.28 & 0.77 & 16.2 & 8.1 & 18.8 \\
\hline 00U30G/0-10 & 680 & 13.8 & 60.9 & 0.37 & 4.2 & 89.4 & 5.06 & 2310 & 0.34 & 1.17 & 26.5 & 16.1 & 34.1 \\
\hline 00U30G/10-30 & 420 & 12.0 & 59.1 & 0.32 & 4.2 & 88.0 & 5.01 & 2230 & 0.31 & 1.10 & 26.6 & 15.0 & 32.6 \\
\hline 00U30G/30-50 & 280 & 12.0 & 58.6 & 0.31 & 4.1 & 93.8 & 4.75 & 2080 & 0.33 & 1.10 & 27.4 & 14.4 & 30.9 \\
\hline 00U30H/0-10 & 610 & 13.7 & 60.8 & 0.37 & 4.4 & 84.8 & 5.53 & 2350 & 0.35 & 1.23 & 28.1 & 16.7 & 41.1 \\
\hline 00U30H/10-30 & 198 & 9.52 & 51.8 & 0.32 & 3.5 & 69.7 & 4.34 & 1200 & 0.34 & 0.99 & 22.2 & 10.6 & 24.2 \\
\hline 00U30H/30-50 & 146 & 9.59 & 50.5 & 0.31 & 3.4 & 68.1 & 4.4 & 1080 & 0.34 & 0.92 & 22.5 & 9.6 & 25.7 \\
\hline 00U30I/0-10 & 679 & 13.3 & 59.7 & 0.35 & 4.1 & 82.7 & 4.98 & 2200 & 0.33 & 1.11 & 25.6 & 15.3 & 31.8 \\
\hline 00U30I/10-30 & 222 & 9.83 & 53 & 0.33 & 3.6 & 70.1 & 4.67 & 1190 & 0.35 & 1.02 & 22.4 & 10.9 & 24.4 \\
\hline 00U30I/30-50 & 195 & 9.82 & 52.8 & 0.29 & 3.5 & 75.3 & 4.76 & 1170 & 0.34 & 1.02 & 23.1 & 10.7 & 25.9 \\
\hline 00U30J/0-10 & 548 & 14.7 & 66.8 & 0.36 & 5.6 & 146 & 6.31 & 2580 & 0.40 & 1.48 & 41.0 & 19.5 & 39.9 \\
\hline $00 \mathrm{U} 30 \mathrm{~J} / 10-30$ & 206 & 11.9 & 60.4 & 0.33 & 4.6 & 96.0 & 5.14 & 2200 & 0.34 & 1.09 & 29.4 & 15.6 & 30.9 \\
\hline $00 \mathrm{U} 30 \mathrm{~J} / 30-50$ & 631 & 13.4 & 63.9 & 038 & 4.5. & 84.5 & 5.75 & 2520 & 0.36 & 1.28 & 29.4 & 17.5 & 35.2 \\
\hline 00U30K/0-10 & 684 & 13.3 & 60.6 & 0.38 & 4.1 & 90.0 & 4.94 & 2250 & 0.33 & 1.13 & 25.8 & 15.8 & 33.2 \\
\hline 00U30K/10-30 & 161 & 9.68 & 52.8 & 0.31 & 3.5 & 72 & 4.85 & 1170 & 0.35 & 1 & 23.2 & 10.7 & 24.4 \\
\hline 00U30K/30-50 & 142 & 9.78 & 52.8 & 0.29 & 3.5 & 72.8 & 4.85 & 1200 & 0.35 & 0.98 & 23.5 & 10.3 & 25.4 \\
\hline $00 \mathrm{U} 30 \mathrm{~L} / 0-10$ & 762 & 13.7 & 63.0 & 042 & 4.6 & 90.7 & 5.45 & 2480 & 0.37 & 1.22 & 28.8 & 16.9 & 33.7 \\
\hline 00U30L/10-30 & 161 & 9.41 & 51.7 & 0.28 & 3.4 & 65.8 & 4.58 & 1040 & 0.34 & 0.95 & 22 & 10 & 24.4 \\
\hline $00 \mathrm{U} 30 \mathrm{~L} / 30-50$ & 142 & 8.91 & 48 & 0.24 & 2.9 & 64.5 & 4.13 & 1010 & 0.31 & 0.9 & 19.9 & 9 & 22.9 \\
\hline 00U30M/0-10 & 654 & 14.4 & 61.5 & 0.39 & 4.4 & 88.1 & 5.55 & 2500 & 0.35 & 1.28 & 27.6 & 17.1 & 39.9 \\
\hline 00U30M/10-30 & 332 & 12.6 & 63.9 & 0.37 & 4.6 & 98.6 & 5.15 & 2390 & 0.34 & 1.20 & 30.0 & 16.4 & 30.3 \\
\hline 00U30M/30-47 & 310 & 13.2 & 63.8 & 0.35 & 4.5 & 103 & 5.10 & 2420 & 0.35 & 1.16 & 29.6 & 16.1 & 30.1 \\
\hline 00U30M/47-61 & 178 & 10 & 50.1 & 0.27 & 3.1 & 71.4 & 4.31 & 1040 & 0.32 & 0.9 & 21.2 & 9.4 & 23.5 \\
\hline 00U30M/61-74 & 189 & 9.36 & 49.8 & 0.28 & 3.2 & 72.3 & 4.47 & 1090 & 0.33 & 0.91 & 22.1 & 9.5 & 23.6 \\
\hline 00U30M/74-89 & 236 & 11.7 & 55.6 & 036 & 4.0 & 87.7 & 4.54 & 1910 & 0.31 & 0.96 & 28.0 & 13.9 & 26.7 \\
\hline 00U30M/89-106 & 232 & 11.3 & 55.2 & 0.38 & 4.3 & 81.1 & 5.62 & 1410 & 0.38 & 1.19 & 30.2 & 12.4 & 30.4 \\
\hline 00U30M/106-118 & 584 & 14.4 & 66.3 & 0.43 & 5.8 & 145 & 6.46 & 2730 & 0.40 & 1.47 & 41.4 & 19.8 & 40.2 \\
\hline 00U30M/118-131 & 359 & 9.91 & 51.2 & 0.31 & 3.9 & 127 & 4.68 & 1170 & 0.35 & 1.26 & 27.7 & 11.2 & 26.9 \\
\hline 00U30M/131-144 & 429 & 12.6 & 61.9 & 0.30 & 4.6 & 137 & 5.16 & 2120 & 0.35 & 1.39 & 34.9 & 16.1 & 33.2 \\
\hline
\end{tabular}


Table 5a-2a. ICP-AES data for the VP-1 soil pit.

\begin{tabular}{llllllllllc}
\hline \multicolumn{1}{c}{ Sample \# } & $\mathbf{A l}(\%)$ & $\mathbf{C a}(\%)$ & $\mathbf{F e}(\%)$ & $\mathbf{K}(\%)$ & $\mathbf{M g}(\%)$ & $\mathbf{N a}(\%)$ & $\mathbf{P}(\%)$ & $\mathbf{T i}(\%)$ & $\mathbf{M n}(\mathbf{p p m})$ & $\mathbf{N d}(\mathbf{p p m})$ \\
\hline VP1/0-6 & 3.28 & 0.513 & 1.16 & 1.95 & 0.481 & 0.386 & 0.0600 & 0.151 & 354 & 15.9 \\
VP1/6-19 & 3.94 & 1.35 & 1.37 & 2.16 & 0.585 & 0.403 & 0.0324 & 0.175 & 396 & 18.4 \\
VP1/19-30 & 3.71 & 1.94 & 1.32 & 2.04 & 0.515 & 0.386 & 0.0194 & 0.173 & 300 & 18.0 \\
VP1/30-55 & 3.76 & 1.26 & 1.42 & 1.94 & 0.513 & 0.372 & 0.0172 & 0.166 & 238 & 18.3 \\
VP1/55-66 & 3.75 & 2.56 & 1.31 & 1.88 & 0.611 & 0.446 & 0.0259 & 0.165 & 228 & 17.7 \\
VP1/66-78 & 3.17 & 6.33 & 1.12 & 1.69 & 0.729 & 0.442 & 0.0385 & 0.138 & 230 & 14.6 \\
VP1/80-90 & 2.96 & 5.75 & 0.911 & 1.59 & 0.758 & 0.422 & 0.0300 & 0.119 & 197 & 12.6 \\
\hline
\end{tabular}

Table 5a-2b. ICP-MS data for soil pit VP-1.

\begin{tabular}{lcccccccccccc}
\hline \multicolumn{1}{c}{ Sample \# } & $\begin{array}{c}\mathbf{A g} \\
(\mathbf{p p m})\end{array}$ & $\begin{array}{c}\mathbf{A l} \\
(\mathbf{p p m})\end{array}$ & $\begin{array}{c}\mathbf{A s} \\
(\mathbf{p p m})\end{array}$ & $\begin{array}{c}\mathbf{B a} \\
(\mathbf{p p m})\end{array}$ & $\begin{array}{c}\mathbf{B e} \\
(\mathbf{p p m})\end{array}$ & $\begin{array}{c}\mathbf{B i} \\
(\mathbf{p p m})\end{array}$ & $\begin{array}{c}\mathbf{C a} \\
(\mathbf{p p m})\end{array}$ & $\begin{array}{c}\mathbf{C d} \\
(\mathbf{p p m})\end{array}$ & $\begin{array}{c}\mathbf{C e} \\
(\mathbf{p p m})\end{array}$ & $\begin{array}{c}\mathbf{C o} \\
(\mathbf{p p m})\end{array}$ & $\begin{array}{c}\mathbf{C r} \\
(\mathbf{p p m})\end{array}$ & $\begin{array}{c}\mathbf{C s} \\
(\mathbf{p p m})\end{array}$ \\
\hline VP1/0-6 & 0.22 & 31900 & 3.2 & 402 & 0.87 & 0.1 & 4800 & 0.17 & 31 & 3.2 & 42.6 & 2.4 \\
VP1/6-19 & $<0.05$ & 37400 & 3.8 & 437 & 1.2 & 0.09 & 12500 & 0.12 & 39.1 & 4.2 & 49.3 & 3.1 \\
VP1/19-30 & $<0.05$ & 36500 & 3.8 & 434 & 1.1 & 0.08 & 18600 & 0.08 & 38 & 4.1 & 46 & 3 \\
VP1/30-55 & $<0.05$ & 38600 & 4.2 & 446 & 1.1 & 0.09 & 12500 & 0.07 & 38.1 & 4.6 & 46 & 3.1 \\
VP1/55-66 & $<0.05$ & 38000 & 4.0 & 460 & 1.2 & 0.09 & 25000 & 0.07 & 37.6 & 4.6 & 43.5 & 2.9 \\
VP1/66-78 & $<0.05$ & 33500 & 3.9 & 440 & 0.98 & 0.07 & 62400 & 0.11 & 33.8 & 4.1 & 34 & 2.5 \\
VP1/80-90 & $<0.05$ & 30100 & 3.7 & 522 & 0.93 & 0.06 & 58400 & 0.09 & 27.8 & 3.6 & 23.3 & 2.3
\end{tabular}

Table 5a-2b. ICP-MS data for soil pit VP-1—Continued.

\begin{tabular}{lcccccccccccc}
\hline \multicolumn{1}{c}{ Sample \# } & $\begin{array}{c}\mathbf{C u} \\
(\mathbf{p p m})\end{array}$ & $\begin{array}{c}\mathbf{F e} \\
(\mathbf{p p m})\end{array}$ & $\begin{array}{c}\mathbf{G a} \\
(\mathbf{p p m})\end{array}$ & $\begin{array}{c}\mathbf{K} \\
(\mathbf{p p m})\end{array}$ & $\begin{array}{c}\mathbf{L a} \\
(\mathbf{p p m})\end{array}$ & $\begin{array}{c}\mathbf{L i} \\
(\mathbf{p p m})\end{array}$ & $\begin{array}{c}\mathbf{M g} \\
(\mathbf{p p m})\end{array}$ & $\begin{array}{c}\mathbf{M n} \\
(\mathbf{p p m})\end{array}$ & $\begin{array}{c}\mathbf{M o} \\
(\mathbf{p p m})\end{array}$ & $\begin{array}{c}\mathbf{N a} \\
(\mathbf{p p m})\end{array}$ & $\begin{array}{c}\mathbf{N b} \\
(\mathbf{p p m})\end{array}$ & $\begin{array}{c}\mathbf{N i} \\
(\mathbf{p p m})\end{array}$ \\
\hline VP1/0-6 & 11.9 & 11200 & 6.2 & 19400 & 17.3 & 16.9 & 5060 & 328 & 0.53 & 3340 & 7.2 & 8.3 \\
VP1/6-19 & 13.9 & 13400 & 7.6 & 20700 & 21.1 & 21.3 & 6060 & 376 & 0.55 & 3360 & 7.6 & 10.7 \\
VP1/19-30 & 13.5 & 12900 & 7.4 & 20200 & 19.9 & 20.3 & 5350 & 284 & 0.46 & 3370 & 6.1 & 10.2 \\
VP1/30-55 & 14.1 & 13900 & 7.8 & 20000 & 20.2 & 20.9 & 5360 & 225 & 0.52 & 3340 & 7.3 & 11.1 \\
VP1/55-66 & 14.1 & 13700 & 7.6 & 19000 & 19.8 & 20.5 & 6660 & 229 & 0.47 & 3940 & 6.9 & 11 \\
VP1/66-78 & 12.1 & 11700 & 6.6 & 16700 & 17.9 & 18.9 & 7760 & 233 & 0.39 & 3810 & 4.3 & 10.5 \\
VP1/80-90 & 22.1 & 9860 & 5.9 & 16100 & 14.9 & 18.2 & 8550 & 206 & 0.26 & 3790 & 3.1 & 9
\end{tabular}

Table 5a-2b. ICP-MS data for soil pit VP-1—Continued.

\begin{tabular}{|c|c|c|c|c|c|c|c|c|c|c|c|c|c|}
\hline Sample \# & $\underset{(\mathbf{p p m})}{P}$ & $\begin{array}{c}\mathrm{Pb} \\
(\mathbf{p p m})\end{array}$ & $\begin{array}{c}\mathbf{R b} \\
(\mathbf{p p m})\end{array}$ & $\underset{(\mathbf{p p m})}{\mathbf{S b}}$ & $\underset{(\mathbf{p p m})}{\mathrm{Sc}}$ & $\begin{array}{c}\mathrm{Sr} \\
(\mathbf{p p m})\end{array}$ & $\begin{array}{c}\text { Th } \\
\text { (ppm) }\end{array}$ & $\underset{(\mathbf{p p m})}{\mathrm{Ti}}$ & $\underset{\text { (ppm) }}{\mathrm{TI}}$ & $\underset{(\mathbf{p p m})}{\mathbf{U}}$ & $\begin{array}{c}\mathbf{V} \\
(\mathbf{p p m})\end{array}$ & $\begin{array}{c}Y \\
(\mathbf{p p m})\end{array}$ & $\underset{(\mathrm{ppm})}{\mathrm{Zn}}$ \\
\hline VP1/0-6 & 640 & 12.8 & 66.6 & 0.41 & 3.8 & 64.3 & 5.42 & 1680 & 0.35 & 1.27 & 24.5 & 12 & 29.8 \\
\hline VP1/6-19 & 333 & 12.1 & 74.9 & 0.44 & 5 & 79.2 & 6.4 & 2000 & 0.4 & 1.38 & 31.9 & 14 & 33.4 \\
\hline VP1/19-30 & 205 & 11.6 & 74 & 0.4 & 4.9 & 84.2 & 5.86 & 1950 & 0.41 & 1.32 & 31.7 & 13.3 & 30.4 \\
\hline VP1/30-55 & 181 & 12.3 & 75.9 & 0.41 & 5.1 & 85.5 & 6.12 & 2010 & 0.42 & 1.27 & 35.1 & 13.9 & 32.8 \\
\hline VP1/55-66 & 276 & 12.1 & 72.2 & 0.4 & 5 & 110 & 5.9 & 1970 & 0.41 & 1.3 & 36.6 & 14 & 31.9 \\
\hline VP1/66-78 & 422 & 10.4 & 62.9 & 0.34 & 4.6 & 135 & 5.36 & 1700 & 0.35 & 1.24 & 32.6 & 12.4 & 28.3 \\
\hline VP1/80-90 & 322 & 9.87 & 59.4 & 0.27 & 3.9 & 157 & 4.37 & 1450 & 0.33 & 1.24 & 30.6 & 10.7 & 26.8 \\
\hline
\end{tabular}


Table 5a-3a. ICP-AES data for soil pit VP-2.

\begin{tabular}{lcccccccccc}
\hline \multicolumn{1}{c}{ Sample \# } & Al (\%) & $\mathbf{C a}(\%)$ & $\mathbf{F e}(\%)$ & $\mathbf{K ~ ( \% )}$ & $\mathbf{M g}(\%)$ & $\mathbf{N a}(\%)$ & $\mathbf{P}(\%)$ & $\mathbf{T i}(\%)$ & $\mathbf{M n}(\mathbf{p p m})$ & $\mathbf{N d}(\mathbf{p p m})$ \\
\hline VP2/0-5 & 3.45 & 1.04 & 1.20 & 1.97 & 0.611 & 0.410 & 0.0773 & 0.171 & 407 & 15.7 \\
VP2/5-14 & 3.73 & 0.592 & 1.33 & 2.06 & 0.572 & 0.392 & 0.0621 & 0.189 & 456 & 18.0 \\
VP2/14-44 & 3.59 & 1.60 & 1.19 & 2.00 & 0.520 & 0.377 & 0.0323 & 0.181 & 341 & 16.2 \\
VP2/44-55 & 3.58 & 2.42 & 1.21 & 1.93 & 0.514 & 0.346 & 0.0312 & 0.164 & 288 & 16.4 \\
VP2/55-85 & 3.92 & 1.40 & 1.27 & 1.99 & 0.462 & 0.380 & 0.0224 & 0.171 & 247 & 16.5 \\
VP2/110-115 & 3.12 & 3.99 & 1.06 & 1.67 & 0.640 & 0.392 & 0.0338 & 0.128 & 216 & 13.1 \\
\hline
\end{tabular}

Table 5a-3b. ICP-MS data for soil pit VP-2.

\begin{tabular}{lcccccccccccc}
\hline \multicolumn{1}{c}{ Sample \# } & $\begin{array}{c}\mathbf{A g} \\
\mathbf{( p p m )}\end{array}$ & $\begin{array}{c}\mathbf{A l} \\
(\mathbf{p p m})\end{array}$ & $\begin{array}{c}\mathbf{A s} \\
(\mathbf{p p m})\end{array}$ & $\begin{array}{c}\mathbf{B a} \\
(\mathbf{p p m})\end{array}$ & $\begin{array}{c}\mathbf{B e} \\
(\mathbf{p p m})\end{array}$ & $\begin{array}{c}\mathbf{B i} \\
(\mathbf{p p m})\end{array}$ & $\begin{array}{c}\mathbf{C a} \\
(\mathbf{p p m})\end{array}$ & $\begin{array}{c}\mathbf{C d} \\
(\mathbf{p p m})\end{array}$ & $\begin{array}{c}\mathbf{C e} \\
(\mathbf{p p m})\end{array}$ & $\begin{array}{c}\mathbf{C o} \\
(\mathbf{p p m})\end{array}$ & $\begin{array}{c}\mathbf{C r} \\
(\mathbf{p p m})\end{array}$ & $\begin{array}{c}\mathbf{C s} \\
(\mathbf{p p m})\end{array}$ \\
\hline VP2/0-5 & $<0.05$ & 35200 & 3.4 & 456 & 0.96 & 0.11 & 10200 & 0.23 & 35.9 & 3.6 & 46.9 & 2.7 \\
VP2/5-14 & $<0.05$ & 38300 & 3.5 & 459 & 1.0 & 0.08 & 5840 & 0.2 & 39.9 & 4 & 41.3 & 3.1 \\
VP2/14-44 & $<0.05$ & 36800 & 3.8 & 450 & 1.1 & 0.08 & 15600 & 0.12 & 36.7 & 3.8 & 43.2 & 2.9 \\
VP2/44-55 & $<0.05$ & 36400 & 3.8 & 443 & 1.2 & 0.08 & 23500 & 0.12 & 35.5 & 3.9 & 41.8 & 2.9 \\
VP2/55-85 & $<0.05$ & 38900 & 4.4 & 455 & 1.1 & 0.08 & 13600 & 0.07 & 38.9 & 4.3 & 44.7 & 3.1 \\
VP2/110-115 & $<0.05$ & 31900 & 3.3 & 430 & 0.97 & 0.07 & 39800 & 0.1 & 29.6 & 3.6 & 34.4 & 2.6
\end{tabular}

Table 5a-3b. ICP-MS data for soil pit VP-2—Continued.

\begin{tabular}{lcccccccccccc}
\hline \multicolumn{1}{c}{ Sample \# } & $\begin{array}{c}\mathbf{C u} \\
(\mathbf{p p m})\end{array}$ & $\begin{array}{c}\mathbf{F e} \\
(\mathbf{p p m})\end{array}$ & $\begin{array}{c}\mathbf{G a} \\
(\mathbf{p p m})\end{array}$ & $\begin{array}{c}\mathbf{K} \\
(\mathbf{p p m})\end{array}$ & $\begin{array}{c}\mathbf{L a} \\
(\mathbf{p p m})\end{array}$ & $\begin{array}{c}\mathbf{L i} \\
\mathbf{( \mathbf { p p m } )}\end{array}$ & $\begin{array}{c}\mathbf{M g} \\
(\mathbf{p p m})\end{array}$ & $\begin{array}{c}\mathbf{M n} \\
(\mathbf{p p m})\end{array}$ & $\begin{array}{c}\mathbf{M o} \\
(\mathbf{p p m})\end{array}$ & $\begin{array}{c}\mathbf{N a} \\
(\mathbf{p p m})\end{array}$ & $\begin{array}{c}\mathbf{N b} \\
(\mathbf{p p m})\end{array}$ & $\begin{array}{c}\mathbf{N i} \\
(\mathbf{p p m})\end{array}$ \\
\hline VP2/0-5 & 12.8 & 12300 & 6.9 & 20400 & 19.1 & 19 & 6820 & 403 & 0.54 & 3830 & 8.2 & 9.3 \\
VP2/5-14 & 12.8 & 13200 & 7.6 & 21400 & 21 & 20.9 & 6040 & 445 & 0.52 & 3580 & 8.3 & 9.9 \\
VP2/14-44 & 12.9 & 12400 & 7.3 & 20600 & 19.4 & 20.4 & 5760 & 348 & 0.44 & 3360 & 9.2 & 9.6 \\
VP2/44-55 & 13.4 & 12400 & 7.2 & 19500 & 18.5 & 20 & 5390 & 281 & 0.43 & 3030 & 8.1 & 9.7 \\
VP2/55-85 & 12.9 & 13100 & 7.8 & 20000 & 20 & 21.2 & 5070 & 244 & 0.48 & 3390 & 8.6 & 9.9 \\
VP2/110-115 & 15.2 & 10900 & 6.2 & 16800 & 15.4 & 18.4 & 6770 & 216 & 0.38 & 3470 & 6 & 9.3
\end{tabular}

Table 5a-3b. ICP-MS data for soil pit VP-2—Continued.

\begin{tabular}{lllllllllllll}
\hline \multicolumn{1}{c}{ Sample \# } & $\begin{array}{c}\mathbf{P b} \\
(\mathbf{p p m})\end{array}$ & $\begin{array}{c}\mathbf{R b} \\
(\mathbf{p p m})\end{array}$ & $\begin{array}{c}\mathbf{S b} \\
(\mathbf{p p m})\end{array}$ & $\begin{array}{c}\mathbf{S c} \\
(\mathbf{p p m})\end{array}$ & $\begin{array}{c}\mathbf{S r} \\
(\mathbf{p p m})\end{array}$ & $\begin{array}{c}\mathbf{T h} \\
(\mathbf{p p m})\end{array}$ & $\begin{array}{c}\mathbf{T i} \\
(\mathbf{p p m})\end{array}$ & $\begin{array}{c}\mathbf{T I} \\
(\mathbf{p p m})\end{array}$ & $\begin{array}{c}\mathbf{U} \\
(\mathbf{p p m})\end{array}$ & $\begin{array}{c}\mathbf{V} \\
(\mathbf{p p m})\end{array}$ & $\begin{array}{c}\mathbf{Y} \\
(\mathbf{p p m})\end{array}$ & $\begin{array}{c}\mathbf{Z n} \\
(\mathbf{p p m})\end{array}$ \\
\hline VP2/0-5 & 14.1 & 72.5 & 0.43 & 4.3 & 81.3 & 5.78 & 2010 & 0.39 & 1.38 & 28.3 & 13.3 & 32.8 \\
VP2/5-14 & 12.2 & 76 & 0.45 & 4.9 & 77.4 & 6.37 & 2160 & 0.4 & 1.42 & 31.4 & 14.8 & 35.2 \\
VP2/14-44 & 11.2 & 73.8 & 0.43 & 4.6 & 84.8 & 5.85 & 2170 & 0.38 & 1.35 & 29.8 & 14.3 & 33.2 \\
VP2/44-55 & 10.9 & 71.6 & 0.37 & 4.6 & 90.9 & 5.57 & 1930 & 0.37 & 1.23 & 29.4 & 12.9 & 33.2 \\
VP2/55-85 & 11.9 & 74.3 & 0.4 & 4.8 & 94.2 & 6.32 & 1930 & 0.41 & 1.27 & 32.7 & 13.1 & 31.3 \\
VP2/110-115 & 10.4 & 63.1 & 0.36 & 4 & 136 & 4.8 & 1500 & 0.34 & 1.17 & 27.8 & 11 & 28.3 \\
\hline
\end{tabular}


Table 5a-4a. ICP-AES data for the 8U-10 auger hole.

\begin{tabular}{|c|c|c|c|c|c|c|c|c|c|c|}
\hline Sample \# & Al (\%) & $\mathrm{Ca}(\%)$ & $\mathrm{Fe}(\%)$ & $\mathrm{K}(\%)$ & $\operatorname{Mg}(\%)$ & $\mathrm{Na}(\%)$ & $\mathbf{P}(\%)$ & $\mathrm{Ti}(\%)$ & Mn (ppm) & Nd (ppm) \\
\hline $8 \mathrm{U}-10 / 0-22$ & 3.01 & 1.87 & 1.00 & 1.80 & 0.607 & 0.423 & 0.0604 & 0.171 & 444 & 17.5 \\
\hline $8 \mathrm{U}-10 / 22-36$ & 3.81 & 1.70 & 1.29 & 2.11 & 0.548 & 0.400 & 0.0321 & 0.154 & 344 & 17.8 \\
\hline $8 \mathrm{U}-10 / 36-47$ & 4.00 & 1.88 & 1.42 & 2.12 & 0.509 & 0.417 & 0.0240 & 0.196 & 292 & 18.7 \\
\hline $8 \mathrm{U}-10 / 47-58$ & 4.26 & 1.52 & 1.48 & 2.09 & 0.556 & 0.440 & 0.0229 & 0.205 & 296 & 20.2 \\
\hline $8 \mathrm{U}-10 / 58-65$ & 4.40 & 1.53 & 1.63 & 2.11 & 0.618 & 0.490 & 0.0242 & 0.217 & 303 & 22.0 \\
\hline $8 U-10 / 65-74$ & 4.14 & 3.13 & 1.44 & 2.02 & 0.714 & 0.498 & 0.0336 & 0.180 & 295 & 21.0 \\
\hline $8 \mathrm{U}-10 / 74-83$ & 3.90 & 5.30 & 1.32 & 1.96 & 0.821 & 0.506 & 0.0399 & 0.169 & 286 & 19.4 \\
\hline $8 \mathrm{U}-10 / 83-90$ & 3.80 & 4.09 & 1.27 & 1.94 & 0.787 & 0.497 & 0.0358 & 0.177 & 283 & 17.8 \\
\hline $8 U-10 / 90-99$ & 3.66 & 4.02 & 1.18 & 1.94 & 0.781 & 0.494 & 0.0343 & 0.166 & 271 & 17.2 \\
\hline 8U-10/99-112 & 3.27 & 3.98 & 1.06 & 1.85 & 0.822 & 0.440 & 0.0316 & 0.154 & 236 & 15.2 \\
\hline $8 \mathrm{U}-10 / 112-121$ & 3.16 & 4.30 & 1.07 & 1.81 & 0.858 & 0.404 & 0.0312 & 0.132 & 228 & 14.7 \\
\hline $8 U-10 / 121-131$ & 3.01 & 3.85 & 0.984 & 1.68 & 0.858 & 0.349 & 0.0291 & 0.104 & 203 & 13.8 \\
\hline $8 \mathrm{U}-10 / 131-142$ & 2.98 & 3.98 & 0.967 & 1.77 & 0.869 & 0.346 & 0.0295 & 0.128 & 193 & 13.4 \\
\hline $8 U-10 / 142-154$ & 2.89 & 3.20 & 0.881 & 1.73 & 0.816 & 0.337 & 0.0281 & 0.104 & 193 & 13.1 \\
\hline $8 U-10 / 154-165$ & 3.06 & 2.76 & 1.01 & 1.80 & 0.806 & 0.359 & 0.0302 & 0.109 & 210 & 13.2 \\
\hline $8 U-10 / 165-175$ & 3.32 & 3.02 & 1.01 & 1.84 & 0.767 & 0.347 & 0.0307 & 0.110 & 221 & 13.4 \\
\hline $8 U-10 / 175-184$ & 3.32 & 2.69 & 0.961 & 1.83 & 0.723 & 0.340 & 0.0290 & 0.106 & 222 & 13.6 \\
\hline 8U-10/184-197 & 3.35 & 2.12 & 0.972 & 1.86 & 0.614 & 0.347 & 0.0261 & 0.112 & 224 & 14.5 \\
\hline $8 U-10 / 197-209$ & 3.03 & 1.96 & 0.965 & 1.77 & 0.539 & 0.315 & 0.0236 & 0.112 & 199 & 12.6 \\
\hline $8 U-10 / 209-220$ & 2.77 & 2.74 & 0.753 & 1.69 & 0.526 & 0.270 & 0.0222 & 0.0873 & 169 & 11.2 \\
\hline $8 U-10 / 220-230$ & 2.46 & 3.21 & 0.633 & 1.58 & 0.509 & 0.217 & 0.0219 & 0.0693 & 141 & 10.6 \\
\hline $8 \mathrm{U}-10 / 230-241$ & 2.22 & 3.66 & 0.520 & 1.49 & 0.491 & 0.170 & 0.0201 & 0.0575 & 113 & 8.67 \\
\hline $8 U-10 / 241-249$ & 2.57 & 2.94 & 0.704 & 1.59 & 0.548 & 0.233 & 0.0227 & 0.0838 & 161 & 10.7 \\
\hline $8 U-10 / 249-261$ & 2.14 & 4.31 & 0.489 & 1.45 & 0.478 & 0.160 & 0.0188 & 0.0637 & 104 & 8.0 \\
\hline $8 \mathrm{U}-10 / 261-270$ & 2.46 & 3.35 & 0.625 & 1.58 & 0.516 & 0.212 & 0.0228 & 0.0735 & 142 & 10.1 \\
\hline $8 U-10 / 270-274$ & 2.20 & 2.96 & 0.650 & 1.47 & 0.480 & 0.178 & 0.0206 & 0.0616 & 128 & 9.16 \\
\hline $8 U-10 / 274-284$ & 2.01 & 2.92 & 0.657 & 1.39 & 0.485 & 0.152 & 0.0177 & 0.0536 & 119 & 8.50 \\
\hline
\end{tabular}


Table 5a-4b. ICP-MS data for auger hole 8U-10.

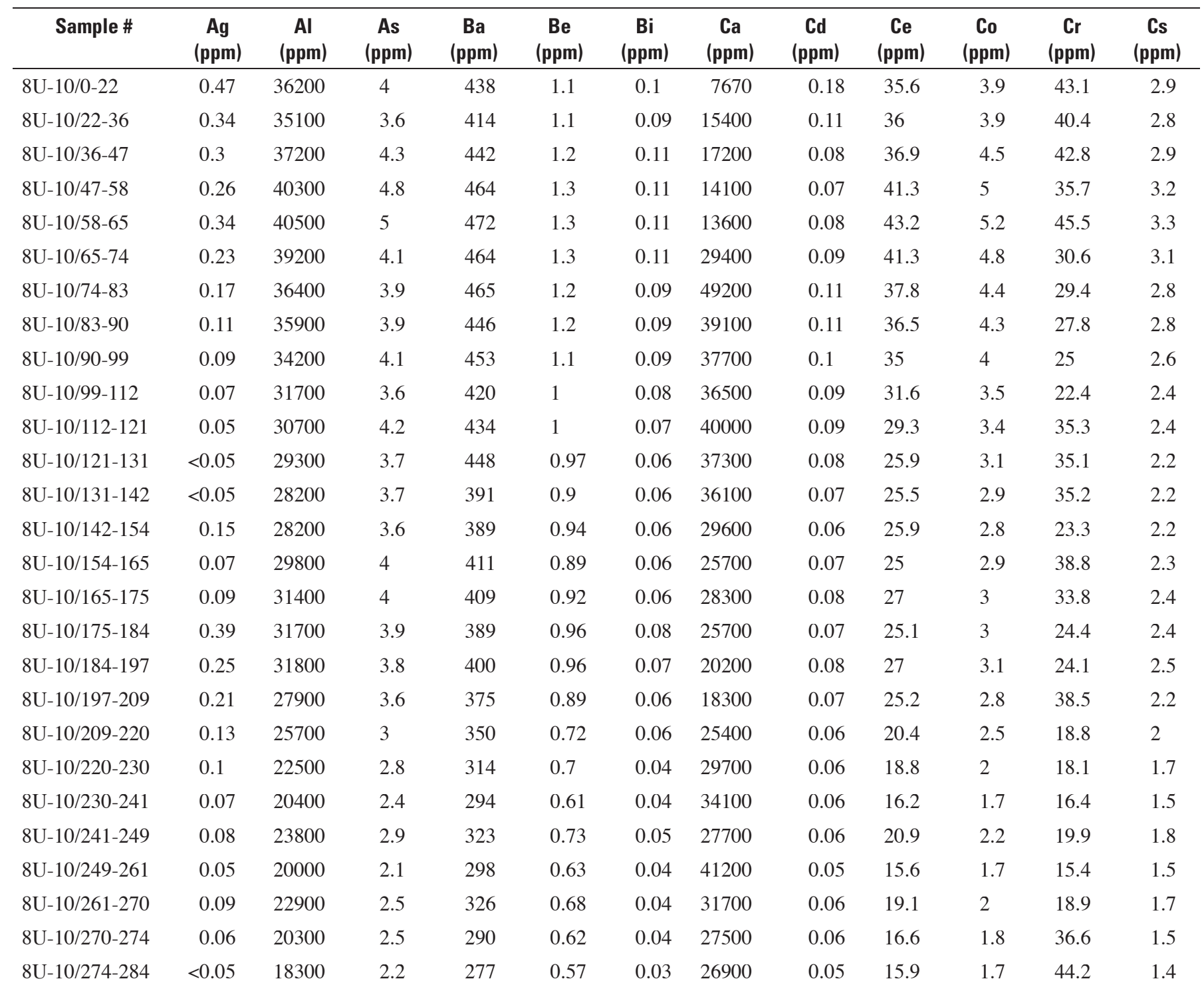


Table 5a-4b. ICP-MS data for auger hole 8U-10—Continued.

\begin{tabular}{|c|c|c|c|c|c|c|c|c|c|c|c|c|}
\hline Sample \# & $\begin{array}{c}\mathrm{Cu} \\
(\mathrm{ppm})\end{array}$ & $\begin{array}{c}\text { Fe } \\
\text { (ppm) }\end{array}$ & $\begin{array}{c}\text { Ga } \\
(\mathrm{ppm})\end{array}$ & $\begin{array}{c}\mathrm{K} \\
(\mathbf{p p m})\end{array}$ & $\begin{array}{c}\text { La } \\
\text { (ppm) }\end{array}$ & $\begin{array}{c}\mathrm{Li} \\
(\mathbf{p p m})\end{array}$ & $\begin{array}{c}\mathrm{Mg} \\
(\mathrm{ppm})\end{array}$ & $\begin{array}{c}\text { Mn } \\
(\mathbf{p p m})\end{array}$ & $\begin{array}{c}\text { Mo } \\
(\text { ppm) }\end{array}$ & $\begin{array}{c}\mathrm{Na} \\
(\mathrm{ppm})\end{array}$ & $\begin{array}{c}\mathbf{N b} \\
\text { (ppm) }\end{array}$ & $\begin{array}{c}\mathbf{N i} \\
\text { (ppm) }\end{array}$ \\
\hline $8 \mathrm{U}-10 / 0-22$ & 12.2 & 12600 & 7.4 & 20800 & 19.3 & 19.8 & 6080 & 406 & 0.62 & 3600 & 7.3 & 9.7 \\
\hline $8 \mathrm{U}-10 / 22-36$ & 12.4 & 12300 & 7.1 & 19900 & 19.4 & 19.4 & 5570 & 313 & 0.54 & 3370 & 6.3 & 9.5 \\
\hline $8 \mathrm{U}-10 / 36-47$ & 13.6 & 13400 & 7.4 & 20000 & 19.8 & 20.2 & 5120 & 263 & 0.55 & 3530 & 6.9 & 10.1 \\
\hline $8 \mathrm{U}-10 / 47-58$ & 14 & 14400 & 8.3 & 20200 & 22.1 & 21.8 & 5860 & 277 & 0.48 & 3840 & 7.3 & 11.2 \\
\hline $8 \mathrm{U}-10 / 58-65$ & 15.3 & 15300 & 8.7 & 19600 & 23.6 & 22.2 & 6300 & 271 & 0.58 & 4150 & 8.8 & 12.3 \\
\hline $8 \mathrm{U}-10 / 65-74$ & 13 & 13900 & 8 & 19100 & 24.4 & 21.3 & 7160 & 272 & 0.43 & 4240 & 3.5 & 11 \\
\hline $8 \mathrm{U}-10 / 74-83$ & 12.7 & 12900 & 7.4 & 18100 & 20.5 & 20 & 8100 & 268 & 0.43 & 4090 & 2.8 & 10.7 \\
\hline $8 \mathrm{U}-10 / 83-90$ & 10.8 & 12400 & 7.2 & 18400 & 19.9 & 20.2 & 7990 & 264 & 0.38 & 4220 & 2.5 & 10.4 \\
\hline 8U-10/90-99 & 9.9 & 11700 & 6.9 & 18500 & 18.9 & 19.8 & 7950 & 257 & 0.36 & 4100 & $<2$ & 9.6 \\
\hline $8 \mathrm{U}-10 / 99-112$ & 10.8 & 10300 & 6.3 & 17000 & 17 & 18.7 & 8340 & 218 & 0.32 & 3620 & $<2$ & 9 \\
\hline $8 \mathrm{U}-10 / 112-121$ & 9.6 & 10600 & 6.2 & 17000 & 15.7 & 18 & 8790 & 216 & 0.44 & 3300 & $<2$ & 9 \\
\hline $8 \mathrm{U}-10 / 121-131$ & 8.9 & 9740 & 5.7 & 16600 & 14.1 & 17 & 8880 & 192 & 0.48 & 3020 & $<2$ & 8.2 \\
\hline $8 \mathrm{U}-10 / 131-142$ & 8.4 & 9340 & 5.5 & 16100 & 14 & 16.5 & 8740 & 180 & 0.56 & 2760 & $<2$ & 8 \\
\hline $8 \mathrm{U}-10 / 142-154$ & 7.2 & 8620 & 5.6 & 16300 & 14 & 16.3 & 8300 & 182 & 0.46 & 2810 & 5.4 & 7.1 \\
\hline $8 \mathrm{U}-10 / 154-165$ & 9.2 & 9790 & 5.8 & 17200 & 13.5 & 17.2 & 8140 & 197 & 0.62 & 2970 & 2.7 & 8.1 \\
\hline $8 \mathrm{U}-10 / 165-175$ & 8.9 & 9920 & 6 & 17400 & 14.8 & 17.8 & 7680 & 206 & 0.56 & 2890 & 3.7 & 8.3 \\
\hline $8 \mathrm{U}-10 / 175-184$ & 8.3 & 9250 & 6 & 17700 & 13.9 & 17.3 & 7140 & 204 & 0.5 & 2790 & 2.5 & 8.3 \\
\hline $8 \mathrm{U}-10 / 184-197$ & 23 & 9430 & 6.2 & 18100 & 14.7 & 17.9 & 6170 & 210 & 0.46 & 2850 & 2.8 & 7.9 \\
\hline $8 U-10 / 197-209$ & 10.3 & 9300 & 5.6 & 16900 & 13.8 & 16.5 & 5460 & 182 & 0.61 & 2610 & 3.6 & 7.9 \\
\hline $8 \mathrm{U}-10 / 209-220$ & 7 & 6630 & 5 & 16000 & 11.5 & 14.6 & 5210 & 155 & 0.33 & 2160 & $<2$ & 6.5 \\
\hline $8 \mathrm{U}-10 / 220-230$ & 6.3 & 5500 & 4.4 & 14500 & 10.7 & 12.6 & 5070 & 122 & 0.28 & 1730 & $<2$ & 5.8 \\
\hline $8 \mathrm{U}-10 / 230-241$ & 5 & 4600 & 3.8 & 13700 & 9 & 11.3 & 5050 & 99 & 0.22 & 1380 & $<2$ & 5.2 \\
\hline $8 \mathrm{U}-10 / 241-249$ & 7.7 & 6730 & 4.6 & 15100 & 11.6 & 13.4 & 5540 & 148 & 0.35 & 1950 & $<2$ & 6.4 \\
\hline $8 \mathrm{U}-10 / 249-261$ & 5.2 & 4440 & 3.7 & 13800 & 8.7 & 11 & 4960 & 91.9 & 0.22 & 1320 & $<2$ & 5.4 \\
\hline $8 \mathrm{U}-10 / 261-270$ & 5.8 & 5580 & 4.4 & 15100 & 10.8 & 12.8 & 5320 & 133 & 0.26 & 1740 & 2.3 & 6.2 \\
\hline $8 \mathrm{U}-10 / 270-274$ & 6.7 & 5710 & 3.8 & 13900 & 9.3 & 11.2 & 4900 & 114 & 0.4 & 1460 & $<2$ & 6.5 \\
\hline $8 \mathrm{U}-10 / 274-284$ & 7.3 & 5790 & 3.5 & 13000 & 8.9 & 10.2 & 4930 & 104 & 0.47 & 1230 & $<2$ & 6.5 \\
\hline
\end{tabular}


Table 5a-4b. ICP-MS data for auger hole 8U-10—Continued.

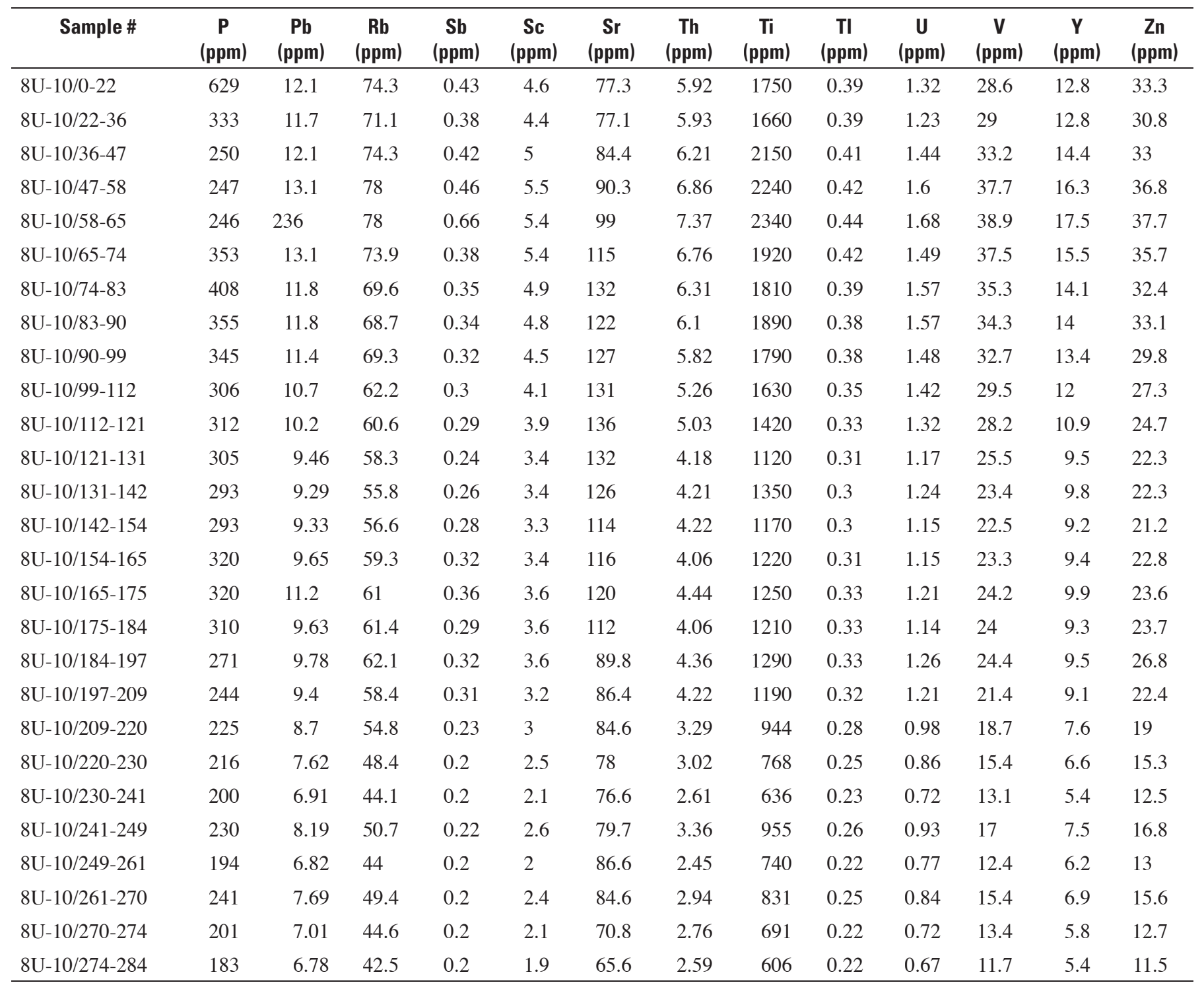


Table 5a-5a. ICP-AES data for auger hole 8U-11.

\begin{tabular}{|c|c|c|c|c|c|c|c|c|c|c|}
\hline Sample \# & Al (\%) & Ca $(\%)$ & $\mathrm{Fe}(\%)$ & K (\%) & $\operatorname{Mg}(\%)$ & $\mathrm{Na}(\%)$ & $\mathbf{P}(\%)$ & Ti (\%) & Mn (ppm) & Nd (ppm) \\
\hline $8 \mathrm{U}-11 / 0-22$ & 3.06 & 0.612 & 1.06 & 1.85 & 0.429 & 0.314 & 0.0317 & 0.119 & 279 & 14.3 \\
\hline $8 \mathrm{U}-11 / 22-38$ & 3.47 & 2.01 & 1.15 & 2.00 & 0.474 & 0.366 & 0.0205 & 0.142 & 277 & 16.4 \\
\hline $8 \mathrm{U}-11 / 38-50$ & 3.51 & 1.21 & 1.20 & 1.85 & 0.455 & 0.340 & 0.0141 & 0.138 & 202 & 17.0 \\
\hline $8 \mathrm{U}-11 / 50-62$ & 3.60 & 1.56 & 1.32 & 1.95 & 0.443 & 0.357 & 0.0156 & 0.145 & 224 & 17.8 \\
\hline $8 \mathrm{U}-11 / 62-71$ & 3.33 & 2.20 & 1.23 & 1.74 & 0.521 & 0.380 & 0.0186 & 0.139 & 192 & 16.8 \\
\hline $8 \mathrm{U}-11 / 71-86$ & 3.11 & 4.23 & 1.02 & 1.66 & 0.618 & 0.409 & 0.0289 & 0.132 & 183 & 15.3 \\
\hline $8 \mathrm{U}-11 / 86-98$ & 3.01 & 4.47 & 0.963 & 1.64 & 0.655 & 0.416 & 0.0308 & 0.136 & 187 & 14.4 \\
\hline 8U-11/98-109 & 2.68 & 4.26 & 0.859 & 1.52 & 0.677 & 0.367 & 0.0289 & 0.101 & 178 & 12.9 \\
\hline 8U-11/109-118 & 2.87 & 3.96 & 0.872 & 1.64 & 0.709 & 0.416 & 0.0301 & 0.0957 & 187 & 12.7 \\
\hline $8 \mathrm{U}-11 / 118-131$ & 2.59 & 3.03 & 0.884 & 1.53 & 0.689 & 0.368 & 0.0252 & 0.0870 & 168 & 11.7 \\
\hline $8 \mathrm{U}-11 / 131-139$ & 2.39 & 2.51 & 0.818 & 1.45 & 0.654 & 0.315 & 0.0228 & 0.0797 & 156 & 10.5 \\
\hline $8 U-11 / 139-151$ & 2.22 & 2.41 & 0.754 & 1.39 & 0.603 & 0.272 & 0.0202 & 0.0720 & 140 & 9.00 \\
\hline $8 \mathrm{U}-11 / 151-161$ & 1.79 & 1.62 & 0.594 & 1.23 & 0.474 & 0.175 & 0.0139 & 0.0488 & 104 & $<8$ \\
\hline $8 \mathrm{U}-11 / 161-172$ & 2.49 & 2.14 & 0.838 & 1.51 & 0.626 & 0.342 & 0.0221 & 0.0928 & 164 & 9.24 \\
\hline $8 \mathrm{U}-11 / 172-183$ & 2.67 & 1.97 & 0.902 & 1.61 & 0.649 & 0.372 & 0.0254 & 0.100 & 184 & 11.8 \\
\hline 8U-11/183-196 & 3.24 & 1.91 & 1.15 & 1.90 & 0.792 & 0.548 & 0.0383 & 0.153 & 264 & 15.0 \\
\hline $8 \mathrm{U}-11 / 196-210$ & 3.24 & 2.04 & 1.12 & 1.81 & 0.740 & 0.453 & 0.0351 & 0.154 & 234 & 14.6 \\
\hline $8 \mathrm{U}-11 / 210-224$ & 2.88 & 2.74 & 0.890 & 1.70 & 0.638 & 0.422 & 0.0303 & 0.111 & 195 & 12.3 \\
\hline $8 \mathrm{U}-11 / 224-237$ & 2.90 & 2.32 & 0.954 & 1.73 & 0.586 & 0.423 & 0.0281 & 0.109 & 198 & 12.3 \\
\hline $8 \mathrm{U}-11 / 237-251$ & 2.96 & 2.04 & 0.962 & 1.78 & 0.561 & 0.435 & 0.0291 & 0.124 & 209 & 12.3 \\
\hline $8 U-11 / 251-265$ & 2.91 & 1.61 & 0.963 & 1.75 & 0.526 & 0.410 & 0.0272 & 0.115 & 208 & 12.8 \\
\hline $8 \mathrm{U}-11 / 265-278$ & 3.01 & 1.90 & 1.02 & 1.76 & 0.534 & 0.422 & 0.0289 & 0.128 & 220 & 12.4 \\
\hline
\end{tabular}


Table 5a-5b. ICP-MS data for auger hole 8U-11.

\begin{tabular}{|c|c|c|c|c|c|c|c|c|c|c|c|c|}
\hline Sample \# & $\begin{array}{c}\mathbf{A g} \\
(\mathrm{ppm})\end{array}$ & $\begin{array}{c}\text { Al } \\
(\mathrm{ppm})\end{array}$ & $\begin{array}{c}\text { As } \\
(\mathrm{ppm})\end{array}$ & $\begin{array}{c}\mathrm{Ba} \\
(\mathrm{ppm})\end{array}$ & $\begin{array}{c}\mathrm{Be} \\
(\mathrm{ppm})\end{array}$ & $\begin{array}{c}\mathrm{Bi} \\
(\mathrm{ppm})\end{array}$ & $\begin{array}{c}\text { Ca } \\
\text { (ppm) }\end{array}$ & $\begin{array}{c}\text { Cd } \\
(\mathrm{ppm})\end{array}$ & $\begin{array}{c}\mathrm{Ce} \\
(\mathrm{ppm})\end{array}$ & $\begin{array}{c}\text { Co } \\
\text { (ppm) }\end{array}$ & $\begin{array}{c}\mathrm{Cr} \\
(\mathrm{ppm})\end{array}$ & $\begin{array}{c}\text { Cs } \\
(\mathbf{p p m})\end{array}$ \\
\hline $8 \mathrm{U}-11 / 0-22$ & 0.16 & 29900 & 2.9 & 380 & 0.85 & 0.07 & 5850 & 0.09 & 28.6 & 3 & 39.3 & 2.3 \\
\hline $8 \mathrm{U}-11 / 22-38$ & 0.12 & 32000 & 3.1 & 391 & 0.99 & 0.08 & 18300 & 0.08 & 32.9 & 3.4 & 37.1 & 2.5 \\
\hline $8 \mathrm{U}-11 / 38-50$ & 0.17 & 33100 & 3.4 & 387 & 1 & 0.08 & 11100 & 0.04 & 32.6 & 4 & 28.1 & 2.6 \\
\hline $8 \mathrm{U}-11 / 50-62$ & 0.12 & 33400 & 3.4 & 390 & 0.98 & 0.09 & 14200 & 0.05 & 34.2 & 4 & 49.5 & 2.6 \\
\hline $8 \mathrm{U}-11 / 62-71$ & 0.15 & 32400 & 3.5 & 400 & 1 & 0.08 & 21200 & 0.05 & 31.4 & 3.9 & 41.8 & 2.4 \\
\hline $8 \mathrm{U}-11 / 71-86$ & 0.11 & 29700 & 3.1 & 392 & 0.9 & 0.07 & 41400 & 0.07 & 29.8 & 3.5 & 23.3 & 2.2 \\
\hline $8 \mathrm{U}-11 / 86-98$ & 0.11 & 28400 & 3.2 & 394 & 0.84 & 0.07 & 43100 & 0.08 & 29.2 & 3.4 & 24.1 & 2 \\
\hline 8U-11/98-109 & 0.06 & 25700 & 3.1 & 454 & 0.76 & 0.06 & 41400 & 0.07 & 23.7 & 3 & 19.4 & 1.9 \\
\hline $8 \mathrm{U}-11 / 109-118$ & 0.32 & 25800 & 3.6 & 402 & 0.78 & 0.08 & 36500 & 0.08 & 22.5 & 2.9 & 19.6 & 1.9 \\
\hline $8 \mathrm{U}-11 / 118-131$ & 0.19 & 23400 & 3 & 360 & 0.67 & 0.06 & 27600 & 0.06 & 21.9 & 2.6 & 32 & 1.8 \\
\hline $8 \mathrm{U}-11 / 131-139$ & 0.17 & 21800 & 2.8 & 331 & 0.63 & 0.05 & 23000 & 0.06 & 18.8 & 2.4 & 34.2 & 1.7 \\
\hline $8 \mathrm{U}-11 / 139-151$ & 0.15 & 20300 & 2.3 & 321 & 0.61 & 0.05 & 22200 & 0.05 & 16.4 & 2.1 & 33.9 & 1.6 \\
\hline $8 \mathrm{U}-11 / 151-161$ & 0.09 & 16300 & 2 & 262 & 0.48 & 0.04 & 15000 & 0.04 & 12.2 & 1.7 & 33.6 & 1.3 \\
\hline $8 \mathrm{U}-11 / 161-172$ & 0.13 & 23300 & 2.6 & 334 & 0.69 & 0.05 & 20000 & 0.06 & 19.4 & 2.5 & 35.6 & 1.8 \\
\hline $8 \mathrm{U}-11 / 172-183$ & 0.1 & 25000 & 2.8 & 353 & 0.72 & 0.06 & 18300 & 0.06 & 22.5 & 2.6 & 34.2 & 1.9 \\
\hline $8 \mathrm{U}-11 / 183-196$ & 0.17 & 32600 & 3.8 & 441 & 0.93 & 0.08 & 18300 & 0.08 & 30.4 & 3.5 & 37.7 & 2.4 \\
\hline $8 \mathrm{U}-11 / 196-210$ & 0.16 & 30400 & 3.6 & 408 & 0.92 & 0.08 & 19100 & 0.08 & 28.4 & 3.4 & 39.8 & 2.4 \\
\hline $8 \mathrm{U}-11 / 210-224$ & 0.08 & 27000 & 3.1 & 378 & 0.83 & 0.07 & 25800 & 0.08 & 27.5 & 2.9 & 22.8 & 2 \\
\hline $8 U-11 / 224-237$ & 0.1 & 27300 & 3.2 & 386 & 0.81 & 0.06 & 21800 & 0.07 & 22.7 & 2.9 & 36 & 2.1 \\
\hline $8 \mathrm{U}-11 / 237-251$ & 0.11 & 29200 & 3.2 & 414 & 0.79 & 0.07 & 19900 & 0.07 & 25.8 & 3 & 36.9 & 2.2 \\
\hline $8 \mathrm{U}-11 / 251-265$ & 0.13 & 28600 & 3.2 & 398 & 0.92 & 0.07 & 15600 & 0.07 & 24.5 & 2.9 & 33.9 & 2.1 \\
\hline $8 U-11 / 265-278$ & 0.17 & 29800 & 3.4 & 400 & 0.83 & 0.07 & 18600 & 0.09 & 26.5 & 3.1 & 37.9 & 2.3 \\
\hline
\end{tabular}


Table 5a-5b. ICP-MS data for auger hole 8U-11-Continued.

\begin{tabular}{|c|c|c|c|c|c|c|c|c|c|c|c|c|}
\hline Sample \# & $\underset{\text { (ppm) }}{\mathrm{Cu}}$ & $\begin{array}{c}\mathrm{Fe} \\
(\mathrm{ppm})\end{array}$ & $\begin{array}{c}\text { Ga } \\
(\mathbf{p p m})\end{array}$ & $\begin{array}{c}\mathrm{K} \\
(\mathbf{p p m})\end{array}$ & $\begin{array}{c}\text { La } \\
(\mathbf{p p m})\end{array}$ & $\underset{(\mathbf{p p m})}{\mathbf{L i}}$ & $\underset{(\mathbf{p p m})}{\mathbf{M g}}$ & $\begin{array}{c}\mathbf{M n} \\
(\mathbf{p p m})\end{array}$ & $\begin{array}{c}\text { Mo } \\
\text { (ppm) }\end{array}$ & $\begin{array}{c}\mathrm{Na} \\
(\mathbf{p p m})\end{array}$ & $\begin{array}{c}\mathbf{N b} \\
(\mathbf{p p m})\end{array}$ & $\begin{array}{c}\mathrm{Ni} \\
\text { (ppm) }\end{array}$ \\
\hline $8 \mathrm{U}-11 / 0-22$ & 10.6 & 10400 & 5.9 & 18600 & 15.9 & 15.9 & 4560 & 261 & 0.44 & 2750 & 5.2 & 7.7 \\
\hline $8 U-11 / 22-38$ & 11.4 & 11200 & 6.5 & 18800 & 18.2 & 17.7 & 4920 & 258 & 0.41 & 3030 & 3.4 & 8.7 \\
\hline $8 U-11 / 38-50$ & 10.8 & 11500 & 6.7 & 17700 & 18.3 & 17.9 & 4670 & 188 & 0.34 & 2850 & 5.5 & 9 \\
\hline $8 U-11 / 50-62$ & 12.7 & 12600 & 6.8 & 18300 & 18.7 & 18 & 4540 & 206 & 0.55 & 2920 & 4.4 & 9.6 \\
\hline $8 U-11 / 62-71$ & 12.2 & 12200 & 6.7 & 17300 & 17.7 & 17.5 & 5330 & 181 & 0.49 & 3250 & 3.9 & 9.8 \\
\hline $8 U-11 / 71-86$ & 9.8 & 10300 & 6 & 16300 & 16.4 & 16.6 & 6460 & 176 & 0.3 & 3520 & 2.1 & 8.7 \\
\hline $8 U-11 / 86-98$ & 8.8 & 9870 & 5.6 & 16000 & 16.3 & 16.6 & 7000 & 184 & 0.32 & 3560 & 2 & 8.3 \\
\hline 8U-11/98-109 & 8.4 & 8490 & 5 & 14900 & 13.5 & 15.6 & 7140 & 169 & 0.27 & 3240 & $<2$ & 7.4 \\
\hline $8 \mathrm{U}-11 / 109-118$ & 7.4 & 8400 & 5.2 & 15000 & 12.7 & 15.7 & 7190 & 173 & 0.37 & 3370 & $<2$ & 7.4 \\
\hline $8 \mathrm{U}-11 / 118-131$ & 8.2 & 8290 & 4.8 & 14000 & 12.3 & 14.3 & 6900 & 153 & 0.46 & 2970 & $<2$ & 7 \\
\hline $8 \mathrm{U}-11 / 131-139$ & 9.3 & 7710 & 4.4 & 13500 & 10.5 & 13.6 & 6610 & 141 & 0.5 & 2610 & 2.4 & 6.7 \\
\hline $8 U-11 / 139-151$ & 8.7 & 7120 & 4.1 & 13100 & 9.1 & 12.8 & 6210 & 121 & 0.48 & 2300 & 2.8 & 6.3 \\
\hline $8 \mathrm{U}-11 / 151-161$ & 6.7 & 5080 & 3.2 & 11600 & 7 & 10.7 & 4850 & 88.6 & 0.41 & 1430 & $<2$ & 5.3 \\
\hline $8 \mathrm{U}-11 / 161-172$ & 9.1 & 8110 & 4.6 & 14500 & 10.9 & 14 & 6500 & 153 & 0.5 & 2860 & 3 & 7 \\
\hline $8 \mathrm{U}-11 / 172-183$ & 8.5 & 8610 & 5 & 15300 & 12.4 & 14.9 & 6610 & 168 & 0.5 & 3140 & 2.6 & 7.3 \\
\hline $8 U-11 / 183-196$ & 10.6 & 11400 & 6.5 & 18700 & 17 & 18.2 & 8310 & 250 & 0.6 & 4720 & 4.7 & 8.6 \\
\hline $8 \mathrm{U}-11 / 196-210$ & 11.3 & 10900 & 6.1 & 17300 & 15.9 & 18 & 7610 & 216 & 0.62 & 3840 & 4.6 & 8.8 \\
\hline $8 \mathrm{U}-11 / 210-224$ & 8.4 & 8730 & 5.3 & 16400 & 13.7 & 15.9 & 6750 & 183 & 0.38 & 3580 & 2.2 & 7.3 \\
\hline $8 U-11 / 224-237$ & 9.3 & 9430 & 5.3 & 16700 & 12.8 & 15.8 & 6150 & 187 & 0.49 & 3590 & 2.4 & 7.8 \\
\hline $8 \mathrm{U}-11 / 237-251$ & 9.9 & 9850 & 5.7 & 17900 & 14.7 & 16.4 & 6110 & 202 & 0.48 & 3850 & 3 & 7.9 \\
\hline $8 \mathrm{U}-11 / 251-265$ & 9.2 & 9480 & 5.6 & 17700 & 13.8 & 16.5 & 5560 & 197 & 0.48 & 3640 & 3.8 & 7.7 \\
\hline $8 \mathrm{U}-11 / 265-278$ & 10.2 & 10200 & 5.9 & 17800 & 14.9 & 17.6 & 5690 & 211 & 0.53 & 3780 & 5 & 8.9 \\
\hline
\end{tabular}


Table 5a-5b. ICP-MS data for auger hole 8U-11-Continued.

\begin{tabular}{|c|c|c|c|c|c|c|c|c|c|c|c|c|c|}
\hline Sample \# & $\begin{array}{c}P \\
\text { (ppm) }\end{array}$ & $\begin{array}{c}\mathrm{Pb} \\
(\mathrm{ppm})\end{array}$ & $\begin{array}{c}\mathbf{R b} \\
(\mathbf{p p m})\end{array}$ & $\begin{array}{c}\text { Sb } \\
(\mathbf{p p m})\end{array}$ & $\begin{array}{c}\text { Sc } \\
(\mathbf{p p m})\end{array}$ & $\begin{array}{c}\text { Sr } \\
\text { (ppm) }\end{array}$ & $\begin{array}{c}\text { Th } \\
\text { (ppm) }\end{array}$ & $\underset{\text { (ppm) }}{\mathrm{Ti}}$ & $\underset{\text { (ppm) }}{\mathrm{TI}}$ & $\underset{(\mathbf{p p m})}{\mathbf{U}}$ & $\underset{(p p m)}{V}$ & $\begin{array}{c}Y \\
\text { (ppm) }\end{array}$ & $\begin{array}{c}\mathrm{Zn} \\
\text { (ppm) }\end{array}$ \\
\hline $8 \mathrm{U}-11 / 0-22$ & 335 & 10.6 & 63.5 & 0.35 & 3.5 & 60.2 & 4.85 & 1360 & 0.33 & 1.06 & 22.6 & 10.1 & 25.2 \\
\hline $8 U-11 / 22-38$ & 204 & 10.8 & 67 & 0.34 & 4.1 & 71.4 & 5.51 & 1500 & 0.36 & 1.19 & 26.5 & 11.3 & 26.8 \\
\hline $8 U-11 / 38-50$ & 137 & 11.2 & 66.2 & 0.34 & 4.3 & 71.9 & 5.71 & 1470 & 0.37 & 1.07 & 29.2 & 11.8 & 27.8 \\
\hline $8 U-11 / 50-62$ & 155 & 11.2 & 67.7 & 0.37 & 4.3 & 72.8 & 5.66 & 1620 & 0.37 & 1.18 & 28.4 & 11.6 & 28.9 \\
\hline $8 U-11 / 62-71$ & 190 & 10.8 & 63.6 & 0.35 & 4.4 & 85 & 5.14 & 1540 & 0.35 & 1.15 & 29.6 & 12 & 28.2 \\
\hline $8 \mathrm{U}-11 / 71-86$ & 299 & 10.2 & 58.9 & 0.3 & 3.9 & 111 & 4.9 & 1460 & 0.33 & 1.16 & 27.5 & 11.1 & 25 \\
\hline $8 U-11 / 86-98$ & 313 & 9.79 & 56.7 & 0.26 & 3.8 & 117 & 4.95 & 1500 & 0.32 & 1.25 & 26.4 & 11 & 24.5 \\
\hline 8U-11/98-109 & 291 & 8.97 & 52.9 & 0.24 & 3.2 & 123 & 4.36 & 1100 & 0.28 & 1.05 & 24.4 & 8.9 & 21.5 \\
\hline $8 \mathrm{U}-11 / 109-118$ & 304 & 9.01 & 55.2 & 0.26 & 3.2 & 121 & 3.69 & 1030 & 0.28 & 1.01 & 25.5 & 8.2 & 20.9 \\
\hline $8 \mathrm{U}-11 / 118-131$ & 258 & 8.67 & 51.6 & 0.24 & 3 & 112 & 3.73 & 976 & 0.26 & 1.01 & 21.5 & 7.9 & 18.6 \\
\hline 8U-11/131-139 & 237 & 8.32 & 48.9 & 0.26 & 2.6 & 91.4 & 3.21 & 868 & 0.26 & 0.88 & 18.9 & 6.8 & 17.5 \\
\hline $8 \mathrm{U}-11 / 139-151$ & 210 & 7.8 & 47 & 0.24 & 2.4 & 88.2 & 2.84 & 776 & 0.24 & 0.82 & 16.6 & 6.2 & 16.3 \\
\hline $8 \mathrm{U}-11 / 151-161$ & 145 & 7.33 & 41.4 & 0.2 & 1.7 & 59.5 & 2.03 & 528 & 0.21 & 0.57 & 11.2 & 4.6 & 11.9 \\
\hline $8 \mathrm{U}-11 / 161-172$ & 228 & 8.5 & 52.1 & 0.28 & 2.8 & 107 & 3.33 & 994 & 0.27 & 0.92 & 18.9 & 7.4 & 18.5 \\
\hline $8 U-11 / 172-183$ & 263 & 9.08 & 54.9 & 0.29 & 3 & 83.1 & 3.83 & 1080 & 0.29 & 1.07 & 20.8 & 8.3 & 19.7 \\
\hline $8 U-11 / 183-196$ & 404 & 11 & 68.2 & 0.38 & 4.1 & 111 & 5.32 & 1740 & 0.36 & 1.48 & 28.7 & 12.1 & 26.8 \\
\hline $8 \mathrm{U}-11 / 196-210$ & 360 & 10.5 & 63.6 & 0.37 & 3.9 & 121 & 4.97 & 1690 & 0.34 & 1.41 & 26.6 & 11.4 & 27 \\
\hline $8 U-11 / 210-224$ & 321 & 9.62 & 58.9 & 0.3 & 3.4 & 90.2 & 4.33 & 1230 & 0.32 & 1.15 & 22.3 & 9.2 & 22 \\
\hline $8 U-11 / 224-237$ & 296 & 9.49 & 59.4 & 0.31 & 3.3 & 83 & 3.92 & 1210 & 0.31 & 1.12 & 22.8 & 9 & 21.2 \\
\hline $8 \mathrm{U}-11 / 237-251$ & 316 & 10.1 & 62.8 & 0.35 & 3.5 & 85.5 & 4.45 & 1420 & 0.34 & 1.25 & 24.3 & 10.2 & 22.6 \\
\hline $8 U-11 / 251-265$ & 288 & 10.1 & 62.2 & 0.33 & 3.4 & 80.9 & 4.18 & 1360 & 0.33 & 1.15 & 23.2 & 9.6 & 22.9 \\
\hline $8 \mathrm{U}-11 / 265-278$ & 313 & 10.3 & 63.6 & 0.35 & 3.6 & 91.7 & 4.66 & 1460 & 0.34 & 1.28 & 24.4 & 10.3 & 23.9 \\
\hline
\end{tabular}


Table 5a-6a. ICP-AES data for auger hole 8U-12.

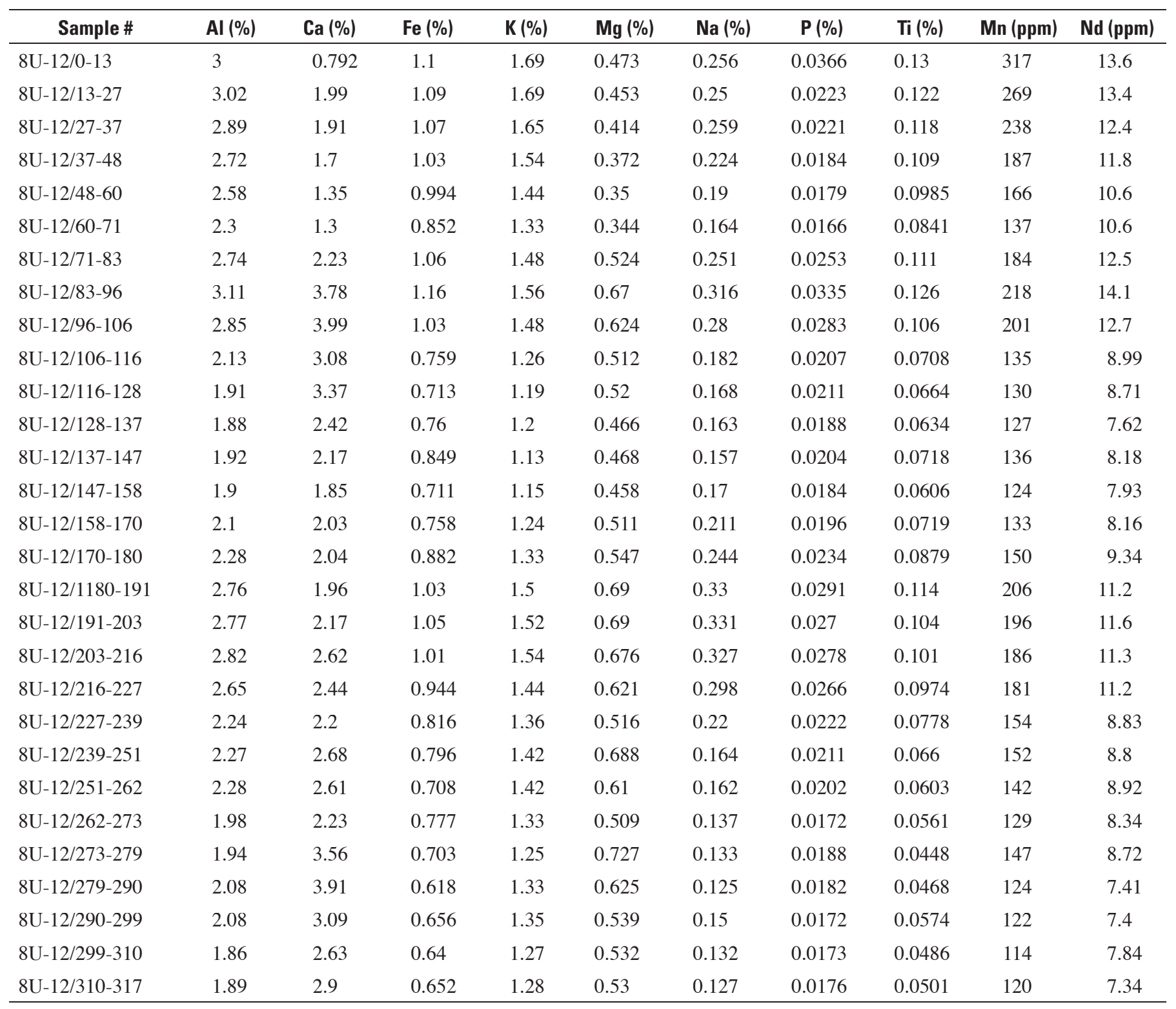


Table 5a-6b. ICP-MS data for auger hole 8U-12.

\begin{tabular}{|c|c|c|c|c|c|c|c|c|c|c|c|c|}
\hline Sample \# & $\underset{(\mathrm{ppm})}{\mathrm{Ag}}$ & $\begin{array}{c}\mathrm{Al} \\
(\mathrm{ppm})\end{array}$ & $\begin{array}{c}\text { As } \\
\text { (ppm) }\end{array}$ & $\begin{array}{c}\mathrm{Ba} \\
\text { (ppm) }\end{array}$ & $\begin{array}{c}\mathrm{Be} \\
(\mathrm{ppm})\end{array}$ & $\underset{(\mathrm{ppm})}{\mathrm{Bi}}$ & $\underset{(\mathrm{ppm})}{\mathrm{Ca}}$ & $\underset{(\mathrm{ppm})}{\mathbf{C d}}$ & $\begin{array}{c}\mathrm{Ce} \\
\text { (ppm) }\end{array}$ & $\begin{array}{c}\text { Co } \\
\text { (ppm) }\end{array}$ & $\underset{(\mathbf{p p m})}{\mathrm{Cr}}$ & $\begin{array}{c}\text { Cs } \\
\text { (ppm) }\end{array}$ \\
\hline $8 \mathrm{U}-12 / 0-13$ & $<2$ & 31700 & 3.3 & 386 & 0.82 & 0.06 & 8280 & 0.31 & 34 & 3.5 & 47.2 & 2.5 \\
\hline $8 \mathrm{U}-12 / 13-27$ & $<2$ & 30600 & 2.8 & 387 & 0.87 & $<0.06$ & 19700 & 0.14 & 31 & 3.5 & 44.8 & 2.5 \\
\hline $8 \mathrm{U}-12 / 27-37$ & $<2$ & 29800 & 3 & 393 & 0.86 & $<0.06$ & 19300 & 0.18 & 32.3 & 3.4 & 48 & 2.4 \\
\hline $8 \mathrm{U}-12 / 37-48$ & $<2$ & 27800 & 2.8 & 375 & 0.86 & $<0.06$ & 17100 & 0.09 & 28.1 & 3.3 & 49.1 & 2.2 \\
\hline $8 \mathrm{U}-12 / 48-60$ & $<2$ & 26700 & 3 & 356 & 0.74 & $<0.06$ & 13900 & 0.05 & 27.3 & 3.2 & 51 & 2.2 \\
\hline $8 \mathrm{U}-12 / 60-71$ & $<2$ & 24300 & 2.8 & 335 & 0.73 & $<0.06$ & 13600 & 0.08 & 26.5 & 2.8 & 39.8 & 2 \\
\hline $8 \mathrm{U}-12 / 71-83$ & $<2$ & 28900 & 3.5 & 386 & 0.88 & $<0.06$ & 23500 & 0.08 & 31.2 & 3.6 & 37.3 & 2.2 \\
\hline $8 \mathrm{U}-12 / 83-96$ & $<2$ & 32900 & 4 & 418 & 0.93 & 0.06 & 39800 & 0.1 & 33.4 & 4.1 & 43.1 & 2.5 \\
\hline 8U-12/96-106 & $<2$ & 30200 & 4.1 & 393 & 0.85 & $<0.06$ & 41900 & 0.1 & 31.2 & 3.7 & 22.8 & 2.3 \\
\hline $8 U-12 / 106-116$ & $<2$ & 21900 & 2.9 & 347 & 0.66 & $<0.06$ & 31500 & 0.12 & 22.5 & 2.5 & 25.2 & 1.6 \\
\hline $8 \mathrm{U}-12 / 116-128$ & $<2$ & 20600 & 2.6 & 326 & 0.62 & $<0.06$ & 34900 & 0.08 & 21.1 & 2.4 & 34.2 & 1.5 \\
\hline $8 \mathrm{U}-12 / 128-137$ & $<2$ & 20300 & 2.2 & 313 & 0.6 & $<0.06$ & 24800 & 0.06 & 18.8 & 2.3 & 47.2 & 1.6 \\
\hline $8 U-12 / 137-147$ & $<2$ & 20900 & 2.5 & 305 & 0.6 & 0.1 & 24400 & 0.14 & 20 & 2.4 & 61.3 & 1.6 \\
\hline $8 \mathrm{U}-12 / 147-158$ & $<2$ & 21200 & 2.3 & 303 & 0.61 & $<0.06$ & 20900 & 0.05 & 18.9 & 2.2 & 41.4 & 1.6 \\
\hline $8 U-12 / 158-170$ & $<2$ & 22900 & 2.4 & 312 & 0.69 & $<0.06$ & 21900 & 0.06 & 20.4 & 2.4 & 40.1 & 1.7 \\
\hline $8 U-12 / 170-180$ & $<2$ & 22100 & 2.8 & 329 & 0.68 & 0.06 & 20300 & 0.06 & 23.3 & 2.5 & 52.8 & 1.8 \\
\hline $8 \mathrm{U}-12 / 1180-191$ & $<2$ & 27900 & 3.6 & 403 & 0.88 & 0.07 & 20000 & 0.07 & 28.9 & 3.2 & 33.3 & 2.1 \\
\hline $8 \mathrm{U}-12 / 191-203$ & $<2$ & 28800 & 3.5 & 418 & 0.82 & 0.07 & 23400 & 0.09 & 27.3 & 3.2 & 55.3 & 2.2 \\
\hline $8 U-12 / 203-216$ & $<2$ & 28300 & 3.3 & 378 & 0.89 & 0.07 & 27300 & 0.08 & 27 & 3.1 & 49.2 & 2.1 \\
\hline $8 U-12 / 216-227$ & $<2$ & 27400 & 3.4 & 361 & 0.85 & 0.07 & 25800 & 0.08 & 26.2 & 3 & 40.9 & 2.1 \\
\hline $8 \mathrm{U}-12 / 227-239$ & $<2$ & 22700 & 3 & 322 & 0.7 & $<0.06$ & 22800 & 0.11 & 22.2 & 2.4 & 48.1 & 1.7 \\
\hline $8 U-12 / 239-251$ & $<2$ & 23600 & 3.1 & 320 & 0.75 & $<0.06$ & 28400 & 0.17 & 20.5 & 2.3 & 49.4 & 1.7 \\
\hline $8 U-12 / 251-262$ & $<2$ & 23300 & 3 & 306 & 0.7 & $<0.06$ & 27100 & 0.08 & 18.7 & 2.1 & 36.3 & 1.7 \\
\hline $8 \mathrm{U}-12 / 262-273$ & $<2$ & 21000 & 2.8 & 288 & 0.69 & $<0.06$ & 23200 & 0.04 & 16.8 & 2 & 60.8 & 1.5 \\
\hline $8 U-12 / 273-279$ & $<2$ & 21000 & 3 & 298 & 0.7 & $<0.06$ & 38900 & 0.06 & 17.2 & 2.2 & 41.4 & 1.5 \\
\hline $8 \mathrm{U}-12 / 279-290$ & $<2$ & 22200 & 3.2 & 293 & 0.72 & $<0.06$ & 41700 & 0.05 & 17.1 & 2 & 32.6 & 1.6 \\
\hline $8 U-12 / 290-299$ & $<2$ & 22600 & 3.1 & 300 & 0.74 & $<0.06$ & 33100 & 0.38 & 18.6 & 2 & 36.4 & 1.6 \\
\hline $8 \mathrm{U}-12 / 299-310$ & $<2$ & 19900 & 2.9 & 275 & 0.72 & $<0.06$ & 27600 & 0.05 & 16.7 & 1.9 & 42.4 & 1.4 \\
\hline $8 \mathrm{U}-12 / 310-317$ & $<2$ & 20300 & 2.9 & 277 & 0.68 & $<0.06$ & 30600 & 0.11 & 16.5 & 1.9 & 44 & 1.5 \\
\hline
\end{tabular}


Table 5a-6b. ICP-MS data for auger hole 8U-12-Continued.

\begin{tabular}{|c|c|c|c|c|c|c|c|c|c|c|c|c|}
\hline Sample \# & $\begin{array}{c}\mathrm{Cu} \\
(\mathrm{ppm})\end{array}$ & $\begin{array}{c}\mathrm{Fe} \\
(\mathrm{ppm})\end{array}$ & $\begin{array}{c}\text { Ga } \\
\text { (ppm) }\end{array}$ & $\begin{array}{c}\mathrm{K} \\
\text { (ppm) }\end{array}$ & $\begin{array}{c}\text { La } \\
\text { (ppm) }\end{array}$ & $\begin{array}{c}\mathrm{Li} \\
\text { (ppm) }\end{array}$ & $\begin{array}{c}\mathrm{Mg} \\
(\mathrm{ppm})\end{array}$ & $\begin{array}{c}\text { Mn } \\
(\mathrm{ppm})\end{array}$ & $\begin{array}{c}\text { Mo } \\
\text { (ppm) }\end{array}$ & $\begin{array}{c}\mathrm{Na} \\
(\mathrm{ppm})\end{array}$ & $\begin{array}{c}\mathrm{Nb} \\
\text { (ppm) }\end{array}$ & $\begin{array}{c}\mathrm{Ni} \\
(\mathrm{ppm})\end{array}$ \\
\hline $8 \mathrm{U}-12 / 0-13$ & 11.7 & 11500 & 6.6 & 18200 & 17.9 & 17.5 & 5290 & 336 & 0.46 & 2720 & 4.5 & 9.6 \\
\hline $8 \mathrm{U}-12 / 27-37$ & 13.1 & 11000 & 6.1 & 17000 & 16.7 & 16 & 4520 & 249 & 0.42 & 2660 & 4.2 & 8.8 \\
\hline $8 \mathrm{U}-12 / 37-48$ & 11.7 & 10500 & 5.7 & 16000 & 14.6 & 14.7 & 4020 & 194 & 0.4 & 2290 & 4.3 & 8.5 \\
\hline $8 \mathrm{U}-12 / 60-71$ & 9.6 & 8920 & 5.1 & 14300 & 14.1 & 13.6 & 3820 & 145 & 0.32 & 1740 & 4.2 & 7.4 \\
\hline $8 \mathrm{U}-12 / 71-83$ & 11.6 & 11100 & 6 & 15900 & 16.4 & 16.9 & 5780 & 195 & 0.43 & 2680 & 5.1 & 8.7 \\
\hline $8 U-12 / 83-96$ & 11.4 & 12400 & 6.6 & 16500 & 17.4 & 19.6 & 7580 & 236 & 0.39 & 3340 & 5.5 & 9.4 \\
\hline $8 U-12 / 96-106$ & 10 & 11100 & 6 & 15600 & 16.2 & 18.1 & 7000 & 216 & 0.32 & 2920 & 5.3 & 8.4 \\
\hline $8 \mathrm{U}-12 / 106-116$ & 7.8 & 7790 & 4.4 & 12900 & 11.8 & 14 & 5720 & 140 & 0.26 & 1850 & 3.2 & 6.1 \\
\hline $8 \mathrm{U}-12 / 137-147$ & 9.9 & 8780 & 4.1 & 12900 & 10.4 & 13.9 & 5280 & 142 & 0.5 & 1770 & 2.8 & 7.4 \\
\hline $8 \mathrm{U}-12 / 147-158$ & 8 & 7530 & 4.1 & 13200 & 10.1 & 14.4 & 5250 & 134 & 0.38 & 1900 & 3 & 6.1 \\
\hline $8 \mathrm{U}-12 / 158-170$ & 8.2 & 8100 & 4.4 & 13700 & 10.8 & 15.1 & 5920 & 143 & 2.2 & 2300 & 3.8 & 6.3 \\
\hline $8 \mathrm{U}-12 / 170-180$ & 10.1 & 8730 & 4.6 & 13300 & 12.3 & 12.2 & 5700 & 150 & 0.67 & 2350 & 4.5 & 7 \\
\hline 8U-12/1180-191 & 10.7 & 10400 & 5.7 & 15800 & 15.2 & 15.7 & 7250 & 212 & 0.63 & 3370 & 5.2 & 8 \\
\hline $8 \mathrm{U}-12 / 191-203$ & 11.3 & 10900 & 5.8 & 16500 & 14.4 & 16.8 & 7620 & 211 & 0.73 & 3460 & 4.7 & 8.6 \\
\hline $8 \mathrm{U}-12 / 203-216$ & 10.9 & 10400 & 5.6 & 16000 & 14.6 & 16.4 & 7470 & 198 & 0.69 & 3310 & 4.2 & 8.2 \\
\hline $8 \mathrm{U}-12 / 216-227$ & 10.2 & 9730 & 5.5 & 15400 & 13.9 & 16.3 & 6890 & 189 & 0.6 & 3100 & 4.4 & 7.6 \\
\hline $8 \mathrm{U}-12 / 227-239$ & 9.3 & 8350 & 4.5 & 14200 & 11.9 & 13.7 & 5680 & 160 & 0.56 & 2240 & 3.1 & 6.9 \\
\hline $8 \mathrm{U}-12 / 290-299$ & 7.1 & 6740 & 4.1 & 14700 & 10.1 & 13.9 & 6050 & 132 & 0.36 & 1590 & 2.6 & 5.6 \\
\hline $8 \mathrm{U}-12 / 299-310$ & 7.2 & 6540 & 3.7 & 13500 & 8.8 & 12.6 & 5940 & 122 & 0.41 & 1340 & 3.2 & 5.5 \\
\hline $8 U-12 / 310-317$ & 7.2 & 6650 & 3.7 & 13600 & 8.7 & 12.7 & 5960 & 126 & 0.46 & 1310 & 2.2 & 6 \\
\hline
\end{tabular}


Table 5a-6b. ICP-MS data for auger hole 8U-12—Continued.

\begin{tabular}{|c|c|c|c|c|c|c|c|c|c|c|c|c|c|}
\hline Sample \# & $\begin{array}{c}P \\
(\mathbf{p p m})\end{array}$ & $\begin{array}{c}\mathrm{Pb} \\
(\mathrm{ppm})\end{array}$ & $\begin{array}{c}\mathbf{R b} \\
\text { (ppm) }\end{array}$ & $\begin{array}{c}\text { Sb } \\
\text { (ppm) }\end{array}$ & $\begin{array}{c}\text { Sc } \\
\text { (ppm) }\end{array}$ & $\begin{array}{c}\mathrm{Sr} \\
(\mathrm{ppm})\end{array}$ & $\begin{array}{c}\text { Th } \\
\text { (ppm) }\end{array}$ & $\underset{\text { (ppm) }}{\mathrm{Ti}}$ & $\begin{array}{c}\mathrm{TI} \\
\text { (ppm) }\end{array}$ & $\underset{(\mathbf{p p m})}{\mathbf{U}}$ & $\underset{(\mathbf{p p m})}{V}$ & $\begin{array}{c}Y \\
\text { (ppm) }\end{array}$ & $\begin{array}{c}\mathrm{Zn} \\
\text { (ppm) }\end{array}$ \\
\hline $8 \mathrm{U}-12 / 0-13$ & 377 & 10.9 & 57.8 & 0.3 & 3.7 & 69.9 & 3.23 & 1390 & 0.3 & 1.09 & 24.7 & 11.4 & 30.8 \\
\hline $8 \mathrm{U}-12 / 13-27$ & 225 & 10.4 & 57.9 & 0.29 & 3.6 & 79.6 & 3.45 & 1170 & 0.31 & 0.88 & 24.5 & 10.6 & 28 \\
\hline $8 U-12 / 27-37$ & 223 & 10.3 & 56.8 & 0.28 & 3.5 & 81.2 & 3.62 & 1130 & 0.3 & 0.96 & 24.4 & 10.4 & 24.8 \\
\hline $8 U-12 / 37-48$ & 188 & 9.91 & 53.4 & 0.27 & 3.1 & 80.4 & 3.33 & 1170 & 0.28 & 0.87 & 22.7 & 9.5 & 24.3 \\
\hline $8 U-12 / 48-60$ & 182 & 9.61 & 50.5 & 0.27 & 3.1 & 74.9 & 3.26 & 1080 & 0.27 & 0.87 & 21.8 & 9 & 22.6 \\
\hline $8 \mathrm{U}-12 / 60-71$ & 174 & 8.93 & 46 & 0.26 & 2.7 & 71.2 & 3.12 & 999 & 0.25 & 0.82 & 20.4 & 8.5 & 20.4 \\
\hline $8 U-12 / 71-83$ & 266 & 10.1 & 51 & 0.3 & 3.5 & 98.3 & 3.82 & 1350 & 0.28 & 1.13 & 26.7 & 11.4 & 24.6 \\
\hline $8 U-12 / 83-96$ & 357 & 10.8 & 53.8 & 0.3 & 4.2 & 120 & 4.04 & 1480 & 0.31 & 1.17 & 32.3 & 11.8 & 32.2 \\
\hline 8U-12/96-106 & 304 & 10.4 & 51 & 0.25 & 3.7 & 114 & 3.61 & 1290 & 0.28 & 1.26 & 29.3 & 11.1 & 27.6 \\
\hline $8 U-12 / 106-116$ & 227 & 8.28 & 41.7 & 0.2 & 2.4 & 91 & 2.54 & 743 & 0.22 & 0.8 & 19.9 & 6.9 & 19.2 \\
\hline $8 \mathrm{U}-12 / 116-128$ & 220 & 7.79 & 39.6 & 0.21 & 2.3 & 100 & 2.32 & 759 & 0.21 & 0.84 & 19 & 6.8 & 15.5 \\
\hline $8 \mathrm{U}-12 / 128-137$ & 200 & 7.85 & 40.4 & 0.2 & 2.1 & 84.6 & 2.08 & 679 & 0.21 & 0.78 & 16.6 & 7 & 17.4 \\
\hline $8 \mathrm{U}-12 / 137-147$ & 207 & 7.87 & 39.8 & 0.21 & 2.2 & 82.5 & 2.19 & 698 & 0.21 & 0.82 & 17.3 & 6.4 & 20.9 \\
\hline $8 \mathrm{U}-12 / 147-158$ & 202 & 7.75 & 40.3 & 0.2 & 2.2 & 80.6 & 2.06 & 738 & 0.2 & 0.82 & 16.9 & 6.6 & 16.7 \\
\hline $8 \mathrm{U}-12 / 158-170$ & 212 & 8.34 & 41.7 & 0.23 & 2.4 & 91.9 & 2.27 & 834 & 0.22 & 0.82 & 18.6 & 6.9 & 20.3 \\
\hline $8 \mathrm{U}-12 / 170-180$ & 199 & 8.85 & 42.7 & 0.27 & 2.5 & 92.7 & 2.18 & 1100 & 0.28 & 0.98 & 18.3 & 7.9 & 17.9 \\
\hline $8 \mathrm{U}-12 / 1180-191$ & 275 & 10.3 & 49.7 & 0.33 & 3.4 & 114 & 3.64 & 1520 & 0.3 & 1.3 & 24.7 & 10.5 & 24.6 \\
\hline $8 \mathrm{U}-12 / 191-203$ & 293 & 10.3 & 50.5 & 0.35 & 3.5 & 114 & 3.56 & 1420 & 0.31 & 1.24 & 24.7 & 10 & 28.8 \\
\hline $8 \mathrm{U}-12 / 203-216$ & 287 & 10 & 48.7 & 0.32 & 3.4 & 103 & 3.57 & 1330 & 0.29 & 1.26 & 23.6 & 9.7 & 22.4 \\
\hline $8 \mathrm{U}-12 / 216-227$ & 281 & 9.68 & 47.2 & 0.29 & 3.2 & 100 & 3.17 & 1250 & 0.28 & 1.18 & 23.8 & 9.2 & 23.9 \\
\hline $8 U-12 / 227-239$ & 223 & 8.36 & 42 & 0.26 & 2.5 & 83.6 & 2.45 & 974 & 0.24 & 0.96 & 17.8 & 7.3 & 17.2 \\
\hline $8 \mathrm{U}-12 / 239-251$ & 218 & 8.14 & 42.1 & 0.22 & 2.4 & 87.3 & 2.11 & 889 & 0.24 & 0.87 & 16.2 & 6.6 & 17.1 \\
\hline $8 \mathrm{U}-12 / 251-262$ & 197 & 8.03 & 40.7 & 0.22 & 2.3 & 78.7 & 1.98 & 733 & 0.23 & 0.75 & 15.4 & 6 & 17.2 \\
\hline $8 U-12 / 262-273$ & 178 & 7.33 & 37.7 & 0.21 & 2 & 71 & 1.82 & 616 & 0.22 & 0.68 & 13.2 & 5.4 & 13.3 \\
\hline $8 \mathrm{U}-12 / 273-279$ & 209 & 7.37 & 37.1 & 0.2 & 2.1 & 87.4 & 1.66 & 681 & 0.21 & 0.74 & 14.2 & 6.3 & 15.1 \\
\hline $8 \mathrm{U}-12 / 279-290$ & 192 & 6.94 & 37.2 & 0.2 & 2 & 94.5 & 1.62 & 647 & 0.2 & 0.74 & 13.7 & 5.6 & 12.9 \\
\hline $8 U-12 / 290-299$ & 183 & 7.48 & 38.8 & 0.2 & 2.1 & 87.4 & 1.77 & 787 & 0.22 & 0.81 & 14.7 & 6.4 & 15.6 \\
\hline $8 \mathrm{U}-12 / 299-310$ & 178 & 7.06 & 34.9 & 0.2 & 1.9 & 73.4 & 1.7 & 725 & 0.2 & 0.85 & 13.4 & 5.7 & 16 \\
\hline $8 \mathrm{U}-12 / 310-317$ & 183 & 6.96 & 35.5 & 0.2 & 1.9 & 74.2 & 1.62 & 689 & 0.2 & 0.68 & 13.2 & 5.5 & 12.4 \\
\hline
\end{tabular}


Table 5a-7a. ICP-AES data for auger hole 00U-27.

\begin{tabular}{|c|c|c|c|c|c|c|c|c|c|c|}
\hline Sample \# & Al (\%) & $\mathrm{Ca}(\%)$ & $\mathrm{Fe}(\%)$ & K (\%) & $\operatorname{Mg}(\%)$ & $\mathrm{Na}(\%)$ & $\mathbf{P}(\%)$ & $\mathrm{Ti}(\%)$ & Mn (ppm) & Nd (ppm) \\
\hline 00U-27/0-10 & 2.91 & 0.507 & 1.04 & 1.77 & 0.441 & 0.329 & 0.0667 & 0.131 & 336 & 14.3 \\
\hline 00U-27/10-30 & 3.26 & 1.51 & 1.16 & 1.87 & 0.514 & 0.34 & 0.0352 & 0.13 & 341 & 16.6 \\
\hline 00U-27/30-50 & 3.12 & 1.69 & 1.06 & 1.83 & 0.451 & 0.32 & 0.029 & 0.129 & 258 & 14.9 \\
\hline 00U-27/50-65 & 3.39 & 1.59 & 1.2 & 1.89 & 0.516 & 0.381 & 0.0336 & 0.144 & 308 & 16.7 \\
\hline 00U-27/65-84 & 3.32 & 1.13 & 1.21 & 1.81 & 0.434 & 0.386 & 0.0236 & 0.151 & 271 & 16.5 \\
\hline 00U-27/84-100 & 3.34 & 0.801 & 1.43 & 1.65 & 0.554 & 0.378 & 0.0294 & 0.168 & 312 & 19.3 \\
\hline 00U-27/100-116 & 3.44 & 0.848 & 1.44 & 1.67 & 0.646 & 0.41 & 0.0326 & 0.157 & 295 & 19 \\
\hline 00U-27/116-128 & 3.51 & 1.7 & 1.32 & 1.77 & 0.696 & 0.446 & 0.0446 & 0.151 & 291 & 16.5 \\
\hline 00U-27/128-141 & 3.48 & 4.17 & 1.2 & 1.78 & 0.736 & 0.481 & 0.0485 & 0.132 & 266 & 16.5 \\
\hline 00U-27/141-156 & 3.04 & 4.01 & 1.05 & 1.66 & 0.637 & 0.395 & 0.0388 & 0.12 & 223 & 13.7 \\
\hline 00U-27/156-168 & 2.68 & 4.22 & 0.937 & 1.51 & 0.652 & 0.334 & 0.0385 & 0.109 & 191 & 12 \\
\hline 00U-27/168-179 & 2.74 & 4.62 & 0.885 & 1.55 & 0.674 & 0.335 & 0.0347 & 0.0976 & 180 & 11.3 \\
\hline 00U-27/179-194 & 2.51 & 4.65 & 0.868 & 1.48 & 0.711 & 0.284 & 0.0353 & 0.0862 & 153 & 10.6 \\
\hline 00U-27/194-208 & 2.36 & 3.55 & 0.793 & 1.42 & 0.708 & 0.254 & 0.0288 & 0.0747 & 156 & 9.89 \\
\hline 00U-27/208-221 & 2.54 & 3.39 & 0.822 & 1.47 & 0.666 & 0.239 & 0.0288 & 0.0802 & 163 & 10.8 \\
\hline 00U-27/221-233 & 2.53 & 3.63 & 0.857 & 1.44 & 0.63 & 0.232 & 0.0293 & 0.0842 & 172 & 11.4 \\
\hline 00U-27/233-242 & 2.42 & 3.46 & 0.783 & 1.4 & 0.561 & 0.208 & 0.0275 & 0.08 & 163 & 10.4 \\
\hline 00U-27/242-252 & 2.45 & 3.76 & 0.765 & 1.44 & 0.593 & 0.21 & 0.0257 & 0.0728 & 162 & 10.8 \\
\hline 00U-27/252-265 & 2.43 & 3.25 & 0.747 & 1.46 & 0.555 & 0.196 & 0.0253 & 0.0729 & 154 & 9.64 \\
\hline 00U-27/265-274 & 2.29 & 2.79 & 0.785 & 1.38 & 0.529 & 0.194 & 0.0238 & 0.0851 & 159 & 9.38 \\
\hline 00U-27/274-283 & 2.25 & 2.31 & 0.763 & 1.38 & 0.489 & 0.184 & 0.0216 & 0.0882 & 159 & 9.49 \\
\hline 00U-27/283-291 & 2.94 & 2.29 & 0.898 & 1.69 & 0.536 & 0.265 & 0.0263 & 0.12 & 199 & 11.9 \\
\hline 00U-27/291-301 & 2.85 & 2.52 & 1.02 & 1.48 & 0.693 & 0.24 & 0.0252 & 0.11 & 237 & 12.2 \\
\hline 00U-27/301-313 & 3.29 & 3.09 & 1.07 & 1.65 & 0.73 & 0.258 & 0.0286 & 0.124 & 246 & 13.3 \\
\hline 00U-27/313-324 & 2.91 & 2.26 & 0.932 & 1.63 & 0.543 & 0.244 & 0.027 & 0.121 & 197 & 12 \\
\hline
\end{tabular}


Table 5a-7b. ICP-MS data for auger hole 00U-27.

\begin{tabular}{|c|c|c|c|c|c|c|c|c|c|c|c|c|}
\hline Sample \# & $\begin{array}{c}\mathbf{A g} \\
(\mathrm{ppm})\end{array}$ & $\begin{array}{c}\text { Al } \\
\text { (ppm) }\end{array}$ & $\begin{array}{c}\text { As } \\
(\mathrm{ppm})\end{array}$ & $\begin{array}{c}\mathrm{Ba} \\
(\mathrm{ppm})\end{array}$ & $\begin{array}{c}\text { Be } \\
\text { (ppm) }\end{array}$ & $\begin{array}{c}\mathbf{B i} \\
\text { (ppm) }\end{array}$ & $\begin{array}{c}\text { Ca } \\
\text { (ppm) }\end{array}$ & $\begin{array}{c}\text { Cd } \\
(\mathrm{ppm})\end{array}$ & $\begin{array}{c}\text { Ce } \\
\text { (ppm) }\end{array}$ & $\begin{array}{c}\text { Co } \\
\text { (ppm) }\end{array}$ & $\begin{array}{c}\mathrm{Cr} \\
(\mathrm{ppm})\end{array}$ & $\begin{array}{c}\text { Cs } \\
\text { (ppm) }\end{array}$ \\
\hline 00U-27/0-10 & $<2$ & 28800 & 2.8 & 425 & 0.74 & 0.1 & 4930 & 0.22 & 34.2 & 3.1 & 37.8 & 2.4 \\
\hline 00U-27/30-50 & $<2$ & 29800 & 2.9 & 410 & 0.84 & 0.08 & 15800 & 0.11 & 33.9 & 3.3 & 34.3 & 2.6 \\
\hline $00 \mathrm{U}-27 / 50-65$ & $<2$ & 33400 & 3.6 & 456 & 0.9 & 0.08 & 15400 & 0.13 & 35.8 & 3.7 & 38.5 & 2.9 \\
\hline 00U-27/84-100 & $<2$ & 39500 & 4.2 & 458 & 1.1 & 0.1 & 9260 & 0.09 & 44.3 & 5.1 & 44.9 & 3.3 \\
\hline 00U-27/100-116 & $<2$ & 39300 & 3.8 & 493 & 1.1 & 0.1 & 9600 & 0.07 & 41.3 & 5.5 & 42.3 & 3.2 \\
\hline 00U-27/116-128 & $<2$ & 36700 & 4.2 & 490 & 1 & 0.1 & 17400 & 0.1 & 37.3 & 4.5 & 33.7 & 3 \\
\hline 00U-27/128-141 & $<2$ & 34100 & 4.4 & 502 & 0.99 & 0.09 & 40100 & 0.12 & 33 & 4.4 & 31.4 & 2.7 \\
\hline 00U-27/141-156 & $<2$ & 29900 & 3.8 & 442 & 0.95 & 0.08 & 39600 & 0.1 & 30.9 & 3.6 & 33.5 & 2.4 \\
\hline 00U-27/179-194 & $<2$ & 26700 & 3.3 & 399 & 0.82 & 0.06 & 48900 & 0.06 & 24.9 & 2.8 & 46.1 & 2 \\
\hline 00U-27/194-208 & $<2$ & 26200 & 2.7 & 380 & 0.73 & $<0.06$ & 39900 & 0.08 & 20.8 & 2.6 & 31.8 & 1.9 \\
\hline 00U-27/208-221 & $<2$ & 27800 & 3.3 & 367 & 0.94 & 0.06 & 36700 & 0.08 & 26.1 & 2.8 & 30.1 & 2.1 \\
\hline 00U-27/221-233 & $<2$ & 29000 & 3.2 & 371 & 0.89 & 0.07 & 41200 & 0.08 & 27.4 & 3 & 31.3 & 2.3 \\
\hline 00U-27/233-242 & $<2$ & 26900 & 3.3 & 347 & 0.81 & 0.06 & 37500 & 0.08 & 24.6 & 2.8 & 28.7 & 2.1 \\
\hline 00U-27/242-252 & $<2$ & 26700 & 3.1 & 347 & 0.93 & $<0.06$ & 40400 & 0.08 & 23 & 2.6 & 27.7 & 2.2 \\
\hline 00U-27/252-265 & $<2$ & 25200 & 3 & 350 & 0.78 & $<0.06$ & 33300 & 0.07 & 20.6 & 2.4 & 30 & 2.1 \\
\hline 00U-27/265-274 & $<2$ & 24500 & 3 & 340 & 0.72 & $<0.06$ & 29600 & 0.07 & 23.2 & 2.5 & 36.6 & 2 \\
\hline 00U-27/274-283 & $<2$ & 24000 & 2.8 & 338 & 0.78 & $<0.06$ & 23800 & 0.07 & 22.2 & 2.4 & 37.3 & 2 \\
\hline
\end{tabular}


Table 5a-7b. ICP-MS data for auger hole 00U-27—Continued.

\begin{tabular}{|c|c|c|c|c|c|c|c|c|c|c|c|c|}
\hline Sample \# & $\begin{array}{c}\text { Cu } \\
\text { (ppm) }\end{array}$ & $\begin{array}{c}\text { Fe } \\
\text { (ppm) }\end{array}$ & $\begin{array}{c}\text { Ga } \\
(\mathrm{ppm})\end{array}$ & $\begin{array}{c}\mathrm{K} \\
\text { (ppm) }\end{array}$ & $\begin{array}{c}\text { La } \\
\text { (ppm) }\end{array}$ & $\begin{array}{c}\text { Li } \\
\text { (ppm) }\end{array}$ & $\begin{array}{c}\mathbf{M g} \\
(\mathbf{p p m})\end{array}$ & $\begin{array}{c}\text { Mn } \\
(\mathbf{p p m})\end{array}$ & $\begin{array}{c}\text { Mo } \\
\text { (ppm) }\end{array}$ & $\begin{array}{c}\mathrm{Na} \\
(\mathrm{ppm})\end{array}$ & $\begin{array}{c}\text { Nb } \\
(\mathrm{ppm})\end{array}$ & $\begin{array}{c}\mathrm{Ni} \\
(\mathrm{ppm})\end{array}$ \\
\hline 00U-27/0-10 & 11 & 10200 & 6.2 & 17300 & 18 & 11.7 & 4510 & 333 & 0.54 & 3100 & 7.8 & 7.9 \\
\hline 00U-27/30-50 & 11 & 10400 & 6.3 & 17300 & 17.4 & 13 & 4440 & 246 & 0.49 & 2910 & 5.9 & 8.2 \\
\hline 00U-27/50-65 & 12.1 & 11800 & 7.2 & 18400 & 19.2 & 15.7 & 5230 & 306 & 0.52 & 3560 & 6.3 & 9.2 \\
\hline 00U-27/84-100 & 15 & 15000 & 8.4 & 19800 & 22.5 & 19.9 & 5930 & 328 & 0.6 & 4260 & 11 & 10.9 \\
\hline 00U-27/100-116 & 14.8 & 14700 & 8.4 & 19200 & 22.1 & 19.5 & 6810 & 304 & 0.56 & 4480 & 9.1 & 11.2 \\
\hline 00U-27/116-128 & 16 & 13400 & 7.8 & 18500 & 20 & 18.3 & 7280 & 298 & 0.54 & 4440 & 6.8 & 10.6 \\
\hline 00U-27/128-141 & 17.7 & 12300 & 7.2 & 17600 & 17.6 & 18 & 7740 & 275 & 0.61 & 4400 & 5.9 & 10.9 \\
\hline 00U-27/141-156 & 14.8 & 10900 & 6.2 & 16500 & 17.6 & 16.7 & 6870 & 234 & 0.53 & 3660 & 5.5 & 8.4 \\
\hline 00U-27/179-194 & 11.9 & 9370 & 5.2 & 15900 & 13 & 15.9 & 8080 & 167 & 0.53 & 2860 & 4.9 & 7.5 \\
\hline 00U-27/194-208 & 11.4 & 8260 & 5.1 & 15900 & 11.2 & 16.1 & 8000 & 162 & 0.44 & 2680 & 3.4 & 6.6 \\
\hline 00U-27/208-221 & 13.4 & 8820 & 5.4 & 16100 & 14.2 & 17.1 & 7590 & 178 & 0.47 & 2510 & 3.5 & 7.3 \\
\hline 00U-27/221-233 & 15.3 & 9110 & 5.6 & 16400 & 14.6 & 17.4 & 7260 & 188 & 0.51 & 2510 & 4.2 & 7.7 \\
\hline 00U-27/233-242 & 12.4 & 8340 & 5.1 & 15400 & 13.1 & 15.9 & 6300 & 176 & 0.5 & 2170 & 4 & 7.2 \\
\hline 00U-27/242-252 & 10.2 & 8000 & 5.2 & 15700 & 12.3 & 15.8 & 6580 & 173 & 0.46 & 2150 & 2.8 & 7 \\
\hline 00U-27/252-265 & 12.1 & 7680 & 4.8 & 15000 & 10.9 & 14.8 & 5990 & 162 & 0.47 & 1910 & 2.9 & 6.8 \\
\hline 00U-27/265-274 & 10.9 & 8080 & 4.8 & 14900 & 12.3 & 14.5 & 5690 & 166 & 0.52 & 1970 & 2.8 & 7.1 \\
\hline 00U-27/274-283 & 10.8 & 7770 & 4.7 & 14800 & 11.8 & 13.8 & 5240 & 161 & 0.54 & 1850 & 3.7 & 7 \\
\hline
\end{tabular}


Table 5a-7b. ICP-MS data for auger hole 00U-27-Continued.

\begin{tabular}{|c|c|c|c|c|c|c|c|c|c|c|c|c|c|}
\hline Sample \# & $\begin{array}{c}\mathbf{P} \\
\text { (ppm) }\end{array}$ & $\begin{array}{c}\mathrm{Pb} \\
(\mathrm{ppm})\end{array}$ & $\begin{array}{c}\mathbf{R b} \\
(\mathbf{p p m})\end{array}$ & $\begin{array}{c}\text { Sb } \\
(\mathbf{p p m})\end{array}$ & $\begin{array}{c}\text { Sc } \\
\text { (ppm) }\end{array}$ & $\begin{array}{c}\mathrm{Sr} \\
(\mathrm{ppm})\end{array}$ & $\begin{array}{c}\text { Th } \\
\text { (ppm) }\end{array}$ & $\begin{array}{c}\mathrm{Ti} \\
(\mathbf{p p m})\end{array}$ & $\begin{array}{c}\mathrm{TI} \\
\text { (ppm) }\end{array}$ & $\underset{\text { (ppm) }}{\mathbf{U}}$ & $\begin{array}{c}V \\
\text { (ppm) }\end{array}$ & $\begin{array}{c}Y \\
\text { (ppm) }\end{array}$ & $\begin{array}{c}\mathrm{Zn} \\
\text { (ppm) }\end{array}$ \\
\hline 00U-27/0-10 & 628 & 12.4 & 59.2 & 0.4 & 3.2 & 69.8 & 4.29 & 1500 & 0.34 & 1.24 & 23.1 & 11.8 & 28.4 \\
\hline 00U-27/10-30 & 332 & 11.4 & 64.2 & 0.4 & 3.9 & 85.3 & 4.97 & 1880 & 0.36 & 1.3 & 27.6 & 13.2 & 29.7 \\
\hline 00U-27/30-50 & 263 & 10.6 & 60 & 0.35 & 3.4 & 85.6 & 4.18 & 1570 & 0.33 & 1.15 & 24.7 & 11.4 & 27.6 \\
\hline 00U-27/50-65 & 318 & 11.2 & 64 & 0.38 & 3.8 & 100 & 4.6 & 1530 & 0.36 & 1.2 & 28.4 & 12 & 30.3 \\
\hline 00U-27/65-84 & 222 & 13.4 & 65.2 & 0.39 & 3.9 & 103 & 5.13 & 1780 & 0.38 & 1.22 & 30 & 11.4 & 35.6 \\
\hline 00U-27/84-100 & 289 & 13.3 & 66.6 & 0.46 & 5 & 106 & 6.48 & 2310 & 0.39 & 1.5 & 36.6 & 15.3 & 39.2 \\
\hline 00U-27/100-116 & 345 & 13.3 & 64.9 & 0.46 & 5 & 112 & 6.42 & 2080 & 0.39 & 1.52 & 38.8 & 14.6 & 37.1 \\
\hline 00U-27/116-128 & 430 & 12.9 & 62.9 & 0.42 & 4.6 & 127 & 4.86 & 1890 & 0.38 & 1.46 & 34.4 & 12.9 & 34.3 \\
\hline 00U-27/128-141 & 495 & 17 & 58.3 & 0.4 & 4.3 & 143 & 4.57 & 1570 & 0.35 & 1.5 & 33.7 & 12.1 & 32.1 \\
\hline 00U-27/141-156 & 410 & 11.8 & 52.1 & 0.37 & 3.8 & 134 & 4.21 & 1580 & 0.32 & 1.42 & 28.9 & 11.2 & 28 \\
\hline 00U-27/156-168 & 374 & 11.6 & 48.6 & 0.31 & 3.4 & 139 & 3.62 & 1420 & 0.29 & 1.3 & 26 & 10.5 & 23.8 \\
\hline 00U-27/168-179 & 342 & 11.9 & 48.3 & 0.32 & 3.3 & 147 & 3.43 & 1410 & 0.28 & 1.16 & 25 & 10.3 & 24 \\
\hline 00U-27/179-194 & 348 & 13.9 & 46.1 & 0.35 & 3 & 170 & 3.12 & 1220 & 0.27 & 1 & 22 & 8.7 & 23.6 \\
\hline 00U-27/194-208 & 300 & 10.3 & 44.8 & 0.27 & 2.8 & 155 & 2.62 & 905 & 0.26 & 0.86 & 20.8 & 7.2 & 19.4 \\
\hline 00U-27/208-221 & 309 & 10.2 & 46.6 & 0.29 & 3.1 & 156 & 3.22 & 1350 & 0.27 & 1.1 & 22.2 & 8.9 & 22.4 \\
\hline 00U-27/221-233 & 294 & 11 & 48.2 & 0.3 & 3.3 & 141 & 3.52 & 1480 & 0.27 & 1.24 & 23.4 & 9.6 & 24.7 \\
\hline 00U-27/233-242 & 253 & 10.1 & 46.3 & 0.27 & 2.9 & 114 & 2.83 & 1190 & 0.26 & 1.11 & 20.2 & 8.6 & 20.4 \\
\hline 00U-27/242-252 & 262 & 9.36 & 46.2 & 0.24 & 2.8 & 120 & 2.66 & 918 & 0.26 & 1.07 & 20.3 & 7.7 & 19.6 \\
\hline 00U-27/252-265 & 247 & 9.32 & 45 & 0.23 & 2.5 & 109 & 2.26 & 810 & 0.25 & 1.02 & 18.3 & 7 & 18.4 \\
\hline 00U-27/265-274 & 243 & 9.16 & 44.8 & 0.24 & 2.6 & 104 & 2.53 & 964 & 0.25 & 1.03 & 18.9 & 7.6 & 19.8 \\
\hline 00U-27/274-283 & 206 & 9.95 & 45.5 & 0.24 & 2.4 & 94.1 & 2.46 & 1040 & 0.24 & 1.03 & 17.9 & 7.8 & 17.9 \\
\hline 00U-27/283-291 & 240 & 11 & 50.6 & 0.28 & 3 & 104 & 2.92 & 1050 & 0.28 & 1.12 & 22 & 8.5 & 27.3 \\
\hline 00U-27/291-301 & 272 & 11.6 & 54.1 & 0.3 & 3.6 & 139 & 3.19 & 1020 & 0.31 & 1.21 & 25.9 & 9.1 & 28.9 \\
\hline 00U-27/301-313 & 268 & 12.4 & 54.8 & 0.31 & 3.8 & 148 & 3.34 & 1490 & 0.3 & 1.39 & 26.7 & 10.5 & 28.4 \\
\hline 00U-27/313-324 & 245 & 11.3 & 52.4 & 0.3 & 3.2 & 113 & 3.39 & 1280 & 0.29 & 1.27 & 22.3 & 9.6 & 26.7 \\
\hline
\end{tabular}


Table 5b-1a. ICP-AES data for the 01U-1 transect.

\begin{tabular}{lcccccccccc}
\hline \multicolumn{1}{c}{ Sample \# } & Al (\%) & $\mathbf{C a}(\%)$ & $\mathbf{F e}(\%)$ & $\mathbf{K}(\%)$ & $\mathbf{M g}(\%)$ & $\mathbf{N a}(\%)$ & $\mathbf{P}(\%)$ & $\mathbf{T i}(\%)$ & $\mathbf{M n}(\mathbf{p p m})$ & $\mathbf{N d}(\mathbf{p p m})$ \\
\hline 01U-1A/0-10 & 2.94 & 3.32 & 1.27 & 1.89 & 1.08 & 0.395 & 0.0392 & 0.117 & 395 & 14.6 \\
01U-1A/10-30 & 2.37 & 2.58 & 1.01 & 1.58 & 0.836 & 0.309 & 0.0256 & 0.0906 & 302 & 10.3 \\
01U-1A/30-50 & 2.75 & 2.96 & 1.20 & 1.61 & 0.699 & 0.326 & 0.0288 & 0.0989 & 343 & 12.6 \\
01U-1B/0-10 & 4.10 & 4.24 & 1.66 & 2.15 & 1.39 & 0.421 & 0.0447 & 0.162 & 484 & 16.8 \\
01U-1B/10-30 & 3.40 & 2.78 & 1.49 & 1.77 & 0.950 & 0.343 & 0.0359 & 0.150 & 396 & 14.7 \\
01U-1B/30-50 & 2.75 & 2.38 & 1.22 & 1.52 & 0.616 & 0.309 & 0.0262 & 0.102 & 319 & 12.4 \\
01U-1C/0-10 & 3.16 & 3.29 & 1.34 & 1.81 & 1.10 & 0.341 & 0.0345 & 0.143 & 386 & 13.2 \\
01U-1C/10-30 & 2.88 & 3.72 & 1.25 & 1.60 & 0.932 & 0.322 & 0.0294 & 0.124 & 437 & 13.4 \\
01U-1C/30-50 & 2.58 & 5.28 & 1.22 & 1.62 & 0.838 & 0.288 & 0.0249 & 0.110 & 358 & 12.6 \\
01U-1D/0-10 & 3.85 & 3.95 & 1.63 & 2.15 & 1.34 & 0.394 & 0.0420 & 0.168 & 488 & 15.6 \\
01U-1D/10-30 & 3.02 & 3.42 & 1.29 & 1.82 & 1.12 & 0.371 & 0.0323 & 0.142 & 394 & 14.1 \\
01U-1D/30-50 & 2.87 & 3.20 & 1.36 & 1.68 & 0.812 & 0.335 & 0.0318 & 0.139 & 389 & 13.7 \\
01U-1E/0-10 & 2.76 & 2.98 & 1.22 & 1.62 & 0.798 & 0.331 & 0.0416 & 0.103 & 378 & 13.5 \\
01U-1E/10-30 & 3.10 & 3.55 & 1.36 & 1.75 & 0.761 & 0.340 & 0.0335 & 0.147 & 398 & 14.6 \\
01U-1E/30-50 & 2.26 & 9.45 & 1.15 & 1.39 & 0.794 & 0.243 & 0.0269 & 0.0972 & 288 & 11.9 \\
01U-1F/0-10 & 2.63 & 3.80 & 1.17 & 1.55 & 0.786 & 0.294 & 0.0340 & 0.118 & 387 & 13.1 \\
01U-1F/10-30 & 2.19 & 6.18 & 0.956 & 1.47 & 0.940 & 0.257 & 0.0241 & 0.0837 & 302 & 11.0 \\
01U-1F/30-50 & 2.32 & 8.04 & 1.06 & 1.55 & 1.50 & 0.292 & 0.0276 & 0.0797 & 310 & 11.5 \\
\hline
\end{tabular}

Table 5b-1b. ICP-MS data for the 01U-1 transect.

\begin{tabular}{|c|c|c|c|c|c|c|c|c|c|c|c|c|}
\hline Sample \# & $\underset{(\mathrm{ppm})}{\mathrm{Ag}}$ & $\begin{array}{c}\text { Al } \\
\text { (ppm) }\end{array}$ & $\begin{array}{c}\text { As } \\
(\mathrm{ppm})\end{array}$ & $\underset{(\mathrm{ppm})}{\mathrm{Ba}}$ & $\begin{array}{c}\mathrm{Be} \\
(\mathrm{ppm})\end{array}$ & $\underset{(\mathrm{ppm})}{\mathbf{B i}}$ & $\underset{(\mathrm{ppm})}{\mathrm{Ca}}$ & $\underset{(\mathrm{ppm})}{\mathrm{Cd}}$ & $\underset{(p p m)}{\mathrm{Ce}}$ & $\underset{\text { (ppm) }}{\text { Co }}$ & $\underset{(\mathrm{ppm})}{\mathrm{Cr}}$ & $\underset{(p p m)}{\text { Cs }}$ \\
\hline $01 \mathrm{U}-1 \mathrm{~A} / 0-10$ & 0.04 & 29700 & 4 & 478 & 0.76 & 0.08 & 33700 & 0.08 & 33 & 4.1 & 61 & 2.1 \\
\hline 01U-1A/10-30 & 0.04 & 26400 & 3.9 & 449 & 0.7 & 0.07 & 30200 & 0.06 & 27.8 & 3.6 & 59 & 1.9 \\
\hline $01 \mathrm{U}-1 \mathrm{~A} / 30-50$ & 0.05 & 28500 & 4.2 & 439 & 0.86 & 0.08 & 33400 & 0.09 & 33.6 & 4.3 & 65.4 & 2.2 \\
\hline $01 \mathrm{U}-1 \mathrm{~B} / 0-10$ & 0.07 & 42200 & 6.2 & 480 & 1.3 & 0.12 & 45100 & 0.11 & 40.8 & 6.4 & 51.2 & 3.4 \\
\hline 01U-1B/10-30 & 0.08 & 36200 & 5.4 & 452 & 1.2 & 0.12 & 31900 & 0.11 & 39 & 5.6 & 57.6 & 3.1 \\
\hline 01U-1B/30-50 & 0.06 & 30800 & 4.1 & 431 & 0.95 & 0.08 & 28400 & 0.08 & 33.9 & 4.4 & 61.3 & 2.4 \\
\hline $01 \mathrm{U}-1 \mathrm{C} / 0-10$ & 0.08 & 33700 & 5.6 & 472 & 0.95 & 0.1 & 37700 & 0.1 & 35.8 & 5.2 & 58.3 & 2.8 \\
\hline $01 \mathrm{U}-1 \mathrm{C} / 10-30$ & 0.08 & 33300 & 5.3 & 466 & 1 & 0.1 & 44700 & 0.12 & 37.9 & 5.2 & 51.5 & 2.6 \\
\hline $01 \mathrm{U}-1 \mathrm{C} / 30-50$ & 0.08 & 24800 & 4.4 & 570 & 0.72 & 0.07 & 54400 & 0.07 & 29.6 & 4 & 62.5 & 1.9 \\
\hline $01 \mathrm{U}-1 \mathrm{D} / 0-10$ & 0.07 & 35900 & 5.9 & 457 & 1.1 & 0.1 & 38300 & 0.08 & 36.9 & 5.5 & 50.8 & 2.9 \\
\hline $01 \mathrm{U}-1 \mathrm{D} / 10-30$ & 0.08 & 31600 & 5.1 & 455 & 0.96 & 0.09 & 37100 & 0.08 & 34 & 4.8 & 53.6 & 2.5 \\
\hline $01 \mathrm{U}-1 \mathrm{D} / 30-50$ & 0.08 & 31000 & 4.7 & 436 & 0.9 & 0.09 & 34400 & 0.09 & 36.7 & 4.5 & 62.8 & 2.3 \\
\hline 01U-1E/0-10 & 0.06 & 30400 & 3.9 & 424 & 0.8 & 0.09 & 32800 & 0.12 & 33.3 & 4.1 & 55.2 & 2.1 \\
\hline $01 \mathrm{U}-1 \mathrm{E} / 10-30$ & 0.07 & 31500 & 4.5 & 434 & 0.86 & 0.09 & 36600 & 0.1 & 37.6 & 4.5 & 52.6 & 2.4 \\
\hline $01 \mathrm{U}-1 \mathrm{E} / 30-50$ & 0.05 & 21400 & 7.2 & 444 & 0.65 & 0.06 & 94800 & 0.07 & 28.4 & 3.8 & 54.8 & 1.7 \\
\hline $01 \mathrm{U}-1 \mathrm{~F} / 0-10$ & 0.09 & 25600 & 4.5 & 420 & 0.72 & 0.09 & 41100 & 0.13 & 34.8 & 4.1 & 55.5 & 2 \\
\hline 01U-1F/10-30 & 0.05 & 22500 & 4.7 & 440 & 0.59 & 0.06 & 66400 & 0.06 & 25.2 & 3.9 & 56.7 & 1.6 \\
\hline $01 \mathrm{U}-1 \mathrm{~F} / 30-50$ & 0.07 & 23400 & 6 & 612 & 0.66 & 0.05 & 81100 & 0.06 & 25 & 3.8 & 52.6 & 1.5 \\
\hline
\end{tabular}


Table 5b-1b. ICP-MS data for the 01U-1 transect-Continued.

\begin{tabular}{lcccccccccccc}
\hline \multicolumn{1}{c}{ Sample \# } & $\begin{array}{c}\mathbf{C u} \\
(\mathbf{p p m})\end{array}$ & $\begin{array}{c}\mathbf{F e} \\
(\mathbf{p p m})\end{array}$ & $\begin{array}{c}\mathbf{G a} \\
(\mathbf{p p m})\end{array}$ & $\begin{array}{c}\mathbf{K} \\
(\mathbf{p p m})\end{array}$ & $\begin{array}{c}\mathbf{L a} \\
(\mathbf{p p m})\end{array}$ & $\begin{array}{c}\mathbf{L i} \\
(\mathbf{p p m})\end{array}$ & $\begin{array}{c}\mathbf{M g} \\
(\mathbf{p p m})\end{array}$ & $\begin{array}{c}\mathbf{M n} \\
(\mathbf{p p m})\end{array}$ & $\begin{array}{c}\mathbf{M o} \\
(\mathbf{p p m})\end{array}$ & $\begin{array}{c}\mathbf{N a} \\
(\mathbf{p p m})\end{array}$ & $\begin{array}{c}\mathbf{N b} \\
(\mathbf{p p m})\end{array}$ & $\begin{array}{c}\mathbf{N i} \\
(\mathbf{p p m})\end{array}$ \\
\hline 01U-1A/0-10 & 15.2 & 12000 & 6.4 & 19300 & 17.3 & 18.3 & 11400 & 385 & 0.68 & 3930 & $<2$ & 10.5 \\
01U-1A/10-30 & 13.9 & 11000 & 5.7 & 18500 & 15 & 16.9 & 9860 & 327 & 0.61 & 3450 & $<2$ & 9.2 \\
01U-1A/30-50 & 16.6 & 13000 & 6.4 & 18500 & 18.2 & 18.6 & 8240 & 375 & 0.74 & 3600 & $<2$ & 11 \\
01U-1B/0-10 & 19.8 & 17000 & 9.4 & 23500 & 21.8 & 28.5 & 14700 & 506 & 0.59 & 4240 & 2 & 14.8 \\
01U-1B/10-30 & 23 & 16000 & 8.5 & 20900 & 20.7 & 25.8 & 11300 & 433 & 0.72 & 3950 & 2.2 & 13.6 \\
01U-1B/30-50 & 16.6 & 14000 & 6.9 & 18100 & 17.9 & 19.3 & 7460 & 353 & 0.76 & 3650 & $<2$ & 11.4 \\
01U-1C/0-10 & 20.8 & 15000 & 7.9 & 21300 & 19.5 & 23.3 & 12400 & 432 & 0.63 & 3810 & 2.5 & 12.6 \\
01U-1C/10-30 & 17.4 & 14000 & 7.5 & 19400 & 21 & 22 & 11500 & 502 & 0.65 & 3840 & 2.1 & 12.6 \\
01U-1C/30-50 & 14.6 & 12000 & 5.6 & 16800 & 15.6 & 17.3 & 8780 & 347 & 0.7 & 2890 & 2.6 & 10.5 \\
01U-1D/0-10 & 17.3 & 15000 & 8.1 & 21300 & 19.8 & 24.7 & 13500 & 451 & 0.57 & 3840 & 2 & 12.9 \\
01U-1D/10-30 & 23 & 13000 & 7.2 & 19800 & 18.6 & 21.4 & 12800 & 410 & 0.57 & 3900 & 2.1 & 11.7 \\
01U-1D/30-50 & 17.7 & 14000 & 6.9 & 17900 & 19.4 & 19.3 & 9110 & 396 & 0.73 & 3620 & 2 & 11.4 \\
01U-1E/0-10 & 15 & 12000 & 6.4 & 17100 & 18.2 & 17.6 & 9290 & 384 & 0.61 & 3940 & $<2$ & 10.2 \\
01U-1E/10-30 & 15.7 & 13000 & 7 & 18100 & 20 & 19.8 & 8440 & 395 & 0.64 & 3770 & 1.9 & 11 \\
01U-1E/30-50 & 12.4 & 11000 & 4.9 & 13200 & 15.3 & 15.4 & 8300 & 277 & 0.61 & 2500 & $<2$ & 10.3 \\
01U-1F/0-10 & 14.2 & 12000 & 6 & 16800 & 19 & 16.8 & 8170 & 406 & 2 & 3280 & 3.6 & 10.3 \\
01U-1F/10-30 & 13.6 & 10000 & 4.9 & 15600 & 13.4 & 16.5 & 10700 & 314 & 0.63 & 2900 & $<2$ & 10.1 \\
01U-1F/30-50 & 13.8 & 10000 & 4.7 & 15000 & 13.4 & 18.5 & 15900 & 302 & 2.3 & 3040 & 2.3 & 11.7
\end{tabular}

Table 5b-1b. ICP-MS data for the 01U-1 transect-Continued.

\begin{tabular}{|c|c|c|c|c|c|c|c|c|c|c|c|c|c|}
\hline Sample \# & $\begin{array}{c}\mathbf{P} \\
\text { (ppm) }\end{array}$ & $\begin{array}{c}\mathrm{Pb} \\
(\mathbf{p p m})\end{array}$ & $\begin{array}{c}\mathbf{R b} \\
(\mathbf{p p m})\end{array}$ & $\begin{array}{c}\text { Sb } \\
\text { (ppm) }\end{array}$ & $\begin{array}{c}\text { Sc } \\
(\mathbf{p p m})\end{array}$ & $\begin{array}{c}\mathrm{Sr} \\
(\mathbf{p p m})\end{array}$ & $\begin{array}{c}\text { Th } \\
\text { (ppm) }\end{array}$ & $\underset{\text { (ppm) }}{\mathrm{Ti}}$ & $\begin{array}{c}\mathrm{Tl} \\
\text { (ppm) }\end{array}$ & $\underset{(\mathbf{p p m})}{\mathbf{U}}$ & $\underset{(\mathbf{p p m})}{\mathbf{V}}$ & $\begin{array}{c}Y \\
\text { (ppm) }\end{array}$ & $\begin{array}{c}\mathrm{Zn} \\
\text { (ppm) }\end{array}$ \\
\hline $01 \mathrm{U}-1 \mathrm{~A} / 0-10$ & 340 & 12 & 62.1 & 0.34 & 4.2 & 182 & 4.7 & 1300 & 0.4 & 1.2 & 25.1 & 11.4 & 25.6 \\
\hline 01U-1A/10-30 & 250 & 10.8 & 59.1 & 0.31 & 3.6 & 162 & 4 & 1200 & 0.4 & 1.1 & 21.4 & 10 & 21 \\
\hline 01U-1A/30-50 & 280 & 11.8 & 59 & 0.34 & 4.2 & 146 & 5 & 1300 & 0.4 & 1.3 & 26.1 & 11.7 & 28.9 \\
\hline $01 \mathrm{U}-1 \mathrm{~B} / 0-10$ & 420 & 14.4 & 76.8 & 0.44 & 6.6 & 254 & 6.1 & 2000 & 0.5 & 1.5 & 41.7 & 15.8 & 40.3 \\
\hline 01U-1B/10-30 & 360 & 14 & 69.8 & 0.42 & 5.7 & 183 & 6.1 & 1900 & 0.4 & 1.6 & 37.3 & 15 & 37.3 \\
\hline 01U-1B/30-50 & 270 & 11.8 & 61 & 0.37 & 4.5 & 133 & 5.2 & 1300 & 0.4 & 1.3 & 27.8 & 11.8 & 28.4 \\
\hline 01U-1C/0-10 & 320 & 13.8 & 69.1 & 0.42 & 5.4 & 216 & 5.4 & 1900 & 0.4 & 1.4 & 34 & 14.1 & 34.1 \\
\hline 01U-1C/10-30 & 310 & 13.7 & 65.5 & 0.38 & 5.2 & 206 & 5.6 & 1700 & 0.4 & 1.6 & 32.8 & 15.6 & 31.2 \\
\hline 01U-1C/30-50 & 200 & 10.6 & 53.2 & 0.34 & 3.7 & 210 & 4.5 & 1300 & 0.3 & 1.2 & 24.5 & 11.4 & 22.3 \\
\hline $01 \mathrm{U}-1 \mathrm{D} / 0-10$ & 340 & 13.4 & 71.3 & 0.41 & 5.5 & 216 & 5.6 & 1800 & 0.4 & 1.4 & 34.2 & 14.1 & 34.1 \\
\hline 01U-1D/10-30 & 280 & 13.1 & 66.2 & 0.38 & 5 & 202 & 5.1 & 1700 & 0.4 & 1.4 & 29.8 & 13.5 & 30 \\
\hline 01U-1D/30-50 & 280 & 12.2 & 61.5 & 0.39 & 4.4 & 157 & 5.6 & 1600 & 0.4 & 1.5 & 28.5 & 13.8 & 28.6 \\
\hline 01U-1E/0-10 & 380 & 12.9 & 60.1 & 0.35 & 3.9 & 146 & 5 & 1300 & 0.4 & 1.3 & 25.9 & 12.2 & 27.4 \\
\hline 01U-1E/10-30 & 280 & 12.2 & 61.4 & 0.34 & 4.6 & 144 & 5.6 & 1600 & 0.4 & 1.5 & 28.1 & 14.2 & 30.4 \\
\hline 01U-1E/30-50 & 220 & 9 & 45.5 & 0.27 & 3.2 & 264 & 4 & 1100 & 0.3 & 1.3 & 23.3 & 10.8 & 19.5 \\
\hline $01 \mathrm{U}-1 \mathrm{~F} / 0-10$ & 310 & 12.1 & 55.4 & 0.35 & 3.9 & 165 & 5.2 & 1500 & 0.3 & 1.4 & 25.5 & 14.1 & 28.3 \\
\hline 01U-1F/10-30 & 220 & 9.8 & 48.1 & 0.28 & 3.2 & 268 & 3.6 & 1100 & 0.3 & 1 & 20 & 10.2 & 20.5 \\
\hline 01U-1F/30-50 & 260 & 9.5 & 46.3 & 0.31 & 3.1 & 498 & 3.7 & 1000 & 0.3 & 1.1 & 21.3 & 9.8 & 18.5 \\
\hline
\end{tabular}


Table 5b-2a. ICP-AES data for the $04 \mathrm{U}$ transect.

\begin{tabular}{llllllllllc}
\hline \multicolumn{1}{c}{ Sample \# } & Al (\%) & $\mathbf{C a}(\%)$ & $\mathbf{F e}(\%)$ & $\mathbf{K}(\%)$ & $\mathbf{M g}(\%)$ & $\mathbf{N a}(\%)$ & $\mathbf{P}(\%)$ & $\mathbf{T i}(\%)$ & $\mathbf{M n}(\mathbf{p p m})$ & $\mathbf{N d}(\mathbf{p p m})$ \\
\hline 04U-21A/0-10 & 3.52 & 3.67 & 1.22 & 1.98 & 1.15 & 0.403 & 0.0396 & 0.113 & 409 & 13.9 \\
04U-21B/10-30 & 3.18 & 3.2 & 1.05 & 1.92 & 0.952 & 0.386 & 0.0329 & 0.0955 & 354 & 13 \\
04U-22A/0-10 & 3.64 & 3.54 & 1.31 & 2.03 & 1.12 & 0.359 & 0.0374 & 0.112 & 406 & 15 \\
04U-22B/10-30 & 3.03 & 3.44 & 1.12 & 1.74 & 0.778 & 0.328 & 0.0294 & 0.102 & 396 & 14.2 \\
04U-23A/0-10 & 3.91 & 4.12 & 1.4 & 2.13 & 1.28 & 0.41 & 0.0392 & 0.128 & 445 & 16 \\
04U-23B/10-30 & 3.75 & 3.81 & 1.37 & 2.02 & 1.23 & 0.405 & 0.0398 & 0.126 & 428 & 17.2 \\
04U-24A/0-10 & 3.62 & 3.99 & 1.26 & 2.04 & 1.24 & 0.419 & 0.0385 & 0.157 & 416 & 14.5 \\
04U-24B/10-30 & 2.56 & 2.61 & 0.892 & 1.65 & 0.778 & 0.295 & 0.025 & 0.0854 & 304 & 10.4 \\
\hline
\end{tabular}

Table 5b-2b. ICP-MS data for the 04U transect.

\begin{tabular}{lcccccccccccc}
\hline \multicolumn{1}{c}{ Sample \# } & $\begin{array}{c}\mathbf{A g} \\
(\mathbf{p p m})\end{array}$ & $\begin{array}{c}\mathbf{A l} \\
\mathbf{( \mathbf { p p m } )}\end{array}$ & $\begin{array}{c}\mathbf{A s} \\
(\mathbf{p p m})\end{array}$ & $\begin{array}{c}\mathbf{B a} \\
(\mathbf{p p m})\end{array}$ & $\begin{array}{c}\mathbf{B e} \\
(\mathbf{p p m})\end{array}$ & $\begin{array}{c}\mathbf{B i} \\
(\mathbf{p p m})\end{array}$ & $\begin{array}{c}\mathbf{C a} \\
(\mathbf{p p m})\end{array}$ & $\begin{array}{c}\mathbf{C d} \\
(\mathbf{p p m})\end{array}$ & $\begin{array}{c}\mathbf{C e} \\
(\mathbf{p p m})\end{array}$ & $\begin{array}{c}\mathbf{C o} \\
(\mathbf{p p m})\end{array}$ & $\begin{array}{c}\mathbf{C r} \\
(\mathbf{p p m})\end{array}$ & $\begin{array}{c}\mathbf{C s} \\
(\mathbf{p p m})\end{array}$ \\
\hline 04U-21A/0-10 & $<2$ & 37300 & 5 & 456 & 1.1 & 0.09 & 39400 & 0.11 & 31.4 & 4.8 & 33.1 & 2.7 \\
04U-21B/10-30 & $<2$ & 33500 & 4.4 & 449 & 0.92 & 0.08 & 34100 & 0.08 & 28.4 & 4 & 31.6 & 2.2 \\
04U-22A/0-10 & $<2$ & 39100 & 10.7 & 460 & 1.3 & 0.09 & 38300 & 0.1 & 31.9 & 5.1 & 33.7 & 2.9 \\
04U-22B/10-30 & $<2$ & 33400 & 17.9 & 459 & 1.1 & 0.08 & 37600 & 0.1 & 31.6 & 4.4 & 27.9 & 2.4 \\
04U-23A/0-10 & $<2$ & 42400 & 5.9 & 491 & 1.4 & 0.14 & 44200 & 0.04 & 34.1 & 5.7 & 36 & 3.2 \\
04U-23B/10-30 & $<2$ & 40300 & 5.4 & 500 & 1.2 & 0.1 & 40300 & 0.09 & 34.9 & 5.3 & 36.5 & 3 \\
04U-24A/0-10 & $<2$ & 38900 & 5.1 & 480 & 1.2 & 0.09 & 42600 & 0.09 & 34.1 & 5.2 & 34.5 & 2.8 \\
04U-24B/10-30 & $<2$ & 28000 & 3.9 & 473 & 0.77 & 0.06 & 28000 & 0.06 & 23.5 & 3.3 & 33.1 & 1.9
\end{tabular}

Table 5b-2b. ICP-MS data for the 04U transect-Continued.

\begin{tabular}{lcccccccccccc}
\hline \multicolumn{1}{c}{ Sample \# } & $\begin{array}{c}\mathbf{C u} \\
(\mathbf{p p m})\end{array}$ & $\begin{array}{c}\mathbf{F e} \\
(\mathbf{p p m})\end{array}$ & $\begin{array}{c}\mathbf{G a} \\
(\mathbf{p p m})\end{array}$ & $\begin{array}{c}\mathbf{K} \\
(\mathbf{p p m})\end{array}$ & $\begin{array}{c}\mathbf{L a} \\
(\mathbf{p p m})\end{array}$ & $\begin{array}{c}\mathbf{L i} \\
(\mathbf{p p m})\end{array}$ & $\begin{array}{c}\mathbf{M g} \\
(\mathbf{p p m})\end{array}$ & $\begin{array}{c}\mathbf{M n} \\
(\mathbf{p p m})\end{array}$ & $\begin{array}{c}\mathbf{M o} \\
(\mathbf{p p m})\end{array}$ & $\begin{array}{c}\mathbf{N a} \\
(\mathbf{p p m})\end{array}$ & $\begin{array}{c}\mathbf{N b} \\
(\mathbf{p p m})\end{array}$ & $\begin{array}{c}\mathbf{N i} \\
(\mathbf{p p m})\end{array}$ \\
\hline 4U-21A/0-10 & 14.4 & 12900 & 7.2 & 22000 & 16.7 & 24.4 & 12800 & 448 & 0.36 & 4290 & 4.4 & 9.9 \\
4U-21B/10-30 & 15.5 & 11000 & 6.3 & 20900 & 15.1 & 21.2 & 10900 & 386 & 0.32 & 4100 & 3.4 & 18.1 \\
4U-22A/0-10 & 14.6 & 13900 & 7.6 & 22500 & 17 & 27 & 12900 & 444 & 0.37 & 3900 & 3.9 & 10.9 \\
4U-22B/10-30 & 12.8 & 11900 & 6.5 & 19700 & 16.7 & 21.4 & 8840 & 437 & 0.45 & 3640 & 3.6 & 20 \\
4U-23A/0-10 & 20.2 & 15000 & 8.2 & 23500 & 17.8 & 28.4 & 14500 & 490 & 0.4 & 4390 & 4 & 12 \\
4U-23B/10-30 & 19.4 & 14300 & 8 & 22000 & 18.5 & 27.1 & 13600 & 460 & 0.38 & 4340 & 4.2 & 12.5 \\
4U-24A/0-10 & 16.9 & 13500 & 7.5 & 22200 & 17.5 & 25.1 & 13900 & 462 & 0.31 & 4500 & 3.7 & 10.9 \\
4U-24B/10-30 & 11.8 & 9440 & 5.3 & 18300 & 12.4 & 17.9 & 8830 & 332 & 0.33 & 3210 & 3 & 7.8
\end{tabular}


Table 5b-2b. ICP-MS data for the 04U transect-Continued.

\begin{tabular}{lccccccccccccc}
\hline \multicolumn{1}{c}{ Sample \# } & $\begin{array}{c}\mathbf{P} \\
(\mathbf{p p m})\end{array}$ & $\begin{array}{c}\mathbf{P b} \\
(\mathbf{p p m})\end{array}$ & $\begin{array}{c}\mathbf{R b} \\
(\mathbf{p p m})\end{array}$ & $\begin{array}{c}\mathbf{S b} \\
(\mathbf{p p m})\end{array}$ & $\begin{array}{c}\mathbf{S c} \\
(\mathbf{p p m})\end{array}$ & $\begin{array}{c}\mathbf{S r} \\
(\mathbf{p p m})\end{array}$ & $\begin{array}{c}\mathbf{T h} \\
(\mathbf{p p m})\end{array}$ & $\begin{array}{c}\mathbf{T i} \\
(\mathbf{p p m})\end{array}$ & $\begin{array}{c}\mathbf{T I} \\
(\mathbf{p p m})\end{array}$ & $\begin{array}{c}\mathbf{U} \\
(\mathbf{p p m})\end{array}$ & $\begin{array}{c}\mathbf{V} \\
(\mathbf{p p m})\end{array}$ & $\begin{array}{c}\mathbf{Y} \\
(\mathbf{p p m})\end{array}$ & $\begin{array}{c}\mathbf{Z n} \\
(\mathbf{p p m})\end{array}$ \\
\hline 4U-21A/0-10 & 420 & 12.7 & 63.2 & 0.39 & 5.2 & 194 & 3.58 & 1480 & 0.39 & 1.15 & 33.3 & 12.1 & 30.2 \\
4U-21B/10-30 & 340 & 11.8 & 58.8 & 0.33 & 4.4 & 166 & 3.24 & 1250 & 0.36 & 1.05 & 27.6 & 10.7 & 24.7 \\
4U-22A/0-10 & 398 & 12.5 & 64.6 & 0.36 & 5.5 & 200 & 3.8 & 1460 & 0.4 & 1.19 & 36.1 & 12 & 32.1 \\
4U-22B/10-30 & 308 & 11.4 & 56.3 & 0.32 & 4.5 & 152 & 3.8 & 1200 & 0.34 & 1.45 & 30.3 & 11.6 & 27.9 \\
4U-23A/0-10 & 409 & 13 & 69.5 & 0.41 & 6.1 & 232 & 4.24 & 1450 & 0.43 & 1.17 & 37.8 & 12.9 & 34.1 \\
4U-23B/10-30 & 410 & 12.7 & 67.6 & 0.37 & 5.5 & 224 & 4.08 & 1610 & 0.42 & 1.31 & 36.6 & 13 & 33 \\
4U-24A/0-10 & 400 & 11.9 & 66.6 & 0.35 & 5.4 & 222 & 3.79 & 1540 & 0.4 & 1.09 & 33.6 & 12.4 & 30.6 \\
4U-24B/10-30 & 264 & 9.9 & 55 & 0.3 & 3.3 & 150 & 2.42 & 971 & 0.32 & 0.94 & 22.4 & 8.8 & 20.1 \\
\hline
\end{tabular}

Table 5c-1a. ICP-AES data for auger hole 00U-38.

\begin{tabular}{lcccccccccc}
\hline \multicolumn{1}{c}{ Sample \# } & $\mathbf{A l}(\%)$ & $\mathbf{C a}(\%)$ & $\mathbf{F e}(\%)$ & $\mathbf{K}(\%)$ & $\mathbf{M g}(\%)$ & $\mathbf{N a}(\%)$ & $\mathbf{P}(\%)$ & $\mathbf{T i}(\%)$ & $\mathbf{M n}(\mathbf{p p m})$ & $\mathbf{N d}(\mathbf{p p m})$ \\
\hline 00U-38/0-10 & 1.61 & 1.60 & 0.557 & 1.25 & 0.348 & 0.0659 & 0.0189 & 0.0410 & 92.0 & 6.89 \\
00U-38/10-30 & 1.61 & 1.63 & 0.874 & 1.28 & 0.340 & 0.0688 & 0.0165 & 0.0455 & 101 & 7.08 \\
00U-38/30-44 & 1.64 & 1.68 & 0.747 & 1.29 & 0.348 & 0.0694 & 0.0156 & 0.0488 & 99.4 & 6.57 \\
00U-38/44-57 & 1.46 & 1.56 & 0.524 & 1.16 & 0.300 & 0.0650 & 0.0148 & 0.0323 & 78.3 & 4.83 \\
00U-38/57-74 & 1.50 & 1.73 & 0.744 & 1.18 & 0.316 & 0.0686 & 0.0146 & 0.0324 & 94.4 & 5.48 \\
00U-38/74-96 & 1.53 & 1.76 & 0.554 & 1.20 & 0.330 & 0.0765 & 0.0156 & 0.0350 & 87.1 & 5.77 \\
00U-38/96-115 & 1.73 & 2.19 & 0.567 & 1.31 & 0.382 & 0.0944 & 0.0193 & 0.0447 & 101 & 6.66 \\
00U-38/115-140 & 1.80 & 2.27 & 0.584 & 1.35 & 0.392 & 0.105 & 0.0198 & 0.0432 & 109 & 7.84 \\
00U-38/140-156 & 1.86 & 2.50 & 1.02 & 1.43 & 0.446 & 0.114 & 0.0222 & 0.0728 & 132 & 8.38 \\
00U-38/156-185 & 1.62 & 2.29 & 0.519 & 1.30 & 0.405 & 0.0962 & 0.0156 & 0.0324 & 86.6 & 5.92 \\
00U-38/185-207 & 1.64 & 2.31 & 0.468 & 1.29 & 0.433 & 0.109 & 0.0161 & 0.0336 & 88.6 & 5.76 \\
00U-38/207-229 & 1.66 & 2.40 & 0.482 & 1.29 & 0.434 & 0.104 & 0.0166 & 0.0307 & 92.8 & 5.77 \\
00U-38/227-242 & 1.66 & 2.54 & 0.483 & 1.28 & 0.469 & 0.102 & 0.0170 & 0.0282 & 96.2 & 6.56 \\
\hline
\end{tabular}


Table 5c-1b. ICP-MS data for auger hole 00U38.

\begin{tabular}{|c|c|c|c|c|c|c|c|c|c|}
\hline Sample \# & $\underset{(\mathrm{ppm})}{\mathbf{A g}}$ & $\begin{array}{c}\text { Al } \\
\text { (ppm) }\end{array}$ & $\begin{array}{c}\text { As } \\
\text { (ppm) }\end{array}$ & $\begin{array}{c}\mathrm{Ba} \\
\text { (ppm) }\end{array}$ & $\begin{array}{c}\mathrm{Be} \\
\text { (ppm) }\end{array}$ & $\begin{array}{c}\mathrm{Bi} \\
(\mathrm{ppm})\end{array}$ & $\begin{array}{c}\mathrm{Ca} \\
\text { (ppm) }\end{array}$ & $\underset{(\mathrm{ppm})}{\mathbf{C d}}$ & $\begin{array}{c}\mathrm{Ce} \\
\text { (ppm) }\end{array}$ \\
\hline 00U-38/0-10 & $<2$ & 20200 & 1.4 & 284 & 0.60 & $<0.06$ & 19400 & 0.06 & 15.6 \\
\hline 00U-38/10-30 & $<2$ & 20400 & 1.7 & 300 & 0.64 & $<0.06$ & 19700 & 0.04 & 14.7 \\
\hline 00U-38/30-44 & $<2$ & 21100 & 1.7 & 299 & 0.53 & $<0.06$ & 20600 & 0.04 & 14.0 \\
\hline 00U-38/44-57 & $<2$ & 14800 & 1.4 & 257 & 0.43 & $<0.06$ & 15000 & 0.03 & 10.6 \\
\hline 00U-38/57-74 & $<2$ & 15200 & 1.6 & 266 & 0.5 & $<0.06$ & 16800 & 0.03 & 11.4 \\
\hline 00U-38/74-96 & $<2$ & 15900 & 1.3 & 278 & 0.5 & $<0.06$ & 17400 & 0.03 & 12.2 \\
\hline 00U-38/96-115 & $<2$ & 18200 & 1.2 & 301 & 0.57 & $<0.06$ & 22100 & 0.04 & 13.7 \\
\hline 00U-38/115-140 & $<2$ & 18800 & 1.3 & 302 & 0.67 & $<0.06$ & 22400 & 0.04 & 16.2 \\
\hline 00U-38/140-156 & $<2$ & 24000 & 2.2 & 334 & 0.86 & $<0.06$ & 31000 & 0.05 & 17.6 \\
\hline 00U-38/156-185 & $<2$ & 16500 & 1.4 & 293 & 0.47 & $<0.06$ & 22200 & 0.03 & 11.2 \\
\hline 00U-38/185-207 & $<2$ & 16600 & 1.2 & 302 & 0.57 & $<0.06$ & 22800 & 0.04 & 11.4 \\
\hline 00U-38/207-229 & $<2$ & 16600 & 1.4 & 304 & 0.54 & $<0.06$ & 23300 & 0.04 & 12.3 \\
\hline 00U-38/227-242 & $<2$ & 16900 & 1.2 & 299 & 0.54 & $<0.06$ & 25100 & 0.04 & 13.5 \\
\hline
\end{tabular}

Table 5c-1b. ICP-MS data for auger hole 00U38—Continued.

\begin{tabular}{|c|c|c|c|c|c|c|c|c|c|c|}
\hline Sample \# & $\begin{array}{c}\text { Co } \\
\text { (ppm) }\end{array}$ & $\underset{(\mathrm{ppm})}{\mathrm{Cr}}$ & $\begin{array}{c}\text { Cs } \\
\text { (ppm) }\end{array}$ & $\underset{(\mathbf{p p m})}{\mathrm{Cu}}$ & $\begin{array}{c}\mathrm{Fe} \\
(\mathrm{ppm})\end{array}$ & $\begin{array}{c}\text { Ga } \\
\text { (ppm) }\end{array}$ & $\underset{(\mathbf{p p m})}{\mathbf{K}}$ & $\begin{array}{c}\text { La } \\
\text { (ppm) }\end{array}$ & $\underset{(\mathbf{p p m})}{\mathbf{L i}}$ & $\underset{\text { (ppm) }}{\mathrm{Mg}}$ \\
\hline 00U-38/0-10 & 1.6 & 28.9 & 1.1 & 10.5 & 5850 & 3.4 & 15600 & 8.9 & 9.7 & 4710 \\
\hline 00U-38/10-30 & 2.0 & 105 & 1.0 & 19.4 & 9580 & 3.4 & 15600 & 8.3 & 10.0 & 4610 \\
\hline 00U-38/30-44 & 1.9 & 81.7 & 1.1 & 16.6 & 8300 & 3.7 & 16200 & 7.8 & 10.4 & 4790 \\
\hline 00U-38/44-57 & 1.2 & 37.5 & 0.91 & 6.2 & 4800 & 2.7 & 12100 & 6.2 & 9.3 & 3060 \\
\hline 00U-38/57-74 & 1.5 & 74.3 & 0.95 & 10.7 & 6900 & 2.7 & 12500 & 6.5 & 9.4 & 3280 \\
\hline 00U-38/74-96 & 1.4 & 40.2 & 0.98 & 6.9 & 5130 & 2.9 & 13000 & 7 & 9.6 & 3420 \\
\hline 00U-38/96-115 & 1.6 & 32.7 & 1.1 & 6.5 & 5250 & 3.3 & 14400 & 7.7 & 10.6 & 3970 \\
\hline 00U-38/115-140 & 1.7 & 32.5 & 1.2 & 6.8 & 5560 & 3.4 & 14500 & 9 & 10.8 & 4100 \\
\hline 00U-38/140-156 & 2.6 & 124 & 1.2 & 16.7 & 11200 & 4.1 & 18100 & 9.9 & 11.4 & 6060 \\
\hline 00U-38/156-185 & 1.4 & 32.7 & 1 & 6.8 & 4820 & 2.9 & 13600 & 6.6 & 9.8 & 4200 \\
\hline 00U-38/185-207 & 1.3 & 20 & 1 & 6.1 & 4130 & 3 & 13600 & 6.6 & 9.7 & 4510 \\
\hline 00U-38/207-229 & 1.5 & 27.5 & 0.98 & 7 & 4610 & 3 & 13600 & 6.9 & 9.8 & 4450 \\
\hline 00U-38/227-242 & 1.5 & 26.4 & 1 & 6.4 & 4550 & 3 & 13800 & 6.7 & 9.9 & 4850 \\
\hline
\end{tabular}


Table 5c-1b. ICP-MS data for auger hole 00U38-Continued.

\begin{tabular}{lcccccccc}
\hline \multicolumn{1}{c}{ Sample \# } & $\begin{array}{c}\mathbf{M n} \\
\mathbf{( p p m )}\end{array}$ & $\begin{array}{c}\mathbf{M} \\
\mathbf{( p p m )}\end{array}$ & $\begin{array}{c}\mathbf{N a} \\
\mathbf{( p p m )}\end{array}$ & $\begin{array}{c}\mathbf{N b} \\
\mathbf{( p p m )}\end{array}$ & $\begin{array}{c}\mathbf{N i} \\
\mathbf{( p p m )}\end{array}$ & $\begin{array}{c}\mathbf{P} \\
\mathbf{( p p m )}\end{array}$ & $\begin{array}{c}\mathbf{P b} \\
\mathbf{( p p m )}\end{array}$ & $\begin{array}{c}\mathbf{R b} \\
(\mathbf{p p m})\end{array}$ \\
\hline 00U-38/0-10 & 115 & 0.22 & 838 & 0.93 & 3.8 & 230 & 8.64 & 38.8 \\
00U-38/10-30 & 132 & 0.85 & 888 & 0.86 & 8.3 & 201 & 8.23 & 38.0 \\
00U-38/30-44 & 131 & 0.65 & 935 & 0.97 & 7.1 & 200 & 8.81 & 38.5 \\
00U-38/44-57 & 69.8 & 0.43 & 638 & 0.1 & 3.7 & 145 & 6.33 & 30.9 \\
00U-38/57-74 & 84.1 & 0.85 & 698 & $<0.1$ & 6 & 149 & 6.42 & 32.1 \\
00U-38/74-96 & 79.8 & 0.48 & 792 & $<0.1$ & 4.2 & 160 & 6.26 & 33 \\
00U-38/96-115 & 93.8 & 0.38 & 958 & 0.25 & 4.1 & 201 & 7 & 37.1 \\
00U-38/115-140 & 102 & 0.37 & 1030 & 0.4 & 4.4 & 201 & 7.19 & 37.3 \\
00U-38/140-156 & 172 & 0.95 & 1510 & 1.3 & 9.8 & 273 & 8.69 & 43.0 \\
00U-38/156-185 & 79.3 & 0.35 & 951 & $<0.1$ & 3.7 & 158 & 6.67 & 35.5 \\
00U-38/185-207 & 80.7 & 0.23 & 1090 & $<0.1$ & 3 & 175 & 6.81 & 35.4 \\
00U-38/207-229 & 84.1 & 0.31 & 1080 & 0.27 & 3.4 & 172 & 6.81 & 35.2 \\
00U-38/227-242 & 88.3 & 0.32 & 1010 & 0.1 & 3.4 & 174 & 6.8 & 35.9
\end{tabular}

Table 5c-1b. ICP-MS data for auger hole 00U38-Continued.

\begin{tabular}{lcccccccccc}
\hline \multicolumn{1}{c}{ Sample \# } & $\begin{array}{c}\mathbf{S b} \\
\text { (ppm) }\end{array}$ & $\begin{array}{c}\mathbf{S c} \\
\text { (ppm) }\end{array}$ & $\begin{array}{c}\mathbf{S r} \\
\mathbf{( p p m )}\end{array}$ & $\begin{array}{c}\text { Th } \\
\text { (ppm) }\end{array}$ & $\begin{array}{c}\text { Ti } \\
\text { (ppm) }\end{array}$ & $\begin{array}{c}\text { TI } \\
\text { (ppm) }\end{array}$ & $\begin{array}{c}\mathbf{U} \\
\text { (ppm) }\end{array}$ & $\begin{array}{c}\mathbf{V} \\
\text { (ppm) }\end{array}$ & $\begin{array}{c}\mathbf{Y} \\
\text { (ppm) }\end{array}$ & $\begin{array}{c}\text { Zn } \\
\text { (ppm) }\end{array}$ \\
\hline 00U-38/0-10 & 0.09 & 1.6 & 53.8 & 2.13 & 665 & 0.20 & 0.48 & 10.9 & 6.4 & 10.9 \\
00U-38/10-30 & 0.2 & 1.7 & 59.1 & 2.28 & 709 & 0.18 & 0.49 & 9.4 & 6.7 & 11.4 \\
00U-38/30-44 & 0.2 & 1.7 & 59.8 & 2.13 & 716 & 0.20 & 0.54 & 8.4 & 6.6 & 11.3 \\
00U-38/44-57 & 0.1 & 1.1 & 43.9 & 1.76 & 311 & 0.2 & 0.38 & 7.1 & 4 & 7.9 \\
00U-38/57-74 & 0.2 & 1.2 & 42.8 & 1.94 & 348 & 0.2 & 0.42 & 7.1 & 4.2 & 8.4 \\
00U-38/74-96 & 0.1 & 1.3 & 45.9 & 1.9 & 373 & 0.2 & 0.48 & 7.4 & 4.6 & 10.3 \\
00U-38/96-115 & 0.2 & 1.6 & 56.5 & 2.2 & 448 & 0.23 & 0.53 & 9.2 & 5.2 & 10.3 \\
00U-38/115-140 & 0.1 & 1.6 & 57.6 & 2.97 & 514 & 0.23 & 0.53 & 10 & 5.3 & 10.8 \\
00U-38/140-156 & 0.26 & 2.0 & 81.7 & 2.52 & 1010 & 0.22 & 0.62 & 11.0 & 8.7 & 14.9 \\
00U-38/156-185 & 0.1 & 1.3 & 59.1 & 1.84 & 351 & 0.22 & 0.45 & 8.6 & 4.4 & 8.8 \\
00U-38/185-207 & 0.1 & 1.3 & 61.2 & 1.9 & 347 & 0.22 & 0.42 & 8.3 & 4.2 & 10.2 \\
00U-38/207-229 & 0.1 & 1.3 & 60 & 2.18 & 340 & 0.22 & 0.45 & 8.2 & 4.1 & 9.2 \\
00U-38/227-242 & 0.1 & 1.4 & 62.5 & 1.94 & 313 & 0.22 & 0.45 & 7.7 & 4.4 & 9.6 \\
\hline
\end{tabular}


Table 5c-2a. ICP-AES data for the 00U-39 transect.

\begin{tabular}{|c|c|c|c|c|c|c|c|c|c|c|}
\hline Sample \# & $\mathrm{Al}(\%)$ & $\mathrm{Ca}(\%)$ & $\mathrm{Fe}(\%)$ & K (\%) & $\mathbf{M g}(\%)$ & $\mathrm{Na}(\%)$ & $\mathbf{P}(\%)$ & Ti (\%) & Mn (ppm) & Nd (ppm) \\
\hline 00U-39/A-0-10 & 1.66 & 1.74 & 0.746 & 1.39 & 0.365 & 0.0740 & 0.0152 & 0.0442 & 98.7 & 7.33 \\
\hline 00U-39A/10-30 & 1.45 & 1.64 & 0.532 & 1.27 & 0.317 & 0.0695 & 0.0119 & 0.0261 & 79.5 & 5.22 \\
\hline 00U-39A/30-50 & 1.67 & 1.76 & 0.616 & 1.31 & 0.377 & 0.0744 & 0.0150 & 0.0321 & 95.4 & 6.42 \\
\hline 00U-39/B-0-10 & 1.80 & 1.69 & 0.964 & 1.52 & 0.352 & 0.0887 & 0.0173 & 0.0455 & 109 & 7.45 \\
\hline 00U-39/B-10-30 & 1.72 & 1.68 & 0.737 & 1.48 & 0.330 & 0.0874 & 0.0149 & 0.0414 & 94.8 & 6.73 \\
\hline 00U-39/B-30-50 & 1.92 & 2.27 & 0.681 & 1.62 & 0.358 & 0.103 & 0.0154 & 0.0444 & 99.7 & 7.07 \\
\hline 00U-39/C-0-10 & 1.82 & 1.82 & 0.682 & 1.52 & 0.362 & 0.0981 & 0.0180 & 0.0437 & 103 & 7.73 \\
\hline 00U-39C/10-30 & 1.72 & 2.20 & 0.544 & 1.44 & 0.347 & 0.0938 & 0.0138 & 0.0329 & 92.8 & 6.01 \\
\hline 00U-39C/30-50 & 1.77 & 2.21 & 0.516 & 1.39 & 0.360 & 0.0930 & 0.0147 & 0.0395 & 93.5 & 6.89 \\
\hline 00U-39/D-0-10 & 1.72 & 1.64 & 0.729 & 1.52 & 0.338 & 0.0842 & 0.0168 & 0.0417 & 101 & 7.07 \\
\hline 00U-39D/10-30 & 1.70 & 2.06 & 0.578 & 1.38 & 0.334 & 0.0914 & 0.0171 & 0.0461 & 98.0 & 7.94 \\
\hline 00U-39D/30-50 & 1.68 & 2.01 & 0.522 & 1.36 & 0.333 & 0.0907 & 0.0158 & 0.0359 & 95.4 & 7.11 \\
\hline 00U-39-E-0-10 & 1.87 & 1.82 & 0.659 & 1.54 & 0.391 & 0.0980 & 0.0228 & 0.0482 & 118 & 7.90 \\
\hline 00U-39E/10-30 & 1.85 & 2.17 & 0.656 & 1.41 & 0.370 & 0.0976 & 0.0188 & 0.0510 & 118 & 6.82 \\
\hline 00U-39E/30-50 & 1.76 & 2.18 & 0.625 & 1.37 & 0.363 & 0.0910 & 0.0163 & 0.0465 & 110 & 6.99 \\
\hline 00U-39/F-0-10 & 1.77 & 1.76 & 0.692 & 1.46 & 0.379 & 0.0842 & 0.0219 & 0.0500 & 113 & 7.95 \\
\hline 00U-39/F-10-30 & 1.78 & 1.78 & 0.663 & 1.46 & 0.371 & 0.0855 & 0.0206 & 0.0534 & 113 & 7.73 \\
\hline 00U-39/F-30-50 & 2.22 & 2.42 & 0.826 & 1.66 & 0.462 & 0.129 & 0.0247 & 0.0810 & 158 & 9.74 \\
\hline 00U-39/G-0-10 & 1.89 & 1.84 & 0.727 & 1.51 & 0.389 & 0.0959 & 0.0256 & 0.0611 & 129 & 7.83 \\
\hline 00U-39G/10-30 & 2.07 & 2.35 & 0.654 & 1.49 & 0.404 & 0.129 & 0.0236 & 0.0626 & 141 & 9.21 \\
\hline 00U-39G/30-50 & 2.08 & 2.46 & 0.610 & 1.50 & 0.407 & 0.128 & 0.0212 & 0.0614 & 135 & 9.74 \\
\hline 00U-39-H-0-10 & 1.80 & 1.79 & 0.679 & 1.49 & 0.380 & 0.103 & 0.0276 & 0.0542 & 123 & 7.81 \\
\hline 00U-39H/10-30 & 2.14 & 2.22 & 0.744 & 1.53 & 0.410 & 0.133 & 0.0255 & 0.0735 & 156 & 9.60 \\
\hline 00U-39H/30-50 & 2.23 & 2.84 & 0.666 & 1.57 & 0.416 & 0.144 & 0.0215 & 0.0651 & 145 & 8.06 \\
\hline 00U-39/I-1-10 & 2.09 & 1.93 & 0.728 & 1.61 & 0.420 & 0.129 & 0.0312 & 0.0663 & 149 & 9.58 \\
\hline 00U-39I/10-30 & 2.53 & 2.81 & 0.828 & 1.73 & 0.460 & 0.164 & 0.0256 & 0.0820 & 181 & 10.1 \\
\hline 00U-39I/30-50 & 2.41 & 3.18 & 0.792 & 1.64 & 0.428 & 0.147 & 0.0180 & 0.0779 & 155 & 9.45 \\
\hline 00U-39/J-0-10 & 2.00 & 1.79 & 0.753 & 1.60 & 0.372 & 0.118 & 0.0297 & 0.0662 & 133 & 9.68 \\
\hline $00 \mathrm{U}-39 \mathrm{~J} / 10-30$ & 2.21 & 2.46 & 0.821 & 1.61 & 0.411 & 0.150 & 0.0242 & 0.0731 & 159 & 9.80 \\
\hline 00U-39J/30-50 & 2.13 & 2.62 & 0.713 & 1.59 & 0.417 & 0.148 & 0.0210 & 0.0688 & 154 & 9.54 \\
\hline 00U-39/K-0-10 & 2.40 & 2.20 & 0.887 & 1.74 & 0.478 & 0.164 & 0.0361 & 0.0908 & 182 & 12.9 \\
\hline 00U-39/K-10-30 & 2.74 & 3.10 & 0.970 & 1.86 & 0.519 & 0.193 & 0.0286 & 0.109 & 208 & 13.5 \\
\hline 00U-39/K-30-50 & 2.77 & 3.55 & 0.952 & 1.87 & 0.537 & 0.192 & 0.0275 & 0.109 & 193 & 14.9 \\
\hline 00U-39/L-0-10 & 2.34 & 2.15 & 0.844 & 1.78 & 0.436 & 0.161 & 0.0350 & 0.0852 & 164 & 12.0 \\
\hline 00U-39L/10-30 & 2.38 & 2.79 & 0.766 & 1.64 & 0.454 & 0.167 & 0.0246 & 0.0786 & 178 & 9.49 \\
\hline 00U-39L/30-50 & 2.44 & 3.26 & 0.809 & 1.65 & 0.430 & 0.172 & 0.0173 & 0.0759 & 160 & 9.60 \\
\hline 00U-39/M-0-10 & 2.80 & 2.95 & 0.998 & 1.88 & 0.553 & 0.192 & 0.0388 & 0.110 & 212 & 15.4 \\
\hline 00U-39M/10-30 & 2.17 & 2.96 & 0.766 & 1.64 & 0.399 & 0.163 & 0.0188 & 0.0754 & 148 & 9.00 \\
\hline 00U-39M/30-50 & 2.05 & 3.09 & 0.740 & 1.56 & 0.381 & 0.139 & 0.0177 & 0.0677 & 123 & 8.15 \\
\hline 00U-39/N-0-10 & 2.75 & 3.22 & 0.846 & 1.94 & 0.772 & 0.151 & 0.0343 & 0.0898 & 171 & 12.7 \\
\hline 00U-39/N-10-30 & 2.69 & 3.35 & 0.888 & 1.91 & 0.727 & 0.171 & 0.0294 & 0.0990 & 181 & 14.2 \\
\hline 00U-39/N-30-50 & 2.89 & 3.80 & 1.00 & 1.88 & 0.592 & 0.208 & 0.0329 & 0.116 & 224 & 16.0 \\
\hline 00U-39/O-0-10 & 2.76 & 3.51 & 0.878 & 1.90 & 0.887 & 0.132 & 0.0283 & 0.0885 & 172 & 12.3 \\
\hline 00U-39O/10-30 & 2.39 & 3.41 & 0.731 & 1.71 & 0.870 & 0.125 & 0.0203 & 0.0606 & 162 & 8.29 \\
\hline 00U-39O/30-50 & 2.93 & 3.12 & 1.05 & 1.78 & 0.589 & 0.185 & 0.0275 & 0.105 & 218 & 11.2 \\
\hline
\end{tabular}


Table 5c-2b. ICP-MS data for the 00U-39 transect.

\begin{tabular}{|c|c|c|c|c|c|c|c|c|c|c|c|c|c|}
\hline Sample \# & $\underset{(\mathrm{ppm})}{\mathbf{A g}}$ & $\begin{array}{c}\text { Al } \\
\text { (ppm) }\end{array}$ & $\begin{array}{c}\text { As } \\
\text { (ppm) }\end{array}$ & $\begin{array}{c}\mathrm{Ba} \\
\text { (ppm) }\end{array}$ & $\begin{array}{c}\mathrm{Be} \\
(\mathrm{ppm})\end{array}$ & $\underset{(p p m)}{\mathbf{B i}}$ & $\begin{array}{c}\mathrm{Ca} \\
\text { (ppm) }\end{array}$ & $\begin{array}{c}\text { Cd } \\
\text { (ppm) }\end{array}$ & $\begin{array}{c}\text { Ce } \\
\text { (ppm) }\end{array}$ & $\begin{array}{c}\text { Co } \\
\text { (ppm) }\end{array}$ & $\begin{array}{c}\mathrm{Cr} \\
(\mathrm{ppm})\end{array}$ & $\begin{array}{c}\text { Cs } \\
\text { (ppm) }\end{array}$ & $\begin{array}{c}\mathrm{Cu} \\
(\mathrm{ppm})\end{array}$ \\
\hline 00U-39/A-0-10 & $<2$ & 21500 & 1.6 & 328 & 0.55 & $<0.06$ & 21300 & 0.04 & 15.2 & 1.8 & 71.5 & 1.1 & 22.0 \\
\hline 00U-39A/30-50 & $<2$ & 16900 & 1.5 & 301 & 0.52 & $<0.06$ & 17400 & 0.04 & 11.9 & 1.5 & 37.8 & 1.1 & 5.9 \\
\hline 00U-39/B-0-10 & $<2$ & 23300 & 1.6 & 368 & 0.62 & $<0.06$ & 20900 & 0.04 & 16.1 & 2.0 & 114 & 1.2 & 17.6 \\
\hline 00U-39/B-10-30 & $<2$ & 22100 & 1.5 & 360 & 0.51 & $<0.06$ & 20600 & 0.03 & 14.6 & 1.7 & 77.3 & 1.1 & 12.6 \\
\hline 00U-39C/10-30 & $<2$ & 17500 & 1.2 & 323 & 0.5 & $<0.06$ & 21200 & 0.04 & 12.8 & 1.6 & 36.3 & 1.1 & 6.7 \\
\hline 00U-39C/30-50 & $<2$ & 18100 & 1.6 & 317 & 0.55 & $<0.06$ & 21900 & 0.04 & 13.5 & 1.5 & 26 & 1.1 & 5.2 \\
\hline 00U-39/D-0-10 & $<2$ & 22400 & 1.2 & 357 & 0.52 & $<0.06$ & 20200 & 0.06 & 14.7 & 1.8 & 71.1 & 1.1 & 11.4 \\
\hline 00U-39D/10-30 & $<2$ & 17500 & 1.5 & 311 & 0.5 & $<0.06$ & 20000 & 0.04 & 12.8 & 1.5 & 37.2 & 1.1 & 6.7 \\
\hline 00U-39D/30-50 & $<2$ & 17400 & 1.2 & 314 & 0.53 & $<0.06$ & 19600 & 0.04 & 12.7 & 1.4 & 29 & 1.1 & 5.8 \\
\hline 00U-39-E-0-10 & $<2$ & 24400 & 1.3 & 364 & 0.70 & $<0.06$ & 22300 & 0.06 & 17.2 & 1.9 & 59.1 & 1.2 & 15.0 \\
\hline 00U-39/F-30-50 & $<2$ & 29400 & 2.3 & 386 & 0.91 & $<0.06$ & 30500 & 0.07 & 21.6 & 2.6 & 65.5 & 1.6 & 15.0 \\
\hline 00U-39/G-0-10 & $<2$ & 24400 & 1.6 & 353 & 0.68 & $<0.06$ & 22800 & 0.07 & 17.1 & 2.0 & 63.2 & 1.2 & 11.7 \\
\hline 00U-39G/10-30 & $<2$ & 21300 & 1.8 & 331 & 0.65 & $<0.06$ & 22900 & 0.05 & 17.6 & 1.9 & 28.4 & 1.4 & 7 \\
\hline 00U-39G/30-50 & $<2$ & 21200 & 1.6 & 340 & 0.68 & $<0.06$ & 24500 & 0.06 & 17.1 & 1.9 & 23.6 & 1.4 & 6.6 \\
\hline 00U-39-H-0-10 & $<2$ & 23100 & 1.5 & 352 & 0.49 & $<0.06$ & 22000 & 0.06 & 16.8 & 1.9 & 61.2 & 1.2 & 11.2 \\
\hline 00U-39H/10-30 & $<2$ & 22300 & 2 & 344 & 0.72 & $<0.06$ & 22100 & 0.07 & 18.1 & 2.1 & 39.1 & 1.5 & 8.6 \\
\hline 00U-39H/30-50 & $<2$ & 23300 & 2.1 & 356 & 0.84 & $<0.06$ & 27900 & 0.06 & 18.2 & 2.1 & 25.2 & 1.6 & 7.4 \\
\hline 00U-39/I-1-10 & $<2$ & 26800 & 1.7 & 374 & 0.71 & $<0.06$ & 24100 & 0.08 & 21.2 & 2.2 & 52.8 & 1.4 & 17.4 \\
\hline 00U-39I/10-30 & $<2$ & 25900 & 2.6 & 358 & 0.86 & $<0.06$ & 29600 & 0.08 & 25.4 & 2.6 & 16.7 & 1.8 & 8.7 \\
\hline 00U-39I/30-50 & $<2$ & 25500 & 2.8 & 361 & 0.77 & $<0.06$ & 34600 & 0.07 & 21.8 & 2.6 & 21.1 & 1.7 & 8.4 \\
\hline 00U-39/J-0-10 & $<2$ & 21300 & 1.6 & 348 & 0.47 & $<0.06$ & 18600 & 0.07 & 17.3 & 1.8 & 53.8 & 1.3 & 13.6 \\
\hline 00U-39L/30-50 & $<2$ & 26400 & 2.8 & 367 & 0.86 & $<0.06$ & 36100 & 0.07 & 23.8 & 2.6 & 20.2 & 1.8 & 8.4 \\
\hline 00U-39/M-0-10 & $<2$ & 32000 & 2.6 & 408 & 0.93 & $<0.06$ & 32500 & 0.10 & 28.8 & 3.0 & 44.0 & 2.1 & 14.3 \\
\hline 00U-39M/10-30 & $<2$ & 23800 & 2.6 & 357 & 0.76 & $<0.06$ & 32000 & 0.06 & 25.4 & 2.4 & 19.8 & 1.6 & 7.7 \\
\hline $00 \mathrm{U}-39 \mathrm{M} / 30-50$ & $<2$ & 10600 & 2.2 & 86.9 & 0.57 & $<0.06$ & 33800 & 0.06 & 19.2 & 2.1 & 23.2 & 1 & 5.9 \\
\hline 00U-39/N-0-10 & $<2$ & 30600 & 2.2 & 379 & 0.90 & $<0.06$ & 34300 & 0.09 & 22.9 & 2.5 & 46.6 & 1.8 & 11.6 \\
\hline $00 \mathrm{U}-39 / \mathrm{N}-10-30$ & $<2$ & 30600 & 2.4 & 382 & 1.1 & $<0.06$ & 36500 & 0.07 & 26.0 & 2.7 & 49.0 & 1.8 & 9.9 \\
\hline $00 \mathrm{U}-39 / \mathrm{N}-30-50$ & $<2$ & 33400 & 2.5 & 412 & 0.99 & $<0.06$ & 42100 & 0.10 & 29.4 & 3.2 & 41.5 & 2.2 & 17.0 \\
\hline 00U-39/O-0-10 & $<2$ & 31500 & 2.2 & 373 & 1.1 & $<0.06$ & 38400 & 0.06 & 24.4 & 2.7 & 51.9 & 1.8 & 9.3 \\
\hline 00U-39O/10-30 & $<2$ & 11000 & 1.9 & 58.6 & 0.77 & $<0.06$ & 36500 & 0.06 & 17.7 & 2.3 & 24.1 & 1.1 & 4.4 \\
\hline $00 \mathrm{U}-39 \mathrm{O} / 30-50$ & $<2$ & 31200 & 3.4 & 364 & 1.1 & $<0.06$ & 33900 & 0.09 & 28 & 3.3 & 34.8 & 2.1 & 10.8 \\
\hline
\end{tabular}


Table 5c-2b. ICP-MS data for the 00U-39 transect-Continued.

\begin{tabular}{|c|c|c|c|c|c|c|c|c|c|c|c|c|c|c|}
\hline Sample \# & $\begin{array}{c}\mathrm{Fe} \\
(\mathrm{ppm})\end{array}$ & $\begin{array}{c}\text { Ga } \\
\text { (ppm) }\end{array}$ & $\begin{array}{c}\mathrm{K} \\
\text { (ppm) }\end{array}$ & $\begin{array}{c}\text { La } \\
(\mathbf{p p m})\end{array}$ & $\begin{array}{c}\mathrm{Li} \\
\text { (ppm) }\end{array}$ & $\begin{array}{c}\mathbf{M g} \\
(\mathbf{p p m})\end{array}$ & $\begin{array}{c}\text { Mn } \\
\text { (ppm) }\end{array}$ & $\begin{array}{c}\text { Mo } \\
\text { (ppm) }\end{array}$ & $\begin{array}{c}\mathrm{Na} \\
(\mathrm{ppm})\end{array}$ & $\begin{array}{c}\mathrm{Nb} \\
(\mathbf{p p m})\end{array}$ & $\begin{array}{c}\mathrm{Ni} \\
(\mathbf{p p m})\end{array}$ & $\begin{array}{c}P \\
(\mathrm{ppm})\end{array}$ & $\begin{array}{c}\mathbf{P b} \\
(\mathbf{p p m})\end{array}$ & $\begin{array}{c}\mathbf{R b} \\
\text { (ppm) }\end{array}$ \\
\hline 00U-39/A-0-10 & 8180 & 3.4 & 17400 & 8.6 & 8.7 & 4920 & 126 & 0.54 & 989 & 0.93 & 6.0 & 189 & 9.83 & 44.0 \\
\hline 00U-39A/10-30 & 5010 & 2.6 & 13400 & 6.3 & 7.5 & 3310 & 72.3 & 0.43 & 699 & $<0.1$ & 3.6 & 129 & 7.85 & 37.3 \\
\hline 00U-39A/30-50 & 5560 & 2.9 & 14200 & 6.8 & 9 & 3940 & 86.3 & 0.45 & 774 & $<0.1$ & 3.9 & 148 & 8.05 & 39.3 \\
\hline 00U-39/B-0-10 & 10500 & 3.7 & 19400 & 9.2 & 9.0 & 4790 & 141 & 0.87 & 1180 & 0.77 & 8.7 & 214 & 11.5 & 50.1 \\
\hline 00U-39/B-10-30 & 8140 & 3.6 & 18800 & 8.3 & 9.0 & 4530 & 125 & 0.57 & 1160 & 1.0 & 63 & 184 & 10.3 & 48.5 \\
\hline 00U-39/B-30-50 & 7600 & 4.0 & 20300 & 9.0 & 9.3 & 4900 & 132 & 0.49 & 1360 & 0.90 & 5.6 & 188 & 11.1 & 53.2 \\
\hline 00U-39/C-0-10 & 7730 & 3.8 & 19300 & 9.1 & 9.3 & 4980 & 136 & 0.49 & 1330 & 1.2 & 5.9 & 225 & 10.8 & 48.6 \\
\hline 00U-39C/10-30 & 4990 & 3 & 15100 & 7.4 & 8.9 & 3590 & 84.2 & 0.39 & 934 & 0.2 & 3.8 & 138 & 8.14 & 40.6 \\
\hline 00U-39C/30-50 & 4720 & 3.2 & 15100 & 7.7 & 9.5. & 3710 & 86 & 0.28 & 941 & 0.2 & 35 & 150 & 7.91 & 41 \\
\hline 00U-39/D-0-10 & 8110 & 3.6 & 19400 & 8.3 & 8.9 & 4700 & 132 & 0.55 & 1140 & 0.97 & 6.1 & 216 & 10.8 & 49.1 \\
\hline 00U-39D/10-30 & 5410 & 3.2 & 14600 & 7.4 & 9.8 & 3490 & 89.8 & 0.41 & 920 & 0.2 & 4.2 & 166 & 7.62 & 39.4 \\
\hline 00U-39D/30-50 & 4970 & 3.1 & 14600 & 7.4 & 9.5 & 3480 & 87.3 & 0.3 & 908 & 0.2 & 3.6 & 163 & 7.76 & 39.2 \\
\hline 00U-39-E-0-10 & 7420 & 3.8 & 19700 & 9.9 & 10.1 & 5400 & 156 & 0.47 & 1320 & 1.2 & 6.0 & 281 & 10.9 & 48.5 \\
\hline 00U-39E/10-30 & 6140 & 3.4 & 15100 & 9 & 10.6 & 3790 & 106 & 0.48 & 986 & 0.64 & 4.8 & 192 & 7.91 & 41.1 \\
\hline 00U-39E/30-50 & 5770 & 3.2 & 14700 & 8.4 & 9.8 & 3730 & 98.3 & 0.5 & 930 & 0.32 & 4.7 & 163 & 7.87 & 39.9 \\
\hline 00U-39/F-0-10 & 7790 & 3.8 & 19100 & 9.7 & $10 . c$ & 5320 & 149 & 0.49 & 1150 & 1.0 & & 283 & 10.2 & 46.8 \\
\hline 00U-39/F-10-30 & 7460 & 3.8 & 19000 & 9.8 & 10.3 & 5130 & 150 & 0.47 & 1180 & 0.88 & 5.8 & 257 & 9.75 & 46.4 \\
\hline 00U-39/F-30-50 & 9440 & 5.1 & 21600 & 12.4 & 12.7 & 6410 & 213 & 0.52 & 1800 & 1.7 & 7.4 & 308 & 10.6 & 53.5 \\
\hline 00U-39/G-0-10 & 8100 & 4.1 & 19200 & 9.7 & 11.2 & 5280 & 170 & 0.50 & 1270 & 1.3 & 6.6 & 328 & 9.89 & 46.3 \\
\hline 00U-39G/10-30 & 6120 & 3.9 & 15900 & 10 & 11.9 & 4150 & 130 & 0.35 & 1290 & 0.74 & 4.6 & 234 & 8.07 & 43 \\
\hline 00U-39G/30-50 & 5720 & 3.9 & 16000 & 9.8 & 12.1 & 4240 & 124 & 0.28 & 1270 & 0.74 & 4.2 & 215 & 8.12 & 42.6 \\
\hline 00U-39-H-0-10 & 7640 & 3.9 & 19400 & 9.6 & 10.8 & 5280 & 162 & 0.52 & 1380 & 1.3 & 6.0 & 355 & 10.1 & 44.7 \\
\hline 00U-39H/10-30 & 7090 & 4.1 & 16500 & 10.2 & 12.9 & 4250 & 146 & 0.45 & 1340 & 0.79 & 5.6 & 265 & 8.52 & 44 \\
\hline 00U-39H/30-50 & 6510 & 4.3 & 16900 & 10.4 & 13.4 & 4360 & 13 & 0.31 & 1470 & 0.6 & 4.8 & 218 & 8.5 & 44.8 \\
\hline 00U-39/I-1-10 & 8280 & 4.5 & 20900 & 11.9 & 12.4 & 5840 & 200 & 0.41 & 1760 & 1.5 & 6.7 & 394 & 11.2 & 49.0 \\
\hline 00U-39I/10-30 & 8520 & 5.1 & 17600 & 13.3 & 14.6 & 5140 & 189 & 0.32 & 1620 & 0.96 & 6.2 & 277 & 8.92 & 49.3 \\
\hline$/ 30-50$ & 8160 & 4.8 & 17300 & 11.9 & 13. & 4 & 160 & 0.26 & 1530 & 0.99 & 5.8 & 183 & 8.86 & 47.6 \\
\hline 00U-39/J-0-10 & 7550 & 3.8 & 17400 & 9.6 & 11. & 4100 & 141 & 0.50 & 1310 & 2.1 & 5.8 & 318 & 9.94 & 44.6 \\
\hline $00 \mathrm{U}-39 \mathrm{~J} / 10-30$ & 8450 & 4.6 & 16900 & 11.4 & 12.6 & 4540 & 168 & 0.44 & 1550 & 0.65 & 6 & 258 & 8.58 & 46.5 \\
\hline 00U-39J/30-50 & 7330 & 45 & 17100 & 11.7 & 12.1 & 4600 & 158 & 0.25 & 1580 & 0.48 & 4.9 & 212 & 8.63 & 46.4 \\
\hline $\mathrm{K}-0-10$ & 9160 & 48 & 19100 & 13. & 14. & 54 & 19 & 0.53 & 18 & 3.1 & 6.9 & 404 & 11.1 & 49.7 \\
\hline $00 \mathrm{U}-39 / \mathrm{K}-10-30$ & 9860 & 5.3 & 19800 & 14.7 & 16.5 & 5800 & 216 & 0.46 & 2130 & 3.5 & 7.7 & 313 & 10.7 & 52.1 \\
\hline 00U-39/K-30-50 & 9830 & 5.5 & 20300 & 14.7 & 16.9 & 6080 & 208 & 0.42 & 2140 & 3.5 & 7.4 & 305 & 11.0 & 53.4 \\
\hline 00U-39/L-0-10 & 8540 & 4.3 & 18800 & 12.6 & 14.0 & 4830 & 174 & 0.48 & 1770 & 2.8 & 6.5 & 394 & 10.6 & 47.6 \\
\hline $00 \mathrm{U}_{-}$ & 775 & 4.8 & 17300 & 11. & 13 & 508 & 184 & 023 & 180 & 0.69 & 5.6 & 260 & 8.75 & 47.1 \\
\hline 00U-39L/30-50 & 8490 & 5.2 & 17900 & 12.8 & 14.3 & 4850 & 167 & 0.28 & 1880 & 0.96 & 6 & 201 & 8.99 & 48.9 \\
\hline 00U-39/M-0-10 & 10400 & 5.6 & 20800 & 16.1 & 16.9 & 6330 & 229 & 0.42 & 2220 & 3.7 & 7.7 & 444 & 12.0 & 55.5 \\
\hline 00U-39M/10-30 & 7900 & 4.7 & 17300 & 13.2 & 12.7 & 4610 & 147 & 0.32 & 1760 & 0.62 & 5.4 & 196 & 9.09 & 48.1 \\
\hline M/30-50 & 6730 & 2.8 & 4330 & 10.2 & 6. & 4310 & 116 & 0.23 & 156 & $<0.1$ & 5.2 & 162 & 3.4 & 14.8 \\
\hline $00 \mathrm{U}-39 / \mathrm{N}-0-10$ & 8820 & 5.2 & 21100 & 12.7 & 15.5 & 8770 & 185 & 0.42 & 1690 & 3.0 & 6.9 & 388 & 10.8 & 51.7 \\
\hline $00 U-39 / \mathrm{N}-10-30$ & 9310 & 5.2 & 21200 & 14.4 & 15.6 & 8360 & 196 & 0.39 & 1960 & 3.1 & 7.2 & 328 & 10.1 & 50.2 \\
\hline $00 \mathrm{U}-39 / \mathrm{N}-30-50$ & 10600 & 5.8 & 21100 & 16.4 & 17.8 & 6960 & 243 & 0.39 & 2450 & 3.7 & 8.2 & 384 & 10.9 & 55.2 \\
\hline $\mathrm{O}-0-10$ & 9320 & 53 & 21200 & 13.4 & 15.1 & 10400 & 188 & 0.45 & 1520 & 2.7 & 7.3 & 329 & 10.3 & 51.0 \\
\hline 00U-39O/10-30 & 6310 & 3 & 4040 & 9.3 & 6.8 & 9900 & 162 & 0.2 & 80.6 & $<0.1$ & 5.3 & 205 & 3.2 & 12.8 \\
\hline 00U-39O/30-50 & 10900 & 6.1 & 19300 & 14.9 & 16.8 & 6740 & 225 & 0.49 & 2000 & 1.1 & 8.2 & 298 & 9.4 & 51.9 \\
\hline
\end{tabular}


Table 5c-2b. ICP-MS data for the 00U-39 transect-Continued.

\begin{tabular}{|c|c|c|c|c|c|c|c|c|c|c|}
\hline Sample \# & $\begin{array}{c}\text { Sb } \\
\text { (ppm) }\end{array}$ & $\begin{array}{c}\text { Sc } \\
(\mathbf{p p m})\end{array}$ & $\begin{array}{c}\mathrm{Sr} \\
\text { (ppm) }\end{array}$ & $\begin{array}{c}\text { Th } \\
\text { (ppm) }\end{array}$ & $\begin{array}{c}\mathrm{Ti} \\
\text { (ppm) }\end{array}$ & $\begin{array}{c}\mathrm{TI} \\
\text { (ppm) }\end{array}$ & $\underset{(\mathbf{p p m})}{\mathbf{U}}$ & $\underset{(\mathbf{p p m})}{V}$ & $\begin{array}{c}Y \\
\text { (ppm) }\end{array}$ & $\begin{array}{c}\mathrm{Zn} \\
\text { (ppm) }\end{array}$ \\
\hline 00U-39/A-0-10 & 0.1 & 1.7 & 57.5 & 2.48 & 675 & 0.23 & 0.52 & 11.0 & 6.7 & 13.6 \\
\hline 00U-39A/10-30 & 0.1 & 1.1 & 41 & 1.97 & 276 & 0.23 & 0.38 & 7.2 & 3.7 & 7.7 \\
\hline 00U-39A/30-50 & 0.1 & 1.3 & 43.4 & 2.06 & 332 & 0.25 & 0.44 & 8.9 & 4.1 & 8.8 \\
\hline 00U-39/B-0-10 & 0.2 & 1.7 & 62.5 & 2.49 & 719 & 0.26 & 0.54 & 10.4 & 7.1 & 11.5 \\
\hline 00U-39/B-10-30 & 0.1 & 1.6 & 62.7 & 2.28 & 648 & 0.24 & 0.51 & 10.2 & 6.5 & 9.6 \\
\hline 00U-39/B-30-50 & 0.1 & 1.8 & 75.3 & 2.53 & 720 & 0.27 & 0.55 & 11.9 & 7.0 & 10.8 \\
\hline 00U-39/C-0-10 & 0.1 & 1.8 & 64.5 & 2.43 & 775 & 0.25 & 0.56 & 11.1 & 7.0 & 13.0 \\
\hline 00U-39C/10-30 & 0.1 & 1.3 & 52.3 & 2.21 & 340 & 0.25 & 0.45 & 8.4 & 4.4 & 8.7 \\
\hline 00U-39C/30-50 & 0.1 & 1.4 & 54.6 & 2.36 & 431 & 0.25 & 0.51 & 9 & 5.3 & 9.5 \\
\hline 00U-39/D-0-10 & 0.1 & 1.6 & 59.6 & 2.26 & 657 & 0.24 & 0.51 & 10.1 & 6.8 & 10.1 \\
\hline 00U-39D/10-30 & 0.1 & 1.5 & 49.3 & 2.15 & 470 & 0.24 & 0.51 & 8.9 & 5.4 & 9.3 \\
\hline 00U-39D/30-50 & 0.1 & 1.4 & 49.4 & 2.23 & 362 & 0.24 & 0.47 & 9.2 & 4.6 & 10.7 \\
\hline 00U-39-E-0-10 & 0.1 & 2.0 & 64.0 & 2.49 & 810 & 0.24 & 0.55 & 10.3 & 7.8 & 13.9 \\
\hline 00U-39E/10-30 & 0.2 & 1.6 & 52.8 & 2.56 & 530 & 0.25 & 0.63 & 10.1 & 5.9 & 11.5 \\
\hline 00U-39E/30-50 & 0.2 & 1.5 & 50.5 & 2.54 & 555 & 0.25 & 0.58 & 9 & 5.7 & 10.6 \\
\hline 00U-39/F-0-10 & 0.1 & 1.8 & 60.7 & 2.57 & 780 & 0.24 & 0.56 & 10.4 & 7.6 & 14.9 \\
\hline 00U-39/F-10-30 & 0.1 & 1.9 & 62.0 & 2.50 & 806 & 0.23 & 0.56 & 10.2 & 7.9 & 12.9 \\
\hline 00U-39/F-30-50 & 0.20 & 2.7 & 80.4 & 3.08 & 1250 & 0.26 & 0.68 & 15.4 & 10.4 & 17.3 \\
\hline 00U-39/G-0-10 & 0.1 & 2.0 & 64.9 & 2.41 & 901 & 0.22 & 0.54 & 11.1 & 8.0 & 14.3 \\
\hline 00U-39G/10-30 & 0.2 & 2 & 58.9 & 2.86 & 658 & 0.26 & 1.17 & 12.5 & 6.8 & 13.1 \\
\hline 00U-39G/30-50 & 0.2 & 2.1 & 61.6 & 2.92 & 684 & 0.26 & 0.69 & 12 & 7.1 & 13.2 \\
\hline 00U-39-H-0-10 & 0.2 & 2.0 & 64.3 & 2.35 & 860 & 0.22 & 0.57 & 10.4 & 7.8 & 14.8 \\
\hline 00U-39H/10-30 & 0.2 & 2.2 & 60.4 & 3.03 & 769 & 0.28 & 0.71 & 13.1 & 7.8 & 15 \\
\hline 00U-39H/30-50 & 0.2 & 2.4 & 70.5 & 3.09 & 744 & 0.28 & 0.72 & 14.8 & 7.5 & 14.7 \\
\hline 00U-39/I-1-10 & 0.2 & 2.5 & 73.4 & 2.98 & 1100 & 0.24 & 0.67 & 14.0 & 9.5 & 19.9 \\
\hline 00U-39I/10-30 & 0.22 & 2.9 & 78.5 & 3.79 & 789 & 0.3 & 0.81 & 17.7 & 8.8 & 19.4 \\
\hline 00U-39I/30-50 & 0.2 & 2.9 & 86.4 & 3.41 & 743 & 0.3 & 0.72 & 17.8 & 8.2 & 18.3 \\
\hline 00U-39/J-0-10 & 0.21 & 1.9 & 60.5 & 2.40 & 929 & 0.25 & 0.64 & 11.2 & 8.3 & 14.4 \\
\hline 00U-39J/10-30 & 0.2 & 2.6 & 73.1 & 3.47 & 695 & 0.28 & 0.73 & 15.8 & 8 & 18 \\
\hline $00 \mathrm{U}-39 \mathrm{~J} / 30-50$ & 0.2 & 2.4 & 75.4 & 3.44 & 684 & 0.29 & 0.71 & 15.7 & 7.8 & 16.1 \\
\hline 00U-39/K-0-10 & 0.26 & 2.6 & 73.9 & 3.58 & 1340 & 0.29 & 0.90 & 16.4 & 11.3 & 17.8 \\
\hline $00 \mathrm{U}-39 / \mathrm{K}-10-30$ & 0.27 & 3.2 & 88.8 & 4.06 & 1500 & 0.29 & 0.96 & 19.0 & 12.2 & 20.1 \\
\hline 00U-39/K-30-50 & 0.26 & 3.1 & 101 & 4.09 & 1450 & 0.30 & 0.96 & 20.2 & 12.0 & 20.9 \\
\hline 00U-39/L-0-10 & 0.22 & 2.4 & 69.6 & 3.48 & 1220 & 0.26 & 0.80 & 14.0 & 10.0 & 16.0 \\
\hline 00U-39L/10-30 & 0.2 & 2.7 & 80.2 & 3.38 & 751 & 0.3 & 0.69 & 16 & 8 & 18.4 \\
\hline 00U-39L/30-50 & 0.2 & 2.9 & 92.9 & 3.84 & 817 & 0.31 & 0.8 & 18.8 & 8.4 & 17.9 \\
\hline 00U-39/M-0-10 & 0.28 & 3.4 & 90.5 & 4.38 & 1580 & 0.32 & 1.02 & 21.7 & 12.8 & 22.5 \\
\hline 00U-39M/10-30 & 0.2 & 2.6 & 82.6 & 3.97 & 733 & 0.3 & 0.79 & 17.4 & 8.1 & 16.2 \\
\hline 00U-39M/30-50 & 0.09 & 3.5 & 59.3 & 2.9 & 197 & 0.1 & 0.4 & 13.1 & 5.5 & 13.1 \\
\hline 00U-39/N-0-10 & 0.24 & 2.8 & 92.4 & 3.30 & 1250 & 0.28 & 0.80 & 15.8 & 10.7 & 17.7 \\
\hline $00 \mathrm{U}-39 / \mathrm{N}-10-30$ & 0.25 & 2.9 & 95.7 & 3.98 & 1380 & 0.28 & 0.92 & 17.5 & 11.8 & 17.4 \\
\hline 00U-39/N-30-50 & 0.29 & 3.6 & 107 & 4.33 & 1650 & 0.31 & 1.04 & 22.4 & 13.6 & 23.7 \\
\hline 00U-39/O-0-10 & 0.20 & 2.9 & 92.8 & 3.64 & 1280 & 0.28 & 0.82 & 16.2 & 10.8 & 18.1 \\
\hline 00U-39O/10-30 & 0.06 & 3.3 & 57.4 & 2.9 & 171 & $<0.08$ & 0.36 & 11.1 & 5.4 & 14.6 \\
\hline 00U-39O/30-50 & 0.22 & 3.6 & 94.2 & 4.38 & 1000 & 0.32 & 0.9 & 22.6 & 10.3 & 25.6 \\
\hline
\end{tabular}


Table 5c-3a. ICP-AES data for auger hole 00U-40.

\begin{tabular}{lcccccccccc}
\hline \multicolumn{1}{c}{ Sample \# } & Al (\%) & $\mathbf{C a}(\%)$ & $\mathbf{F e}(\%)$ & $\mathbf{K ~ ( \% )}$ & $\mathbf{M g}(\%)$ & $\mathbf{N a}(\%)$ & $\mathbf{P}(\%)$ & $\mathbf{T i}(\%)$ & $\mathbf{M n}(\mathbf{p p m})$ & $\mathbf{N d}(\mathbf{p p m})$ \\
\hline 00U-40/0-10 & 3.98 & 4.42 & 1.26 & 2.26 & 0.992 & 0.203 & 0.0426 & 0.149 & 253 & 17.2 \\
00U-40/10-30 & 4.28 & 5.10 & 1.29 & 2.42 & 0.998 & 0.217 & 0.0372 & 0.144 & 240 & 17.3 \\
00U-40/30-48 & 3.78 & 4.55 & 1.16 & 2.20 & 0.920 & 0.207 & 0.0339 & 0.130 & 220 & 17.0 \\
00U-40/48-64 & 3.27 & 4.06 & 0.990 & 1.92 & 0.879 & 0.188 & 0.0276 & 0.101 & 190 & 10.2 \\
00U-40/64-82 & 3.92 & 4.96 & 1.20 & 2.15 & 1.13 & 0.270 & 0.0318 & 0.133 & 228 & 13.3 \\
00U-40/82-102 & 4.31 & 5.62 & 1.40 & 2.22 & 1.27 & 0.284 & 0.0393 & 0.163 & 237 & 18.4 \\
00U-40/102-121 & 3.49 & 3.42 & 1.19 & 2.09 & 0.899 & 0.299 & 0.0391 & 0.144 & 247 & 17.2 \\
OOU40/121-142 & 2.26 & 2.01 & 0.865 & 1.59 & 0.439 & 0.184 & 0.0189 & 0.0830 & 166 & 8.81 \\
00U-40/142-154 & 2.14 & 2.03 & 0.748 & 1.54 & 0.398 & 0.164 & 0.0171 & 0.0691 & 130 & 7.50 \\
00U-40/154-168 & 1.96 & 2.62 & 0.677 & 1.50 & 0.391 & 0.147 & 0.0160 & 0.0567 & 108 & 7.14 \\
00U-40/168-183 & 1.62 & 2.49 & 0.545 & 1.38 & 0.312 & 0.112 & 0.0118 & 0.0408 & 77.6 & 6.36 \\
00U-40/183-193 & 1.81 & 3.54 & 0.633 & 1.39 & 0.413 & 0.133 & 0.0140 & 0.0478 & 91.5 & 5.93 \\
00U-40/192-204 & 1.60 & 12.3 & 0.548 & 1.16 & 0.670 & 0.171 & 0.0130 & 0.0389 & 77.2 & 5.06 \\
00U-40/204-217 & 1.62 & 9.36 & 0.500 & 1.24 & 0.863 & 0.200 & 0.00897 & 0.0330 & 96.1 & 5.62 \\
\hline
\end{tabular}

Table 5c-3b. ICP-MS data for auger hole 00U-40.

\begin{tabular}{lccccccccc}
\hline \multicolumn{1}{c}{ Sample\# } & $\begin{array}{c}\mathbf{A g} \\
(\mathbf{p p m})\end{array}$ & $\begin{array}{c}\mathbf{A l} \\
(\mathbf{p p m})\end{array}$ & $\begin{array}{c}\mathbf{A s} \\
(\mathbf{p p m})\end{array}$ & $\begin{array}{c}\mathbf{B a} \\
(\mathbf{p p m})\end{array}$ & $\begin{array}{c}\mathbf{B e} \\
(\mathbf{p p m})\end{array}$ & $\begin{array}{c}\mathbf{B i} \\
(\mathbf{p p m})\end{array}$ & $\begin{array}{c}\mathbf{C a} \\
(\mathbf{p p m})\end{array}$ & $\begin{array}{c}\mathbf{C d} \\
(\mathbf{p p m})\end{array}$ & $\begin{array}{c}\mathbf{C e} \\
(\mathbf{p p m})\end{array}$ \\
\hline 00U-40/0-10 & $<2$ & 45800 & 3.5 & 407 & 1.6 & $<0.06$ & 49000 & 0.10 & 34.4 \\
00U-40/10-30 & $<2$ & 49500 & 4.1 & 441 & 1.7 & $<0.06$ & 56700 & 0.10 & 35.1 \\
00U-40/30-48 & $<2$ & 43000 & 3.3 & 420 & 1.3 & $<0.06$ & 49400 & 0.07 & 32.4 \\
00U-40/48-64 & $<2$ & 35500 & 3.7 & 379 & 1.3 & $<0.06$ & 45900 & 0.07 & 27.2 \\
00U-40/64-82 & $<2$ & 41100 & 4.1 & 386 & 1.6 & $<0.06$ & 54400 & 0.08 & 33.2 \\
00U-40/82-102 & $<2$ & 50100 & 4.5 & 434 & 1.7 & $<0.06$ & 62300 & 0.08 & 36.8 \\
00U-40/102-121 & $<2$ & 40100 & 3.4 & 437 & 1.4 & $<0.06$ & 37500 & 0.08 & 34.5 \\
00U-40/121-142 & $<2$ & 24800 & 2.4 & 353 & 0.8 & $<0.06$ & 21900 & 0.06 & 21.6 \\
00U-40/142-154 & $<2$ & 23400 & 2.3 & 338 & 0.85 & $<0.06$ & 22100 & 0.06 & 18.4 \\
00U-40/154-168 & $<2$ & 21600 & 2.2 & 336 & 0.66 & $<0.06$ & 28600 & 0.05 & 17.4 \\
00U-40/168-183 & $<2$ & 17500 & 1.6 & 320 & 0.45 & $<0.06$ & 27100 & 0.04 & 14.3 \\
00U-40/183-193 & $<2$ & 19900 & 2.9 & 323 & 0.64 & $<0.06$ & 39100 & 0.04 & 16.6 \\
00U-40/192-204 & $<2$ & 17000 & 3.3 & 345 & 0.49 & $<0.06$ & 141000 & 0.06 & 15.4 \\
00U-40/204-217 & $<2$ & 16900 & 2.3 & 361 & 0.56 & $<0.06$ & 104000 & 0.03 & 13.2
\end{tabular}


Table 5c-3b. ICP-MS data for auger hole 00U-40—Continued.

\begin{tabular}{|c|c|c|c|c|c|c|c|c|c|c|}
\hline Sample \# & $\begin{array}{c}\text { Co } \\
\text { (ppm) }\end{array}$ & $\underset{(\mathbf{p p m})}{\mathrm{Cr}}$ & $\underset{\text { (ppm) }}{\text { Cs }}$ & $\underset{(\mathbf{p p m})}{\mathrm{Cu}}$ & $\begin{array}{c}\mathrm{Fe} \\
(\mathrm{ppm})\end{array}$ & $\underset{\text { (ppm) }}{\mathbf{G a}}$ & $\underset{(\mathbf{p p m})}{\mathrm{K}}$ & $\begin{array}{c}\text { La } \\
\text { (ppm) }\end{array}$ & $\underset{(\mathbf{p p m})}{\mathbf{L i}}$ & $\underset{\text { (ppm) }}{\mathbf{M g}}$ \\
\hline 00U-40/0-10 & 4.1 & 61.4 & 2.9 & 13.0 & 13900 & 7.9 & 25400 & 19.1 & 23.1 & 11600 \\
\hline 00U-40/10-30 & 4.5 & 62.7 & 3.0 & 13.6 & 14800 & 8.6 & 27300 & 19.7 & 25.1 & 12100 \\
\hline 00U-40/30-48 & 3.8 & 49.9 & 2.6 & 16.6 & 12200 & 7.5 & 24400 & 18.0 & 21.7 & 10600 \\
\hline 00U-40/48-64 & 3.4 & 18.5 & 2.2 & 10.4 & 10400 & 6.8 & 21400 & 14.3 & 18.8 & 10000 \\
\hline 00U-40/64-82 & 4.2 & 30.8 & 2.6 & 12.8 & 12700 & 8 & 23100 & 17.3 & 22.3 & 12700 \\
\hline 00U-40/82-102 & 4.8 & 61.5 & 3.2 & 12.7 & 15700 & 8.9 & 25100 & 20.2 & 26.8 & 14800 \\
\hline 00U-40/102-121 & 3.9 & 51.5 & 2.5 & 9.3 & 12500 & 7.1 & 23900 & 19.2 & 21.4 & 10500 \\
\hline 00U-40/121-142 & 2.5 & 25.7 & 1.7 & 8.5 & 8760 & 4.8 & 17400 & 11.4 & 13.4 & 5000 \\
\hline 00U-40/142-154 & 2.2 & 16.5 & 1.6 & 6.9 & 7440 & 4.4 & 16700 & 9.8 & 11.8 & 4510 \\
\hline 00U-40/154-168 & 2 & 20.8 & 1.4 & 6.6 & 7090 & 4.1 & 16300 & 9.3 & 11 & 4490 \\
\hline 00U-40/168-183 & 1.5 & 23.5 & 1.1 & 5.1 & 5430 & 3.2 & 14900 & 7.7 & 8.8 & 3580 \\
\hline 00U-40/183-193 & 1.9 & 19.8 & 1.3 & 7.6 & 6670 & 3.7 & 15300 & 8 & 10.3 & 4820 \\
\hline 00U-40/192-204 & 2 & 10.4 & 1 & 5.6 & 5440 & 3.3 & 12000 & 8.4 & 8.8 & 7360 \\
\hline 00U-40/204-217 & 1.9 & 15.4 & 0.81 & 5.5 & 4910 & 3.1 & 12900 & 7.2 & 7.6 & 9390 \\
\hline
\end{tabular}

Table 5c-3b. ICP-MS data for auger hole 00U-40—Continued.

\begin{tabular}{|c|c|c|c|c|c|c|c|c|c|c|}
\hline Sample \# & $\begin{array}{c}\text { Mn } \\
\text { (ppm) }\end{array}$ & $\begin{array}{c}\text { Mo } \\
\text { (ppm) }\end{array}$ & $\begin{array}{c}\mathrm{Na} \\
\text { (ppm) }\end{array}$ & $\begin{array}{c}\mathbf{N b} \\
(\mathrm{ppm})\end{array}$ & $\begin{array}{c}\mathrm{Ni} \\
\text { (ppm) }\end{array}$ & $\begin{array}{c}P \\
\text { (ppm) }\end{array}$ & $\begin{array}{c}\mathrm{Pb} \\
(\mathrm{ppm})\end{array}$ & $\begin{array}{c}\mathbf{R b} \\
\text { (ppm) }\end{array}$ & $\begin{array}{c}\text { Sb } \\
\text { (ppm) }\end{array}$ & $\begin{array}{c}\text { Sc } \\
\text { (ppm) }\end{array}$ \\
\hline 00U-40/0-10 & 283 & 0.51 & 2370 & 5.1 & 11.5 & 490 & 11.6 & 60.5 & 0.32 & 4.5 \\
\hline 00U-40/10-30 & 280 & 0.50 & 2560 & 5.2 & 12.6 & 444 & 11.9 & 64.0 & 0.33 & 4.8 \\
\hline 00U-40/30-48 & 239 & 0.40 & 2400 & 4.3 & 10.3 & 396 & 11.0 & 58.2 & 0.30 & 4.3 \\
\hline 00U-40/48-64 & 204 & 0.33 & 2060 & 1.4 & 8.1 & 299 & 10.1 & 53.4 & 0.2 & 3.8 \\
\hline 00U-40/64-82 & 244 & 0.36 & 2890 & 1.1 & 10 & 347 & 9.81 & 57.6 & 0.24 & 4.7 \\
\hline 00U-40/82-102 & 266 & 0.54 & 3310 & 5.8 & 13.2 & 454 & 11.5 & 61.4 & 0.34 & 5.1 \\
\hline 00U-40/102-121 & 267 & 0.49 & 3540 & 3.6 & 9.9 & 459 & 11.6 & 58.9 & 0.31 & 4.2 \\
\hline 00U-40/121-142 & 171 & 0.44 & 2030 & 0.93 & 6.6 & 202 & 9.18 & 48.5 & 0.2 & 2.8 \\
\hline 00U-40/142-154 & 126 & 0.24 & 1850 & 0.65 & 5.4 & 177 & 8.71 & 46.8 & 0.1 & 2.5 \\
\hline 00U-40/154-168 & 108 & 0.3 & 1640 & 0.42 & 5.1 & 174 & 8.51 & 45.3 & 0.2 & 2.2 \\
\hline 00U-40/168-183 & 74.4 & 0.24 & 1260 & 0.1 & 3.5 & 133 & 8.16 & 41.2 & 0.1 & 1.6 \\
\hline 00U-40/183-193 & 102 & 0.31 & 1490 & 0.28 & 5 & 151 & 8.96 & 41.8 & 0.1 & 2 \\
\hline 00U-40/192-204 & 79.6 & 0.2 & 1730 & 0.34 & 1.9 & 151 & 6.1 & 31.1 & 0.1 & 2 \\
\hline 00U-40/204-217 & 93 & 0.2 & 2040 & $<0.1$ & 2.3 & 93.5 & 5.99 & 31.4 & 0.1 & 1.8 \\
\hline
\end{tabular}


Table 5c-3b. ICP-MS data for auger hole 00U-40-Continued.

\begin{tabular}{|c|c|c|c|c|c|c|c|c|}
\hline Sample \# & $\begin{array}{c}\mathrm{Sr} \\
(\mathbf{p p m})\end{array}$ & $\begin{array}{c}\text { Th } \\
(\mathbf{p p m})\end{array}$ & $\begin{array}{c}\mathrm{Ti} \\
(\mathbf{p p m})\end{array}$ & $\begin{array}{c}\mathrm{TI} \\
(\mathrm{ppm})\end{array}$ & $\underset{(\mathbf{p p m})}{\mathbf{U}}$ & $\begin{array}{c}\mathbf{V} \\
(\mathbf{p p m})\end{array}$ & $\begin{array}{c}Y \\
\text { (ppm) }\end{array}$ & $\underset{(p p m)}{Z n}$ \\
\hline 00U-40/0-10 & 128 & 4.88 & 2080 & 0.34 & 1.11 & 26.3 & 15.1 & 27.5 \\
\hline 00U-40/10-30 & 158 & 5.06 & 2220 & 0.36 & 1.20 & 29.1 & 15.9 & 30.3 \\
\hline 00U-40/30-48 & 145 & 4.70 & 1880 & 0.31 & 1.08 & 25.5 & 14.1 & 26.3 \\
\hline 00U-40/48-64 & 138 & 4.12 & 1020 & 0.32 & 0.86 & 22.7 & 9.7 & 24.5 \\
\hline 00U-40/64-82 & 178 & 5.02 & 1290 & 0.35 & 1.1 & 29.6 & 11.9 & 29.3 \\
\hline 00U-40/82-102 & 184 & 5.41 & 2310 & 0.36 & 1.33 & 34.4 & 16.0 & 33.0 \\
\hline 00U-40/102-121 & 122 & 5.34 & 2030 & 0.33 & 1.34 & 28.1 & 15.2 & 26.2 \\
\hline 00U-40/121-142 & 75.7 & 3.88 & 778 & 0.3 & 0.84 & 17.8 & 8 & 18 \\
\hline 00U-40/142-154 & 75.4 & 2.9 & 647 & 0.3 & 0.65 & 15.6 & 6.4 & 15.7 \\
\hline 00U-40/154-168 & 80.7 & 2.99 & 564 & 0.28 & 0.62 & 14.8 & 6.1 & 13.9 \\
\hline 00U-40/168-183 & 67.7 & 2.31 & 385 & 0.26 & 0.54 & 11.2 & 4.7 & 12 \\
\hline 00U-40/183-193 & 94 & 2.35 & 506 & 0.26 & 0.59 & 15 & 5.4 & 15.8 \\
\hline 00U-40/192-204 & 218 & 2.48 & 443 & 0.19 & 0.62 & 15.3 & 5.5 & 10.2 \\
\hline 00U-40/204-217 & 239 & 1.99 & 356 & 0.19 & 0.52 & 12.4 & 4.8 & 8.1 \\
\hline
\end{tabular}


Table 5d-1a. ICP-AES data for the 00U-35 transect.

\begin{tabular}{lcccccccccc}
\hline \multicolumn{1}{c}{ Sample \# } & Al (\%) & $\mathbf{C a}(\%)$ & $\mathbf{F e}(\%)$ & $\mathbf{K}(\%)$ & $\mathbf{M g}(\%)$ & $\mathbf{N a}(\%)$ & $\mathbf{P}(\%)$ & $\mathbf{T}(\%)$ & $\mathbf{M n}(\mathbf{p p m})$ & $\mathbf{N d}(\mathbf{p p m})$ \\
\hline 00U-35A/0-10 & 1.66 & 2.72 & 0.689 & 0.982 & 0.277 & 0.120 & 0.0238 & 0.0600 & 131 & 7.64 \\
00U-35A/10-30 & 1.55 & 2.95 & 0.715 & 0.931 & 0.254 & 0.116 & 0.0165 & 0.0558 & 117 & 7.05 \\
00U-35A/30-50 & 1.55 & 3.12 & 0.693 & 0.934 & 0.261 & 0.117 & 0.0173 & 0.0552 & 116 & 7.81 \\
00U-35B/0-10 & 1.32 & 1.40 & 0.631 & 0.871 & 0.188 & 0.100 & 0.0200 & 0.0472 & 107 & 5.24 \\
00U-35B/10-30 & 1.64 & 2.16 & 0.656 & 0.973 & 0.252 & 0.119 & 0.0164 & 0.0602 & 122 & 7.72 \\
00U-35B/30-50 & 1.64 & 2.52 & 0.660 & 0.982 & 0.236 & 0.116 & 0.0140 & 0.0590 & 106 & 7.14 \\
00U-35C/0-10 & 1.54 & 1.89 & 0.757 & 0.973 & 0.253 & 0.115 & 0.0264 & 0.0590 & 138 & 7.92 \\
00U-35C/10-30 & 1.72 & 2.24 & 0.766 & 1.04 & 0.279 & 0.134 & 0.0185 & 0.0644 & 138 & 8.02 \\
00U-35C/30-50 & 1.72 & 2.52 & 0.832 & 1.05 & 0.273 & 0.136 & 0.0174 & 0.0656 & 126 & 8.08 \\
00U-35D/0-10 & 1.65 & 1.75 & 0.831 & 1.05 & 0.269 & 0.129 & 0.0328 & 0.0659 & 152 & 7.68 \\
00U-35D/10-30 & 2.01 & 2.22 & 0.859 & 1.22 & 0.330 & 0.162 & 0.0222 & 0.0812 & 172 & 9.79 \\
00U-35D/30-50 & 1.93 & 2.43 & 0.803 & 1.14 & 0.318 & 0.149 & 0.0203 & 0.0754 & 158 & 9.88 \\
00U-35E/0-10 & 2.43 & 2.14 & 0.957 & 1.43 & 0.436 & 0.191 & 0.0443 & 0.0942 & 230 & 11.9 \\
00U-35E/10-30 & 2.43 & 2.75 & 0.969 & 1.42 & 0.419 & 0.195 & 0.0269 & 0.0897 & 216 & 11.4 \\
00U-35E/30-50 & 2.07 & 3.18 & 0.764 & 1.22 & 0.324 & 0.161 & 0.0207 & 0.0710 & 141 & 9.57 \\
00U-35F/0-10 & 3.52 & 2.88 & 1.32 & 1.86 & 0.672 & 0.278 & 0.0575 & 0.128 & 370 & 16.2 \\
00U-35F/10-30 & 3.48 & 3.41 & 1.32 & 1.85 & 0.612 & 0.275 & 0.0322 & 0.127 & 316 & 15.5 \\
00U-35F/30-50 & 3.25 & 3.14 & 1.28 & 1.68 & 0.563 & 0.247 & 0.0280 & 0.116 & 271 & 14.0 \\
00U-35G/0-10 & 3.03 & 2.30 & 1.13 & 1.72 & 0.567 & 0.234 & 0.0548 & 0.115 & 317 & 13.3 \\
00U-35G/10-30 & 3.12 & 2.85 & 1.21 & 1.71 & 0.550 & 0.246 & 0.0311 & 0.123 & 291 & 13.9 \\
00U-35G/30-50 & 2.95 & 2.94 & 1.09 & 1.62 & 0.478 & 0.221 & 0.0254 & 0.106 & 220 & 12.5 \\
00U-35H/0-10 & 4.20 & 2.89 & 1.48 & 2.04 & 0.846 & 0.318 & 0.0927 & 0.155 & 490 & 18.1 \\
00U-35H/10-30 & 4.74 & 3.94 & 1.62 & 2.15 & 0.845 & 0.326 & 0.0543 & 0.168 & 446 & 19.0 \\
00U-35H/30-50 & 4.52 & 3.17 & 1.55 & 1.98 & 0.732 & 0.291 & 0.0436 & 0.160 & 358 & 17.9 \\
\hline
\end{tabular}


Table 5d-1b. ICP-MS data for the 00U-35 transect.

\begin{tabular}{|c|c|c|c|c|c|c|c|c|}
\hline Sample \# & $\begin{array}{c}\mathrm{Ag} \\
(\mathrm{ppm})\end{array}$ & $\begin{array}{c}\text { Al } \\
(\mathbf{p p m})\end{array}$ & $\begin{array}{c}\text { As } \\
\text { (ppm) }\end{array}$ & $\begin{array}{c}\text { Ba } \\
\text { (ppm) }\end{array}$ & $\begin{array}{c}\mathrm{Be} \\
(\mathrm{ppm})\end{array}$ & $\begin{array}{c}\mathrm{Bi} \\
(\mathbf{p p m})\end{array}$ & $\begin{array}{c}\mathrm{Ca} \\
(\mathbf{p p m})\end{array}$ & $\underset{(p p m)}{\text { Cd }}$ \\
\hline 00U-35A/0-10 & $<2$ & 17200 & 3.8 & 252 & 0.54 & 0.04 & 29900 & 0.08 \\
\hline 00U-35A/10-30 & $<2$ & 16100 & 4.1 & 247 & 0.54 & 0.03 & 32100 & 0.06 \\
\hline 00U-35A/30-50 & $<2$ & 15700 & 3.8 & 251 & 0.52 & 0.02 & 33900 & 0.06 \\
\hline 00U-35B/0-10 & $<2$ & 12600 & 3 & 217 & 0.42 & 0.03 & 14800 & 0.08 \\
\hline 00U-35B/10-30 & $<2$ & 16100 & 3.8 & 235 & 0.53 & 0.02 & 23100 & 0.06 \\
\hline 00U-35B/30-50 & $<2$ & 15800 & 3.6 & 240 & 0.59 & 0.02 & 26700 & 0.03 \\
\hline 00U-35C/0-10 & $<2$ & 15200 & 3.6 & 226 & 0.5 & 0.03 & 20200 & 0.08 \\
\hline 00U-35C/10-30 & $<2$ & 17000 & 4.2 & 251 & 0.54 & 0.02 & 24200 & 0.06 \\
\hline 00U-35C/30-50 & $<2$ & 16900 & 4.2 & 257 & 0.63 & 0.02 & 26800 & 0.06 \\
\hline 00U-35D/0-10 & $<2$ & 16100 & 3.4 & 249 & 0.53 & 0.04 & 18500 & 0.1 \\
\hline 00U-35D/10-30 & $<2$ & 19800 & 3.9 & 284 & 0.7 & 0.03 & 23000 & 0.08 \\
\hline 00U-35D/30-50 & $<2$ & 19300 & 3.8 & 280 & 0.69 & 0.04 & 26300 & 0.08 \\
\hline 00U-35E/0-10 & $<2$ & 23800 & 3.5 & 326 & 0.84 & 0.06 & 22400 & 0.14 \\
\hline 00U-35E/10-30 & $<2$ & 24000 & 3.9 & 332 & 0.87 & 0.05 & 29100 & 0.1 \\
\hline 00U-35E/30-50 & $<2$ & 20100 & 4.4 & 302 & 0.76 & 0.03 & 32600 & 0.08 \\
\hline 00U-35F/0-10 & $<2$ & 35100 & 4.4 & 399 & 1.2 & 0.17 & 29000 & 0.2 \\
\hline 00U-35F/10-30 & $<2$ & 34500 & 4.9 & 402 & 1.2 & 0.12 & 33600 & 0.14 \\
\hline 00U-35F/30-50 & $<2$ & 32700 & 5 & 386 & 1.1 & 0.11 & 31800 & 0.11 \\
\hline 00U-35G/0-10 & $<2$ & 29700 & 3.4 & 366 & 0.92 & 0.1 & 22400 & 0.18 \\
\hline 00U-35G/10-30 & $<2$ & 30800 & 3.9 & 379 & 1 & 0.1 & 28100 & 0.12 \\
\hline 00U-35G/30-50 & $<2$ & 29900 & 3.8 & 365 & 0.98 & 0.09 & 29700 & 0.09 \\
\hline 00U-35H/0-10 & $<2$ & 42900 & 4.5 & 438 & 1.4 & 0.15 & 29300 & 0.31 \\
\hline 00U-35H/10-30 & $<2$ & 47000 & 5.3 & 444 & 1.4 & 0.13 & 39300 & 0.22 \\
\hline 00U-35H/30-50 & $<2$ & 46000 & 5.2 & 428 & 1.4 & 0.13 & 31800 & 0.17 \\
\hline
\end{tabular}


Table 5d-1b. ICP-MS data for the 00U-35 transect-Continued.

\begin{tabular}{|c|c|c|c|c|c|c|c|c|}
\hline Sample \# & $\underset{(\mathbf{p p m})}{\mathrm{Ce}}$ & $\begin{array}{c}\text { Co } \\
\text { (ppm) }\end{array}$ & $\underset{(\mathbf{p p m})}{\mathrm{Cr}}$ & $\underset{(\mathbf{p p m})}{\text { Cs }}$ & $\underset{(\mathrm{ppm})}{\mathrm{Cu}}$ & $\underset{(\mathrm{ppm})}{\mathrm{Fe}}$ & $\underset{(\mathbf{p p m})}{\mathrm{Ga}}$ & $\underset{(\mathbf{p p m})}{\mathrm{K}}$ \\
\hline 00U-35A/0-10 & 20.4 & 2.5 & 50.7 & 1.2 & 10.6 & 7400 & 3.8 & 10900 \\
\hline 00U-35A/10-30 & 18.7 & 2.4 & 62 & 1.1 & 11.4 & 7600 & 3.5 & 10200 \\
\hline 00U-35A/30-50 & 18.1 & 2.6 & 59.9 & 1.1 & 10.9 & 7500 & 3.6 & 10500 \\
\hline 00U-35B/0-10 & 15 & 1.8 & 61.1 & 0.92 & 9.9 & 6700 & 2.9 & 9610 \\
\hline 00U-35B/10-30 & 20 & 2.3 & 44.3 & 1.2 & 9.1 & 7100 & 3.7 & 10700 \\
\hline $00 \mathrm{U}-35 \mathrm{~B} / 30-50$ & 17.4 & 2.3 & 44.6 & 1.2 & 9 & 7000 & 3.6 & 10600 \\
\hline 00U-35C/0-10 & 19.2 & 2.4 & 66.2 & 1.1 & 11 & 8100 & 3.4 & 10600 \\
\hline 00U-35C/10-30 & 20.3 & 2.6 & 58.6 & 1.3 & 10.7 & 8400 & 3.9 & 11600 \\
\hline 00U-35C/30-50 & 19.3 & 2.6 & 70.8 & 1.3 & 12.1 & 8900 & 3.8 & 11400 \\
\hline 00U-35D/0-10 & 20.8 & 2.4 & 74.9 & 1.2 & 13 & 9000 & 3.7 & 11600 \\
\hline 00U-35D/10-30 & 24.3 & 2.8 & 60.1 & 1.5 & 12.6 & 9400 & 4.5 & 13100 \\
\hline 00U-35D/30-50 & 23.3 & 2.8 & 51.8 & 1.5 & 11.2 & 8700 & 4.3 & 12700 \\
\hline 00U-35E/0-10 & 29.9 & 3.3 & 47.7 & 1.9 & 12.2 & 10000 & 5.4 & 15700 \\
\hline 00U-35E/10-30 & 28.5 & 3.4 & 50.2 & 1.9 & 12.5 & 11000 & 5.5 & 15600 \\
\hline 00U-35E/30-50 & 25.3 & 2.9 & 38.4 & 1.6 & 10 & 8300 & 4.6 & 13100 \\
\hline 00U-35F/0-10 & 35.4 & 4.6 & 46.6 & 2.9 & 15.8 & 14000 & 7.6 & 19000 \\
\hline 00U-35F/10-30 & 36.4 & 4.6 & 49 & 2.9 & 16 & 14000 & 7.5 & 18700 \\
\hline 00U-35F/30-50 & 34.3 & 4.5 & 53.1 & 2.7 & 14.8 & 13000 & 7.2 & 17300 \\
\hline 00U-35G/0-10 & 33.8 & 3.7 & 41.8 & 2.5 & 12.8 & 12000 & 6.4 & 17200 \\
\hline 00U-35G/10-30 & 32.9 & 4 & 48.4 & 2.6 & 14.7 & 12000 & 6.8 & 17000 \\
\hline 00U-35G/30-50 & 30.6 & 3.7 & 40.4 & 2.4 & 12.1 & 11000 & 6.3 & 16600 \\
\hline 00U-35H/0-10 & 43 & 5.3 & 42.1 & 3.5 & 16.9 & 16000 & 9.1 & 21000 \\
\hline 00U-35H/10-30 & 44.3 & 5.9 & 44.7 & 3.9 & 17.7 & 17000 & 10 & 22100 \\
\hline 00U-35H/30-50 & 46.3 & 5.6 & 41.5 & 3.7 & 16.1 & 16000 & 9.8 & 20400 \\
\hline
\end{tabular}


Table 5d-1b. ICP-MS data for the 00U-35 transect-Continued.

\begin{tabular}{|c|c|c|c|c|c|c|c|c|}
\hline Sample \# & $\begin{array}{c}\text { La } \\
\text { (ppm) }\end{array}$ & $\begin{array}{c}\mathrm{Li} \\
\text { (ppm) }\end{array}$ & $\begin{array}{c}\text { Mg } \\
(\mathrm{ppm})\end{array}$ & $\begin{array}{c}\mathrm{Mn} \\
(\mathrm{ppm})\end{array}$ & $\begin{array}{c}\text { Mo } \\
\text { (ppm) }\end{array}$ & $\begin{array}{c}\mathrm{Na} \\
(\mathrm{ppm})\end{array}$ & $\begin{array}{c}\mathbf{N b} \\
(\mathrm{ppm})\end{array}$ & $\begin{array}{c}\mathrm{Ni} \\
(\mathrm{ppm})\end{array}$ \\
\hline 00U-35A/0-10 & 11.2 & 11.6 & 2900 & 140 & n.d. & 1270 & 2.5 & 8.1 \\
\hline 00U-35A/30-50 & 9.9 & 11.2 & 2720 & 122 & n.d. & 1260 & 2.1 & 8.3 \\
\hline $00 \mathrm{U}-35 \mathrm{~B} / 0-10$ & 8.5 & 9.2 & 2040 & 112 & n.d. & 1130 & $<2$ & 7.1 \\
\hline 00U-35B/30-50 & 9.4 & 11.1 & 2520 & 111 & n.d. & 1260 & 2.4 & 7.5 \\
\hline $00 \mathrm{U}-35 \mathrm{C} / 0-10$ & 10.6 & 10.6 & 2710 & 144 & n.d. & 1260 & 2 & 8.4 \\
\hline 00U-35C/10-30 & 11 & 11.7 & 2970 & 147 & n.d. & 1490 & 2.3 & 8.6 \\
\hline 00U-35C/30-50 & 10.6 & 11.8 & 2890 & 134 & n.d. & 1480 & 2.3 & 9.2 \\
\hline 00U-35D/0-10 & 12.5 & 11.2 & 2870 & 163 & n.d. & 1430 & 2.5 & 8.8 \\
\hline 00U-35E/0-10 & 16.1 & 15.3 & 5090 & 249 & n.d. & 2070 & 2.9 & 9.6 \\
\hline 00U-35E/10-30 & 15.4 & 15.5 & 4930 & 234 & n.d. & 2180 & 3 & 10.5 \\
\hline 00U-35E/30-50 & 13.7 & 13.4 & 3560 & 148 & n.d. & 1840 & 2.8 & 8.8 \\
\hline 00U-35F/0-10 & 19.4 & 20.9 & 7270 & 388 & n.d. & 2880 & 4.6 & 12.5 \\
\hline 00U-35F/10-30 & 20 & 21.3 & 6760 & 326 & n.d. & 2890 & 5.4 & 12.6 \\
\hline 00U-35F/30-50 & 18.9 & 20.1 & 6190 & 280 & n.d. & 2680 & 4.5 & 12.2 \\
\hline 00U-35G/0-10 & 16.9 & 18.2 & 6330 & 319 & n.d. & 2510 & 4 & 10.1 \\
\hline 00U-35G/10-30 & 18.2 & 19.2 & 6000 & 298 & n.d. & 2650 & 4.5 & 11.2 \\
\hline 00U-35G/30-50 & 16.9 & 18.1 & 5300 & 229 & n.d. & 2340 & 4.8 & 9.8 \\
\hline
\end{tabular}


Table 5d-1b. ICP-MS data for the 00U-35 transect-Continued.

\begin{tabular}{|c|c|c|c|c|c|c|c|c|c|c|c|c|}
\hline Sample \# & $\begin{array}{c}\mathrm{Pb} \\
(\mathbf{p p m})\end{array}$ & $\begin{array}{c}\mathbf{R b} \\
(\mathbf{p p m})\end{array}$ & $\begin{array}{c}\text { Sb } \\
(\mathbf{p p m})\end{array}$ & $\begin{array}{c}\text { Sc } \\
(\mathbf{p p m})\end{array}$ & $\begin{array}{c}\mathrm{Sr} \\
(\mathrm{ppm})\end{array}$ & $\begin{array}{c}\text { Th } \\
\text { (ppm) }\end{array}$ & $\begin{array}{c}\mathrm{Ti} \\
(\mathrm{ppm})\end{array}$ & $\begin{array}{c}\mathrm{TI} \\
(\mathbf{p p m})\end{array}$ & $\underset{\text { (ppm) }}{\mathbf{U}}$ & $\begin{array}{c}\mathbf{V} \\
\text { (ppm) }\end{array}$ & $\begin{array}{c}Y \\
\text { (ppm) }\end{array}$ & $\begin{array}{c}\mathrm{Zn} \\
\text { (ppm) }\end{array}$ \\
\hline 00U-35A/0-10 & 8.5 & 32.7 & 0.23 & 2.2 & 75.2 & 2.8 & 860 & 0.2 & 0.76 & 12.6 & 8.3 & 17.7 \\
\hline 00U-35A/10-30 & 7.7 & 30.8 & 0.22 & 2 & 81.8 & 2.8 & 710 & 0.2 & 0.74 & 10.5 & 6.2 & 15.5 \\
\hline 00U-35A/30-50 & 7.8 & 30.8 & 0.22 & 2 & 85.8 & 2.6 & 650 & 0.2 & 0.67 & 11.5 & 6.1 & 15 \\
\hline 00U-35B/0-10 & 7.8 & 28.5 & 0.2 & 1.5 & 43.3 & 2.2 & 490 & 0.2 & 0.53 & 8.3 & 4.5 & 13.6 \\
\hline 00U-35B/10-30 & 7.8 & 32.3 & 0.22 & 2 & 57.2 & 2.9 & 650 & 0.2 & 0.68 & 12.1 & 6.2 & 16.5 \\
\hline 00U-35B/30-50 & 7.8 & 31.9 & 0.22 & 2 & 60.2 & 2.6 & 680 & 0.2 & 0.61 & 12 & 5.7 & 15.9 \\
\hline 00U-35C/0-10 & 8.2 & 32 & 0.24 & 1.8 & 51.9 & 2.8 & 610 & 0.2 & 0.66 & 10.6 & 6.1 & 16.4 \\
\hline 00U-35C/10-30 & 7.9 & 34.8 & 0.23 & 2.1 & 61.7 & 3.2 & 700 & 0.2 & 0.77 & 13.2 & 8.2 & 17.6 \\
\hline 00U-35C/30-50 & 7.8 & 34.3 & 0.24 & 2.1 & 69.8 & 3 & 800 & 0.2 & 0.71 & 12.6 & 6.6 & 16.8 \\
\hline 00U-35D/0-10 & 8.6 & 35.3 & 0.24 & 2 & 53.6 & 3.1 & 840 & 0.2 & 0.83 & 11.6 & 7.2 & 17.8 \\
\hline 00U-35D/10-30 & 8.5 & 40.9 & 0.26 & 2.6 & 66.2 & 3.8 & 1100 & 0.2 & 0.88 & 15.8 & 8.4 & 20.4 \\
\hline 00U-35D/30-50 & 8.4 & 39.4 & 0.22 & 2.5 & 70.1 & 3.5 & 1000 & 0.2 & 0.84 & 15.7 & 8.3 & 20.2 \\
\hline 00U-35E/0-10 & 10.5 & 48.7 & 0.29 & 3.2 & 80.3 & 4.7 & 980 & 0.3 & 0.94 & 19.5 & 9.8 & 26.4 \\
\hline 00U-35E/10-30 & 9.8 & 48.8 & 0.3 & 3.1 & 87.1 & 4.5 & 1000 & 0.3 & 0.9 & 19.6 & 9.5 & 24.5 \\
\hline 00U-35E/30-50 & 9 & 41.8 & 0.24 & 2.5 & 89.9 & 3.7 & 750 & 0.3 & 0.88 & 16.8 & 7.8 & 20.2 \\
\hline 00U-35F/0-10 & 13.1 & 58.9 & 0.31 & 4.7 & 98.6 & 5.8 & 1400 & 0.4 & 1.3 & 28.5 & 12.9 & 37.9 \\
\hline 00U-35F/10-30 & 12.6 & 57.8 & 0.37 & 4.5 & 107 & 5.9 & 1500 & 0.4 & 1.3 & 28.2 & 12.7 & 35.7 \\
\hline 00U-35F/30-50 & 12.1 & 55.9 & 0.35 & 4.2 & 112 & 5.4 & 1500 & 0.4 & 1.2 & 26.9 & 11.9 & 34.5 \\
\hline 00U-35G/0-10 & 11.6 & 53.8 & 0.31 & 3.8 & 84.3 & 4.9 & 1200 & 0.4 & 1.1 & 22.7 & 10.8 & 31.1 \\
\hline 00U-35G/10-30 & 11.1 & 54.6 & 0.32 & 4 & 96.8 & 5.2 & 1400 & 0.4 & 1.2 & 24.3 & 11.7 & 31.2 \\
\hline 00U-35G/30-50 & 10.8 & 52.4 & 0.3 & 3.8 & 98.1 & 5.1 & 1400 & 0.4 & 1.2 & 23.8 & 11.4 & 29.1 \\
\hline 00U-35H/0-10 & 14.4 & 67.9 & 0.44 & 5.5 & 116 & 7.3 & 2000 & 0.4 & 1.6 & 34 & 15.6 & 46.5 \\
\hline 00U-35H/10-30 & 14 & 71.4 & 0.45 & 6.1 & 138 & 7.3 & 1900 & 0.5 & 1.6 & 38.8 & 15.5 & 49.7 \\
\hline 00U-35H/30-50 & 13.7 & 67.9 & 0.4 & 5.8 & 136 & 7 & 2000 & 0.4 & 1.5 & 37.6 & 14.9 & 46.5 \\
\hline
\end{tabular}


Table 5d-2a. ICP-AES data for auger hole 00U-36.

\begin{tabular}{lcccccccccc}
\hline \multicolumn{1}{c}{ Sample \# } & Al (\%) & $\mathbf{C a}(\%)$ & $\mathbf{F e}(\%)$ & $\mathbf{K}(\%)$ & $\mathbf{M g}(\%)$ & $\mathbf{N a}(\%)$ & $\mathbf{P}(\%)$ & $\mathbf{T i}(\%)$ & $\mathbf{M n}(\mathbf{p p m})$ & $\mathbf{N d}(\mathbf{p p m})$ \\
\hline 00U-36/0-10 & 1.47 & 1.97 & 0.554 & 0.936 & 0.241 & 0.112 & 0.0245 & 0.0494 & 123 & 7.62 \\
00U-36/10-30 & 1.74 & 2.64 & 0.607 & 1.05 & 0.289 & 0.136 & 0.0190 & 0.0570 & 134 & 7.58 \\
00U-36/30-46 & 1.70 & 2.80 & 0.618 & 1.08 & 0.289 & 0.140 & 0.0184 & 0.0621 & 131 & 8.62 \\
00U-36/46-68 & 1.72 & 2.68 & 0.661 & 1.11 & 0.270 & 0.139 & 0.0173 & 0.0715 & 124 & 7.55 \\
00U-36/68-86 & 1.83 & 2.87 & 0.671 & 1.14 & 0.308 & 0.159 & 0.0188 & 0.0735 & 119 & 8.34 \\
00U-36/86-97 & 2.74 & 4.84 & 0.964 & 1.50 & 0.517 & 0.304 & 0.0281 & 0.115 & 167 & 11.2 \\
00U-36/97-108 & 2.06 & 5.45 & 0.778 & 1.15 & 0.483 & 0.243 & 0.0222 & 0.0889 & 133 & 9.33 \\
00U-36/108-120 & 2.40 & 4.16 & 0.865 & 1.34 & 0.453 & 0.272 & 0.0242 & 0.0986 & 160 & 10.8 \\
00U-36/120-130 & 2.30 & 4.20 & 0.821 & 1.28 & 0.466 & 0.282 & 0.0232 & 0.0968 & 146 & 10.4 \\
\hline
\end{tabular}

Table 5d-2b. ICP-MS data for auger hole 00U-36-Continued.

\begin{tabular}{llccccccc}
\hline \multicolumn{1}{c}{ Sample \# } & $\begin{array}{c}\mathbf{A g} \\
(\mathbf{p p m})\end{array}$ & $\begin{array}{c}\mathbf{A l} \\
(\mathbf{p p m})\end{array}$ & $\begin{array}{c}\mathbf{A s} \\
\mathbf{( p p m )}\end{array}$ & $\begin{array}{c}\mathbf{B a} \\
(\mathbf{p p m})\end{array}$ & $\begin{array}{c}\mathbf{B e} \\
(\mathbf{p p m})\end{array}$ & $\begin{array}{c}\mathbf{B i} \\
(\mathbf{p p m})\end{array}$ & $\begin{array}{c}\mathbf{C a} \\
(\mathbf{p p m})\end{array}$ & $\begin{array}{c}\mathbf{C d} \\
(\mathbf{p p m})\end{array}$ \\
\hline 00U-36/0-10 & $<2$ & 14400 & 3.5 & 234 & 0.53 & 0.06 & 19200 & 0.08 \\
00U-36/10-30 & $<2$ & 16500 & 4.3 & 262 & 0.58 & 0.05 & 24900 & 0.06 \\
00U-36/30-46 & $<2$ & 16700 & 4.7 & 261 & 0.58 & 0.05 & 26500 & 0.07 \\
00U-36/46-68 & $<2$ & 18800 & 4.4 & 246 & 0.56 & $<0.06$ & 26800 & 0.06 \\
00U-36/68-86 & $<2$ & 19900 & 4.4 & 271 & 0.61 & $<0.06$ & 28600 & 0.05 \\
00U-36/86-97 & $<2$ & 28500 & 5 & 392 & 0.82 & $<0.06$ & 49000 & 0.07 \\
00U-36/97-108 & $<2$ & 21500 & 4.3 & 404 & 0.63 & $<0.06$ & 53900 & 0.07 \\
00U-36/108-120 & $<2$ & 23900 & 3.4 & 339 & 0.66 & $<0.06$ & 41000 & 0.07 \\
00U-36/120-130 & $<2$ & 24100 & 3.8 & 382 & 0.72 & $<0.06$ & 43300 & 0.06
\end{tabular}

Table 5d-2b. ICP-MS data for auger hole 00U-36-Continued.

\begin{tabular}{|c|c|c|c|c|c|c|c|c|}
\hline Sample \# & $\begin{array}{c}\mathrm{Ce} \\
(\mathbf{p p m})\end{array}$ & $\begin{array}{c}\text { Co } \\
(\mathrm{ppm})\end{array}$ & $\begin{array}{c}\mathrm{Cr} \\
(\mathbf{p p m})\end{array}$ & $\begin{array}{c}\text { Cs } \\
(\mathrm{ppm})\end{array}$ & $\underset{(\mathrm{ppm})}{\mathrm{Cu}}$ & $\begin{array}{c}\mathrm{Fe} \\
(\mathrm{ppm})\end{array}$ & $\underset{(\mathrm{ppm})}{\mathbf{G a}}$ & $\begin{array}{c}\mathbf{K} \\
(\mathbf{p p m})\end{array}$ \\
\hline 00U-36/0-10 & 19.1 & 1.9 & 30.3 & 1.1 & 7.1 & 5600 & 3.2 & 9360 \\
\hline 00U-36/10-30 & 18.8 & 2.4 & 25.1 & 1.3 & 7.5 & 6000 & 3.6 & 10200 \\
\hline 00U-36/30-46 & 20.4 & 2.4 & 26 & 1.3 & 8.3 & 6100 & 3.7 & 10400 \\
\hline 00U-36/46-68 & 16.8 & 2.4 & 35.8 & 1.2 & 8.7 & 6560 & 3.7 & 11300 \\
\hline 00U-36/68-86 & 18.3 & 2.4 & 32.1 & 1.2 & 8.5 & 6720 & 3.8 & 11600 \\
\hline 00U-36/86-97 & 27.1 & 3.5 & 31.7 & 1.8 & 13.2 & 9670 & 5.4 & 15400 \\
\hline 00U-36/97-108 & 21.1 & 2.7 & 32.1 & 1.4 & 9.8 & 7680 & 4.1 & 11400 \\
\hline 00U-36/108-120 & 25.8 & 3 & 31.8 & 1.6 & 10.6 & 8500 & 4.7 & 13200 \\
\hline 00U-36/120-130 & 22.9 & 2.9 & 28.7 & 1.5 & 9.5 & 8240 & 4.7 & 13400 \\
\hline
\end{tabular}


Table 5d-2b. ICP-MS data for auger hole 00U-36—Continued.

\begin{tabular}{|c|c|c|c|c|c|c|c|c|}
\hline Sample \# & $\begin{array}{c}\text { La } \\
\text { (ppm) }\end{array}$ & $\begin{array}{c}\mathbf{L i} \\
(\mathbf{p p m})\end{array}$ & $\begin{array}{c}\mathrm{Mg} \\
\text { (ppm) }\end{array}$ & $\underset{(\mathrm{ppm})}{\mathrm{Mn}}$ & $\begin{array}{c}\text { Mo } \\
\text { (ppm) }\end{array}$ & $\begin{array}{c}\mathrm{Na} \\
(\mathrm{ppm})\end{array}$ & $\begin{array}{c}\mathbf{N b} \\
(\mathbf{p p m})\end{array}$ & $\begin{array}{c}\mathrm{Ni} \\
\text { (ppm) }\end{array}$ \\
\hline 00U-36/0-10 & 10.8 & 10 & 2480 & 123 & $\mathrm{nr}$ & 1240 & 2.2 & 6.1 \\
\hline 00U-36/10-30 & 10.4 & 11.5 & 2970 & 128 & $\mathrm{nr}$ & 1530 & 2.7 & 7.1 \\
\hline 00U-36/30-46 & 11.1 & 11.8 & 2980 & 128 & $\mathrm{nr}$ & 1540 & 2.5 & 7.1 \\
\hline 00U-36/46-68 & 9.3 & 10.8 & 2950 & 124 & 0.45 & 1400 & 0.66 & 7.3 \\
\hline 00U-36/68-86 & 10 & 11.8 & 3380 & 116 & 0.4 & 1600 & 0.99 & 6.6 \\
\hline 00U-36/86-97 & 14.5 & 15.8 & 5600 & 162 & 0.47 & 3230 & 2 & 8.8 \\
\hline 00U-36/97-108 & 11.5 & 13.8 & 5290 & 129 & 0.45 & 2540 & 1.3 & 6.7 \\
\hline 00U-36/108-120 & 13.9 & 14.2 & 4980 & 152 & 0.44 & 2860 & 1.5 & 7 \\
\hline 00U-36/120-130 & 12.3 & 14 & 5190 & 143 & 0.37 & 3010 & 0.76 & 6.9 \\
\hline
\end{tabular}

Table 5d-2b. ICP-MS data for auger hole 00U-36-Continued.

\begin{tabular}{lccccccccccccc}
\hline \multicolumn{1}{c}{ Sample \# } & $\begin{array}{c}\mathbf{P} \\
(\mathbf{p p m})\end{array}$ & $\begin{array}{c}\mathbf{P b} \\
(\mathbf{p p m})\end{array}$ & $\begin{array}{c}\mathbf{R b} \\
(\mathbf{p p m})\end{array}$ & $\begin{array}{c}\mathbf{S b} \\
(\mathbf{p p m})\end{array}$ & $\begin{array}{c}\mathbf{S c} \\
\mathbf{( \mathbf { p p m } )}\end{array}$ & $\begin{array}{c}\mathbf{S r} \\
(\mathbf{p p m})\end{array}$ & $\begin{array}{c}\mathbf{T h} \\
(\mathbf{p p m})\end{array}$ & $\begin{array}{c}\mathbf{T i} \\
(\mathbf{p p m})\end{array}$ & $\begin{array}{c}\mathbf{T I} \\
(\mathbf{p p m})\end{array}$ & $\begin{array}{c}\mathbf{U} \\
(\mathbf{p p m})\end{array}$ & $\begin{array}{c}\mathbf{V} \\
(\mathbf{p p m})\end{array}$ & $\begin{array}{c}\mathbf{Y} \\
(\mathbf{p p m})\end{array}$ & $\begin{array}{c}\mathbf{Z n} \\
(\mathbf{p p m})\end{array}$ \\
\hline 00U-36/0-10 & 220 & 8.2 & 29.4 & 0.2 & 1.6 & 50.3 & 2.7 & 600 & 0.2 & 0.68 & 9.8 & 5.9 & 15.7 \\
00U-36/10-30 & 160 & 8.8 & 32.6 & 0.2 & 2 & 70 & 2.7 & 780 & 0.2 & 0.71 & 12.2 & 6.4 & 16.1 \\
00U-36/30-46 & 160 & 8.6 & 32.5 & 0.2 & 2 & 72 & 2.9 & 800 & 0.2 & 0.74 & 12.4 & 6.5 & 16.6 \\
00U-36/46-68 & 178 & 7.72 & 31.6 & 0.24 & 2.1 & 66.7 & 2.2 & 603 & 0.19 & 0.63 & 13 & 5.8 & 15.4 \\
00U-36/68-86 & 202 & 7.88 & 33.4 & 0.21 & 2.1 & 79.9 & 2.62 & 651 & 0.22 & 0.7 & 14.2 & 6 & 17 \\
00U-36/86-97 & 307 & 9.99 & 46.2 & 0.28 & 3.3 & 121 & 4.28 & 1030 & 0.3 & 1.02 & 25.6 & 9.2 & 25.3 \\
00U-36/97-108 & 237 & 8.23 & 34 & 0.22 & 2.6 & 134 & 3.2 & 767 & 0.22 & 0.96 & 18.2 & 7.2 & 18.5 \\
00U-36/108-120 & 256 & 8.54 & 40.1 & 0.25 & 2.8 & 99.5 & 3.96 & 889 & 0.25 & 1 & 23.3 & 8.1 & 21.3 \\
00U-36/120-130 & 244 & 8.54 & 39.1 & 0.22 & 2.6 & 116 & 3.46 & 844 & 0.25 & 0.91 & 21.2 & 7.7 & 21 \\
\hline
\end{tabular}


Table 5d-3a. ICP-AES data for auger hole 00U-37.

\begin{tabular}{lcccccccccc}
\hline \multicolumn{1}{c}{ Sample \# } & $\mathbf{A l}(\%)$ & $\mathbf{C a}(\%)$ & $\mathbf{F e}(\%)$ & $\mathbf{K}(\%)$ & $\mathbf{M g}(\%)$ & $\mathbf{N a}(\%)$ & $\mathbf{P}(\%)$ & $\mathbf{T i}(\%)$ & $\mathbf{M n}(\mathbf{p p m})$ & $\mathbf{N d}(\mathbf{p p m})$ \\
\hline 00U-37/0-10 & 4.66 & 3.65 & 1.61 & 2.18 & 0.861 & 0.324 & 0.0744 & 0.163 & 486 & 18.5 \\
00U-37/10-30 & 4.36 & 3.81 & 1.52 & 2.00 & 0.742 & 0.317 & 0.0470 & 0.158 & 387 & 19.3 \\
00U-37/30-43 & 4.29 & 3.41 & 1.48 & 1.93 & 0.705 & 0.301 & 0.0438 & 0.157 & 361 & 16.8 \\
00U-37/43-55 & 4.04 & 2.41 & 1.39 & 1.90 & 0.540 & 0.266 & 0.0239 & 0.154 & 243 & 15.0 \\
00U-37/55-68 & 3.32 & 4.69 & 1.14 & 1.62 & 0.536 & 0.241 & 0.0316 & 0.121 & 164 & 12.8 \\
00U-37/68-80 & 2.57 & 2.79 & 1.01 & 1.42 & 0.417 & 0.214 & 0.0229 & 0.0922 & 147 & 10.1 \\
00U-37/80-94 & 2.39 & 2.38 & 0.960 & 1.36 & 0.412 & 0.220 & 0.0205 & 0.0957 & 149 & 10.2 \\
00U-37/94-110 & 3.16 & 11.7 & 0.949 & 1.56 & 0.634 & 0.223 & 0.0292 & 0.102 & 153 & 11.7 \\
00U-37/110-120 & 3.03 & 7.28 & 0.938 & 1.55 & 0.667 & 0.236 & 0.0263 & 0.100 & 149 & 11.8 \\
00U-37/120-131 & 2.04 & 4.62 & 0.613 & 1.25 & 0.395 & 0.131 & 0.0214 & 0.0678 & 85.5 & 7.84 \\
00U-37/131-139 & 2.13 & 4.56 & 0.655 & 1.33 & 0.400 & 0.143 & 0.0213 & 0.0729 & 104 & 8.11 \\
00U-37/139-148 & 2.67 & 6.57 & 0.717 & 1.47 & 0.521 & 0.169 & 0.0221 & 0.0804 & 112 & 8.67 \\
00U-37/148-155 & 2.75 & 4.30 & 0.880 & 1.48 & 0.490 & 0.183 & 0.0338 & 0.0967 & 192 & 10.3 \\
\hline
\end{tabular}

Table 5d-3b. ICP-MS data for auger hole 00U-37.

\begin{tabular}{|c|c|c|c|c|c|c|c|c|}
\hline Sample \# & $\begin{array}{c}\mathrm{Ag} \\
(\mathrm{ppm})\end{array}$ & $\begin{array}{c}\mathrm{Al} \\
\text { (ppm) }\end{array}$ & $\begin{array}{c}\text { As } \\
\text { (ppm) }\end{array}$ & $\begin{array}{c}\mathrm{Ba} \\
\text { (ppm) }\end{array}$ & $\begin{array}{c}\text { Be } \\
(\mathrm{ppm})\end{array}$ & $\begin{array}{c}\mathbf{B i} \\
\text { (ppm) }\end{array}$ & $\begin{array}{c}\mathrm{Ca} \\
\text { (ppm) }\end{array}$ & $\begin{array}{c}\text { Cd } \\
\text { (ppm) }\end{array}$ \\
\hline 00U-37/0-10 & $<2$ & 44800 & 4.8 & 437 & 1.5 & 0.13 & 35000 & 0.27 \\
\hline 00U-37/10-30 & $<2$ & 43200 & 4.8 & 435 & 1.4 & 0.12 & 37400 & 0.19 \\
\hline 00U-37/30-43 & $<2$ & 42800 & 4.7 & 419 & 1.4 & 0.12 & 33000 & 0.17 \\
\hline 00U-37/43-55 & $<2$ & 40700 & 4.3 & 376 & 1.3 & $<0.06$ & 23700 & 0.09 \\
\hline 00U-37/55-68 & $<2$ & 32900 & 3.5 & 334 & 1 & $<0.06$ & 45600 & 0.11 \\
\hline 00U-37/68-80 & $<2$ & 26300 & 3.1 & 305 & 0.8 & $<0.06$ & 27600 & 0.06 \\
\hline 00U-37/80-94 & $<2$ & 24900 & 3.2 & 302 & 0.7 & $<0.06$ & 23600 & 0.06 \\
\hline 00U-37/94-110 & $<2$ & 31600 & 5.5 & 418 & 1 & $<0.06$ & 117000 & 0.13 \\
\hline 00U-37/110-120 & $<2$ & 29800 & 4.6 & 417 & 0.9 & $<0.06$ & 71700 & 0.08 \\
\hline 00U-37/120-131 & $<2$ & 21200 & 3.5 & 319 & 0.59 & $<0.06$ & 46700 & 0.06 \\
\hline 00U-37/131-139 & $<2$ & 22100 & 3.1 & 308 & 0.76 & $<0.06$ & 44800 & 0.06 \\
\hline 00U-37/139-148 & $<2$ & 26300 & 3.7 & 430 & 0.81 & $<0.06$ & 64500 & 0.08 \\
\hline 00U-37/148-155 & $<2$ & 28000 & 3.4 & 328 & 0.77 & $<0.06$ & 42900 & 0.11 \\
\hline
\end{tabular}


Table 5d-3b. ICP-MS data for auger hole 00U-37-Continued.

\begin{tabular}{|c|c|c|c|c|c|c|c|c|}
\hline Sample \# & $\begin{array}{c}\text { Ce } \\
\text { (ppm) }\end{array}$ & $\begin{array}{c}\text { Co } \\
\text { (ppm) }\end{array}$ & $\begin{array}{c}\mathrm{Cr} \\
(\mathrm{ppm})\end{array}$ & $\begin{array}{c}\text { Cs } \\
(\mathbf{p p m})\end{array}$ & $\begin{array}{c}\text { Cu } \\
(\mathbf{p p m})\end{array}$ & $\begin{array}{c}\mathrm{Fe} \\
(\mathrm{ppm})\end{array}$ & $\begin{array}{c}\text { Ga } \\
(\mathbf{p p m})\end{array}$ & $\underset{\text { (ppm) }}{\mathbf{K}}$ \\
\hline 00U-37/0-10 & 44.5 & 5.7 & 47.4 & 3.8 & 18.1 & 17000 & 9.8 & 21800 \\
\hline 00U-37/10-30 & 42.2 & 5.5 & 52 & 3.5 & 17.3 & 16000 & 9.4 & 20500 \\
\hline 00U-37/30-43 & 41.5 & 5.2 & 47.6 & 3.4 & 15.7 & 15000 & 9.1 & 19300 \\
\hline 00U-37/43-55 & 34.8 & 4.8 & 36.7 & 2.9 & 13.7 & 13900 & 8 & 19100 \\
\hline 00U-37/55-68 & 30 & 4 & 31.8 & 2.4 & 12.3 & 11400 & 6.4 & 15900 \\
\hline 00U-37/68-80 & 22.5 & 3.2 & 50.6 & 1.8 & 11 & 10200 & 4.9 & 14500 \\
\hline 00U-37/80-94 & 22.6 & 2.9 & 42.1 & 1.7 & 10.1 & 9460 & 4.8 & 14100 \\
\hline 00U-37/94-110 & 26.2 & 3.2 & 27.4 & 2.2 & 8.4 & 9390 & 5.7 & 15400 \\
\hline 00U-37/110-120 & 24.1 & 3.1 & 28.2 & 2 & 8.4 & 9060 & 5.5 & 15400 \\
\hline 00U-37/120-131 & 17.2 & 1.8 & 24.2 & 1.3 & 6.2 & 6000 & 3.9 & 12900 \\
\hline 00U-37/131-139 & 20.6 & 1.9 & 23.2 & 1.4 & 6.6 & 6300 & 4.1 & 13200 \\
\hline 00U-37/139-148 & 19.2 & 2.4 & 22.8 & 1.6 & 6.4 & 7060 & 4.6 & 14500 \\
\hline 00U-37/148-155 & 22.8 & 2.8 & 29.1 & 1.9 & 10.8 & 8690 & 5.2 & 15200 \\
\hline
\end{tabular}

Table 5d-3b. ICP-MS data for auger hole 00U-37—Continued.

\begin{tabular}{lcccccccc}
\hline \multicolumn{1}{c}{ Sample \# } & $\begin{array}{c}\text { La } \\
\mathbf{( p p m )}\end{array}$ & $\begin{array}{c}\mathbf{L i} \\
\mathbf{( p p m )}\end{array}$ & $\begin{array}{c}\mathbf{M g} \\
\mathbf{( p p m )}\end{array}$ & $\begin{array}{c}\mathbf{M n} \\
\mathbf{( \mathbf { p m } )}\end{array}$ & $\begin{array}{c}\mathbf{M o} \\
\mathbf{( p p m )}\end{array}$ & $\begin{array}{c}\mathbf{N a} \\
\mathbf{( p p m )}\end{array}$ & $\begin{array}{c}\mathbf{N b} \\
\mathbf{( p p m )}\end{array}$ & $\begin{array}{c}\mathbf{N i} \\
\mathbf{( p p m )}\end{array}$ \\
\hline 00U-37/0-10 & 24.4 & 27.4 & 9470 & 491 & $\mathrm{nr}$ & 3440 & 9.2 & 14.8 \\
00U-37/10-30 & 23.2 & 26 & 8260 & 401 & $\mathrm{nr}$ & 3470 & 6.9 & 14.5 \\
00U-37/30-43 & 22.5 & 25.3 & 7820 & 354 & $\mathrm{nr}$ & 3240 & 6.6 & 13.5 \\
00U-37/43-55 & 18.5 & 21.6 & 5900 & 235 & 0.49 & 2760 & 2.6 & 11.9 \\
00U-37/55-68 & 16.4 & 18.3 & 5810 & 161 & 0.47 & 2420 & 1.5 & 9.3 \\
00U-37/68-80 & 12.4 & 14.3 & 4660 & 145 & 0.62 & 2260 & 1 & 8.7 \\
00U-37/80-94 & 12.4 & 13.6 & 4520 & 144 & 0.54 & 2300 & 1.4 & 8 \\
00U-37/94-110 & 14.4 & 17.4 & 6560 & 150 & 0.38 & 2210 & 1.5 & 6.3 \\
00U-37/110-120 & 13.1 & 18.1 & 7240 & 139 & 0.34 & 2450 & 1.6 & 6.8 \\
00U-37/120-131 & 9.4 & 12.6 & 4320 & 83.2 & 0.2 & 1380 & 0.92 & 4.9 \\
00U-37/131-139 & 11.3 & 13 & 4430 & 98.8 & 0.2 & 1480 & 0.98 & 5.3 \\
00U-37/139-148 & 11.1 & 15.4 & 5630 & 107 & 0.21 & 1730 & 1.4 & 5.7 \\
00U-37/148-155 & 12.6 & 15.8 & 5510 & 187 & 0.32 & 1910 & 1.2 & 7.3
\end{tabular}


Table 5d-3b. ICP-MS data for auger hole 00U-37-Continued.

\begin{tabular}{|c|c|c|c|c|c|c|c|c|c|c|c|c|c|}
\hline Sample \# & $\underset{\text { (ppm) }}{P}$ & $\begin{array}{c}\mathrm{Pb} \\
(\mathrm{ppm})\end{array}$ & $\begin{array}{c}\mathbf{R b} \\
\text { (ppm) }\end{array}$ & $\begin{array}{c}\text { Sb } \\
(\mathbf{p p m})\end{array}$ & $\begin{array}{c}\text { Sc } \\
\text { (ppm) }\end{array}$ & $\begin{array}{c}\mathrm{Sr} \\
(\mathbf{p p m})\end{array}$ & $\begin{array}{c}\text { Th } \\
\text { (ppm) }\end{array}$ & $\underset{(\mathrm{ppm})}{\mathrm{Ti}}$ & $\begin{array}{c}\mathrm{TI} \\
\text { (ppm) }\end{array}$ & $\underset{(\mathbf{p p m})}{\mathbf{U}}$ & $\underset{\text { (ppm) }}{V}$ & $\begin{array}{c}Y \\
\text { (ppm) }\end{array}$ & $\begin{array}{c}\mathrm{Zn} \\
\text { (ppm) }\end{array}$ \\
\hline 00U-37/0-10 & 690 & 14.1 & 69.7 & 0.45 & 5.9 & 126 & 7.4 & 1800 & 0.4 & 1.6 & 36.9 & 16.1 & 47.9 \\
\hline 00U-37/10-30 & 450 & 13.2 & 66.4 & 0.42 & 5.5 & 132 & 7 & 1900 & 0.4 & 1.5 & 34.7 & 15 & 43.6 \\
\hline 00U-37/30-43 & 410 & 13.1 & 65 & 0.41 & 5.1 & 131 & 6.7 & 1800 & 0.4 & 1.4 & 33.5 & 14.2 & 42.3 \\
\hline 00U-37/43-55 & 255 & 11.1 & 60 & 0.33 & 5 & 104 & 5.4 & 1370 & 0.37 & 1.1 & 32.1 & 11.8 & 36.7 \\
\hline 00U-37/55-68 & 336 & 9.39 & 49.2 & 0.29 & 4 & 106 & 4.51 & 1060 & 0.32 & 1.04 & 27.1 & 10.2 & 29.3 \\
\hline 00U-37/68-80 & 252 & 8.46 & 43.1 & 0.26 & 3 & 78.6 & 3.63 & 781 & 0.27 & 0.82 & 20.4 & 7.8 & 24.3 \\
\hline 00U-37/80-94 & 219 & 8.2 & 42 & 0.24 & 2.8 & 74.6 & 3.48 & 929 & 0.27 & 0.81 & 20 & 7.5 & 22.1 \\
\hline 00U-37/94-110 & 308 & 8.14 & 44 & 0.28 & 3.7 & 218 & 4.06 & 955 & 0.28 & 0.94 & 24.5 & 9.5 & 21.9 \\
\hline 00U-37/110-120 & 280 & 8.41 & 43.5 & 0.27 & 3.6 & 200 & 3.7 & 895 & 0.28 & 0.91 & 23.2 & 8.4 & 22.2 \\
\hline 00U-37/120-131 & 226 & 6.92 & 35.5 & 0.2 & 2 & 130 & 2.64 & 589 & 0.22 & 0.67 & 15.4 & 6 & 13.6 \\
\hline 00U-37/131-139 & 219 & 7.02 & 36.4 & 0.2 & 2.1 & 123 & 3.33 & 612 & 0.23 & 0.71 & 16.2 & 6.5 & 14.8 \\
\hline 00U-37/139-148 & 229 & 7.41 & 40 & 0.21 & 2.9 & 177 & 2.85 & 724 & 0.24 & 0.72 & 18.5 & 6.8 & 17.2 \\
\hline 00U-37/148-155 & 372 & 8.29 & 43.7 & 0.22 & 2.9 & 117 & 3.47 & 844 & 0.27 & 0.8 & 21.2 & 8.2 & 24.4 \\
\hline
\end{tabular}


Table 5e-1a. ICP-AES data for the GP transect.

\begin{tabular}{|c|c|c|c|c|c|c|c|c|c|c|}
\hline Sample \# & Al (\%) & $\mathrm{Ca}(\%)$ & $\mathrm{Fe}(\%)$ & $K(\%)$ & $\mathbf{M g}(\%)$ & $\mathrm{Na}(\%)$ & $\mathbf{P}(\%)$ & Ti (\%) & Mn (ppm) & Nd (ppm) \\
\hline GP-A/0-10 & 2.02 & 0.480 & 0.494 & 1.73 & 0.205 & 0.107 & 0.0205 & 0.0598 & 163 & 6.11 \\
\hline GP-A/10-30 & 1.98 & 0.528 & 0.509 & 1.70 & 0.196 & 0.0986 & 0.0170 & 0.0608 & 167 & 6.18 \\
\hline GP-A/30-50 & 1.97 & 0.722 & 0.492 & 1.70 & 0.185 & 0.0891 & 0.0143 & 0.0567 & 156 & 5.94 \\
\hline GP-B/0-10 & 2.36 & 0.361 & 0.642 & 1.80 & 0.268 & 0.127 & 0.0220 & 0.0745 & 189 & 8.23 \\
\hline GP-B/10-30 & 2.19 & 0.820 & 0.602 & 1.75 & 0.242 & 0.123 & 0.0167 & 0.0686 & 156 & 7.63 \\
\hline GP-B/30-50 & 1.87 & 1.64 & 0.394 & 1.64 & 0.175 & 0.0771 & 0.0131 & 0.0469 & 108 & 5.53 \\
\hline GP-C/0-10 & 2.16 & 0.162 & 0.601 & 1.72 & 0.226 & 0.129 & 0.0213 & 0.0710 & 180 & 7.47 \\
\hline GP-C/10-30 & 2.34 & 0.417 & 0.653 & 1.80 & 0.259 & 0.121 & 0.0188 & 0.0753 & 190 & 8.05 \\
\hline GP-C/30-50 & 2.18 & 0.941 & 0.584 & 1.78 & 0.240 & 0.124 & 0.0173 & 0.0716 & 157 & 7.34 \\
\hline GP-D/0-10 & 2.31 & 0.172 & 0.645 & 1.80 & 0.250 & 0.139 & 0.0230 & 0.0825 & 207 & 8.16 \\
\hline GP-D/10-30 & 2.26 & 0.391 & 0.634 & 1.80 & 0.252 & 0.129 & 0.0176 & 0.0710 & 190 & 7.81 \\
\hline GP-D/30-50 & 2.16 & 0.788 & 0.574 & 1.71 & 0.246 & 0.121 & 0.0170 & 0.0683 & 173 & 7.25 \\
\hline GP-E/0-10 & 2.21 & 0.184 & 0.622 & 1.83 & 0.229 & 0.148 & 0.0258 & 0.0748 & 185 & 7.42 \\
\hline GP-E/10-30 & 2.39 & 0.389 & 0.736 & 1.82 & 0.282 & 0.140 & 0.0218 & 0.0824 & 231 & 8.30 \\
\hline GP-E/30-50 & 2.22 & 0.740 & 0.553 & 1.77 & 0.253 & 0.126 & 0.0182 & 0.0683 & 186 & 7.36 \\
\hline GP-F/0-10 & 2.38 & 0.312 & 0.710 & 1.77 & 0.280 & 0.133 & 0.0274 & 0.0786 & 235 & 8.32 \\
\hline GP-F/10-30 & 2.26 & 0.765 & 0.635 & 1.77 & 0.266 & 0.124 & 0.0205 & 0.0689 & 195 & 7.69 \\
\hline GP-F/30-50 & 2.04 & 1.01 & 0.538 & 1.67 & 0.226 & 0.108 & 0.0171 & 0.0595 & 157 & 6.84 \\
\hline GP-G/0-10 & 2.40 & 0.377 & 0.648 & 1.81 & 0.291 & 0.138 & 0.0293 & 0.0814 & 214 & 8.69 \\
\hline GP-G/10-30 & 2.31 & 0.798 & 0.589 & 1.82 & 0.266 & 0.133 & 0.0206 & 0.0769 & 180 & 7.79 \\
\hline GP-G/30-50 & 2.20 & 1.05 & 0.582 & 1.78 & 0.247 & 0.119 & 0.0175 & 0.0662 & 156 & 7.40 \\
\hline GP-H/0-10 & 2.39 & 0.340 & 0.740 & 1.78 & 0.282 & 0.137 & 0.0254 & 0.0814 & 221 & 8.61 \\
\hline GP-H/10-30 & 2.40 & 0.915 & 0.619 & 1.87 & 0.288 & 0.145 & 0.0212 & 0.0709 & 188 & 7.89 \\
\hline GP-H/30-50 & 2.13 & 1.12 & 0.567 & 1.75 & 0.244 & 0.117 & 0.0172 & 0.0632 & 145 & 7.08 \\
\hline GP-I/0-10 & 2.40 & 0.431 & 0.709 & 1.86 & 0.304 & 0.154 & 0.0320 & 0.0916 & 225 & 8.57 \\
\hline GP-I/10-30 & 2.36 & 0.630 & 0.680 & 1.89 & 0.283 & 0.145 & 0.0240 & 0.0904 & 197 & 8.45 \\
\hline GP-I/30-50 & 2.32 & 0.920 & 0.676 & 1.84 & 0.292 & 0.140 & 0.0218 & 0.0857 & 188 & 8.71 \\
\hline GP-J/0-10 & 2.52 & 0.523 & 0.756 & 1.90 & 0.308 & 0.147 & 0.0298 & 0.0822 & 230 & 8.88 \\
\hline GP-J/10-30 & 2.45 & 0.897 & 0.653 & 1.91 & 0.309 & 0.150 & 0.0250 & 0.0826 & 201 & 8.90 \\
\hline GP-J/30-50 & 2.42 & 1.11 & 0.682 & 1.93 & 0.302 & 0.157 & 0.0249 & 0.0795 & 185 & 8.85 \\
\hline GP-K/0-10 & 2.85 & 0.212 & 0.888 & 2.06 & 0.330 & 0.214 & 0.0429 & 0.129 & 280 & 11.3 \\
\hline GP-K/10-30 & 2.66 & 0.341 & 0.828 & 1.95 & 0.316 & 0.184 & 0.0293 & 0.114 & 237 & 10.3 \\
\hline GP-K/30-50 & 2.64 & 0.749 & 0.785 & 2.00 & 0.321 & 0.194 & 0.0239 & 0.110 & 213 & 10.0 \\
\hline
\end{tabular}


Table 5e-1b. ICP-MS data for the GP transect.

\begin{tabular}{|c|c|c|c|c|c|c|c|c|c|c|c|c|}
\hline Sample \# & $\underset{\text { (ppm) }}{\mathrm{Ag}}$ & $\begin{array}{c}\text { Al } \\
\text { (ppm) }\end{array}$ & $\begin{array}{c}\text { As } \\
(\mathbf{p p m})\end{array}$ & $\begin{array}{c}\mathrm{Ba} \\
\text { (ppm) }\end{array}$ & $\begin{array}{c}\mathrm{Be} \\
(\mathbf{p p m})\end{array}$ & $\begin{array}{c}\mathbf{B i} \\
(\mathbf{p p m})\end{array}$ & $\underset{\text { (ppm) }}{\mathrm{Ca}}$ & $\underset{(\mathbf{p p m})}{\mathbf{C d}}$ & $\begin{array}{c}\mathrm{Ce} \\
(\mathrm{ppm})\end{array}$ & $\begin{array}{c}\text { Co } \\
\text { (ppm) }\end{array}$ & $\underset{(\mathbf{p p m})}{\mathrm{Cr}}$ & $\underset{(p p m)}{\text { Cs }}$ \\
\hline GP-A/0-10 & 0.17 & 21200 & 6.48 & 329 & 0.79 & $<0.06$ & 4760 & 0.08 & 13.8 & 1.6 & 26.1 & 1.59 \\
\hline GP-A/10-30 & 0.15 & 20700 & 6.96 & 321 & 0.79 & $<0.06$ & 5300 & 0.07 & 13.8 & 1.6 & 30.8 & 1.59 \\
\hline GP-A/30-50 & 0.14 & 20500 & 8.64 & 310 & 0.83 & $<0.06$ & 7110 & 0.06 & 12.6 & 1.7 & 30.9 & 1.59 \\
\hline GP-B/0-10 & 0.16 & 24600 & 6 & 339 & 0.78 & $<0.06$ & 3580 & 0.08 & 18.7 & 2.1 & 22.9 & 2.014 \\
\hline GP-B/10-30 & 0.14 & 23100 & 4.68 & 339 & 0.71 & $<0.06$ & 8160 & 0.05 & 16.4 & 2 & 27.4 & 1.802 \\
\hline GP-B/30-50 & 0.1 & 19000 & 6.48 & 303 & 0.61 & $<0.06$ & 16400 & 0.05 & 13.3 & 1.5 & 16 & 1.484 \\
\hline GP-C/0-10 & 0.14 & 23100 & 3.84 & 342 & 0.59 & $<0.06$ & 1620 & 0.08 & 17.8 & 1.9 & 25.6 & 1.802 \\
\hline GP-C/10-30 & 0.15 & 24600 & 4.8 & 338 & 0.78 & $<0.06$ & 4160 & 0.06 & 18.3 & 2.2 & 25 & 2.014 \\
\hline GP-C/30-50 & 0.13 & 22800 & 4.56 & 336 & 0.73 & $<0.06$ & 9370 & 0.06 & 17.3 & 2 & 24.2 & 1.802 \\
\hline GP-D/0-10 & 0.2 & 24100 & 3.6 & 342 & 0.7 & $<0.06$ & 1700 & 0.08 & 18.5 & 2 & 21.5 & 1.908 \\
\hline GP-D/10-30 & 0.14 & 23600 & 3.6 & 340 & 0.71 & $<0.06$ & 3790 & 0.06 & 17.8 & 2.1 & 23.1 & 1.908 \\
\hline GP-D/30-50 & 0.14 & 22500 & 3.6 & 331 & 0.68 & $<0.06$ & 7760 & 0.06 & 16.3 & 1.9 & 20.2 & 1.802 \\
\hline GP-E/0-10 & 0.15 & 23300 & 2.4 & 354 & 0.7 & $<0.06$ & 1880 & 0.09 & 17.5 & 1.9 & 28 & 1.802 \\
\hline GP-E/10-30 & 0.15 & 25100 & 4.2 & 340 & 0.72 & $<0.06$ & 3740 & 0.08 & 19.2 & 2.4 & 30.2 & 2.014 \\
\hline GP-E/30-50 & 0.23 & 23100 & 4.32 & 333 & 0.63 & $<0.06$ & 7070 & 0.06 & 17.8 & 1.9 & 14.5 & 1.802 \\
\hline GP-F/0-10 & 0.14 & 24900 & 4.44 & 338 & 0.69 & $<0.06$ & 3010 & 0.09 & 19.1 & 2.4 & 28.5 & 2.014 \\
\hline GP-F/10-30 & 0.14 & 23600 & 5.28 & 333 & 0.74 & $<0.06$ & 7480 & 0.07 & 18.7 & 2.1 & 24.4 & 1.908 \\
\hline GP-F/30-50 & 0.11 & 21400 & 6 & 317 & 0.65 & $<0.06$ & 10200 & 0.06 & 15 & 1.8 & 23.6 & 1.696 \\
\hline GP-G/0-10 & 0.24 & 25600 & 3.6 & 343 & 0.76 & $<0.06$ & 3780 & 0.08 & 18.5 & 2.2 & 16.9 & 2.014 \\
\hline GP-G/10-30 & 0.2 & 23900 & 2.4 & 337 & 0.69 & $<0.06$ & 7710 & 0.05 & 17.3 & 1.9 & 16.6 & 1.908 \\
\hline GP-G/30-50 & 0.16 & 23300 & 2.4 & 336 & 0.62 & $<0.06$ & 10400 & 0.05 & 16.6 & 1.9 & 26 & 1.908 \\
\hline GP-H/0-10 & 0.19 & 25600 & 2.4 & 340 & 0.69 & $<0.06$ & 3310 & 0.08 & 19.9 & 2.3 & 30.8 & 2.12 \\
\hline GP-H/10-30 & 0.18 & 25400 & 2.4 & 357 & 0.73 & $<0.06$ & 8890 & 0.06 & 19 & 2.1 & 16.9 & 2.014 \\
\hline GP-H/30-50 & 0.13 & 22100 & 2.4 & 329 & 0.59 & $<0.06$ & 10900 & 0.04 & 16.4 & 1.8 & 25.5 & 1.696 \\
\hline GP-I/0-10 & 0.17 & 25600 & 2.4 & 354 & 0.74 & $<0.06$ & 4290 & 0.09 & 18.8 & 2.2 & 28.4 & 2.014 \\
\hline GP-I/10-30 & 0.15 & 24400 & 2.4 & 344 & 0.73 & $<0.06$ & 6030 & 0.07 & 18.4 & 2.1 & 30.8 & 1.908 \\
\hline GP-I/30-50 & 0.16 & 24600 & 2.4 & 342 & 0.71 & $<0.06$ & 9090 & 0.06 & 18.4 & 2.2 & 28.3 & 2.014 \\
\hline GP-J/0-10 & 0.18 & 26200 & 2.4 & 351 & 0.75 & $<0.06$ & 5040 & 0.1 & 21 & 2.4 & 32 & 2.12 \\
\hline GP-J/10-30 & 0.15 & 26600 & 2.4 & 364 & 0.81 & $<0.06$ & 9110 & 0.07 & 19.8 & 2.3 & 17.9 & 2.12 \\
\hline GP-J/30-50 & 0.15 & 26000 & 2.4 & 363 & 0.72 & $<0.06$ & 11100 & 0.06 & 19.4 & 2.2 & 28.9 & 2.014 \\
\hline GP-K/0-10 & 0.21 & 31300 & 2.4 & 395 & 0.79 & $<0.06$ & 2160 & 0.11 & 25.3 & 3 & 25.7 & 2.544 \\
\hline GP-K/10-30 & 0.2 & 28800 & 1.2 & 374 & 0.76 & $<0.06$ & 3370 & 0.08 & 23.6 & 2.7 & 27.8 & 2.332 \\
\hline GP-K/30-50 & 0.18 & 28200 & 1.2 & 372 & 0.69 & $<0.06$ & 7320 & 0.06 & 22.9 & 2.6 & 26.6 & 2.226 \\
\hline
\end{tabular}


Table 5e-1b. ICP-MS data for the GP transect-Continued.

\begin{tabular}{|c|c|c|c|c|c|c|c|c|c|c|c|c|}
\hline Sample \# & $\begin{array}{c}\mathrm{Cu} \\
(\mathbf{p p m})\end{array}$ & $\begin{array}{c}\mathrm{Fe} \\
(\mathrm{ppm})\end{array}$ & $\begin{array}{c}\text { Ga } \\
(\mathbf{p p m})\end{array}$ & $\begin{array}{c}\mathrm{K} \\
(\mathbf{p p m})\end{array}$ & $\begin{array}{c}\text { La } \\
\text { (ppm) }\end{array}$ & $\begin{array}{c}\mathrm{Li} \\
\text { (ppm) }\end{array}$ & $\begin{array}{c}\mathbf{M g} \\
\text { (ppm) }\end{array}$ & $\begin{array}{c}\text { Mn } \\
\text { (ppm) }\end{array}$ & $\begin{array}{c}\text { Mo } \\
\text { (ppm) }\end{array}$ & $\begin{array}{c}\mathrm{Na} \\
\text { (ppm) }\end{array}$ & $\begin{array}{c}\mathbf{N b} \\
\text { (ppm) }\end{array}$ & $\begin{array}{c}\mathrm{Ni} \\
(\mathbf{p p m})\end{array}$ \\
\hline GP-A/0-10 & 6.4 & 4970 & 3.7 & 17900 & 7.5 & 8.2 & 2230 & 158 & 0.28 & 1120 & 2.03 & 4.2 \\
\hline GP-A/10-30 & 7.2 & 5150 & 3.7 & 17400 & 7.6 & 8 & 2170 & 166 & 0.35 & 1290 & 1.75 & 4.2 \\
\hline GP-A/30-50 & 5.3 & 4900 & 3.6 & 17200 & 7 & 7.7 & 2020 & 151 & 0.31 & 941 & 1.54 & 4.3 \\
\hline GP-B/0-10 & 7.6 & 6510 & 4.5 & 18600 & 10.1 & 10.1 & 2950 & 186 & 0.3 & 1370 & 2.38 & 5 \\
\hline GP-B/10-30 & 6.7 & 6120 & 4.2 & 18100 & 8.8 & 9.2 & 2650 & 156 & 0.33 & 1270 & 2.03 & 5.1 \\
\hline GP-B/30-50 & 5.1 & 3920 & 3.4 & 16400 & 7.2 & 6.9 & 1840 & 99.4 & 0.2 & 836 & $<2$ & 3.7 \\
\hline GP-C/0-10 & 7.1 & 6130 & 4.1 & 18100 & 9.5 & 9.4 & 2470 & 180 & 0.3 & 2180 & 2.38 & 4.6 \\
\hline GP-C/10-30 & 6.8 & 6540 & 4.5 & 18500 & 9.9 & 9.9 & 2780 & 189 & 0.29 & 1490 & 2.24 & 5.2 \\
\hline GP-C/30-50 & 6.6 & 5890 & 4.1 & 18200 & 9 & 9 & 2520 & 154 & 0.28 & 1250 & 1.68 & 4.8 \\
\hline GP-D/0-10 & 7.4 & 6370 & 4.3 & 18300 & 9.9 & 10.1 & 2610 & 202 & 0.31 & 1420 & 3.43 & 4.8 \\
\hline GP-D/10-30 & 6.9 & 6270 & 4.3 & 18100 & 9.4 & 9.9 & 2650 & 188 & 0.29 & 1340 & 2.24 & 5.1 \\
\hline GP-D/30-50 & 6 & 5740 & 4 & 17400 & 8.7 & 9.5 & 2610 & 172 & 0.24 & 1340 & 2.1 & 4.5 \\
\hline GP-E/0-10 & 7.5 & 6170 & 4.2 & 18800 & 9.4 & 9.4 & 2420 & 184 & 0.33 & 1580 & 2.38 & 4.7 \\
\hline GP-E/10-30 & 8 & 7270 & 4.6 & 18500 & 10 & 10.5 & 2930 & 227 & 0.37 & 1380 & 2.45 & 6 \\
\hline GP-E/30-50 & 6.4 & 5450 & 4.1 & 18000 & 9.5 & 9.5 & 2600 & 183 & 0.2 & 1530 & 4.55 & 4.3 \\
\hline GP-F/0-10 & 8.5 & 7100 & 4.5 & 18000 & 10.1 & 10.8 & 2970 & 238 & 0.35 & 1340 & 2.31 & 5.8 \\
\hline GP-F/10-30 & 7.2 & 6390 & 4.3 & 18100 & 9.8 & 9.9 & 2830 & 193 & 0.29 & 1280 & 1.96 & 5.3 \\
\hline GP-F/30-50 & 7 & 5360 & 3.8 & 17300 & 8.1 & 8.5 & 2370 & 150 & 0.32 & 1130 & 1.61 & 4.5 \\
\hline GP-G/0-10 & 6.5 & 6540 & 4.6 & 19000 & 10 & 10.6 & 3120 & 218 & 0.24 & 1430 & 2.31 & 5 \\
\hline GP-G/10-30 & 6.2 & 5780 & 4.3 & 18600 & 9.2 & 9.6 & 2780 & 180 & 0.21 & 1330 & 2.1 & 4.6 \\
\hline GP-G/30-50 & 7.2 & 5970 & 4.1 & 18700 & 9 & 9.2 & 2630 & 153 & 0.28 & 1220 & 1.96 & 5 \\
\hline GP-H/0-10 & 8 & 7510 & 4.7 & 18700 & 10.5 & 10.9 & 3000 & 224 & 0.36 & 1530 & 2.66 & 5.8 \\
\hline GP-H/10-30 & 6.5 & 6190 & 4.5 & 19400 & 10.1 & 10.3 & 2950 & 188 & 0.2 & 1460 & 2.38 & 5.1 \\
\hline GP-H/30-50 & 6.7 & 5670 & 4 & 17800 & 8.8 & 8.9 & 2490 & 139 & 0.28 & 1160 & 1.75 & 5 \\
\hline GP-I/0-10 & 8.1 & 7060 & 4.6 & 19200 & 10 & 10.7 & 3150 & 227 & 0.34 & 1540 & 2.59 & 5.7 \\
\hline GP-I/10-30 & 7.8 & 6740 & 4.3 & 18900 & 9.8 & 9.9 & 2900 & 198 & 0.34 & 1400 & 2.31 & 5.6 \\
\hline GP-I/30-50 & 8.2 & 6700 & 4.4 & 18900 & 10 & 10.2 & 2980 & 188 & 0.37 & 1500 & 2.45 & 5.6 \\
\hline GP-J/0-10 & 9.3 & 7600 & 4.8 & 19200 & 11.3 & 11.1 & 3210 & 234 & 0.37 & 1590 & 2.73 & 6.3 \\
\hline GP-J/10-30 & 7.2 & 6620 & 4.8 & 20000 & 10.7 & 11.1 & 3180 & 206 & 0.21 & 1540 & 2.38 & 5.3 \\
\hline GP-J/30-50 & 7.8 & 6900 & 4.7 & 20100 & 10.3 & 10.7 & 3100 & 190 & 0.36 & 1570 & 2.45 & 5.9 \\
\hline GP-K/0-10 & 9.1 & 9330 & 5.7 & 21900 & 13.1 & 13.6 & 3480 & 290 & 0.37 & 2540 & 3.78 & 6.8 \\
\hline GP-K/10-30 & 8.6 & 8630 & 5.2 & 20700 & 12.5 & 12.1 & 3260 & 242 & 0.34 & 2050 & 3.29 & 6.6 \\
\hline GP-K/30-50 & 8.6 & 7890 & 5 & 20400 & 11.9 & 12.1 & 3290 & 219 & 0.36 & 1940 & 2.94 & 6.2 \\
\hline
\end{tabular}


Table 5e-1b. ICP-MS data for the GP transect-Continued.

\begin{tabular}{|c|c|c|c|c|c|c|c|c|c|c|c|c|c|}
\hline Sample \# & $\underset{(\mathbf{p p m})}{\mathbf{P}}$ & $\begin{array}{c}\mathrm{Pb} \\
(\mathrm{ppm})\end{array}$ & $\begin{array}{c}\mathbf{R b} \\
(\mathbf{p p m})\end{array}$ & $\underset{\text { (ppm) }}{\text { Sb }}$ & $\begin{array}{c}\text { Sc } \\
(\mathbf{p p m})\end{array}$ & $\begin{array}{c}\mathrm{Sr} \\
(\mathbf{p p m})\end{array}$ & $\begin{array}{c}\text { Th } \\
\text { (ppm) }\end{array}$ & $\underset{(\mathbf{p p m})}{\mathrm{Ti}}$ & $\begin{array}{c}\mathrm{TI} \\
\text { (ppm) }\end{array}$ & $\underset{(\mathbf{p p m})}{\mathbf{U}}$ & $\begin{array}{c}\mathbf{V} \\
(\mathbf{p p m})\end{array}$ & $\begin{array}{c}Y \\
\text { (ppm) }\end{array}$ & $\underset{(p p m)}{Z n}$ \\
\hline GP-A/0-10 & 216 & 10.2 & 49.7 & 0.33 & 1.8 & 67.6 & 1.86 & 705 & 0.32 & 0.5 & 11.1 & 5.52 & 25.6 \\
\hline GP-A/10-30 & 238 & 9.78 & 48.3 & 0.49 & 1.8 & 71 & 1.94 & 2300 & 0.32 & 0.49 & 11 & 5.52 & 26.8 \\
\hline GP-A/30-50 & 149 & 9.21 & 47.5 & 0.35 & 1.8 & 80.4 & 1.7 & 642 & 0.32 & 0.48 & 10.4 & 5.04 & 22.7 \\
\hline GP-B/0-10 & 235 & 10.8 & 53.3 & 0.35 & 2.5 & 71.9 & 2.75 & 865 & 0.35 & 0.63 & 14.9 & 7.08 & 27.4 \\
\hline GP-B/10-30 & 171 & 9.17 & 51.3 & 0.3 & 2.2 & 71.6 & 2.26 & 801 & 0.33 & 0.6 & 13.3 & 6.36 & 22.5 \\
\hline GP-B/30-50 & 132 & 8.46 & 45.6 & 0.23 & 1.6 & 80.6 & 1.49 & 538 & 0.3 & 0.41 & 10.4 & 4.44 & 18.8 \\
\hline GP-C/0-10 & 228 & 10.5 & 50.9 & 0.31 & 2.2 & 63.6 & 2.79 & 853 & 0.33 & 0.65 & 13.5 & 6.6 & 24.1 \\
\hline GP-C/10-30 & 198 & 9.69 & 52.4 & 0.33 & 2.5 & 68.2 & 2.65 & 861 & 0.34 & 0.59 & 14.7 & 7.2 & 28.2 \\
\hline GP-C/30-50 & 172 & 9.43 & 51.3 & 0.37 & 2.1 & 73 & 2.33 & 788 & 0.34 & 0.57 & 13.4 & 6.48 & 21.4 \\
\hline GP-D/0-10 & 233 & 10.3 & 52.5 & 0.33 & 2.4 & 64.4 & 2.82 & 924 & 0.34 & 0.66 & 14.3 & 7.2 & 25.5 \\
\hline GP-D/10-30 & 190 & 9.37 & 52.2 & 0.29 & 2.4 & 64.9 & 2.68 & 847 & 0.34 & 0.6 & 13.8 & 7.08 & 23.7 \\
\hline GP-D/30-50 & 177 & 8.87 & 49.4 & 0.28 & 2.2 & 65.9 & 2.31 & 805 & 0.32 & 0.62 & 13.3 & 6.6 & 21.6 \\
\hline GP-E/0-10 & 276 & 10.4 & 52.2 & 0.3 & 2.2 & 63.7 & 2.66 & 876 & 0.34 & 0.62 & 12.9 & 7.2 & 23.9 \\
\hline GP-E/10-30 & 228 & 9.66 & 52.5 & 0.33 & 2.6 & 69.1 & 2.76 & 931 & 0.34 & 0.66 & 15.5 & 8.04 & 26.8 \\
\hline GP-E/30-50 & 188 & 9.1 & 50.8 & 0.28 & 2.3 & 68.9 & 2.47 & 832 & 0.32 & 0.63 & 13.8 & 6.96 & 23.4 \\
\hline GP-F/0-10 & 287 & 10.4 & 52.5 & 0.32 & 2.6 & 67.3 & 2.79 & 917 & 0.34 & 0.7 & 14.8 & 7.92 & 28 \\
\hline GP-F/10-30 & 214 & 9.48 & 51.1 & 0.31 & 2.4 & 70.2 & 2.63 & 848 & 0.33 & 0.64 & 14.3 & 6.96 & 24.5 \\
\hline GP-F/30-50 & 181 & 8.8 & 48.2 & 0.29 & 2 & 71.5 & 2.07 & 753 & 0.3 & 0.52 & 12.7 & 6 & 21.7 \\
\hline GP-G/0-10 & 320 & 10.5 & 53.1 & 0.29 & 2.7 & 69.1 & 2.61 & 908 & 0.35 & 0.66 & 15.7 & 7.8 & 26.4 \\
\hline GP-G/10-30 & 213 & 9.28 & 51.6 & 0.26 & 2.3 & 69.7 & 2.53 & 840 & 0.33 & 0.61 & 13.6 & 6.72 & 21.4 \\
\hline GP-G/30-50 & 186 & 9.17 & 51.7 & 0.28 & 2.2 & 74.3 & 2.26 & 846 & 0.33 & 0.55 & 13.6 & 6.36 & 20.2 \\
\hline GP-H/0-10 & 269 & 10.7 & 53.5 & 0.3 & 2.7 & 68.1 & 2.98 & 1000 & 0.34 & 0.67 & 15.6 & 7.92 & 25.5 \\
\hline GP-H/10-30 & 223 & 9.73 & 54.7 & 0.27 & 2.5 & 75.7 & 2.67 & 912 & 0.34 & 0.64 & 14.9 & 7.56 & 22 \\
\hline GP-H/30-50 & 178 & 8.84 & 49.5 & 0.26 & 2.1 & 74.3 & 2.26 & 808 & 0.32 & 0.52 & 12.2 & 6.24 & 19 \\
\hline GP-I/0-10 & 345 & 10.5 & 55 & 0.29 & 2.6 & 72 & 2.93 & 995 & 0.35 & 0.7 & 15.2 & 7.92 & 25 \\
\hline GP-I/10-30 & 251 & 9.49 & 52.9 & 0.27 & 2.5 & 72.2 & 2.65 & 942 & 0.33 & 0.62 & 14 & 7.44 & 22 \\
\hline GP-I/30-50 & 232 & 9.6 & 52.8 & 0.29 & 2.4 & 76.1 & 2.7 & 956 & 0.33 & 0.65 & 14.9 & 7.44 & 22.2 \\
\hline GP-J/0-10 & 314 & 10.7 & 54.5 & 0.31 & 2.8 & 72.6 & 3.11 & 990 & 0.35 & 0.72 & 15.7 & 8.28 & 25.8 \\
\hline GP-J/10-30 & 265 & 9.94 & 56.1 & 0.28 & 2.7 & 78.6 & 2.84 & 1030 & 0.35 & 0.64 & 16 & 7.8 & 24.2 \\
\hline GP-J/30-50 & 259 & 9.69 & 55.7 & 0.28 & 2.5 & 80.4 & 2.88 & 994 & 0.35 & 0.65 & 15.4 & 7.8 & 22.6 \\
\hline GP-K/0-10 & 465 & 11.9 & 61.6 & 0.35 & 3.4 & 77.4 & 3.97 & 1370 & 0.39 & 0.94 & 20.6 & 10.44 & 30.2 \\
\hline GP-K/10-30 & 314 & 10.2 & 58 & 0.31 & 3.2 & 69.6 & 3.61 & 1160 & 0.37 & 0.82 & 18.3 & 11.64 & 27 \\
\hline GP-K/30-50 & 258 & 9.86 & 56.5 & 0.28 & 2.9 & 71.8 & 3.33 & 1160 & 0.36 & 0.77 & 18.1 & 9.12 & 25.6 \\
\hline
\end{tabular}


Table 5f-1a. ICP-AES data for the 00U-41 transect.

\begin{tabular}{|c|c|c|c|c|c|c|c|c|c|c|}
\hline Sample \# & Al (\%) & $\mathrm{Ca}(\%)$ & $\mathrm{Fe}(\%)$ & $K(\%)$ & $\operatorname{Mg}(\%)$ & $\mathrm{Na}(\%)$ & $\mathbf{P}(\%)$ & $\mathrm{Ti}(\%)$ & Mn (ppm) & Nd (ppm) \\
\hline 00U-41A/0-10 & 2.26 & 2.42 & 1.05 & 1.43 & 0.610 & 0.399 & 0.0186 & 0.0714 & 232 & 11.8 \\
\hline 00U-41A/10-25 & 2.05 & 2.52 & 0.782 & 1.34 & 0.614 & 0.374 & 0.0159 & 0.0551 & 230 & 7.76 \\
\hline 00U-41B/0-10 & 2.37 & 2.27 & 0.911 & 1.50 & 0.477 & 0.434 & 0.0202 & 0.0799 & 195 & 11.9 \\
\hline 00U-41B/10-25 & 3.50 & 3.82 & 1.34 & 2.00 & 0.703 & 0.709 & 0.0243 & 0.116 & 236 & 16.8 \\
\hline $00 \mathrm{U}-41 \mathrm{~B} / 25-45$ & 4.87 & 6.97 & 1.60 & 2.66 & 0.892 & 1.18 & 0.0267 & 0.143 & 288 & 22.4 \\
\hline 00U-41C/0-10 & 3.60 & 1.96 & 1.30 & 2.19 & 0.632 & 0.773 & 0.0438 & 0.130 & 271 & 17.3 \\
\hline 00U-41C/10-20 & 2.89 & 2.32 & 1.05 & 1.80 & 0.420 & 0.612 & 0.0209 & 0.0827 & 192 & 9.69 \\
\hline 00U-41C/20-30 & 2.80 & 2.33 & 0.975 & 1.78 & 0.380 & 0.654 & 0.0204 & 0.0780 & 179 & 9.69 \\
\hline 00U-41D/0-10 & 2.95 & 2.32 & 1.17 & 1.82 & 0.494 & 0.600 & 0.0241 & 0.0973 & 214 & 13.7 \\
\hline 00U-41D/10-30 & 3.46 & 2.40 & 1.20 & 2.05 & 0.611 & 0.714 & 0.0351 & 0.113 & 261 & 12.5 \\
\hline 00U-41D/30-50 & 3.60 & 2.70 & 1.23 & 2.10 & 0.665 & 0.744 & 0.0362 & 0.123 & 267 & 14.7 \\
\hline 00U-41E/0-10 & 3.89 & 2.09 & 1.52 & 2.27 & 0.724 & 0.748 & 0.0474 & 0.151 & 320 & 21.0 \\
\hline 00U-41E/10-30 & 3.50 & 2.23 & 1.25 & 2.05 & 0.634 & 0.673 & 0.0347 & 0.124 & 278 & 13.9 \\
\hline 00U-41E/30-50 & 3.79 & 3.24 & 1.30 & 2.18 & 0.684 & 0.738 & 0.0336 & 0.134 & 272 & 14.1 \\
\hline 00U-41F/0-10 & 3.95 & 2.11 & 1.38 & 2.30 & 0.717 & 0.728 & 0.0440 & 0.146 & 327 & 18.0 \\
\hline 00U-41F/10-30 & 3.92 & 2.72 & 1.40 & 2.32 & 0.733 & 0.718 & 0.0376 & 0.160 & 307 & 20.2 \\
\hline $00 \mathrm{U}-41 \mathrm{~F} / 30-50$ & 4.20 & 3.89 & 1.43 & 2.44 & 0.792 & 0.803 & 0.0360 & 0.155 & 279 & 20.0 \\
\hline 00U-41G/0-10 & 3.54 & 1.80 & 1.20 & 2.21 & 0.634 & 0.749 & 0.0403 & 0.134 & 272 & 15.6 \\
\hline 00U-41G/10-30 & 3.90 & 2.36 & 1.40 & 2.33 & 0.748 & 0.734 & 0.0414 & 0.163 & 318 & 18.5 \\
\hline 00U-41G/30-50 & 4.11 & 3.20 & 1.41 & 2.45 & 0.804 & 0.786 & 0.0370 & 0.172 & 303 & 20.8 \\
\hline 00U-41H1/0-10 & 3.99 & 2.16 & 1.49 & 2.38 & 0.766 & 0.717 & 0.0458 & 0.155 & 358 & 20.8 \\
\hline 00U-41H1/10-30 & 3.71 & 2.94 & 1.38 & 2.13 & 0.697 & 0.646 & 0.0294 & 0.142 & 291 & 15.6 \\
\hline 00U-41H1/30-50 & 3.68 & 4.34 & 1.26 & 2.12 & 0.742 & 0.711 & 0.0304 & 0.118 & 245 & 13.8 \\
\hline 00U-41H2/0-10 & 3.94 & 2.22 & 1.51 & 2.18 & 0.794 & 0.634 & 0.0512 & 0.172 & 366 & 19.9 \\
\hline 00U-41H2/10-30 & 3.66 & 2.21 & 1.36 & 2.11 & 0.702 & 0.627 & 0.0385 & 0.132 & 328 & 15.5 \\
\hline 00U-41H2/30-50 & 3.80 & 2.54 & 1.32 & 2.20 & 0.676 & 0.702 & 0.0291 & 0.143 & 277 & 14.9 \\
\hline
\end{tabular}


Table 5f-1b. ICP-MS data for the 00U-41 transect.

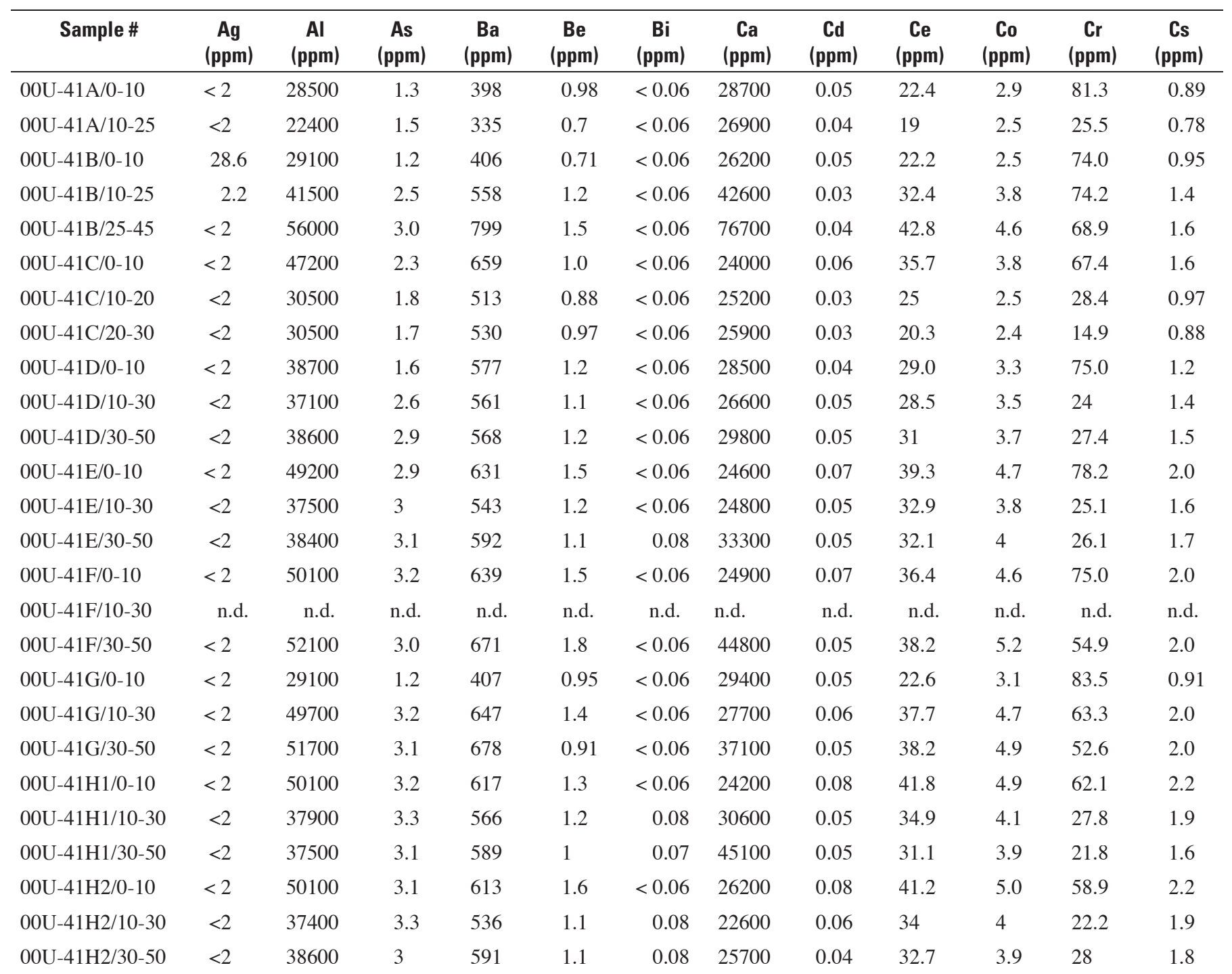


Table 5f-1b. ICP-MS data for the 00U-41 transect-Continued.

\begin{tabular}{|c|c|c|c|c|c|c|c|c|c|c|c|c|}
\hline Sample \# & $\begin{array}{c}\text { Cu } \\
\text { (ppm) }\end{array}$ & $\begin{array}{c}\mathrm{Fe} \\
(\mathrm{ppm})\end{array}$ & $\begin{array}{c}\text { Ga } \\
\text { (ppm) }\end{array}$ & $\begin{array}{c}\text { K } \\
\text { (ppm) }\end{array}$ & $\begin{array}{c}\text { La } \\
\text { (ppm) }\end{array}$ & $\begin{array}{c}\mathbf{L i} \\
\text { (ppm) }\end{array}$ & $\begin{array}{c}\mathbf{M g} \\
(\mathbf{p p m})\end{array}$ & $\begin{array}{c}\mathrm{Mn} \\
(\mathrm{ppm})\end{array}$ & $\begin{array}{c}\text { Mo } \\
\text { (ppm) }\end{array}$ & $\begin{array}{c}\mathrm{Na} \\
(\mathrm{ppm})\end{array}$ & $\begin{array}{c}\mathbf{N b} \\
\text { (ppm) }\end{array}$ & $\begin{array}{c}\mathrm{Ni} \\
(\mathbf{p p m})\end{array}$ \\
\hline $00 \mathrm{U} 41 \mathrm{~A} / 0-10$ & 11.3 & 11800 & 4.8 & 17600 & 12.8 & 8.8 & 7690 & 272 & 0.62 & 5270 & 3.1 & 7.3 \\
\hline 00U41A/10-25 & 7.1 & 7930 & 4.3 & 14200 & 11 & 7 & 7120 & 234 & 0.23 & 4140 & 0.84 & 3.8 \\
\hline $00 \mathrm{U} 41 \mathrm{~B} / 0-10$ & 12.9 & 10000 & 5.0 & 18300 & 12.3 & 9.5 & 6090 & 222 & 0.46 & 5640 & 2.9 & 6.2 \\
\hline $00 \mathrm{U} 41 \mathrm{~B} / 10-25$ & 19.8 & 15300 & 7.6 & 23100 & 18.5 & 13.3 & 8670 & 267 & 0.58 & 8800 & 4.7 & 8.7 \\
\hline $00 \mathrm{U} 41 \mathrm{~B} / 25-45$ & 10.7 & 18000 & 10.5 & 29600 & 24.4 & 15.4 & 10600 & 327 & 0.53 & 13900 & 7.1 & 9.6 \\
\hline $00 \mathrm{U} 41 \mathrm{C} / 0-10$ & 11.5 & 16000 & 8.0 & 28000 & 19.9 & 14.7 & 8520 & 338 & 0.49 & 10700 & 5.8 & 9.1 \\
\hline 00U41C/10-20 & 9 & 10800 & 6.1 & 19600 & 13.1 & 8.4 & 4840 & 197 & 0.43 & 7010 & 1.6 & 5.5 \\
\hline $00 \mathrm{U} 41 \mathrm{C} / 20-30$ & 8.4 & 10100 & 6 & 20000 & 10.9 & 7.8 & 4390 & 183 & 0.37 & 7820 & 1.9 & 4.9 \\
\hline 00U41D/0-10 & 12.8 & 13800 & 6.8 & 23900 & 16.3 & 11.1 & 6610 & 260 & 0.57 & 8320 & 4.6 & 7.8 \\
\hline 00U41D/10-30 & 11.3 & 12600 & 7.2 & 22600 & 15.2 & 12.7 & 7350 & 271 & 0.38 & 8160 & 2 & 7.4 \\
\hline 00U41D/30-50 & 12.2 & 12800 & 7.4 & 23200 & 16.5 & 13.2 & 7980 & 281 & 0.39 & 8520 & 2.2 & 7.7 \\
\hline 00U41E/0-10 & 15.2 & 18100 & 8.4 & 28200 & 22.0 & 16.9 & 9390 & 384 & 0.61 & 9840 & 6.6 & 11.3 \\
\hline 00U41E/10-30 & 12.1 & 13100 & 7.3 & 22800 & 17.4 & 14.1 & 7600 & 291 & 0.43 & 7750 & 2.1 & 8.1 \\
\hline 00U41E/30-50 & 12 & 12900 & 7.7 & 22300 & 17.6 & 14.3 & 7310 & 272 & 0.47 & 7750 & 2.3 & 7.9 \\
\hline $00 \mathrm{U} 41 \mathrm{~F} / 0-10$ & 17.9 & 16400 & 8.6 & 28700 & 20.1 & 17.7 & 9350 & 390 & 0.46 & 9650 & 5.6 & 10.7 \\
\hline 00U41F/10-30 & n.d. & n.d. & n.d. & n.d. & n.d. & n.d. & n.d. & n.d. & n.d. & n.d. & n.d. & n.d. \\
\hline $00 \mathrm{U} 41 \mathrm{~F} / 30-50$ & 19.3 & 17200 & 9.0 & 29500 & 21.0 & 18.2 & 10100 & 340 & 0.38 & 10400 & 5.7 & 10.8 \\
\hline $00 \mathrm{U} 41 \mathrm{G} / 0-10$ & 11.5 & 12200 & 4.9 & 18200 & 13.0 & 8.8 & 7920 & 278 & 0.63 & 5360 & 3.2 & 7.5 \\
\hline 00U41G/10-30 & 13.8 & 17000 & 8.3 & 28700 & 20.9 & 17.9 & 9640 & 388 & 0.52 & 9690 & 5.9 & 10.5 \\
\hline 00U41G/30-50 & 16.2 & 17100 & 8.6 & 30000 & 21.5 & 18.6 & 10300 & 370 & 0.43 & 10200 & 5.9 & 10.6 \\
\hline 00U41H1/0-10 & 16.2 & 17600 & 8.4 & 28300 & 22.9 & 18.8 & 9680 & 426 & 0.52 & 8940 & 6.8 & 11.2 \\
\hline 00U41H1/10-30 & 13.1 & 13600 & 7.6 & 22100 & 18.9 & 16.1 & 7480 & 287 & 0.56 & 6860 & 2.3 & 8.8 \\
\hline 00U41H1/30-50 & 13.6 & 12200 & 7.4 & 21800 & 16.9 & 14.6 & 7930 & 246 & 0.39 & 7420 & 2.5 & 7.6 \\
\hline 00U41H2/0-10 & 14.3 & 17700 & 8.6 & 28600 & 22.9 & 19.5 & 10500 & 434 & 0.49 & 9000 & 7.1 & 11.4 \\
\hline 00U41H2/10-30 & 12.3 & 13200 & 7.4 & 21700 & 18.5 & 15.6 & 7590 & 318 & 0.55 & 6670 & 2.6 & 8.6 \\
\hline 00U41H2/30-50 & 11.2 & 12800 & 7.5 & 22600 & 17.8 & 15.4 & 7270 & 264 & 0.42 & 7490 & 2.4 & 8 \\
\hline
\end{tabular}


Table 5f-1b. ICP-MS data for the 00U-41 transect-Continued.

\begin{tabular}{|c|c|c|c|c|c|c|c|c|c|c|c|c|c|}
\hline Sample \# & $\begin{array}{c}P \\
(p p m)\end{array}$ & $\begin{array}{c}\mathrm{Pb} \\
(\mathrm{ppm})\end{array}$ & $\begin{array}{c}\mathbf{R b} \\
(\mathbf{p p m})\end{array}$ & $\begin{array}{c}\text { Sb } \\
(\mathbf{p p m})\end{array}$ & $\begin{array}{c}\text { Sc } \\
(\mathbf{p p m})\end{array}$ & $\begin{array}{c}\mathrm{Sr} \\
(\mathrm{ppm})\end{array}$ & $\begin{array}{c}\text { Th } \\
\text { (ppm) }\end{array}$ & $\begin{array}{c}\mathrm{Ti} \\
(\mathrm{ppm})\end{array}$ & $\begin{array}{c}\mathrm{TI} \\
\text { (ppm) }\end{array}$ & $\underset{(\mathbf{p p m})}{\mathbf{U}}$ & $\underset{(\mathbf{p p m})}{\mathbf{V}}$ & $\begin{array}{c}Y \\
\text { (ppm) }\end{array}$ & $\begin{array}{c}\mathrm{Zn} \\
(\mathrm{ppm})\end{array}$ \\
\hline $00 \mathrm{U} 41 \mathrm{~A} / 0-10$ & 237 & 9.35 & 39.9 & 0.20 & 2.5 & 74.1 & 2.98 & 1110 & 0.20 & 0.81 & 23.5 & 10.5 & 13.3 \\
\hline $00 \mathrm{U} 41 \mathrm{~A} / 10-25$ & 179 & 6.86 & 34.1 & 0.1 & 2.2 & 71 & 2.69 & 587 & 0.2 & 0.59 & 20 & 7.2 & 12.4 \\
\hline $00 \mathrm{U} 41 \mathrm{~B} / 0-10$ & 260 & 9.65 & 39.6 & 0.2 & 2.6 & 79.1 & 3.82 & 1120 & 0.20 & 0.78 & 20.7 & 10.5 & 13.6 \\
\hline 00U41B/10-25 & 289 & 10.4 & 50.0 & 0.28 & 4.2 & 152 & 4.51 & 1630 & 0.24 & 0.96 & 35.1 & 13.8 & 19.5 \\
\hline 00U41B/25-45 & 305 & 12.1 & 62.5 & 0.29 & 5.6 & 273 & 5.18 & 2080 & 0.29 & 1.18 & 46.9 & 17.9 & 22.3 \\
\hline 00U41C/0-10 & 572 & 13.0 & 59.9 & 0.32 & 4.5 & 112 & 5.00 & 2160 & 0.30 & 1.21 & 32.0 & 17.4 & 21.6 \\
\hline 00U41C/10-20 & 224 & 8.91 & 44 & 0.2 & 2.8 & 96 & 3.72 & 796 & 0.25 & 0.88 & 23.1 & 8.9 & 13.9 \\
\hline $00 \mathrm{U} 41 \mathrm{C} / 20-30$ & 242 & 8.66 & 44.1 & 0.2 & 2.6 & 101 & 2.82 & 798 & 0.25 & 0.69 & 22 & 8.1 & 12.5 \\
\hline $00 \mathrm{U} 41 \mathrm{D} / 0-10$ & 323 & 11.0 & 51.4 & 0.25 & 3.5 & 104 & 3.89 & 1490 & 0.25 & 0.95 & 28.1 & 12.9 & 17.3 \\
\hline 00U41D/10-30 & 387 & 10 & 51.9 & 0.22 & 4.1 & 116 & 4.12 & 1110 & 0.3 & 0.97 & 26.9 & 12 & 21.3 \\
\hline $00 \mathrm{U} 41 \mathrm{D} / 30-50$ & 369 & 10.1 & 53.7 & 0.23 & 4.4 & 132 & 4.52 & 1160 & 0.31 & 1 & 29.3 & 12.4 & 21.5 \\
\hline $00 \mathrm{U} 41 \mathrm{E} / 0-10$ & 613 & 13.4 & 62.3 & 0.38 & 5.3 & 117 & 5.51 & 2390 & 0.32 & 1.37 & 35.6 & 19.2 & 27.0 \\
\hline 00U41E/10-30 & 387 & 10.2 & 53.3 & 0.26 & 4.4 & 118 & 4.93 & 1200 & 0.32 & 1.09 & 28.8 & 13.1 & 23.2 \\
\hline 00U41E/30-50 & 326 & 10.4 & 54.9 & 0.24 & 4.8 & 140 & 4.62 & 1220 & 0.32 & 1.05 & 30.5 & 12.9 & 23.9 \\
\hline $00 \mathrm{U} 41 \mathrm{~F} / 0-10$ & 569 & 13.1 & 63.2 & 0.33 & 5.3 & 116 & 4.84 & 2220 & 0.33 & 1.14 & 33.7 & 16.8 & 27.7 \\
\hline $00 \mathrm{U} 41 \mathrm{~F} / 10-30$ & n.d. & n.d. & n.d. & n.d. & n.d. & n.d. & n.d. & n.d. & n.d. & n.d. & n.d. & n.d. & n.d. \\
\hline $00 \mathrm{U} 41 \mathrm{~F} / 30-50$ & 451 & 12.7 & 63.3 & 0.31 & 6.0 & 166 & 5.04 & 2420 & 0.33 & 1.26 & 38.7 & 18.2 & 27.4 \\
\hline 00U41G/0-10 & 243 & 9.48 & 40.5 & 0.20 & 2.5 & 76.0 & 3.01 & 1130 & 0.20 & 0.81 & 24.4 & 10.7 & 13.3 \\
\hline 00U41G/10-30 & 531 & 12.9 & 62.7 & 0.34 & 5.5 & 134 & 5.20 & 2370 & 0.32 & 1.28 & 35.3 & 18.1 & 26.2 \\
\hline $00 \mathrm{U} 41 \mathrm{G} / 30-50$ & 474 & 12.6 & 64.4 & 0.34 & 5.8 & 179 & 5.24 & 2450 & 0.34 & 1.34 & 38.8 & 19.0 & 28.0 \\
\hline 00U41H1/0-10 & 588 & 13.6 & 63.7 & 0.39 & 5.6 & 122 & 5.93 & 2760 & 0.33 & 1.53 & 36.7 & 20.7 & 29.2 \\
\hline 00U41H1/10-30 & 303 & 11 & 55.9 & 0.27 & 4.8 & 143 & 5.44 & 1340 & 0.34 & 1.22 & 29.4 & 13.6 & 25.3 \\
\hline 00U41H1/30-50 & 321 & 10.4 & 53.2 & 0.24 & 4.6 & 172 & 4.48 & 1180 & 0.32 & 1.01 & 30.8 & 11.9 & 22.6 \\
\hline 98145.45189 & 648 & 13.7 & 63.9 & 0.40 & 5.9 & 125 & 6.04 & 2830 & 0.34 & 1.47 & 37.8 & 20.6 & 30.4 \\
\hline 00U41H2/10-30 & 395 & 10.7 & 55 & 0.28 & 4.6 & 119 & 5.26 & 1270 & 0.33 & 1.08 & 29.7 & 13.4 & 24.9 \\
\hline OOU41H2/30-50 & 288 & 10.7 & 55.9 & 0.26 & 4.8 & 150 & 4.96 & 1420 & 0.33 & 1.24 & 30.5 & 14 & 23.8 \\
\hline
\end{tabular}


Table 5f-2a. ICP-AES data for auger hole 00U-42.

\begin{tabular}{lcccccccccc}
\hline \multicolumn{1}{c}{ Sample \# } & Al (\%) & $\mathbf{C a}(\%)$ & $\mathbf{F e}(\%)$ & $\mathbf{K}(\%)$ & $\mathbf{M g}(\%)$ & $\mathbf{N a}(\%)$ & $\mathbf{P}(\%)$ & $\mathbf{T i}(\%)$ & $\mathbf{M n}(\mathbf{p p m})$ & $\mathbf{N d}(\mathbf{p p m})$ \\
\hline 00U-42/0-10 & 3.26 & 2.11 & 1.08 & 1.99 & 0.459 & 0.711 & 0.0303 & 0.105 & 220 & 12.5 \\
00U-42/10-30 & 3.49 & 2.37 & 1.11 & 2.06 & 0.484 & 0.738 & 0.0282 & 0.107 & 230 & 13.5 \\
00U-42/30-50 & 4.02 & 2.87 & 1.23 & 2.35 & 0.559 & 0.817 & 0.0303 & 0.124 & 262 & 16.6 \\
00U-42/50-63 & 3.84 & 2.20 & 1.26 & 2.27 & 0.503 & 0.829 & 0.0248 & 0.126 & 249 & 12.6 \\
00U-42/63-74 & 3.86 & 2.58 & 1.18 & 2.29 & 0.526 & 0.842 & 0.0261 & 0.119 & 240 & 12.6 \\
00U-42/74-87 & 3.86 & 3.46 & 1.16 & 2.26 & 0.615 & 0.865 & 0.0274 & 0.111 & 226 & 14.0 \\
00U-42/87-95 & 3.82 & 3.43 & 1.23 & 2.22 & 0.701 & 0.839 & 0.0298 & 0.117 & 237 & 13.6 \\
00U-42/95-106 & 4.48 & 4.87 & 1.40 & 2.55 & 1.01 & 0.952 & 0.0360 & 0.151 & 283 & 20.8 \\
00U-42/106-119 & 3.95 & 3.70 & 1.31 & 2.26 & 0.867 & 0.860 & 0.0307 & 0.127 & 259 & 13.7 \\
00U-42/119-125 & 4.26 & 4.26 & 1.31 & 2.47 & 0.841 & 0.909 & 0.0322 & 0.141 & 272 & 18.0 \\
00U-42/125-133 & 3.80 & 3.99 & 1.19 & 2.20 & 0.774 & 0.843 & 0.0280 & 0.112 & 244 & 12.2 \\
\hline
\end{tabular}

Table 5f-2b. ICP-MS data for auger hole 00U-42.

\begin{tabular}{lcccccccccccc}
\hline \multicolumn{1}{c}{ Sample \# } & $\begin{array}{c}\mathbf{A g} \\
(\mathbf{p p m})\end{array}$ & $\begin{array}{c}\mathbf{A l} \\
(\mathbf{p p m})\end{array}$ & $\begin{array}{c}\mathbf{A s} \\
(\mathbf{p p m})\end{array}$ & $\begin{array}{c}\mathbf{B a} \\
(\mathbf{p p m})\end{array}$ & $\begin{array}{c}\mathbf{B e} \\
(\mathbf{p p m})\end{array}$ & $\begin{array}{c}\mathbf{B i} \\
(\mathbf{p p m})\end{array}$ & $\begin{array}{c}\mathbf{C a} \\
(\mathbf{p p m})\end{array}$ & $\begin{array}{c}\mathbf{C d} \\
(\mathbf{p p m})\end{array}$ & $\begin{array}{c}\mathbf{C e} \\
(\mathbf{p p m})\end{array}$ & $\begin{array}{c}\mathbf{C o} \\
(\mathbf{p p m})\end{array}$ & $\begin{array}{c}\mathbf{C r} \\
(\mathbf{p p m})\end{array}$ & $\begin{array}{c}\mathbf{C s} \\
(\mathbf{p p m})\end{array}$ \\
\hline 00U-42/0-10 & $<2$ & 42200 & 1.4 & 646 & 1.2 & $<0.06$ & 25600 & 0.05 & 26.9 & 3.0 & 56.3 & 1.2 \\
00U-42/10-30 & $<2$ & 45000 & 1.5 & 669 & 1.2 & $<0.06$ & 28600 & 0.04 & 28.3 & 3.4 & 56.3 & 1.4 \\
00U-42/30-50 & $<2$ & 53800 & 2.6 & 696 & 1.6 & $<0.06$ & 54200 & 0.04 & 38.1 & 4.9 & 46.5 & 1.7 \\
00U-42/50-63 & $<2$ & 39700 & 2.6 & 656 & 1.1 & 0.06 & 23000 & 0.04 & 31.8 & 3.5 & 28.2 & 1.5 \\
00U-42/63-74 & $<2$ & 40200 & 2.4 & 663 & 1.1 & 0.06 & 27000 & 0.04 & 29.6 & 3.5 & 17 & 1.5 \\
00U-42/74-87 & $<2$ & 38800 & 2.5 & 613 & 1.2 & 0.06 & 35200 & 0.04 & 32.8 & 3.5 & 20.6 & 1.4 \\
00U-42/87-95 & $<2$ & 39200 & 2.6 & 619 & 1.1 & 0.07 & 35800 & 0.04 & 31.2 & 3.6 & 29.8 & 1.4 \\
00U-42/95-106 & $<2$ & 55200 & 2.2 & 697 & 1.4 & $<0.06$ & 54600 & 0.04 & 38.1 & 5.0 & 46.6 & 1.7 \\
00U-42/106-119 & $<2$ & 42400 & 3.2 & 657 & 1.1 & 0.06 & 40200 & 0.03 & 32.3 & 4 & 28.2 & 1.5 \\
00U-42/119-125 & $<2$ & 53600 & 2.6 & 717 & 1.5 & $<0.06$ & 49500 & 0.04 & 35.7 & 4.6 & 48.0 & 1.7 \\
00U-42/125-133 & $<2$ & 38700 & 2.8 & 609 & 1.1 & 0.06 & 41100 & 0.04 & 28 & 3.5 & 21.3 & 1.4
\end{tabular}


Table 5f-2b. ICP-MS data for auger hole 00U-42-Continued.

\begin{tabular}{lcccccccccccc}
\hline \multicolumn{1}{c}{ Sample \# } & $\begin{array}{c}\mathbf{C u} \\
(\mathbf{p p m})\end{array}$ & $\begin{array}{c}\mathbf{F e} \\
(\mathbf{p p m})\end{array}$ & $\begin{array}{c}\mathbf{G a} \\
(\mathbf{p p m})\end{array}$ & $\begin{array}{c}\mathbf{K} \\
(\mathbf{p p m})\end{array}$ & $\begin{array}{c}\mathbf{L a} \\
(\mathbf{p p m})\end{array}$ & $\begin{array}{c}\mathbf{L i} \\
(\mathbf{p p m})\end{array}$ & $\begin{array}{c}\mathbf{M g} \\
(\mathbf{p p m})\end{array}$ & $\begin{array}{c}\mathbf{M n} \\
(\mathbf{p p m})\end{array}$ & $\begin{array}{c}\mathbf{M o} \\
(\mathbf{p p m})\end{array}$ & $\begin{array}{c}\mathbf{N a} \\
(\mathbf{p p m})\end{array}$ & $\begin{array}{c}\mathbf{N b} \\
(\mathbf{p p m})\end{array}$ & $\begin{array}{c}\mathbf{N i} \\
(\mathbf{p p m})\end{array}$ \\
\hline 00U42/0-10 & 13.7 & 12200 & 7.1 & 26600 & 15.6 & 11.1 & 6120 & 258 & 0.45 & 10200 & 5.3 & 7.2 \\
00U42/10-30 & 11.3 & 12700 & 7.6 & 27200 & 15.9 & 12.7 & 6560 & 273 & 0.42 & 10700 & 5.3 & 7.9 \\
00U42/30-50 & 31.0 & 16500 & 9.2 & 29900 & 21.0 & 17.0 & 12500 & 336 & 0.30 & 12100 & 5.4 & 10.0 \\
00U42/50-63 & 13 & 12200 & 7.8 & 23700 & 17.5 & 13.1 & 5520 & 242 & 0.58 & 8940 & 2.1 & 7.9 \\
00U42/63-74 & 12.6 & 11400 & 7.8 & 24400 & 16.4 & 12.8 & 5820 & 233 & 0.47 & 9250 & 2 & 7 \\
00U42/74-87 & 15.7 & 11300 & 7.6 & 22800 & 17.9 & 12.7 & 6660 & 224 & 0.4 & 8940 & 1.5 & 6.8 \\
00U42/87-95 & 16.2 & 11900 & 7.9 & 23000 & 17.1 & 12.9 & 7600 & 232 & 0.43 & 8940 & 2.3 & 7.1 \\
00U42/95-106 & 31.2 & 16600 & 9.3 & 30100 & 21.2 & 17.2 & 12700 & 339 & 0.30 & 12200 & 5.2 & 10.1 \\
00U42/106-119 & 14.6 & 12900 & 8.2 & 24500 & 17.8 & 14.4 & 9410 & 255 & 0.49 & 9510 & 3.4 & 8.4 \\
00U42/119-125 & 13.8 & 16300 & 9.0 & 30400 & 19.7 & 16.5 & 10900 & 334 & 0.34 & 12000 & 6.3 & 10.0 \\
00U42/125-133 & 19 & 11500 & 7.5 & 22300 & 15.4 & 12.8 & 8260 & 236 & 0.51 & 8830 & 1.2 & 7.2
\end{tabular}

Table 5f-2b. ICP-MS data for auger hole 00U-42—Continued.

\begin{tabular}{|c|c|c|c|c|c|c|c|c|c|c|c|c|c|}
\hline Sample \# & $\underset{(p p m)}{P}$ & $\begin{array}{c}\mathrm{Pb} \\
(\mathrm{ppm})\end{array}$ & $\begin{array}{c}\mathbf{R b} \\
(\mathbf{p p m})\end{array}$ & $\begin{array}{c}\text { Sb } \\
(\mathbf{p p m})\end{array}$ & $\begin{array}{c}\text { Sc } \\
(\mathbf{p p m})\end{array}$ & $\begin{array}{c}\mathrm{Sr} \\
(\mathbf{p p m})\end{array}$ & $\begin{array}{c}\text { Th } \\
\text { (ppm) }\end{array}$ & $\begin{array}{c}\mathrm{Ti} \\
(\mathbf{p p m})\end{array}$ & $\begin{array}{c}\mathrm{TI} \\
(\mathrm{ppm})\end{array}$ & $\underset{(\mathbf{p p m})}{\mathbf{U}}$ & $\underset{(\mathbf{p p m})}{V}$ & $\begin{array}{c}Y \\
\text { (ppm) }\end{array}$ & $\begin{array}{c}\mathrm{Zn} \\
(\mathrm{ppm})\end{array}$ \\
\hline 00U42/0-10 & 386 & 11.5 & 54.6 & 0.25 & 3.4 & 117 & 3.59 & 1710 & 0.28 & 0.99 & 24.7 & 14.0 & 16.3 \\
\hline 00U42/10-30 & 372 & 11.4 & 56.2 & 0.26 & 3.9 & 127 & 3.70 & 1820 & 0.28 & 0.98 & 26.9 & 14.4 & 19.0 \\
\hline 00U42/30-50 & 435 & 12.2 & 61.7 & 0.28 & 5.9 & 170 & 4.91 & 2520 & 0.32 & 1.35 & 41.1 & 18.6 & 29.4 \\
\hline 00U42/50-63 & 259 & 10.7 & 56.6 & 0.24 & 4.2 & 130 & 4.58 & 1190 & 0.33 & 1.03 & 27 & 12.6 & 21.3 \\
\hline 00U42/63-74 & 258 & 10.6 & 57.3 & 0.22 & 4.1 & 134 & 4.16 & 1020 & 0.33 & 0.89 & 27.3 & 11.1 & 20.7 \\
\hline 00U42/74-87 & 289 & 10.2 & 54.2 & 0.21 & 4.2 & 138 & 4.98 & 1040 & 0.31 & 1.1 & 27.6 & 12 & 22.3 \\
\hline 00U42/87-95 & 286 & 10.4 & 54.1 & 0.22 & 4.2 & 137 & 4.41 & 1210 & 0.31 & 1.01 & 30 & 11.9 & 21 \\
\hline 00U42/95-106 & 434 & 12.3 & 61.6 & 0.31 & 6.0 & 169 & 4.99 & 2530 & 0.32 & 1.37 & 41.0 & 18.6 & 30.1 \\
\hline 00U42/106-119 & 312 & 10.8 & 57.6 & 0.27 & 4.8 & 161 & 4.79 & 1140 & 0.32 & 1.05 & 32.4 & 12.2 & 23 \\
\hline 00U42/119-125 & 416 & 12.4 & 63.2 & 0.27 & 5.6 & 174 & 4.62 & 2500 & 0.32 & 1.32 & 39.8 & 18.8 & 25.2 \\
\hline 00U42/125-133 & 292 & 10.1 & 52.8 & 0.21 & 4.2 & 154 & 3.76 & 1040 & 0.31 & 0.98 & 28.8 & 11.3 & 20.6 \\
\hline
\end{tabular}


Table 5f-3a. ICP-AES data for auger hole 00U-43.

\begin{tabular}{llllllllllc}
\hline \multicolumn{1}{c}{ Sample \# } & $\mathbf{A l}(\%)$ & $\mathbf{C a}(\%)$ & $\mathbf{F e}(\%)$ & $\mathbf{K}(\%)$ & $\mathbf{M g}(\%)$ & $\mathbf{N a}(\%)$ & $\mathbf{P ~ ( \% )}$ & $\mathbf{T i}(\%)$ & $\mathbf{M n}(\mathbf{p p m})$ & $\mathbf{N d}(\mathbf{p p m})$ \\
\hline 00U-43/0-10 & 3.92 & 2.24 & 1.27 & 2.38 & 0.768 & 0.759 & 0.0457 & 0.149 & 344 & 18.0 \\
00U-43/10-25 & 3.96 & 2.85 & 1.50 & 2.33 & 0.838 & 0.693 & 0.0421 & 0.173 & 350 & 20.9 \\
00U-43/25-34 & 3.76 & 2.74 & 1.35 & 2.19 & 0.746 & 0.684 & 0.0298 & 0.122 & 307 & 14.8 \\
00U-43/34-48 & 4.13 & 3.56 & 1.39 & 2.45 & 0.736 & 0.812 & 0.0304 & 0.156 & 264 & 19.1 \\
00U-43/48-55 & 3.65 & 4.69 & 1.30 & 2.14 & 0.937 & 0.716 & 0.0310 & 0.109 & 288 & 12.8 \\
00U-43/55-64 & 3.91 & 11.2 & 1.44 & 2.17 & 1.68 & 0.664 & 0.0395 & 0.143 & 381 & 21.0 \\
00U-43/64-76 & 3.61 & 7.54 & 1.44 & 1.97 & 1.43 & 0.634 & 0.0403 & 0.118 & 370 & 14.8 \\
00U-43/76-86 & 3.75 & 7.37 & 1.38 & 2.05 & 1.62 & 0.658 & 0.0387 & 0.134 & 340 & 13.2 \\
00U-43/86-96 & 3.54 & 7.47 & 1.33 & 1.94 & 1.89 & 0.673 & 0.0363 & 0.117 & 351 & 14.5 \\
00U-43/96-108 & 4.11 & 5.68 & 1.44 & 2.35 & 1.88 & 0.821 & 0.0422 & 0.146 & 396 & 20.0 \\
00U-43/108-114 & 4.12 & 4.99 & 1.65 & 2.36 & 1.72 & 0.831 & 0.0403 & 0.155 & 385 & 19.1 \\
\hline
\end{tabular}

Table 5f-3b. ICP-MS data for auger hole 00U-43.

\begin{tabular}{|c|c|c|c|c|c|c|c|c|c|c|c|c|}
\hline Sample \# & 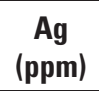 & $\begin{array}{c}\text { Al } \\
\text { (ppm) }\end{array}$ & $\begin{array}{c}\text { As } \\
\text { (ppm) }\end{array}$ & $\begin{array}{c}\mathrm{Ba} \\
\text { (ppm) }\end{array}$ & $\begin{array}{c}\text { Be } \\
\text { (ppm) }\end{array}$ & $\underset{(\mathrm{ppm})}{\mathrm{Bi}}$ & $\begin{array}{c}\mathrm{Ca} \\
\text { (ppm) }\end{array}$ & $\underset{(\mathbf{p p m})}{\mathbf{C d}}$ & $\begin{array}{c}\mathrm{Ce} \\
\text { (ppm) }\end{array}$ & $\begin{array}{c}\text { Co } \\
\text { (ppm) }\end{array}$ & $\underset{(\mathbf{p p m})}{\mathrm{Cr}}$ & $\begin{array}{c}\text { Cs } \\
(\mathbf{p p m})\end{array}$ \\
\hline 00U-43/0-10 & $<2$ & 51300 & 2.6 & 700 & 1.4 & $<0.06$ & 26600 & 0.08 & 36.0 & 4.6 & 49.2 & 2.0 \\
\hline 00U-43/10-25 & $<2$ & 53000 & 4.7 & 673 & 1.4 & $<0.06$ & 34700 & 0.07 & 42.7 & 5.6 & 62.6 & 2.3 \\
\hline 00U-43/25-34 & $<2$ & 37800 & 3.2 & 578 & 1.1 & 0.08 & 27800 & 0.04 & 31.6 & 4 & 24 & 1.8 \\
\hline 00U-43/34-48 & $<2$ & 52600 & 3.1 & 708 & 1.5 & $<0.06$ & 41500 & 0.04 & 36.6 & 5.0 & 54.6 & 2.0 \\
\hline 00U-43/48-55 & $<2$ & 36900 & 3.2 & 592 & 1.1 & 0.07 & 48600 & 0.05 & 29.7 & 4.1 & 20.2 & 1.6 \\
\hline 00U-43/55-64 & $<2$ & 46300 & 4.3 & 629 & 1.5 & $<0.06$ & 125000 & 0.05 & 39.9 & 5.9 & 47.7 & 2.0 \\
\hline 00U-43/64-76 & $<2$ & 36200 & 4.2 & 568 & 1.2 & 0.08 & 79500 & 0.04 & 34 & 5 & 18.8 & 1.9 \\
\hline 00U-43/76-86 & $<2$ & 36100 & 4.2 & 768 & 1.1 & 0.07 & 73400 & 0.04 & 30.8 & 4.5 & 25.6 & 1.7 \\
\hline 00U-43/86-96 & $<2$ & 34400 & 4.6 & 882 & 1 & 0.07 & 74900 & 0.04 & 31.7 & 4.3 & 40.2 & 1.6 \\
\hline 00U-43/96-108 & $<2$ & 49800 & 4.6 & 1010 & 1.0 & $<0.06$ & 64100 & 0.03 & 36.9 & 5.5 & 51.0 & 2.0 \\
\hline 00U-43/108-114 & $<2$ & 50700 & 4.8 & 894 & 1.3 & $<0.06$ & 57100 & 0.04 & 36.1 & 5.8 & 99.8 & 1.9 \\
\hline
\end{tabular}


Table 5f-3b. ICP-MS data for auger hole 00U-43-Continued.

\begin{tabular}{|c|c|c|c|c|c|c|c|c|c|c|c|c|}
\hline Sample \# & $\underset{(p p m)}{\mathrm{Cu}}$ & $\begin{array}{c}\mathrm{Fe} \\
(\mathrm{ppm})\end{array}$ & $\underset{(\mathrm{ppm})}{\mathbf{G a}}$ & $\underset{(\mathbf{p p m})}{\mathrm{K}}$ & $\begin{array}{c}\text { La } \\
(\mathbf{p p m})\end{array}$ & $\begin{array}{c}\mathrm{Li} \\
(\mathrm{ppm})\end{array}$ & $\begin{array}{c}\mathrm{Mg} \\
(\mathrm{ppm})\end{array}$ & $\underset{(\mathrm{ppm})}{\mathrm{Mn}}$ & $\begin{array}{c}\text { Mo } \\
(\mathrm{ppm})\end{array}$ & $\begin{array}{c}\mathrm{Na} \\
(\mathrm{ppm})\end{array}$ & $\begin{array}{c}\mathbf{N b} \\
(\mathbf{p p m})\end{array}$ & $\underset{(\mathrm{ppm})}{\mathrm{Ni}}$ \\
\hline 00U-43/0-10 & 11.7 & 15000 & 8.3 & 30000 & 20.2 & 17.2 & 10100 & 427 & 0.41 & 10300 & 6.5 & 9.9 \\
\hline 00U-43/10-25 & 18.6 & 18800 & 8.9 & 30600 & 23.6 & 20.1 & 11300 & 440 & 0.54 & 9590 & 7.3 & 11.9 \\
\hline 00U-43/25-34 & 13.1 & 12800 & 7.3 & 22100 & 17.4 & 15.5 & 8240 & 292 & 0.53 & 7370 & 2.5 & 8.6 \\
\hline 00U-43/34-48 & 21.8 & 17100 & 8.9 & 30500 & 20.3 & 17.4 & 9540 & 313 & 0.47 & 10800 & 5.9 & 10.4 \\
\hline 00U-43/48-55 & 11.5 & 12600 & 7.2 & 22000 & 16.6 & 14.2 & 10300 & 278 & 0.39 & 7580 & 1.5 & 7.6 \\
\hline 00U-43/55-64 & 22.8 & 17300 & 8.0 & 24700 & 22.1 & 19.2 & 20800 & 457 & 0.36 & 8120 & 5.2 & 10.4 \\
\hline 00U-43/64-76 & 16.1 & 14000 & 7.2 & 19800 & 18.6 & 17.4 & 15300 & 360 & 0.42 & 6750 & 1 & 8.9 \\
\hline 00U-43/76-86 & 13.7 & 13100 & 7.1 & 19800 & 16.8 & 17.3 & 17100 & 322 & 0.4 & 6720 & 1.7 & 8.1 \\
\hline 00U-43/86-96 & 12.8 & 12600 & 6.9 & 19200 & 17.2 & 16.9 & 19700 & 334 & 1.2 & 6860 & 1.1 & 7.9 \\
\hline 00U-43/96-108 & 19.1 & 17200 & 8.2 & 27700 & 20.4 & 20.9 & 23500 & 474 & 0.62 & 10500 & 5.3 & 11.1 \\
\hline 00U-43/108-114 & 18.1 & 20100 & 8.6 & 28500 & 20.0 & 20.5 & 22200 & 469 & 0.89 & 10800 & 4.9 & 13.8 \\
\hline
\end{tabular}

Table 5f-3b. ICP-MS data for auger hole 00U-43-Continued.

\begin{tabular}{lccccccccccccc}
\hline \multicolumn{1}{c}{ Sample \# } & $\begin{array}{c}\mathbf{P} \\
(\mathbf{p p m})\end{array}$ & $\begin{array}{c}\mathbf{P b} \\
(\mathbf{p p m})\end{array}$ & $\begin{array}{c}\mathbf{R b} \\
(\mathbf{p p m})\end{array}$ & $\begin{array}{c}\mathbf{S b} \\
(\mathbf{p p m})\end{array}$ & $\begin{array}{c}\mathbf{S c} \\
(\mathbf{p p m})\end{array}$ & $\begin{array}{c}\mathbf{S r} \\
(\mathbf{p p m})\end{array}$ & $\begin{array}{c}\mathbf{T h} \\
(\mathbf{p p m})\end{array}$ & $\begin{array}{c}\mathbf{T i} \\
(\mathbf{p p m})\end{array}$ & $\begin{array}{c}\mathbf{T I} \\
(\mathbf{p p m})\end{array}$ & $\begin{array}{c}\mathbf{U} \\
\mathbf{( p p m})\end{array}$ & $\begin{array}{c}\mathbf{V} \\
(\mathbf{p p m})\end{array}$ & $\begin{array}{c}\mathbf{Y} \\
(\mathbf{p p m})\end{array}$ & $\begin{array}{c}\mathbf{Z n} \\
(\mathbf{p p m})\end{array}$ \\
\hline 00U-43/0-10 & 611 & 14.0 & 63.7 & 0.36 & 5.3 & 142 & 4.81 & 2500 & 0.32 & 1.32 & 34.2 & 18.6 & 27.5 \\
00U-43/10-25 & 572 & 14.1 & 66.6 & 0.39 & 6.1 & 162 & 5.96 & 2950 & 0.34 & 1.67 & 41.8 & 22.3 & 31.2 \\
00U-43/25-34 & 308 & 11 & 54.5 & 0.28 & 4.7 & 167 & 4.76 & 1140 & 0.34 & 1.13 & 29.6 & 12.5 & 23.9 \\
00U-43/34-48 & 392 & 12.6 & 63.7 & 0.32 & 5.9 & 204 & 4.85 & 2500 & 0.34 & 1.30 & 39.6 & 18.7 & 27.4 \\
00U-43/48-55 & 313 & 10.3 & 52.3 & 0.25 & 4.8 & 238 & 4.35 & 1060 & 0.31 & 1.03 & 31.6 & 12.6 & 21.7 \\
00U-43/55-64 & 540 & 11.5 & 53.3 & 0.32 & 6.6 & 603 & 5.37 & 2320 & 0.27 & 1.52 & 43.5 & 20.9 & 33.8 \\
00U-43/64-76 & 412 & 10 & 48.6 & 0.27 & 5.2 & 585 & 5.07 & 1110 & 0.29 & 1.21 & 36.8 & 13.2 & 25.6 \\
00U-43/76-86 & 366 & 10 & 47.8 & 0.24 & 5 & 787 & 4.49 & 1250 & 0.28 & 1.24 & 36.3 & 13 & 24.7 \\
00U-43/86-96 & 367 & 9.6 & 45.9 & 0.26 & 4.8 & 835 & 4.75 & 1140 & 0.27 & 1.32 & 35.9 & 12.9 & 22.9 \\
00U-43/96-108 & 549 & 12.0 & 57.7 & 0.33 & 6.1 & 704 & 4.85 & 2370 & 0.30 & 1.64 & 49.3 & 19.0 & 29.1 \\
00U-43/108-114 & 536 & 12.0 & 58.8 & 0.38 & 6.2 & 865 & 4.86 & 2500 & 0.31 & 1.68 & 48.0 & 19.6 & 28.4 \\
\hline
\end{tabular}




\section{Table 6. Magnetic properties}

[Sample \#: Unique sample number (e.g. 00U-41A/0-10); presented as sample designation (00U-41), transect sampling location (A), and depth in centimeters $(0-10)$.

MSlf/MShf: Low frequency (lf) and high frequency (hf) magnetic susceptibility in $\mathrm{m}^{3} / \mathrm{kg}$.

FDMS: Frequency-dependent magnetic susceptibility. Expressed in percent and $\mathrm{m}^{3} / \mathrm{kg}$.

ARM: Anhysteretic remanent magnetization in $\mathrm{Am}^{2} / \mathrm{kg}$.

IRM 1.2: Isothermal remanent magnetization from induction in a 1.2 tesla field at room temperature. Expressed in $\mathrm{Am}^{2} / \mathrm{kg}$.

IRM -0.3: Isothermal remanent magnetization from induction in a -0.3 tesla field at room temperature. Expressed in $\mathrm{Am}^{2} / \mathrm{kg}$.

HIRM: Hard isothermal remanent magnetization: HIRM is calculated as: (IRM1.2T + IRM-0.3T)/2.

S: (S ratio) calculated as IRM-0.3T/IRM1.2T.

n.d.: not determined]

Table 6a-1. Magnetic properties of the less than 2-mm size fraction for the 00U-29 transect.

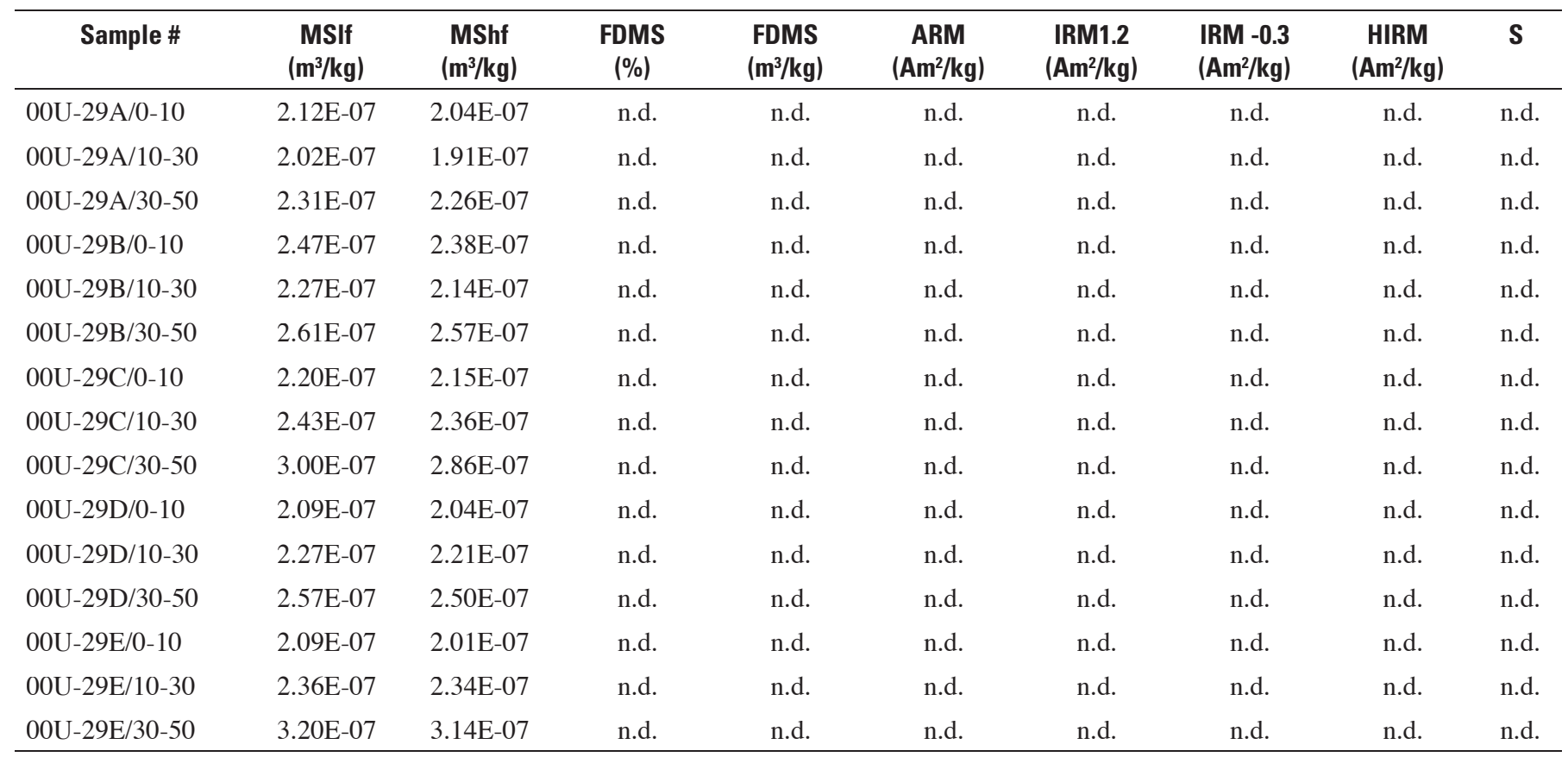


Table 6a-2a. Magnetic properties of the less than $2 \mathrm{~mm}$ size fraction for the $00 \mathrm{U}-30$ transect.

\begin{tabular}{|c|c|c|c|c|c|c|c|c|c|}
\hline Sample \# & $\begin{array}{c}\text { MSIf } \\
\left(\mathbf{m}^{3} / \mathbf{k g}\right)\end{array}$ & $\begin{array}{c}\text { MShf } \\
\left(\mathbf{m}^{3} / \mathbf{k g}\right)\end{array}$ & $\begin{array}{c}\text { FDMS } \\
(\%)\end{array}$ & $\begin{array}{l}\text { FDMS } \\
\left(\mathrm{m}^{3} / \mathbf{k g}\right)\end{array}$ & $\begin{array}{c}\text { ARM } \\
\left(\mathrm{Am}^{2} / \mathbf{k g}\right)\end{array}$ & $\begin{array}{c}\text { IRM1.2 } \\
\left(\mathrm{Am}^{2} / \mathrm{kg}\right)\end{array}$ & $\begin{array}{l}\text { IRM -0.3 } \\
\left(\mathrm{Am}^{2} / \mathbf{k g}\right)\end{array}$ & $\begin{array}{c}\text { HIRM } \\
\left(\mathrm{Am}^{2} / \mathbf{k g}\right)\end{array}$ & $\mathbf{S}$ \\
\hline 00U-30A/0-10 & 3.03E-08 & $2.98 \mathrm{E}-08$ & 1.68 & $5.08 \mathrm{E}-10$ & 1.19E-05 & 5.27E-04 & $2.64 \mathrm{E}-04$ & 1.31E-04 & 0.50 \\
\hline 00U-30A/10-30 & $1.34 \mathrm{E}-08$ & 1.33E-08 & 0.76 & $1.02 \mathrm{E}-10$ & 5.91E-06 & $5.22 \mathrm{E}-04$ & $5.38 \mathrm{E}-05$ & 2.34E-04 & 0.10 \\
\hline 00U-30A/30-40 & $1.87 \mathrm{E}-08$ & $1.96 \mathrm{E}-08$ & -4.60 & $-8.63 \mathrm{E}-10$ & 5.34E-06 & 3.78E-04 & $9.04 \mathrm{E}-05$ & 1.44E-04 & 0.24 \\
\hline 00U-30B/0-10 & 7.72E-08 & 7.80E-08 & -1.11 & $-8.59 \mathrm{E}-10$ & 3.47E-05 & $1.32 \mathrm{E}-03$ & $8.27 \mathrm{E}-04$ & 2.46E-04 & 0.63 \\
\hline $00 \mathrm{U}-30 \mathrm{~B} / 10-30$ & $5.09 \mathrm{E}-08$ & $5.25 \mathrm{E}-08$ & -3.25 & $-1.65 \mathrm{E}-09$ & $1.99 \mathrm{E}-05$ & 8.23E-04 & $5.00 \mathrm{E}-04$ & 1.61E-04 & 0.61 \\
\hline 00U-30B/30-50 & $4.27 \mathrm{E}-08$ & 4.61E-08 & -7.91 & $-3.38 \mathrm{E}-09$ & $1.82 \mathrm{E}-05$ & $6.87 \mathrm{E}-04$ & $3.74 \mathrm{E}-04$ & 1.57E-04 & 0.54 \\
\hline 00U-30C/0-10 & $1.03 \mathrm{E}-07$ & $9.86 \mathrm{E}-08$ & 4.40 & 4.53E-09 & $4.25 \mathrm{E}-05$ & $1.56 \mathrm{E}-03$ & $1.00 \mathrm{E}-03$ & 2.81E-04 & 0.64 \\
\hline 00U-30C/10-30 & $1.15 \mathrm{E}-07$ & 1.09E-07 & 5.32 & $6.12 \mathrm{E}-09$ & $5.30 \mathrm{E}-05$ & $1.71 \mathrm{E}-03$ & $1.21 \mathrm{E}-03$ & 2.52E-04 & 0.70 \\
\hline 00U-30C/30-46 & $1.28 \mathrm{E}-07$ & $1.22 \mathrm{E}-07$ & 4.99 & $6.38 \mathrm{E}-09$ & $6.89 \mathrm{E}-05$ & $1.90 \mathrm{E}-03$ & $1.38 \mathrm{E}-03$ & 2.59E-04 & 0.73 \\
\hline 00U-30C/46-62 & $1.08 \mathrm{E}-07$ & $1.05 \mathrm{E}-07$ & 2.24 & 2.42E-09 & 7.41E-05 & $1.61 \mathrm{E}-03$ & $1.25 \mathrm{E}-03$ & $1.76 \mathrm{E}-04$ & 0.78 \\
\hline 00U-30C/62-81 & $1.06 \mathrm{E}-07$ & 1.04E-07 & 2.54 & $2.70 \mathrm{E}-09$ & $6.60 \mathrm{E}-05$ & $1.65 \mathrm{E}-03$ & $1.30 \mathrm{E}-03$ & $1.78 \mathrm{E}-04$ & 0.78 \\
\hline 00U-30C/81-93 & $1.08 \mathrm{E}-07$ & $1.02 \mathrm{E}-07$ & 5.59 & $6.06 \mathrm{E}-09$ & $5.00 \mathrm{E}-05$ & $1.58 \mathrm{E}-03$ & $1.27 \mathrm{E}-03$ & $1.57 \mathrm{E}-04$ & 0.80 \\
\hline 00U-30C/93-104 & $1.19 \mathrm{E}-07$ & $1.17 \mathrm{E}-07$ & 1.77 & $2.11 \mathrm{E}-09$ & 4.11E-05 & $1.77 \mathrm{E}-03$ & $1.41 \mathrm{E}-03$ & $1.81 \mathrm{E}-04$ & 0.80 \\
\hline 00U-30C/104-118 & $1.44 \mathrm{E}-07$ & 1.40E-07 & 2.65 & 3.81E-09 & 3.87E-05 & 2.07E-03 & $1.70 \mathrm{E}-03$ & 1.84E-04 & 0.82 \\
\hline 00U-30C/118-128 & $1.75 \mathrm{E}-07$ & 1.71E-07 & 1.81 & $3.16 \mathrm{E}-09$ & 4.42E-05 & $2.55 \mathrm{E}-03$ & $2.01 \mathrm{E}-03$ & $2.25 \mathrm{E}-04$ & 0.82 \\
\hline 00U-30C/128-142 & 2.03E-07 & 2.01E-07 & 0.77 & $1.57 \mathrm{E}-09$ & $4.68 \mathrm{E}-05$ & $2.99 \mathrm{E}-03$ & $2.44 \mathrm{E}-03$ & $2.75 \mathrm{E}-04$ & 0.82 \\
\hline 00U-30D/0-10 & $8.82 \mathrm{E}-08$ & 8.02E-08 & 9.08 & $8.00 \mathrm{E}-09$ & 4.19E-05 & $1.35 \mathrm{E}-03$ & $9.39 \mathrm{E}-04$ & 2.07E-04 & 0.69 \\
\hline 00U-30D/10-30 & $1.12 \mathrm{E}-07$ & $1.03 \mathrm{E}-07$ & 7.41 & $8.27 \mathrm{E}-09$ & $4.30 \mathrm{E}-05$ & $1.48 \mathrm{E}-03$ & $1.06 \mathrm{E}-03$ & $2.10 \mathrm{E}-04$ & 0.72 \\
\hline $00 \mathrm{U}-30 \mathrm{D} / 30-50$ & $9.70 \mathrm{E}-08$ & $9.06 \mathrm{E}-08$ & 6.65 & $6.46 \mathrm{E}-09$ & 4.17E-05 & $1.40 \mathrm{E}-03$ & $9.58 \mathrm{E}-04$ & 2.23E-04 & 0.68 \\
\hline 00U-30E/0-10 & $1.19 \mathrm{E}-07$ & $1.15 \mathrm{E}-07$ & 4.06 & 4.85E-09 & 4.19E-05 & $1.33 \mathrm{E}-03$ & $1.06 \mathrm{E}-03$ & 1.34E-04 & 0.80 \\
\hline 00U-30E/10-30 & $1.34 \mathrm{E}-07$ & $1.27 \mathrm{E}-07$ & 5.02 & $6.71 \mathrm{E}-09$ & $5.68 \mathrm{E}-05$ & $1.96 \mathrm{E}-03$ & $1.38 \mathrm{E}-03$ & 2.91E-04 & 0.70 \\
\hline 00U-30E/30-50 & $1.21 \mathrm{E}-07$ & 1.16E-07 & 3.90 & 4.73E-09 & $5.34 \mathrm{E}-05$ & $1.74 \mathrm{E}-03$ & $1.27 \mathrm{E}-03$ & 2.33E-04 & 0.73 \\
\hline 00U-30F/0-10 & $1.47 \mathrm{E}-07$ & $1.43 \mathrm{E}-07$ & 2.44 & $3.59 \mathrm{E}-09$ & $5.84 \mathrm{E}-05$ & $1.90 \mathrm{E}-03$ & $1.40 \mathrm{E}-03$ & 2.46E-04 & 0.74 \\
\hline 00U-30F/10-30 & $1.01 \mathrm{E}-07$ & $1.21 \mathrm{E}-07$ & -10.16 & $-1.11 \mathrm{E}-08$ & $5.70 \mathrm{E}-05$ & $1.86 \mathrm{E}-03$ & $1.36 \mathrm{E}-03$ & $2.47 \mathrm{E}-04$ & 0.73 \\
\hline 00U-30F/30-50 & $1.29 \mathrm{E}-07$ & $1.25 \mathrm{E}-07$ & 2.58 & 3.32E-09 & $5.55 \mathrm{E}-05$ & $1.83 \mathrm{E}-03$ & $1.37 \mathrm{E}-03$ & 2.31E-04 & 0.75 \\
\hline 00U-30G/0-10 & $1.78 \mathrm{E}-07$ & $1.64 \mathrm{E}-07$ & 7.58 & $1.35 \mathrm{E}-08$ & $7.88 \mathrm{E}-05$ & $2.45 \mathrm{E}-03$ & $1.98 \mathrm{E}-03$ & 2.34E-04 & 0.81 \\
\hline 00U-30G/10-30 & $1.71 \mathrm{E}-07$ & 1.61E-07 & 6.00 & $1.03 \mathrm{E}-08$ & $8.18 \mathrm{E}-05$ & $2.62 \mathrm{E}-03$ & $1.89 \mathrm{E}-03$ & $3.65 \mathrm{E}-04$ & 0.72 \\
\hline 00U-30G/30-50 & $1.80 \mathrm{E}-07$ & $1.66 \mathrm{E}-07$ & 7.86 & $1.42 \mathrm{E}-08$ & $9.20 \mathrm{E}-05$ & $2.44 \mathrm{E}-03$ & $1.84 \mathrm{E}-03$ & 2.98E-04 & 0.76 \\
\hline 00U-30H/0-10 & $1.95 \mathrm{E}-07$ & $1.87 \mathrm{E}-07$ & 4.24 & $8.27 \mathrm{E}-09$ & $6.72 \mathrm{E}-05$ & $2.26 \mathrm{E}-03$ & $1.69 \mathrm{E}-03$ & 2.83E-04 & 0.75 \\
\hline 00U-30H/10-30 & $1.80 \mathrm{E}-07$ & 1.77E-07 & 1.77 & $3.18 \mathrm{E}-09$ & $8.30 \mathrm{E}-05$ & $2.50 \mathrm{E}-03$ & $1.89 \mathrm{E}-03$ & 3.09E-04 & 0.75 \\
\hline $00 \mathrm{U}-30 \mathrm{H} / 30-50$ & $2.44 \mathrm{E}-07$ & 2.43E-07 & 0.68 & $1.65 \mathrm{E}-09$ & $1.19 \mathrm{E}-04$ & $2.91 \mathrm{E}-03$ & $2.64 \mathrm{E}-03$ & 1.35E-04 & 0.91 \\
\hline 00U-30I/0-10 & $1.83 \mathrm{E}-07$ & $1.81 \mathrm{E}-07$ & 1.20 & $2.19 \mathrm{E}-09$ & 7.7 & 2.5 & $1.94 \mathrm{I}$ & $3.16 \mathrm{E}-04$ & 0.75 \\
\hline 00U-30I/10-30 & $1.95 \mathrm{E}-07$ & $1.95 \mathrm{E}-07$ & 0.26 & $5.12 \mathrm{E}-10$ & $8.72 \mathrm{E}-05$ & $2.84 \mathrm{E}-03$ & $2.12 \mathrm{E}-03$ & 3.59E-04 & 0.75 \\
\hline 00U-30I/30-50 & $2.31 \mathrm{E}-07$ & 2.19E-07 & 5.48 & $1.27 \mathrm{E}-08$ & $1.21 \mathrm{E}-04$ & $3.20 \mathrm{E}-03$ & $2.62 \mathrm{E}-03$ & $2.90 \mathrm{E}-04$ & 0.82 \\
\hline 00U-30J/0-10 & $1.83 \mathrm{E}-07$ & 1.77E-07 & 3.21 & $5.87 \mathrm{E}-09$ & $8.10 \mathrm{E}-05$ & $2.88 \mathrm{E}-03$ & $2.15 \mathrm{E}-03$ & $3.62 \mathrm{E}-04$ & 0.75 \\
\hline $00 \mathrm{U}-30 \mathrm{~J} / 10-30$ & $1.77 \mathrm{E}-07$ & 1.71E-07 & 3.60 & $6.39 \mathrm{E}-09$ & $8.62 \mathrm{E}-05$ & $2.59 \mathrm{E}-03$ & $1.93 \mathrm{E}-03$ & 3.31E-04 & 0.75 \\
\hline 00U-30J/30-50 & $1.82 \mathrm{E}-07$ & $1.70 \mathrm{E}-07$ & 6.27 & $1.14 \mathrm{E}-08$ & $1.01 \mathrm{E}-04$ & $2.54 \mathrm{E}-03$ & $1.97 \mathrm{E}-03$ & 2.83E-04 & 0.78 \\
\hline 00U-30K/0-10 & $1.90 \mathrm{E}-07$ & $1.85 \mathrm{E}-07$ & 2.51 & $4.78 \mathrm{E}-09$ & $8.77 \mathrm{E}-05$ & $3.02 \mathrm{E}-03$ & $2.20 \mathrm{E}-03$ & 4.08E-04 & 0.73 \\
\hline 00U-30K/10-30 & 2.13E-07 & 2.04E-07 & 4.24 & $9.05 \mathrm{E}-09$ & $1.08 \mathrm{E}-04$ & $3.04 \mathrm{E}-03$ & $2.38 \mathrm{E}-03$ & 3.32E-04 & 0.78 \\
\hline 00U-30K/30-50 & 2.53E-07 & $2.48 \mathrm{E}-07$ & 1.94 & $4.89 \mathrm{E}-09$ & $1.53 \mathrm{E}-04$ & $3.51 \mathrm{E}-03$ & $2.91 \mathrm{E}-03$ & $3.02 \mathrm{E}-04$ & 0.83 \\
\hline 00U-30L/0-10 & $1.73 \mathrm{E}-07$ & 1.73E-07 & 0.37 & $6.46 \mathrm{E}-10$ & 7.43E-05 & 2.87E-03 & $2.14 \mathrm{E}-03$ & $3.65 \mathrm{E}-04$ & 0.75 \\
\hline 00U-30L/10-30 & $1.68 \mathrm{E}-07$ & 1.66E-07 & 1.08 & $1.82 \mathrm{E}-09$ & 8.67E-05 & $2.43 \mathrm{E}-03$ & $1.81 \mathrm{E}-03$ & 3.01E-04 & 0.75 \\
\hline 00U-30L/30-50 & $1.70 \mathrm{E}-07$ & $1.62 \mathrm{E}-07$ & 4.54 & 7.70E-09 & $1.07 \mathrm{E}-04$ & $2.43 \mathrm{E}-03$ & $1.95 \mathrm{E}-03$ & $2.42 \mathrm{E}-04$ & 0.80 \\
\hline 00U-30M/0-10 & $1.95 \mathrm{E}-07$ & 1.89E-07 & 3.31 & $6.46 \mathrm{E}-09$ & $9.73 \mathrm{E}-05$ & 3.03E-03 & $2.30 \mathrm{E}-03$ & 3.66E-04 & 0.76 \\
\hline 00U-30M/10-30 & $1.91 \mathrm{E}-07$ & $1.80 \mathrm{E}-07$ & 5.5 & $1.06 \mathrm{E}-08$ & $9.90 \mathrm{E}-05$ & $2.77 \mathrm{E}-03$ & 2.11E-03 & $3.29 \mathrm{E}-04$ & 0.76 \\
\hline 00U-30M/30-47 & 2.11E-07 & $1.95 \mathrm{E}-07$ & 7.71 & $1.63 \mathrm{E}-08$ & $1.08 \mathrm{E}-04$ & $2.94 \mathrm{E}-03$ & $2.32 \mathrm{E}-03$ & $3.10 \mathrm{E}-04$ & 0.79 \\
\hline 00U-30M/47-61 & $2.22 \mathrm{E}-07$ & 2.11E-07 & 5.02 & $1.12 \mathrm{E}-08$ & $1.28 \mathrm{E}-04$ & $3.14 \mathrm{E}-03$ & $2.51 \mathrm{E}-03$ & 3.13E-04 & 0.80 \\
\hline 00U-30M/61-74 & $2.41 \mathrm{E}-07$ & 2.29E-07 & 4.94 & $1.19 \mathrm{E}-08$ & $1.59 \mathrm{E}-04$ & $3.38 \mathrm{E}-03$ & $2.87 \mathrm{E}-03$ & $2.55 \mathrm{E}-04$ & 0.85 \\
\hline 00U-30M/74-89 & $2.31 \mathrm{E}-07$ & 2.26E-07 & 2.40 & $5.54 \mathrm{E}-09$ & $1.34 \mathrm{E}-04$ & $3.17 \mathrm{E}-03$ & $2.75 \mathrm{E}-03$ & $2.11 \mathrm{E}-04$ & 0.87 \\
\hline 00U-30M/89-106 & $3.51 \mathrm{E}-07$ & $3.41 \mathrm{E}-07$ & 2.86 & $1.01 \mathrm{E}-08$ & $1.12 \mathrm{E}-04$ & $4.76 \mathrm{E}-03$ & $4.01 \mathrm{E}-03$ & 3.33E-04 & 0.86 \\
\hline 00U-30M/106-118 & 4.19E-07 & 4.09E-07 & 2.54 & $1.06 \mathrm{E}-08$ & $1.05 \mathrm{E}-04$ & $6.13 \mathrm{E}-03$ & $5.36 \mathrm{E}-03$ & $3.87 \mathrm{E}-04$ & 0.87 \\
\hline 00U-30M/118-131 & $3.02 \mathrm{E}-07$ & 2.93E-07 & 2.98 & $9.01 \mathrm{E}-09$ & $8.39 \mathrm{E}-05$ & $4.38 \mathrm{E}-03$ & $3.74 \mathrm{E}-03$ & 3.19E-04 & 0.85 \\
\hline 00U-30M/131-144 & 2.74E-07 & 2.65E-07 & 3.33 & $9.12 \mathrm{E}-09$ & $7.85 \mathrm{E}-05$ & $3.75 \mathrm{E}-03$ & $3.17 \mathrm{E}-03$ & 2.87E-04 & 0.85 \\
\hline
\end{tabular}


Table 6a-2b. Magnetic properties of the less than 63-micron size fraction for the 00U-30 transect.

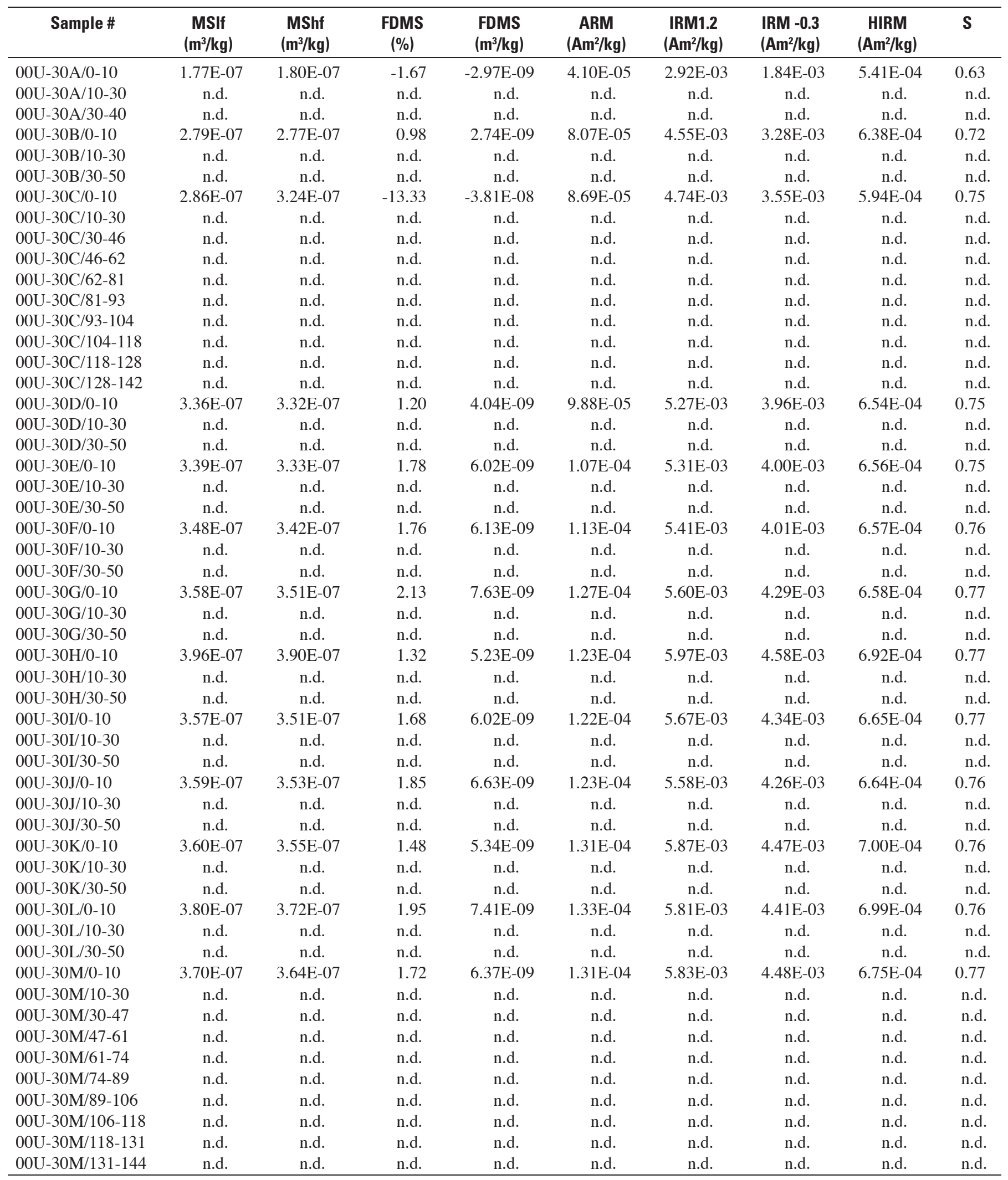


Table 6a-3a. Magnetic properties of the less than 2-mm size fraction for soil pit VP-1.

\begin{tabular}{|c|c|c|c|c|c|c|c|c|c|}
\hline Sample \# & $\begin{array}{c}\text { MSIf } \\
\left(\mathbf{m}^{3} / \mathbf{k g}\right)\end{array}$ & $\begin{array}{c}\text { MShf } \\
\left(\mathbf{m}^{3} / \mathbf{k g}\right)\end{array}$ & $\begin{array}{c}\text { FDMS } \\
(\%)\end{array}$ & $\begin{array}{l}\text { FDMS } \\
\left(\mathrm{m}^{3} / \mathbf{k g}\right)\end{array}$ & $\begin{array}{c}\text { ARM } \\
\left(\mathrm{Am}^{2} / \mathbf{k g}\right)\end{array}$ & $\begin{array}{c}\text { IRM1.2 } \\
\left(\mathrm{Am}^{2} / \mathrm{kg}\right)\end{array}$ & $\begin{array}{l}\text { IRM -0.3 } \\
\left(\mathrm{Am}^{2} / \mathrm{kg}\right)\end{array}$ & $\begin{array}{c}\text { HIRM } \\
\left(\mathrm{Am}^{2} / \mathbf{k g}\right)\end{array}$ & $\mathbf{S}$ \\
\hline VP-1/0-6 & 2.03E-07 & $2.06 \mathrm{E}-07$ & -1.46 & $-2.96 \mathrm{E}-09$ & $8.66 \mathrm{E}-05$ & $3.25 \mathrm{E}-03$ & $2.48 \mathrm{E}-03$ & $3.84 \mathrm{E}-04$ & 0.76 \\
\hline VP-1/6-19 & $2.23 \mathrm{E}-07$ & $2.26 \mathrm{E}-07$ & -1.62 & $-3.62 \mathrm{E}-09$ & $9.83 \mathrm{E}-05$ & $3.28 \mathrm{E}-03$ & $2.48 \mathrm{E}-03$ & 4.03E-04 & 0.75 \\
\hline VP-1/19-30 & $2.43 \mathrm{E}-07$ & $2.49 \mathrm{E}-07$ & -2.27 & $-5.52 \mathrm{E}-09$ & 1.19E-04 & $3.55 \mathrm{E}-03$ & $2.81 \mathrm{E}-03$ & $3.74 \mathrm{E}-04$ & 0.79 \\
\hline VP-1/30-55 & 3.32E-07 & $3.37 \mathrm{E}-07$ & -1.57 & $-5.21 \mathrm{E}-09$ & $1.57 \mathrm{E}-04$ & $4.74 \mathrm{E}-03$ & $3.96 \mathrm{E}-03$ & $3.90 \mathrm{E}-04$ & 0.84 \\
\hline VP-1/55-66 & $3.82 \mathrm{E}-07$ & $3.87 \mathrm{E}-07$ & -1.20 & $-4.59 \mathrm{E}-09$ & $1.10 \mathrm{E}-04$ & $5.59 \mathrm{E}-03$ & $4.85 \mathrm{E}-03$ & 3.67E-04 & 0.87 \\
\hline VP-1/66-78 & $3.42 \mathrm{E}-07$ & $3.42 \mathrm{E}-07$ & 0.16 & $5.64 \mathrm{E}-10$ & $9.97 \mathrm{E}-05$ & $5.01 \mathrm{E}-03$ & 4.30E-03 & $3.59 \mathrm{E}-04$ & 0.86 \\
\hline VP-1/80-90 & 2.50E-07 & 2.51E-07 & -0.19 & $-4.77 \mathrm{E}-10$ & 7.09E-05 & $3.57 \mathrm{E}-03$ & 3.04E-03 & 2.62E-04 & 0.85 \\
\hline
\end{tabular}

Table 6a-3b. Magnetic properties of the less than 63-micron size fraction for soil pit VP-1.

\begin{tabular}{|c|c|c|c|c|c|c|c|c|c|}
\hline Sample \# & $\begin{array}{c}\text { MSIf } \\
\left(\mathbf{m}^{3} / \mathbf{k g}\right)\end{array}$ & $\begin{array}{c}\text { MShf } \\
\left(\mathrm{m}^{3} / \mathrm{kg}\right)\end{array}$ & $\begin{array}{c}\text { FDMS } \\
(\%)\end{array}$ & $\begin{array}{l}\text { FDMS } \\
\left(\mathrm{m}^{3} / \mathbf{k g}\right)\end{array}$ & $\begin{array}{c}\text { ARM } \\
\left(\mathbf{A m}^{2} / \mathbf{k g}\right)\end{array}$ & $\begin{array}{c}\text { IRM1.2 } \\
\left(\mathrm{Am}^{2} / \mathrm{kg}\right)\end{array}$ & $\begin{array}{l}\text { IRM }-0.3 \\
\left(\mathrm{Am}^{2} / \mathrm{kg}\right)\end{array}$ & $\begin{array}{c}\text { HIRM } \\
\left(\mathrm{Am}^{2} / \mathbf{k g}\right)\end{array}$ & $S$ \\
\hline VP-1/0-6 & 4.63E-07 & 4.71E-07 & -1.89 & $-8.75 \mathrm{E}-09$ & $1.41 \mathrm{E}-04$ & 7.03E-03 & $5.41 \mathrm{E}-03$ & $8.12 \mathrm{E}-04$ & 0.77 \\
\hline VP-1/6-19 & $4.08 \mathrm{E}-07$ & 4.15E-07 & -1.80 & $-7.36 \mathrm{E}-09$ & $1.34 \mathrm{E}-04$ & $5.93 \mathrm{E}-03$ & 4.49E-03 & $7.21 \mathrm{E}-04$ & 0.76 \\
\hline VP-1/19-30 & 4.70E-07 & $4.78 \mathrm{E}-07$ & -1.68 & $-7.88 \mathrm{E}-09$ & $1.65 \mathrm{E}-04$ & $6.67 \mathrm{E}-03$ & $5.27 \mathrm{E}-03$ & $6.99 \mathrm{E}-04$ & 0.79 \\
\hline VP-1/30-55 & $7.00 \mathrm{E}-07$ & $7.11 \mathrm{E}-07$ & -1.55 & $-1.09 \mathrm{E}-08$ & $2.32 \mathrm{E}-04$ & $9.60 \mathrm{E}-03$ & $8.11 \mathrm{E}-03$ & 7.47E-04 & 0.84 \\
\hline VP-1/55-66 & $7.78 \mathrm{E}-07$ & $7.86 \mathrm{E}-07$ & -0.95 & $-7.36 \mathrm{E}-09$ & $1.88 \mathrm{E}-04$ & $1.08 \mathrm{E}-02$ & $9.55 \mathrm{E}-03$ & $6.47 \mathrm{E}-04$ & 0.88 \\
\hline VP-1/66-78 & $6.26 \mathrm{E}-07$ & $6.30 \mathrm{E}-07$ & -0.65 & $-4.09 \mathrm{E}-09$ & $1.51 \mathrm{E}-04$ & 8.79E-03 & 7.72E-03 & $5.38 \mathrm{E}-04$ & 0.88 \\
\hline VP-1/80-90 & $5.47 \mathrm{E}-07$ & $5.52 \mathrm{E}-07$ & -0.79 & $-4.30 \mathrm{E}-09$ & $1.26 \mathrm{E}-04$ & $7.51 \mathrm{E}-03$ & $6.42 \mathrm{E}-03$ & $5.44 \mathrm{E}-04$ & 0.86 \\
\hline
\end{tabular}


Table 6a-4a. Magnetic properties of the less than 2-mm size fraction for soil pit VP-2.

\begin{tabular}{|c|c|c|c|c|c|c|c|c|c|}
\hline Sample \# & $\begin{array}{c}\text { MSlf } \\
\left(\mathbf{m}^{3} / \mathbf{k g}\right)\end{array}$ & $\begin{array}{l}\text { MShf } \\
\left(\mathbf{m}^{3} / \mathbf{k g}\right)\end{array}$ & $\begin{array}{c}\text { FDMS } \\
(\%)\end{array}$ & $\begin{array}{l}\text { FDMS } \\
\left(\mathbf{m}^{3} / \mathbf{k g}\right)\end{array}$ & $\begin{array}{c}\text { ARM } \\
\left(\mathrm{Am}^{2} / \mathbf{k g}\right)\end{array}$ & $\begin{array}{c}\text { IRM1.2 } \\
\left(\mathrm{Am}^{2} / \mathrm{kg}\right)\end{array}$ & $\begin{array}{l}\text { IRM -0.3 } \\
\left(\mathrm{Am}^{2} / \mathbf{k g}\right)\end{array}$ & $\begin{array}{c}\text { HIRM } \\
\left(\mathbf{A m}^{2} / \mathbf{k g}\right)\end{array}$ & S \\
\hline VP-2/0-5 & $2.23 \mathrm{E}-07$ & $2.27 \mathrm{E}-07$ & -2.01 & $-4.47 \mathrm{E}-09$ & $1.05 \mathrm{E}-04$ & $3.59 \mathrm{E}-03$ & $2.75 \mathrm{E}-03$ & 4.19E-04 & 0.77 \\
\hline VP-2/5-14 & $2.27 \mathrm{E}-07$ & $2.32 \mathrm{E}-07$ & -2.11 & $-4.79 \mathrm{E}-09$ & $1.09 \mathrm{E}-04$ & $3.59 \mathrm{E}-03$ & $2.73 \mathrm{E}-03$ & 4.31E-04 & 0.76 \\
\hline VP-2/14-44 & $2.15 \mathrm{E}-07$ & 2.19E-07 & -1.61 & $-3.46 \mathrm{E}-09$ & $1.06 \mathrm{E}-04$ & $3.21 \mathrm{E}-03$ & $2.45 \mathrm{E}-03$ & $3.81 \mathrm{E}-04$ & 0.76 \\
\hline VP-2/44-55 & $2.31 \mathrm{E}-07$ & $2.34 \mathrm{E}-07$ & -1.21 & $-2.79 \mathrm{E}-09$ & $1.25 \mathrm{E}-04$ & $3.42 \mathrm{E}-03$ & $2.70 \mathrm{E}-03$ & $3.62 \mathrm{E}-04$ & 0.79 \\
\hline VP-2/55-85 & $3.19 \mathrm{E}-07$ & $3.27 \mathrm{E}-07$ & -2.34 & $-7.47 \mathrm{E}-09$ & $1.92 \mathrm{E}-04$ & 4.67E-03 & $3.87 \mathrm{E}-03$ & 4.01E-04 & 0.83 \\
\hline VP-2/110-115 & 2.47E-07 & 2.49E-07 & -1.12 & $-2.76 \mathrm{E}-09$ & $8.75 \mathrm{E}-05$ & 3.47E-03 & $2.86 \mathrm{E}-03$ & 3.07E-04 & 0.82 \\
\hline
\end{tabular}

Table 6a-4b. Magnetic properties of the less than 63-micron size fraction for soil pit VP-2.

\begin{tabular}{|c|c|c|c|c|c|c|c|c|c|}
\hline Sample \# & $\begin{array}{c}\text { MSIf } \\
\left(\mathbf{m}^{3} / \mathbf{k g}\right)\end{array}$ & $\begin{array}{c}\text { MShf } \\
\left(\mathbf{m}^{3} / \mathbf{k g}\right)\end{array}$ & $\begin{array}{l}\text { FDMS } \\
(\%)\end{array}$ & $\begin{array}{l}\text { FDMS } \\
\left(\mathrm{m}^{3} / \mathrm{kg}\right)\end{array}$ & $\begin{array}{c}\text { ARM } \\
\left(\mathbf{A m}^{2} / \mathbf{k g}\right)\end{array}$ & $\begin{array}{c}\text { IRM1.2 } \\
\left(\mathrm{Am}^{2} / \mathrm{kg}\right)\end{array}$ & $\begin{array}{l}\text { IRM }-0.3 \\
\left(\mathrm{Am}^{2} / \mathrm{kg}\right)\end{array}$ & $\begin{array}{c}\text { HIRM } \\
\left(\mathrm{Am}^{2} / \mathrm{kg}\right)\end{array}$ & $S$ \\
\hline VP-2/0-5 & $3.81 \mathrm{E}-07$ & $3.90 \mathrm{E}-07$ & -2.36 & $-8.97 \mathrm{E}-09$ & $1.39 \mathrm{E}-04$ & 5.91E-03 & $4.54 \mathrm{E}-03$ & $6.84 \mathrm{E}-04$ & 0.77 \\
\hline VP-2/5-14 & 4.14E-07 & 4.22E-07 & -2.04 & -8.46E-09 & $1.45 \mathrm{E}-04$ & $6.34 \mathrm{E}-03$ & $4.86 \mathrm{E}-03$ & 7.40E-04 & 0.77 \\
\hline VP-2/14-44 & $3.94 \mathrm{E}-07$ & $4.00 \mathrm{E}-07$ & -1.64 & $-6.45 \mathrm{E}-09$ & $1.50 \mathrm{E}-04$ & $5.79 \mathrm{E}-03$ & 4.46E-03 & $6.67 \mathrm{E}-04$ & 0.77 \\
\hline VP-2/44-55 & 4.73E-07 & $4.82 \mathrm{E}-07$ & -1.80 & $-8.52 \mathrm{E}-09$ & $1.86 \mathrm{E}-04$ & $6.72 \mathrm{E}-03$ & $5.39 \mathrm{E}-03$ & $6.62 \mathrm{E}-04$ & 0.80 \\
\hline VP-2/55-85 & $6.06 \mathrm{E}-07$ & $6.19 \mathrm{E}-07$ & -2.21 & $-1.34 \mathrm{E}-08$ & $2.76 \mathrm{E}-04$ & 8.63E-03 & 7.24E-03 & $6.97 \mathrm{E}-04$ & 0.84 \\
\hline VP-2/110-115 & $5.63 \mathrm{E}-07$ & 5.70E-07 & -1.38 & $-7.75 \mathrm{E}-09$ & $1.57 \mathrm{E}-04$ & 7.57E-03 & $6.23 \mathrm{E}-03$ & $6.68 \mathrm{E}-04$ & 0.82 \\
\hline
\end{tabular}


Table 6a-5. Magnetic properties of the less than 2-mm size fraction for auger hole 8U-10.

\begin{tabular}{|c|c|c|c|c|c|c|c|c|c|}
\hline Sample \# & $\begin{array}{c}\text { MSlf } \\
\left(\mathbf{m}^{3} / \mathbf{k g}\right)\end{array}$ & $\begin{array}{c}\text { MShf } \\
\left(\mathbf{m}^{3} / \mathbf{k g}\right)\end{array}$ & $\begin{array}{c}\text { FDMS } \\
(\%)\end{array}$ & $\begin{array}{l}\text { FDMS } \\
\left(\mathrm{m}^{3} / \mathrm{kg}\right)\end{array}$ & $\begin{array}{c}\text { ARM } \\
\left(\mathrm{Am}^{2} / \mathrm{kg}\right)\end{array}$ & $\begin{array}{c}\text { IRM1.2 } \\
\left(\mathrm{Am}^{2} / \mathrm{kg}\right)\end{array}$ & $\begin{array}{l}\text { IRM -0.3 } \\
\left(\mathrm{Am}^{2} / \mathrm{kg}\right) \\
\end{array}$ & $\begin{array}{c}\text { HIRM } \\
\left(\mathrm{Am}^{2} / \mathbf{k g}\right)\end{array}$ & S \\
\hline $8 U-10 / 0-22$ & $2.52 \mathrm{E}-07$ & $2.44 \mathrm{E}-07$ & 3.35 & 8.43E-09 & $1.22 \mathrm{E}-04$ & $3.87 \mathrm{E}-03$ & $3.01 \mathrm{E}-03$ & $4.30 \mathrm{E}-04$ & 0.78 \\
\hline $8 U-10 / 22-36$ & $2.50 \mathrm{E}-07$ & $2.42 \mathrm{E}-07$ & 3.23 & 8.09E-09 & $1.17 \mathrm{E}-04$ & $3.56 \mathrm{E}-03$ & $2.70 \mathrm{E}-03$ & 4.27E-04 & 0.76 \\
\hline $8 \mathrm{U}-10 / 36-47$ & $3.57 \mathrm{E}-07$ & $3.44 \mathrm{E}-07$ & 3.77 & $1.35 \mathrm{E}-08$ & $1.88 \mathrm{E}-04$ & $4.84 \mathrm{E}-03$ & $4.00 \mathrm{E}-03$ & 4.17E-04 & 0.83 \\
\hline $8 \mathrm{U}-10 / 47-58$ & $4.16 \mathrm{E}-07$ & 4.02E-07 & 3.24 & $1.35 \mathrm{E}-08$ & $1.77 \mathrm{E}-04$ & $5.70 \mathrm{E}-03$ & $4.81 \mathrm{E}-03$ & 4.42E-04 & 0.84 \\
\hline $8 \mathrm{U}-10 / 58-65$ & 4.61E-07 & 4.47E-07 & 3.00 & $1.38 \mathrm{E}-08$ & $1.65 \mathrm{E}-04$ & $6.36 \mathrm{E}-03$ & $5.42 \mathrm{E}-03$ & $4.70 \mathrm{E}-04$ & 0.85 \\
\hline $8 \mathrm{U}-10 / 65-74$ & $4.39 \mathrm{E}-07$ & $4.29 \mathrm{E}-07$ & 2.29 & $1.00 \mathrm{E}-08$ & $1.43 \mathrm{E}-04$ & $6.16 \mathrm{E}-03$ & $5.29 \mathrm{E}-03$ & $4.36 \mathrm{E}-04$ & 0.86 \\
\hline $8 \mathrm{U}-10 / 74-83$ & $4.05 \mathrm{E}-07$ & $3.95 \mathrm{E}-07$ & 2.64 & $1.07 \mathrm{E}-08$ & $1.17 \mathrm{E}-04$ & $5.63 \mathrm{E}-03$ & 4.79E-03 & 4.22E-04 & 0.85 \\
\hline $8 \mathrm{U}-10 / 83-90$ & $3.85 \mathrm{E}-07$ & 3.73E-07 & 2.89 & 1.11E-08 & $1.07 \mathrm{E}-04$ & $5.24 \mathrm{E}-03$ & 4.43E-03 & 4.02E-04 & 0.85 \\
\hline $8 \mathrm{U}-10 / 90-99$ & $3.42 \mathrm{E}-07$ & $3.33 \mathrm{E}-07$ & 2.63 & 8.98E-09 & $9.33 \mathrm{E}-05$ & $4.59 \mathrm{E}-03$ & $3.83 \mathrm{E}-03$ & $3.79 \mathrm{E}-04$ & 0.83 \\
\hline 8U-10/99-112 & $2.79 \mathrm{E}-07$ & $2.70 \mathrm{E}-07$ & 3.14 & 8.75E-09 & 7.77E-05 & $3.70 \mathrm{E}-03$ & $3.01 \mathrm{E}-03$ & $3.47 \mathrm{E}-04$ & 0.81 \\
\hline $8 \mathrm{U}-10 / 112-121$ & $2.32 \mathrm{E}-07$ & $2.23 \mathrm{E}-07$ & 3.55 & $8.23 \mathrm{E}-09$ & $6.94 \mathrm{E}-05$ & $3.15 \mathrm{E}-03$ & $2.47 \mathrm{E}-03$ & $3.39 \mathrm{E}-04$ & 0.79 \\
\hline $8 \mathrm{U}-10 / 121-131$ & $1.87 \mathrm{E}-07$ & $1.80 \mathrm{E}-07$ & 3.69 & 6.91E-09 & $5.75 \mathrm{E}-05$ & $2.54 \mathrm{E}-03$ & $1.94 \mathrm{E}-03$ & 3.01E-04 & 0.76 \\
\hline $8 \mathrm{U}-10 / 131-142$ & $1.61 \mathrm{E}-07$ & $1.57 \mathrm{E}-07$ & 2.92 & 4.71E-09 & 4.97E-05 & $2.29 \mathrm{E}-03$ & $1.65 \mathrm{E}-03$ & $3.17 \mathrm{E}-04$ & 0.72 \\
\hline $8 \mathrm{U}-10142-154$ & $1.60 \mathrm{E}-07$ & $1.55 \mathrm{E}-07$ & 3.41 & $5.46 \mathrm{E}-09$ & $4.76 \mathrm{E}-05$ & $2.24 \mathrm{E}-03$ & $1.63 \mathrm{E}-03$ & $3.04 \mathrm{E}-04$ & 0.73 \\
\hline $8 \mathrm{U}-10 / 154-165$ & $1.72 \mathrm{E}-07$ & $1.67 \mathrm{E}-07$ & 3.05 & $5.25 \mathrm{E}-09$ & $5.32 \mathrm{E}-05$ & $2.38 \mathrm{E}-03$ & $1.75 \mathrm{E}-03$ & $3.16 \mathrm{E}-04$ & 0.73 \\
\hline $8 \mathrm{U}-10 / 165-175$ & $1.66 \mathrm{E}-07$ & $1.63 \mathrm{E}-07$ & 1.64 & 2.72E-09 & $5.10 \mathrm{E}-05$ & $2.21 \mathrm{E}-03$ & $1.63 \mathrm{E}-03$ & $2.92 \mathrm{E}-04$ & 0.74 \\
\hline $8 \mathrm{U}-10 / 175-184$ & $1.75 \mathrm{E}-07$ & $1.71 \mathrm{E}-07$ & 2.00 & $3.50 \mathrm{E}-09$ & $6.00 \mathrm{E}-05$ & $2.53 \mathrm{E}-03$ & $1.85 \mathrm{E}-03$ & $3.43 \mathrm{E}-04$ & 0.73 \\
\hline $8 \mathrm{U}-10 / 184-197$ & $1.67 \mathrm{E}-07$ & $1.61 \mathrm{E}-07$ & 3.66 & $6.11 \mathrm{E}-09$ & $5.78 \mathrm{E}-05$ & $2.40 \mathrm{E}-03$ & $1.72 \mathrm{E}-03$ & $3.41 \mathrm{E}-04$ & 0.72 \\
\hline $8 \mathrm{U}-10 / 197-209$ & $1.52 \mathrm{E}-07$ & $1.51 \mathrm{E}-07$ & 1.11 & $1.69 \mathrm{E}-09$ & $5.21 \mathrm{E}-05$ & $2.14 \mathrm{E}-03$ & $1.55 \mathrm{E}-03$ & $2.94 \mathrm{E}-04$ & 0.73 \\
\hline $8 \mathrm{U}-10 / 209-220$ & $1.26 \mathrm{E}-07$ & $1.23 \mathrm{E}-07$ & 2.37 & 2.99E-09 & $4.46 \mathrm{E}-05$ & $1.77 \mathrm{E}-03$ & $1.28 \mathrm{E}-03$ & $2.43 \mathrm{E}-04$ & 0.72 \\
\hline $8 \mathrm{U}-10 / 220-230$ & $9.38 \mathrm{E}-08$ & $9.24 \mathrm{E}-08$ & 1.57 & $1.47 \mathrm{E}-09$ & $3.39 \mathrm{E}-05$ & $1.35 \mathrm{E}-03$ & $9.43 \mathrm{E}-04$ & $2.05 \mathrm{E}-04$ & 0.70 \\
\hline $8 \mathrm{U}-10 / 230-241$ & $6.20 \mathrm{E}-08$ & $6.16 \mathrm{E}-08$ & 0.71 & 4.42E-10 & $2.25 \mathrm{E}-05$ & $9.87 \mathrm{E}-04$ & $6.21 \mathrm{E}-04$ & $1.83 \mathrm{E}-04$ & 0.63 \\
\hline $8 \mathrm{U}-10 / 241-249$ & $1.14 \mathrm{E}-07$ & $1.13 \mathrm{E}-07$ & 0.79 & $9.06 \mathrm{E}-10$ & $4.20 \mathrm{E}-05$ & $1.69 \mathrm{E}-03$ & $1.21 \mathrm{E}-03$ & $2.37 \mathrm{E}-04$ & 0.72 \\
\hline $8 \mathrm{U}-10 / 249-261$ & $5.49 \mathrm{E}-08$ & $5.52 \mathrm{E}-08$ & -0.62 & $-3.40 \mathrm{E}-10$ & $1.92 \mathrm{E}-05$ & $9.00 \mathrm{E}-04$ & $5.50 \mathrm{E}-04$ & $1.75 \mathrm{E}-04$ & 0.61 \\
\hline $8 \mathrm{U}-10 / 261-270$ & $9.55 \mathrm{E}-08$ & $9.44 \mathrm{E}-08$ & 1.19 & $1.14 \mathrm{E}-09$ & $3.20 \mathrm{E}-05$ & $1.46 \mathrm{E}-03$ & $1.04 \mathrm{E}-03$ & $2.09 \mathrm{E}-04$ & 0.71 \\
\hline $8 \mathrm{U}-10 / 270-274$ & 7.65E-08 & 7.57E-08 & 1.00 & $7.61 \mathrm{E}-10$ & $2.90 \mathrm{E}-05$ & $1.20 \mathrm{E}-03$ & 8.40E-04 & $1.78 \mathrm{E}-04$ & 0.70 \\
\hline $8 \mathrm{U}-10 / 274-284$ & $6.38 \mathrm{E}-08$ & $6.35 \mathrm{E}-08$ & 0.35 & $2.20 \mathrm{E}-10$ & $2.50 \mathrm{E}-05$ & $1.05 \mathrm{E}-03$ & 7.03E-04 & $1.72 \mathrm{E}-04$ & 0.67 \\
\hline
\end{tabular}


Table 6a-6. Magnetic properties of the less than 2-mm size fraction for auger hole 8U-11.

\begin{tabular}{|c|c|c|c|c|c|c|c|c|c|}
\hline Sample \# & $\begin{array}{c}\text { MSIf } \\
\left(\mathbf{m}^{3} / \mathbf{k g}\right)\end{array}$ & $\begin{array}{c}\text { MShf } \\
\left(\mathrm{m}^{3} / \mathbf{k g}\right)\end{array}$ & $\begin{array}{c}\text { FDMS } \\
(\%)\end{array}$ & $\begin{array}{l}\text { FDMS } \\
\left(\mathrm{m}^{3} / \mathrm{kg}\right)\end{array}$ & $\begin{array}{c}\text { ARM } \\
\left(\mathbf{A m}^{2} / \mathbf{k g}\right)\end{array}$ & $\begin{array}{c}\text { IRM1.2 } \\
\left(\mathrm{Am}^{2} / \mathrm{kg}\right)\end{array}$ & $\begin{array}{l}\text { IRM -0.3 } \\
\left(\mathrm{Am}^{2} / \mathrm{kg}\right)\end{array}$ & $\begin{array}{c}\text { HIRM } \\
\left(\mathrm{Am}^{2} / \mathbf{k g}\right)\end{array}$ & $\mathbf{S}$ \\
\hline $8 \mathrm{U}-11 / 0-22$ & $1.97 \mathrm{E}-07$ & $1.92 \mathrm{E}-07$ & 0.00 & $2.54 \mathrm{E}+00$ & $8.04 \mathrm{E}-05$ & $2.84 \mathrm{E}-03$ & $2.11 \mathrm{E}-03$ & $3.66 \mathrm{E}-04$ & 0.74 \\
\hline $8 U-11 / 22-38$ & $2.15 \mathrm{E}-07$ & 2.07E-07 & 0.00 & $3.42 \mathrm{E}+00$ & $9.15 \mathrm{E}-05$ & $2.98 \mathrm{E}-03$ & $2.21 \mathrm{E}-03$ & $3.85 \mathrm{E}-04$ & 0.74 \\
\hline $8 U-11 / 38-50$ & $2.87 \mathrm{E}-07$ & $2.79 \mathrm{E}-07$ & 0.00 & $2.64 \mathrm{E}+00$ & $1.39 \mathrm{E}-04$ & $3.93 \mathrm{E}-03$ & $3.17 \mathrm{E}-03$ & $3.81 \mathrm{E}-04$ & 0.81 \\
\hline $8 U-11 / 50-62$ & $3.07 \mathrm{E}-07$ & $3.01 \mathrm{E}-07$ & 0.00 & $1.92 \mathrm{E}+00$ & $1.22 \mathrm{E}-04$ & $4.23 \mathrm{E}-03$ & $3.53 \mathrm{E}-03$ & $3.46 \mathrm{E}-04$ & 0.84 \\
\hline $8 U-11 / 62-71$ & $3.51 \mathrm{E}-07$ & 3.44E-07 & 0.00 & $1.88 \mathrm{E}+00$ & $1.00 \mathrm{E}-04$ & 4.82E-03 & 4.15E-03 & 3.37E-04 & 0.86 \\
\hline $8 U-11 / 71-86$ & $3.48 \mathrm{E}-07$ & $3.41 \mathrm{E}-07$ & 0.00 & $1.85 \mathrm{E}+00$ & 8.92E-05 & $4.84 \mathrm{E}-03$ & 4.18E-03 & $3.27 \mathrm{E}-04$ & 0.86 \\
\hline $8 U-11 / 86-98$ & $3.30 \mathrm{E}-07$ & $3.24 \mathrm{E}-07$ & 0.00 & $1.77 \mathrm{E}+00$ & $8.58 \mathrm{E}-05$ & 4.42E-03 & $3.96 \mathrm{E}-03$ & $2.29 \mathrm{E}-04$ & 0.90 \\
\hline $8 \mathrm{U}-11 / 98-109$ & 2.60E-07 & $2.59 \mathrm{E}-07$ & 0.00 & $5.82 \mathrm{E}-01$ & 7.20E-05 & $3.64 \mathrm{E}-03$ & $3.01 \mathrm{E}-03$ & 2.73E-04 & 0.85 \\
\hline $8 \mathrm{U}-11 / 109-118$ & $2.70 \mathrm{E}-07$ & $2.65 \mathrm{E}-07$ & 0.00 & $1.79 \mathrm{E}+00$ & 7.45E-05 & $3.70 \mathrm{E}-03$ & $3.14 \mathrm{E}-03$ & $2.83 \mathrm{E}-04$ & 0.85 \\
\hline $8 \mathrm{U}-11 / 118-131$ & $2.20 \mathrm{E}-07$ & $2.17 \mathrm{E}-07$ & 0.00 & $1.27 \mathrm{E}+00$ & $6.48 \mathrm{E}-05$ & $3.05 \mathrm{E}-03$ & $2.51 \mathrm{E}-03$ & $2.70 \mathrm{E}-04$ & 0.82 \\
\hline $8 \mathrm{U}-11 / 131-139$ & $1.63 \mathrm{E}-07$ & $1.61 \mathrm{E}-07$ & 0.00 & $7.29 \mathrm{E}-01$ & $5.35 \mathrm{E}-05$ & $2.28 \mathrm{E}-03$ & $1.79 \mathrm{E}-03$ & $2.47 \mathrm{E}-04$ & 0.78 \\
\hline $8 \mathrm{U}-11 / 139-151$ & $1.18 \mathrm{E}-07$ & $1.17 \mathrm{E}-07$ & 0.00 & $1.00 \mathrm{E}+00$ & $4.38 \mathrm{E}-05$ & $1.65 \mathrm{E}-03$ & $1.24 \mathrm{E}-03$ & $2.05 \mathrm{E}-04$ & 0.75 \\
\hline $8 \mathrm{U}-11 / 151-161$ & $1.05 \mathrm{E}-07$ & $1.04 \mathrm{E}-07$ & 0.00 & $5.88 \mathrm{E}-01$ & 4.04E-05 & $1.46 \mathrm{E}-03$ & $1.09 \mathrm{E}-03$ & $1.87 \mathrm{E}-04$ & 0.74 \\
\hline $8 \mathrm{U}-11 / 161-172$ & $1.70 \mathrm{E}-07$ & $1.66 \mathrm{E}-07$ & 0.00 & $2.08 \mathrm{E}+00$ & $5.50 \mathrm{E}-05$ & $2.31 \mathrm{E}-03$ & $1.79 \mathrm{E}-03$ & $2.57 \mathrm{E}-04$ & 0.78 \\
\hline $8 \mathrm{U}-11 / 172-183$ & $1.87 \mathrm{E}-07$ & $1.83 \mathrm{E}-07$ & 0.00 & $2.14 \mathrm{E}+00$ & $5.68 \mathrm{E}-05$ & $2.52 \mathrm{E}-03$ & $1.97 \mathrm{E}-03$ & $2.75 \mathrm{E}-04$ & 0.78 \\
\hline 8U-11/183-196 & $3.12 \mathrm{E}-07$ & $3.07 \mathrm{E}-07$ & 0.00 & $1.49 \mathrm{E}+00$ & 7.46E-05 & $4.06 \mathrm{E}-03$ & $3.33 \mathrm{E}-03$ & $3.62 \mathrm{E}-04$ & 0.82 \\
\hline $8 \mathrm{U}-11 / 196-210$ & 2.19E-07 & $2.15 \mathrm{E}-07$ & 0.00 & $1.78 \mathrm{E}+00$ & $6.27 \mathrm{E}-05$ & $2.92 \mathrm{E}-03$ & $2.25 \mathrm{E}-03$ & 3.35E-04 & 0.77 \\
\hline $8 \mathrm{U}-11 / 210-224$ & $2.00 \mathrm{E}-07$ & $1.96 \mathrm{E}-07$ & 0.00 & $1.90 \mathrm{E}+00$ & $6.08 \mathrm{E}-05$ & $2.64 \mathrm{E}-03$ & $2.02 \mathrm{E}-03$ & $3.07 \mathrm{E}-04$ & 0.77 \\
\hline $8 \mathrm{U}-11 / 224-237$ & $1.90 \mathrm{E}-07$ & $1.85 \mathrm{E}-07$ & 0.00 & $2.38 \mathrm{E}+00$ & $5.99 \mathrm{E}-05$ & $2.51 \mathrm{E}-03$ & $1.90 \mathrm{E}-03$ & $3.02 \mathrm{E}-04$ & 0.76 \\
\hline $8 \mathrm{U}-11 / 237-251$ & $1.91 \mathrm{E}-07$ & $1.89 \mathrm{E}-07$ & 0.00 & $1.24 \mathrm{E}+00$ & $5.92 \mathrm{E}-05$ & $2.48 \mathrm{E}-03$ & $1.88 \mathrm{E}-03$ & $3.02 \mathrm{E}-04$ & 0.76 \\
\hline $8 U-11 / 251-265$ & $1.96 \mathrm{E}-07$ & $1.93 \mathrm{E}-07$ & 0.00 & $1.80 \mathrm{E}+00$ & $6.32 \mathrm{E}-05$ & $2.58 \mathrm{E}-03$ & $1.96 \mathrm{E}-03$ & $3.12 \mathrm{E}-04$ & 0.76 \\
\hline $8 \mathrm{U}-11 / 265-278$ & $1.99 \mathrm{E}-07$ & $1.96 \mathrm{E}-07$ & 0.00 & $1.58 \mathrm{E}+00$ & $6.67 \mathrm{E}-05$ & $2.69 \mathrm{E}-03$ & $2.03 \mathrm{E}-03$ & 3.34E-04 & 0.75 \\
\hline
\end{tabular}


Table 6a-7. Magnetic properties of the less than 2-mm size fraction for auger hole 8U-12.

\begin{tabular}{|c|c|c|c|c|c|c|c|c|c|}
\hline Sample \# & $\begin{array}{c}\text { MSIf } \\
\left(\mathbf{m}^{3} / \mathbf{k g}\right)\end{array}$ & $\begin{array}{c}\text { MShf } \\
\left(\mathrm{m}^{3} / \mathrm{kg}\right)\end{array}$ & $\begin{array}{c}\text { FDMS } \\
(\%)\end{array}$ & $\begin{array}{l}\text { FDMS } \\
\left(\mathrm{m}^{3} / \mathrm{kg}\right)\end{array}$ & $\begin{array}{c}\text { ARM } \\
\left(\mathrm{Am}^{2} / \mathbf{k g}\right)\end{array}$ & $\begin{array}{c}\text { IRM1.2 } \\
\left(\mathrm{Am}^{2} / \mathrm{kg}\right)\end{array}$ & $\begin{array}{l}\text { IRM }-0.3 \\
\left(\mathrm{Am}^{2} / \mathrm{kg}\right)\end{array}$ & $\begin{array}{c}\text { HIRM } \\
\left(\mathbf{A m}^{2} / \mathbf{k g}\right)\end{array}$ & S \\
\hline $8 \mathrm{U}-12 / 0-13$ & $1.98 \mathrm{E}-07$ & $1.92 \mathrm{E}-07$ & 0.00 & $2.81 \mathrm{E}+00$ & $9.59 \mathrm{E}-05$ & $2.93 \mathrm{E}-03$ & $2.15 \mathrm{E}-03$ & $3.91 \mathrm{E}-04$ & 0.73 \\
\hline $8 \mathrm{U}-12 / 13-27$ & $2.05 \mathrm{E}-07$ & $1.99 \mathrm{E}-07$ & 0.00 & $3.31 \mathrm{E}+00$ & $1.02 \mathrm{E}-04$ & $2.89 \mathrm{E}-03$ & $2.13 \mathrm{E}-03$ & $3.80 \mathrm{E}-04$ & 0.74 \\
\hline $8 \mathrm{U}-12 / 27-37$ & $2.11 \mathrm{E}-07$ & $2.04 \mathrm{E}-07$ & 0.00 & $3.56 \mathrm{E}+00$ & $1.08 \mathrm{E}-04$ & $2.99 \mathrm{E}-03$ & $2.25 \mathrm{E}-03$ & $3.68 \mathrm{E}-04$ & 0.75 \\
\hline $8 \mathrm{U}-12 / 37-48$ & $2.30 \mathrm{E}-07$ & $2.24 \mathrm{E}-07$ & 0.00 & $2.82 \mathrm{E}+00$ & $1.30 \mathrm{E}-04$ & $3.20 \mathrm{E}-03$ & $2.52 \mathrm{E}-03$ & $3.41 \mathrm{E}-04$ & 0.79 \\
\hline $8 \mathrm{U}-12 / 48-60$ & $2.05 \mathrm{E}-07$ & $1.99 \mathrm{E}-07$ & 0.00 & $3.34 \mathrm{E}+00$ & $1.18 \mathrm{E}-04$ & $2.87 \mathrm{E}-03$ & $2.27 \mathrm{E}-03$ & $3.01 \mathrm{E}-04$ & 0.79 \\
\hline $8 \mathrm{U}-12 / 60-71$ & $1.85 \mathrm{E}-07$ & $1.82 \mathrm{E}-07$ & 0.00 & $1.70 \mathrm{E}+00$ & $8.42 \mathrm{E}-05$ & $2.56 \mathrm{E}-03$ & $2.01 \mathrm{E}-03$ & $2.34 \mathrm{E}-04$ & 0.82 \\
\hline $8 \mathrm{U}-12 / 71-83$ & $2.88 \mathrm{E}-07$ & $2.83 \mathrm{E}-07$ & 0.00 & $1.88 \mathrm{E}+00$ & $8.45 \mathrm{E}-05$ & $3.98 \mathrm{E}-03$ & 3.37E-03 & $3.06 \mathrm{E}-04$ & 0.85 \\
\hline $8 \mathrm{U}-12 / 83-96$ & $3.61 \mathrm{E}-07$ & $3.56 \mathrm{E}-07$ & 0.00 & $1.54 \mathrm{E}+00$ & $9.07 \mathrm{E}-05$ & $5.07 \mathrm{E}-03$ & 4.37E-03 & $3.46 \mathrm{E}-04$ & 0.86 \\
\hline $8 \mathrm{U}-12 / 96-106$ & $3.02 \mathrm{E}-07$ & $2.95 \mathrm{E}-07$ & 0.00 & $2.15 \mathrm{E}+00$ & $8.29 \mathrm{E}-05$ & $4.35 \mathrm{E}-03$ & $3.74 \mathrm{E}-03$ & $3.04 \mathrm{E}-04$ & 0.86 \\
\hline $8 \mathrm{U}-12 / 106-116$ & $1.67 \mathrm{E}-07$ & $1.63 \mathrm{E}-07$ & 0.00 & $1.91 \mathrm{E}+00$ & $5.29 \mathrm{E}-05$ & $2.45 \mathrm{E}-03$ & $2.01 \mathrm{E}-03$ & $2.22 \mathrm{E}-04$ & 0.82 \\
\hline $8 \mathrm{U}-12 / 116-128$ & $1.54 \mathrm{E}-07$ & $1.52 \mathrm{E}-07$ & 0.00 & $1.16 \mathrm{E}+00$ & $4.89 \mathrm{E}-05$ & $2.27 \mathrm{E}-03$ & $1.83 \mathrm{E}-03$ & $2.17 \mathrm{E}-04$ & 0.81 \\
\hline $8 \mathrm{U}-12 / 128-137$ & $1.44 \mathrm{E}-07$ & $1.42 \mathrm{E}-07$ & 0.00 & $1.26 \mathrm{E}+00$ & 4.62E-05 & $2.13 \mathrm{E}-03$ & $1.70 \mathrm{E}-03$ & $2.12 \mathrm{E}-04$ & 0.80 \\
\hline $8 \mathrm{U}-12 / 137-147$ & $1.44 \mathrm{E}-07$ & $1.42 \mathrm{E}-07$ & 0.00 & $1.40 \mathrm{E}+00$ & $4.78 \mathrm{E}-05$ & $2.14 \mathrm{E}-03$ & $1.72 \mathrm{E}-03$ & $2.12 \mathrm{E}-04$ & 0.80 \\
\hline $8 \mathrm{U}-12 / 147-158$ & $1.30 \mathrm{E}-07$ & $1.28 \mathrm{E}-07$ & 0.00 & $1.13 \mathrm{E}+00$ & $4.06 \mathrm{E}-05$ & $1.88 \mathrm{E}-03$ & $1.47 \mathrm{E}-03$ & $2.06 \mathrm{E}-04$ & 0.78 \\
\hline $8 \mathrm{U}-12 / 158-170$ & $1.53 \mathrm{E}-07$ & $1.50 \mathrm{E}-07$ & 0.00 & $2.19 \mathrm{E}+00$ & 4.69E-05 & $2.17 \mathrm{E}-03$ & $1.68 \mathrm{E}-03$ & $2.46 \mathrm{E}-04$ & 0.77 \\
\hline $8 \mathrm{U}-12 / 170-180$ & $1.73 \mathrm{E}-07$ & $1.70 \mathrm{E}-07$ & 0.00 & $1.95 \mathrm{E}+00$ & $5.13 \mathrm{E}-05$ & 2.44E-03 & $1.90 \mathrm{E}-03$ & $2.67 \mathrm{E}-04$ & 0.78 \\
\hline $8 \mathrm{U}-12 / 1180-191$ & $2.62 \mathrm{E}-07$ & $2.58 \mathrm{E}-07$ & 0.00 & $1.65 \mathrm{E}+00$ & $6.57 \mathrm{E}-05$ & $3.52 \mathrm{E}-03$ & $2.88 \mathrm{E}-03$ & $3.20 \mathrm{E}-04$ & 0.82 \\
\hline $8 \mathrm{U}-12 / 191-203$ & $2.17 \mathrm{E}-07$ & $2.12 \mathrm{E}-07$ & 0.00 & $2.32 \mathrm{E}+00$ & $6.02 \mathrm{E}-05$ & $2.97 \mathrm{E}-03$ & $2.31 \mathrm{E}-03$ & $3.26 \mathrm{E}-04$ & 0.78 \\
\hline $8 \mathrm{U}-12 / 203-216$ & $2.04 \mathrm{E}-07$ & $2.01 \mathrm{E}-07$ & 0.00 & $1.21 \mathrm{E}+00$ & $5.94 \mathrm{E}-05$ & $2.78 \mathrm{E}-03$ & 2.13E-03 & $3.27 \mathrm{E}-04$ & 0.77 \\
\hline $8 \mathrm{U}-12 / 216-227$ & $1.78 \mathrm{E}-07$ & $1.76 \mathrm{E}-07$ & 0.00 & $1.41 \mathrm{E}+00$ & $5.45 \mathrm{E}-05$ & $2.48 \mathrm{E}-03$ & $1.89 \mathrm{E}-03$ & 2.93E-04 & 0.76 \\
\hline $8 \mathrm{U}-12 / 227-239$ & $1.36 \mathrm{E}-07$ & $1.36 \mathrm{E}-07$ & 0.00 & 5.65E-01 & $4.20 \mathrm{E}-05$ & $1.94 \mathrm{E}-03$ & $1.45 \mathrm{E}-03$ & $2.48 \mathrm{E}-04$ & 0.75 \\
\hline $8 \mathrm{U}-12 / 239-251$ & $9.63 \mathrm{E}-08$ & $9.59 \mathrm{E}-08$ & 0.00 & 3.94E-01 & $3.05 \mathrm{E}-05$ & $1.47 \mathrm{E}-03$ & $9.59 \mathrm{E}-04$ & $2.53 \mathrm{E}-04$ & 0.65 \\
\hline $8 \mathrm{U}-12 / 251-262$ & $9.47 \mathrm{E}-08$ & $9.44 \mathrm{E}-08$ & 0.00 & 3.61E-01 & $3.14 \mathrm{E}-05$ & $1.47 \mathrm{E}-03$ & 9.91E-04 & $2.38 \mathrm{E}-04$ & 0.68 \\
\hline $8 \mathrm{U}-12 / 262-273$ & $7.98 \mathrm{E}-08$ & 7.78E-08 & 0.00 & $2.54 \mathrm{E}+00$ & $2.45 \mathrm{E}-05$ & $1.22 \mathrm{E}-03$ & $7.88 \mathrm{E}-04$ & $2.16 \mathrm{E}-04$ & 0.65 \\
\hline $8 \mathrm{U}-12 / 273-279$ & $8.24 \mathrm{E}-08$ & 7.92E-08 & 0.00 & $3.94 \mathrm{E}+00$ & 2.73E-05 & $1.31 \mathrm{E}-03$ & 8.72E-04 & $2.18 \mathrm{E}-04$ & 0.67 \\
\hline $8 \mathrm{U}-12 / 279-290$ & $5.65 \mathrm{E}-08$ & $5.67 \mathrm{E}-08$ & -0.00 & $-3.90 \mathrm{E}-01$ & $2.02 \mathrm{E}-05$ & $9.51 \mathrm{E}-04$ & $5.38 \mathrm{E}-04$ & $2.06 \mathrm{E}-04$ & 0.57 \\
\hline $8 \mathrm{U}-12 / 290-299$ & 7.30E-08 & $7.10 \mathrm{E}-08$ & 0.00 & $2.77 \mathrm{E}+00$ & $2.76 \mathrm{E}-05$ & $1.16 \mathrm{E}-03$ & 7.31E-04 & $2.16 \mathrm{E}-04$ & 0.63 \\
\hline $8 \mathrm{U}-12 / 299-310$ & $6.88 \mathrm{E}-08$ & $6.52 \mathrm{E}-08$ & 0.00 & $5.22 \mathrm{E}+00$ & $2.46 \mathrm{E}-05$ & $1.11 \mathrm{E}-03$ & $7.18 \mathrm{E}-04$ & $1.99 \mathrm{E}-04$ & 0.64 \\
\hline $8 \mathrm{U}-12 / 310-317$ & 7.53E-08 & 7.14E-08 & 0.00 & $5.15 \mathrm{E}+00$ & $2.74 \mathrm{E}-05$ & $1.22 \mathrm{E}-03$ & 8.32E-04 & $1.96 \mathrm{E}-04$ & 0.68 \\
\hline
\end{tabular}


Table 6a-8. Magnetic properties of the less than 2-mm size fraction for auger hole 9U-22.

\begin{tabular}{|c|c|c|c|c|c|c|c|c|c|}
\hline Sample \# & $\begin{array}{c}\text { MSlf } \\
\left(\mathbf{m}^{3} / \mathbf{k g}\right)\end{array}$ & $\begin{array}{c}\text { MShf } \\
\left(\mathbf{m}^{3} / \mathbf{k g}\right)\end{array}$ & $\begin{array}{c}\text { FDMS } \\
(\%)\end{array}$ & $\begin{array}{l}\text { FDMS } \\
\left(\mathrm{m}^{3} / \mathrm{kg}\right)\end{array}$ & $\begin{array}{c}\text { ARM } \\
\left(\mathrm{Am}^{2} / \mathrm{kg}\right)\end{array}$ & $\begin{array}{c}\text { IRM1.2 } \\
\left(\mathrm{Am}^{2} / \mathrm{kg}\right)\end{array}$ & $\begin{array}{l}\text { IRM -0.3 } \\
\left(\mathrm{Am}^{2} / \mathrm{kg}\right)\end{array}$ & $\begin{array}{c}\text { HIRM } \\
\left(\mathrm{Am}^{2} / \mathrm{kg}\right)\end{array}$ & $\mathbf{S}$ \\
\hline $9 \mathrm{U}-22 / 0-15$ & $2.21 \mathrm{E}-07$ & $2.14 \mathrm{E}-07$ & 3.04 & $6.72 \mathrm{E}-09$ & 9.99E-05 & 3.19E-03 & $2.38 \mathrm{E}-03$ & 4.07E-04 & 0.75 \\
\hline $9 \mathrm{U}-22 / 15-27$ & $2.28 \mathrm{E}-07$ & $2.24 \mathrm{E}-07$ & 2.03 & 4.63E-09 & $1.08 \mathrm{E}-04$ & $3.28 \mathrm{E}-03$ & $2.50 \mathrm{E}-03$ & 3.93E-04 & 0.76 \\
\hline $9 \mathrm{U}-22 / 27-41$ & $2.80 \mathrm{E}-07$ & $2.74 \mathrm{E}-07$ & 2.01 & $5.88 \mathrm{E}-09$ & $1.37 \mathrm{E}-04$ & $3.80 \mathrm{E}-03$ & $3.01 \mathrm{E}-03$ & 3.51E-04 & 0.82 \\
\hline $9 \mathrm{U}-22 / 41-53$ & $3.48 \mathrm{E}-07$ & 3.39E-07 & 2.43 & $8.45 \mathrm{E}-09$ & $1.81 \mathrm{E}-04$ & 4.79E-03 & $4.08 \mathrm{E}-03$ & 3.55E-04 & 0.85 \\
\hline 9U-22/53-67 & $3.68 \mathrm{E}-07$ & $3.59 \mathrm{E}-07$ & 2.43 & 8.94E-09 & $1.41 \mathrm{E}-04$ & $5.00 \mathrm{E}-03$ & $4.32 \mathrm{E}-03$ & $3.41 \mathrm{E}-04$ & 0.86 \\
\hline $9 \mathrm{U}-22 / 67-75$ & 3.33E-07 & $3.28 \mathrm{E}-07$ & 1.63 & 5.42E-09 & $1.20 \mathrm{E}-04$ & 4.91E-03 & $4.19 \mathrm{E}-03$ & 3.61E-04 & 0.85 \\
\hline $9 \mathrm{U}-22 / 75-87$ & $2.62 \mathrm{E}-07$ & $2.57 \mathrm{E}-07$ & 1.85 & 4.84E-09 & $8.92 \mathrm{E}-05$ & $3.85 \mathrm{E}-03$ & $3.24 \mathrm{E}-03$ & $3.05 \mathrm{E}-04$ & 0.84 \\
\hline 9U-22/87-97 & $2.40 \mathrm{E}-07$ & $2.34 \mathrm{E}-07$ & 2.83 & $6.81 \mathrm{E}-09$ & 7.35E-05 & $3.38 \mathrm{E}-03$ & $2.85 \mathrm{E}-03$ & $2.68 \mathrm{E}-04$ & 0.84 \\
\hline 9U-22/97-107 & $1.95 \mathrm{E}-07$ & $1.91 \mathrm{E}-07$ & 2.26 & 4.41E-09 & 5.82E-05 & $2.74 \mathrm{E}-03$ & $2.24 \mathrm{E}-03$ & $2.48 \mathrm{E}-04$ & 0.82 \\
\hline $9 \mathrm{U}-22 / 107-116$ & $1.67 \mathrm{E}-07$ & $1.63 \mathrm{E}-07$ & 2.40 & $4.02 \mathrm{E}-09$ & 4.93E-05 & $2.44 \mathrm{E}-03$ & $1.95 \mathrm{E}-03$ & $2.44 \mathrm{E}-04$ & 0.80 \\
\hline $9 \mathrm{U}-22 / 116-124$ & $1.61 \mathrm{E}-07$ & $1.59 \mathrm{E}-07$ & 1.35 & 2.18E-09 & $5.90 \mathrm{E}-05$ & $2.22 \mathrm{E}-03$ & $1.77 \mathrm{E}-03$ & $2.22 \mathrm{E}-04$ & 0.80 \\
\hline $9 \mathrm{U}-22 / 124-136$ & $1.45 \mathrm{E}-07$ & $1.40 \mathrm{E}-07$ & 3.44 & 4.99E-09 & 4.68E-05 & $2.06 \mathrm{E}-03$ & $1.61 \mathrm{E}-03$ & $2.25 \mathrm{E}-04$ & 0.78 \\
\hline $9 \mathrm{U}-22 / 136-146$ & $1.12 \mathrm{E}-07$ & 1.11E-07 & 0.98 & $1.10 \mathrm{E}-09$ & 3.93E-05 & $1.58 \mathrm{E}-03$ & $1.21 \mathrm{E}-03$ & 1.81E-04 & 0.77 \\
\hline $9 \mathrm{U}-22 / 146-156$ & $1.16 \mathrm{E}-07$ & $1.17 \mathrm{E}-07$ & -0.78 & $-9.08 \mathrm{E}-10$ & 4.24E-05 & $1.63 \mathrm{E}-03$ & $1.28 \mathrm{E}-03$ & $1.80 \mathrm{E}-04$ & 0.78 \\
\hline $9 \mathrm{U}-22 / 156-165$ & $1.61 \mathrm{E}-07$ & $1.57 \mathrm{E}-07$ & 2.92 & 4.72E-09 & $3.17 \mathrm{E}-05$ & $2.26 \mathrm{E}-03$ & $1.83 \mathrm{E}-03$ & 2.13E-04 & 0.81 \\
\hline $9 \mathrm{U}-22 / 165-178$ & $2.67 \mathrm{E}-07$ & $2.63 \mathrm{E}-07$ & 1.35 & 3.61E-09 & 5.71E-05 & $3.35 \mathrm{E}-03$ & $2.83 \mathrm{E}-03$ & 2.60E-04 & 0.85 \\
\hline 9U-22/178-190 & $2.30 \mathrm{E}-07$ & $2.25 \mathrm{E}-07$ & 2.45 & 5.65E-09 & $5.30 \mathrm{E}-05$ & $3.18 \mathrm{E}-03$ & $2.60 \mathrm{E}-03$ & $2.89 \mathrm{E}-04$ & 0.82 \\
\hline $9 \mathrm{U}-22 / 190-204$ & $1.99 \mathrm{E}-07$ & $1.97 \mathrm{E}-07$ & 1.06 & $2.11 \mathrm{E}-09$ & 5.39E-05 & $2.72 \mathrm{E}-03$ & 2.11E-03 & 3.04E-04 & 0.78 \\
\hline $9 \mathrm{U}-22 / 204-216$ & $2.07 \mathrm{E}-07$ & $2.04 \mathrm{E}-07$ & 1.27 & 2.62E-09 & 4.84E-05 & $2.85 \mathrm{E}-03$ & $2.25 \mathrm{E}-03$ & 3.00E-04 & 0.79 \\
\hline $9 \mathrm{U}-22 / 216-229$ & $2.21 \mathrm{E}-07$ & 2.19E-07 & 0.95 & 2.01E-09 & $5.66 \mathrm{E}-05$ & $3.15 \mathrm{E}-03$ & $2.43 \mathrm{E}-03$ & 3.60E-04 & 0.77 \\
\hline $9 \mathrm{U}-22 / 229-240$ & $2.06 \mathrm{E}-07$ & $2.04 \mathrm{E}-07$ & 1.23 & 2.53E-09 & $6.22 \mathrm{E}-05$ & $2.84 \mathrm{E}-03$ & $2.19 \mathrm{E}-03$ & 3.23E-04 & 0.77 \\
\hline $9 \mathrm{U}-22 / 240-254$ & $1.90 \mathrm{E}-07$ & $1.88 \mathrm{E}-07$ & 1.32 & 2.50E-09 & $5.29 \mathrm{E}-05$ & $2.51 \mathrm{E}-03$ & $1.94 \mathrm{E}-03$ & $2.85 \mathrm{E}-04$ & 0.77 \\
\hline $9 \mathrm{U}-22 / 254-265$ & $2.05 \mathrm{E}-07$ & $1.89 \mathrm{E}-07$ & 7.65 & 1.57E-08 & $5.55 \mathrm{E}-05$ & $2.61 \mathrm{E}-03$ & $2.03 \mathrm{E}-03$ & $2.87 \mathrm{E}-04$ & 0.78 \\
\hline $9 \mathrm{U}-22 / 265-274$ & $1.87 \mathrm{E}-07$ & $1.85 \mathrm{E}-07$ & 0.97 & $1.82 \mathrm{E}-09$ & $5.98 \mathrm{E}-05$ & $2.65 \mathrm{E}-03$ & $2.02 \mathrm{E}-03$ & 3.17E-04 & 0.76 \\
\hline $9 \mathrm{U}-22 / 274-283$ & $1.81 \mathrm{E}-07$ & $1.77 \mathrm{E}-07$ & 2.00 & 3.61E-09 & 7.01E-05 & $2.65 \mathrm{E}-03$ & $1.95 \mathrm{E}-03$ & 3.53E-04 & 0.73 \\
\hline $9 U-22 / 283-295$ & $1.75 \mathrm{E}-07$ & $1.71 \mathrm{E}-07$ & 2.38 & 4.16E-09 & $6.84 \mathrm{E}-05$ & $2.36 \mathrm{E}-03$ & $1.67 \mathrm{E}-03$ & 3.43E-04 & 0.71 \\
\hline $9 \mathrm{U}-22 / 295-305$ & $1.83 \mathrm{E}-07$ & $1.73 \mathrm{E}-07$ & 5.66 & $1.04 \mathrm{E}-08$ & 7.77E-05 & $2.58 \mathrm{E}-03$ & $1.92 \mathrm{E}-03$ & 3.31E-04 & 0.74 \\
\hline $9 \mathrm{U}-22 / 305-314$ & $1.87 \mathrm{E}-07$ & $1.84 \mathrm{E}-07$ & 1.96 & 3.68E-09 & $8.88 \mathrm{E}-05$ & $2.66 \mathrm{E}-03$ & $2.03 \mathrm{E}-03$ & $3.14 \mathrm{E}-04$ & 0.76 \\
\hline $9 \mathrm{U}-22 / 314-325$ & $1.70 \mathrm{E}-07$ & $1.68 \mathrm{E}-07$ & 1.21 & 2.05E-09 & $9.00 \mathrm{E}-05$ & $2.33 \mathrm{E}-03$ & $1.77 \mathrm{E}-03$ & $2.78 \mathrm{E}-04$ & 0.76 \\
\hline $9 \mathrm{U}-22 / 325-335$ & $1.55 \mathrm{E}-07$ & $1.52 \mathrm{E}-07$ & 1.99 & 3.09E-09 & $8.30 \mathrm{E}-05$ & $2.16 \mathrm{E}-03$ & $1.64 \mathrm{E}-03$ & $2.58 \mathrm{E}-04$ & 0.76 \\
\hline $9 \mathrm{U}-22 / 335-343$ & $1.33 \mathrm{E}-07$ & $1.31 \mathrm{E}-07$ & 1.81 & 2.40E-09 & 7.20E-05 & $1.88 \mathrm{E}-03$ & $1.42 \mathrm{E}-03$ & 2.30E-04 & 0.76 \\
\hline $9 \mathrm{U}-22 / 343-354$ & $1.20 \mathrm{E}-07$ & $1.18 \mathrm{E}-07$ & 1.10 & 1.32E-09 & $5.54 \mathrm{E}-05$ & $1.71 \mathrm{E}-03$ & $1.27 \mathrm{E}-03$ & 2.20E-04 & 0.74 \\
\hline $9 \mathrm{U}-22 / 354-364$ & $1.19 \mathrm{E}-07$ & $1.19 \mathrm{E}-07$ & 0.28 & $3.32 \mathrm{E}-10$ & 5.79E-05 & $1.73 \mathrm{E}-03$ & $1.30 \mathrm{E}-03$ & 2.13E-04 & 0.75 \\
\hline $9 \mathrm{U}-22 / 364-372$ & $1.05 \mathrm{E}-07$ & $1.06 \mathrm{E}-07$ & -0.41 & $-4.36 \mathrm{E}-10$ & 4.98E-05 & $1.55 \mathrm{E}-03$ & $1.16 \mathrm{E}-03$ & $1.98 \mathrm{E}-04$ & 0.75 \\
\hline $9 \mathrm{U}-22 / 372-383$ & 8.33E-08 & $8.45 \mathrm{E}-08$ & -1.44 & $-1.20 \mathrm{E}-09$ & 2.92E-05 & $1.20 \mathrm{E}-03$ & $8.82 \mathrm{E}-04$ & $1.58 \mathrm{E}-04$ & 0.74 \\
\hline
\end{tabular}


Table 6a-9. Magnetic properties of the less than 2-mm size fraction for auger hole 9U-23.

\begin{tabular}{|c|c|c|c|c|c|c|c|c|c|}
\hline Sample \# & $\begin{array}{l}\text { MSIf } \\
\left(\mathbf{m}^{3} / \mathbf{k g}\right)\end{array}$ & $\begin{array}{l}\text { MShf } \\
\left(\mathbf{m}^{3} / \mathbf{k g}\right)\end{array}$ & $\begin{array}{c}\text { FDMS } \\
(\%)\end{array}$ & $\begin{array}{l}\text { FDMS } \\
\left(\mathrm{m}^{3} / \mathrm{kg}\right)\end{array}$ & $\begin{array}{c}\text { ARM } \\
\left(\mathrm{Am}^{2} / \mathrm{kg}\right)\end{array}$ & $\begin{array}{c}\text { IRM1.2 } \\
\left(\mathrm{Am}^{2} / \mathrm{kg}\right)\end{array}$ & $\begin{array}{l}\text { IRM -0.3 } \\
\left(\mathrm{Am}^{2} / \mathrm{kg}\right)\end{array}$ & $\begin{array}{c}\text { HIRM } \\
\left(\mathrm{Am}^{2} / \mathrm{kg}\right)\end{array}$ & S \\
\hline $9 \mathrm{U}-23 / 0-22$ & $1.44 \mathrm{E}-07$ & $1.44 \mathrm{E}-07$ & 0.52 & $7.50 \mathrm{E}-10$ & $6.66 \mathrm{E}-05$ & $2.08 \mathrm{E}-03$ & $1.53 \mathrm{E}-03$ & $2.80 \mathrm{E}-04$ & 0.73 \\
\hline $9 U-23 / 22-23$ & $1.67 \mathrm{E}-07$ & $1.66 \mathrm{E}-07$ & 0.76 & $1.26 \mathrm{E}-09$ & $6.82 \mathrm{E}-05$ & $2.11 \mathrm{E}-03$ & $1.57 \mathrm{E}-03$ & $2.73 \mathrm{E}-04$ & 0.74 \\
\hline $9 U-23 / 33-46$ & $1.94 \mathrm{E}-07$ & $1.91 \mathrm{E}-07$ & 1.55 & $3.00 \mathrm{E}-09$ & 8.77E-05 & $2.73 \mathrm{E}-03$ & $2.01 \mathrm{E}-03$ & $3.18 \mathrm{E}-04$ & 0.77 \\
\hline $9 U-23 / 46-58$ & $2.48 \mathrm{E}-07$ & $2.44 \mathrm{E}-07$ & 1.34 & 3.33E-09 & $1.11 \mathrm{E}-04$ & $3.39 \mathrm{E}-03$ & $2.84 \mathrm{E}-03$ & 2.73E-04 & 0.84 \\
\hline $9 U-23 / 58-67$ & $2.58 \mathrm{E}-07$ & $2.54 \mathrm{E}-07$ & 1.30 & 3.34E-09 & $1.09 \mathrm{E}-04$ & $3.61 \mathrm{E}-03$ & $3.01 \mathrm{E}-03$ & $2.54 \mathrm{E}-04$ & 0.86 \\
\hline $9 U-23 / 67-75$ & $2.41 \mathrm{E}-07$ & $2.38 \mathrm{E}-07$ & 1.36 & $3.27 \mathrm{E}-09$ & $9.86 \mathrm{E}-05$ & $3.44 \mathrm{E}-03$ & $2.89 \mathrm{E}-03$ & $2.73 \mathrm{E}-04$ & 0.84 \\
\hline $9 U-23 / 75-83$ & $2.30 \mathrm{E}-07$ & $2.29 \mathrm{E}-07$ & 0.81 & $1.88 \mathrm{E}-09$ & $8.29 \mathrm{E}-05$ & 3.33E-03 & $2.79 \mathrm{E}-03$ & $2.71 \mathrm{E}-04$ & 0.84 \\
\hline $9 U-23 / 83-96$ & $1.93 \mathrm{E}-07$ & $1.93 \mathrm{E}-07$ & 0.04 & $7.96 \mathrm{E}-11$ & $6.89 \mathrm{E}-05$ & $2.75 \mathrm{E}-03$ & $2.24 \mathrm{E}-03$ & $2.52 \mathrm{E}-04$ & 0.82 \\
\hline 9U-23/96-105 & $1.64 \mathrm{E}-07$ & $1.62 \mathrm{E}-07$ & 0.95 & $1.55 \mathrm{E}-09$ & $6.47 \mathrm{E}-05$ & $2.35 \mathrm{E}-03$ & $1.90 \mathrm{E}-03$ & $2.24 \mathrm{E}-04$ & 0.81 \\
\hline $9 U-23 / 105-115$ & $1.24 \mathrm{E}-07$ & $1.24 \mathrm{E}-07$ & 0.02 & $2.35 \mathrm{E}-11$ & $5.31 \mathrm{E}-05$ & $1.74 \mathrm{E}-03$ & $1.37 \mathrm{E}-03$ & $1.84 \mathrm{E}-04$ & 0.79 \\
\hline $9 U-23 / 115-125$ & $1.26 \mathrm{E}-07$ & $1.26 \mathrm{E}-07$ & 0.46 & $5.81 \mathrm{E}-10$ & 5.19E-05 & $1.77 \mathrm{E}-03$ & $1.37 \mathrm{E}-03$ & $2.00 \mathrm{E}-04$ & 0.77 \\
\hline $9 U-23 / 125-138$ & $1.23 \mathrm{E}-07$ & $1.23 \mathrm{E}-07$ & -0.15 & $-1.84 \mathrm{E}-10$ & 4.78E-05 & $1.68 \mathrm{E}-03$ & $1.28 \mathrm{E}-03$ & $2.00 \mathrm{E}-04$ & 0.76 \\
\hline $9 U-23 / 138-148$ & $1.37 \mathrm{E}-07$ & $1.37 \mathrm{E}-07$ & -0.19 & $-2.63 \mathrm{E}-10$ & $5.27 \mathrm{E}-05$ & $1.94 \mathrm{E}-03$ & $1.49 \mathrm{E}-03$ & $2.23 \mathrm{E}-04$ & 0.77 \\
\hline $9 U-23 / 148-159$ & $1.25 \mathrm{E}-07$ & $1.25 \mathrm{E}-07$ & -0.49 & $-6.08 \mathrm{E}-10$ & $4.81 \mathrm{E}-05$ & $1.72 \mathrm{E}-03$ & $1.31 \mathrm{E}-03$ & $2.05 \mathrm{E}-04$ & 0.76 \\
\hline $9 U-23 / 159-171$ & $1.22 \mathrm{E}-07$ & $1.22 \mathrm{E}-07$ & 0.19 & $2.31 \mathrm{E}-10$ & $5.55 \mathrm{E}-05$ & $1.72 \mathrm{E}-03$ & $1.23 \mathrm{E}-03$ & $2.47 \mathrm{E}-04$ & 0.71 \\
\hline $9 U-23 / 171-182$ & $1.63 \mathrm{E}-07$ & $1.62 \mathrm{E}-07$ & 0.98 & $1.60 \mathrm{E}-09$ & 7.61E-05 & $2.26 \mathrm{E}-03$ & $1.67 \mathrm{E}-03$ & $2.96 \mathrm{E}-04$ & 0.74 \\
\hline 9U-23/182-192 & $1.72 \mathrm{E}-07$ & $1.70 \mathrm{E}-07$ & 1.26 & 2.17E-09 & 7.92E-05 & $2.35 \mathrm{E}-03$ & $1.76 \mathrm{E}-03$ & $2.94 \mathrm{E}-04$ & 0.75 \\
\hline 9U-23/192-201 & $1.63 \mathrm{E}-07$ & $1.61 \mathrm{E}-07$ & 1.14 & $1.85 \mathrm{E}-09$ & $7.89 \mathrm{E}-05$ & $2.26 \mathrm{E}-03$ & $1.68 \mathrm{E}-03$ & $2.92 \mathrm{E}-04$ & 0.74 \\
\hline $9 U-23 / 201-208$ & $1.43 \mathrm{E}-07$ & $1.42 \mathrm{E}-07$ & 0.96 & 1.38E-09 & $7.16 \mathrm{E}-05$ & $2.05 \mathrm{E}-03$ & $1.51 \mathrm{E}-03$ & $2.70 \mathrm{E}-04$ & 0.74 \\
\hline $9 U-23 / 208-218$ & $1.17 \mathrm{E}-07$ & $1.17 \mathrm{E}-07$ & 0.09 & $1.06 \mathrm{E}-10$ & $5.38 \mathrm{E}-05$ & $1.64 \mathrm{E}-03$ & $1.23 \mathrm{E}-03$ & $2.07 \mathrm{E}-04$ & 0.75 \\
\hline $9 U-23 / 218-228$ & $9.39 \mathrm{E}-08$ & $9.49 \mathrm{E}-08$ & -1.09 & $-1.02 \mathrm{E}-09$ & $3.98 \mathrm{E}-05$ & $1.34 \mathrm{E}-03$ & $10.00 \mathrm{E}-04$ & $1.69 \mathrm{E}-04$ & 0.75 \\
\hline $9 \mathrm{U}-23 / 228-236$ & $1.00 \mathrm{E}-07$ & $1.01 \mathrm{E}-07$ & -0.89 & $-8.92 \mathrm{E}-10$ & 4.01E-05 & $1.40 \mathrm{E}-03$ & $1.06 \mathrm{E}-03$ & $1.68 \mathrm{E}-04$ & 0.76 \\
\hline $9 \mathrm{U}-23 / 236-248$ & $9.34 \mathrm{E}-08$ & $9.45 \mathrm{E}-08$ & -1.24 & $-1.16 \mathrm{E}-09$ & $3.62 \mathrm{E}-05$ & $1.32 \mathrm{E}-03$ & $9.98 \mathrm{E}-04$ & $1.60 \mathrm{E}-04$ & 0.76 \\
\hline $9 \mathrm{U}-23 / 248-260$ & 7.91E-08 & $8.03 \mathrm{E}-08$ & -1.61 & $-1.27 \mathrm{E}-09$ & $3.14 \mathrm{E}-05$ & $1.11 \mathrm{E}-03$ & 8.13E-04 & $1.48 \mathrm{E}-04$ & 0.73 \\
\hline $9 U-23 / 260-270$ & $8.51 \mathrm{E}-08$ & 8.67E-08 & -1.85 & $-1.57 \mathrm{E}-09$ & $3.35 \mathrm{E}-05$ & $1.12 \mathrm{E}-03$ & $8.13 \mathrm{E}-04$ & $1.51 \mathrm{E}-04$ & 0.73 \\
\hline $9 \mathrm{U}-23 / 270-280$ & $1.22 \mathrm{E}-07$ & $1.23 \mathrm{E}-07$ & -0.38 & $-4.70 \mathrm{E}-10$ & 4.43E-05 & $1.73 \mathrm{E}-03$ & $1.34 \mathrm{E}-03$ & $1.92 \mathrm{E}-04$ & 0.78 \\
\hline $9 U-23 / 280-290$ & $9.71 \mathrm{E}-08$ & $9.84 \mathrm{E}-08$ & -1.32 & $-1.28 \mathrm{E}-09$ & 4.04E-05 & $1.39 \mathrm{E}-03$ & $1.09 \mathrm{E}-03$ & $1.50 \mathrm{E}-04$ & 0.78 \\
\hline $9 \mathrm{U}-23 / 290-298$ & $2.43 \mathrm{E}-08$ & $2.67 \mathrm{E}-08$ & -9.95 & $-2.41 \mathrm{E}-09$ & $1.64 \mathrm{E}-05$ & $3.47 \mathrm{E}-04$ & 2.57E-04 & 4.48E-05 & 0.74 \\
\hline
\end{tabular}


Table 6a-10a. Magnetic properties of the less than 2-mm size fraction for auger hole 00U-27.

\begin{tabular}{|c|c|c|c|c|c|c|c|c|c|}
\hline Sample \# & $\begin{array}{c}\text { MSIf } \\
\left(\mathrm{m}^{3} / \mathrm{kg}\right)\end{array}$ & $\begin{array}{c}\text { MShf } \\
\left(\mathbf{m}^{3} / \mathbf{k g}\right)\end{array}$ & $\begin{array}{c}\text { FDMS } \\
(\%)\end{array}$ & $\begin{array}{l}\text { FDMS } \\
\left(\mathrm{m}^{3} / \mathrm{kg}\right)\end{array}$ & $\begin{array}{c}\text { ARM } \\
\left(\mathrm{Am}^{2} / \mathbf{k g}\right)\end{array}$ & $\begin{array}{c}\text { IRM1.2 } \\
\left(\mathrm{Am}^{2} / \mathrm{kg}\right)\end{array}$ & $\begin{array}{l}\text { IRM -0.3 } \\
\left(\mathrm{Am}^{2} / \mathrm{kg}\right) \\
\end{array}$ & $\begin{array}{c}\text { HIRM } \\
\left(\mathrm{Am}^{2} / \mathbf{k g}\right) \\
\end{array}$ & S \\
\hline 00U-27/0-10 & $2.19 \mathrm{E}-07$ & $2.12 \mathrm{E}-07$ & 3.11 & $6.79 \mathrm{E}-09$ & $9.54 \mathrm{E}-05$ & $3.25 \mathrm{E}-03$ & $2.46 \mathrm{E}-03$ & $3.97 \mathrm{E}-04$ & 0.76 \\
\hline 00U-27/10-30 & $2.36 \mathrm{E}-07$ & $2.29 \mathrm{E}-07$ & 2.98 & 7.03E-09 & $1.06 \mathrm{E}-04$ & 3.30E-03 & $2.51 \mathrm{E}-03$ & 3.97E-04 & 0.76 \\
\hline 00U-27/30-50 & $2.39 \mathrm{E}-07$ & $2.32 \mathrm{E}-07$ & 2.68 & $6.40 \mathrm{E}-09$ & $1.09 \mathrm{E}-04$ & $3.28 \mathrm{E}-03$ & $2.57 \mathrm{E}-03$ & $3.55 \mathrm{E}-04$ & 0.78 \\
\hline 00U-27/50-65 & $2.88 \mathrm{E}-07$ & $2.81 \mathrm{E}-07$ & 2.45 & 7.05E-09 & $1.43 \mathrm{E}-04$ & 4.07E-03 & $3.25 \mathrm{E}-03$ & $4.08 \mathrm{E}-04$ & 0.80 \\
\hline 00U-27/65-84 & $3.83 \mathrm{E}-07$ & $3.70 \mathrm{E}-07$ & 3.51 & $1.34 \mathrm{E}-08$ & $2.29 \mathrm{E}-04$ & $5.49 \mathrm{E}-03$ & 4.67E-03 & 4.01E-04 & 0.85 \\
\hline 00U-27/84-100 & 4.40E-07 & $4.24 \mathrm{E}-07$ & 3.68 & $1.62 \mathrm{E}-08$ & $2.54 \mathrm{E}-04$ & $6.01 \mathrm{E}-03$ & $5.14 \mathrm{E}-03$ & $4.80 \mathrm{E}-04$ & 0.84 \\
\hline 00U-27/100-116 & $4.68 \mathrm{E}-07$ & $4.56 \mathrm{E}-07$ & 2.57 & $1.20 \mathrm{E}-08$ & $1.82 \mathrm{E}-04$ & $6.47 \mathrm{E}-03$ & $5.60 \mathrm{E}-03$ & $4.36 \mathrm{E}-04$ & 0.87 \\
\hline 00U-27/116-128 & 4.17E-07 & 4.09E-07 & 1.99 & $8.28 \mathrm{E}-09$ & $1.48 \mathrm{E}-04$ & $5.81 \mathrm{E}-03$ & 4.98E-03 & 4.17E-04 & 0.86 \\
\hline 00U-27/128-141 & 4.17E-07 & $4.12 \mathrm{E}-07$ & 1.28 & 5.35E-09 & $1.21 \mathrm{E}-04$ & $5.76 \mathrm{E}-03$ & $5.06 \mathrm{E}-03$ & $3.48 \mathrm{E}-04$ & 0.88 \\
\hline 00U-27/141-156 & $3.18 \mathrm{E}-07$ & $3.14 \mathrm{E}-07$ & 1.11 & $3.54 \mathrm{E}-09$ & 8.92E-05 & 4.22E-03 & $3.53 \mathrm{E}-03$ & $3.47 \mathrm{E}-04$ & 0.84 \\
\hline 00U-27/156-168 & $2.49 \mathrm{E}-07$ & $2.47 \mathrm{E}-07$ & 0.66 & $1.64 \mathrm{E}-09$ & $6.80 \mathrm{E}-05$ & $3.26 \mathrm{E}-03$ & $2.66 \mathrm{E}-03$ & $2.99 \mathrm{E}-04$ & 0.82 \\
\hline 00U-27/168-179 & $2.14 \mathrm{E}-07$ & $2.11 \mathrm{E}-07$ & 1.38 & 2.96E-09 & $6.46 \mathrm{E}-05$ & $2.85 \mathrm{E}-03$ & $2.26 \mathrm{E}-03$ & $2.97 \mathrm{E}-04$ & 0.79 \\
\hline 00U-27/179-194 & $1.56 \mathrm{E}-07$ & $1.56 \mathrm{E}-07$ & 0.36 & $5.58 \mathrm{E}-10$ & 4.79E-05 & $2.11 \mathrm{E}-03$ & $1.61 \mathrm{E}-03$ & $2.53 \mathrm{E}-04$ & 0.76 \\
\hline 00U-27/194-208 & $1.50 \mathrm{E}-07$ & $1.49 \mathrm{E}-07$ & 0.55 & $8.26 \mathrm{E}-10$ & $4.58 \mathrm{E}-05$ & $2.05 \mathrm{E}-03$ & $1.50 \mathrm{E}-03$ & $2.73 \mathrm{E}-04$ & 0.73 \\
\hline 00U-27/208-221 & $1.52 \mathrm{E}-07$ & $1.51 \mathrm{E}-07$ & 0.99 & $1.50 \mathrm{E}-09$ & $5.17 \mathrm{E}-05$ & $2.09 \mathrm{E}-03$ & $1.52 \mathrm{E}-03$ & $2.85 \mathrm{E}-04$ & 0.73 \\
\hline 00U-27/221-233 & $1.55 \mathrm{E}-07$ & $1.55 \mathrm{E}-07$ & 0.13 & $2.06 \mathrm{E}-10$ & $5.58 \mathrm{E}-05$ & $2.19 \mathrm{E}-03$ & $1.60 \mathrm{E}-03$ & 2.93E-04 & 0.73 \\
\hline 00U-27/233-242 & $1.41 \mathrm{E}-07$ & $1.41 \mathrm{E}-07$ & -0.39 & $-5.55 \mathrm{E}-10$ & $5.01 \mathrm{E}-05$ & $2.00 \mathrm{E}-03$ & $1.47 \mathrm{E}-03$ & $2.66 \mathrm{E}-04$ & 0.73 \\
\hline 00U-27/242-252 & $1.39 \mathrm{E}-07$ & $1.39 \mathrm{E}-07$ & 0.36 & 4.94E-10 & 4.94E-05 & $1.97 \mathrm{E}-03$ & $1.46 \mathrm{E}-03$ & $2.54 \mathrm{E}-04$ & 0.74 \\
\hline 00U-27/242-265 & $1.23 \mathrm{E}-07$ & $1.24 \mathrm{E}-07$ & -0.98 & $-1.20 \mathrm{E}-09$ & $4.69 \mathrm{E}-05$ & $1.74 \mathrm{E}-03$ & $1.28 \mathrm{E}-03$ & $2.30 \mathrm{E}-04$ & 0.74 \\
\hline 00U-27/265-274 & $1.41 \mathrm{E}-07$ & $1.40 \mathrm{E}-07$ & 0.78 & $1.01 \mathrm{E}-09$ & $5.30 \mathrm{E}-05$ & $1.94 \mathrm{E}-03$ & $1.48 \mathrm{E}-03$ & $2.29 \mathrm{E}-04$ & 0.76 \\
\hline 00U-27/274-283 & $1.31 \mathrm{E}-07$ & $1.31 \mathrm{E}-07$ & 0.08 & $1.09 \mathrm{E}-10$ & $4.84 \mathrm{E}-05$ & $1.81 \mathrm{E}-03$ & $1.36 \mathrm{E}-03$ & $2.23 \mathrm{E}-04$ & 0.75 \\
\hline 00U-27/283-291 & $1.92 \mathrm{E}-07$ & $1.89 \mathrm{E}-07$ & 1.43 & 2.75E-09 & $7.04 \mathrm{E}-05$ & $2.61 \mathrm{E}-03$ & $2.03 \mathrm{E}-03$ & $2.91 \mathrm{E}-04$ & 0.78 \\
\hline 00U-27/291-301 & $1.89 \mathrm{E}-07$ & $1.85 \mathrm{E}-07$ & 2.08 & 3.94E-09 & $8.07 \mathrm{E}-05$ & $2.75 \mathrm{E}-03$ & $2.11 \mathrm{E}-03$ & $3.22 \mathrm{E}-04$ & 0.77 \\
\hline 00U-27/301-313 & $1.73 \mathrm{E}-07$ & $1.71 \mathrm{E}-07$ & 1.02 & 1.77E-09 & $8.68 \mathrm{E}-05$ & $2.64 \mathrm{E}-03$ & $2.03 \mathrm{E}-03$ & 3.03E-04 & 0.77 \\
\hline 00U-27/313-324 & $1.68 \mathrm{E}-07$ & $1.66 \mathrm{E}-07$ & 1.45 & 2.43E-09 & 7.07E-05 & $2.36 \mathrm{E}-03$ & $1.77 \mathrm{E}-03$ & $2.94 \mathrm{E}-04$ & 0.75 \\
\hline
\end{tabular}


Table 6a-10b. Magnetic properties of the less than 63-micron size fraction for auger hole 00U-27.

\begin{tabular}{|c|c|c|c|c|c|c|c|c|c|}
\hline Sample \# & $\begin{array}{c}\text { MSIf } \\
\left(\mathrm{m}^{3} / \mathrm{kg}\right)\end{array}$ & $\begin{array}{c}\text { MShf } \\
\left(\mathrm{m}^{3} / \mathrm{kg}\right)\end{array}$ & $\begin{array}{c}\text { FDMS } \\
(\%)\end{array}$ & $\begin{array}{c}\text { FDMS } \\
\left(\mathrm{m}^{3} / \mathrm{kg}\right)\end{array}$ & $\begin{array}{c}\text { ARM } \\
\left(\mathrm{Am}^{2} / \mathbf{k g}\right)\end{array}$ & $\begin{array}{c}\text { IRM1.2 } \\
\left(\mathrm{Am}^{2} / \mathrm{kg}\right)\end{array}$ & $\begin{array}{l}\text { IRM }-0.3 \\
\left(\mathrm{Am}^{2} / \mathbf{k g}\right) \\
\end{array}$ & $\begin{array}{c}\text { HIRM } \\
\left(\mathrm{Am}^{2} / \mathbf{k g}\right) \\
\end{array}$ & $\mathbf{S}$ \\
\hline 00U-27/0-10 & $4.38 \mathrm{E}-07$ & $4.24 \mathrm{E}-07$ & 3.16 & $1.39 \mathrm{E}-08$ & 1.44E-04 & $6.49 \mathrm{E}-03$ & $5.00 \mathrm{E}-03$ & 7.41E-04 & 0.77 \\
\hline 00U-27/10-30 & $4.58 \mathrm{E}-07$ & 4.42E-07 & 3.60 & $1.65 \mathrm{E}-08$ & $1.53 \mathrm{E}-04$ & $6.28 \mathrm{E}-03$ & $4.90 \mathrm{E}-03$ & $6.90 \mathrm{E}-04$ & 0.78 \\
\hline 00U-27/30-50 & 4.71E-07 & $4.59 \mathrm{E}-07$ & 2.39 & $1.12 \mathrm{E}-08$ & $1.69 \mathrm{E}-04$ & $6.54 \mathrm{E}-03$ & $5.18 \mathrm{E}-03$ & $6.84 \mathrm{E}-04$ & 0.79 \\
\hline 00U-27/50-65 & $5.05 \mathrm{E}-07$ & 4.92E-07 & 2.60 & $1.31 \mathrm{E}-08$ & $2.05 \mathrm{E}-04$ & $7.12 \mathrm{E}-03$ & $5.78 \mathrm{E}-03$ & $6.71 \mathrm{E}-04$ & 0.81 \\
\hline 00U-27/65-84 & $6.43 \mathrm{E}-07$ & $6.22 \mathrm{E}-07$ & 3.35 & $2.16 \mathrm{E}-08$ & $3.12 \mathrm{E}-04$ & $9.04 \mathrm{E}-03$ & 7.74E-03 & $6.47 \mathrm{E}-04$ & 0.86 \\
\hline 00U-27/84-100 & $7.56 \mathrm{E}-07$ & $7.30 \mathrm{E}-07$ & 3.50 & $2.65 \mathrm{E}-08$ & $3.45 \mathrm{E}-04$ & $1.04 \mathrm{E}-02$ & $8.96 \mathrm{E}-03$ & 7.05E-04 & 0.86 \\
\hline 00U-27/100-116 & 8.03E-07 & 7.79E-07 & 3.03 & $2.44 \mathrm{E}-08$ & $2.50 \mathrm{E}-04$ & $1.10 \mathrm{E}-02$ & $9.56 \mathrm{E}-03$ & $7.31 \mathrm{E}-04$ & 0.87 \\
\hline 00U-27/116-128 & 7.17E-07 & $7.00 \mathrm{E}-07$ & 2.39 & $1.71 \mathrm{E}-08$ & $2.09 \mathrm{E}-04$ & $1.00 \mathrm{E}-02$ & $8.65 \mathrm{E}-03$ & $6.77 \mathrm{E}-04$ & 0.86 \\
\hline 00U-27/128-141 & 7.63E-07 & 7.43E-07 & 2.59 & $1.98 \mathrm{E}-08$ & $1.90 \mathrm{E}-04$ & $1.06 \mathrm{E}-02$ & $9.30 \mathrm{E}-03$ & $6.49 \mathrm{E}-04$ & 0.88 \\
\hline 00U-27/141-156 & $6.59 \mathrm{E}-07$ & $6.43 \mathrm{E}-07$ & 2.43 & $1.60 \mathrm{E}-08$ & $1.59 \mathrm{E}-04$ & 8.67E-03 & 7.39E-03 & $6.39 \mathrm{E}-04$ & 0.85 \\
\hline 00U-27/156-168 & $5.78 \mathrm{E}-07$ & $5.67 \mathrm{E}-07$ & 1.89 & $1.09 \mathrm{E}-08$ & $1.29 \mathrm{E}-04$ & 7.46E-03 & $6.24 \mathrm{E}-03$ & $6.13 \mathrm{E}-04$ & 0.84 \\
\hline 00U-27/168-179 & $4.82 \mathrm{E}-07$ & 4.71E-07 & 2.25 & $1.08 \mathrm{E}-08$ & $1.19 \mathrm{E}-04$ & $6.31 \mathrm{E}-03$ & $5.17 \mathrm{E}-03$ & $5.68 \mathrm{E}-04$ & 0.82 \\
\hline 00U-27/179-194 & $3.84 \mathrm{E}-07$ & $3.78 \mathrm{E}-07$ & 1.53 & $5.86 \mathrm{E}-09$ & $9.17 \mathrm{E}-05$ & $5.09 \mathrm{E}-03$ & 4.01E-03 & $5.42 \mathrm{E}-04$ & 0.79 \\
\hline 00U-27/194-208 & 4.17E-07 & $4.09 \mathrm{E}-07$ & 2.00 & 8.33E-09 & $9.14 \mathrm{E}-05$ & 5.43E-03 & $4.25 \mathrm{E}-03$ & 5.87E-04 & 0.78 \\
\hline 00U-27/208-221 & $4.45 \mathrm{E}-07$ & 4.37E-07 & 1.69 & 7.52E-09 & $1.08 \mathrm{E}-04$ & $5.86 \mathrm{E}-03$ & $4.58 \mathrm{E}-03$ & $6.43 \mathrm{E}-04$ & 0.78 \\
\hline 00U-27/221-235 & 4.17E-07 & $4.08 \mathrm{E}-07$ & 2.04 & $8.52 \mathrm{E}-09$ & $1.16 \mathrm{E}-04$ & $5.60 \mathrm{E}-03$ & $4.36 \mathrm{E}-03$ & $6.18 \mathrm{E}-04$ & 0.78 \\
\hline 00U-27/233-242 & $4.21 \mathrm{E}-07$ & $4.11 \mathrm{E}-07$ & 2.27 & $9.55 \mathrm{E}-09$ & 1.17E-04 & $5.70 \mathrm{E}-03$ & 4.44E-03 & $6.32 \mathrm{E}-04$ & 0.78 \\
\hline 00U-27/242-252 & $3.81 \mathrm{E}-07$ & $3.71 \mathrm{E}-07$ & 2.66 & $1.01 \mathrm{E}-08$ & $1.11 \mathrm{E}-04$ & $5.20 \mathrm{E}-03$ & 4.03E-03 & $5.83 \mathrm{E}-04$ & 0.78 \\
\hline 00U-27/242-265 & $3.93 \mathrm{E}-07$ & $3.85 \mathrm{E}-07$ & 1.81 & 7.08E-09 & $1.13 \mathrm{E}-04$ & $5.39 \mathrm{E}-03$ & 4.17E-03 & $6.09 \mathrm{E}-04$ & 0.77 \\
\hline 00U-27/265-274 & $4.52 \mathrm{E}-07$ & 4.43E-07 & 2.01 & $9.07 \mathrm{E}-09$ & $1.28 \mathrm{E}-04$ & $6.09 \mathrm{E}-03$ & $4.82 \mathrm{E}-03$ & $6.38 \mathrm{E}-04$ & 0.79 \\
\hline 00U-27/274-283 & $4.80 \mathrm{E}-07$ & 4.71E-07 & 1.92 & $9.22 \mathrm{E}-09$ & $1.28 \mathrm{E}-04$ & $6.42 \mathrm{E}-03$ & $5.09 \mathrm{E}-03$ & $6.65 \mathrm{E}-04$ & 0.79 \\
\hline 00U-27/283-291 & $5.03 \mathrm{E}-07$ & 4.92E-07 & 2.09 & $1.05 \mathrm{E}-08$ & $1.45 \mathrm{E}-04$ & $6.71 \mathrm{E}-03$ & $5.37 \mathrm{E}-03$ & $6.67 \mathrm{E}-04$ & 0.80 \\
\hline 00U-27/291-301 & 4.39E-07 & $4.29 \mathrm{E}-07$ & 2.19 & $9.60 \mathrm{E}-09$ & $1.54 \mathrm{E}-04$ & $6.26 \mathrm{E}-03$ & 4.98E-03 & $6.40 \mathrm{E}-04$ & 0.80 \\
\hline 00U-27/301-313 & $4.25 \mathrm{E}-07$ & 4.15E-07 & 2.28 & $9.70 \mathrm{E}-09$ & $1.66 \mathrm{E}-04$ & $6.14 \mathrm{E}-03$ & 4.84E-03 & $6.50 \mathrm{E}-04$ & 0.79 \\
\hline 00U-27/313-324 & 4.61E-07 & 4.51E-07 & 2.08 & $9.59 \mathrm{E}-09$ & $1.40 \mathrm{E}-04$ & $6.16 \mathrm{E}-03$ & 4.84E-03 & $6.62 \mathrm{E}-04$ & 0.79 \\
\hline
\end{tabular}


Table 6a-11a. Magnetic properties of the less than 2-mm size fraction for auger hole 00U-31.

\begin{tabular}{|c|c|c|c|c|c|c|c|c|}
\hline Sample \# & $\begin{array}{c}\text { MShf } \\
\left(\mathrm{m}^{3} / \mathrm{kg}\right)\end{array}$ & $\begin{array}{c}\text { FDMS } \\
(\%)\end{array}$ & $\begin{array}{l}\text { FDMS } \\
\left(\mathrm{m}^{3} / \mathbf{k g}\right)\end{array}$ & $\begin{array}{c}\text { ARM } \\
\left(\mathrm{Am}^{2} / \mathbf{k g}\right)\end{array}$ & $\begin{array}{c}\text { IRM1.2 } \\
\left(\mathrm{Am}^{2} / \mathrm{kg}\right)\end{array}$ & $\begin{array}{l}\text { IRM }-0.3 \\
\left(\mathrm{Am}^{2} / \mathrm{kg}\right)\end{array}$ & $\begin{array}{c}\text { HIRM } \\
\left(\mathrm{Am}^{2} / \mathrm{kg}\right)\end{array}$ & $\mathbf{S}$ \\
\hline 00U-31/0-10 & $1.48 \mathrm{E}-07$ & 3.00 & $4.58 \mathrm{E}-09$ & $2.54 \mathrm{E}-04$ & $9.29 \mathrm{E}-03$ & $6.93 \mathrm{E}-03$ & $2.71 \mathrm{E}-04$ & 0.75 \\
\hline 00U-31/10-30 & $1.46 \mathrm{E}-07$ & -5.89 & $-8.09 \mathrm{E}-09$ & $2.39 \mathrm{E}-04$ & $8.74 \mathrm{E}-03$ & $6.55 \mathrm{E}-03$ & $2.55 \mathrm{E}-04$ & 0.75 \\
\hline 00U-31/30-47 & $1.41 \mathrm{E}-07$ & 0.94 & $1.34 \mathrm{E}-09$ & $2.39 \mathrm{E}-04$ & 8.73E-03 & $6.36 \mathrm{E}-03$ & $2.68 \mathrm{E}-04$ & 0.73 \\
\hline 00U-31/47-57 & $1.54 \mathrm{E}-07$ & 2.51 & $3.96 \mathrm{E}-09$ & $2.88 \mathrm{E}-04$ & 9.73E-03 & $7.11 \mathrm{E}-03$ & $2.96 \mathrm{E}-04$ & 0.73 \\
\hline 00U-31/57-70 & $1.60 \mathrm{E}-07$ & 3.71 & $6.16 \mathrm{E}-09$ & $2.98 \mathrm{E}-04$ & $9.46 \mathrm{E}-03$ & $6.97 \mathrm{E}-03$ & $2.94 \mathrm{E}-04$ & 0.74 \\
\hline 00U-31/70-80 & $1.60 \mathrm{E}-07$ & 3.48 & 5.75E-09 & $3.23 \mathrm{E}-04$ & $1.01 \mathrm{E}-02$ & 7.34E-03 & 2.99E-04 & 0.73 \\
\hline 00U-31/80-91 & $1.47 \mathrm{E}-07$ & 5.94 & 9.32E-09 & 3.04E-04 & $9.16 \mathrm{E}-03$ & $6.56 \mathrm{E}-03$ & 2.90E-04 & 0.72 \\
\hline 00U-31/91-100 & $1.47 \mathrm{E}-07$ & 1.58 & $2.36 \mathrm{E}-09$ & $3.01 \mathrm{E}-04$ & 8.95E-03 & $6.69 \mathrm{E}-03$ & 2.49E-04 & 0.75 \\
\hline 00U-31/100-113 & $1.24 \mathrm{E}-07$ & 0.80 & $9.96 \mathrm{E}-10$ & $2.55 \mathrm{E}-04$ & 8.06E-03 & $6.00 \mathrm{E}-03$ & $2.24 \mathrm{E}-04$ & 0.74 \\
\hline 00U-31/113-124 & $1.04 \mathrm{E}-07$ & 2.02 & 2.14E-09 & $1.95 \mathrm{E}-04$ & 7.03E-03 & $5.29 \mathrm{E}-03$ & $1.82 \mathrm{E}-04$ & 0.75 \\
\hline 00U-31/124-134 & $1.00 \mathrm{E}-07$ & 1.02 & $1.03 \mathrm{E}-09$ & $1.58 \mathrm{E}-04$ & $6.31 \mathrm{E}-03$ & $4.71 \mathrm{E}-03$ & $1.75 \mathrm{E}-04$ & 0.75 \\
\hline 00U-31/134-146 & $8.18 \mathrm{E}-08$ & -1.28 & $-1.03 \mathrm{E}-09$ & $1.19 \mathrm{E}-04$ & $5.06 \mathrm{E}-03$ & 3.73E-03 & $1.50 \mathrm{E}-04$ & 0.74 \\
\hline
\end{tabular}

Table 6a-11b. Magnetic properties of the less than 63-micron size fraction for auger hole 00U-31.

\begin{tabular}{|c|c|c|c|c|c|c|c|c|c|}
\hline Sample \# & $\begin{array}{c}\text { MSIf } \\
\left(\mathrm{m}^{3} / \mathbf{k g}\right)\end{array}$ & $\begin{array}{l}\text { MShf } \\
\left(\mathbf{m}^{3} / \mathbf{k g}\right)\end{array}$ & $\begin{array}{l}\text { FDMS } \\
(\%)\end{array}$ & $\begin{array}{l}\text { FDMS } \\
\left(\mathbf{m}^{3} / \mathbf{k g}\right)\end{array}$ & $\begin{array}{c}\text { ARM } \\
\left(\mathrm{Am}^{2} / \mathbf{k g}\right)\end{array}$ & $\begin{array}{c}\text { IRM1.2 } \\
\left(\mathrm{Am}^{2} / \mathrm{kg}\right)\end{array}$ & $\begin{array}{l}\text { IRM -0.3 } \\
\left(\mathrm{Am}^{2} / \mathrm{kg}\right)\end{array}$ & $\begin{array}{c}\text { HIRM } \\
\left(\mathrm{Am}^{2} / \mathbf{k g}\right)\end{array}$ & $\mathbf{S}$ \\
\hline 00U-31/0-10 & $5.15 \mathrm{E}-07$ & $5.08 \mathrm{E}-07$ & 1.25 & $6.45 \mathrm{E}-09$ & $4.51 \mathrm{E}-04$ & $2.89 \mathrm{E}-02$ & $2.24 \mathrm{E}-02$ & 7.67E-04 & 0.77 \\
\hline 00U-31/10-30 & 4.12E-07 & 4.09E-07 & 0.84 & 3.47E-09 & 4.07E-04 & $2.18 \mathrm{E}-02$ & $1.71 \mathrm{E}-02$ & $5.86 \mathrm{E}-04$ & 0.79 \\
\hline 00U-31/30-47 & 4.24E-07 & 4.19E-07 & 0.99 & 4.19E-09 & $3.96 \mathrm{E}-04$ & $2.12 \mathrm{E}-02$ & $1.65 \mathrm{E}-02$ & $6.15 \mathrm{E}-04$ & 0.78 \\
\hline 00U-31/47-57 & 4.73E-07 & 4.67E-07 & 1.35 & 6.39E-09 & $4.88 \mathrm{E}-04$ & $2.46 \mathrm{E}-02$ & $1.93 \mathrm{E}-02$ & $6.66 \mathrm{E}-04$ & 0.78 \\
\hline 00U-31/57-70 & 4.91E-07 & $4.83 \mathrm{E}-07$ & 1.50 & 7.38E-09 & $5.48 \mathrm{E}-04$ & $2.52 \mathrm{E}-02$ & $1.97 \mathrm{E}-02$ & $7.00 \mathrm{E}-04$ & 0.78 \\
\hline 00U-31/70-80 & $5.09 \mathrm{E}-07$ & $5.00 \mathrm{E}-07$ & 1.79 & $9.12 \mathrm{E}-09$ & $6.07 \mathrm{E}-04$ & $2.52 \mathrm{E}-02$ & 1.96E-02 & 7.35E-04 & 0.78 \\
\hline 00U-31/80-91 & $4.88 \mathrm{E}-07$ & 4.79E-07 & 1.95 & $9.54 \mathrm{E}-09$ & $6.11 \mathrm{E}-04$ & $2.41 \mathrm{E}-02$ & $1.87 \mathrm{E}-02$ & 7.04E-04 & 0.78 \\
\hline 00U-31/91-100 & 4.65E-07 & $4.56 \mathrm{E}-07$ & 1.95 & $9.06 \mathrm{E}-09$ & $5.90 \mathrm{E}-04$ & $2.29 \mathrm{E}-02$ & $1.80 \mathrm{E}-02$ & $6.44 \mathrm{E}-04$ & 0.79 \\
\hline 00U-31/100-113 & $3.77 \mathrm{E}-07$ & $3.70 \mathrm{E}-07$ & 1.92 & 7.25E-09 & 4.37E-04 & $1.70 \mathrm{E}-02$ & $1.34 \mathrm{E}-02$ & $5.13 \mathrm{E}-04$ & 0.79 \\
\hline 00U-31/113-124 & $2.86 \mathrm{E}-07$ & $2.80 \mathrm{E}-07$ & 2.14 & 6.13E-09 & $3.07 \mathrm{E}-04$ & $1.30 \mathrm{E}-02$ & 1.02E-02 & 4.07E-04 & 0.79 \\
\hline 00U-31/124-134 & $3.67 \mathrm{E}-07$ & $3.63 \mathrm{E}-07$ & 1.24 & 4.55E-09 & $3.52 \mathrm{E}-04$ & $1.81 \mathrm{E}-02$ & 1.44E-02 & 4.82E-04 & 0.80 \\
\hline 00U-31/134-146 & $3.28 \mathrm{E}-07$ & $3.26 \mathrm{E}-07$ & 0.46 & $1.50 \mathrm{E}-09$ & $2.91 \mathrm{E}-04$ & $1.55 \mathrm{E}-02$ & $1.24 \mathrm{E}-02$ & 4.32E-04 & 0.80 \\
\hline
\end{tabular}


Table 6a-12a. Magnetic properties of the less than 2-mm size fraction for auger hole 00U-33.

\begin{tabular}{|c|c|c|c|c|c|c|c|c|c|}
\hline Sample \# & $\begin{array}{c}\text { MSIf } \\
\left(\mathbf{m}^{3} / \mathbf{k g}\right)\end{array}$ & $\begin{array}{c}\text { MShf } \\
\left(\mathbf{m}^{3} / \mathbf{k g}\right)\end{array}$ & $\begin{array}{l}\text { FDMS } \\
(\%)\end{array}$ & $\begin{array}{l}\text { FDMS } \\
\left(\mathrm{m}^{3} / \mathrm{kg}\right)\end{array}$ & $\begin{array}{c}\text { ARM } \\
\left(\mathrm{Am}^{2} / \mathrm{kg}\right)\end{array}$ & $\begin{array}{c}\text { IRM1.2 } \\
\left(\mathrm{Am}^{2} / \mathrm{kg}\right)\end{array}$ & $\begin{array}{l}\text { IRM -0.3 } \\
\left(\mathrm{Am}^{2} / \mathrm{kg}\right)\end{array}$ & $\begin{array}{c}\text { HIRM } \\
\left(\mathrm{Am}^{2} / \mathbf{k g}\right)\end{array}$ & $\mathbf{S}$ \\
\hline 00U-33/0-10 & $1.44 \mathrm{E}-07$ & $1.44 \mathrm{E}-07$ & -0.56 & $-8.08 \mathrm{E}-10$ & $3.27 \mathrm{E}-04$ & $1.00 \mathrm{E}-02$ & $7.60 \mathrm{E}-03$ & $2.60 \mathrm{E}-04$ & 0.76 \\
\hline 00U-33/10-30 & $1.66 \mathrm{E}-07$ & 1.64E-07 & 0.82 & $1.35 \mathrm{E}-09$ & 3.01E-04 & 9.93E-03 & 7.42E-03 & $2.88 \mathrm{E}-04$ & 0.75 \\
\hline 00U-33/30-47 & $1.70 \mathrm{E}-07$ & 1.71E-07 & -0.58 & $-9.91 \mathrm{E}-10$ & $3.12 \mathrm{E}-04$ & $9.50 \mathrm{E}-03$ & $7.26 \mathrm{E}-03$ & $2.72 \mathrm{E}-04$ & 0.76 \\
\hline 00U-33/47-61 & $2.05 \mathrm{E}-07$ & 2.02E-07 & 1.20 & 2.46E-09 & $3.95 \mathrm{E}-04$ & $1.16 \mathrm{E}-02$ & $9.32 \mathrm{E}-03$ & $2.70 \mathrm{E}-04$ & 0.80 \\
\hline 00U-33/61-73 & $1.47 \mathrm{E}-07$ & 1.41E-07 & 3.85 & 5.67E-09 & $2.62 \mathrm{E}-04$ & $8.08 \mathrm{E}-03$ & $6.44 \mathrm{E}-03$ & 2.03E-04 & 0.80 \\
\hline 00U-33/73-89 & 7.32E-08 & 1.42E-07 & -93.64 & $-6.86 \mathrm{E}-08$ & $1.03 \mathrm{E}-04$ & 4.42E-03 & 3.33E-03 & $1.32 \mathrm{E}-04$ & 0.75 \\
\hline
\end{tabular}

Table 6a-12b. Magnetic properties of the less than 63-micron size fraction for auger hole 00U-33.

\begin{tabular}{|c|c|c|c|c|c|c|c|c|c|}
\hline Sample \# & $\begin{array}{c}\text { MSIf } \\
\left(\mathrm{m}^{3} / \mathbf{k g}\right)\end{array}$ & $\begin{array}{l}\text { MShf } \\
\left(\mathrm{m}^{3} / \mathrm{kg}\right)\end{array}$ & $\begin{array}{c}\text { FDMS } \\
(\%)\end{array}$ & $\begin{array}{l}\text { FDMS } \\
\left(\mathbf{m}^{3} / \mathbf{k g}\right)\end{array}$ & $\begin{array}{c}\text { ARM } \\
\left(\mathrm{Am}^{2} / \mathrm{kg}\right)\end{array}$ & $\begin{array}{c}\text { IRM1.2 } \\
\left(\mathrm{Am}^{2} / \mathrm{kg}\right)\end{array}$ & $\begin{array}{l}\text { IRM }-0.3 \\
\left(\mathrm{Am}^{2} / \mathrm{kg}\right)\end{array}$ & $\begin{array}{c}\text { HIRM } \\
\left(\mathrm{Am}^{2} / \mathrm{kg}\right)\end{array}$ & $\mathbf{S}$ \\
\hline 00U-33/0-10 & $4.38 \mathrm{E}-07$ & $4.30 \mathrm{E}-07$ & 1.82 & 7.98E-09 & $5.60 \mathrm{E}-04$ & $2.85 \mathrm{E}-02$ & $2.20 \mathrm{E}-02$ & 7.22E-04 & 0.77 \\
\hline 00U-33/10-30 & 4.40E-07 & 4.33E-07 & 1.48 & $6.50 \mathrm{E}-09$ & 4.63E-04 & $2.40 \mathrm{E}-02$ & $1.86 \mathrm{E}-02$ & $6.74 \mathrm{E}-04$ & 0.77 \\
\hline 00U-33/30-47 & $4.80 \mathrm{E}-07$ & 4.74E-07 & 1.12 & 5.37E-09 & $5.37 \mathrm{E}-04$ & $2.50 \mathrm{E}-02$ & $1.99 \mathrm{E}-02$ & $6.57 \mathrm{E}-04$ & 0.80 \\
\hline 00U-33/47-61 & $6.27 \mathrm{E}-07$ & $6.17 \mathrm{E}-07$ & 1.46 & 9.13E-09 & $6.90 \mathrm{E}-04$ & $3.16 \mathrm{E}-02$ & 2.64E-02 & $6.63 \mathrm{E}-04$ & 0.84 \\
\hline 00U-33/61-73 & 4.35E-07 & $4.31 \mathrm{E}-07$ & 1.01 & 4.37E-09 & $4.22 \mathrm{E}-04$ & $1.85 \mathrm{E}-02$ & $1.53 \mathrm{E}-02$ & $5.05 \mathrm{E}-04$ & 0.83 \\
\hline 00U-33/73-89 & $2.42 \mathrm{E}-07$ & $3.01 \mathrm{E}-08$ & 87.56 & $2.12 \mathrm{E}-07$ & $2.06 \mathrm{E}-04$ & $1.15 \mathrm{E}-02$ & $9.25 \mathrm{E}-03$ & $3.23 \mathrm{E}-04$ & 0.81 \\
\hline
\end{tabular}


Table 6b-1a. Magnetic properties of the less than 2-mm size fraction for the 01U-1 transect.

\begin{tabular}{|c|c|c|c|c|c|c|c|c|c|}
\hline Sample \# & $\begin{array}{c}\text { MSIf } \\
\left(\mathbf{m}^{3} / \mathbf{k g}\right)\end{array}$ & $\begin{array}{c}\text { MShf } \\
\left(\mathrm{m}^{3} / \mathbf{k g}\right)\end{array}$ & $\begin{array}{c}\text { FDMS } \\
(\%)\end{array}$ & $\begin{array}{l}\text { FDMS } \\
\left(\mathrm{m}^{3} / \mathbf{k g}\right)\end{array}$ & $\begin{array}{c}\text { ARM } \\
\left(\mathrm{Am}^{2} / \mathrm{kg}\right)\end{array}$ & $\begin{array}{c}\text { IRM1.2 } \\
\left(\mathrm{Am}^{2} / \mathrm{kg}\right) \\
\end{array}$ & $\begin{array}{l}\text { IRM }-0.3 \\
\left(\mathrm{Am}^{2} / \mathrm{kg}\right) \\
\end{array}$ & $\begin{array}{c}\text { HIRM } \\
\left(\mathrm{Am}^{2} / \mathrm{kg}\right) \\
\end{array}$ & S \\
\hline $01 \mathrm{U}-1 \mathrm{~A} / 0-10$ & 6.97E-08 & 7.01E-08 & -0.58 & $-4.04 \mathrm{E}-10$ & $2.39 \mathrm{E}-05$ & $1.40 \mathrm{E}-03$ & $5.68 \mathrm{E}-04$ & 4.14E-04 & 0.41 \\
\hline 01U-1A/10-30 & $5.85 \mathrm{E}-08$ & $5.89 \mathrm{E}-08$ & -0.80 & $-4.65 \mathrm{E}-10$ & $1.89 \mathrm{E}-05$ & $1.17 \mathrm{E}-03$ & 4.98E-04 & $3.34 \mathrm{E}-04$ & 0.43 \\
\hline 01U-1A/30-50 & $9.65 \mathrm{E}-08$ & $9.51 \mathrm{E}-08$ & 1.46 & $1.41 \mathrm{E}-09$ & 4.47E-05 & $1.71 \mathrm{E}-03$ & 8.38E-04 & 4.35E-04 & 0.49 \\
\hline $01 \mathrm{U}-1 \mathrm{~B} / 0-10$ & $1.05 \mathrm{E}-07$ & $1.03 \mathrm{E}-07$ & 1.62 & $1.70 \mathrm{E}-09$ & 4.53E-05 & $1.89 \mathrm{E}-03$ & 7.76E-04 & $5.56 \mathrm{E}-04$ & 0.41 \\
\hline 01U-1B/10-30 & $1.14 \mathrm{E}-07$ & $1.12 \mathrm{E}-07$ & 1.61 & $1.83 \mathrm{E}-09$ & $5.57 \mathrm{E}-05$ & $1.97 \mathrm{E}-03$ & $9.12 \mathrm{E}-04$ & $5.31 \mathrm{E}-04$ & 0.46 \\
\hline 01U-1B/30-50 & $1.13 \mathrm{E}-07$ & $1.11 \mathrm{E}-07$ & 1.28 & $1.44 \mathrm{E}-09$ & $5.64 \mathrm{E}-05$ & $1.91 \mathrm{E}-03$ & $1.00 \mathrm{E}-03$ & 4.52E-04 & 0.53 \\
\hline $01 \mathrm{U}-1 \mathrm{C} / 0-10$ & $8.28 \mathrm{E}-08$ & $8.23 \mathrm{E}-08$ & 0.56 & 4.62E-10 & $3.16 \mathrm{E}-05$ & $1.53 \mathrm{E}-03$ & $6.02 \mathrm{E}-04$ & 4.64E-04 & 0.39 \\
\hline 01U-1C/10-30 & $9.26 \mathrm{E}-08$ & 9.13E-08 & 1.46 & $1.35 \mathrm{E}-09$ & 4.00E-05 & $1.66 \mathrm{E}-03$ & 7.49E-04 & $4.58 \mathrm{E}-04$ & 0.45 \\
\hline 01U-1C/30-50 & $6.62 \mathrm{E}-08$ & $6.65 \mathrm{E}-08$ & -0.43 & $-2.83 \mathrm{E}-10$ & $2.95 \mathrm{E}-05$ & $1.24 \mathrm{E}-03$ & $5.21 \mathrm{E}-04$ & 3.57E-04 & 0.42 \\
\hline 01U-1D/0-10 & 7.43E-08 & 7.44E-08 & -0.24 & $-1.77 \mathrm{E}-10$ & $3.24 \mathrm{E}-05$ & $1.38 \mathrm{E}-03$ & 4.91E-04 & 4.43E-04 & 0.36 \\
\hline 01U-1D/10-30 & 7.14E-08 & $7.26 \mathrm{E}-08$ & -1.65 & $-1.18 \mathrm{E}-09$ & $2.59 \mathrm{E}-05$ & $1.35 \mathrm{E}-03$ & 5.30E-04 & 4.09E-04 & 0.39 \\
\hline 01U-1D/30-50 & $9.88 \mathrm{E}-08$ & 9.77E-08 & 1.10 & $1.09 \mathrm{E}-09$ & $4.58 \mathrm{E}-05$ & $1.74 \mathrm{E}-03$ & 8.69E-04 & 4.33E-04 & 0.50 \\
\hline $01 \mathrm{U}-1 \mathrm{E} / 0-10$ & $9.88 \mathrm{E}-08$ & $9.85 \mathrm{E}-08$ & 0.35 & $3.46 \mathrm{E}-10$ & 4.67E-05 & $1.74 \mathrm{E}-03$ & 8.71E-04 & $4.32 \mathrm{E}-04$ & 0.50 \\
\hline 01U-1E/10-30 & $1.16 \mathrm{E}-07$ & $1.14 \mathrm{E}-07$ & 1.30 & $1.50 \mathrm{E}-09$ & $5.70 \mathrm{E}-05$ & $1.93 \mathrm{E}-03$ & $1.05 \mathrm{E}-03$ & 4.42E-04 & 0.54 \\
\hline 01U-1E/30-50 & $6.31 \mathrm{E}-08$ & $6.69 \mathrm{E}-08$ & -6.03 & $-3.81 \mathrm{E}-09$ & $3.28 \mathrm{E}-05$ & $1.16 \mathrm{E}-03$ & $5.35 \mathrm{E}-04$ & $3.14 \mathrm{E}-04$ & 0.46 \\
\hline $01 \mathrm{U}-1 \mathrm{~F} / 0-10$ & $6.39 \mathrm{E}-08$ & $9.85 \mathrm{E}-08$ & -54.15 & $-3.46 \mathrm{E}-08$ & 4.84E-05 & $1.71 \mathrm{E}-03$ & $8.56 \mathrm{E}-04$ & $4.28 \mathrm{E}-04$ & 0.50 \\
\hline 01U-1F/10-30 & $9.91 \mathrm{E}-08$ & 5.84E-08 & 41.10 & 4.07E-08 & $2.57 \mathrm{E}-05$ & $1.11 \mathrm{E}-03$ & 4.75E-04 & $3.19 \mathrm{E}-04$ & 0.43 \\
\hline $01 \mathrm{U}-1 \mathrm{~F} / 30-50$ & $3.81 \mathrm{E}-08$ & 4.11E-08 & -7.83 & $-2.99 \mathrm{E}-09$ & $1.54 \mathrm{E}-05$ & $9.45 \mathrm{E}-04$ & $2.11 \mathrm{E}-04$ & 3.67E-04 & 0.22 \\
\hline
\end{tabular}


Table $\mathbf{6 b}-\mathbf{1 b}$. Magnetic properties of the less than 63 -micron size fraction for the $01 \mathrm{U}-1$ transect.

\begin{tabular}{|c|c|c|c|c|c|c|c|c|c|}
\hline Sample \# & $\begin{array}{c}\text { MSIf } \\
\left(\mathrm{m}^{3} / \mathrm{kg}\right)\end{array}$ & $\begin{array}{l}\text { MShf } \\
\left(\mathrm{m}^{3} / \mathbf{k g}\right)\end{array}$ & $\begin{array}{c}\text { FDMS } \\
(\%)\end{array}$ & $\begin{array}{l}\text { FDMS } \\
\left(\mathrm{m}^{3} / \mathrm{kg}\right)\end{array}$ & $\begin{array}{c}\text { ARM } \\
\left(\mathrm{Am}^{2} / \mathrm{kg}\right)\end{array}$ & $\begin{array}{c}\text { IRM1.2 } \\
\left(\mathrm{Am}^{2} / \mathrm{kg}\right)\end{array}$ & $\begin{array}{l}\text { IRM }-0.3 \\
\left(\mathrm{Am}^{2} / \mathrm{kg}\right)\end{array}$ & $\begin{array}{l}\text { HIRM } \\
\left(\mathrm{Am}^{2} / \mathbf{k g}\right)\end{array}$ & S \\
\hline 01U-1A/0-10 & $1.27 \mathrm{E}-07$ & $1.27 \mathrm{E}-07$ & 0.65 & $8.22 \mathrm{E}-10$ & $3.78 \mathrm{E}-05$ & $2.37 \mathrm{E}-03$ & $1.13 \mathrm{E}-03$ & $6.23 \mathrm{E}-04$ & 0.47 \\
\hline $01 \mathrm{U}-1 \mathrm{~A} / 10-30$ & $1.17 \mathrm{E}-07$ & $1.16 \mathrm{E}-07$ & 1.02 & 1.19E-09 & 3.30E-05 & $2.23 \mathrm{E}-03$ & $9.95 \mathrm{E}-04$ & $6.17 \mathrm{E}-04$ & 0.45 \\
\hline $01 \mathrm{U}-1 \mathrm{~A} / 30-50$ & $1.93 \mathrm{E}-07$ & $1.90 \mathrm{E}-07$ & 1.45 & 2.80E-09 & 7.06E-05 & $3.41 \mathrm{E}-03$ & $1.90 \mathrm{E}-03$ & $7.56 \mathrm{E}-04$ & 0.56 \\
\hline 01U-1B/0-10 & $1.32 \mathrm{E}-07$ & $1.31 \mathrm{E}-07$ & 1.05 & 1.39E-09 & $5.35 \mathrm{E}-05$ & $2.37 \mathrm{E}-03$ & $1.03 \mathrm{E}-03$ & $6.70 \mathrm{E}-04$ & 0.43 \\
\hline 01U-1B/10-30 & $1.77 \mathrm{E}-07$ & $1.74 \mathrm{E}-07$ & 1.63 & 2.89E-09 & 7.33E-05 & $3.08 \mathrm{E}-03$ & $1.58 \mathrm{E}-03$ & 7.48E-04 & 0.51 \\
\hline 01U-1B/30-50 & $2.25 \mathrm{E}-07$ & $2.21 \mathrm{E}-07$ & 1.52 & $3.42 \mathrm{E}-09$ & $9.13 \mathrm{E}-05$ & $3.73 \mathrm{E}-03$ & $2.16 \mathrm{E}-03$ & 7.84E-04 & 0.58 \\
\hline 01U-1C/0-10 & $1.17 \mathrm{E}-07$ & $1.17 \mathrm{E}-07$ & 0.30 & $3.56 \mathrm{E}-10$ & 4.16E-05 & $2.21 \mathrm{E}-03$ & $9.66 \mathrm{E}-04$ & $6.21 \mathrm{E}-04$ & 0.44 \\
\hline 01U-1C/10-30 & $1.55 \mathrm{E}-07$ & $1.54 \mathrm{E}-07$ & 0.51 & $7.87 \mathrm{E}-10$ & $5.61 \mathrm{E}-05$ & $2.84 \mathrm{E}-03$ & $1.49 \mathrm{E}-03$ & $6.77 \mathrm{E}-04$ & 0.52 \\
\hline 01U-1C/30-50 & $1.56 \mathrm{E}-07$ & $1.56 \mathrm{E}-07$ & 0.34 & $5.26 \mathrm{E}-10$ & $5.62 \mathrm{E}-05$ & $2.77 \mathrm{E}-03$ & $1.41 \mathrm{E}-03$ & $6.80 \mathrm{E}-04$ & 0.51 \\
\hline 01U-1D/0-10 & $1.20 \mathrm{E}-07$ & $1.20 \mathrm{E}-07$ & 0.13 & $1.53 \mathrm{E}-10$ & $4.52 \mathrm{E}-05$ & $2.20 \mathrm{E}-03$ & $8.70 \mathrm{E}-04$ & $6.63 \mathrm{E}-04$ & 0.40 \\
\hline 01U-1D/10-30 & $1.14 \mathrm{E}-07$ & $1.14 \mathrm{E}-07$ & -0.50 & $-5.71 \mathrm{E}-10$ & $3.71 \mathrm{E}-05$ & $2.23 \mathrm{E}-03$ & $9.45 \mathrm{E}-04$ & $6.43 \mathrm{E}-04$ & 0.42 \\
\hline 01U-1D/30-50 & $2.00 \mathrm{E}-07$ & $1.97 \mathrm{E}-07$ & 1.46 & $2.92 \mathrm{E}-09$ & 7.34E-05 & $3.53 \mathrm{E}-03$ & $1.98 \mathrm{E}-03$ & $7.76 \mathrm{E}-04$ & 0.56 \\
\hline 01U-1E/0-10 & $1.94 \mathrm{E}-07$ & $1.90 \mathrm{E}-07$ & 2.18 & 4.23E-09 & $7.32 \mathrm{E}-05$ & $3.37 \mathrm{E}-03$ & $1.87 \mathrm{E}-03$ & 7.49E-04 & 0.56 \\
\hline 01U-1E/10-30 & 2.32E-07 & 2.23E-07 & 3.80 & 8.80E-09 & $9.17 \mathrm{E}-05$ & $3.88 \mathrm{E}-03$ & $2.27 \mathrm{E}-03$ & 8.03E-04 & 0.59 \\
\hline 01U-1E/30-50 & $1.53 \mathrm{E}-07$ & $1.48 \mathrm{E}-07$ & 3.67 & $5.62 \mathrm{E}-09$ & $6.20 \mathrm{E}-05$ & $2.56 \mathrm{E}-03$ & $1.41 \mathrm{E}-03$ & 5.73E-04 & 0.55 \\
\hline 01U-1F/0-10 & $2.24 \mathrm{E}-07$ & $2.20 \mathrm{E}-07$ & 1.85 & 4.15E-09 & 8.19E-05 & $3.85 \mathrm{E}-03$ & $2.22 \mathrm{E}-03$ & $8.15 \mathrm{E}-04$ & 0.58 \\
\hline 01U-1F/10-30 & $1.44 \mathrm{E}-07$ & $1.45 \mathrm{E}-07$ & -0.24 & $-3.49 \mathrm{E}-10$ & $5.03 \mathrm{E}-05$ & $2.64 \mathrm{E}-03$ & $1.30 \mathrm{E}-03$ & $6.72 \mathrm{E}-04$ & 0.49 \\
\hline 01U-1F/30-50 & 8.86E-08 & $9.12 \mathrm{E}-08$ & -2.88 & $-2.55 \mathrm{E}-09$ & $2.90 \mathrm{E}-05$ & $1.92 \mathrm{E}-03$ & 6.64E-04 & $6.28 \mathrm{E}-04$ & 0.35 \\
\hline
\end{tabular}

Table 6b-2. Magnetic properties of the less than 63-micron size fraction for the $04 \mathrm{U}$ transect.

\begin{tabular}{|c|c|c|c|c|c|c|c|c|c|}
\hline Sample \# & $\begin{array}{c}\text { MSIf } \\
\left(\mathbf{m}^{3} / \mathbf{k g}\right)\end{array}$ & $\begin{array}{c}\text { MShf } \\
\left(\mathrm{m}^{3} / \mathrm{kg}\right)\end{array}$ & $\begin{array}{c}\text { FDMS } \\
(\%)\end{array}$ & $\begin{array}{l}\text { FDMS } \\
\left(\mathrm{m}^{3} / \mathrm{kg}\right)\end{array}$ & $\begin{array}{c}\text { ARM } \\
\left(\mathrm{Am}^{2} / \mathrm{kg}\right)\end{array}$ & $\begin{array}{c}\text { IRM1.2 } \\
\left(\mathrm{Am}^{2} / \mathrm{kg}\right)\end{array}$ & $\begin{array}{l}\text { IRM -0.3 } \\
\left(\mathrm{Am}^{2} / \mathbf{k g}\right)\end{array}$ & $\begin{array}{c}\text { HIRM } \\
\left(\mathrm{Am}^{2} / \mathbf{k g}\right)\end{array}$ & $\mathbf{S}$ \\
\hline 04U-21A & 8.33E-08 & $8.46 \mathrm{E}-08$ & -1.61 & $-1.34 \mathrm{E}-09$ & $3.01 \mathrm{E}-05$ & $1.55 \mathrm{E}-03$ & 7.07E-04 & $4.21 \mathrm{E}-04$ & 0.46 \\
\hline 04U-21B & $1.43 \mathrm{E}-07$ & $1.46 \mathrm{E}-07$ & -1.80 & $-2.58 \mathrm{E}-09$ & $3.76 \mathrm{E}-05$ & $2.27 \mathrm{E}-03$ & $1.53 \mathrm{E}-03$ & $3.70 \mathrm{E}-04$ & 0.67 \\
\hline $04 \mathrm{U}-22 \mathrm{~A}$ & $8.48 \mathrm{E}-08$ & $8.46 \mathrm{E}-08$ & 0.22 & $1.88 \mathrm{E}-10$ & $3.77 \mathrm{E}-05$ & $1.57 \mathrm{E}-03$ & $6.69 \mathrm{E}-04$ & 4.49E-04 & 0.43 \\
\hline 04U-22B & $1.09 \mathrm{E}-07$ & $1.08 \mathrm{E}-07$ & 0.58 & $6.36 \mathrm{E}-10$ & $5.55 \mathrm{E}-05$ & $1.91 \mathrm{E}-03$ & $1.03 \mathrm{E}-03$ & $4.39 \mathrm{E}-04$ & 0.54 \\
\hline 04U-23A & 8.64E-08 & 8.68E-08 & -0.46 & $-4.01 \mathrm{E}-10$ & $3.36 \mathrm{E}-04$ & $1.62 \mathrm{E}-03$ & $6.30 \mathrm{E}-04$ & $4.96 \mathrm{E}-04$ & 0.39 \\
\hline 04U-23B & $8.84 \mathrm{E}-08$ & 8.89E-08 & -0.62 & $-5.44 \mathrm{E}-10$ & $3.70 \mathrm{E}-05$ & $1.68 \mathrm{E}-03$ & $7.28 \mathrm{E}-04$ & 4.73E-04 & 0.43 \\
\hline 04U-24A & 7.61E-08 & $7.66 \mathrm{E}-08$ & -0.69 & $-5.27 \mathrm{E}-10$ & $3.00 \mathrm{E}-05$ & $1.46 \mathrm{E}-03$ & $5.69 \mathrm{E}-04$ & $4.48 \mathrm{E}-04$ & 0.39 \\
\hline 04U-24B & $5.16 \mathrm{E}-08$ & $5.38 \mathrm{E}-08$ & -4.29 & $-2.22 \mathrm{E}-09$ & 6.47E-05 & $1.11 \mathrm{E}-03$ & $5.21 \mathrm{E}-04$ & 2.95E-04 & 0.47 \\
\hline
\end{tabular}


Table 6c-1. Magnetic properties of the less than 2-mm size fraction for auger hole 00U-38.

\begin{tabular}{|c|c|c|c|c|c|c|c|c|c|}
\hline Sample \# & $\begin{array}{c}\text { MSIf } \\
\left(\mathrm{m}^{3} / \mathbf{k g}\right)\end{array}$ & $\begin{array}{c}\text { MShf } \\
\left(\mathbf{m}^{3} / \mathbf{k g}\right)\end{array}$ & $\begin{array}{c}\text { FDMS } \\
(\%)\end{array}$ & $\begin{array}{l}\text { FDMS } \\
\left(\mathrm{m}^{3} / \mathrm{kg}\right)\end{array}$ & $\begin{array}{c}\text { ARM } \\
\left(\mathrm{Am}^{2} / \mathrm{kg}\right)\end{array}$ & $\begin{array}{c}\text { IRM1.2 } \\
\left(\mathrm{Am}^{2} / \mathrm{kg}\right)\end{array}$ & $\begin{array}{l}\text { IRM -0.3 } \\
\left(\mathrm{Am}^{2} / \mathbf{k g}\right)\end{array}$ & $\begin{array}{c}\text { HIRM } \\
\left(\mathrm{Am}^{2} / \mathrm{kg}\right)\end{array}$ & S \\
\hline 00U-38/0-10 & $2.01 \mathrm{E}-08$ & $2.19 \mathrm{E}-08$ & -8.84 & $-1.78 \mathrm{E}-09$ & $7.75 \mathrm{E}-05$ & 4.94E-04 & $1.66 \mathrm{E}-04$ & $1.64 \mathrm{E}-04$ & 0.34 \\
\hline 00U-38/10-30 & $1.75 \mathrm{E}-08$ & $1.86 \mathrm{E}-08$ & -6.47 & $-1.13 \mathrm{E}-09$ & 8.36E-05 & 4.52E-04 & $1.47 \mathrm{E}-04$ & $1.53 \mathrm{E}-04$ & 0.32 \\
\hline 00U-38/30-44 & $1.98 \mathrm{E}-08$ & $2.01 \mathrm{E}-08$ & -1.79 & $-3.54 \mathrm{E}-10$ & $5.31 \mathrm{E}-05$ & 4.54E-04 & $1.66 \mathrm{E}-04$ & $1.44 \mathrm{E}-04$ & 0.37 \\
\hline 00U-38/44-57 & $1.90 \mathrm{E}-08$ & $2.00 \mathrm{E}-08$ & -5.11 & $-9.72 \mathrm{E}-10$ & $5.59 \mathrm{E}-05$ & 4.34E-04 & $1.60 \mathrm{E}-04$ & $1.37 \mathrm{E}-04$ & 0.37 \\
\hline 00U-38/57-74 & $2.47 \mathrm{E}-08$ & $2.65 \mathrm{E}-08$ & -7.41 & $-1.83 \mathrm{E}-09$ & $2.57 \mathrm{E}-05$ & 4.64E-04 & $1.95 \mathrm{E}-04$ & $1.34 \mathrm{E}-04$ & 0.42 \\
\hline 00U-38/74-96 & 2.62E-08 & $2.69 \mathrm{E}-08$ & -2.80 & $-7.35 \mathrm{E}-10$ & 7.76E-05 & $5.24 \mathrm{E}-04$ & $2.35 \mathrm{E}-04$ & $1.44 \mathrm{E}-04$ & 0.45 \\
\hline 00U-38/96-115 & 2.99E-08 & $3.19 \mathrm{E}-08$ & -6.37 & $-1.91 \mathrm{E}-09$ & 7.37E-05 & $6.17 \mathrm{E}-04$ & 3.02E-04 & $1.57 \mathrm{E}-04$ & 0.49 \\
\hline 00U-38/115-140 & 3.93E-08 & $3.86 \mathrm{E}-08$ & 1.74 & $6.83 \mathrm{E}-10$ & $8.25 \mathrm{E}-05$ & 7.46E-04 & 3.83E-04 & $1.81 \mathrm{E}-04$ & 0.51 \\
\hline 00U-38/140-156 & 3.17E-08 & $3.48 \mathrm{E}-08$ & -9.66 & $-3.06 \mathrm{E}-09$ & $7.18 \mathrm{E}-05$ & $6.16 \mathrm{E}-04$ & $2.86 \mathrm{E}-04$ & $1.65 \mathrm{E}-04$ & 0.46 \\
\hline 00U-38/156-185 & 2.72E-08 & 2.92E-08 & -7.65 & $-2.08 \mathrm{E}-09$ & $9.81 \mathrm{E}-05$ & $5.46 \mathrm{E}-04$ & $2.38 \mathrm{E}-04$ & $1.54 \mathrm{E}-04$ & 0.44 \\
\hline 00U-38/185-207 & $2.85 \mathrm{E}-08$ & $3.16 \mathrm{E}-08$ & -11.03 & $-3.14 \mathrm{E}-09$ & $8.80 \mathrm{E}-05$ & $5.46 \mathrm{E}-04$ & $2.82 \mathrm{E}-04$ & $1.32 \mathrm{E}-04$ & 0.52 \\
\hline 00U-38/207-229 & $3.53 \mathrm{E}-08$ & $3.60 \mathrm{E}-08$ & -2.00 & $-7.01 \mathrm{E}-10$ & 7.91E-05 & 7.17E-04 & 3.82E-04 & $1.67 \mathrm{E}-04$ & 0.53 \\
\hline 00U-38/229-242 & $5.75 \mathrm{E}-08$ & $5.82 \mathrm{E}-08$ & -1.26 & $-7.27 \mathrm{E}-10$ & $6.71 \mathrm{E}-05$ & $1.05 \mathrm{E}-03$ & $7.46 \mathrm{E}-04$ & $1.53 \mathrm{E}-04$ & 0.71 \\
\hline
\end{tabular}


Table 6c-2a. Magnetic properties of the less than 2-mm size fraction for the 00U-39 transect.

\begin{tabular}{|c|c|c|c|c|c|c|c|c|c|}
\hline Sample \# & $\begin{array}{c}\text { MSIf } \\
\left(\mathrm{m}^{3} / \mathrm{kg}\right)\end{array}$ & $\begin{array}{c}\text { MShf } \\
\left(\mathrm{m}^{3} / \mathrm{kg}\right)\end{array}$ & $\begin{array}{c}\text { FDMS } \\
(\%)\end{array}$ & $\begin{array}{l}\text { FDMS } \\
\left(\mathbf{m}^{3} / \mathbf{k g}\right)\end{array}$ & $\begin{array}{c}\text { ARM } \\
\left(\mathrm{Am}^{2} / \mathbf{k g}\right)\end{array}$ & $\begin{array}{c}\text { IRM1.2 } \\
\left(\mathrm{Am}^{2} / \mathrm{kg}\right) \\
\end{array}$ & $\begin{array}{l}\text { IRM -0.3 } \\
\left(\mathrm{Am}^{2} / \mathrm{kg}\right) \\
\end{array}$ & $\begin{array}{c}\text { HIRM } \\
\left(\mathrm{Am}^{2} / \mathbf{k g}\right)\end{array}$ & S \\
\hline 00U-39A/0-10 & $1.91 \mathrm{E}-08$ & $1.94 \mathrm{E}-08$ & -1.84 & $-3.50 \mathrm{E}-10$ & $1.37 \mathrm{E}-05$ & $5.01 \mathrm{E}-04$ & $1.02 \mathrm{E}-04$ & $1.99 \mathrm{E}-04$ & 0.20 \\
\hline 00U-39A/10-30 & $1.73 \mathrm{E}-08$ & $1.81 \mathrm{E}-08$ & -5.05 & $-8.71 \mathrm{E}-10$ & $1.26 \mathrm{E}-05$ & 4.50E-04 & $9.30 \mathrm{E}-05$ & $1.78 \mathrm{E}-04$ & 0.21 \\
\hline 00U-39A/30-50 & $2.60 \mathrm{E}-08$ & $2.51 \mathrm{E}-08$ & 3.48 & $9.04 \mathrm{E}-10$ & $1.72 \mathrm{E}-05$ & $5.59 \mathrm{E}-04$ & $1.70 \mathrm{E}-04$ & $1.95 \mathrm{E}-04$ & 0.30 \\
\hline 00U-39B/0-10 & $2.05 \mathrm{E}-08$ & 2.37E-08 & -15.47 & $-3.18 \mathrm{E}-09$ & $1.34 \mathrm{E}-05$ & 4.22E-04 & $1.25 \mathrm{E}-04$ & $1.49 \mathrm{E}-04$ & 0.30 \\
\hline 00U-39B/10-30 & $1.60 \mathrm{E}-08$ & $1.88 \mathrm{E}-08$ & -17.90 & $-2.86 \mathrm{E}-09$ & $1.28 \mathrm{E}-05$ & 4.17E-04 & $7.58 \mathrm{E}-05$ & $1.71 \mathrm{E}-04$ & 0.18 \\
\hline 00U-39B/30-50 & $2.06 \mathrm{E}-08$ & $2.41 \mathrm{E}-08$ & -16.81 & $-3.46 \mathrm{E}-09$ & $1.46 \mathrm{E}-05$ & 4.42E-04 & $1.32 \mathrm{E}-04$ & $1.55 \mathrm{E}-04$ & 0.30 \\
\hline 00U-39C/0-10 & $2.42 \mathrm{E}-08$ & 3.03E-08 & -25.06 & $-6.07 \mathrm{E}-09$ & $1.23 \mathrm{E}-05$ & 4.75E-04 & $1.28 \mathrm{E}-04$ & $1.74 \mathrm{E}-04$ & 0.27 \\
\hline 00U-39C/10-30 & $2.29 \mathrm{E}-08$ & $2.57 \mathrm{E}-08$ & -12.16 & $-2.79 \mathrm{E}-09$ & $1.41 \mathrm{E}-05$ & 4.76E-04 & $1.58 \mathrm{E}-04$ & $1.59 \mathrm{E}-04$ & 0.33 \\
\hline 00U-39C/30-50 & $2.68 \mathrm{E}-08$ & $2.58 \mathrm{E}-08$ & 3.86 & $1.04 \mathrm{E}-09$ & $1.56 \mathrm{E}-05$ & 5.17E-04 & $1.90 \mathrm{E}-04$ & $1.63 \mathrm{E}-04$ & 0.37 \\
\hline 00U-39D/0-10 & $2.52 \mathrm{E}-08$ & $2.68 \mathrm{E}-08$ & -6.26 & $-1.58 \mathrm{E}-09$ & $1.46 \mathrm{E}-05$ & $5.24 \mathrm{E}-04$ & $1.42 \mathrm{E}-04$ & $1.91 \mathrm{E}-04$ & 0.27 \\
\hline 00U-39D/10-30 & $2.72 \mathrm{E}-08$ & $3.10 \mathrm{E}-08$ & -14.02 & $-3.81 \mathrm{E}-09$ & $1.64 \mathrm{E}-05$ & $5.30 \mathrm{E}-04$ & $1.73 \mathrm{E}-04$ & $1.78 \mathrm{E}-04$ & 0.33 \\
\hline 00U-39D/30-50 & $2.71 \mathrm{E}-08$ & $2.52 \mathrm{E}-08$ & 7.27 & $1.97 \mathrm{E}-09$ & $1.53 \mathrm{E}-05$ & 4.70E-04 & $1.67 \mathrm{E}-04$ & $1.51 \mathrm{E}-04$ & 0.36 \\
\hline 00U-39E/0-10 & $2.63 \mathrm{E}-08$ & $2.83 \mathrm{E}-08$ & -7.75 & $-2.04 \mathrm{E}-09$ & $1.62 \mathrm{E}-05$ & $5.25 \mathrm{E}-04$ & $1.87 \mathrm{E}-04$ & $1.69 \mathrm{E}-04$ & 0.36 \\
\hline 00U-39E/10-30 & $3.41 \mathrm{E}-08$ & $3.60 \mathrm{E}-08$ & -5.48 & $-1.87 \mathrm{E}-09$ & $2.08 \mathrm{E}-05$ & $6.63 \mathrm{E}-04$ & $2.60 \mathrm{E}-04$ & 2.01E-04 & 0.39 \\
\hline 00U-39E/30-50 & $3.35 \mathrm{E}-08$ & 3.48E-08 & -3.72 & $-1.25 \mathrm{E}-09$ & $1.48 \mathrm{E}-05$ & 4.73E-04 & $1.88 \mathrm{E}-04$ & $1.42 \mathrm{E}-04$ & 0.40 \\
\hline 00U-39F/0-10 & $2.16 \mathrm{E}-08$ & $2.46 \mathrm{E}-08$ & -13.64 & $-2.95 \mathrm{E}-09$ & $1.62 \mathrm{E}-05$ & 4.86E-04 & $1.55 \mathrm{E}-04$ & $1.66 \mathrm{E}-04$ & 0.32 \\
\hline 00U-39F/10-30 & $2.53 \mathrm{E}-08$ & $3.18 \mathrm{E}-08$ & -25.32 & $-6.42 \mathrm{E}-09$ & $1.68 \mathrm{E}-05$ & $5.22 \mathrm{E}-04$ & $1.86 \mathrm{E}-04$ & $1.68 \mathrm{E}-04$ & 0.36 \\
\hline 00U-39F/30-50 & 4.72E-08 & 4.72E-08 & -0.14 & $-6.57 \mathrm{E}-11$ & $2.45 \mathrm{E}-05$ & 7.27E-04 & $3.43 \mathrm{E}-04$ & $1.92 \mathrm{E}-04$ & 0.47 \\
\hline 00U-39G/0-10 & 3.04E-08 & $3.06 \mathrm{E}-08$ & -0.61 & $-1.85 \mathrm{E}-10$ & 2.09E-05 & $6.09 \mathrm{E}-04$ & $2.41 \mathrm{E}-04$ & $1.84 \mathrm{E}-04$ & 0.40 \\
\hline 00U-39G/10-30 & $5.25 \mathrm{E}-08$ & $5.36 \mathrm{E}-08$ & -2.16 & $-1.14 \mathrm{E}-09$ & 2.83E-05 & $9.29 \mathrm{E}-04$ & 4.45E-04 & $2.42 \mathrm{E}-04$ & 0.48 \\
\hline 00U-39G/30-50 & $2.81 \mathrm{E}-08$ & 2.72E-08 & 3.23 & $9.01 \mathrm{E}-10$ & $1.61 \mathrm{E}-05$ & 5.33E-04 & $1.85 \mathrm{E}-04$ & $1.74 \mathrm{E}-04$ & 0.35 \\
\hline 00U-39H/0-10 & $5.55 \mathrm{E}-08$ & $5.16 \mathrm{E}-08$ & 7.03 & $3.90 \mathrm{E}-09$ & $3.10 \mathrm{E}-05$ & $9.06 \mathrm{E}-04$ & $5.20 \mathrm{E}-04$ & $1.93 \mathrm{E}-04$ & 0.57 \\
\hline 00U-39H/10-30 & n.d & n.d & n.d & n.d & n.d & n.d & n.d & n.d & n.d \\
\hline 00U-39H/30-50 & n.d. & n.d. & n.d. & n.d. & n.d. & n.d. & n.d. & n.d. & n.d. \\
\hline 00U-39I/0-10 & 4.45E-08 & 4.82E-08 & -8.38 & $-3.73 \mathrm{E}-09$ & $2.26 \mathrm{E}-05$ & 7.21E-04 & $3.52 \mathrm{E}-04$ & $1.85 \mathrm{E}-04$ & 0.49 \\
\hline 00U-39I/10-30 & $7.59 \mathrm{E}-08$ & 7.67E-08 & -1.09 & $-8.26 \mathrm{E}-10$ & $2.89 \mathrm{E}-05$ & $9.46 \mathrm{E}-04$ & 4.67E-04 & $2.40 \mathrm{E}-04$ & 0.49 \\
\hline 00U-39I/30-50 & $3.83 \mathrm{E}-08$ & $3.88 \mathrm{E}-08$ & -1.17 & $-4.50 \mathrm{E}-10$ & $2.42 \mathrm{E}-05$ & $7.89 \mathrm{E}-04$ & $3.50 \mathrm{E}-04$ & 2.19E-04 & 0.44 \\
\hline 00U-39J/0-10 & 4.13E-08 & $4.19 \mathrm{E}-08$ & -1.43 & $-5.91 \mathrm{E}-10$ & 2.37E-05 & 7.99E-04 & 3.32E-04 & 2.33E-04 & 0.42 \\
\hline $00 \mathrm{U}-39 \mathrm{~J} / 10-30$ & 7.03E-08 & $6.94 \mathrm{E}-08$ & 1.15 & $8.11 \mathrm{E}-10$ & $3.58 \mathrm{E}-05$ & $1.18 \mathrm{E}-03$ & 6.49E-04 & 2.67E-04 & 0.55 \\
\hline $00 \mathrm{U}-39 \mathrm{~J} / 30-50$ & $6.70 \mathrm{E}-08$ & $6.78 \mathrm{E}-08$ & -1.21 & $-8.07 \mathrm{E}-10$ & 3.53E-05 & $1.17 \mathrm{E}-03$ & $6.12 \mathrm{E}-04$ & 2.79E-04 & 0.52 \\
\hline 00U-39K/0-10 & $6.83 \mathrm{E}-08$ & $6.74 \mathrm{E}-08$ & 1.33 & $9.05 \mathrm{E}-10$ & $3.38 \mathrm{E}-05$ & $1.17 \mathrm{E}-03$ & $5.86 \mathrm{E}-04$ & $2.95 \mathrm{E}-04$ & 0.50 \\
\hline 00U-39K/10-30 & $8.72 \mathrm{E}-08$ & $9.02 \mathrm{E}-08$ & -3.47 & $-3.03 \mathrm{E}-09$ & $4.22 \mathrm{E}-05$ & $1.50 \mathrm{E}-03$ & 8.87E-04 & 3.07E-04 & 0.59 \\
\hline 00U-39K/30-50 & 7.92E-08 & 7.87E-08 & 0.55 & $4.32 \mathrm{E}-10$ & $3.92 \mathrm{E}-05$ & $1.33 \mathrm{E}-03$ & $6.90 \mathrm{E}-04$ & $3.22 \mathrm{E}-04$ & 0.52 \\
\hline 00U-39L/0-10 & $5.49 \mathrm{E}-08$ & $5.52 \mathrm{E}-08$ & -0.54 & $-2.98 \mathrm{E}-10$ & 3.03E-05 & 7.48E-04 & 3.61E-04 & $1.93 \mathrm{E}-04$ & 0.48 \\
\hline 00U-39L/10-30 & $8.30 \mathrm{E}-08$ & 8.17E-08 & 1.62 & $1.35 \mathrm{E}-09$ & 4.40E-05 & $1.42 \mathrm{E}-03$ & 8.07E-04 & $3.06 \mathrm{E}-04$ & 0.57 \\
\hline 00U-39L/30-50 & $8.52 \mathrm{E}-08$ & $8.29 \mathrm{E}-08$ & 2.67 & $2.28 \mathrm{E}-09$ & 4.01E-05 & $1.37 \mathrm{E}-03$ & 7.34E-04 & $3.17 \mathrm{E}-04$ & 0.54 \\
\hline 00U-39M/0-10 & $8.95 \mathrm{E}-08$ & $8.65 \mathrm{E}-08$ & 3.35 & 2.99E-09 & $4.74 \mathrm{E}-05$ & $1.53 \mathrm{E}-03$ & 8.83E-04 & $3.21 \mathrm{E}-04$ & 0.58 \\
\hline 00U-39M/10-30 & 7.93E-08 & 8.11E-08 & -2.24 & $-1.77 \mathrm{E}-09$ & 3.91E-05 & $1.35 \mathrm{E}-03$ & $7.50 \mathrm{E}-04$ & 3.02E-04 & 0.55 \\
\hline 00U-39M/30-50 & 7.84E-08 & 7.76E-08 & 1.02 & $8.04 \mathrm{E}-10$ & $3.78 \mathrm{E}-05$ & $1.26 \mathrm{E}-03$ & 7.41E-04 & 2.61E-04 & 0.59 \\
\hline 00U-39N/0-10 & $5.08 \mathrm{E}-08$ & $5.40 \mathrm{E}-08$ & -6.16 & $-3.13 \mathrm{E}-09$ & $2.65 \mathrm{E}-05$ & $9.79 \mathrm{E}-04$ & $4.54 \mathrm{E}-04$ & 2.63E-04 & 0.46 \\
\hline 00U-39N/10-30 & $6.21 \mathrm{E}-08$ & 5.97E-08 & 3.91 & 2.43E-09 & $2.90 \mathrm{E}-05$ & $1.13 \mathrm{E}-03$ & $6.38 \mathrm{E}-04$ & $2.45 \mathrm{E}-04$ & 0.57 \\
\hline $00 U-39 N / 30-50$ & $9.02 \mathrm{E}-08$ & $8.90 \mathrm{E}-08$ & 1.33 & $1.20 \mathrm{E}-09$ & $4.55 \mathrm{E}-05$ & $1.51 \mathrm{E}-03$ & $9.08 \mathrm{E}-04$ & $3.00 \mathrm{E}-04$ & 0.60 \\
\hline 00U-39O/0-10 & 4.37E-08 & 4.70E-08 & -7.70 & $-3.36 \mathrm{E}-09$ & $2.22 \mathrm{E}-05$ & 9.17E-04 & $3.52 \mathrm{E}-04$ & $2.83 \mathrm{E}-04$ & 0.38 \\
\hline 00U-39O/10-30 & $5.08 \mathrm{E}-08$ & $5.41 \mathrm{E}-08$ & -6.43 & $-3.26 \mathrm{E}-09$ & 2.62E-05 & $1.05 \mathrm{E}-03$ & 4.01E-04 & $3.19 \mathrm{E}-04$ & 0.39 \\
\hline 00U-39O/30-50 & 9.94E-08 & $9.66 \mathrm{E}-08$ & 2.82 & $2.80 \mathrm{E}-09$ & $4.90 \mathrm{E}-05$ & $1.71 \mathrm{E}-03$ & $9.35 \mathrm{E}-04$ & $3.90 \mathrm{E}-04$ & 0.55 \\
\hline
\end{tabular}


Table 6c-2b. Magnetic properties of the less than 63-micron size fraction for the 00U-39 transect.

\begin{tabular}{|c|c|c|c|c|c|c|c|c|c|}
\hline Sample \# & $\begin{array}{c}\text { MSlf } \\
\left(\mathbf{m}^{3} / \mathbf{k g}\right)\end{array}$ & $\begin{array}{c}\text { MShf } \\
\left(\mathrm{m}^{3} / \mathbf{k g}\right)\end{array}$ & $\begin{array}{c}\text { FDMS } \\
(\%)\end{array}$ & $\begin{array}{l}\text { FDMS } \\
\left(\mathrm{m}^{3} / \mathbf{k g}\right)\end{array}$ & $\begin{array}{c}\text { ARM } \\
\left(\mathrm{Am}^{2} / \mathbf{k g}\right)\end{array}$ & $\begin{array}{c}\text { IRM1.2 } \\
\left(\mathrm{Am}^{2} / \mathrm{kg}\right)\end{array}$ & $\begin{array}{l}\text { IRM -0.3 } \\
\left(\mathrm{Am}^{2} / \mathbf{k g}\right)\end{array}$ & $\begin{array}{c}\text { HIRM } \\
\left(\mathrm{Am}^{2} / \mathbf{k g}\right)\end{array}$ & $\mathbf{S}$ \\
\hline 00U-39A/0-10 & $2.01 \mathrm{E}-07$ & $2.03 \mathrm{E}-07$ & -1.03 & $-2.07 \mathrm{E}-09$ & $5.79 \mathrm{E}-05$ & $3.72 \mathrm{E}-03$ & $2.05 \mathrm{E}-03$ & 8.35E-04 & 0.55 \\
\hline 00U-39A/30-50 & n.d. & n.d. & n.d. & n.d. & n.d. & n.d. & n.d. & n.d. & n.d. \\
\hline 00U-39B/0-10 & 2.19E-07 & $2.18 \mathrm{E}-07$ & 0.11 & 2.39E-10 & $6.32 \mathrm{E}-05$ & $3.77 \mathrm{E}-03$ & 2.19E-03 & 7.88E-04 & 0.58 \\
\hline 00U-39B/10-30 & n.d. & n.d. & n.d. & n.d. & n.d. & n.d. & n.d. & n.d. & n.d. \\
\hline 00U-39C/10-30 & n.d. & n.d. & n.d. & n.d. & n.d. & n.d. & n.d. & n.d. & n.d. \\
\hline 00U-39C/30-50 & n.d. & n.d. & n.d. & n.d. & n.d. & n.d. & n.d. & n.d. & n.d. \\
\hline 00U-39D/0-10 & n.d. & n.d. & n.d. & n.d. & n.d. & n.d. & n.d. & n.d. & n.d. \\
\hline 00U-39D/10-30 & n.d. & n.d. & n.d. & n.d. & n.d. & n.d. & n.d. & n.d. & n.d. \\
\hline 00U-39D/30-50 & n.d. & n.d. & n.d. & n.d. & n.d. & n.d. & n.d. & n.d. & n.d. \\
\hline 00U-39E/0-10 & $1.93 \mathrm{E}-07$ & $1.92 \mathrm{E}-07$ & 0.56 & $1.08 \mathrm{E}-09$ & $6.75 \mathrm{E}-05$ & $3.38 \mathrm{E}-03$ & 2.03E-03 & $6.75 \mathrm{E}-04$ & 0.60 \\
\hline 00U-39F/30-50 & n.d. & n.d. & n.d. & n.d. & n.d. & n.d. & n.d. & n.d. & n.d. \\
\hline 00U-39G/0-10 & $2.07 \mathrm{E}-07$ & $2.07 \mathrm{E}-07$ & 0.10 & $2.07 \mathrm{E}-10$ & $6.45 \mathrm{E}-05$ & $3.57 \mathrm{E}-03$ & $2.16 \mathrm{E}-03$ & 7.08E-04 & 0.60 \\
\hline 00U-39G/10-30 & n.d. & n.d. & n.d. & n.d. & n.d. & n.d. & n.d. & n.d. & n.d. \\
\hline 00U-39G/30-50 & n.d. & n.d. & n.d. & n.d. & n.d. & n.d. & n.d. & n.d. & n.d. \\
\hline 00U-39H/0-10 & $1.96 \mathrm{E}-07$ & $1.93 \mathrm{E}-07$ & 1.79 & $3.52 \mathrm{E}-09$ & $5.82 \mathrm{E}-05$ & 3.33E-03 & $1.97 \mathrm{E}-03$ & $6.80 \mathrm{E}-04$ & 0.59 \\
\hline 00U-39H/10-30 & n.d. & n.d. & n.d. & n.d. & n.d. & n.d. & n.d. & n.d. & n.d. \\
\hline 00U-39H/30-50 & n.d. & n.d. & n.d. & n.d. & n.d. & n.d. & n.d. & n.d. & n.d. \\
\hline 00U-39I/0-10 & 0.00000 & 0.00000 & 0.27915 & 0.00000 & 0.00006 & 0.00310 & 0.00185 & 0.00062 & 0.59808 \\
\hline 00U-39I/10-30 & n.d. & n.d. & n.d. & n.d. & n.d. & n.d. & n.d. & n.d. & n.d. \\
\hline 00U-39I/30-50 & n.d. & n.d. & n.d. & n.d. & n.d. & n.d. & n.d. & n.d. & n.d. \\
\hline $00 \mathrm{U}-39 \mathrm{~J} / 0-10$ & $1.96 \mathrm{E}-07$ & $1.97 \mathrm{E}-07$ & -0.23 & $-4.58 \mathrm{E}-10$ & $6.38 \mathrm{E}-05$ & $3.36 \mathrm{E}-03$ & $1.98 \mathrm{E}-03$ & $6.94 \mathrm{E}-04$ & 0.59 \\
\hline 00U-39M/0-10 & $2.28 \mathrm{E}-07$ & $2.26 \mathrm{E}-07$ & 0.72 & $1.63 \mathrm{E}-09$ & $8.00 \mathrm{E}-05$ & $3.74 \mathrm{E}-03$ & $2.42 \mathrm{E}-03$ & $6.59 \mathrm{E}-04$ & 0.65 \\
\hline 00U-39M/10-30 & n.d. & n.d. & n.d. & n.d. & n.d. & n.d. & n.d. & n.d. & n.d. \\
\hline $00 \mathrm{U}-39 \mathrm{M} / 30-50$ & n.d. & n.d. & n.d. & n.d. & n.d. & n.d. & n.d. & n.d. & n.d. \\
\hline 00U-39N/0-10 & $1.64 \mathrm{E}-07$ & $1.64 \mathrm{E}-07$ & 0.35 & $5.79 \mathrm{E}-10$ & $5.64 \mathrm{E}-05$ & $2.92 \mathrm{E}-03$ & $1.68 \mathrm{E}-03$ & $6.20 \mathrm{E}-04$ & 0.58 \\
\hline 00U-39N/10-30 & n.d. & n.d. & n.d. & n.d. & n.d. & n.d. & n.d. & n.d. & n.d. \\
\hline 00U-39N/30-50 & n.d. & n.d. & n.d. & n.d. & n.d. & n.d. & n.d. & n.d. & n.d. \\
\hline 00U-39O/0-10 & $1.60 \mathrm{E}-07$ & $1.59 \mathrm{E}-07$ & 0.60 & $9.57 \mathrm{E}-10$ & $5.54 \mathrm{E}-05$ & $2.90 \mathrm{E}-03$ & $1.63 \mathrm{E}-03$ & $6.32 \mathrm{E}-04$ & 0.56 \\
\hline 00U-39O/10-30 & n.d. & n.d. & n.d. & n.d. & n.d. & n.d. & n.d. & n.d. & n.d. \\
\hline 00U-39O/30-50 & n.d. & n.d. & n.d. & n.d. & n.d. & n.d. & n.d. & n.d. & n.d. \\
\hline
\end{tabular}


Table 6c-3a. Magnetic properties of the less than 2-mm size fraction for auger hole 00U-40.

\begin{tabular}{|c|c|c|c|c|c|c|c|c|c|}
\hline Sample \# & $\begin{array}{c}\text { MSIf } \\
\left(\mathbf{m}^{3} / \mathbf{k g}\right)\end{array}$ & $\begin{array}{c}\text { MShf } \\
\left(\mathrm{m}^{3} / \mathrm{kg}\right)\end{array}$ & $\begin{array}{c}\text { FDMS } \\
(\%)\end{array}$ & $\begin{array}{l}\text { FDMS } \\
\left(\mathrm{m}^{3} / \mathrm{kg}\right)\end{array}$ & $\begin{array}{c}\text { ARM } \\
\left(\mathrm{Am}^{2} / \mathbf{k g}\right)\end{array}$ & $\begin{array}{c}\text { IRM1.2 } \\
\left(\mathrm{Am}^{2} / \mathbf{k g}\right)\end{array}$ & $\begin{array}{l}\text { IRM -0.3 } \\
\left(\mathrm{Am}^{2} / \mathrm{kg}\right)\end{array}$ & $\begin{array}{c}\text { HIRM } \\
\left(\mathrm{Am}^{2} / \mathrm{kg}\right)\end{array}$ & $\mathbf{S}$ \\
\hline 00U-40/0-10 & $8.78 \mathrm{E}-08$ & $8.88 \mathrm{E}-08$ & -1.09 & $-9.58 \mathrm{E}-10$ & $1.98 \mathrm{E}-04$ & $1.72 \mathrm{E}-03$ & 8.57E-04 & 4.31E-04 & 0.50 \\
\hline 00U-40/10-30 & $8.58 \mathrm{E}-08$ & 8.63E-08 & -0.55 & $-4.74 \mathrm{E}-10$ & 7.18E-04 & $1.75 \mathrm{E}-03$ & 7.27E-04 & $5.10 \mathrm{E}-04$ & 0.42 \\
\hline 00U-40/30-48 & 8.02E-08 & 7.98E-08 & 0.55 & $4.41 \mathrm{E}-10$ & $1.95 \mathrm{E}-04$ & $1.53 \mathrm{E}-03$ & $7.21 \mathrm{E}-04$ & 4.06E-04 & 0.47 \\
\hline 00U-40/48-64 & $8.06 \mathrm{E}-08$ & 7.96E-08 & 1.21 & $9.78 \mathrm{E}-10$ & $1.92 \mathrm{E}-04$ & $1.55 \mathrm{E}-03$ & $7.55 \mathrm{E}-04$ & 4.00E-04 & 0.49 \\
\hline 00U-40/64-82 & $1.03 \mathrm{E}-07$ & $1.04 \mathrm{E}-07$ & -0.30 & $-3.13 \mathrm{E}-10$ & 2.44E-04 & $2.02 \mathrm{E}-03$ & $1.02 \mathrm{E}-03$ & $5.00 \mathrm{E}-04$ & 0.50 \\
\hline 00U-40/82-102 & $9.92 \mathrm{E}-08$ & $9.67 \mathrm{E}-08$ & 2.55 & $2.53 \mathrm{E}-09$ & $2.51 \mathrm{E}-04$ & $1.97 \mathrm{E}-03$ & 8.95E-04 & 5.39E-04 & 0.45 \\
\hline 00U-40/102-121 & $1.02 \mathrm{E}-07$ & $1.06 \mathrm{E}-07$ & -3.70 & $-3.78 \mathrm{E}-09$ & $1.63 \mathrm{E}-04$ & $1.80 \mathrm{E}-03$ & $1.03 \mathrm{E}-03$ & $3.85 \mathrm{E}-04$ & 0.57 \\
\hline 00U-40/121-142 & $9.29 \mathrm{E}-08$ & 9.43E-08 & -1.57 & $-1.46 \mathrm{E}-09$ & $9.24 \mathrm{E}-05$ & $1.52 \mathrm{E}-03$ & $9.27 \mathrm{E}-04$ & $2.96 \mathrm{E}-04$ & 0.61 \\
\hline 00U-40/142-154 & 7.91E-08 & 8.34E-08 & -5.39 & $-4.26 \mathrm{E}-09$ & $9.73 \mathrm{E}-05$ & $1.33 \mathrm{E}-03$ & $8.18 \mathrm{E}-04$ & $2.58 \mathrm{E}-04$ & 0.61 \\
\hline 00U-40/154-168 & $6.24 \mathrm{E}-08$ & $6.10 \mathrm{E}-08$ & 2.19 & 1.37E-09 & 8.39E-05 & $1.09 \mathrm{E}-03$ & $6.51 \mathrm{E}-04$ & $2.22 \mathrm{E}-04$ & 0.60 \\
\hline 00U-40/168-183 & $4.29 \mathrm{E}-08$ & $4.53 \mathrm{E}-08$ & -5.60 & $-2.40 \mathrm{E}-09$ & $2.64 \mathrm{E}-05$ & $8.02 \mathrm{E}-04$ & $4.22 \mathrm{E}-04$ & $1.90 \mathrm{E}-04$ & 0.53 \\
\hline 00U-40/183-193 & $6.11 \mathrm{E}-08$ & $6.14 \mathrm{E}-08$ & -0.58 & $-3.56 \mathrm{E}-10$ & $9.46 \mathrm{E}-05$ & $1.07 \mathrm{E}-03$ & $6.44 \mathrm{E}-04$ & $2.14 \mathrm{E}-04$ & 0.60 \\
\hline 00U-40/192-204 & $3.25 \mathrm{E}-08$ & $3.45 \mathrm{E}-08$ & -6.09 & $-1.98 \mathrm{E}-09$ & $9.61 \mathrm{E}-05$ & $6.98 \mathrm{E}-04$ & 3.11E-04 & $1.94 \mathrm{E}-04$ & 0.45 \\
\hline 00U-40/204-217 & $2.08 \mathrm{E}-08$ & $2.27 \mathrm{E}-08$ & -9.31 & $-1.93 \mathrm{E}-09$ & $1.19 \mathrm{E}-04$ & $5.72 \mathrm{E}-04$ & $1.84 \mathrm{E}-04$ & 1.94E-04 & 0.32 \\
\hline
\end{tabular}

Table 6c-3b. Magnetic properties of the less than 63 -micron size fraction for the $00 \mathrm{U}-40$ transect.

\begin{tabular}{|c|c|c|c|c|c|c|c|c|c|}
\hline Sample \# & $\begin{array}{c}\text { MSIf } \\
\left(\mathrm{m}^{3} / \mathbf{k g}\right)\end{array}$ & $\begin{array}{c}\text { MShf } \\
\left(\mathrm{m}^{3} / \mathrm{kg}\right)\end{array}$ & $\begin{array}{c}\text { FDMS } \\
(\%)\end{array}$ & $\begin{array}{l}\text { FDMS } \\
\left(\mathbf{m}^{3} / \mathbf{k g}\right)\end{array}$ & $\begin{array}{c}\text { ARM } \\
\left(\mathrm{Am}^{2} / \mathrm{kg}\right)\end{array}$ & $\begin{array}{l}\text { IRM1.2 } \\
\left(\mathrm{Am}^{2} / \mathbf{k g}\right)\end{array}$ & $\begin{array}{l}\text { IRM -0.3 } \\
\left(\mathrm{Am}^{2} / \mathbf{k g}\right)\end{array}$ & $\begin{array}{c}\text { HIRM } \\
\left(\mathrm{Am}^{2} / \mathbf{k g}\right)\end{array}$ & $\mathbf{S}$ \\
\hline 00U-40/0-10 & $1.68 \mathrm{E}-07$ & $1.67 \mathrm{E}-07$ & 0.42 & $7.01 \mathrm{E}-10$ & $6.39 \mathrm{E}-05$ & $3.02 \mathrm{E}-03$ & $1.77 \mathrm{E}-03$ & $6.26 \mathrm{E}-04$ & 0.59 \\
\hline 00U-40/10-30 & n.d. & n.d. & n.d. & n.d. & n.d. & n.d. & n.d. & n.d. & n.d. \\
\hline 00U-40/30-48 & n.d. & n.d. & n.d. & n.d. & n.d. & n.d. & n.d. & n.d. & n.d. \\
\hline 00U-40/48-64 & n.d. & n.d. & n.d. & n.d. & n.d. & n.d. & n.d. & n.d. & n.d. \\
\hline 00U-40/64-82 & n.d. & n.d. & n.d. & n.d. & n.d. & n.d. & n.d. & n.d. & n.d. \\
\hline 00U-40/82-102 & n.d. & n.d. & n.d. & n.d. & n.d. & n.d. & n.d. & n.d. & n.d. \\
\hline 00U-40/102-121 & n.d. & n.d. & n.d. & n.d. & n.d. & n.d. & n.d. & n.d. & n.d. \\
\hline 00U-40/121-142 & n.d. & n.d. & n.d. & n.d. & n.d. & n.d. & n.d. & n.d. & n.d. \\
\hline 00U-40/142-154 & n.d. & n.d. & n.d. & n.d. & n.d. & n.d. & n.d. & n.d. & n.d. \\
\hline 00U-40/154-168 & n.d. & n.d. & n.d. & n.d. & n.d. & n.d. & n.d. & n.d. & n.d. \\
\hline 00U-40/168-183 & n.d. & n.d. & n.d. & n.d. & n.d. & n.d. & n.d. & n.d. & n.d. \\
\hline 00U-40/183-193 & n.d. & n.d. & n.d. & n.d. & n.d. & n.d. & n.d. & n.d. & n.d. \\
\hline 00U-40/192-204 & n.d. & n.d. & n.d. & n.d. & n.d. & n.d. & n.d. & n.d. & n.d. \\
\hline 00U-40/204-217 & n.d. & n.d. & n.d. & n.d. & n.d. & n.d. & n.d. & n.d. & n.d. \\
\hline
\end{tabular}


Table 6d-1a. Magnetic properties of the less than 2-mm size fraction for the 00U-35 transect.

\begin{tabular}{|c|c|c|c|c|c|c|c|c|c|}
\hline Sample \# & $\begin{array}{c}\text { MSIf } \\
\left(\mathbf{m}^{3} / \mathrm{kg}\right)\end{array}$ & $\begin{array}{c}\text { MShf } \\
\left(\mathbf{m}^{3} / \mathbf{k g}\right)\end{array}$ & $\begin{array}{c}\text { FDMS } \\
(\%)\end{array}$ & $\begin{array}{l}\text { FDMS } \\
\left(\mathrm{m}^{3} / \mathrm{kg}\right)\end{array}$ & $\begin{array}{c}\text { ARM } \\
\left(\mathrm{Am}^{2} / \mathbf{k g}\right)\end{array}$ & $\begin{array}{l}\text { IRM1.2 } \\
\left(\mathrm{Am}^{2} / \mathrm{kg}\right)\end{array}$ & $\begin{array}{l}\text { IRM -0.3 } \\
\left(\mathrm{Am}^{2} / \mathrm{kg}\right)\end{array}$ & $\begin{array}{c}\text { HIRM } \\
\left(\mathrm{Am}^{2} / \mathrm{kg}\right)\end{array}$ & $S$ \\
\hline 00U-35A/0-10 & $3.00 \mathrm{E}-07$ & $2.96 \mathrm{E}-07$ & 1.55 & 4.67E-09 & 3.90E-05 & $1.17 \mathrm{E}-03$ & 8.30E-04 & $1.71 \mathrm{E}-04$ & 0.71 \\
\hline 00U-35A/10-30 & 2.83E-07 & $2.76 \mathrm{E}-07$ & 2.40 & $6.81 \mathrm{E}-09$ & 3.64E-05 & $1.09 \mathrm{E}-03$ & $7.76 \mathrm{E}-04$ & $1.57 \mathrm{E}-04$ & 0.71 \\
\hline 00U-35A/30-50 & 2.64E-07 & $2.61 \mathrm{E}-07$ & 1.14 & $3.01 \mathrm{E}-09$ & $3.68 \mathrm{E}-05$ & $1.06 \mathrm{E}-03$ & 7.72E-04 & $1.42 \mathrm{E}-04$ & 0.73 \\
\hline 00U-35B/0-10 & $2.66 \mathrm{E}-07$ & $2.63 \mathrm{E}-07$ & 1.26 & $3.36 \mathrm{E}-09$ & 3.17E-05 & $8.71 \mathrm{E}-04$ & $6.07 \mathrm{E}-04$ & $1.32 \mathrm{E}-04$ & 0.70 \\
\hline 00U-35B/10-30 & $3.06 \mathrm{E}-07$ & $3.01 \mathrm{E}-07$ & 1.44 & 4.39E-09 & $3.88 \mathrm{E}-05$ & $1.11 \mathrm{E}-03$ & 8.01E-04 & $1.57 \mathrm{E}-04$ & 0.72 \\
\hline 00U-35B/30-50 & $3.36 \mathrm{E}-07$ & $3.33 \mathrm{E}-07$ & 0.77 & $2.59 \mathrm{E}-09$ & 4.33E-05 & $1.26 \mathrm{E}-03$ & $9.28 \mathrm{E}-04$ & $1.64 \mathrm{E}-04$ & 0.74 \\
\hline 00U-35C/0-10 & $3.10 \mathrm{E}-07$ & $3.03 \mathrm{E}-07$ & 2.42 & $7.50 \mathrm{E}-09$ & 3.93E-05 & $1.13 \mathrm{E}-03$ & $8.45 \mathrm{E}-04$ & $1.40 \mathrm{E}-04$ & 0.75 \\
\hline 00U-35C/10-30 & $3.44 \mathrm{E}-07$ & $3.39 \mathrm{E}-07$ & 1.44 & 4.97E-09 & 4.19E-05 & $1.30 \mathrm{E}-03$ & $9.29 \mathrm{E}-04$ & $1.83 \mathrm{E}-04$ & 0.72 \\
\hline $00 \mathrm{U}-35 \mathrm{C} / 30-50$ & 3.19E-07 & $3.12 \mathrm{E}-07$ & 2.16 & $6.88 \mathrm{E}-09$ & 4.21E-05 & $1.30 \mathrm{E}-03$ & $9.63 \mathrm{E}-04$ & $1.70 \mathrm{E}-04$ & 0.74 \\
\hline 00U-35D/0-10 & $3.22 \mathrm{E}-07$ & $3.18 \mathrm{E}-07$ & 1.29 & 4.15E-09 & $4.28 \mathrm{E}-05$ & $1.24 \mathrm{E}-03$ & 8.82E-04 & $1.78 \mathrm{E}-04$ & 0.71 \\
\hline 00U-35D/10-30 & $3.24 \mathrm{E}-07$ & $2.97 \mathrm{E}-07$ & 8.41 & $2.73 \mathrm{E}-08$ & $5.18 \mathrm{E}-05$ & $1.53 \mathrm{E}-03$ & $1.12 \mathrm{E}-03$ & $2.05 \mathrm{E}-04$ & 0.73 \\
\hline 00U-35D/30-50 & 3.09E-07 & $3.28 \mathrm{E}-07$ & -6.28 & $-1.94 \mathrm{E}-08$ & 4.93E-05 & $1.42 \mathrm{E}-03$ & $1.06 \mathrm{E}-03$ & $1.76 \mathrm{E}-04$ & 0.75 \\
\hline 00U-35E/0-10 & $3.57 \mathrm{E}-07$ & $3.46 \mathrm{E}-07$ & 3.08 & $1.01 \mathrm{E}-08$ & $6.45 \mathrm{E}-05$ & $1.95 \mathrm{E}-03$ & $1.39 \mathrm{E}-03$ & $2.81 \mathrm{E}-04$ & 0.71 \\
\hline 00U-35E/10-30 & $3.38 \mathrm{E}-07$ & $3.34 \mathrm{E}-07$ & 1.18 & $3.98 \mathrm{E}-09$ & 5.90E-05 & $1.88 \mathrm{E}-03$ & $1.37 \mathrm{E}-03$ & $2.53 \mathrm{E}-04$ & 0.73 \\
\hline $00 \mathrm{U}-35 \mathrm{E} / 30-50$ & $3.61 \mathrm{E}-07$ & $3.58 \mathrm{E}-07$ & 0.93 & 3.37E-09 & 5.13E-05 & $1.69 \mathrm{E}-03$ & $1.28 \mathrm{E}-03$ & $2.07 \mathrm{E}-04$ & 0.76 \\
\hline 00U-35F/0-10 & $3.42 \mathrm{E}-07$ & $3.32 \mathrm{E}-07$ & 2.90 & $9.92 \mathrm{E}-09$ & 9.91E-05 & 2.91E-03 & $2.11 \mathrm{E}-03$ & $3.97 \mathrm{E}-04$ & 0.73 \\
\hline $00 \mathrm{U}-35 \mathrm{~F} / 10-30$ & $3.41 \mathrm{E}-07$ & 3.33E-07 & 2.38 & 8.12E-09 & $10.00 \mathrm{E}-05$ & 2.82E-03 & $2.09 \mathrm{E}-03$ & $3.66 \mathrm{E}-04$ & 0.74 \\
\hline 00U-35F/30-50 & $3.53 \mathrm{E}-07$ & 3.47E-07 & 1.71 & $6.05 \mathrm{E}-09$ & $1.05 \mathrm{E}-04$ & $2.90 \mathrm{E}-03$ & $2.18 \mathrm{E}-03$ & $3.61 \mathrm{E}-04$ & 0.75 \\
\hline 00U-35G/0-10 & 3.53E-07 & $3.44 \mathrm{E}-07$ & 2.55 & $9.01 \mathrm{E}-09$ & $8.51 \mathrm{E}-05$ & $2.43 \mathrm{E}-03$ & $1.73 \mathrm{E}-03$ & $3.50 \mathrm{E}-04$ & 0.71 \\
\hline 00U-35G/10-30 & $3.05 \mathrm{E}-07$ & 2.97E-07 & 2.53 & 7.70E-09 & $9.06 \mathrm{E}-05$ & $2.55 \mathrm{E}-03$ & $1.83 \mathrm{E}-03$ & $3.59 \mathrm{E}-04$ & 0.72 \\
\hline 00U-35G/30-50 & $3.43 \mathrm{E}-07$ & $3.35 \mathrm{E}-07$ & 2.54 & $8.72 \mathrm{E}-09$ & $9.34 \mathrm{E}-05$ & 2.47E-03 & $1.84 \mathrm{E}-03$ & $3.15 \mathrm{E}-04$ & 0.74 \\
\hline 00U-35H/0-10 & $3.87 \mathrm{E}-07$ & $3.73 \mathrm{E}-07$ & 3.60 & $1.39 \mathrm{E}-08$ & $1.29 \mathrm{E}-04$ & $3.79 \mathrm{E}-03$ & $2.82 \mathrm{E}-03$ & $4.82 \mathrm{E}-04$ & 0.75 \\
\hline 00U-35H/10-30 & $3.75 \mathrm{E}-07$ & $3.64 \mathrm{E}-07$ & 2.77 & $1.04 \mathrm{E}-08$ & 1.45E-04 & 3.91E-03 & $2.94 \mathrm{E}-03$ & $4.88 \mathrm{E}-04$ & 0.75 \\
\hline 00U-35H/30-50 & $4.15 \mathrm{E}-07$ & 4.02E-07 & 3.17 & $1.32 \mathrm{E}-08$ & $1.62 \mathrm{E}-04$ & 4.05E-03 & $3.12 \mathrm{E}-03$ & 4.69E-04 & 0.77 \\
\hline
\end{tabular}


Table $6 \mathbf{d}-1 \mathbf{b}$. Magnetic properties of the less than 63 -micron size fraction for the $00 \mathrm{U}-35$ transect.

\begin{tabular}{|c|c|c|c|c|c|c|c|c|c|}
\hline Sample \# & $\begin{array}{c}\text { MSIf } \\
\left(\mathbf{m}^{3} / \mathbf{k g}\right)\end{array}$ & $\begin{array}{c}\text { MShf } \\
\left(\mathrm{m}^{3} / \mathrm{kg}\right)\end{array}$ & $\begin{array}{l}\text { FDMS } \\
(\%)\end{array}$ & $\begin{array}{l}\text { FDMS } \\
\left(\mathrm{m}^{3} / \mathbf{k g}\right)\end{array}$ & $\begin{array}{c}\text { ARM } \\
\left(\mathrm{Am}^{2} / \mathrm{kg}\right)\end{array}$ & $\begin{array}{c}\text { IRM1.2 } \\
\left(\mathrm{Am}^{2} / \mathrm{kg}\right)\end{array}$ & $\begin{array}{l}\text { IRM -0.3 } \\
\left(\mathrm{Am}^{2} / \mathrm{kg}\right)\end{array}$ & $\begin{array}{c}\text { HIRM } \\
\left(\mathrm{Am}^{2} / \mathbf{k g}\right)\end{array}$ & $\mathbf{S}$ \\
\hline 00U-35A/0-10 & $3.00 \mathrm{E}-07$ & $2.96 \mathrm{E}-07$ & 1.55 & 4.67E-09 & $8.24 \mathrm{E}-05$ & $4.48 \mathrm{E}-03$ & $3.25 \mathrm{E}-03$ & $6.17 \mathrm{E}-04$ & 0.72 \\
\hline 00U-35A/10-30 & $2.83 \mathrm{E}-07$ & $2.76 \mathrm{E}-07$ & 2.40 & $6.81 \mathrm{E}-09$ & 8.32E-05 & 4.15E-03 & $3.11 \mathrm{E}-03$ & $5.18 \mathrm{E}-04$ & 0.75 \\
\hline 00U-35A/30-50 & $2.64 \mathrm{E}-07$ & $2.61 \mathrm{E}-07$ & 1.14 & 3.01E-09 & $8.24 \mathrm{E}-05$ & $3.89 \mathrm{E}-03$ & 2.93E-03 & 4.84E-04 & 0.75 \\
\hline 00U-35B/0-10 & $2.66 \mathrm{E}-07$ & $2.63 \mathrm{E}-07$ & 1.26 & 3.36E-09 & 7.93E-05 & 4.07E-03 & $2.90 \mathrm{E}-03$ & $5.83 \mathrm{E}-04$ & 0.71 \\
\hline 00U-35B/10-30 & $3.06 \mathrm{E}-07$ & $3.01 \mathrm{E}-07$ & 1.44 & 4.39E-09 & $9.26 \mathrm{E}-05$ & $4.48 \mathrm{E}-03$ & 3.33E-03 & $5.75 \mathrm{E}-04$ & 0.74 \\
\hline 00U-35B/30-50 & $3.36 \mathrm{E}-07$ & 3.33E-07 & 0.77 & 2.59E-09 & 9.97E-05 & 4.69E-03 & $3.57 \mathrm{E}-03$ & $5.60 \mathrm{E}-04$ & 0.76 \\
\hline 00U-35C/0-10 & $3.10 \mathrm{E}-07$ & 3.03E-07 & 2.42 & 7.50E-09 & $8.98 \mathrm{E}-05$ & $4.59 \mathrm{E}-03$ & $3.37 \mathrm{E}-03$ & $6.10 \mathrm{E}-04$ & 0.73 \\
\hline 00U-35C/10-30 & $3.44 \mathrm{E}-07$ & 3.39E-07 & 1.44 & 4.97E-09 & $9.25 \mathrm{E}-05$ & 4.94E-03 & 3.70E-03 & $6.21 \mathrm{E}-04$ & 0.75 \\
\hline 00U-35C/30-50 & 3.19E-07 & $3.12 \mathrm{E}-07$ & 2.16 & $6.88 \mathrm{E}-09$ & $8.81 \mathrm{E}-05$ & $4.48 \mathrm{E}-03$ & $3.40 \mathrm{E}-03$ & $5.43 \mathrm{E}-04$ & 0.76 \\
\hline 00U-35D/0-10 & $3.22 \mathrm{E}-07$ & $3.18 \mathrm{E}-07$ & 1.29 & 4.15E-09 & 8.94E-05 & $4.70 \mathrm{E}-03$ & $3.42 \mathrm{E}-03$ & $6.39 \mathrm{E}-04$ & 0.73 \\
\hline 00U-35D/10-30 & $3.24 \mathrm{E}-07$ & 2.97E-07 & 8.41 & 2.73E-08 & $1.01 \mathrm{E}-04$ & $4.75 \mathrm{E}-03$ & $3.53 \mathrm{E}-03$ & $6.09 \mathrm{E}-04$ & 0.74 \\
\hline 00U-35D/30-50 & 3.09E-07 & $3.28 \mathrm{E}-07$ & -6.28 & $-1.94 \mathrm{E}-08$ & $9.55 \mathrm{E}-05$ & 4.42E-03 & 3.30E-03 & $5.57 \mathrm{E}-04$ & 0.75 \\
\hline 00U-35E/0-10 & 3.57E-07 & $3.46 \mathrm{E}-07$ & 3.08 & $1.01 \mathrm{E}-08$ & $1.01 \mathrm{E}-04$ & $5.21 \mathrm{E}-03$ & $3.87 \mathrm{E}-03$ & $6.70 \mathrm{E}-04$ & 0.74 \\
\hline 00U-35E/10-30 & $3.38 \mathrm{E}-07$ & 3.34E-07 & 1.18 & 3.98E-09 & $1.08 \mathrm{E}-04$ & 4.99E-03 & $3.77 \mathrm{E}-03$ & $6.12 \mathrm{E}-04$ & 0.76 \\
\hline 00U-35E/30-50 & $3.61 \mathrm{E}-07$ & $3.58 \mathrm{E}-07$ & 0.93 & 3.37E-09 & $1.06 \mathrm{E}-04$ & $5.06 \mathrm{E}-03$ & 4.04E-03 & $5.06 \mathrm{E}-04$ & 0.80 \\
\hline 00U-35F/0-10 & $3.42 \mathrm{E}-07$ & 3.32E-07 & 2.90 & 9.92E-09 & $1.28 \mathrm{E}-04$ & $5.15 \mathrm{E}-03$ & $3.76 \mathrm{E}-03$ & 6.97E-04 & 0.73 \\
\hline 00U-35F/10-30 & $3.41 \mathrm{E}-07$ & 3.33E-07 & 2.38 & $8.12 \mathrm{E}-09$ & $1.38 \mathrm{E}-04$ & $5.05 \mathrm{E}-03$ & $3.77 \mathrm{E}-03$ & $6.42 \mathrm{E}-04$ & 0.75 \\
\hline 00U-35F/30-50 & $3.53 \mathrm{E}-07$ & $3.47 \mathrm{E}-07$ & 1.71 & $6.05 \mathrm{E}-09$ & $1.59 \mathrm{E}-04$ & $5.25 \mathrm{E}-03$ & 4.03E-03 & $6.09 \mathrm{E}-04$ & 0.77 \\
\hline 00U-35G/0-10 & 3.53E-07 & 3.44E-07 & 2.55 & $9.01 \mathrm{E}-09$ & $1.43 \mathrm{E}-04$ & $5.32 \mathrm{E}-03$ & 3.92E-03 & 7.03E-04 & 0.74 \\
\hline 00U-35G/10-30 & $3.05 \mathrm{E}-07$ & 2.97E-07 & 2.53 & 7.70E-09 & $1.36 \mathrm{E}-04$ & $4.58 \mathrm{E}-03$ & $3.42 \mathrm{E}-03$ & $5.81 \mathrm{E}-04$ & 0.75 \\
\hline 00U-35G/30-50 & $3.43 \mathrm{E}-07$ & $3.35 \mathrm{E}-07$ & 2.54 & $8.72 \mathrm{E}-09$ & $1.49 \mathrm{E}-04$ & $5.02 \mathrm{E}-03$ & $3.87 \mathrm{E}-03$ & $5.75 \mathrm{E}-04$ & 0.77 \\
\hline 00U-35H/0-10 & 3.87E-07 & $3.73 \mathrm{E}-07$ & 3.60 & $1.39 \mathrm{E}-08$ & $1.72 \mathrm{E}-04$ & $6.07 \mathrm{E}-03$ & 4.57E-03 & 7.47E-04 & 0.75 \\
\hline 00U-35H/10-30 & 3.75E-07 & $3.64 \mathrm{E}-07$ & 2.77 & $1.04 \mathrm{E}-08$ & $1.88 \mathrm{E}-04$ & $5.84 \mathrm{E}-03$ & 4.39E-03 & $7.24 \mathrm{E}-04$ & 0.75 \\
\hline 00U-35H/30-50 & 4.15E-07 & $4.02 \mathrm{E}-07$ & 3.17 & 1.32E-08 & $2.19 \mathrm{E}-04$ & $6.35 \mathrm{E}-03$ & 4.94E-03 & $7.05 \mathrm{E}-04$ & 0.78 \\
\hline
\end{tabular}


Table 6d-2a. Magnetic properties of the less than 2-mm size fraction for auger hole 00U-36.

\begin{tabular}{|c|c|c|c|c|c|c|c|c|c|}
\hline Sample \# & $\begin{array}{c}\text { MSIf } \\
\left(\mathrm{m}^{3} / \mathbf{k g}\right)\end{array}$ & $\begin{array}{c}\text { MShf } \\
\left(\mathbf{m}^{3} / \mathbf{k g}\right)\end{array}$ & $\begin{array}{c}\text { FDMS } \\
(\%)\end{array}$ & $\begin{array}{l}\text { FDMS } \\
\left(\mathrm{m}^{3} / \mathrm{kg}\right)\end{array}$ & $\begin{array}{c}\text { ARM } \\
\left(\mathrm{Am}^{2} / \mathrm{kg}\right)\end{array}$ & $\begin{array}{l}\text { IRM1.2 } \\
\left(\mathrm{Am}{ }^{2} / \mathrm{kg}\right)\end{array}$ & $\begin{array}{l}\text { IRM -0.3 } \\
\left(\mathrm{Am}^{2} / \mathbf{k g}\right)\end{array}$ & $\begin{array}{c}\text { HIRM } \\
\left(\mathrm{Am}^{2} / \mathbf{k g}\right)\end{array}$ & S \\
\hline 00U-36/0-10 & $8.22 \mathrm{E}-08$ & $8.05 \mathrm{E}-08$ & 2.05 & $1.68 \mathrm{E}-09$ & $3.62 \mathrm{E}-05$ & $1.11 \mathrm{E}-03$ & $8.15 \mathrm{E}-04$ & $1.49 \mathrm{E}-04$ & 0.73 \\
\hline 00U-36/10-30 & $9.77 \mathrm{E}-08$ & $9.66 \mathrm{E}-08$ & 1.09 & 1.07E-09 & 3.97E-05 & $1.36 \mathrm{E}-03$ & $1.01 \mathrm{E}-03$ & $1.71 \mathrm{E}-04$ & 0.75 \\
\hline 00U-36/30-46 & $9.75 \mathrm{E}-08$ & $9.71 \mathrm{E}-08$ & 0.38 & $3.73 \mathrm{E}-10$ & $5.50 \mathrm{E}-05$ & $1.34 \mathrm{E}-03$ & $1.01 \mathrm{E}-03$ & $1.69 \mathrm{E}-04$ & 0.75 \\
\hline 00U-36/46-68 & $9.65 \mathrm{E}-08$ & $9.63 \mathrm{E}-08$ & 0.23 & $2.21 \mathrm{E}-10$ & $5.08 \mathrm{E}-05$ & $1.33 \mathrm{E}-03$ & $1.00 \mathrm{E}-03$ & $1.65 \mathrm{E}-04$ & 0.75 \\
\hline 00U-36/68-86 & $1.37 \mathrm{E}-07$ & $1.35 \mathrm{E}-07$ & 1.71 & 2.34E-09 & 3.89E-05 & $1.80 \mathrm{E}-03$ & $1.46 \mathrm{E}-03$ & $1.68 \mathrm{E}-04$ & 0.81 \\
\hline 00U-36/86-97 & $3.22 \mathrm{E}-07$ & $3.14 \mathrm{E}-07$ & 2.36 & 7.58E-09 & 4.07E-05 & $4.18 \mathrm{E}-03$ & $3.74 \mathrm{E}-03$ & $2.18 \mathrm{E}-04$ & 0.90 \\
\hline 00U-36/97-108 & 2.77E-07 & $2.73 \mathrm{E}-07$ & 1.44 & 3.99E-09 & 4.62E-05 & $3.72 \mathrm{E}-03$ & $3.24 \mathrm{E}-03$ & $2.36 \mathrm{E}-04$ & 0.87 \\
\hline 00U-36/108-120 & 2.44E-07 & $2.42 \mathrm{E}-07$ & 0.74 & $1.81 \mathrm{E}-09$ & 7.19E-05 & $3.24 \mathrm{E}-03$ & $2.85 \mathrm{E}-03$ & $1.97 \mathrm{E}-04$ & 0.88 \\
\hline 00U-36/120-130 & 2.03E-07 & 2.02E-07 & 0.27 & $5.46 \mathrm{E}-10$ & $6.56 \mathrm{E}-05$ & $2.74 \mathrm{E}-03$ & $2.37 \mathrm{E}-03$ & 1.84E-04 & 0.87 \\
\hline
\end{tabular}

Table 6d-2b. Magnetic properties of the less than 63-micron size fraction for auger hole 00U-36.

\begin{tabular}{|c|c|c|c|c|c|c|c|c|c|}
\hline Sample \# & $\begin{array}{c}\text { MSIf } \\
\left(\mathrm{m}^{3} / \mathrm{kg}\right)\end{array}$ & $\begin{array}{c}\text { MShf } \\
\left(\mathrm{m}^{3} / \mathrm{kg}\right)\end{array}$ & $\begin{array}{l}\text { FDMS } \\
(\%)\end{array}$ & $\begin{array}{l}\text { FDMS } \\
\left(\mathrm{m}^{3} / \mathrm{kg}\right)\end{array}$ & $\begin{array}{c}\text { ARM } \\
\left(\mathrm{Am}^{2} / \mathrm{kg}\right)\end{array}$ & $\begin{array}{c}\text { IRM1.2 } \\
\left(\mathrm{Am}^{2} / \mathrm{kg}\right)\end{array}$ & $\begin{array}{l}\text { IRM -0.3 } \\
\left(\mathrm{Am}^{2} / \mathrm{kg}\right)\end{array}$ & $\begin{array}{c}\text { HIRM } \\
\left(\mathrm{Am}^{2} / \mathbf{k g}\right)\end{array}$ & $\mathbf{S}$ \\
\hline 00U-36/0-10 & 3.19E-07 & 3.11E-07 & 2.67 & 8.53E-09 & $8.67 \mathrm{E}-05$ & 4.49E-03 & $3.31 \mathrm{E}-03$ & $5.90 \mathrm{E}-04$ & 0.74 \\
\hline 00U-36/10-30 & $3.51 \mathrm{E}-07$ & $3.41 \mathrm{E}-07$ & 2.78 & $9.75 \mathrm{E}-09$ & $1.00 \mathrm{E}-04$ & 4.90E-03 & 3.84E-03 & 5.30E-04 & 0.78 \\
\hline 00U-36/30-46 & $3.62 \mathrm{E}-07$ & $3.53 \mathrm{E}-07$ & 2.43 & $8.79 \mathrm{E}-09$ & $1.36 \mathrm{E}-04$ & $5.02 \mathrm{E}-03$ & 3.82E-03 & $5.99 \mathrm{E}-04$ & 0.76 \\
\hline 00U-36/46-68 & $4.32 \mathrm{E}-07$ & $4.20 \mathrm{E}-07$ & 2.70 & $1.17 \mathrm{E}-08$ & $1.08 \mathrm{E}-04$ & $5.77 \mathrm{E}-03$ & 4.41E-03 & $6.81 \mathrm{E}-04$ & 0.76 \\
\hline 00U-36/68-86 & $5.47 \mathrm{E}-07$ & $5.34 \mathrm{E}-07$ & 2.36 & $1.29 \mathrm{E}-08$ & $1.02 \mathrm{E}-04$ & 7.01E-03 & $5.83 \mathrm{E}-03$ & $6.33 \mathrm{E}-04$ & 0.82 \\
\hline 00U-36/86-97 & $8.56 \mathrm{E}-07$ & $8.38 \mathrm{E}-07$ & 2.09 & $1.79 \mathrm{E}-08$ & $1.15 \mathrm{E}-04$ & $1.10 \mathrm{E}-02$ & $9.84 \mathrm{E}-03$ & 5.95E-04 & 0.89 \\
\hline 00U-36/97-108 & $8.76 \mathrm{E}-07$ & $8.59 \mathrm{E}-07$ & 1.87 & $1.63 \mathrm{E}-08$ & $1.01 \mathrm{E}-04$ & $1.12 \mathrm{E}-02$ & $1.00 \mathrm{E}-02$ & $5.77 \mathrm{E}-04$ & 0.90 \\
\hline 00U-36/108-120 & $8.34 \mathrm{E}-07$ & 8.19E-07 & 1.72 & $1.44 \mathrm{E}-08$ & $1.29 \mathrm{E}-04$ & $1.07 \mathrm{E}-02$ & $9.58 \mathrm{E}-03$ & $5.51 \mathrm{E}-04$ & 0.90 \\
\hline 00U-36/120-130 & $6.45 \mathrm{E}-07$ & $6.35 \mathrm{E}-07$ & 1.66 & $1.07 \mathrm{E}-08$ & $1.46 \mathrm{E}-04$ & $8.56 \mathrm{E}-03$ & 7.57E-03 & $4.95 \mathrm{E}-04$ & 0.88 \\
\hline
\end{tabular}


Table 6d-3a. Magnetic properties of the less than 2-mm size fraction for auger hole 00U-37.

\begin{tabular}{|c|c|c|c|c|c|c|c|c|c|}
\hline Sample \# & $\begin{array}{c}\text { MSIf } \\
\left(\mathbf{m}^{3} / \mathbf{k g}\right)\end{array}$ & $\begin{array}{l}\text { MShf } \\
\left(\mathbf{m}^{3} / \mathbf{k g}\right)\end{array}$ & $\begin{array}{c}\text { FDMS } \\
(\%)\end{array}$ & $\begin{array}{l}\text { FDMS } \\
\left(\mathrm{m}^{3} / \mathbf{k g}\right)\end{array}$ & $\begin{array}{c}\text { ARM } \\
\left(\mathrm{Am}^{2} / \mathbf{k g}\right)\end{array}$ & $\begin{array}{c}\text { IRM1.2 } \\
\text { (Am²/kg) }\end{array}$ & $\begin{array}{l}\text { IRM -0.3 } \\
\left(\mathrm{Am}^{2} / \mathrm{kg}\right)\end{array}$ & $\begin{array}{c}\text { HIRM } \\
\left(\mathrm{Am}^{2} / \mathbf{k g}\right)\end{array}$ & S \\
\hline 00U-37/0-10 & $2.46 \mathrm{E}-07$ & $2.37 \mathrm{E}-07$ & 3.73 & $9.16 \mathrm{E}-09$ & $1.22 \mathrm{E}-04$ & $3.58 \mathrm{E}-03$ & $2.59 \mathrm{E}-03$ & 4.96E-04 & 0.72 \\
\hline 00U-37/10-30 & $2.45 \mathrm{E}-07$ & 2.36E-07 & 3.69 & $9.05 \mathrm{E}-09$ & $1.23 \mathrm{E}-04$ & $3.59 \mathrm{E}-03$ & $2.67 \mathrm{E}-03$ & 4.63E-04 & 0.74 \\
\hline 00U-37/30-43 & 2.49E-07 & $2.41 \mathrm{E}-07$ & 3.37 & 8.40E-09 & $6.00 \mathrm{E}-05$ & $3.70 \mathrm{E}-03$ & $2.78 \mathrm{E}-03$ & 4.62E-04 & 0.75 \\
\hline 00U-37/43-55 & 2.40E-07 & 2.32E-07 & 3.14 & 7.51E-09 & $3.78 \mathrm{E}-05$ & $3.40 \mathrm{E}-03$ & $2.68 \mathrm{E}-03$ & 3.59E-04 & 0.79 \\
\hline 00U-37/55-68 & $2.07 \mathrm{E}-07$ & 2.04E-07 & 1.87 & $3.89 \mathrm{E}-09$ & $2.88 \mathrm{E}-05$ & 2.99E-03 & $2.42 \mathrm{E}-03$ & 2.84E-04 & 0.81 \\
\hline 00U-37/68-80 & $1.83 \mathrm{E}-07$ & $1.80 \mathrm{E}-07$ & 1.55 & $2.83 \mathrm{E}-09$ & $3.28 \mathrm{E}-05$ & $2.48 \mathrm{E}-03$ & $2.07 \mathrm{E}-03$ & $2.01 \mathrm{E}-04$ & 0.84 \\
\hline 00U-37/80-94 & $1.64 \mathrm{E}-07$ & $1.60 \mathrm{E}-07$ & 2.41 & $3.95 \mathrm{E}-09$ & $5.89 \mathrm{E}-05$ & $2.21 \mathrm{E}-03$ & $1.86 \mathrm{E}-03$ & $1.77 \mathrm{E}-04$ & 0.84 \\
\hline 00U-37/94-110 & $7.76 \mathrm{E}-08$ & 7.89E-08 & -1.74 & $-1.35 \mathrm{E}-09$ & $1.25 \mathrm{E}-04$ & $1.10 \mathrm{E}-03$ & $6.90 \mathrm{E}-04$ & 2.07E-04 & 0.62 \\
\hline 00U-37/110-120 & $1.23 \mathrm{E}-07$ & $1.23 \mathrm{E}-07$ & -0.05 & $-6.19 \mathrm{E}-11$ & $1.39 \mathrm{E}-04$ & $1.83 \mathrm{E}-03$ & $1.34 \mathrm{E}-03$ & $2.42 \mathrm{E}-04$ & 0.74 \\
\hline 00U-37/120-131 & 7.63E-08 & 7.75E-08 & -1.56 & $-1.19 \mathrm{E}-09$ & $9.30 \mathrm{E}-05$ & 1.19E-03 & 7.63E-04 & $2.12 \mathrm{E}-04$ & 0.64 \\
\hline 00U-37/131-139 & $5.83 \mathrm{E}-08$ & 5.93E-08 & -1.79 & $-1.04 \mathrm{E}-09$ & $5.49 \mathrm{E}-05$ & $9.38 \mathrm{E}-04$ & $5.88 \mathrm{E}-04$ & $1.75 \mathrm{E}-04$ & 0.63 \\
\hline 00U-37/139-148 & $7.00 \mathrm{E}-08$ & 7.12E-08 & -1.70 & $-1.19 \mathrm{E}-09$ & 4.79E-05 & $1.07 \mathrm{E}-03$ & 7.14E-04 & $1.77 \mathrm{E}-04$ & 0.67 \\
\hline 00U-37/148-155 & $1.21 \mathrm{E}-07$ & $1.19 \mathrm{E}-07$ & 1.55 & $1.87 \mathrm{E}-09$ & 3.19E-05 & $1.81 \mathrm{E}-03$ & $1.30 \mathrm{E}-03$ & $2.54 \mathrm{E}-04$ & 0.72 \\
\hline
\end{tabular}

Table 6d-3b. Magnetic properties of the less than 63-micron size fraction for auger hole 00U-37.

\begin{tabular}{|c|c|c|c|c|c|c|c|c|c|}
\hline Sample \# & $\begin{array}{c}\text { MSIf } \\
\left(\mathbf{m}^{3} / \mathbf{k g}\right)\end{array}$ & $\begin{array}{l}\text { MShf } \\
\left(\mathrm{m}^{3} / \mathrm{kg}\right)\end{array}$ & $\begin{array}{c}\text { FDMS } \\
(\%)\end{array}$ & $\begin{array}{l}\text { FDMS } \\
\left(\mathbf{m}^{3} / \mathbf{k g}\right)\end{array}$ & $\begin{array}{c}\text { ARM } \\
\left(\mathrm{Am}^{2} / \mathrm{kg}\right)\end{array}$ & $\begin{array}{l}\text { IRM1.2 } \\
\left(\mathrm{Am}^{2} / \mathrm{kg}\right)\end{array}$ & $\begin{array}{l}\text { IRM -0.3 } \\
\left(\mathrm{Am}^{2} / \mathrm{kg}\right)\end{array}$ & $\begin{array}{l}\text { HIRM } \\
\left(\mathrm{Am}^{2} / \mathrm{kg}\right)\end{array}$ & $\mathbf{S}$ \\
\hline 00U-37/0-10 & $3.95 \mathrm{E}-07$ & $3.81 \mathrm{E}-07$ & 3.58 & $1.42 \mathrm{E}-08$ & $1.55 \mathrm{E}-04$ & $5.69 \mathrm{E}-03$ & 4.19E-03 & 7.53E-04 & 0.74 \\
\hline 00U-37/10-30 & 4.04E-07 & 3.91E-07 & 3.20 & $1.29 \mathrm{E}-08$ & $1.77 \mathrm{E}-04$ & $5.90 \mathrm{E}-03$ & 4.43E-03 & 7.37E-04 & 0.75 \\
\hline 00U-37/30-43 & 4.45E-07 & $4.28 \mathrm{E}-07$ & 3.74 & $1.66 \mathrm{E}-08$ & 8.33E-05 & $6.49 \mathrm{E}-03$ & 4.97E-03 & 7.62E-04 & 0.77 \\
\hline 00U-37/43-55 & $5.65 \mathrm{E}-07$ & $5.45 \mathrm{E}-07$ & 3.52 & $1.99 \mathrm{E}-08$ & $7.55 \mathrm{E}-05$ & 7.91E-03 & $6.40 \mathrm{E}-03$ & $7.55 \mathrm{E}-04$ & 0.81 \\
\hline 00U-37/55-68 & 5.67E-07 & $5.52 \mathrm{E}-07$ & 2.56 & $1.45 \mathrm{E}-08$ & $8.31 \mathrm{E}-05$ & 7.83E-03 & $6.58 \mathrm{E}-03$ & $6.23 \mathrm{E}-04$ & 0.84 \\
\hline 00U-37/68-80 & 7.99E-07 & $7.80 \mathrm{E}-07$ & 2.35 & $1.88 \mathrm{E}-08$ & $9.23 \mathrm{E}-05$ & 1.04E-02 & $9.03 \mathrm{E}-03$ & $6.70 \mathrm{E}-04$ & 0.87 \\
\hline 00U-37/80-94 & $7.48 \mathrm{E}-07$ & 7.39E-07 & 1.27 & $9.51 \mathrm{E}-09$ & $1.08 \mathrm{E}-04$ & $9.58 \mathrm{E}-03$ & $8.31 \mathrm{E}-03$ & $6.36 \mathrm{E}-04$ & 0.87 \\
\hline 00U-37/94-110 & $1.44 \mathrm{E}-07$ & $1.44 \mathrm{E}-07$ & 0.04 & $6.43 \mathrm{E}-11$ & $1.98 \mathrm{E}-04$ & $1.98 \mathrm{E}-03$ & $1.34 \mathrm{E}-03$ & $3.19 \mathrm{E}-04$ & 0.68 \\
\hline 00U-37/110-120 & $2.58 \mathrm{E}-07$ & $2.54 \mathrm{E}-07$ & 1.50 & $3.88 \mathrm{E}-09$ & $2.57 \mathrm{E}-04$ & $3.66 \mathrm{E}-03$ & $2.81 \mathrm{E}-03$ & $4.25 \mathrm{E}-04$ & 0.77 \\
\hline 00U-37/120-131 & $2.03 \mathrm{E}-07$ & $2.01 \mathrm{E}-07$ & 0.89 & $1.81 \mathrm{E}-09$ & $1.65 \mathrm{E}-04$ & $2.95 \mathrm{E}-03$ & $2.06 \mathrm{E}-03$ & 4.47E-04 & 0.70 \\
\hline 00U-37/131-139 & $2.32 \mathrm{E}-07$ & $2.30 \mathrm{E}-07$ & 1.10 & $2.56 \mathrm{E}-09$ & $1.52 \mathrm{E}-04$ & $3.30 \mathrm{E}-03$ & $2.31 \mathrm{E}-03$ & 4.95E-04 & 0.70 \\
\hline 00U-37/139-148 & $2.48 \mathrm{E}-07$ & $2.45 \mathrm{E}-07$ & 1.27 & $3.16 \mathrm{E}-09$ & $1.34 \mathrm{E}-04$ & $3.57 \mathrm{E}-03$ & $2.57 \mathrm{E}-03$ & 4.98E-04 & 0.72 \\
\hline 00U-37/148-155 & $3.16 \mathrm{E}-07$ & $3.07 \mathrm{E}-07$ & 2.63 & $8.29 \mathrm{E}-09$ & 4.86E-05 & $4.54 \mathrm{E}-03$ & $3.38 \mathrm{E}-03$ & $5.80 \mathrm{E}-04$ & 0.74 \\
\hline
\end{tabular}


Table 6d-4a. Magnetic properties of the less than 2-mm size fraction for soil pit JN-1.

\begin{tabular}{|c|c|c|c|c|c|c|c|c|c|}
\hline Sample \# & $\begin{array}{c}\text { MSIf } \\
\left(\mathbf{m}^{3} / \mathbf{k g}\right)\end{array}$ & $\begin{array}{c}\text { MShf } \\
\left(\mathrm{m}^{3} / \mathbf{k g}\right)\end{array}$ & $\begin{array}{c}\text { FDMS } \\
(\%)\end{array}$ & $\begin{array}{c}\text { FDMS } \\
\left(\mathrm{m}^{3} / \mathbf{k g}\right)\end{array}$ & $\begin{array}{c}\text { ARM } \\
\left(\mathrm{Am}^{2} / \mathbf{k g}\right)\end{array}$ & $\begin{array}{c}\text { IRM1.2 } \\
\left(\mathrm{Am}^{2} / \mathbf{k g}\right)\end{array}$ & $\begin{array}{l}\text { IRM }-0.3 \\
\left(\mathrm{Am}^{2} / \mathrm{kg}\right)\end{array}$ & $\begin{array}{c}\text { HIRM } \\
\left(\mathrm{Am}^{2} / \mathbf{k g}\right)\end{array}$ & S \\
\hline $\mathrm{JN}-1 / 0-10$ & $9.27 \mathrm{E}-08$ & $9.21 \mathrm{E}-08$ & n.d. & n.d. & n.d. & n.d. & n.d. & n.d. & n.d. \\
\hline JN-1/ 26-77 & $1.12 \mathrm{E}-07$ & $1.11 \mathrm{E}-07$ & n.d. & n.d. & n.d. & n.d. & n.d. & n.d. & n.d. \\
\hline JN-1/ 77-93 & $9.98 \mathrm{E}-08$ & $9.91 \mathrm{E}-08$ & n.d. & n.d. & n.d. & n.d. & n.d. & n.d. & n.d. \\
\hline
\end{tabular}

Table 6d-4b. Magnetic properties of the less than 63-micron size fraction for soil pit JN-1.

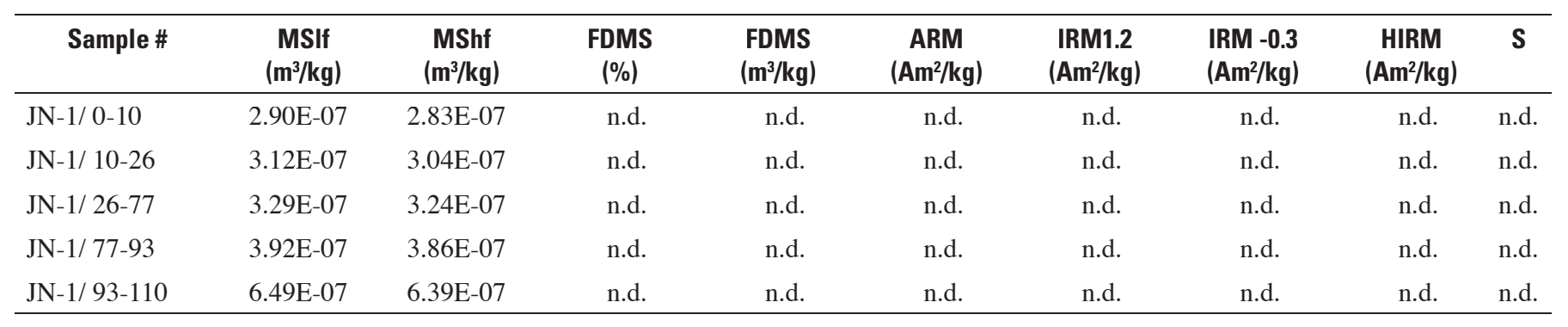


Table 6d-5a. Magnetic properties of the less than 2-mm size fraction for soil pit JN-2.

\begin{tabular}{|c|c|c|c|c|c|c|c|c|c|}
\hline Sample \# & $\underset{\left(\mathbf{m}^{3} / \mathbf{k g}\right)}{\text { MSIf }}$ & $\begin{array}{c}\text { MShf } \\
\left(\mathrm{m}^{3} / \mathrm{kg}\right)\end{array}$ & $\begin{array}{c}\text { FDMS } \\
(\%)\end{array}$ & $\begin{array}{c}\text { FDMS } \\
\left(\mathrm{m}^{3} / \mathrm{kg}\right)\end{array}$ & $\begin{array}{c}\text { ARM } \\
\left(\mathrm{Am}^{2} / \mathbf{k g}\right)\end{array}$ & $\begin{array}{c}\text { IRM1.2 } \\
\left(\mathrm{Am}^{2} / \mathrm{kg}\right)\end{array}$ & $\begin{array}{l}\text { IRM }-0.3 \\
\left(\mathrm{Am}^{2} / \mathrm{kg}\right)\end{array}$ & $\begin{array}{c}\text { HIRM } \\
\left(\mathrm{Am}^{2} / \mathrm{kg}\right)\end{array}$ & $S$ \\
\hline JN-2/ 0-7 & $8.22 \mathrm{E}-08$ & $8.14 \mathrm{E}-08$ & n.d. & n.d. & n.d. & n.d. & n.d. & n.d. & n.d. \\
\hline $\mathrm{JN}-2 / 7-14$ & $1.03 \mathrm{E}-07$ & $1.02 \mathrm{E}-07$ & n.d. & n.d. & n.d. & n.d. & n.d. & n.d. & n.d. \\
\hline $\mathrm{JN}-2 / 14-41$ & $1.47 \mathrm{E}-07$ & $1.44 \mathrm{E}-07$ & n.d. & n.d. & n.d. & n.d. & n.d. & n.d. & n.d. \\
\hline JN-2/ 41-64 & $1.63 \mathrm{E}-07$ & $1.61 \mathrm{E}-07$ & n.d. & n.d. & n.d. & n.d. & n.d. & n.d. & n.d. \\
\hline JN-2/ 64-86 & $1.75 \mathrm{E}-07$ & $1.73 \mathrm{E}-07$ & n.d. & n.d. & n.d. & n.d. & n.d. & n.d. & n.d. \\
\hline JN-2/ 86-100 & $1.98 \mathrm{E}-07$ & $1.97 \mathrm{E}-07$ & n.d. & n.d. & n.d. & n.d. & n.d. & n.d. & n.d. \\
\hline
\end{tabular}

Table $6 \mathbf{d}-\mathbf{5 b}$. Magnetic properties of the less than 63 -micron size fraction for soil pit JN-2.

\begin{tabular}{|c|c|c|c|c|c|c|c|c|c|}
\hline Sample \# & $\begin{array}{c}\text { MSIf } \\
\left(\mathbf{m}^{3} / \mathbf{k g}\right)\end{array}$ & $\begin{array}{c}\text { MShf } \\
\left(\mathbf{m}^{3} / \mathbf{k g}\right)\end{array}$ & $\begin{array}{c}\text { FDMS } \\
(\%)\end{array}$ & $\begin{array}{l}\text { FDMS } \\
\left(\mathrm{m}^{3} / \mathrm{kg}\right)\end{array}$ & $\begin{array}{c}\text { ARM } \\
\left(\mathrm{Am}^{2} / \mathbf{k g}\right)\end{array}$ & $\begin{array}{l}\text { IRM1.2 } \\
\left(\mathrm{Am}^{2} / \mathrm{kg}\right)\end{array}$ & $\begin{array}{l}\text { IRM -0.3 } \\
\left(\mathrm{Am}^{2} / \mathbf{k g}\right)\end{array}$ & $\begin{array}{c}\text { HIRM } \\
\left(\mathrm{Am}^{2} / \mathbf{k g}\right)\end{array}$ & $\mathbf{S}$ \\
\hline $\mathrm{JN}-2 / 0-7$ & $3.18 \mathrm{E}-07$ & $3.16 \mathrm{E}-07$ & n.d. & n.d. & n.d. & n.d. & n.d. & n.d. & n.d. \\
\hline $\mathrm{JN}-2 / 7-14$ & $3.27 \mathrm{E}-07$ & $3.25 \mathrm{E}-07$ & n.d. & n.d. & n.d. & n.d. & n.d. & n.d. & n.d. \\
\hline $\mathrm{JN}-2 / 14-41$ & 3.63E-07 & $3.52 \mathrm{E}-07$ & n.d. & n.d. & n.d. & n.d. & n.d. & n.d. & n.d. \\
\hline $\mathrm{JN}-2 / 41-64$ & 4.74E-07 & 4.63E-07 & n.d. & n.d. & n.d. & n.d. & n.d. & n.d. & n.d. \\
\hline JN-2/ 64-86 & $6.14 \mathrm{E}-07$ & $6.06 \mathrm{E}-07$ & n.d. & n.d. & n.d. & n.d. & n.d. & n.d. & n.d. \\
\hline $\mathrm{JN}-2 / 86-100$ & $6.78 \mathrm{E}-07$ & $6.64 \mathrm{E}-07$ & n.d. & n.d. & n.d. & n.d. & n.d. & n.d. & n.d. \\
\hline
\end{tabular}


Table 6d-6a. Magnetic properties of the less than 2-mm size fraction for soil pit JN-3.

\begin{tabular}{|c|c|c|c|c|c|c|c|c|c|}
\hline Sample \# & $\begin{array}{c}\text { MSIf } \\
\left(\mathrm{m}^{3} / \mathbf{k g}\right)\end{array}$ & $\begin{array}{l}\text { MShf } \\
\left(\mathrm{m}^{3} / \mathbf{k g}\right)\end{array}$ & $\begin{array}{c}\text { FDMS } \\
(\%)\end{array}$ & $\begin{array}{l}\text { FDMS } \\
\left(\mathrm{m}^{3} / \mathrm{kg}\right)\end{array}$ & $\begin{array}{c}\text { ARM } \\
\left(\mathrm{Am}^{2} / \mathrm{kg}\right)\end{array}$ & $\begin{array}{c}\text { IRM1.2 } \\
\left(\mathrm{Am}^{2} / \mathrm{kg}\right)\end{array}$ & $\begin{array}{l}\text { IRM }-0.3 \\
\left(\mathrm{Am}^{2} / \mathrm{kg}\right)\end{array}$ & $\begin{array}{c}\text { HIRM } \\
\left(\mathrm{Am}^{2} / \mathbf{k g}\right)\end{array}$ & S \\
\hline $\mathrm{JN}-3 / 0-7$ & $1.95 \mathrm{E}-07$ & $1.89 \mathrm{E}-07$ & n.d. & n.d. & n.d. & n.d. & n.d. & n.d. & n.d. \\
\hline JN-3/ 36-52 & 2.19E-07 & $2.16 \mathrm{E}-07$ & n.d. & n.d. & n.d. & n.d. & n.d. & n.d. & n.d. \\
\hline JN-3/ 52-83 & $1.33 \mathrm{E}-07$ & $1.33 \mathrm{E}-07$ & n.d. & n.d. & n.d. & n.d. & n.d. & n.d. & n.d. \\
\hline
\end{tabular}

Table 6d-6b. Magnetic properties of the less than 63-micron size fraction for soil pit JN-3.

\begin{tabular}{|c|c|c|c|c|c|c|c|c|c|}
\hline Sample \# & $\begin{array}{c}\text { MSIf } \\
\left(\mathrm{m}^{3} / \mathbf{k g}\right)\end{array}$ & $\begin{array}{c}\text { MShf } \\
\left(\mathrm{m}^{3} / \mathrm{kg}\right)\end{array}$ & $\begin{array}{c}\text { FDMS } \\
(\%)\end{array}$ & $\begin{array}{l}\text { FDMS } \\
\left(\mathrm{m}^{3} / \mathrm{kg}\right)\end{array}$ & $\begin{array}{c}\text { ARM } \\
\left(\mathrm{Am}^{2} / \mathrm{kg}\right)\end{array}$ & $\begin{array}{c}\text { IRM1.2 } \\
\left(\mathrm{Am}^{2} / \mathrm{kg}\right)\end{array}$ & $\begin{array}{l}\text { IRM -0.3 } \\
\left(\mathrm{Am}^{2} / \mathrm{kg}\right)\end{array}$ & $\begin{array}{c}\text { HIRM } \\
\left(\mathrm{Am}^{2} / \mathrm{kg}\right)\end{array}$ & $\mathbf{S}$ \\
\hline $\mathrm{JN}-3 / 0-7$ & $3.26 \mathrm{E}-07$ & $3.13 \mathrm{E}-07$ & n.d. & n.d. & n.d. & n.d. & n.d. & n.d. & n.d. \\
\hline $\mathrm{JN}-3 / 36-52$ & 4.97E-07 & $4.84 \mathrm{E}-07$ & n.d. & n.d. & n.d. & n.d. & n.d. & n.d. & n.d. \\
\hline JN-3/ 52-83 & $5.35 \mathrm{E}-07$ & $5.25 \mathrm{E}-07$ & n.d. & n.d. & n.d. & n.d. & n.d. & n.d. & n.d. \\
\hline
\end{tabular}


Table 6e-1. Magnetic properties of the less than 2-mm size fraction for the GP transect.

\begin{tabular}{|c|c|c|c|c|c|c|c|c|c|}
\hline Sample \# & $\begin{array}{c}\text { MSIf } \\
\left(\mathbf{m}^{3} / \mathbf{k g}\right)\end{array}$ & $\begin{array}{c}\text { MShf } \\
\left(\mathbf{m}^{3} / \mathbf{k g}\right)\end{array}$ & $\begin{array}{c}\text { FDMS } \\
(\%)\end{array}$ & $\begin{array}{l}\text { FDMS } \\
\left(\mathrm{m}^{3} / \mathrm{kg}\right)\end{array}$ & $\begin{array}{c}\text { ARM } \\
\left(\mathrm{Am}^{2} / \mathbf{k g}\right)\end{array}$ & $\begin{array}{c}\text { IRM1.2 } \\
\left(\mathrm{Am}^{2} / \mathrm{kg}\right) \\
\end{array}$ & $\begin{array}{l}\text { IRM }-0.3 \\
\left(\mathrm{Am}^{2} / \mathrm{kg}\right) \\
\end{array}$ & $\begin{array}{c}\text { HIRM } \\
\left(\mathrm{Am}^{2} / \mathbf{k g}\right) \\
\end{array}$ & S \\
\hline GP-A/0-10 & $3.59 \mathrm{E}-08$ & $3.88 \mathrm{E}-08$ & -8.32 & $-2.98 \mathrm{E}-09$ & $1.97 \mathrm{E}-05$ & $6.43 \mathrm{E}-04$ & $3.20 \mathrm{E}-04$ & $1.62 \mathrm{E}-04$ & 0.50 \\
\hline GP-A/10-30 & $3.64 \mathrm{E}-08$ & $3.96 \mathrm{E}-08$ & -8.72 & $-3.18 \mathrm{E}-09$ & $1.96 \mathrm{E}-05$ & $6.15 \mathrm{E}-04$ & $3.02 \mathrm{E}-04$ & $1.57 \mathrm{E}-04$ & 0.49 \\
\hline GP-A/30-50 & 3.39E-08 & $3.62 \mathrm{E}-08$ & -6.81 & $-2.31 \mathrm{E}-09$ & $1.86 \mathrm{E}-05$ & $5.91 \mathrm{E}-04$ & $2.67 \mathrm{E}-04$ & $1.62 \mathrm{E}-04$ & 0.45 \\
\hline GP-B/0-10 & $6.07 \mathrm{E}-08$ & $6.20 \mathrm{E}-08$ & -2.10 & $-1.28 \mathrm{E}-09$ & $3.50 \mathrm{E}-05$ & $1.03 \mathrm{E}-03$ & $3.78 \mathrm{E}-04$ & $3.26 \mathrm{E}-04$ & 0.37 \\
\hline GP-B/10-30 & $5.43 \mathrm{E}-08$ & $5.60 \mathrm{E}-08$ & -3.19 & $-1.73 \mathrm{E}-09$ & 2.84E-05 & $9.23 \mathrm{E}-04$ & $3.16 \mathrm{E}-04$ & $3.04 \mathrm{E}-04$ & 0.34 \\
\hline GP-B/30-50 & $3.00 \mathrm{E}-08$ & $3.40 \mathrm{E}-08$ & -13.36 & $-4.01 \mathrm{E}-09$ & $1.65 \mathrm{E}-05$ & $5.25 \mathrm{E}-04$ & $1.76 \mathrm{E}-04$ & $1.75 \mathrm{E}-04$ & 0.33 \\
\hline GP-C/0-10 & $6.22 \mathrm{E}-08$ & $6.79 \mathrm{E}-08$ & -9.30 & $-5.78 \mathrm{E}-09$ & $3.50 \mathrm{E}-05$ & 7.27E-04 & $6.80 \mathrm{E}-04$ & $2.36 \mathrm{E}-05$ & 0.94 \\
\hline GP-C/10-30 & $5.96 \mathrm{E}-08$ & $6.16 \mathrm{E}-08$ & -3.22 & $-1.92 \mathrm{E}-09$ & $3.13 \mathrm{E}-05$ & $6.74 \mathrm{E}-04$ & $5.83 \mathrm{E}-04$ & $4.58 \mathrm{E}-05$ & 0.86 \\
\hline GP-C/30-50 & 4.77E-08 & $5.08 \mathrm{E}-08$ & -6.45 & $-3.08 \mathrm{E}-09$ & $2.44 \mathrm{E}-05$ & $5.54 \mathrm{E}-04$ & $4.78 \mathrm{E}-04$ & $3.79 \mathrm{E}-05$ & 0.86 \\
\hline GP-D/0-10 & $6.52 \mathrm{E}-08$ & $6.71 \mathrm{E}-08$ & -2.84 & $-1.85 \mathrm{E}-09$ & $3.68 \mathrm{E}-05$ & $1.08 \mathrm{E}-03$ & 7.03E-04 & $1.87 \mathrm{E}-04$ & 0.65 \\
\hline GP-D/10-30 & $5.86 \mathrm{E}-08$ & $6.13 \mathrm{E}-08$ & -4.60 & $-2.69 \mathrm{E}-09$ & $2.96 \mathrm{E}-05$ & $9.09 \mathrm{E}-04$ & $5.75 \mathrm{E}-04$ & $1.67 \mathrm{E}-04$ & 0.63 \\
\hline GP-D/30-50 & $5.20 \mathrm{E}-08$ & $5.54 \mathrm{E}-08$ & -6.42 & $-3.34 \mathrm{E}-09$ & $2.60 \mathrm{E}-05$ & $8.30 \mathrm{E}-04$ & $5.08 \mathrm{E}-04$ & $1.61 \mathrm{E}-04$ & 0.61 \\
\hline GP-E/0-10 & $5.80 \mathrm{E}-08$ & $5.92 \mathrm{E}-08$ & -2.01 & $-1.17 \mathrm{E}-09$ & $3.45 \mathrm{E}-05$ & $1.00 \mathrm{E}-03$ & $6.63 \mathrm{E}-04$ & $1.69 \mathrm{E}-04$ & 0.66 \\
\hline GP-E/10-30 & $6.82 \mathrm{E}-08$ & 7.04E-08 & -3.22 & $-2.20 \mathrm{E}-09$ & $3.75 \mathrm{E}-05$ & $1.01 \mathrm{E}-03$ & 7.19E-04 & $1.89 \mathrm{E}-04$ & 0.66 \\
\hline GP-E/30-50 & $5.48 \mathrm{E}-08$ & $5.76 \mathrm{E}-08$ & -5.09 & $-2.79 \mathrm{E}-09$ & $2.51 \mathrm{E}-05$ & $8.90 \mathrm{E}-04$ & $5.63 \mathrm{E}-04$ & $1.63 \mathrm{E}-04$ & 0.63 \\
\hline GP-F/0-10 & $6.88 \mathrm{E}-08$ & 7.11E-08 & -3.34 & $-2.30 \mathrm{E}-09$ & $3.53 \mathrm{E}-05$ & $1.09 \mathrm{E}-03$ & $7.16 \mathrm{E}-04$ & $1.86 \mathrm{E}-04$ & 0.66 \\
\hline GP-F/10-30 & $5.67 \mathrm{E}-08$ & 5.87E-08 & -3.50 & $-1.98 \mathrm{E}-09$ & $2.57 \mathrm{E}-05$ & $8.41 \mathrm{E}-04$ & $5.01 \mathrm{E}-04$ & $1.66 \mathrm{E}-04$ & 0.61 \\
\hline GP-F/30-50 & 4.61E-08 & $4.85 \mathrm{E}-08$ & -5.23 & $-2.41 \mathrm{E}-09$ & $2.30 \mathrm{E}-05$ & 7.43E-04 & $4.75 \mathrm{E}-04$ & $1.34 \mathrm{E}-04$ & 0.64 \\
\hline GP-G/0-10 & 7.38E-08 & 7.53E-08 & -2.01 & $-1.48 \mathrm{E}-09$ & $3.60 \mathrm{E}-05$ & $1.21 \mathrm{E}-03$ & 7.72E-04 & $2.19 \mathrm{E}-04$ & 0.64 \\
\hline GP-G/10-30 & 5.93E-08 & $6.27 \mathrm{E}-08$ & -5.74 & $-3.40 \mathrm{E}-09$ & $3.01 \mathrm{E}-05$ & $1.01 \mathrm{E}-03$ & $6.45 \mathrm{E}-04$ & $1.80 \mathrm{E}-04$ & 0.64 \\
\hline GP-G/30-50 & $5.09 \mathrm{E}-08$ & $5.26 \mathrm{E}-08$ & -3.34 & $-1.70 \mathrm{E}-09$ & $2.34 \mathrm{E}-05$ & $8.25 \mathrm{E}-04$ & $5.01 \mathrm{E}-04$ & $1.62 \mathrm{E}-04$ & 0.61 \\
\hline GP-H/0-10 & 7.24E-08 & 7.40E-08 & -2.22 & $-1.60 \mathrm{E}-09$ & $3.24 \mathrm{E}-05$ & $1.18 \mathrm{E}-03$ & 7.64E-04 & $2.09 \mathrm{E}-04$ & 0.65 \\
\hline GP-H/10-30 & $6.31 \mathrm{E}-08$ & $6.61 \mathrm{E}-08$ & -4.73 & $-2.98 \mathrm{E}-09$ & 2.71E-05 & $1.01 \mathrm{E}-03$ & $6.61 \mathrm{E}-04$ & $1.72 \mathrm{E}-04$ & 0.66 \\
\hline GP-H/30-50 & $4.53 \mathrm{E}-08$ & $4.73 \mathrm{E}-08$ & -4.45 & $-2.01 \mathrm{E}-09$ & 2.13E-05 & 7.67E-04 & $4.68 \mathrm{E}-04$ & $1.50 \mathrm{E}-04$ & 0.61 \\
\hline GP-I/0-10 & 7.62E-08 & 7.69E-08 & -0.94 & $-7.16 \mathrm{E}-10$ & $4.00 \mathrm{E}-05$ & $1.27 \mathrm{E}-03$ & 8.39E-04 & $2.15 \mathrm{E}-04$ & 0.66 \\
\hline GP-I/10-30 & $6.88 \mathrm{E}-08$ & $6.93 \mathrm{E}-08$ & -0.69 & $-4.77 \mathrm{E}-10$ & $9.40 \mathrm{E}-05$ & $1.15 \mathrm{E}-03$ & 7.64E-04 & $1.95 \mathrm{E}-04$ & 0.66 \\
\hline GP-I/30-50 & $6.24 \mathrm{E}-08$ & $6.23 \mathrm{E}-08$ & 0.13 & 7.92E-11 & 2.92E-05 & $9.82 \mathrm{E}-04$ & $6.51 \mathrm{E}-04$ & $1.65 \mathrm{E}-04$ & 0.66 \\
\hline GP-J/0-10 & $6.86 \mathrm{E}-08$ & $6.87 \mathrm{E}-08$ & -0.21 & $-1.42 \mathrm{E}-10$ & $3.48 \mathrm{E}-05$ & $1.05 \mathrm{E}-03$ & $7.18 \mathrm{E}-04$ & $1.68 \mathrm{E}-04$ & 0.68 \\
\hline GP-J/10-30 & $6.99 \mathrm{E}-08$ & 7.02E-08 & -0.37 & $-2.61 \mathrm{E}-10$ & 3.39E-05 & $1.12 \mathrm{E}-03$ & $7.21 \mathrm{E}-04$ & $1.98 \mathrm{E}-04$ & 0.65 \\
\hline GP-J/30-50 & 7.23E-08 & $7.28 \mathrm{E}-08$ & -0.70 & $-5.09 \mathrm{E}-10$ & $3.16 \mathrm{E}-05$ & $1.12 \mathrm{E}-03$ & 7.33E-04 & 1.92E-04 & 0.66 \\
\hline GP-K/0-10 & $1.13 \mathrm{E}-07$ & $1.13 \mathrm{E}-07$ & -0.54 & $-6.05 \mathrm{E}-10$ & 5.54E-05 & $1.73 \mathrm{E}-03$ & $1.23 \mathrm{E}-03$ & $2.51 \mathrm{E}-04$ & 0.71 \\
\hline GP-K/10-30 & $9.79 \mathrm{E}-08$ & $9.79 \mathrm{E}-08$ & 0.06 & $5.87 \mathrm{E}-11$ & 4.15E-05 & $1.40 \mathrm{E}-03$ & $1.05 \mathrm{E}-03$ & $1.78 \mathrm{E}-04$ & 0.75 \\
\hline GP-K/30-50 & $9.48 \mathrm{E}-08$ & $9.51 \mathrm{E}-08$ & -0.27 & $-2.57 \mathrm{E}-10$ & $4.27 \mathrm{E}-05$ & $1.44 \mathrm{E}-03$ & $9.96 \mathrm{E}-04$ & $2.24 \mathrm{E}-04$ & 0.69 \\
\hline
\end{tabular}


Table 6f-1a. Magnetic properties of the less than 2-mm size fraction for the 00U-41 transect.

\begin{tabular}{|c|c|c|c|c|c|c|c|c|c|}
\hline Sample \# & $\begin{array}{c}\text { MSIf } \\
\left(\mathrm{m}^{3} / \mathrm{kg}\right)\end{array}$ & $\begin{array}{c}\text { MShf } \\
\left(\mathbf{m}^{3} / \mathrm{kg}\right)\end{array}$ & $\begin{array}{c}\text { FDMS } \\
(\%)\end{array}$ & $\begin{array}{l}\text { FDMS } \\
\left(\mathrm{m}^{3} / \mathrm{kg}\right)\end{array}$ & $\begin{array}{c}\text { ARM } \\
\left(\mathrm{Am}^{2} / \mathbf{k g}\right)\end{array}$ & $\begin{array}{c}\text { IRM1.2 } \\
\left(\mathrm{Am}^{2} / \mathrm{kg}\right)\end{array}$ & $\begin{array}{l}\text { IRM -0.3 } \\
\left(\mathrm{Am}^{2} / \mathbf{k g}\right) \\
\end{array}$ & $\begin{array}{c}\text { HIRM } \\
\left(\mathrm{Am}^{2} / \mathrm{kg}\right)\end{array}$ & $\mathbf{S}$ \\
\hline 00U-41A/0-10 & $2.89 \mathrm{E}-08$ & $3.14 \mathrm{E}-08$ & -8.51 & $-2.46 \mathrm{E}-09$ & $1.06 \mathrm{E}-05$ & $9.21 \mathrm{E}-04$ & $1.30 \mathrm{E}-04$ & $3.95 \mathrm{E}-04$ & 0.14 \\
\hline $00 \mathrm{U}-41 \mathrm{~A} / 10-25$ & $2.82 \mathrm{E}-08$ & $2.60 \mathrm{E}-08$ & 7.72 & 2.18E-09 & $1.04 \mathrm{E}-05$ & $9.70 \mathrm{E}-04$ & $7.18 \mathrm{E}-05$ & 4.49E-04 & 0.07 \\
\hline 00U-41B/0-10 & $2.87 \mathrm{E}-08$ & $3.12 \mathrm{E}-08$ & -8.39 & $-2.41 \mathrm{E}-09$ & $1.16 \mathrm{E}-05$ & 8.33E-04 & $1.52 \mathrm{E}-04$ & $3.40 \mathrm{E}-04$ & 0.18 \\
\hline $00 \mathrm{U}-41 \mathrm{~B} / 10-25$ & $4.83 \mathrm{E}-08$ & 4.91E-08 & -1.68 & $-8.11 \mathrm{E}-10$ & $1.76 \mathrm{E}-05$ & $1.30 \mathrm{E}-03$ & $1.56 \mathrm{E}-04$ & $5.71 \mathrm{E}-04$ & 0.12 \\
\hline 00U-41B/25-45 & 5.61E-08 & $5.66 \mathrm{E}-08$ & -0.85 & $-4.75 \mathrm{E}-10$ & $1.99 \mathrm{E}-05$ & $1.64 \mathrm{E}-03$ & $6.63 \mathrm{E}-05$ & $7.88 \mathrm{E}-04$ & 0.04 \\
\hline 00U-41C/0-10 & 4.66E-08 & 4.72E-08 & -1.33 & $-6.18 \mathrm{E}-10$ & $2.15 \mathrm{E}-05$ & $1.17 \mathrm{E}-03$ & $2.32 \mathrm{E}-04$ & 4.67E-04 & 0.20 \\
\hline 00U-41C/10-20 & 4.87E-08 & 4.89E-08 & -0.43 & $-2.10 \mathrm{E}-10$ & $2.00 \mathrm{E}-05$ & $1.27 \mathrm{E}-03$ & $2.05 \mathrm{E}-04$ & $5.35 \mathrm{E}-04$ & 0.16 \\
\hline $00 \mathrm{U}-41 \mathrm{C} / 20-30$ & 4.43E-08 & $4.56 \mathrm{E}-08$ & -2.92 & $-1.29 \mathrm{E}-09$ & $1.66 \mathrm{E}-05$ & $1.27 \mathrm{E}-03$ & 1.77E-04 & $5.48 \mathrm{E}-04$ & 0.14 \\
\hline 00U-41D/0-10 & $6.27 \mathrm{E}-08$ & $6.27 \mathrm{E}-08$ & -0.07 & $-4.11 \mathrm{E}-11$ & 2.72E-05 & $1.35 \mathrm{E}-03$ & 4.45E-04 & 4.55E-04 & 0.33 \\
\hline 00U-41D/10-30 & 7.79E-08 & $7.82 \mathrm{E}-08$ & -0.40 & $-3.14 \mathrm{E}-10$ & $3.36 \mathrm{E}-05$ & $1.83 \mathrm{E}-03$ & $5.92 \mathrm{E}-04$ & $6.21 \mathrm{E}-04$ & 0.32 \\
\hline 00U-41D/30-50 & 8.04E-08 & $7.90 \mathrm{E}-08$ & 1.77 & $1.42 \mathrm{E}-09$ & $3.34 \mathrm{E}-05$ & $1.84 \mathrm{E}-03$ & $5.50 \mathrm{E}-04$ & $6.44 \mathrm{E}-04$ & 0.30 \\
\hline 00U-41E/0-10 & 8.59E-08 & 8.32E-08 & 3.18 & 2.73E-09 & $3.61 \mathrm{E}-05$ & $1.93 \mathrm{E}-03$ & $6.71 \mathrm{E}-04$ & $6.27 \mathrm{E}-04$ & 0.35 \\
\hline 00U-41E/10-30 & $7.78 \mathrm{E}-08$ & 7.69E-08 & 1.25 & $9.75 \mathrm{E}-10$ & $3.28 \mathrm{E}-05$ & $1.80 \mathrm{E}-03$ & $5.46 \mathrm{E}-04$ & $6.27 \mathrm{E}-04$ & 0.30 \\
\hline 00U-41E/30-50 & $8.81 \mathrm{E}-08$ & $8.79 \mathrm{E}-08$ & 0.24 & $2.16 \mathrm{E}-10$ & $3.58 \mathrm{E}-05$ & $1.94 \mathrm{E}-03$ & $5.82 \mathrm{E}-04$ & $6.77 \mathrm{E}-04$ & 0.30 \\
\hline 00U-41F/0-10 & 7.67E-08 & $7.75 \mathrm{E}-08$ & -0.97 & $-7.46 \mathrm{E}-10$ & $3.83 \mathrm{E}-05$ & $1.68 \mathrm{E}-03$ & $5.82 \mathrm{E}-04$ & $5.51 \mathrm{E}-04$ & 0.35 \\
\hline 00U-41F/10-30 & 8.39E-08 & $8.16 \mathrm{E}-08$ & 2.76 & 2.31E-09 & $3.54 \mathrm{E}-05$ & $1.78 \mathrm{E}-03$ & $5.82 \mathrm{E}-04$ & $5.98 \mathrm{E}-04$ & 0.33 \\
\hline $00 \mathrm{U}-41 \mathrm{~F} / 30-50$ & $8.66 \mathrm{E}-08$ & $8.35 \mathrm{E}-08$ & 3.66 & 3.17E-09 & $3.47 \mathrm{E}-05$ & $1.87 \mathrm{E}-03$ & $5.63 \mathrm{E}-04$ & $6.52 \mathrm{E}-04$ & 0.30 \\
\hline 00U-41G/0-10 & $6.02 \mathrm{E}-08$ & $5.74 \mathrm{E}-08$ & 4.68 & 2.82E-09 & $2.94 \mathrm{E}-05$ & $1.36 \mathrm{E}-03$ & 4.37E-04 & 4.64E-04 & 0.32 \\
\hline 00U-41G/10-30 & $8.50 \mathrm{E}-08$ & $8.27 \mathrm{E}-08$ & 2.64 & $2.24 \mathrm{E}-09$ & $3.81 \mathrm{E}-05$ & $1.83 \mathrm{E}-03$ & $5.76 \mathrm{E}-04$ & $6.25 \mathrm{E}-04$ & 0.32 \\
\hline $00 \mathrm{U}-41 \mathrm{G} / 30-50$ & 8.57E-08 & $8.51 \mathrm{E}-08$ & 0.73 & $6.26 \mathrm{E}-10$ & $3.66 \mathrm{E}-05$ & $1.85 \mathrm{E}-03$ & $5.64 \mathrm{E}-04$ & $6.44 \mathrm{E}-04$ & 0.30 \\
\hline 00U-41H1/0-10 & $9.29 \mathrm{E}-08$ & $9.05 \mathrm{E}-08$ & 2.58 & 2.40E-09 & 4.39E-05 & $1.87 \mathrm{E}-03$ & $7.22 \mathrm{E}-04$ & $5.76 \mathrm{E}-04$ & 0.39 \\
\hline 00U-41H1/10-30 & $9.79 \mathrm{E}-08$ & $9.62 \mathrm{E}-08$ & 1.73 & $1.69 \mathrm{E}-09$ & 4.43E-05 & $2.07 \mathrm{E}-03$ & $7.28 \mathrm{E}-04$ & $6.72 \mathrm{E}-04$ & 0.35 \\
\hline 00U-41H1/30-50 & $9.03 \mathrm{E}-08$ & $8.75 \mathrm{E}-08$ & 3.11 & $2.81 \mathrm{E}-09$ & $3.89 \mathrm{E}-05$ & $1.91 \mathrm{E}-03$ & $6.24 \mathrm{E}-04$ & $6.44 \mathrm{E}-04$ & 0.33 \\
\hline 00U-41H2/0-10 & $9.23 \mathrm{E}-08$ & $9.65 \mathrm{E}-08$ & -4.50 & $-4.15 \mathrm{E}-09$ & $4.30 \mathrm{E}-05$ & $2.00 \mathrm{E}-03$ & $7.55 \mathrm{E}-04$ & $6.22 \mathrm{E}-04$ & 0.38 \\
\hline 00U-41H2/10-30 & $1.00 \mathrm{E}-07$ & $9.67 \mathrm{E}-08$ & 3.57 & $3.58 \mathrm{E}-09$ & $4.86 \mathrm{E}-05$ & $2.11 \mathrm{E}-03$ & $8.15 \mathrm{E}-04$ & $6.49 \mathrm{E}-04$ & 0.39 \\
\hline 00U-41H2/30-50 & 9.37E-08 & 9.31E-08 & 0.68 & $6.32 \mathrm{E}-10$ & $3.96 \mathrm{E}-05$ & $2.00 \mathrm{E}-03$ & $6.54 \mathrm{E}-04$ & $6.72 \mathrm{E}-04$ & 0.33 \\
\hline
\end{tabular}


Table 6f-1b. Magnetic properties of the less than 63-micron size fraction for the 00U-41 transect.

\begin{tabular}{|c|c|c|c|c|c|c|c|c|c|}
\hline Sample \# & $\begin{array}{c}\text { MSIf } \\
\left(\mathrm{m}^{3} / \mathrm{kg}\right)\end{array}$ & $\begin{array}{c}\text { MShf } \\
\left(\mathrm{m}^{3} / \mathrm{kg}\right)\end{array}$ & $\begin{array}{c}\text { FDMS } \\
(\%)\end{array}$ & $\begin{array}{l}\text { FDMS } \\
\left(\mathrm{m}^{3} / \mathrm{kg}\right)\end{array}$ & $\begin{array}{c}\text { ARM } \\
\left(\mathrm{Am}^{2} / \mathbf{k g}\right)\end{array}$ & $\begin{array}{c}\text { IRM1.2 } \\
\left(\mathrm{Am}^{2} / \mathrm{kg}\right)\end{array}$ & $\begin{array}{l}\text { IRM -0.3 } \\
\left(\mathrm{Am}^{2} / \mathrm{kg}\right)\end{array}$ & $\begin{array}{c}\text { HIRM } \\
\left(\mathrm{Am}^{2} / \mathbf{k g}\right)\end{array}$ & $\mathbf{S}$ \\
\hline 00U-41A/0-10 & $1.52 \mathrm{E}-07$ & $1.52 \mathrm{E}-07$ & n.d. & n.d. & n.d. & n.d. & n.d. & n.d. & n.d. \\
\hline 00U-41A/10-25 & n.d. & n.d. & n.d. & n.d. & n.d. & n.d. & n.d. & n.d. & n.d. \\
\hline 00U-41B/0-10 & $1.72 \mathrm{E}-07$ & $1.72 \mathrm{E}-07$ & n.d. & n.d. & n.d. & n.d. & n.d. & n.d. & n.d. \\
\hline 00U-41B/10-25 & n.d. & n.d. & n.d. & n.d. & n.d. & n.d. & n.d. & n.d. & n.d. \\
\hline 00U-41B/25-45 & n.d. & n.d. & n.d. & n.d. & n.d. & n.d. & n.d. & n.d. & n.d. \\
\hline 00U-41C/0-10 & $1.90 \mathrm{E}-07$ & $1.91 \mathrm{E}-07$ & n.d. & n.d. & n.d. & n.d. & n.d. & n.d. & n.d. \\
\hline 00U-41C/10-20 & n.d. & n.d. & n.d. & n.d. & n.d. & n.d. & n.d. & n.d. & n.d. \\
\hline 00U-41C/20-30 & n.d. & n.d. & n.d. & n.d. & n.d. & n.d. & n.d. & n.d. & n.d. \\
\hline 00U-41D/0-10 & $1.81 \mathrm{E}-07$ & $1.80 \mathrm{E}-07$ & n.d. & n.d. & n.d. & n.d. & n.d. & n.d. & n.d. \\
\hline 00U-41D/10-30 & n.d. & n.d. & n.d. & n.d. & n.d. & n.d. & n.d. & n.d. & n.d. \\
\hline 00U-41D/30-50 & n.d. & n.d. & n.d. & n.d. & n.d. & n.d. & n.d. & n.d. & n.d. \\
\hline 00U-41E/0-10 & $1.91 \mathrm{E}-07$ & $1.90 \mathrm{E}-07$ & n.d. & n.d. & n.d. & n.d. & n.d. & n.d. & n.d. \\
\hline 00U-41E/10-30 & n.d. & n.d. & n.d. & n.d. & n.d. & n.d. & n.d. & n.d. & n.d. \\
\hline 00U-41E/30-50 & n.d. & n.d. & n.d. & n.d. & n.d. & n.d. & n.d. & n.d. & n.d. \\
\hline 00U-41F/0-10 & $1.80 \mathrm{E}-07$ & $1.78 \mathrm{E}-07$ & n.d. & n.d. & n.d. & n.d. & n.d. & n.d. & n.d. \\
\hline 00U-41F/10-30 & n.d. & n.d. & n.d. & n.d. & n.d. & n.d. & n.d. & n.d. & n.d. \\
\hline 00U-41F/30-50 & n.d. & n.d. & n.d. & n.d. & n.d. & n.d. & n.d. & n.d. & n.d. \\
\hline 00U-41G/0-10 & $1.80 \mathrm{E}-07$ & $1.80 \mathrm{E}-07$ & n.d. & n.d. & n.d. & n.d. & n.d. & n.d. & n.d. \\
\hline 00U-41G/10-30 & n.d. & n.d. & n.d. & n.d. & n.d. & n.d. & n.d. & n.d. & n.d. \\
\hline 00U-41G/30-50 & n.d. & n.d. & n.d. & n.d. & n.d. & n.d. & n.d. & n.d. & n.d. \\
\hline 00U-41H1/0-10 & $1.90 \mathrm{E}-07$ & $1.88 \mathrm{E}-07$ & n.d. & n.d. & n.d. & n.d. & n.d. & n.d. & n.d. \\
\hline 00U-41H1/10-30 & n.d. & n.d. & n.d. & n.d. & n.d. & n.d. & n.d. & n.d. & n.d. \\
\hline 00U-41H1/30-50 & n.d. & n.d. & n.d. & n.d. & n.d. & n.d. & n.d. & n.d. & n.d. \\
\hline 00U-41H2/0-10 & $1.87 \mathrm{E}-07$ & $1.85 \mathrm{E}-07$ & n.d. & n.d. & n.d. & n.d. & n.d. & n.d. & n.d. \\
\hline 00U-41H2/10-30 & n.d. & n.d. & n.d. & n.d. & n.d. & n.d. & n.d. & n.d. & n.d. \\
\hline 00U-41H2/30-50 & n.d. & n.d. & n.d. & n.d. & n.d. & n.d. & n.d. & n.d. & n.d. \\
\hline
\end{tabular}


Table 6f-2a. Magnetic properties of the less than 2-mm size fraction for auger hole 00U-42.

\begin{tabular}{|c|c|c|c|c|c|c|c|c|c|}
\hline Sample \# & $\begin{array}{c}\text { MSIf } \\
\left(\mathbf{m}^{3} / \mathbf{k g}\right)\end{array}$ & $\begin{array}{c}\text { MShf } \\
\left(\mathrm{m}^{3} / \mathrm{kg}\right)\end{array}$ & $\begin{array}{l}\text { FDMS } \\
(\%)\end{array}$ & $\begin{array}{l}\text { FDMS } \\
\left(\mathbf{m}^{3} / \mathrm{kg}\right)\end{array}$ & $\begin{array}{c}\text { ARM } \\
\left(\mathrm{Am}^{2} / \mathrm{kg}\right)\end{array}$ & $\begin{array}{c}\text { IRM1.2 } \\
\left(\mathrm{Am}^{2} / \mathrm{kg}\right)\end{array}$ & $\begin{array}{l}\text { IRM }-0.3 \\
\left(\mathrm{Am}^{2} / \mathrm{kg}\right)\end{array}$ & $\begin{array}{c}\text { HIRM } \\
\left(\mathrm{Am}^{2} / \mathbf{k g}\right)\end{array}$ & $\mathbf{S}$ \\
\hline 00U-42/0-10 & 4.92E-08 & 4.83E-08 & 1.90 & $9.36 \mathrm{E}-10$ & 2.72E-04 & $1.15 \mathrm{E}-03$ & $3.50 \mathrm{E}-04$ & 4.00E-04 & 0.30 \\
\hline 00U-42/10-30 & $5.66 \mathrm{E}-08$ & $5.84 \mathrm{E}-08$ & -3.13 & $-1.77 \mathrm{E}-09$ & 2.73E-04 & $1.30 \mathrm{E}-03$ & $3.94 \mathrm{E}-04$ & $4.55 \mathrm{E}-04$ & 0.30 \\
\hline 00U-42/30-50 & $6.98 \mathrm{E}-08$ & $6.87 \mathrm{E}-08$ & 1.56 & 1.09E-09 & $2.72 \mathrm{E}-04$ & $1.42 \mathrm{E}-03$ & $4.56 \mathrm{E}-04$ & 4.80E-04 & 0.32 \\
\hline 00U-42/50-63 & $8.54 \mathrm{E}-08$ & $8.29 \mathrm{E}-08$ & 2.90 & 2.47E-09 & $3.20 \mathrm{E}-04$ & $1.70 \mathrm{E}-03$ & $6.42 \mathrm{E}-04$ & 5.31E-04 & 0.38 \\
\hline 00U-42/63-74 & $9.12 \mathrm{E}-08$ & $8.90 \mathrm{E}-08$ & 2.43 & 2.22E-09 & $3.21 \mathrm{E}-04$ & $1.83 \mathrm{E}-03$ & $1.79 \mathrm{E}-03$ & $1.97 \mathrm{E}-05$ & 0.98 \\
\hline 00U-42/74-87 & $1.03 \mathrm{E}-07$ & $9.95 \mathrm{E}-08$ & 3.09 & 3.17E-09 & 3.33E-04 & $1.97 \mathrm{E}-03$ & $8.55 \mathrm{E}-04$ & $5.59 \mathrm{E}-04$ & 0.43 \\
\hline 00U-42/87-95 & $8.90 \mathrm{E}-08$ & $8.98 \mathrm{E}-08$ & -0.91 & $-8.06 \mathrm{E}-10$ & $3.34 \mathrm{E}-04$ & $1.80 \mathrm{E}-03$ & 7.04E-04 & $5.50 \mathrm{E}-04$ & 0.39 \\
\hline 00U-42/95-106 & 7.89E-08 & $7.86 \mathrm{E}-08$ & 0.43 & $3.41 \mathrm{E}-10$ & 3.79E-04 & $1.81 \mathrm{E}-03$ & $5.44 \mathrm{E}-04$ & $6.31 \mathrm{E}-04$ & 0.30 \\
\hline 00U-42/106-119 & $9.01 \mathrm{E}-08$ & $9.17 \mathrm{E}-08$ & -1.84 & $-1.66 \mathrm{E}-09$ & 4.17E-04 & $2.00 \mathrm{E}-03$ & $6.88 \mathrm{E}-04$ & $6.57 \mathrm{E}-04$ & 0.34 \\
\hline 00U-42/119-125 & $1.04 \mathrm{E}-07$ & $1.05 \mathrm{E}-07$ & -1.14 & $-1.18 \mathrm{E}-09$ & $3.26 \mathrm{E}-04$ & $2.11 \mathrm{E}-03$ & 8.83E-04 & $6.13 \mathrm{E}-04$ & 0.42 \\
\hline 00U-42/125-133 & $9.54 \mathrm{E}-08$ & $9.31 \mathrm{E}-08$ & 2.39 & 2.27E-09 & 3.67E-04 & $1.91 \mathrm{E}-03$ & 7.47E-04 & $5.81 \mathrm{E}-04$ & 0.39 \\
\hline
\end{tabular}

Table 6f-2b. Magnetic properties of the less than 63-micron size fraction for auger hole 00U-42.

\begin{tabular}{|c|c|c|c|c|c|c|c|c|c|}
\hline Sample \# & $\begin{array}{c}\text { MSIf } \\
\left(\mathbf{m}^{3} / \mathbf{k g}\right)\end{array}$ & $\begin{array}{c}\text { MShf } \\
\left(\mathrm{m}^{3} / \mathrm{kg}\right)\end{array}$ & $\begin{array}{c}\text { FDMS } \\
(\%)\end{array}$ & $\begin{array}{l}\text { FDMS } \\
\left(\mathrm{m}^{3} / \mathbf{k g}\right)\end{array}$ & $\begin{array}{c}\text { ARM } \\
\left(\mathrm{Am}^{2} / \mathbf{k g}\right)\end{array}$ & $\begin{array}{l}\text { IRM1.2 } \\
\left(\mathrm{Am}^{2} / \mathrm{kg}\right)\end{array}$ & $\begin{array}{l}\text { IRM -0.3 } \\
\left(\mathrm{Am}^{2} / \mathrm{kg}\right)\end{array}$ & $\begin{array}{c}\text { HIRM } \\
\left(\mathrm{Am}^{2} / \mathbf{k g}\right)\end{array}$ & $\mathbf{S}$ \\
\hline 00U-42/0-10 & $1.70 \mathrm{E}-07$ & $1.70 \mathrm{E}-07$ & n.d. & n.d. & n.d. & n.d. & n.d. & n.d. & n.d. \\
\hline 00U-42/10-30 & n.d. & n.d. & n.d. & n.d. & n.d. & n.d. & n.d. & n.d. & n.d. \\
\hline 00U-42/30-50 & n.d. & n.d. & n.d. & n.d. & n.d. & n.d. & n.d. & n.d. & n.d. \\
\hline 00U-42/50-63 & n.d. & n.d. & n.d. & n.d. & n.d. & n.d. & n.d. & n.d. & n.d. \\
\hline 00U-42/63-74 & n.d. & n.d. & n.d. & n.d. & n.d. & n.d. & n.d. & n.d. & n.d. \\
\hline 00U-42/74-87 & n.d. & n.d. & n.d. & n.d. & n.d. & n.d. & n.d. & n.d. & n.d. \\
\hline 00U-42/87-95 & n.d. & n.d. & n.d. & n.d. & n.d. & n.d. & n.d. & n.d. & n.d. \\
\hline 00U-42/95-106 & n.d. & n.d. & n.d. & n.d. & n.d. & n.d. & n.d. & n.d. & n.d. \\
\hline 00U-42/106-119 & n.d. & n.d. & n.d. & n.d. & n.d. & n.d. & n.d. & n.d. & n.d. \\
\hline 00U-42/119-125 & n.d. & n.d. & n.d. & n.d. & n.d. & n.d. & n.d. & n.d. & n.d. \\
\hline 00U-42/125-133 & n.d. & n.d. & n.d. & n.d. & n.d. & n.d. & n.d. & n.d. & n.d. \\
\hline
\end{tabular}


Table 6f-3a. Magnetic properties of the less than 2-mm size fraction for auger hole 00U-43.

\begin{tabular}{|c|c|c|c|c|c|c|c|c|c|}
\hline Sample \# & $\begin{array}{c}\text { MSIf } \\
\left(\mathrm{m}^{3} / \mathrm{kg}\right)\end{array}$ & $\begin{array}{c}\text { MShf } \\
\left(\mathrm{m}^{3} / \mathrm{kg}\right)\end{array}$ & $\begin{array}{l}\text { FDMS } \\
(\%)\end{array}$ & $\begin{array}{l}\text { FDMS } \\
\left(\mathrm{m}^{3} / \mathrm{kg}\right)\end{array}$ & $\begin{array}{c}\text { ARM } \\
\left(\mathrm{Am}^{2} / \mathrm{kg}\right)\end{array}$ & $\begin{array}{c}\text { IRM1.2 } \\
\left(\mathrm{Am}^{2} / \mathrm{kg}\right)\end{array}$ & $\begin{array}{l}\text { IRM }-0.3 \\
\left(\mathrm{Am}^{2} / \mathrm{kg}\right)\end{array}$ & $\begin{array}{c}\text { HIRM } \\
\left(\mathrm{Am}^{2} / \mathrm{kg}\right)\end{array}$ & $\mathbf{S}$ \\
\hline 00U-43/0-10 & 7.53E-08 & 7.57E-08 & -0.56 & $-4.22 \mathrm{E}-10$ & $6.34 \mathrm{E}-04$ & $1.53 \mathrm{E}-03$ & $6.09 \mathrm{E}-04$ & 4.59E-04 & 0.40 \\
\hline 00U-43/10-25 & $8.80 \mathrm{E}-08$ & 8.52E-08 & 3.22 & 2.84E-09 & $2.71 \mathrm{E}-04$ & $1.79 \mathrm{E}-03$ & 7.39E-04 & $5.27 \mathrm{E}-04$ & 0.41 \\
\hline 00U-43/25-34 & 8.63E-08 & 8.62E-08 & 0.04 & $3.21 \mathrm{E}-11$ & 3.03E-04 & $1.81 \mathrm{E}-03$ & $6.98 \mathrm{E}-04$ & $5.57 \mathrm{E}-04$ & 0.39 \\
\hline 00U-43/34-48 & 8.97E-08 & $8.80 \mathrm{E}-08$ & 1.83 & $1.64 \mathrm{E}-09$ & $2.68 \mathrm{E}-04$ & $1.88 \mathrm{E}-03$ & $7.15 \mathrm{E}-04$ & $5.84 \mathrm{E}-04$ & 0.38 \\
\hline 00U-43/48-55 & $8.22 \mathrm{E}-08$ & $8.15 \mathrm{E}-08$ & 0.88 & $7.25 \mathrm{E}-10$ & $3.43 \mathrm{E}-04$ & $1.80 \mathrm{E}-03$ & $6.55 \mathrm{E}-04$ & 5.73E-04 & 0.36 \\
\hline 00U-43/55-64 & $5.78 \mathrm{E}-08$ & $5.44 \mathrm{E}-08$ & 5.91 & $3.42 \mathrm{E}-09$ & $3.52 \mathrm{E}-04$ & $1.56 \mathrm{E}-03$ & $3.23 \mathrm{E}-04$ & $6.20 \mathrm{E}-04$ & 0.21 \\
\hline 00U-43/64-76 & $5.75 \mathrm{E}-08$ & $5.65 \mathrm{E}-08$ & 1.82 & $1.04 \mathrm{E}-09$ & $3.95 \mathrm{E}-04$ & $1.68 \mathrm{E}-03$ & $2.56 \mathrm{E}-04$ & 7.12E-04 & 0.15 \\
\hline 00U-43/76-86 & $5.31 \mathrm{E}-08$ & $5.37 \mathrm{E}-08$ & -1.10 & $-5.87 \mathrm{E}-10$ & $3.61 \mathrm{E}-04$ & $1.51 \mathrm{E}-03$ & $2.10 \mathrm{E}-04$ & $6.51 \mathrm{E}-04$ & 0.14 \\
\hline 00U-43/86-96 & $5.02 \mathrm{E}-08$ & $5.12 \mathrm{E}-08$ & -2.06 & $-1.03 \mathrm{E}-09$ & $3.25 \mathrm{E}-04$ & $1.37 \mathrm{E}-03$ & $1.95 \mathrm{E}-04$ & $5.89 \mathrm{E}-04$ & 0.14 \\
\hline 00U-43/96-108 & 4.92E-08 & $5.08 \mathrm{E}-08$ & -3.14 & $-1.54 \mathrm{E}-09$ & $3.38 \mathrm{E}-04$ & $1.32 \mathrm{E}-03$ & $1.66 \mathrm{E}-04$ & $5.78 \mathrm{E}-04$ & 0.13 \\
\hline 00U-43/108-114 & $5.17 \mathrm{E}-08$ & $5.23 \mathrm{E}-08$ & -1.20 & $-6.22 \mathrm{E}-10$ & $3.46 \mathrm{E}-04$ & $1.40 \mathrm{E}-03$ & $2.16 \mathrm{E}-04$ & $5.94 \mathrm{E}-04$ & 0.15 \\
\hline
\end{tabular}

Table 6f-3b. Magnetic properties of the less than 63-micron size fraction for auger hole 00U-43.

\begin{tabular}{|c|c|c|c|c|c|c|c|c|c|}
\hline Sample \# & $\begin{array}{c}\text { MSIf } \\
\left(\mathbf{m}^{3} / \mathbf{k g}\right)\end{array}$ & $\begin{array}{c}\text { MShf } \\
\left(\mathrm{m}^{3} / \mathrm{kg}\right)\end{array}$ & $\begin{array}{c}\text { FDMS } \\
(\%)\end{array}$ & $\begin{array}{c}\text { FDMS } \\
\left(\mathrm{m}^{3} / \mathrm{kg}\right)\end{array}$ & $\begin{array}{c}\text { ARM } \\
\left(\mathrm{Am}^{2} / \mathrm{kg}\right)\end{array}$ & $\begin{array}{c}\text { IRM1.2 } \\
\left(\mathrm{Am}^{2} / \mathrm{kg}\right)\end{array}$ & $\begin{array}{l}\text { IRM -0.3 } \\
\left(\mathrm{Am}^{2} / \mathrm{kg}\right)\end{array}$ & $\begin{array}{c}\text { HIRM } \\
\left(\mathbf{A m}^{2} / \mathbf{k g}\right)\end{array}$ & $\mathbf{S}$ \\
\hline 00U-43/0-10 & $1.92 \mathrm{E}-07$ & $1.90 \mathrm{E}-07$ & n.d. & n.d. & n.d. & n.d. & n.d. & n.d. & n.d. \\
\hline 00U-43/10-25 & n.d. & n.d. & n.d. & n.d. & n.d. & n.d. & n.d. & n.d. & n.d. \\
\hline 00U-43/25-34 & n.d. & n.d. & n.d. & n.d. & n.d. & n.d. & n.d. & n.d. & n.d. \\
\hline 00U-43/34-48 & n.d. & n.d. & n.d. & n.d. & n.d. & n.d. & n.d. & n.d. & n.d. \\
\hline 00U-43/48-55 & n.d. & n.d. & n.d. & n.d. & n.d. & n.d. & n.d. & n.d. & n.d. \\
\hline 00U-43/55-64 & n.d. & n.d. & n.d. & n.d. & n.d. & n.d. & n.d. & n.d. & n.d. \\
\hline 00U-43/64-76 & n.d. & n.d. & n.d. & n.d. & n.d. & n.d. & n.d. & n.d. & n.d. \\
\hline 00U-43/76-86 & n.d. & n.d. & n.d. & n.d. & n.d. & n.d. & n.d. & n.d. & n.d. \\
\hline 00U-43/86-96 & n.d. & n.d. & n.d. & n.d. & n.d. & n.d. & n.d. & n.d. & n.d. \\
\hline 00U-43/96-108 & n.d. & n.d. & n.d. & n.d. & n.d. & n.d. & n.d. & n.d. & n.d. \\
\hline 00U-43/108-114 & n.d. & n.d. & n.d. & n.d. & n.d. & n.d. & n.d. & n.d. & n.d. \\
\hline
\end{tabular}


Table 6g-1. Magnetic properties of the less than 2-mm size fraction for auger hole 8U-18.

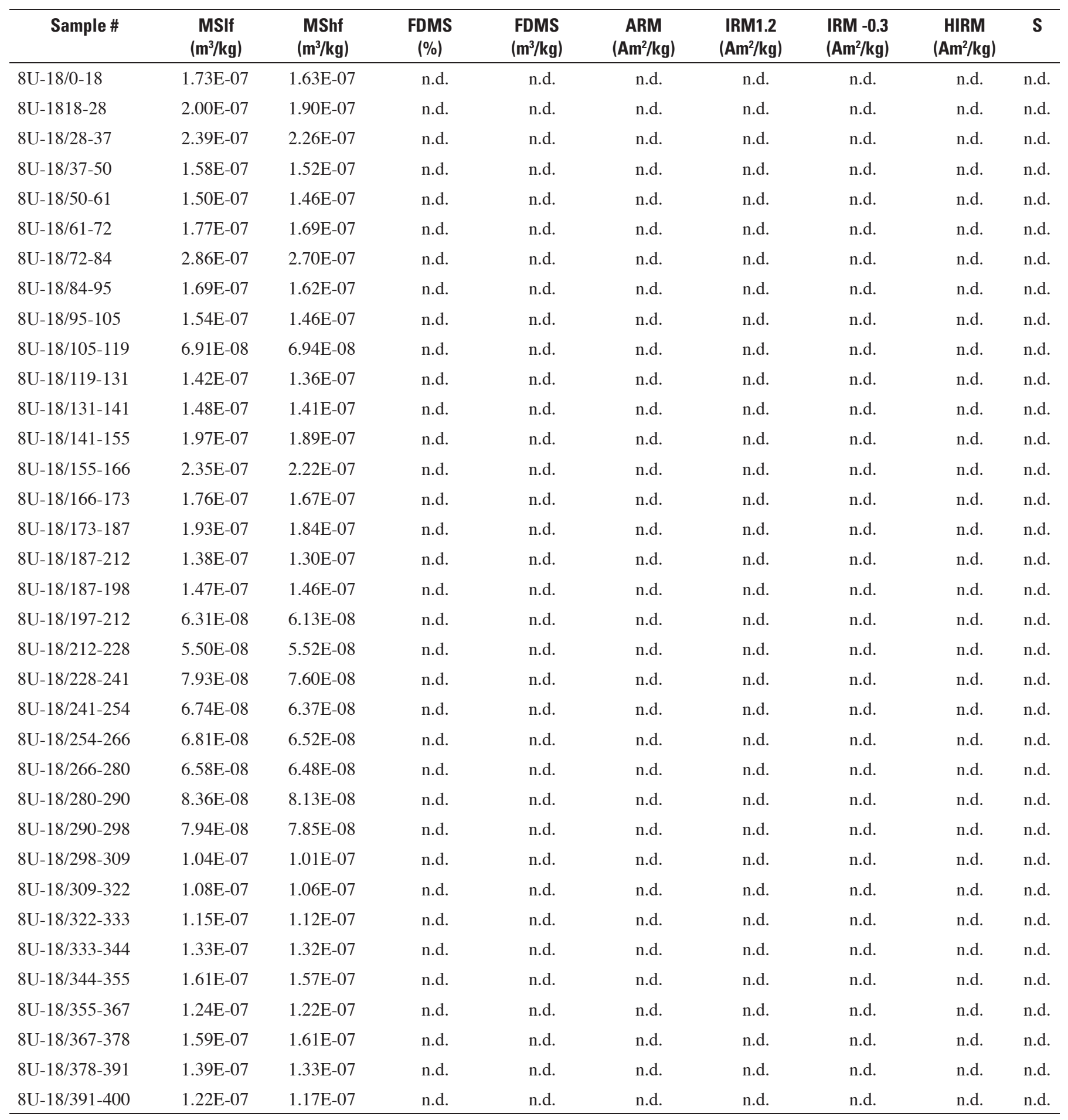


Table 6g-2. Magnetic properties of the less than 2-mm size fraction for auger hole 8U-20.

\begin{tabular}{|c|c|c|c|c|c|c|c|c|c|}
\hline Sample \# & $\begin{array}{c}\text { MSIf } \\
\left(\mathbf{m}^{3} / \mathbf{k g}\right)\end{array}$ & $\begin{array}{c}\text { MShf } \\
\left(\mathbf{m}^{3} / \mathbf{k g}\right)\end{array}$ & $\begin{array}{l}\text { FDMS } \\
(\%)\end{array}$ & $\begin{array}{c}\text { FDMS } \\
\left(\mathrm{m}^{3} / \mathbf{k g}\right)\end{array}$ & $\begin{array}{c}\text { ARM } \\
\left(\mathrm{Am}^{2} / \mathbf{k g}\right)\end{array}$ & $\begin{array}{c}\text { IRM1.2 } \\
\left(\mathrm{Am}^{2} / \mathbf{k g}\right)\end{array}$ & $\begin{array}{l}\text { IRM -0.3 } \\
\left(\mathrm{Am}^{2} / \mathrm{kg}\right)\end{array}$ & $\begin{array}{c}\text { HIRM } \\
\left(\mathrm{Am}^{2} / \mathrm{kg}\right)\end{array}$ & $\mathbf{S}$ \\
\hline $8 \mathrm{U}-20 / 0-18$ & $2.11 \mathrm{E}-07$ & $2.00 \mathrm{E}-07$ & n.d. & n.d. & n.d. & n.d. & n.d. & n.d. & n.d. \\
\hline $8 \mathrm{U}-20 / 18-26$ & 1.79E-07 & $1.72 \mathrm{E}-07$ & n.d. & n.d. & n.d. & n.d. & n.d. & n.d. & n.d. \\
\hline $8 \mathrm{U}-2026-35$ & 1.69E-07 & $1.63 \mathrm{E}-07$ & n.d. & n.d. & n.d. & n.d. & n.d. & n.d. & n.d. \\
\hline $8 U-20 / 35-45$ & $1.65 \mathrm{E}-07$ & $1.58 \mathrm{E}-07$ & n.d. & n.d. & n.d. & n.d. & n.d. & n.d. & n.d. \\
\hline $8 U-20 / 45-54$ & $1.64 \mathrm{E}-07$ & $1.57 \mathrm{E}-07$ & n.d. & n.d. & n.d. & n.d. & n.d. & n.d. & n.d. \\
\hline $8 \mathrm{U}-20 / 54-65$ & $1.75 \mathrm{E}-07$ & $1.66 \mathrm{E}-07$ & n.d. & n.d. & n.d. & n.d. & n.d. & n.d. & n.d. \\
\hline $8 \mathrm{U}-20 / 65-76$ & 6.69E-08 & $6.61 \mathrm{E}-08$ & n.d. & n.d. & n.d. & n.d. & n.d. & n.d. & n.d. \\
\hline 8U-20/76-88 & 8.43E-08 & 8.32E-08 & n.d. & n.d. & n.d. & n.d. & n.d. & n.d. & n.d. \\
\hline 8U-20/88-102 & 5.30E-08 & $5.39 \mathrm{E}-08$ & n.d. & n.d. & n.d. & n.d. & n.d. & n.d. & n.d. \\
\hline $8 \mathrm{U}-20 / 102-124$ & 3.07E-08 & $3.20 \mathrm{E}-08$ & n.d. & n.d. & n.d. & n.d. & n.d. & n.d. & n.d. \\
\hline $8 \mathrm{U}-20 / 124-136$ & 9.87E-09 & $1.19 \mathrm{E}-08$ & n.d. & n.d. & n.d. & n.d. & n.d. & n.d. & n.d. \\
\hline $8 \mathrm{U}-20 / 136-147$ & 1.19E-08 & $1.24 \mathrm{E}-08$ & n.d. & n.d. & n.d. & n.d. & n.d. & n.d. & n.d. \\
\hline $8 U-20 / 147-159$ & $1.77 \mathrm{E}-08$ & $1.95 \mathrm{E}-08$ & n.d. & n.d. & n.d. & n.d. & n.d. & n.d. & n.d. \\
\hline $8 U-20 / 159-168$ & $6.47 \mathrm{E}-08$ & $6.49 \mathrm{E}-08$ & n.d. & n.d. & n.d. & n.d. & n.d. & n.d. & n.d. \\
\hline $8 U-20 / 168-182$ & 8.91E-08 & $8.84 \mathrm{E}-08$ & n.d. & n.d. & n.d. & n.d. & n.d. & n.d. & n.d. \\
\hline 8U-20/182-194 & $5.00 \mathrm{E}-08$ & $5.12 \mathrm{E}-08$ & n.d. & n.d. & n.d. & n.d. & n.d. & n.d. & n.d. \\
\hline 8U-20/194-207 & $5.84 \mathrm{E}-08$ & $5.86 \mathrm{E}-08$ & n.d. & n.d. & n.d. & n.d. & n.d. & n.d. & n.d. \\
\hline $8 \mathrm{U}-20 / 207-218$ & $1.15 \mathrm{E}-07$ & $1.13 \mathrm{E}-07$ & n.d. & n.d. & n.d. & n.d. & n.d. & n.d. & n.d. \\
\hline $8 U-20 / 218-230$ & 1.67E-07 & $1.61 \mathrm{E}-07$ & n.d. & n.d. & n.d. & n.d. & n.d. & n.d. & n.d. \\
\hline $8 \mathrm{U}-20 / 230-240$ & 2.03E-07 & $1.98 \mathrm{E}-07$ & n.d. & n.d. & n.d. & n.d. & n.d. & n.d. & n.d. \\
\hline $8 U-20 / 240-251$ & $1.90 \mathrm{E}-07$ & $1.83 \mathrm{E}-07$ & n.d. & n.d. & n.d. & n.d. & n.d. & n.d. & n.d. \\
\hline $8 \mathrm{U}-20 / 251-261$ & 1.70E-07 & $1.65 \mathrm{E}-07$ & n.d. & n.d. & n.d. & n.d. & n.d. & n.d. & n.d. \\
\hline $8 U-20 / 261-272$ & 2.33E-07 & $2.26 \mathrm{E}-07$ & n.d. & n.d. & n.d. & n.d. & n.d. & n.d. & n.d. \\
\hline $8 U-20 / 272-282$ & $2.79 \mathrm{E}-07$ & $2.69 \mathrm{E}-07$ & n.d. & n.d. & n.d. & n.d. & n.d. & n.d. & n.d. \\
\hline $8 U-20 / 282-290$ & 2.98E-07 & $2.86 \mathrm{E}-07$ & n.d. & n.d. & n.d. & n.d. & n.d. & n.d. & n.d. \\
\hline $8 U-20 / 290-298$ & 2.92E-07 & $2.79 \mathrm{E}-07$ & n.d. & n.d. & n.d. & n.d. & n.d. & n.d. & n.d. \\
\hline $8 U-20 / 298-311$ & $3.01 \mathrm{E}-07$ & $2.97 \mathrm{E}-07$ & n.d. & n.d. & n.d. & n.d. & n.d. & n.d. & n.d. \\
\hline $8 \mathrm{U}-20 / 311-321$ & 2.69E-07 & $2.58 \mathrm{E}-07$ & n.d. & n.d. & n.d. & n.d. & n.d. & n.d. & n.d. \\
\hline $8 U-20 / 321-335$ & $2.56 \mathrm{E}-07$ & $2.47 \mathrm{E}-07$ & n.d. & n.d. & n.d. & n.d. & n.d. & n.d. & n.d. \\
\hline $8 \mathrm{U}-20 / 335-346$ & $3.24 \mathrm{E}-07$ & $3.13 \mathrm{E}-07$ & n.d. & n.d. & n.d. & n.d. & n.d. & n.d. & n.d. \\
\hline $8 U-20 / 346-358$ & 3.07E-07 & $2.92 \mathrm{E}-07$ & n.d. & n.d. & n.d. & n.d. & n.d. & n.d. & n.d. \\
\hline $8 U-20 / 358-370$ & $1.71 \mathrm{E}-07$ & $1.65 \mathrm{E}-07$ & n.d. & n.d. & n.d. & n.d. & n.d. & n.d. & n.d. \\
\hline $8 \mathrm{U}-20 / 370-380$ & $1.48 \mathrm{E}-07$ & $1.45 \mathrm{E}-07$ & n.d. & n.d. & n.d. & n.d. & n.d. & n.d. & n.d. \\
\hline 8U-20/380-389 & $1.21 \mathrm{E}-07$ & $1.19 \mathrm{E}-07$ & n.d. & n.d. & n.d. & n.d. & n.d. & n.d. & n.d. \\
\hline 8U-20/389-400 & $1.74 \mathrm{E}-07$ & $1.70 \mathrm{E}-07$ & n.d. & n.d. & n.d. & n.d. & n.d. & n.d. & n.d. \\
\hline
\end{tabular}


Table 6h-1. Magnetic properties of the less than 2-mm size fraction for auger hole 8U-15.

\begin{tabular}{|c|c|c|c|c|c|c|c|c|c|}
\hline Sample \# & $\begin{array}{c}\text { MSIf } \\
\left(\mathrm{m}^{3} / \mathbf{k g}\right)\end{array}$ & $\begin{array}{c}\text { MShf } \\
\left(\mathrm{m}^{3} / \mathbf{k g}\right)\end{array}$ & $\begin{array}{c}\text { FDMS } \\
(\%)\end{array}$ & $\begin{array}{c}\text { FDMS } \\
\left(\mathrm{m}^{3} / \mathbf{k g}\right)\end{array}$ & $\begin{array}{c}\text { ARM } \\
\left(\mathrm{Am}^{2} / \mathrm{kg}\right)\end{array}$ & $\begin{array}{c}\text { IRM1.2 } \\
\left(\mathrm{Am}^{2} / \mathrm{kg}\right)\end{array}$ & $\begin{array}{l}\text { IRM -0.3 } \\
\left(\mathrm{Am}^{2} / \mathrm{kg}\right)\end{array}$ & $\begin{array}{c}\text { HIRM } \\
\left(\mathrm{Am}^{2} / \mathbf{k g}\right)\end{array}$ & $\mathbf{S}$ \\
\hline $8 \mathrm{U}-15 / 0-19$ & $3.04 \mathrm{E}-07$ & $2.93 \mathrm{E}-07$ & n.d. & n.d. & n.d. & n.d. & n.d. & n.d. & n.d. \\
\hline $8 \mathrm{U}-15 / 29-42$ & $3.57 \mathrm{E}-07$ & $3.43 \mathrm{E}-07$ & n.d. & n.d. & n.d. & n.d. & n.d. & n.d. & n.d. \\
\hline $8 U-15 / 42-53$ & $3.68 \mathrm{E}-07$ & $3.65 \mathrm{E}-07$ & n.d. & n.d. & n.d. & n.d. & n.d. & n.d. & n.d. \\
\hline $8 U-15 / 64-77$ & $3.96 \mathrm{E}-07$ & $3.81 \mathrm{E}-07$ & n.d. & n.d. & n.d. & n.d. & n.d. & n.d. & n.d. \\
\hline $8 \mathrm{U}-15 / 77-94$ & 4.46E-07 & 4.29E-07 & n.d. & n.d. & n.d. & n.d. & n.d. & n.d. & n.d. \\
\hline $8 \mathrm{U}-15 / 94-103$ & $4.50 \mathrm{E}-07$ & $4.32 \mathrm{E}-07$ & n.d. & n.d. & n.d. & n.d. & n.d. & n.d. & n.d. \\
\hline $8 \mathrm{U}-15 / 103-114$ & 4.17E-07 & 3.99E-07 & n.d. & n.d. & n.d. & n.d. & n.d. & n.d. & n.d. \\
\hline $8 \mathrm{U}-15 / 114-124$ & $3.87 \mathrm{E}-07$ & $3.73 \mathrm{E}-07$ & n.d. & n.d. & n.d. & n.d. & n.d. & n.d. & n.d. \\
\hline $8 \mathrm{U}-15 / 143-152$ & $4.26 \mathrm{E}-07$ & $4.15 \mathrm{E}-07$ & n.d. & n.d. & n.d. & n.d. & n.d. & n.d. & n.d. \\
\hline $8 \mathrm{U}-15 / 152-162$ & 5.12E-07 & 4.99E-07 & n.d. & n.d. & n.d. & n.d. & n.d. & n.d. & n.d. \\
\hline $8 \mathrm{U}-15 / 162-171$ & $5.24 \mathrm{E}-07$ & $5.01 \mathrm{E}-07$ & n.d. & n.d. & n.d. & n.d. & n.d. & n.d. & n.d. \\
\hline $8 \mathrm{U}-15 / 171-182$ & $5.48 \mathrm{E}-07$ & $5.38 \mathrm{E}-07$ & n.d. & n.d. & n.d. & n.d. & n.d. & n.d. & n.d. \\
\hline $8 \mathrm{U}-15 / 182-192$ & 5.61E-07 & $5.52 \mathrm{E}-07$ & n.d. & n.d. & n.d. & n.d. & n.d. & n.d. & n.d. \\
\hline 8U-15/192-202 & 4.61E-07 & $4.52 \mathrm{E}-07$ & n.d. & n.d. & n.d. & n.d. & n.d. & n.d. & n.d. \\
\hline $8 U-15 / 202-213$ & $2.81 \mathrm{E}-07$ & $2.78 \mathrm{E}-07$ & n.d. & n.d. & n.d. & n.d. & n.d. & n.d. & n.d. \\
\hline $8 \mathrm{U}-15 / 213-221$ & $2.89 \mathrm{E}-07$ & $2.83 \mathrm{E}-07$ & n.d. & n.d. & n.d. & n.d. & n.d. & n.d. & n.d. \\
\hline $8 \mathrm{U}-15 / 221-228$ & $1.89 \mathrm{E}-07$ & $1.85 \mathrm{E}-07$ & n.d. & n.d. & n.d. & n.d. & n.d. & n.d. & n.d. \\
\hline $8 \mathrm{U}-15 / 278-286$ & $1.03 \mathrm{E}-07$ & $1.02 \mathrm{E}-07$ & n.d. & n.d. & n.d. & n.d. & n.d. & n.d. & n.d. \\
\hline $8 U-15 / 286-300$ & $1.22 \mathrm{E}-07$ & $1.21 \mathrm{E}-07$ & n.d. & n.d. & n.d. & n.d. & n.d. & n.d. & n.d. \\
\hline $8 \mathrm{U}-15 / 300-308$ & 1.17E-07 & $1.15 \mathrm{E}-07$ & n.d. & n.d. & n.d. & n.d. & n.d. & n.d. & n.d. \\
\hline $8 \mathrm{U}-15 / 308-319$ & $1.16 \mathrm{E}-07$ & $1.15 \mathrm{E}-07$ & n.d. & n.d. & n.d. & n.d. & n.d. & n.d. & n.d. \\
\hline $8 \mathrm{U}-15 / 319-330$ & $1.02 \mathrm{E}-07$ & $10.00 \mathrm{E}-08$ & n.d. & n.d. & n.d. & n.d. & n.d. & n.d. & n.d. \\
\hline $8 \mathrm{U}-15 / 330-339$ & $9.03 \mathrm{E}-08$ & 8.97E-08 & n.d. & n.d. & n.d. & n.d. & n.d. & n.d. & n.d. \\
\hline 8U-15/339-349 & $8.18 \mathrm{E}-08$ & $8.16 \mathrm{E}-08$ & n.d. & n.d. & n.d. & n.d. & n.d. & n.d. & n.d. \\
\hline $8 U-15 / 349-360$ & $6.97 \mathrm{E}-08$ & $6.95 \mathrm{E}-08$ & n.d. & n.d. & n.d. & n.d. & n.d. & n.d. & n.d. \\
\hline $8 U-15 / 360-369$ & $1.04 \mathrm{E}-07$ & $1.04 \mathrm{E}-07$ & n.d. & n.d. & n.d. & n.d. & n.d. & n.d. & n.d. \\
\hline $8 U-15 / 369-378$ & $8.51 \mathrm{E}-08$ & 8.44E-08 & n.d. & n.d. & n.d. & n.d. & n.d. & n.d. & n.d. \\
\hline $8 U-15 / 379-387$ & $1.08 \mathrm{E}-07$ & $1.07 \mathrm{E}-07$ & n.d. & n.d. & n.d. & n.d. & n.d. & n.d. & n.d. \\
\hline $8 \mathrm{U}-15 / 387-395$ & $1.13 \mathrm{E}-07$ & $1.12 \mathrm{E}-07$ & n.d. & n.d. & n.d. & n.d. & n.d. & n.d. & n.d. \\
\hline
\end{tabular}


Table 6h-2. Magnetic properties of the less than 2-mm size fraction for auger hole 8U-16.

\begin{tabular}{|c|c|c|c|c|c|c|c|c|c|}
\hline Sample \# & $\begin{array}{c}\text { MSIf } \\
\left(\mathbf{m}^{3} / \mathbf{k g}\right)\end{array}$ & $\begin{array}{c}\text { MShf } \\
\left(\mathbf{m}^{3} / \mathbf{k g}\right)\end{array}$ & $\begin{array}{c}\text { FDMS } \\
(\%)\end{array}$ & $\begin{array}{l}\text { FDMS } \\
\left(\mathbf{m}^{3} / \mathbf{k g}\right)\end{array}$ & $\begin{array}{c}\text { ARM } \\
\left(\mathrm{Am}^{2} / \mathbf{k g}\right)\end{array}$ & $\begin{array}{c}\text { IRM1.2 } \\
\left(\mathrm{Am}^{2} / \mathbf{k g}\right)\end{array}$ & $\begin{array}{l}\text { IRM -0.3 } \\
\left(\mathrm{Am}^{2} / \mathrm{kg}\right)\end{array}$ & $\begin{array}{c}\text { HIRM } \\
\left(\mathrm{Am}^{2} / \mathbf{k g}\right)\end{array}$ & $\mathbf{S}$ \\
\hline $8 U-16 / 0-22$ & $2.48 \mathrm{E}-07$ & $2.41 \mathrm{E}-07$ & n.d. & n.d. & n.d. & n.d. & n.d. & n.d. & n.d. \\
\hline $8 \mathrm{U}-16 / 22-36$ & $2.52 \mathrm{E}-07$ & $2.45 \mathrm{E}-07$ & n.d. & n.d. & n.d. & n.d. & n.d. & n.d. & n.d. \\
\hline $8 \mathrm{U}-16 / 36-51$ & $2.60 \mathrm{E}-07$ & $2.54 \mathrm{E}-07$ & n.d. & n.d. & n.d. & n.d. & n.d. & n.d. & n.d. \\
\hline $8 \mathrm{U}-16 / 51-63$ & $2.51 \mathrm{E}-07$ & $2.45 \mathrm{E}-07$ & n.d. & n.d. & n.d. & n.d. & n.d. & n.d. & n.d. \\
\hline $8 \mathrm{U}-16 / 63-76$ & $2.14 \mathrm{E}-07$ & $2.08 \mathrm{E}-07$ & n.d. & n.d. & n.d. & n.d. & n.d. & n.d. & n.d. \\
\hline $8 \mathrm{U}-16 / 76-89$ & $1.59 \mathrm{E}-07$ & $1.56 \mathrm{E}-07$ & n.d. & n.d. & n.d. & n.d. & n.d. & n.d. & n.d. \\
\hline $8 \mathrm{U}-16 / 89-103$ & $1.70 \mathrm{E}-07$ & $1.68 \mathrm{E}-07$ & n.d. & n.d. & n.d. & n.d. & n.d. & n.d. & n.d. \\
\hline $8 \mathrm{U}-16 / 103-117$ & $1.64 \mathrm{E}-07$ & $1.65 \mathrm{E}-07$ & n.d. & n.d. & n.d. & n.d. & n.d. & n.d. & n.d. \\
\hline $8 \mathrm{U}-16 / 117-131$ & $1.10 \mathrm{E}-07$ & $1.01 \mathrm{E}-07$ & n.d. & n.d. & n.d. & n.d. & n.d. & n.d. & n.d. \\
\hline $8 \mathrm{U}-16 / 131-148$ & $1.65 \mathrm{E}-07$ & $1.63 \mathrm{E}-07$ & n.d. & n.d. & n.d. & n.d. & n.d. & n.d. & n.d. \\
\hline $8 U-16 / 148-158$ & 1.47E-07 & 1.46E-07 & n.d. & n.d. & n.d. & n.d. & n.d. & n.d. & n.d. \\
\hline
\end{tabular}

Table 6h-3. Magnetic properties of the less than 2-mm size fraction for arroyo exposure 9U-21.

\begin{tabular}{|c|c|c|c|c|c|c|c|c|c|}
\hline Sample \# & $\begin{array}{c}\text { MSIf } \\
\left(\mathrm{m}^{3} / \mathrm{kg}\right)\end{array}$ & $\begin{array}{c}\text { MShf } \\
\left(\mathrm{m}^{3} / \mathrm{kg}\right)\end{array}$ & $\begin{array}{c}\text { FDMS } \\
(\%)\end{array}$ & $\begin{array}{l}\text { FDMS } \\
\left(\mathrm{m}^{3} / \mathrm{kg}\right)\end{array}$ & $\begin{array}{c}\text { ARM } \\
\left(\mathrm{Am}^{2} / \mathbf{k g}\right)\end{array}$ & $\begin{array}{c}\text { IRM1.2 } \\
\left(\mathrm{Am}^{2} / \mathrm{kg}\right)\end{array}$ & $\begin{array}{l}\text { IRM -0.3 } \\
\left(\mathrm{Am}^{2} / \mathrm{kg}\right)\end{array}$ & $\begin{array}{c}\text { HIRM } \\
\left(\mathrm{Am}^{2} / \mathrm{kg}\right)\end{array}$ & $S$ \\
\hline $9 U-21 / 0-5$ & $2.17 \mathrm{E}-07$ & $2.12 \mathrm{E}-07$ & 0.00 & $2.17 \mathrm{E}+00$ & $8.95 \mathrm{E}-05$ & $3.01 \mathrm{E}-03$ & $2.44 \mathrm{E}-03$ & $2.81 \mathrm{E}-04$ & 0.81 \\
\hline $9 U-21 / 5-25$ & $1.58 \mathrm{E}-07$ & $1.54 \mathrm{E}-07$ & 0.00 & $2.38 \mathrm{E}+00$ & $8.88 \mathrm{E}-05$ & $2.37 \mathrm{E}-03$ & $1.82 \mathrm{E}-03$ & 2.77E-04 & 0.77 \\
\hline $9 U-21 / 25-70$ & $1.82 \mathrm{E}-07$ & $1.80 \mathrm{E}-07$ & 0.00 & $9.76 \mathrm{E}-01$ & $6.64 \mathrm{E}-05$ & $2.71 \mathrm{E}-03$ & $2.30 \mathrm{E}-03$ & 2.03E-04 & 0.85 \\
\hline $9 U-21 / 70-90$ & $2.16 \mathrm{E}-07$ & $2.14 \mathrm{E}-07$ & 0.00 & $1.10 \mathrm{E}+00$ & $6.85 \mathrm{E}-05$ & $3.16 \mathrm{E}-03$ & $2.71 \mathrm{E}-03$ & $2.24 \mathrm{E}-04$ & 0.86 \\
\hline 9U-21/90-115 & $2.67 \mathrm{E}-07$ & $2.65 \mathrm{E}-07$ & 0.00 & $9.83 \mathrm{E}-01$ & $7.48 \mathrm{E}-05$ & $4.00 \mathrm{E}-03$ & $3.45 \mathrm{E}-03$ & 2.74E-04 & 0.86 \\
\hline $9 U-21 / 115-128$ & $2.08 \mathrm{E}-07$ & $2.05 \mathrm{E}-07$ & 0.00 & $1.37 \mathrm{E}+00$ & $6.55 \mathrm{E}-05$ & $3.01 \mathrm{E}-03$ & $2.51 \mathrm{E}-03$ & $2.51 \mathrm{E}-04$ & 0.83 \\
\hline $9 \mathrm{U}-21 / 128-148$ & $1.48 \mathrm{E}-07$ & $1.47 \mathrm{E}-07$ & 0.00 & $3.85 \mathrm{E}-01$ & $4.78 \mathrm{E}-05$ & $2.15 \mathrm{E}-03$ & $1.76 \mathrm{E}-03$ & 1.94E-04 & 0.82 \\
\hline $9 U-21 / 148-168$ & $1.77 \mathrm{E}-07$ & $1.77 \mathrm{E}-07$ & -0.00 & $-9.81 \mathrm{E}-02$ & $5.80 \mathrm{E}-05$ & $2.48 \mathrm{E}-03$ & $1.99 \mathrm{E}-03$ & $2.46 \mathrm{E}-04$ & 0.80 \\
\hline $9 U-21 / 168-201$ & $1.49 \mathrm{E}-07$ & $1.48 \mathrm{E}-07$ & 0.00 & $6.72 \mathrm{E}-01$ & $4.81 \mathrm{E}-05$ & $2.11 \mathrm{E}-03$ & $1.68 \mathrm{E}-03$ & 2.19E-04 & 0.79 \\
\hline $9 \mathrm{U}-21 / 210-225$ & $1.01 \mathrm{E}-07$ & $1.01 \mathrm{E}-07$ & -0.00 & $-4.22 \mathrm{E}-01$ & $3.78 \mathrm{E}-05$ & $1.38 \mathrm{E}-03$ & $1.02 \mathrm{E}-03$ & $1.78 \mathrm{E}-04$ & 0.74 \\
\hline $9 \mathrm{U}-21 / 225-250$ & $7.08 \mathrm{E}-08$ & $6.97 \mathrm{E}-08$ & 0.00 & $1.55 \mathrm{E}+00$ & $3.24 \mathrm{E}-05$ & $9.87 \mathrm{E}-04$ & $7.01 \mathrm{E}-04$ & $1.38 \mathrm{E}-04$ & 0.72 \\
\hline $9 \mathrm{U}-21 / 250-300$ & $8.54 \mathrm{E}-08$ & $8.48 \mathrm{E}-08$ & 0.00 & $6.75 \mathrm{E}-01$ & $3.53 \mathrm{E}-05$ & $1.16 \mathrm{E}-03$ & 8.33E-04 & $1.62 \mathrm{E}-04$ & 0.72 \\
\hline $9 \mathrm{U}-21 / 300-345$ & $9.27 \mathrm{E}-08$ & $9.21 \mathrm{E}-08$ & 0.00 & $6.22 \mathrm{E}-01$ & 4.36E-05 & $1.33 \mathrm{E}-03$ & $9.93 \mathrm{E}-04$ & $1.67 \mathrm{E}-04$ & 0.75 \\
\hline $9 U-21 / 345-375$ & $9.17 \mathrm{E}-08$ & $9.07 \mathrm{E}-08$ & 0.00 & $1.15 \mathrm{E}+00$ & 4.41E-05 & $1.34 \mathrm{E}-03$ & $9.78 \mathrm{E}-04$ & $1.82 \mathrm{E}-04$ & 0.73 \\
\hline $9 \mathrm{U}-21 / 375-440$ & $9.36 \mathrm{E}-08$ & $9.18 \mathrm{E}-08$ & 0.00 & $1.97 \mathrm{E}+00$ & 4.47E-05 & $1.36 \mathrm{E}-03$ & $1.01 \mathrm{E}-03$ & $1.74 \mathrm{E}-04$ & 0.74 \\
\hline
\end{tabular}




\section{Table 7. Age data}

This table presents age data from ${ }^{14} \mathrm{C}$ and optically stimulated luminescence (OSL) techniques. See text for field and analytical methodology.

Table 7-1. ${ }^{14} \mathrm{C}$ age from Virginia Park

\begin{tabular}{|c|c|c|c|c|c|c|}
\hline Field Sample \# & Lab Sample \# ${ }^{1}$ & Material dated & Stratigraphic unit & $\begin{array}{c}\text { Depth below } \\
\text { surface }(\mathrm{m})\end{array}$ & ${ }^{14} \mathrm{Cage}^{2}$ & Calibrated age $^{3}$ \\
\hline $8 \mathrm{U}-14$ & WW3004 & Charcoal in pebbly lens & $\begin{array}{l}\text { Base of alluvial fill inset } \\
\text { into older dune Sand }\end{array}$ & 0.75 & $4,580 \pm 50$ & $5250+/-200$ \\
\hline
\end{tabular}

${ }^{1}$ Samples processed and lab numbers assigned at ${ }^{14} \mathrm{C}$ laboratory of the U.S. Geological Survey in Reston, Virgina

${ }^{2}$ Ages expressed as years before present (1950 A.D.) with 1-sigma uncertainty

${ }^{3}$ Calibration performed using CALIB program (http://radiocarbon.pa.qub.ac.uk/calib/; Stuiver et al., 2003); expressed as cal yr B.P. with 2-sigma uncertainty. 


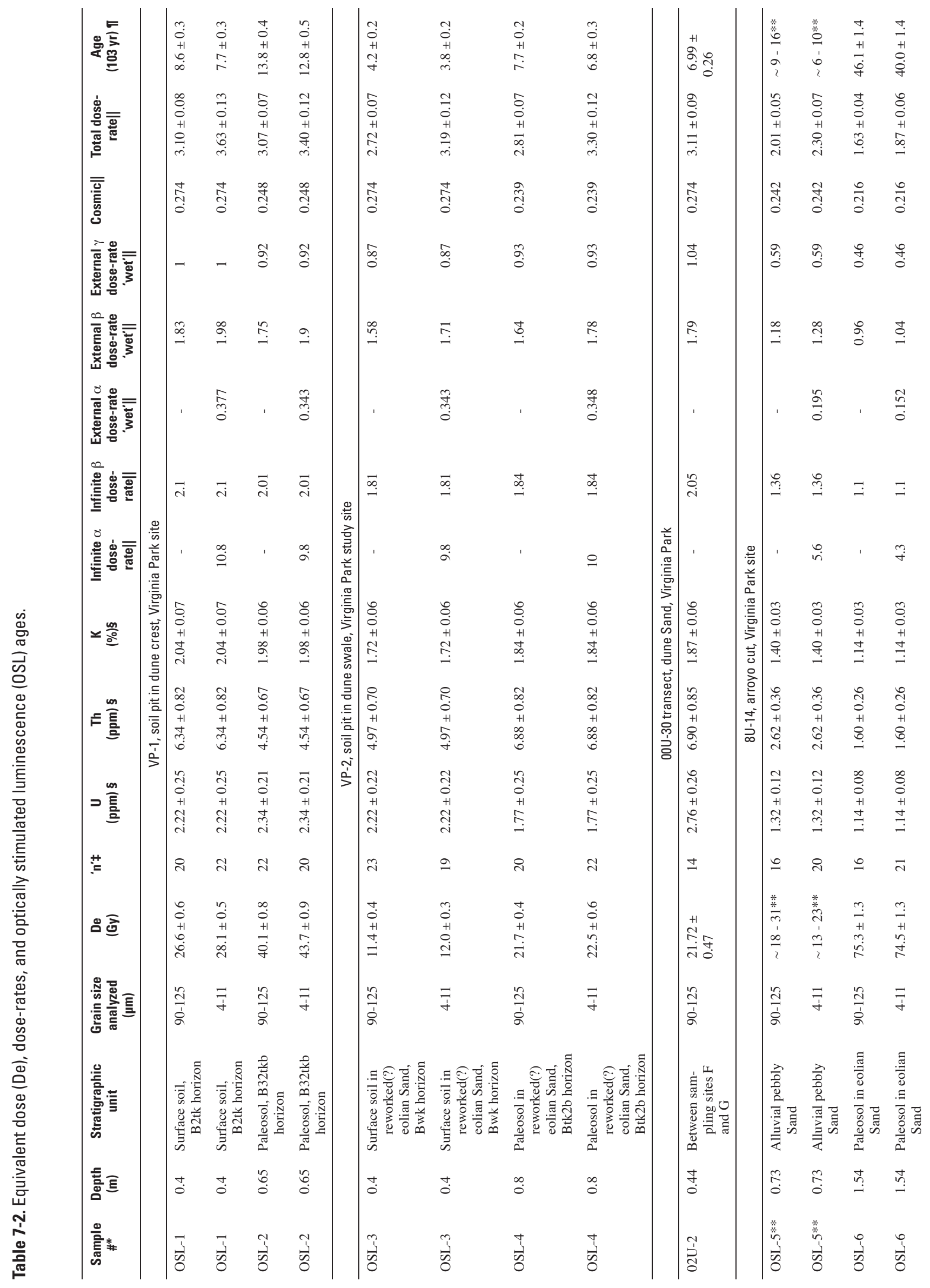




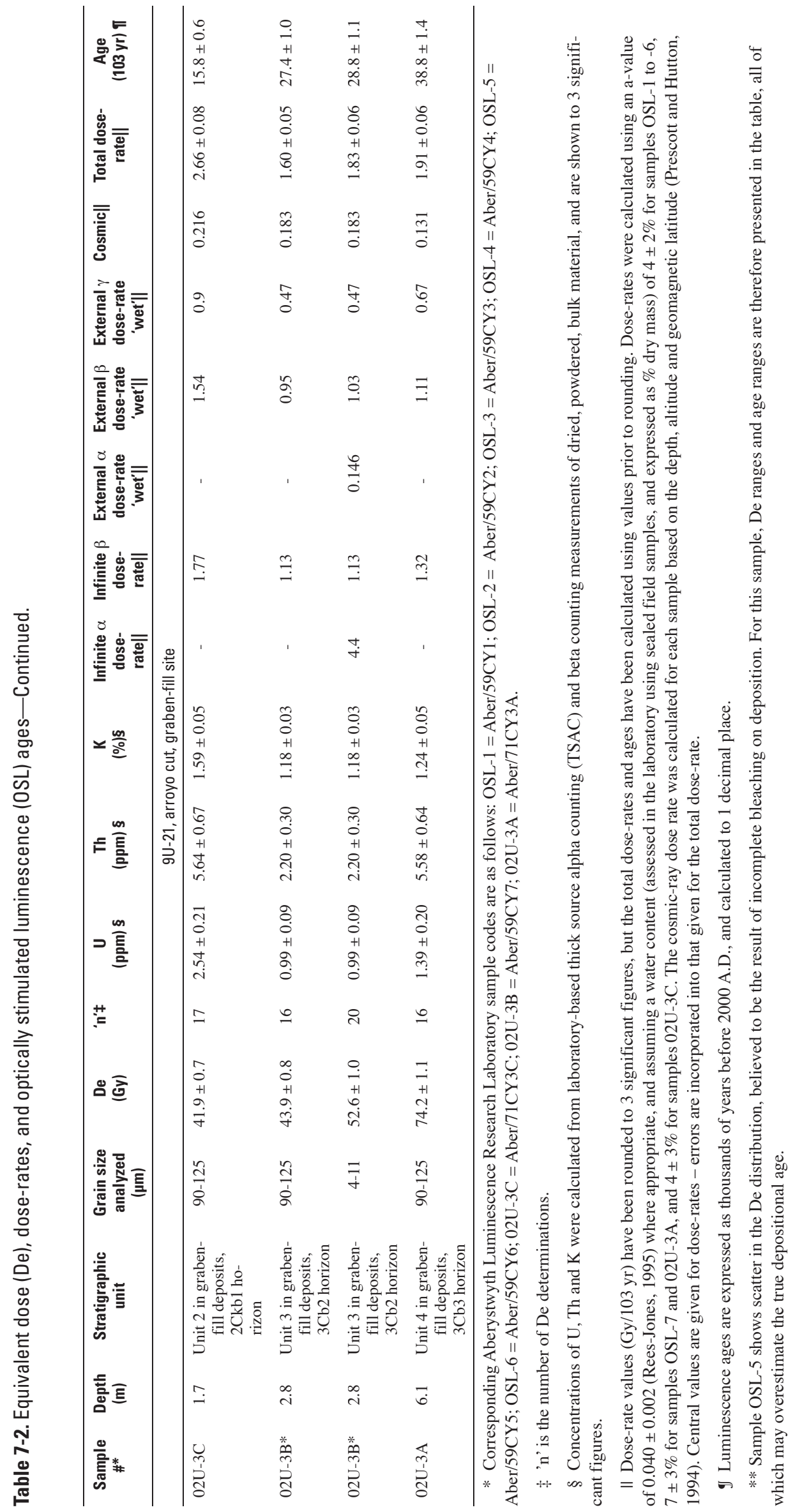

